Final Report

FHWA/IN/JTRP- 2008/4

\title{
EXPERT SYSTEM TO SUPPORT SITE INVESTIGATION FOR SAFETY IMPROVEMENT
}

\author{
By \\ Andrew P. Tarko \\ Professor of Civil Engineering \\ and \\ Andrew Kwasniak \\ Graduate Research Assistant \\ Jorge Ramos \\ Graduate Research Assistant \\ School of Civil Engineering \\ Purdue University \\ Joint Transportation Research Program \\ Project No. CD-36-17AAA \\ File No. A-5-53 \\ SPR- 3103 \\ Conducted in Cooperation with the \\ Indiana Department of Transportation and the \\ U.S. Department of Transportation \\ Federal Highway Administration
}

The contents of this report reflect the views of the authors, who are responsible for the facts and the accuracy of the data presented herein. The contents do not necessarily reflect the official views or policies of the Indiana Department of Transportation or the Federal Highway Administration at the time of publication. The report does not constitute a standard, specification, or regulation.

Purdue University

West Lafayette, Indiana

May 2008 


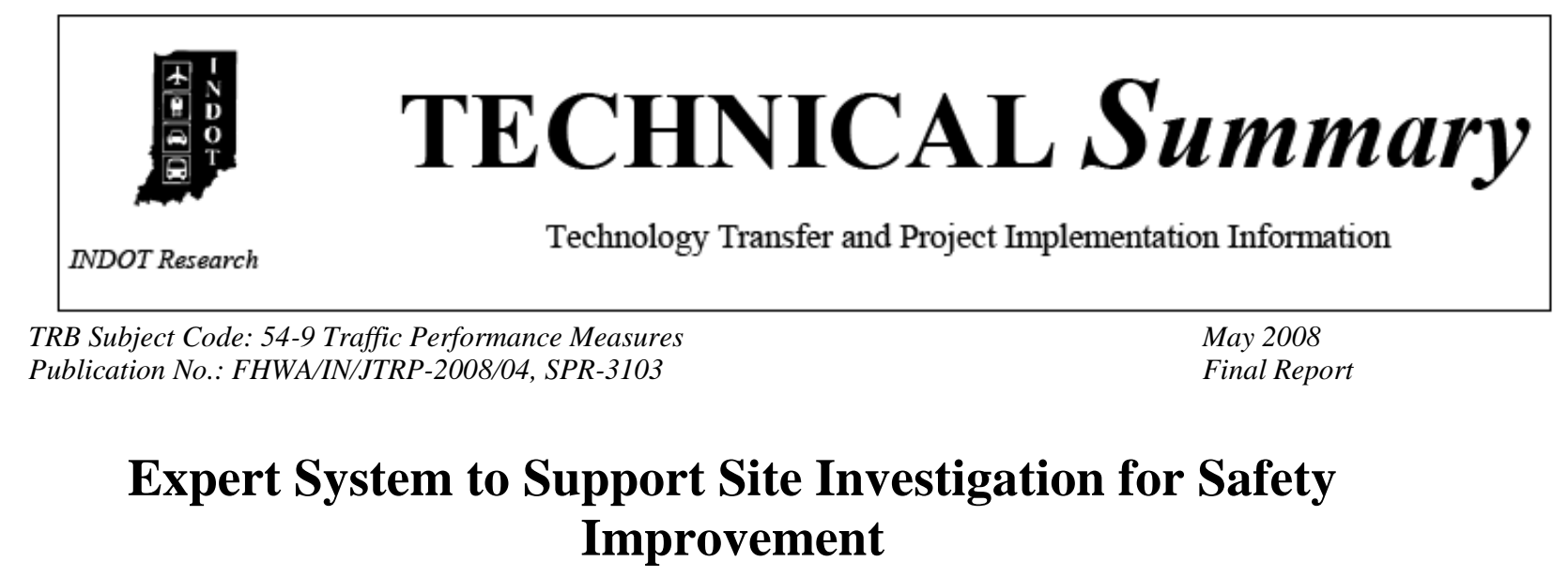

\section{Introduction}

Specialists in transportation and other areas of engineering are trying to improve road safety. The most difficult part is determining the road features, driver behaviors, and vehicular failures that unduly increase the risk of crash. To help INDOT safety investigation teams, a previous research project developed a prototype Road Safety Investigation Tool (RSIT) for two-way stop-controlled intersections. The research summarized here has evaluated the prototype RSIT and has developed a new version RSIT2 that has incorporated the findings of the evaluation.

\section{Findings}

The evaluation of the RSIT prototype confirmed usefulness of the proposed methodology. The tool has been applied to two two-way stop-controlled intersections by a team of non-experts. The two sites have been previously investigated by experts using the standard approach. The safety investigation outcomes obtained by both the teams indicate strong similarities

The proposed RSIT method helped nonexperts identify additional road hazards confirmed later by the experts' team.
RSIT has been found decreasing the required investigation team size and time as well as being useful as a training tool. RSIT presents the logical connections between driver behaviors, road elements and environment conditions.

RSIT2 is the next version of the RSIT method. The knowledge base developed for RSIT 2 includes all the major types of roads in Indiana: a) allway stop-controlled intersection, b) road segment, c) railroad crossing, and d) signalized intersection, and e) improved two-way stop-controlled intersections. The RSIT 2 method uses the rule-based structure converted to a dynamic checklist that adjusts in real-time to the investigation progress. A friendly graphic-user interface has been developed together with a knowledge base editor to allow updating the knowledge base by the user. Finally, a novel technique of ranking safety countermeasures was developed and applied.

\section{Implementation}

The RSIT 2 is ready for implementation. The agency may easily update/modify the default knowledge base to fit its local needs. A series of workshops may be 
needed to help in the implementation phase.

\section{Contacts}

For more information:

Prof. Andrew Tarko

Principal Investigator

School of Civil Engineering

Purdue University

West Lafayette IN 47907

Phone: (765) 494-5027

Fax: (765) 496-7996

E-mail: tarko@ecn.purdue.edu

\section{Indiana Department of} Transportation

Division of Research

1205 Montgomery Street

P.O. Box 2279

West Lafayette, IN 47906

Phone: (765) 463-1521

Fax: (765) 497-1665

\section{Purdue University}

Joint Transportation Research Program

School of Civil Engineering

West Lafayette, IN 47907-1284

Phone: (765) 494-9310

Fax: (765) 496-7996

E-mail: jtrp@ecn.purdue.edu 


\begin{tabular}{|c|c|c|c|c|}
\hline $\begin{array}{l}\text { 1. Report No. } \\
\text { FHWA/IN/JTRP-2008/4 }\end{array}$ & \multicolumn{2}{|c|}{ 2. Government Accession No. } & \multicolumn{2}{|l|}{ 3. Recipient's Catalog No. } \\
\hline \multicolumn{3}{|l|}{ 4. Title and Subtitle } & \multicolumn{2}{|l|}{$\begin{array}{r}\text { 5. Report Date } \\
\text { May } 2008\end{array}$} \\
\hline \multicolumn{3}{|c|}{$\begin{array}{l}\text { Expert System to Support Site Investigation for Safety } \\
\text { Improvement }\end{array}$} & \multicolumn{2}{|c|}{ 6. Performing Organization Code } \\
\hline \multicolumn{3}{|c|}{$\begin{array}{l}\text { 7. Author(s) } \\
\text { Andrew P. Tarko, Andrew M. Kwasniak, Jorge Ramos }\end{array}$} & \multicolumn{2}{|c|}{$\begin{array}{l}\text { 8. Performing Organization Report No. } \\
\text { FHWA/IN/JTRP-2008/4 }\end{array}$} \\
\hline \multirow{2}{*}{\multicolumn{3}{|c|}{$\begin{array}{l}\text { 9. Performing Organization Name and Address } \\
\text { Joint Transportation Research Program } \\
550 \text { Stadium Mall Drive } \\
\text { Purdue University } \\
\text { West Lafayette, IN 47907-2051 }\end{array}$}} & \multicolumn{2}{|c|}{ 10. Work Unit No. (TRAIS) } \\
\hline & & & \multicolumn{2}{|c|}{$\begin{array}{l}\text { 11. Contract or Grant No. } \\
\text { SPR-3103 }\end{array}$} \\
\hline \multirow{2}{*}{\multicolumn{3}{|c|}{$\begin{array}{l}\text { 12. Sponsoring Agency Name and Address } \\
\text { Indiana Department of Transportation } \\
\text { State Office Building } \\
100 \text { North Senate Avenue } \\
\text { Indianapolis. IN } 46204\end{array}$}} & \multicolumn{2}{|c|}{$\begin{array}{l}\text { 13. Type of Report and Period Covered } \\
\text { Draft Final Report }\end{array}$} \\
\hline & & & \multicolumn{2}{|c|}{ 14. Sponsoring Agency Code } \\
\hline \multicolumn{5}{|c|}{$\begin{array}{l}\text { 15. Supplementary Notes } \\
\text { Prepared in cooperation with the Department of Transportation and Federal Highway Administration. }\end{array}$} \\
\hline \multicolumn{5}{|c|}{$\begin{array}{l}\text { 16. Abstract } \\
\text { Specialists in transportation and other areas of engineering are trying to improve road safety. The most difficult part is } \\
\text { determining the road features, driver behaviors, and vehicular failures that unduly increase the risk of crash. To help INDOT } \\
\text { safety investigation teams, a previous research project developed a prototype Road Safety Investigation Tool (RSIT) for two- } \\
\text { way stop-controlled intersections. The research summarized here has evaluated the prototype RSIT and has developed a new } \\
\text { version RSIT2 that has incorporated the findings of the evaluation. } \\
\text { The evaluation of the RSIT prototype confirmed usefulness of the proposed methodology. The tool has been } \\
\text { applied to two two-way stop-controlled intersections by a team of non-experts. The two sites have been previously } \\
\text { investigated by experts using the standard approach. The safety investigation outcomes obtained by both the teams indicate } \\
\text { strong similarities The proposed RSIT method helped non-experts identify additional road hazards confirmed later by the } \\
\text { experts' team. RSIT has been found decreasing the required investigation team size and time as well as being useful as a } \\
\text { training tool. RSIT presents the logical connections between driver behaviors, road elements and environment conditions. } \\
\text { RSIT2 is the next version of the RSIT method. The knowledge base developed for RSIT } 2 \text { includes all the major } \\
\text { types of roads in Indiana: a) all-way stop-controlled intersection, b) road segment, c) railroad crossing, and d) signalized } \\
\text { intersection, and e) improved two-way stop-controlled intersections. The RSIT } 2 \text { method uses the rule-based structure } \\
\text { converted to a dynamic checklist that adjusts in real-time to the investigation progress. A friendly graphic-user interface has } \\
\text { been developed together with a knowledge base editor to allow updating the knowledge base by the user. Finally, a novel } \\
\text { technique of ranking safety countermeasures was developed and applied. }\end{array}$} \\
\hline \multicolumn{2}{|c|}{$\begin{array}{l}\text { 17. Key Words } \\
\text { high-crash locations, safety countermeasures, safety } \\
\text { improvements, knowledge-based system, road safety } \\
\text { audits }\end{array}$} & \multicolumn{3}{|c|}{$\begin{array}{l}\text { 18. Distribution Statement } \\
\text { No restriction. This document is available to the } \\
\text { public from the National Information Service, } \\
\text { Springfield, VA } 22161\end{array}$} \\
\hline $\begin{array}{l}\text { 19. Security Classif. (of this report } \\
\text { Unclassified }\end{array}$ & $\begin{array}{r}\text { 20. Security Clas } \\
\mathrm{U}\end{array}$ & $\begin{array}{l}\text { (of this page) } \\
\text { lassified }\end{array}$ & \begin{tabular}{|c|} 
21. No. of Pages \\
298
\end{tabular} & 22. Price \\
\hline
\end{tabular}




\title{
INDOT Research Project Implementation Plan
}

\author{
Date: August 12, 2009
}

Research Project Number: SPR-3103

Project Title: Knowledge-Based System to Support Site Investigation for Safety Improvement

Principal Investigator (PI): Andrew Tarko

Project Advisor (PA): Shuo Li Signature:

Principal Implementor (PIM): Mike Holowaty Signature:

INDOT Strategic Goal Impact Areas (select all that the implementation of this project will impact):

$\square$ Mobility $\quad \square$ Safety $\square$ Economic Development $\square$ CustomerService

$\square$ Resource Management $\square$ Training

Summary of Implementation Plan: The RSIT 2 tool is ready for implementation. The graphic userfriendly interface has been updated from the prototype of RSIT. The developed knowledge base for the RSIT 2 needs to be evaluated and improved as part of implementation and regular use. The implementation effort may include a half-a-day training with participation of the PI.

Note: If more than one implementor recommended, please fill in the information on each implementor's implementation items: Name of Implementor/User: Mike Holowaty Signature:

Responsible for Implementing.

Help or resources needed for implementation (e.g., help from PI, funding, equipment, etc.):

Name of Implementor/User:

Signature:

Responsible for Implementing:

Help or resources needed for implementation (e.g., help from PI, funding, equipment, etc.):

Name of Implementor/User:

Signature:

Responsible for Implementing:

Help or resources needed for implementation (e.g., help from PI, funding, equipment, etc.):

Signatures of SAC members: 


\section{TABLE OF CONTENTS}

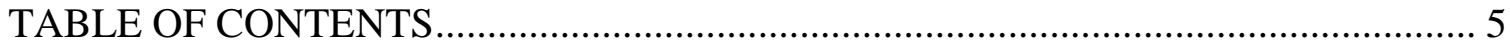

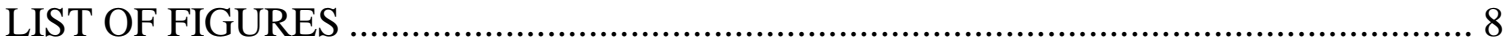

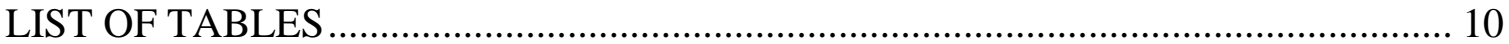

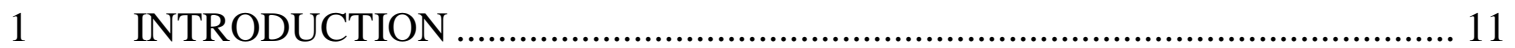

2 SAFETY INVESTIGATION TOOLS AND METHODS................................... 14

2.1 Road Safety Investigation Process and Its Issues ............................................... 14

2.2 Frequency and Severity Rating Method ................................................................. 17

2.3 Interactive Highway Safety Design Model ............................................................. 19

$2.4 \quad$ Road Safety Audit Software........................................................................................ 22

2.5 Summary ...................................................................................................................... 25

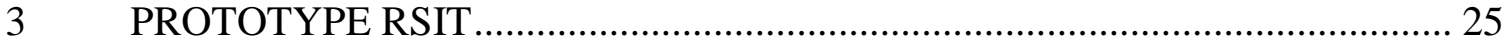

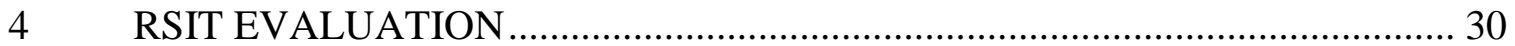

4.1 Deficiencies-based Evaluation...................................................................31

4.2 Countermeasures-based Evaluation........................................................................37

$4.3 \quad$ Summary ...................................................................................................................... 38

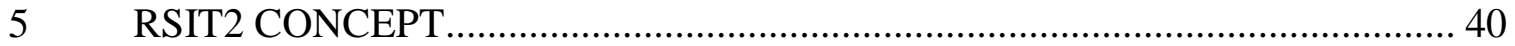

5.1 Checklist Optimization Problem ......................................................................... 40

$5.2 \quad$ RSIT2 Knowledge Representation ........................................................................... 43

$5.3 \quad$ Summary of RSIT2 Components .............................................................................. 47

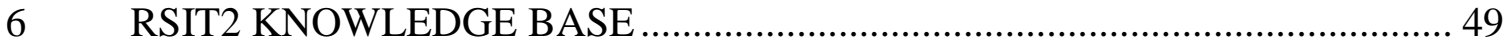

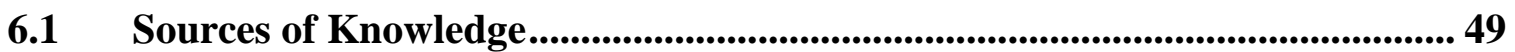

6.2 Knowledge Base Development .................................................................................... 51 
6.3 CRF Study ............................................................................................................. 53

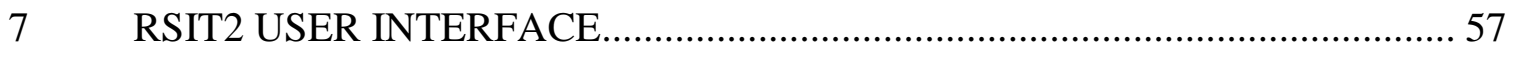

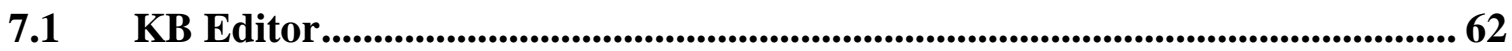

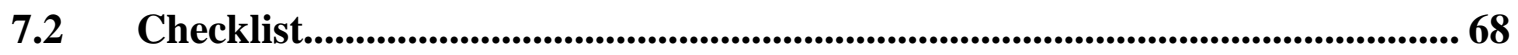

7.3 Ranking Countermeasures and Report .............................................................. 80

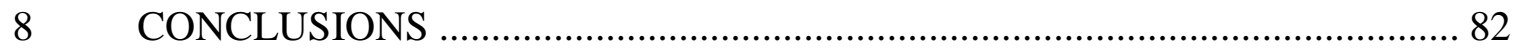

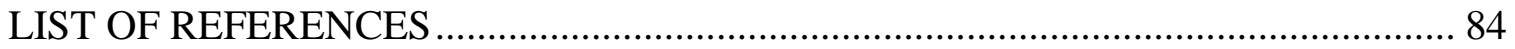

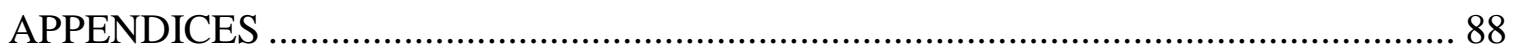

Appendix A ................................................................................................................................... 89

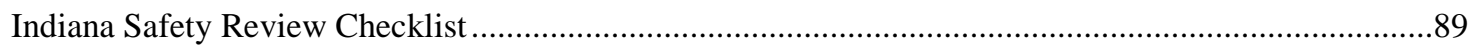

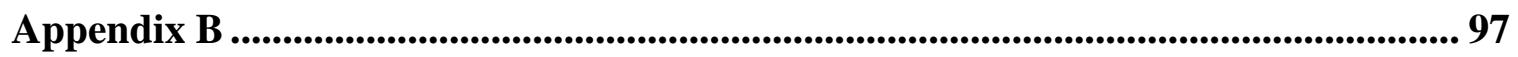

Countermeasures List …………………………………………………………………………...97

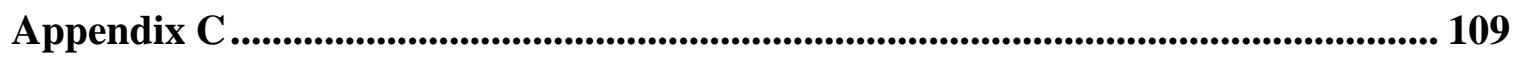

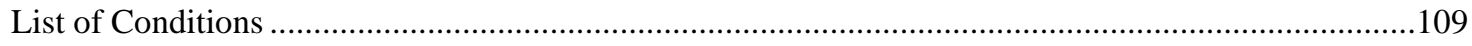

Appendix D .............................................................................................................................. 118

RSIT 2 Knowledge Base ........................................................................................................... 118

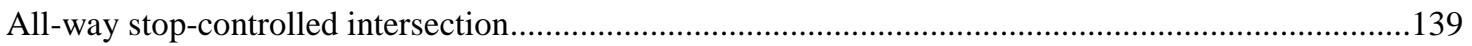

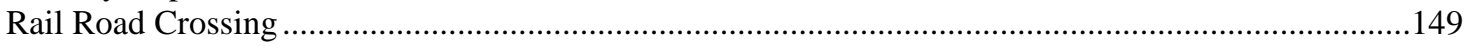

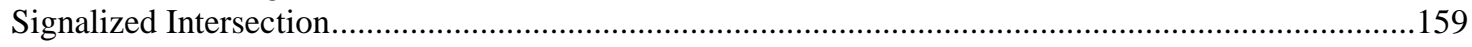

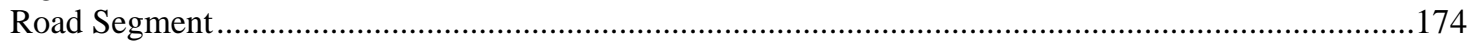

Appendix E ................................................................................................................................. 197

CRF study .................................................................................................................................... 197

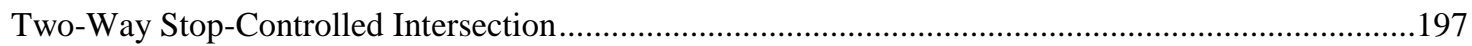

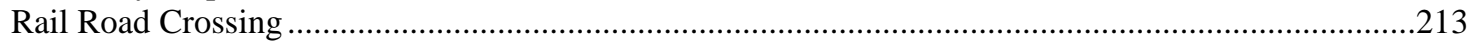

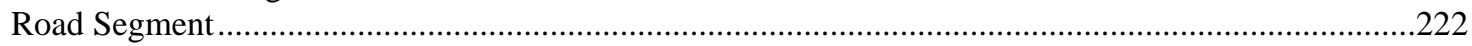

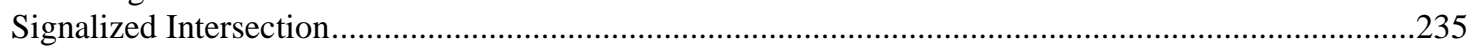

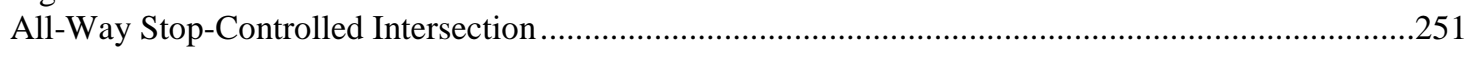

The final CRF values ............................................................................................................... 262 


\section{LIST OF FIGURES}

Figure 2-1 Collision Diagram (LTAP Hazard Elimination Study) ................................ 15

Figure 2-2 Example of the Checklist (Hazard Elimination Study for Smith and Rogers

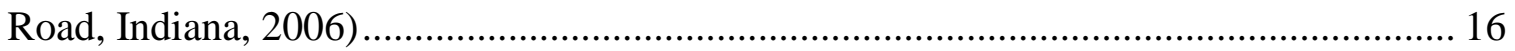

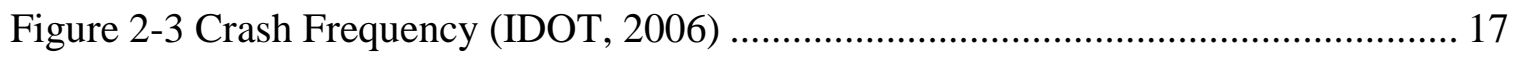

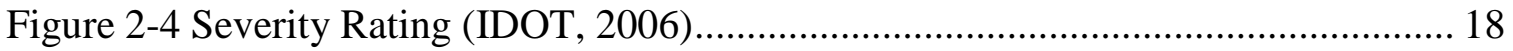

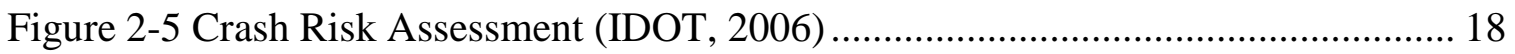

Figure 2-6 Insufficient Sight Distances (Williamson County RSA, Illinois 2006) .......... 19

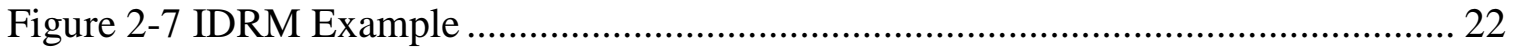

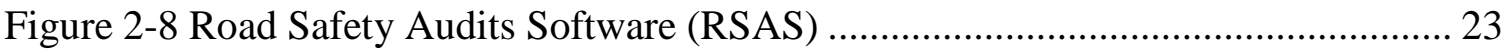

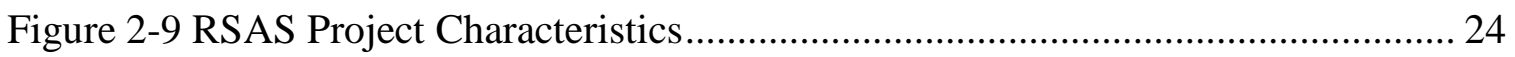

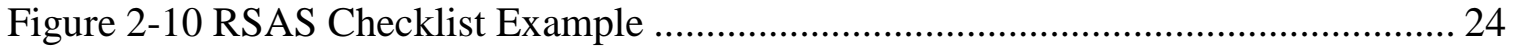

Figure 3-1 Knowledge Base for Right-Angle Collision (using HDS tool)..................... 28

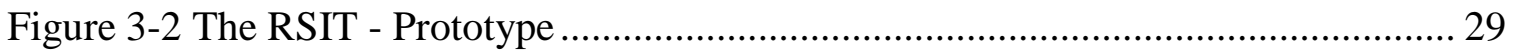

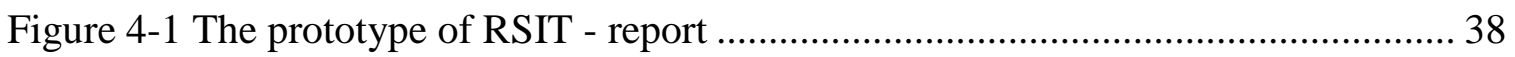

Figure 5-1 RSIT Prototype Knowledge Structure ........................................................ 44

Figure 5-2 The Prototype of RSIT Knowledge Base ................................................. 44

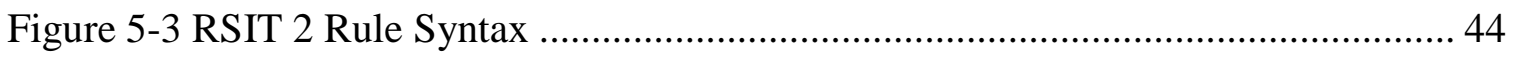

Figure 7-1 RSIT 2 Editor ..............................................Error! Bookmark not defined.

Figure 7-2 RSIT 2 Editor Menus ....................................Error! Bookmark not defined.

Figure 7-3 RSIT 2 Editor Rules/Conditions/Countermeasures ....... Error! Bookmark not defined.

Figure 7-4 RSIT 2 Editor: Adding new rule ......................Error! Bookmark not defined.

Figure 7-5 RSIT 2 Editor - New Rule ..............................Error! Bookmark not defined.

Figure 7-6 RSIT 2 Editor - Updating ...............................Error! Bookmark not defined.

Figure 7-7 RSIT 2 Editor Updating part II .......................Error! Bookmark not defined. 
Figure 7-8 RSIT2 intersection diagram ........................Error! Bookmark not defined.

Figure 7-9 RSIT2 checklist interface with an example checklist .............................. 76 


\section{LIST OF TABLES}

Table 2-1 Example of the Rash Risk Assessment (IDOT, Williamson County RSA) ..... 19

Table 4-1 Prototype evaluation: Identified site deficiencies and proposed safety countermeasures at Stadium Avenue and Grand Street, West Lafayette, Tippecanoe

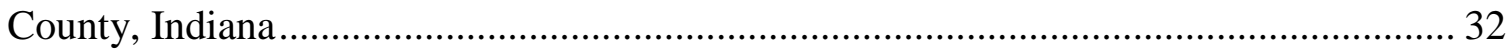

Table 4-2 Prototype evaluation: Identified site deficiencies and proposed safety countermeasures at $6^{\text {th }}$ Street and Salem Street, Lafayette, Tippecanoe County, Indiana 33 Table 4-3 Roadway Deficiencies Identified at the Two Investigated Sites ..................... 36

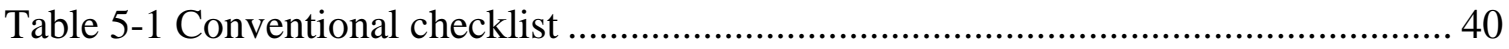

Table 5-2 Example checks with corresponding attributes .......................................... 46

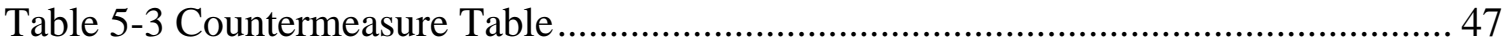

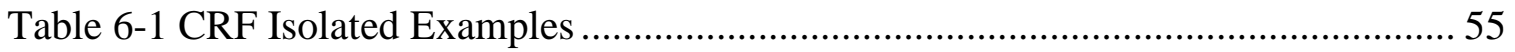

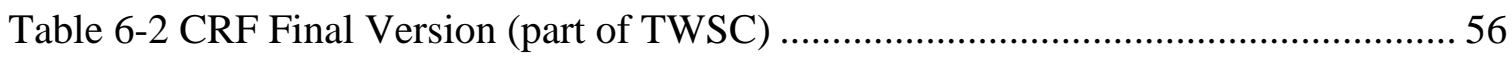




\section{INTRODUCTION}

Road safety has become a critical transportation issue around the world including the United States. Year 2005 brought the highest total number of traffic fatalities since 1990. Safety specialists are continuously striving for improvement in this area. The toughest part is determining the road features, driver behavior, and vehicular failure that unduly increase the risk of crash. Investigating roads that experience excessive number of crashes provides of an opportunity to identify specific road factors contributing to some of these crashes. Even experienced road investigators may have difficulties in connecting various pieces of information and knowledge due to the high level of uncertainty, the great complexity of safety impacts, and the gaps in what is known about driver performance during the crash. Due to the lack of standard techniques, investigative teams rely greatly on their experience and judgment. Moreover, in some cases an investigative team adheres to its past experience, routine actions, and past findings that do not necessarily reflect the causes at the currently investigated site.

Lack of adequate information, time pressure as well as lack of in-depth knowledge of the actual circumstances leading to past crashes increases the complexity of the problem. Current practice, despite the best effort of investigating teams, relies a great deal on the teams' experience and judgment, which may not be sufficient given the limited data, the complexity of roadway-driver-vehicle interactions, and the large number of possible scenarios leading to crashes. One of the tempting alternatives is to focus the investigation on roadway deficiencies that are understood to be a diversion from the current design standards. Although this may bring about sound solutions, it may also reduce the chance of discovering unusual and dangerous combinations of local conditions.

Road Safety Audit (RSA) was first time introduced in the U.S. in the early nineties. RSA was meant to address safety problems in the planning and design stages of highway project. The current RSA is implemented also to the existing road infrastructure. The Road Safety Audit may contain three steps. 
(1) Pre-investigation, where available information related to the crash history on the investigated roadway entity is collected and analyzed;

(2) Site investigation, where the safety team investigates the road site to determine the road deficiencies; and

(3) Post-investigation, where the safety team evaluates the findings of the site investigation and identifies a relevant set of countermeasures.

The common practice of the safety team during investigation is using of safety checklists that organize an audit and serves as the outcome documentation. The Purdue team has developed a comprehensive checklist (Tarko and Kanoida, 2004) as a part of the project on developing the Indiana guidelines for safety improvements (Appendix A). Unfortunately, the length of the checklist and its structure bring the following issues:

(1) The length of the checklist can be as long as 40 pages.

(2) The user has to browse through the entire checklist to find the question/checklist that he/she wants to address.

(3) There is a lack of methodology to indicate a set of relevant countermeasures to address the identified road deficiencies.

(4) The checklist neglects uncertainty of the results.

Despite its deficiencies, a checklist is a frequently used tool as a practical aid in high-crash site investigation. The objective of this research is to develop a computerbased tool to support a high-crash site investigation that provides both a better tool than a conventional checklist. The developed tool should be practical and applicable to the majority of roads in Indiana. It should include a knowledge base applicable to two-way stop-controlled intersections, all-way stop-controlled intersections, road segments, signalized intersections, and railroad crossings.

The presented report is a continuation of previous JTRP research on a prototype Road Site Investigation Tool (RSIT) limited to two-way stop-controlled intersections. In the presented current research, the developed prototype RIST is to be evaluated and improved based on the evaluation outcome. Another important objective is to expand the knowledge base to incorporate other types of roads present in Indiana. 
The first chapter provides an overview of the motivation, scope, and objective of the research. In the second chapter the state-of-the-art and practice, and an overview of actual and propose safety investigation tools are provided. Chapter 3 focuses on the prototype of RSIT while Chapter 4 provides its evaluation. Chapter 5 presents the RSIT 2 concept while Chapter 6 describes the development of the new knowledge base for RSIT 2. An important study of the existing crash reduction factor providing a complete set of the values for the knowledge base is also presented. Chapter 7 provides a description of the RSIT 2 user graphical interface. Conclusions are provided in Chapter 8. 


\section{SAFETY INVESTIGATION TOOLS AND METHODS}

\subsection{Road Safety Investigation Process and Its Issues}

The Road Safety Investigation process is a safety examination of selected roadway entities by teams which should include specialists in highway safety engineering (NCHRP 336, 2004). To improve the investigative process, the safety team should incorporate additional experts outside highway engineering (i.e., human factors experts).

The safety investigation process can be divided into three major stages. The first stage is called preliminary analysis and it helps in the planning of site investigation. During this stage, the safety team collects information about the investigated roadway entity, type of roadway entity, the exact location of the entity, environmental conditions (i.e., school, shopping center, etc.), and any additional data. Probably the most important element in the preliminary stage is the crash data analysis. The crash data are used to determine the predominant crash pattern, weather condition, time of accident, crash severity, and place of crash, as well as information about the primary contributing circumstances and other useful information.

One of the required steps during the preliminary analysis is to prepare a collision diagram (Figure 2-1). This helps find the predominant crash pattern. The time of the accident (for example, night time) is important for site investigation planning. A wellplanned site investigation should take place during the identified periods with frequent crashes. During the preliminary analysis, after all the available data have been prepared, the investigative team can identify possible crash scenarios that may provide guidance during the site investigation by focusing the investigative team on certain periods, weather conditions, traffic movements, driver behaviors, etc. 


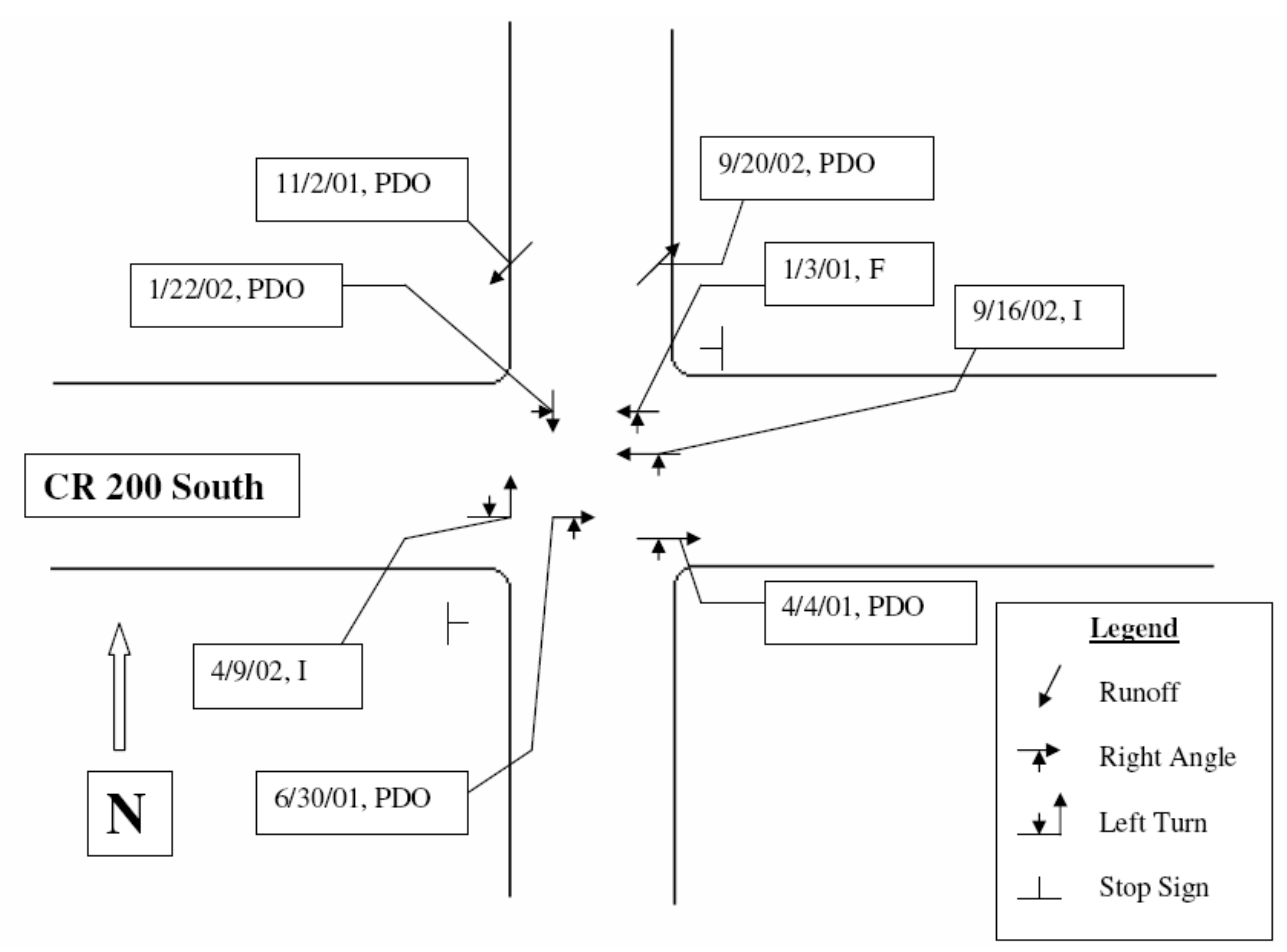

Figure 2-1 Collision Diagram (LTAP Hazard Elimination Study)

The next stage is the site investigation. The investigation team goes to the specific location and determines the existing local conditions. The purpose of this stage is to collect actual information about road conditions, geometry, traffic conditions, and traffic characteristics. In the case of a speed study, the investigator can determine if speed violations are frequent. Collection of the data often requires photographing or videotaping the site. The team may also briefly discuss funding and decide to collect additional data or prepare additional site investigation studies. One of the common practices by the investigation team during this stage of analysis is to follow the checklist (Tarko et al. 2004). An example of the checklist is available in Appendix A. This checklist is an extensive collection of possible roadway deficiencies structured in such a way that helps customize it to the type of roadway location studied. A sample checklist prepared during site investigation is shown in Figure 2-2. 


\section{Safety Review Check List}

\section{Roadway Segment}

Facality Type:

Page: 1 of 3

Location: Smith Road / Rogers Road

Date: Jan 18,200 Time

1,30pm Weather: Sunny

Moving Lanes

Lane widths are inadequate for vehicle classes common to location $20^{\prime}$

Number of lanes are inadequate for traffic

Lanes abrubtly end

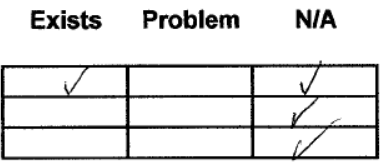

Auxiliary / Turning Lanes

Inadequate advance warning of lane drop

Auxiliary lane lengths are inadequate

Taper lanes and not properly marked or inadequate length

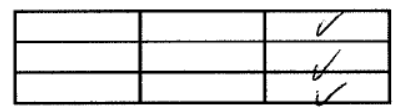

Driveways and Parking

Driveways too closely spaced

Inadequate visibilty of driveways

Parked cars limit visiblity

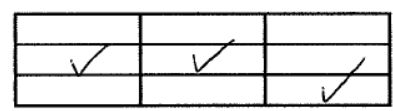

\section{Figure 2-2 Example of the Checklist (Hazard Elimination Study for Smith and} Rogers Road, Indiana, 2006)

The last stage of the safety investigation process is a post-analysis study. The safety team, upon completion of the site investigation, discusses all the findings and determines the safety deficiencies. A common issue faced in the post-analysis study is frequent identification of multiple road deficiencies and corresponding safety countermeasures. The investigative team must decide which of these countermeasures should be recommended.

Use of checklists is a common practice in a Road Safety Audit (RSA). Although the checklist helps in organizing an investigation process and provides an overview of all possible roadway deficiencies, there are also weaknesses in the checklist concept. A safety checklist is customized to a specific site beforehand based on a general knowledge of the site and the pattern of crashes at this site. The checks and their order are not modified at the site during an investigation as the knowledge of the site grows. The most relevant and promising checks may remain further down the list and by the time the investigation reaches them, the investigators may be "worn down" by the job and time pressure. These conditions can negatively affect the efficiency and effectiveness of a site 
investigation. As a result, in some observed RSA investigations, the team put the checklist aside and tried to suggest potential safety candidates for improvement based on their own experience, knowledge, and judgment. In such a case, it is easy to overlook important safety deficiencies and provide inadequate safety improvements.

\subsection{Frequency and Severity Rating Method}

To help investigators classify the road deficiencies, a frequency and severity rating can be used (practiced by the Illinois Department of Transportation (IDOT) in the developing and existing condition). By using a frequency rating, the total number of crashes that occur on specific intersections or road segments is classified as shown in Figure 2-3.

\begin{tabular}{|c|c|c|c|}
\hline \multicolumn{2}{|c|}{ ESTIMATED } & \multirow{2}{*}{$\begin{array}{c}\text { EXPECTED CRASH FREQUENCY (per } \\
\text { audit item) }\end{array}$} & \multirow{2}{*}{$\begin{array}{c}\text { FREQUENCY } \\
\text { RATING }\end{array}$} \\
\hline EXPOSURE & PROBABILITY & & \\
\hline high & High & \multirow{2}{*}{10 or more crashes per year } & \multirow{2}{*}{ Frequent } \\
\hline medium & High & & \\
\hline high & Medium & \multirow{3}{*}{1 to 9 crashes per year } & \multirow{3}{*}{ Occasional } \\
\hline medium & Medium & & \\
\hline low & High & & \\
\hline high & Low & \multirow{2}{*}{$\begin{array}{c}\text { less than } 1 \text { crash per year, but more than } \\
1 \text { crash every } 10 \text { years }\end{array}$} & \multirow{2}{*}{ Infrequent } \\
\hline low & Medium & & \\
\hline medium & Low & \multirow{2}{*}{ less than 1 crash every 10 years } & \multirow{2}{*}{ Rare } \\
\hline low & Low & & \\
\hline
\end{tabular}

Figure 2-3 Crash Frequency (IDOT, 2006)

The severity rating is obtained by combining the typical crashes expected with the expected crash severity; for example, a crash that involves high speed and a heavy vehicle can have an expected crash severity of probable fatality or incapacitating injury (Figure 2-4). 


\begin{tabular}{|c|c|c|}
\hline $\begin{array}{c}\text { TYPICAL CRASHES EXPECTED } \\
\text { (per audit item) }\end{array}$ & $\begin{array}{c}\text { EXPECTED CRASH } \\
\text { SEVERITY }\end{array}$ & $\begin{array}{c}\text { SEVERITY } \\
\text { RATING }\end{array}$ \\
\hline \hline $\begin{array}{c}\text { crashes involving high speeds or heavy } \\
\text { vehicles, pedestrians, or bicycles }\end{array}$ & $\begin{array}{c}\text { probable fatality or } \\
\text { incapacitating injury }\end{array}$ & High \\
\hline $\begin{array}{c}\text { crashes involving medium to high speed; } \\
\text { head-on, crossing, or off-road crashes }\end{array}$ & moderate to severe injury & High Moderate \\
\hline $\begin{array}{c}\text { crashes involving medium to low speeds; } \\
\text { left-turn and right-turn crashes }\end{array}$ & minor to moderate injury & Low Moderate \\
\hline $\begin{array}{c}\text { crashes involving low to medium speeds; } \\
\text { rear-end or sideswipe crashes }\end{array}$ & $\begin{array}{c}\text { property damage only or } \\
\text { minor injury }\end{array}$ & Low \\
\hline
\end{tabular}

\section{Figure 2-4 Severity Rating (IDOT, 2006)}

By combining the severity rating and the frequency rating, the crash risk assessment is obtained. Levels D, E, and F represent the highest crash risk, where safety implementation is needed as shown in Figure 2-5.

\begin{tabular}{|c|c|c|c|c|}
\hline \multirow{2}{*}{$\begin{array}{c}\text { FREQUENCY } \\
\text { RATING }\end{array}$} & \multicolumn{4}{|c|}{ SEVERITY RATING } \\
\hline & Low & Low Moderate & High Moderate & High \\
\hline Frequent & $\mathrm{C}$ & $\mathrm{D}$ & $E$ & $\mathbf{F}$ \\
\hline Occasional & $B$ & $\mathrm{C}$ & $\mathrm{D}$ & $E$ \\
\hline Infrequent & A & B & C & D \\
\hline Rare & $A$ & $A$ & $\mathrm{~B}$ & $\mathrm{C}$ \\
\hline Crash Risk Ratings: & $\begin{array}{l}A: / C \\
B: / 1 \\
C: n\end{array}$ & $\begin{array}{l}\text { risk level } \\
\text { k level } \\
\text { rate-low risk level }\end{array}$ & $\begin{array}{l}\text { D: moderate-high ris } \\
\text { E: high risk level } \\
\text { F: highest risk level }\end{array}$ & \\
\hline
\end{tabular}

Figure 2-5 Crash Risk Assessment (IDOT, 2006)

During site investigation and post-analysis, the safety team develops a list of all possible safety issues. This list is evaluated according to the expected safety risk of associated crashes. Risk is defined by the degree of frequency and the severity of the expected crashes for each safety issue and is given an overall rating level as represented in Table 2-1. 
Following is an example of using the crash risk assessment technique. The observations were made by the IDOT safety team during road safety audits in Williamson County, Illinois (Illinois, 2006). One of the observations during the site investigation was insufficient sight distance triangles (Figure 2-6). The expected frequency and severity were classified as occasional, severe; (Table 2-1) what constitutes a high crash rating and level E.

Table 2-1 Example of the Rash Risk Assessment (IDOT, Williamson County RSA)

\begin{tabular}{|c|c|c|c|}
\hline Expected Frequency & Expected Severity & Risk Rating & E \\
\hline Occasional & Severe & High & . \\
\hline
\end{tabular}

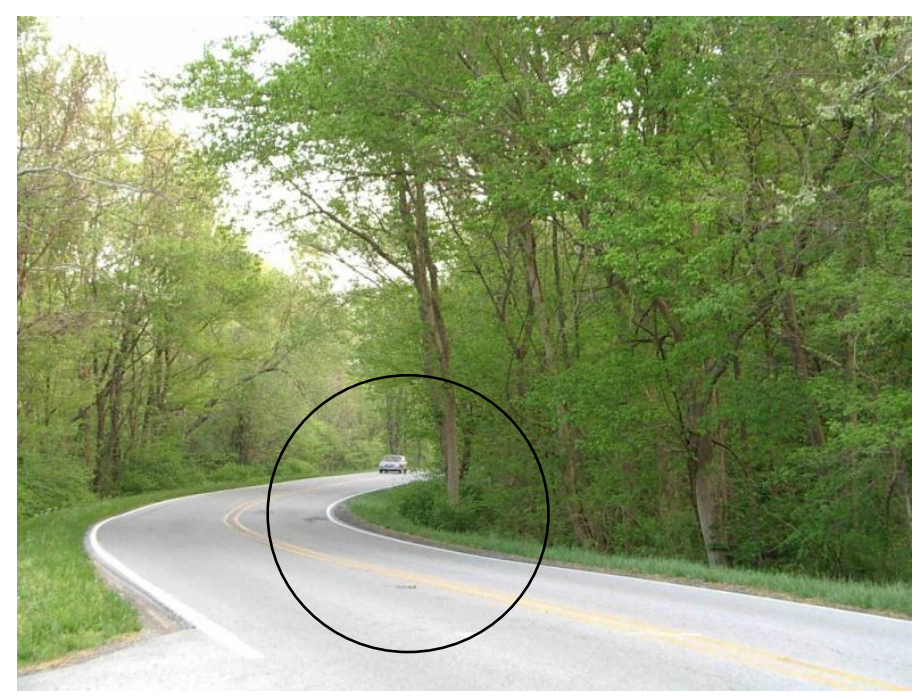

Figure 2-6 Insufficient Sight Distances (Williamson County RSA, Illinois 2006)

Because the insufficient sight distances represent level E, this observation had to be considered. Therefore, the following suggestion was proposed: "Clear vegetation, including strategic tree removal, relocate signs, and regrade slopes or reduce crests to establish sight distance.

\subsection{Interactive Highway Safety Design Model}

The IHSDM (Interactive Highway Safety Design Model) is an advanced public domain decision support system developed to evaluate the safety and operational effects of geometry design on rural two-lane roads (Kindler et al. 2006). The IHSDM released in 
2003 is the cumulative work of researchers and developers over a number of years (Krammes et al. 2003). The current available version of this software provides six modules:

(1) Crash Prediction. The crash prediction module predicts the expected frequency of crashes based on geometry design and traffic characteristics. The base statistical model used to predict crash frequency is derived from negative binomial regression analysis. Moreover, the Accident Modification Factors (AMF) was used to adjust the base model for individual roadway characteristics. The Empirical Bayesian (EB) technique was used to adjust the model to locationspecific data.

(2) Design Consistency. This module was developed to evaluate safety on horizontal curves. In addition, for evaluation purposes the speed-profile was used. The output of this module provides information that helps diagnose safety issues on roadway entities and checks for alignment design.

(3) Driver/Vehicle. This model will be added to the future version of IHSDM to evaluate driver performance on the road and its interaction with the vehicle.

(4) Intersection Review. This module was developed to evaluate intersection safety based on verification in current geometry design. This module focuses on intersection configuration and horizontal/vertical alignment. Because this model is closely related to improving safety on roadway entities (intersection - similar to RSIT), it will be discussed in more detail below.

(5) Policy Review. This module reviews and checks four categories: cross section, horizontal alignment vertical alignment, and sight distance with design standards specified by AASHTO.

(6) Traffic Analysis. This module "uses the TWOPAS traffic simulation module to estimate traffic quality of service measures for an existing or proposed design under current or projected future traffic flows." (FHWA/IDOT, 2005)

The Intersection Diagnostic Review Model (IDRN) described in p. 4 is discussed below in more detail. The IDRM is designated to be used after other modules (crash prediction, policy review, and design consistency) are performed. The output from the previous modules is used as an input into IDRM. The IDRM provides a 
comprehensive review of the intersection design. The knowledge base for at-grade intersections on two-lane highways was developed. The knowledge base is organized into a set of engineering models that are selected based on geometry and safety concerns at the intersections. As a result, 111 potential design problems were identified and 21 models were developed for the IDRM knowledge base. These models include: intersection sight distance (ISD) - based models, stopping sight distance (SSD) -based models, decision sight distance (DSD) based models, clearance time models, turn-lane models, and miscellaneous models. As a result, the IHSDM (IDRM) contains a sufficient knowledge base to check the geometry design for each approach as well as for all intersections. The knowledge base is supported in the engineering models that consist of the most importance factors, which have to be checked for geometry design standards. Information about the actual obstruction of the intersection sight distance is derived by the user. This approach creates a more effective tool and gives more adequate findings

The example below presents the model for intersection sight distance on the left turn minor road. This model defines intersection sight distance by Equation 2.1

$I S D=0.276 V t$

\section{Equation 2.1}

where:

ISD - intersection sight distance (m), $\mathrm{V}$ - initial speed $(\mathrm{km} / \mathrm{h})$, $\mathrm{t}$ - time gap (s).

The initial speed is set up to the actual 85th percentile speed of traffic on the selected major road. To evaluate ISD on the specific intersection, the following procedure applies:

(1) Select a pair of major - and - minor road approaches.

(2) Determine the actual $85^{\text {th }}$ percentile speed on the elected major road approach.

(3) Select an appropriate time gap.

(4) Address the sight triangle issue. In the case of sight obstruction such as: vegetation, man-made obstacles, etc. ask the user if region $\mathrm{X}$ is clear of roadside sight obstruction.

After all the information is provided, the ISD is compared with the calculated threshold and is adequate if a needed advisory message is displayed. 
In Figure 2-7, the example of the IHSDM IDRM is presented including: a configuration of the intersection, potential safety concern and design improvements.

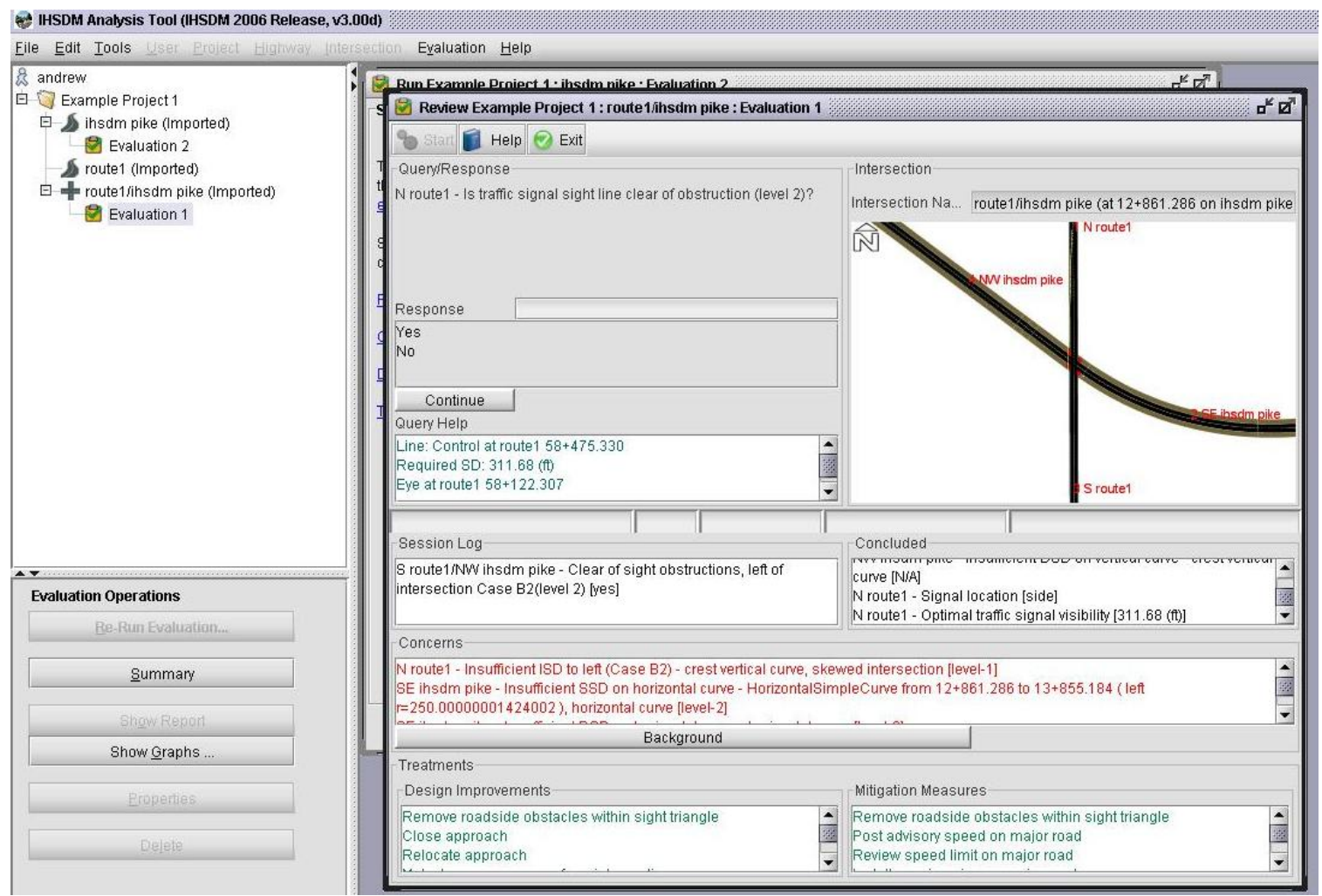

Figure 2-7 IDRM Example

The IHSDM was developed for use in engineering studies, functional/conceptual design, preliminary design, and detailed design stages.

\subsection{Road Safety Audit Software}

The Federal Highway Administration (FHWA) in cooperation with software development and transportation companies developed a prototype of RSA Software (RSAS). Currently, the evaluation version of this software (Beta Version 1.00.0002, January 2006) is available.

FHWA RSAS allows the development of a customized list of checks (prompt or detailed) provided by the auditor information. The welcome windows presented in Figure 2-8 Road Safety Audits Software (RSAS) allows the provision of basic background 
information related to the investigated entity i.e. project number, project description, start/end date, etc.

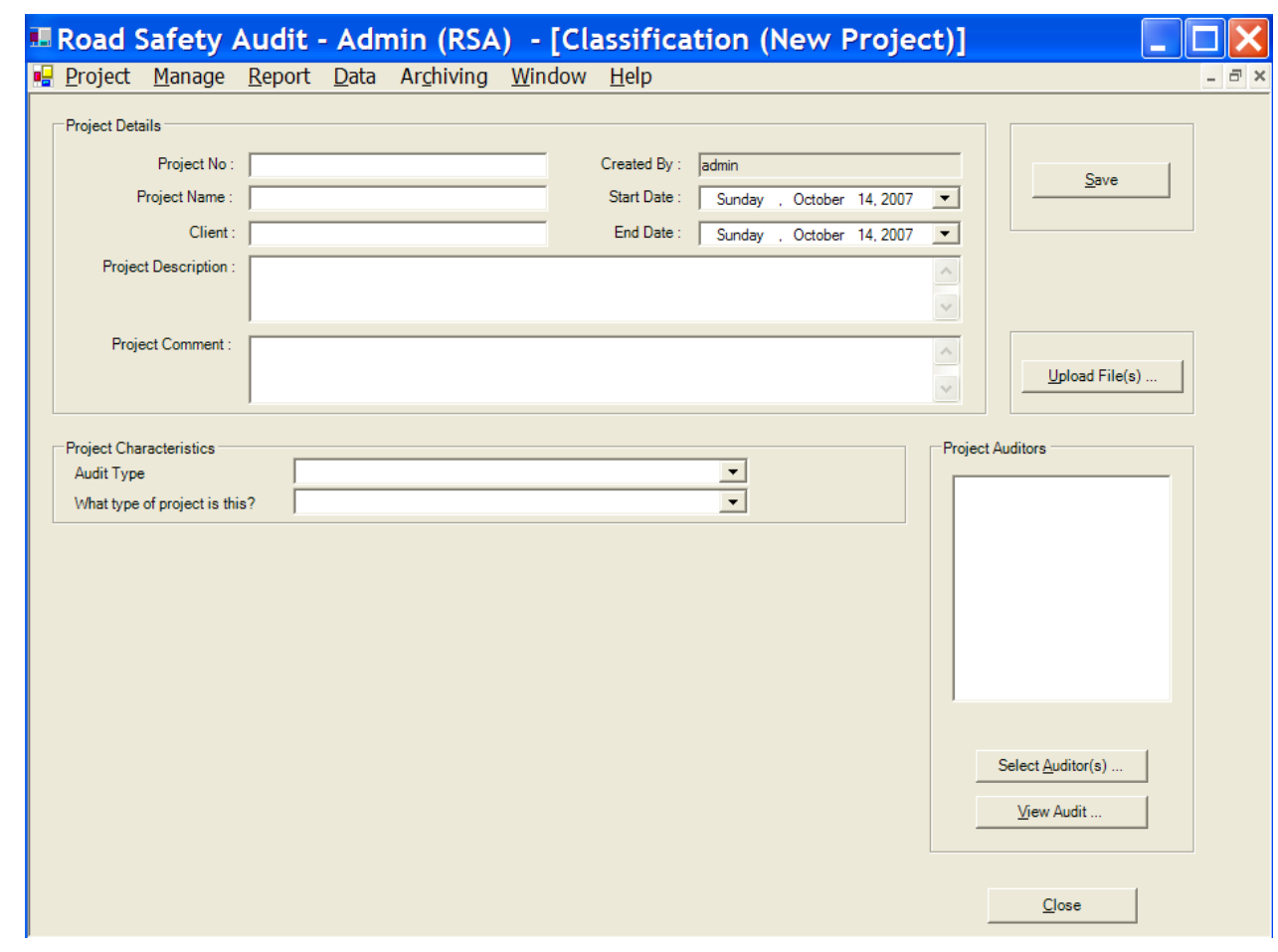

Figure 2-8 Road Safety Audits Software (RSAS)

In addition, the presented software allows a user to choose between different audit types: planning stage, preliminary stage, final decision stage, work zone traffic scheme, preopening stage, existing roads, and land use development proposals. In the next step, based on the project characteristics provided such as: service function, climatic conditions, design speed, adjacent land use, etc. (Figure 2-9), the software generates the checklist (prompt or detailed) as well as a summary list. An example of a detailed generated checklist is presented in Figure 2-10.

Moreover, the RSAS allows multiple users accessibility and administration functions. The final report as well as the current stage can be saved and opened later for further investigation.

The RSAS is currently in the development process; therefore, the beta version will be improved in the near future and additional functionality will be added. 


Project Characteristics
Audit Type
Which Units of Measure will be
used for this project?
What is the Adjacent Land Use? Urban
What is the Design Speed?
Is the roadway divided or
undivided?
What is the Service Function?
Please characterize the
surrounding terrain.
What are the climatic conditions
with respect to temperature?
What are the climatic conditions
with respect to snow?
Which of the following best
describes the roadway scheme? Road section including interchange(s) and non-signalized at-grade ir
What type of project is this?

\section{Figure 2-9 RSAS Project Characteristics}

Road Safety Audit

Checklist: (Detailed)

\begin{tabular}{|l|l|l|}
\hline \multicolumn{1}{|c|}{ ROAD FUNCTION, CLASSIFICATION, } & Yes / No & \\
ENVIRONMENT & Comment \\
\hline General road function, classification, environment & \\
\hline $\begin{array}{l}\text { Is the road function and classification the same as } \\
\text { it was when the road was designed and } \\
\text { constructed? }\end{array}$ & \\
\hline $\begin{array}{l}\text { Is the road environment the same as it was when } \\
\text { the road was designed and constructed (no new } \\
\text { developments, no new pedestrian/bicyclists } \\
\text { activities, special events, scenic vistas etc) }\end{array}$ & \\
\hline
\end{tabular}

国

\begin{tabular}{|l|l|l|}
\hline ROAD ALIGNMENT AND CROSS SECTION & Yes / No & \\
\hline Visibility, sight distance & Comment \\
\hline $\begin{array}{l}\text { Is sight distance adequate for the speed of traffic } \\
\text { using the route? }\end{array}$ & & \\
\hline $\begin{array}{l}\text { Is adequate sight distance provided for } \\
\text { intersections and crossings? (e.g., pedestrian, } \\
\text { bicyclist, cattle, rail crossings) }\end{array}$ & \\
\hline $\begin{array}{l}\text { Is adequate sight distance provided at all private } \\
\text { driveways and property entrances? }\end{array}$ & \\
\hline Design speed & \\
\hline
\end{tabular}

Figure 2-10 RSAS Checklist Example 


\subsection{Summary}

This chapter presented an overview of road safety investigation methods and tools. Available tools dedicated to road safety investigation were discussed. The review of available tools and methods pointed out lack of an adequate approach that can be efficiently during road safety investigation. The lack of in-depth knowledge of the actual circumstances leading to past crashes increases the complexity of the problem. Current practice, despite the best effort of investigating teams, relies a great deal on the teams' experience and judgment, which may not be sufficient given the limited data, the complexity of roadway-driver-vehicle interactions, and the large number of possible scenarios leading to crashes.

A computer-based method is needed to organize an efficient site investigation as well as to utilize all available pieces of information. This tool should be user-friendly and allows for flexibility to update or change the implemented knowledge by a user to customize it to the user needs.

\section{Prototype RSIT}

A prototype of the Road Safety Investigation Tool (RSIT) was developed at Purdue University (Tarko et al. 2006). This prototype is applicable to two-way stop-controlled (TWSC) intersections. The RSIT proposed a new method that utilizes a tree-like knowledge base of the safety countermeasures and their connection with the roadway conditions and crash patterns. The implemented knowledge takes the form of an "intelligent" checklist that leads the investigator through relevant questions in an interactive process. The sequence of affirmative responses forms the set of conditions that point out the corresponding countermeasure.

The development of the prototype of RSIT was accomplished in three phases:

(1) Knowledge acquisition,

(2) Knowledge representation, and

(3) Knowledge implementation. 
In the first phase, available sources of information about safety and countermeasures at two-way stop-controlled intersections were identified. In the second phase, the relevant knowledge was extracted and combined in a tree-like knowledge base. In the last phase, a graphic user interface was developed to facilitate forward chaining applied to determine suitable safety countermeasures during site investigation.

The acquisition process of the developed knowledge includes information found in the following sources: text on human factors (Senders et al. 1991; Wiener at el. 1984; and others), publication on safety facts (Traffic Safety Facts), guidance and manuals for Road Safety Investigation, final reports (LTAP reports; Treat et al. 1979), observation safety specialist at work, and other relevant sources. The protocol analysis technique was used to identify relevant facts and logical relationships in the available information. By using the protocol generation technique and particularly interviewing safety experts and observing their work during two road safety audits by INDOT, IDOT, and TDOT teams, additional rules and facts were obtained. The applied protocol generation technique allowed reorganizing, updating, and improving the finding obtained in the protocol analysis phase. Direct observations of the safety team at work in the field in particular turned out to be an important part of the knowledge base development. The developed knowledge based is limited to two-way stop-controlled intersection.

In the knowledge representation part, the hierarchical generation technique was used to develop the final structure of the acquired knowledge. This structure is a decision tree with the forward chaining technique for solution seeking. Because this tool was developed to help investigators determine potential roadway deficiencies during a site investigation, it must mimic the most effective decision-making process during a site investigation. This process can be summarized from the top to the bottom and it includes a sequence of multiple-choice questions. The starting point is to specify all applicable crash patterns. Therefore, the top level of the knowledge is represented by five choices:

(1) Crash types (six types),

(2) Time of collision,

(3) Weather conditions,

(4) Pedestrians/bicycle collisions, and

(5) Intersection conspicuity. 
This representation was established for the limited (two-way stop-controlled intersection) knowledge base.

The most significant different between the prototype of RSIT and other available methods discussed in the previous chapter is the logical connection among the three stages of driver information processing with driver errors and crash occurrence that RSIT proposed. Such connections are presented in Figure 3-1.

In the three stages of driver information processing, the starting point is reception of a stimulus. In Figure 3-1a, this stimulus is generated by the presence of a STOP sign. If the STOP sign is missing, then a driver unfamiliar with the intersection will not consider stopping. Even if the STOP sign is not missing, it is not guaranteed that the driver will stop because the STOP sign, although existing, may not be visible (Figure 3-1b). A driver will probably fail to perceive the need for stopping if the STOP sign is not visible (Figure 3-1c). Adequate safety countermeasures to improve visibility are needed. If the STOP sign is visible, then the driver will probably recognize the sign and will consider stopping (Figure 3-1d).

At this point, adequate safety improvements depend on the actual roadway conditions (Figure 3-1e). The knowledge tree presented in Figure 3-1 has been built around the sequence of driving sub-tasks aimed to avoid collision (perception, cognition, action) and performed repeatedly in two stages of approaching an intersection (preapproach and approach).

The established knowledge base for the prototype of the RSIT tool was facilitated to be used during field investigation by developing the Graphic User Interface (GUI).

The GUI is a flexible tool for browsing the knowledge base and preparing the final report where the proposed safety countermeasures are described, including complete conditions that imply the proposed improvements and user comments. The GUI of the proposed prototype of the RSIT is presented in Figure 3-2.

The welcome window of the prototype of RSIT is presented in Figure 3-2 (A). The user is allowed to start new investigation by pre-loading developed knowledge base or edit existing knowledge base. The editing is implemented and is allowed by commercial free available software (HDS). The next step of RSA is to determine the type of accidents that 
need to be investigated (Figure 3-2 (B)). The structure of the investigation process, based on proposed prototype of RSIT is presented in Figure 3-2 (C)). The user by answering questions defines deficiencies to finally have a chance to point out adequate countermeasures and provided if necessary own comments. In the last step the report is automatically generated (Figure 3-2 (D)). For more information related to the presented prototype of RSIT (including user manual) the user is referred to Tarko et al. (2006).

The last step of developing the prototype of RSIT was evaluation. The evaluation part is described in details in the next chapter.

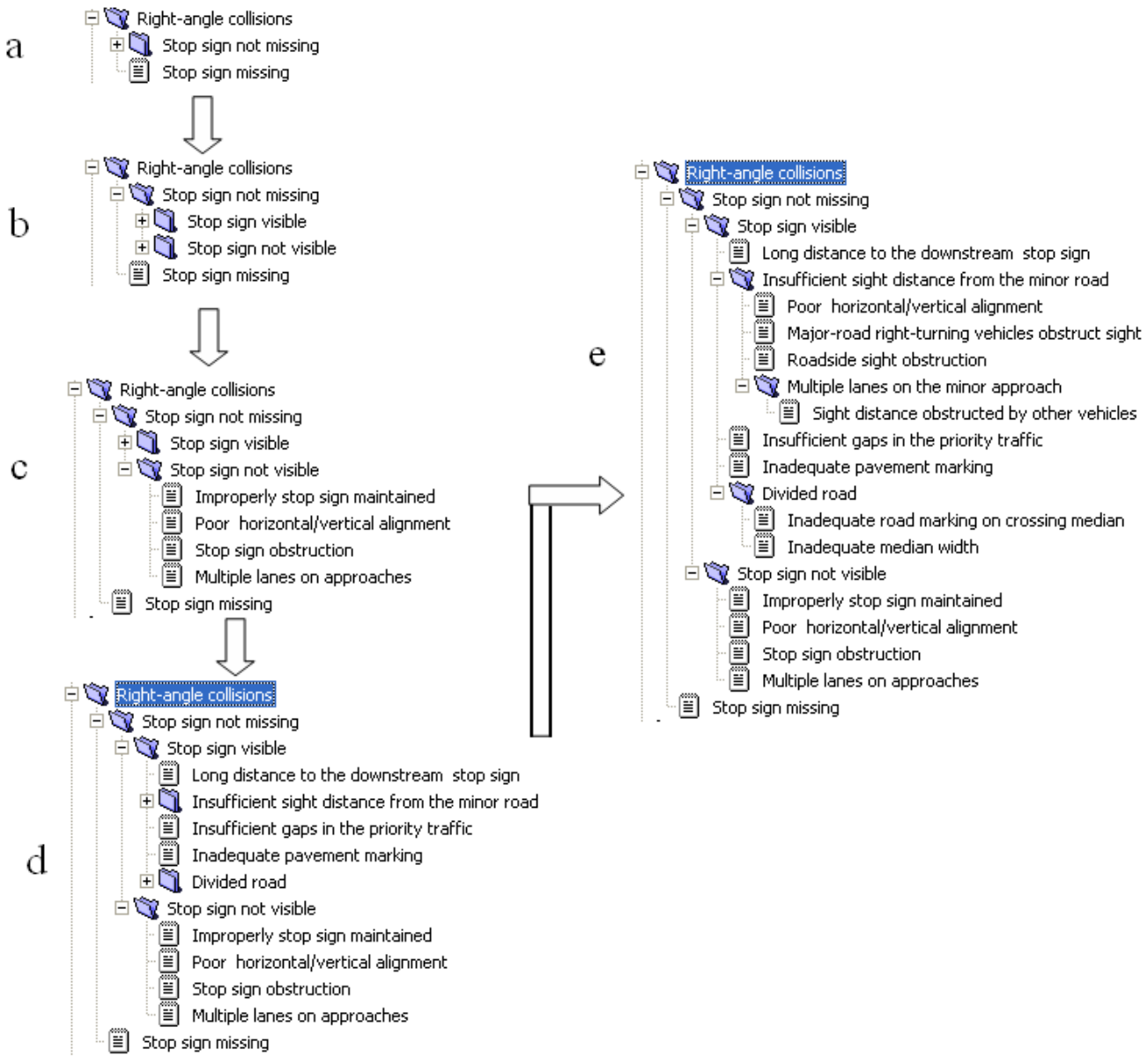

Figure 3-1 Knowledge Base for Right-Angle Collision (using HDS tool) 
RSIT - Road Safety Investigation Tool
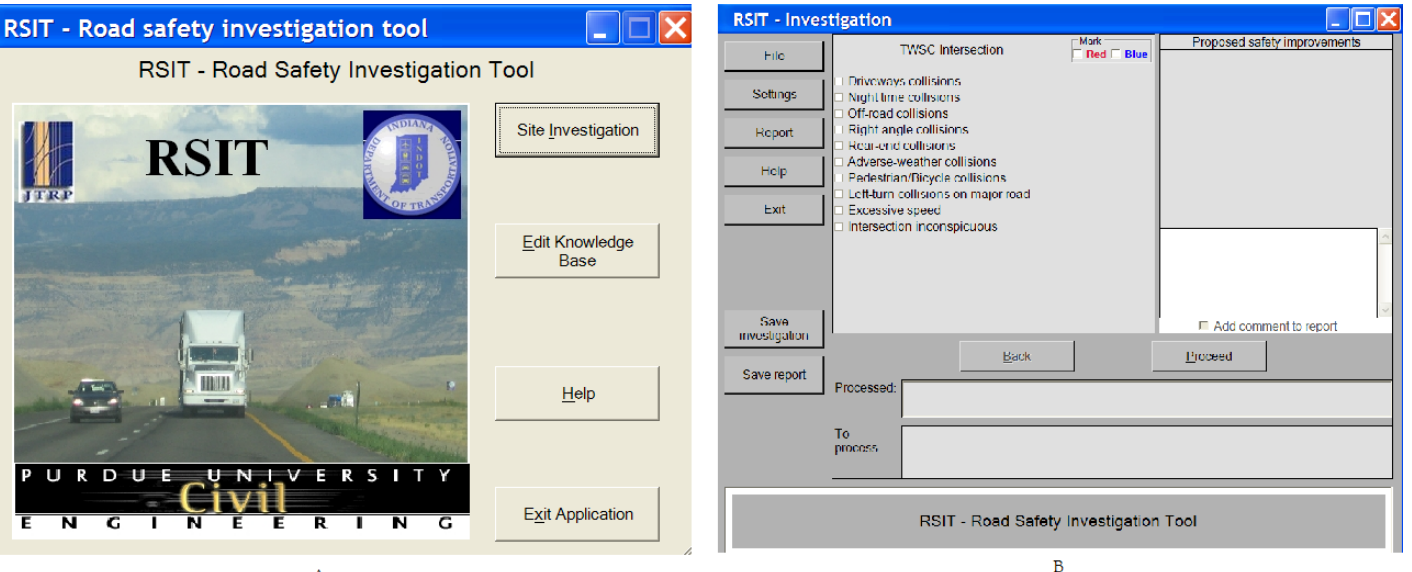

A
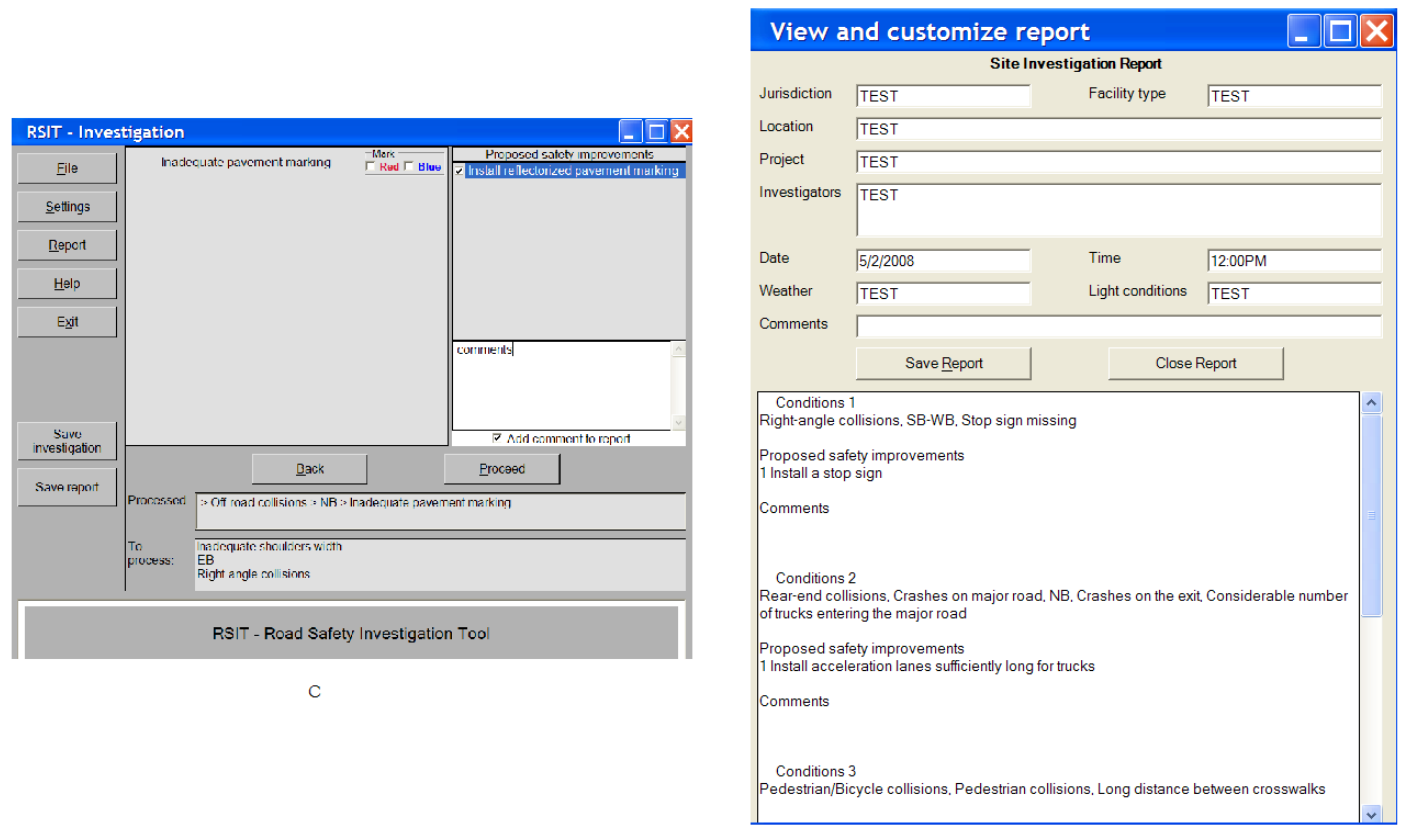

Figure 3-2 The RSIT - Prototype 


\section{RSIT EVALUATION}

The developed prototype was evaluated in two ways: (1) In the field by user of the tool applied to two intersections, and (2) Through a software presentation and following up discussion of experts (members of the Study Advisory Committee for the project). The evaluation part was applied to the prototype with the knowledge base developed for twoway stop-controlled intersections.

In the first evaluation phase, inexperienced site investigators used the RSIT and worked independently from each other and obtained similar solutions. Their solutions agree well with the solutions found by the team of site investigation experts who used traditional checklists. Surprisingly, some of the inexperienced investigators pointed out relevant improvements that were overlooked by the experts. On average, the time needed for a RSIT-supported site investigation was shorter than for the checklist-supported investigation. In the second phase, the feedback from the Study advisory Committee members involved in this research has helped identify additional needs for the tool improvements and led to a new version of RSIT - RSIT2.

The original prototype of RSIT was evaluated to verify its usefulness. The evaluation compared findings obtained at the same locations with two methods:

1) The standard method of formal road safety investigations conducted by an experienced team, and

2) The proposed prototype of RSIT method applied to safety investigations conducted by several groups of "non-experts" (transportation professionals who do not participate in high-crash site investigations)

Two two-way stop-controlled intersections were included in the evaluation:

Stadium Avenue and Grant Street, West Lafayette, Tippecanoe County, Indiana

Sixth Street and Salem Street, Lafayette, Tippecanoe County, Indiana

On June 28, 2006, a road safety review was conducted at the two intersections. The follow-up The follow-up evaluation session was held on August 22, 2006. The roadway and weather conditions weather conditions on that day were similar to the conditions at the time when the formal investigations took place. Seventeen participants included county engineers, city engineers, and 
engineers, and transportation planning specialists. The participants were divided into six teams of teams of two or three persons and then introduced to Tablets PC and to the RSIT method. Before the Before the investigation was conducted the general description of the intersections, the statistics and statistics and details of crashes including collisions diagrams were presented. To assure

independences of the results the participants were requested not to communicate with other teams. other teams. Members of the same team were encouraged to work together. After the introductory introductory part, the investigation of the two sites was conducted in two phases. In the first phase, first phase, three teams were assigned to the Stadium Avenue and Grant Street intersection and intersection and three teams to the Sixth Street and Salem Street intersection. In the second phase, second phase, the teams switched the intersections. This procedure has yielded investigation results investigation results for each of the two sites from six evaluation teams. The results are presented in presented in Table 4-1 and Table 4-2.

Table 4-2

To interpret the results let us focus on one isolated example. During Road Safety Investigation on the intersection: Stadium Avenue and Grand Street, West Lafayette, Tippecanoe County, Indiana experts team found the following safety issue: "Inadequate intersection warning." The proposed countermeasures include: "Install intersection warning signs (Adding a yellow flasher to the warning sign, street name)." Non-Expert teams that investigated the same location found the same safety issue and proposed the following countermeasure: "Install warning signs in advance of intersections (5 teams)." This countermeasure was proposed by five teams out of six. In some cases i.e. Truck movement non-experts did not identify this specific problem as a safety concern compare with expert. The reason for that was non truck movement present during investigation done by non-experts.

\subsection{Deficiencies-based Evaluation}

The evaluated method of proposed prototype of RSIT generates two types of outcomes: roadway deficiencies and safety countermeasures. Out of these two outcomes, the road deficiencies are much more difficult to identify. There are countless ways the site geometry, traffic control, drivers, and combination of the three can contribute to a high 
risk of crashes at investigated sites. On the other hand, the safety countermeasures are relatively easy to point out once the roadway deficiencies are known. 
Table 4-1 Prototype evaluation: Identified site deficiencies and proposed safety countermeasures at Stadium Avenue and Grand Street, West Lafayette, Tippecanoe County, Indiana

\begin{tabular}{|c|c|}
\hline Formal Road Safety Audit & Prototype of Proposed Method (RSIT) \\
\hline $\begin{array}{l}\text { Inadequate intersection visibility } \\
\text { - Trim trees and shrubs } \\
\text { - Relocate stop bars closer to the intersection. }\end{array}$ & $\begin{array}{l}\text { Inadequate intersection visibility } \\
\text { - Remove the object obstructing the sight triangle / } \\
\text { Trim vegetation ( } 5 \text { teams })^{1} \\
\text { - Relocate stop bars closer to the intersection ( } 3 \\
\text { teams) }\end{array}$ \\
\hline $\begin{array}{l}\text { Inadequate intersection warning } \\
\text { - Install intersection warning signs (Adding a } \\
\text { yellow flasher to the warning sign, street name) }\end{array}$ & $\begin{array}{l}\text { Inadequate intersection warning } \\
\text { - Install warning signs in advance of intersections } \\
\text { (5 teams) }\end{array}$ \\
\hline $\begin{array}{l}\text { Pedestrians collision } \\
\text { - Install handicap ramps } \\
\text { - Mark crosswalks }\end{array}$ & $\begin{array}{l}\text { Pedestrians collision } \\
\text { - Improve signage and/or marking of the crosswalk } \\
\text { (4 teams) } \\
\text { - Install lighting ( } 3 \text { teams) }\end{array}$ \\
\hline $\begin{array}{l}\text { Speed limit violation } \\
\text { - Increase speed enforcement. Add changeable } \\
\text { message signs to the speed limit sign that } \\
\text { indicates drivers speed. }\end{array}$ & $\begin{array}{l}\text { Speed limit violation } \\
\text { - Provide targeted speed enforcement ( } 4 \text { teams) } \\
\text { - Provide traffic calming on intersection approaches } \\
\text { through a combination of geometric and traffic } \\
\text { control devices ( } 3 \text { teams) }\end{array}$ \\
\hline $\begin{array}{l}\text { Possible inadequate winter pavement } \\
\text { condition } \\
\text { - Include the intersections as "first response" } \\
\text { areas for winter highway maintenance. }\end{array}$ & $\begin{array}{l}\text { Possible inadequate winter pavement condition } \\
\text { - Improve winter maintenance preparedness } \\
\text { (shorter response time) ( } 3 \text { teams) } \\
\text { - Use salt to prevent snow or ice from forming or } \\
\text { from sticking to the road surface ( } 3 \text { teams) }\end{array}$ \\
\hline $\begin{array}{l}\text { Parking enforcement } \\
\text { - Extend the no parking zones. Post and } \\
\text { enforce the no parking area as a "tow away" } \\
\text { zone }\end{array}$ & Not identified \\
\hline Not identified & $\begin{array}{l}\text { Bike collision } \\
\text { - Install lighting ( } 2 \text { teams) } \\
\text { - Widen the outside through lanes or add bike lanes. } \\
\text { Replace poorly designed drain grates with bicycle- } \\
\text { safe types ( } 2 \text { teams) }\end{array}$ \\
\hline Not identified & $\begin{array}{l}\text { Nighttime collision } \\
\text { - Install lighting (4 teams) } \\
\text { - Improve channelization/delineation (1 team) }\end{array}$ \\
\hline
\end{tabular}


Table 4-2 Prototype evaluation: Identified site deficiencies and proposed safety countermeasures at $6^{\text {th }}$ Street and Salem Street, Lafayette, Tippecanoe County,

Indiana

\begin{tabular}{|c|c|}
\hline Formal Road Safety Audit & Prototype of Proposed Method (RSIT) \\
\hline $\begin{array}{l}\text { Inadequate intersection sight triangle } \\
\text { - Relocate stop bars. Check turning radii }\end{array}$ & $\begin{array}{l}\text { Inadequate intersection sight triangle } \\
\text { - } \quad \text { Relocate stop bar ( } 3 \text { teams })^{1} \\
\text { - Install corner mirrors ( } 2 \text { teams) } \\
\text { - Install traffic signals ( } 1 \text { team) } \\
\text { - } \text { Remove the object obstructing the sight of the } \\
\text { stop sign ( } 3 \text { teams) } \\
\text { - } \quad \text { Double Post Stop Signs ( } 2 \text { teams) }\end{array}$ \\
\hline $\begin{array}{l}\text { Excessive speed } \\
\text { - Perform a speed study on Salem Street, } \\
\text { add changeable speed message signs } \\
\text { indicating driver speed }\end{array}$ & $\begin{array}{l}\text { Excessive speed } \\
\text { - Provide targeted speed enforcement ( } 5 \text { teams) } \\
\text { - Post dynamic message sign to display the speed } \\
\text { of approaching vehicles ( } 2 \text { teams) } \\
\text { - Conduct speed Study ( } 2 \text { teams) } \\
\text { - Provide traffic calming on intersection approaches } \\
\text { through a combination of geometric and traffic } \\
\text { control devices ( } 3 \text { teams) }\end{array}$ \\
\hline $\begin{array}{l}\text { Inadequate pavement marking/ signing } \\
\text { - Provide pavement marking (guidance } \\
\text { for drivers, arrows) }\end{array}$ & $\begin{array}{l}\text { Inadequate pavement marking/ signing } \\
\text { - Provide adequate lanes signing or marking ( } 5 \\
\text { teams) } \\
\text { - Improve pavement marking (arrows on pavement) } \\
\text { (4 teams) } \\
\text { - Provide adequate turning markers or pavement } \\
\text { markings ( } 4 \text { teams) } \\
\text { - Install left /right turn bays if turning volume is } \\
\text { considerable ( } 2 \text { teams) }\end{array}$ \\
\hline $\begin{array}{l}\text { Inadequate warning of the intersection } \\
\text { - Add intersection warning signs (added } \\
\text { street name) }\end{array}$ & $\begin{array}{l}\text { Inadequate warning of the intersection } \\
\text { - Install warning signs in advance of intersections } \\
\text { (5 teams) } \\
\text { - Install larger regulatory and warning signs ( } 2 \\
\text { teams) }\end{array}$ \\
\hline $\begin{array}{l}\text { Parking zone } \\
\text { - Relocate double yellow lane line to the } \\
\text { east to accommodate left turning vehicles. } \\
\text { Eliminate parking }\end{array}$ & $\begin{array}{l}\text { Parking zone } \\
\text { - Eliminate parking ( } 2 \text { teams) }\end{array}$ \\
\hline $\begin{array}{l}\text { Inadequate location of the stop sign } \\
\text { - Remove a portion of sidewalk in front } \\
\text { of the tavern in order to place the stop sign }\end{array}$ & $\begin{array}{l}\text { Inadequate location of the stop sign } \\
\text { - Double Post Stop Signs ( } 2 \text { teams) }\end{array}$ \\
\hline
\end{tabular}




\begin{tabular}{|l|l|}
\hline \multicolumn{1}{|c|}{ Formal Road Safety Audit } & \multicolumn{1}{c|}{ Prototype of Proposed Method (RSIT) } \\
\hline closer to the intersection & Not identified \\
\hline $\begin{array}{l}\text { Inadequate space for left turn vehicles } \\
\text { - Reconstruct the radius at the southwest } \\
\text { corner to provide adequate area for left } \\
\text { turns }\end{array}$ & Not identified \\
\hline $\begin{array}{l}\text { Truck movement } \\
\text { - Pass an ordinance and post "No thru } \\
\text { trucks" }\end{array}$ & $\begin{array}{l}\text { Nighttime collision } \\
\text { - Improve street lights (4 teams). Remove } \\
\text { distracting commercial (2 teams) lights. Upgrade } \\
\text { signing (3 teams) }\end{array}$ \\
\hline $\begin{array}{l}\text { Not identified } \\
\text { Pedestrians block left turn } \\
\text { - Increase the crosswalk setback (3 teams) }\end{array}$ \\
\hline Not identified & $\begin{array}{l}\text { Adverse-weather collisions } \\
\text { - Install static or variable message signs displaying } \\
\text { weather information (2 teams) } \\
\text { - Use sand to increase pavement friction. Use salt } \\
\text { to prevent snow or ice from forming or from sticking } \\
\text { to the road surface. Groove pavement surface (2 } \\
\text { teams) }\end{array}$ \\
\hline
\end{tabular}

The difference in complexity between the two identification tasks is reflected in the knowledge base. The branches of the knowledge tree represent alternative local conditions and the site investigator spends most of the time to identify these conditions. Once the set of local conditions are known, a short list of relevant countermeasures is proposed by the tool. In some cases, the same safety countermeasures apply to different roadway conditions. It is obvious, that the proposed method should be evaluated by checking the identified roadway deficiencies rather than checking the identified safety countermeasures.

The evaluation criteria of the method included consistency and validity of the identified roadway deficiencies and efficiency of the method. The consistency was determined by comparing the results obtained by the non-experts while the validity was checked by comparing the non-experts results with the results obtained in formal safety 
audits. The method efficiency was measured with the time needed to complete a site investigation.

Consistency of the results is the first indication that the method may be valid. Obvious contradictions among results obtained by different investigative teams indicate that some of the solutions produced with the method must be invalid (there cannot be several contradicting answers to the same question). Different but not obviously contradicting results indicate that the method produces incomplete solutions (different teams arrive at different parts of the same solution). Consistency of the investigation results was measured with the similarity of site deficiencies identified by the three evaluation teams and is presented in Table 4-3.

The number of deficiencies identified by any of the non-expert team out of many possible is limited ( 7 at the first intersection and 9 at the second one). All of the identified deficiencies were pointed out by at least two teams. Only four out of 16 were pointed out by two teams while nine were identified by four or five teams. Although the consistency could be better, the one reached at the two studied intersections was satisfactory given the lack of experience of the investigative teams. Some team members using the Tablet PC for the first time reported difficulties. No obvious contradictions were found in the results obtained by the six teams at the two investigated sites.

Validity of the results is more difficult to evaluate than consistency because the correct solutions (ground truth) are not known. The method available and used by us was comparing the results obtained by experts with the results obtained by non-experts. The notion was that the expert results are of better quality than the ones obtained by inexperienced investigators. The following discussion is based on the results shown in Table 4-3. For the first intersection, the experts pointed out six deficiencies, when nonexperts indicated five of them. Missing the parking issue by the non-experts might be caused by different parking situation during their site visit compared to the conditions during the format safety audit (daytime conditions, weather, and geometry conditions were identical or similar). Furthermore, $70 \%$ of the non-experts teams pointed out the same findings as the experts (in average four out of six teams). 
Table 4-3 Roadway Deficiencies Identified at the Two Investigated Sites

\begin{tabular}{|c|c|c|c|c|c|c|c|c|c|c|c|}
\hline \multicolumn{12}{|c|}{ Stadium Ave. and Grand Street, West Lafayette, Tippecanoe County, Indiana } \\
\hline & $\mathbf{A}$ & \multicolumn{2}{|c|}{\begin{tabular}{|c|}
$\mathbf{B}$ \\
\end{tabular}} & $\mathbf{C}$ & D & \multicolumn{2}{|c|}{ E } & $\mathbf{F}$ & G & \multirow{2}{*}{\multicolumn{2}{|c|}{$\begin{array}{l}\mathbf{H} \\
\mathrm{X}\end{array}$}} \\
\hline Team 1 & $\mathrm{X}$ & \multicolumn{2}{|r|}{$\mathrm{X}$} & $\mathrm{X}$ & $\mathrm{X}$ & & & & & & \\
\hline Team 2 & & & $\mathrm{X}$ & $\mathrm{X}$ & & \multicolumn{2}{|r|}{$\mathrm{X}$} & & \multicolumn{2}{|l|}{$\mathrm{X}$} & \\
\hline Team 3 & $\mathrm{X}$ & \multicolumn{2}{|r|}{$\mathrm{X}$} & & $\mathrm{X}$ & \multirow{2}{*}{\multicolumn{3}{|c|}{$X$}} & & & \\
\hline Team 4 & $\mathrm{X}$ & & & & $\mathrm{X}$ & & & & \multirow[b]{2}{*}{$\mathrm{X}$} & & \\
\hline Team 5 & $\mathrm{X}$ & \multicolumn{2}{|r|}{$\mathrm{X}$} & $\mathrm{X}$ & & & & & & \multirow{2}{*}{\multicolumn{2}{|c|}{$\begin{array}{l}X \\
X\end{array}$}} \\
\hline Team 6 & $\mathrm{X}$ & \multicolumn{2}{|r|}{$X$} & $\mathrm{X}$ & $\mathrm{X}$ & \multicolumn{2}{|r|}{$\mathrm{X}$} & & & & \\
\hline Experts & $\mathrm{X}$ & \multicolumn{2}{|r|}{$\mathrm{X}$} & $\mathrm{X}$ & $\mathrm{X}$ & \multicolumn{2}{|r|}{$\mathrm{X}$} & $\mathrm{X}$ & & \multicolumn{2}{|r|}{$\underline{\mathbf{x}}$} \\
\hline \multicolumn{12}{|c|}{$\begin{array}{l}\text { X - Identified deficiencies } \\
\underline{\mathbf{X}} \text { - Deficiency added by the experts after reviewing solutions proposed by the } \\
\text { RSIT } \\
\text { Deficiencies: A - Inadequate intersection visibility; B - Inadequate intersection } \\
\text { warning; C - Pedestrians collision; D - Speed limit violation; } \mathbf{E} \text { - Possible } \\
\text { inadequate winter pavement condition; F - Parking enforcement; G - Bike collision; } \\
\text { H - Night time collision. }\end{array}$} \\
\hline \multicolumn{12}{|c|}{$6^{\text {th }}$ Street and Salem Street, Lafayette, Tippecanoe County, Indiana } \\
\hline & A & B & $\mathbf{C}$ & D & $\mathbf{E}$ & $\mathbf{F}$ & G & H & I & $\mathbf{J}$ & K \\
\hline Team 1 & $\mathrm{X}$ & $\mathrm{X}$ & & $\mathrm{X}$ & $\mathrm{X}$ & & & & $\mathrm{X}$ & & $\mathrm{X}$ \\
\hline Team 2 & & $\mathrm{X}$ & $\mathrm{X}$ & $\mathrm{X}$ & & $\mathrm{X}$ & & & & $\mathrm{X}$ & \\
\hline Team 3 & $\mathrm{X}$ & & $\mathrm{X}$ & $\mathrm{X}$ & $\mathrm{X}$ & $\mathrm{X}$ & & & $\mathrm{X}$ & $\mathrm{X}$ & \\
\hline Team 4 & & $\mathrm{X}$ & $\mathrm{X}$ & $\mathrm{X}$ & & & & & & & \\
\hline Team 5 & & $\mathrm{X}$ & $\mathrm{X}$ & $\mathrm{X}$ & & & & & $\mathrm{X}$ & $\mathrm{X}$ & $\mathrm{X}$ \\
\hline Team 6 & $X$ & $\mathrm{X}$ & $X$ & & & & & & $X$ & & \\
\hline Experts & $\mathrm{X}$ & $\mathrm{X}$ & $\mathrm{X}$ & $X$ & $X$ & $X$ & $X$ & $X$ & $\underline{\mathbf{X}}$ & & \\
\hline \multicolumn{12}{|c|}{$\begin{array}{l}\text { X-Identified deficiencies } \\
\underline{\mathbf{X}} \text { - Deficiency added by the experts after reviewing solutions proposed by the } \\
\text { RSIT } \\
\text { Deficiencies: A - Inadequate intersection sight triangle; } \mathbf{B} \text { - Excessive speed; } \\
\mathbf{C} \text { - Inadequate pavement marking/ signing; D - Inadequate warning of the } \\
\text { intersection; } \mathbf{E} \text { - Parking zone; } \mathbf{F} \text { - Inadequate location of the stop sign; } \\
\text { G - Inadequate space for left turn vehicles; } \mathbf{H} \text { - Truck movement; I - Night time } \\
\text { collision; } \mathbf{J} \text { - Pedestrians block left turn; } \mathbf{K} \text { - Adverse-weather collisions }\end{array}$} \\
\hline
\end{tabular}

Additionally the non-experts (four out of six non-experts teams) using the prototype of RSIT pointed out on the both investigated intersections, nighttime collisions issue which was not considered by the experts. The experts, after reviewing the prototype 
findings, updated they final report. For the second intersection, nearly four out of six nonexperts teams indicate the same deficiencies as the experts. Two expert findings where not present in the non-experts results. One of them: "truck movement" could not be identified by the non-experts because neither past truck accidents nor considerable truck traffic during their visit took place. The results obtained by experts and non-experts are strikingly similar which is an important positive evaluation outcome.

The time needed for a RSIT-supported prototype site investigation was shorter than for the checklist-supported investigation. Further reduction in a RSIT-based prototype site investigating is expected after the investigative team equipped with the prototype of RSIT was two or three members while the typical safety audit involves three to four members.

The most spectacular result of the evaluation was helping inexperienced investigators properly determine safety deficiencies as well as preventing overlooking some important safety issues. Experts investigated both intersections overlooked one of the safety concern: nighttime collisions (almost 30\% related crashes). The non-experts using the prototype of RSIT indicate nighttime collisions as an important safety issue (four teams out of six).

\subsection{Countermeasures-based Evaluation}

The presented above evaluation results have confirmed that site deficiencies were satisfactorily identified. These positive results confirm the concept of the method and its implementation. The next step in the presented evaluation was to check the identified countermeasures. This phase is of lesser weight because any needed modifications of the countermeasures in the tool can be easily implemented and they do not affect the knowledge. Nevertheless, the consistency and validity of the safety countermeasures have been evaluated based on the results presented in Table 4-1 and Table 4-2. The results exhibit similar consistency and a sign of validity as the identified site deficiencies. Some discrepancies could also be found. An example is presented in Figure 4-1. Group 1 proposed installing a corner mirror which is not proposed by Group 4 . 


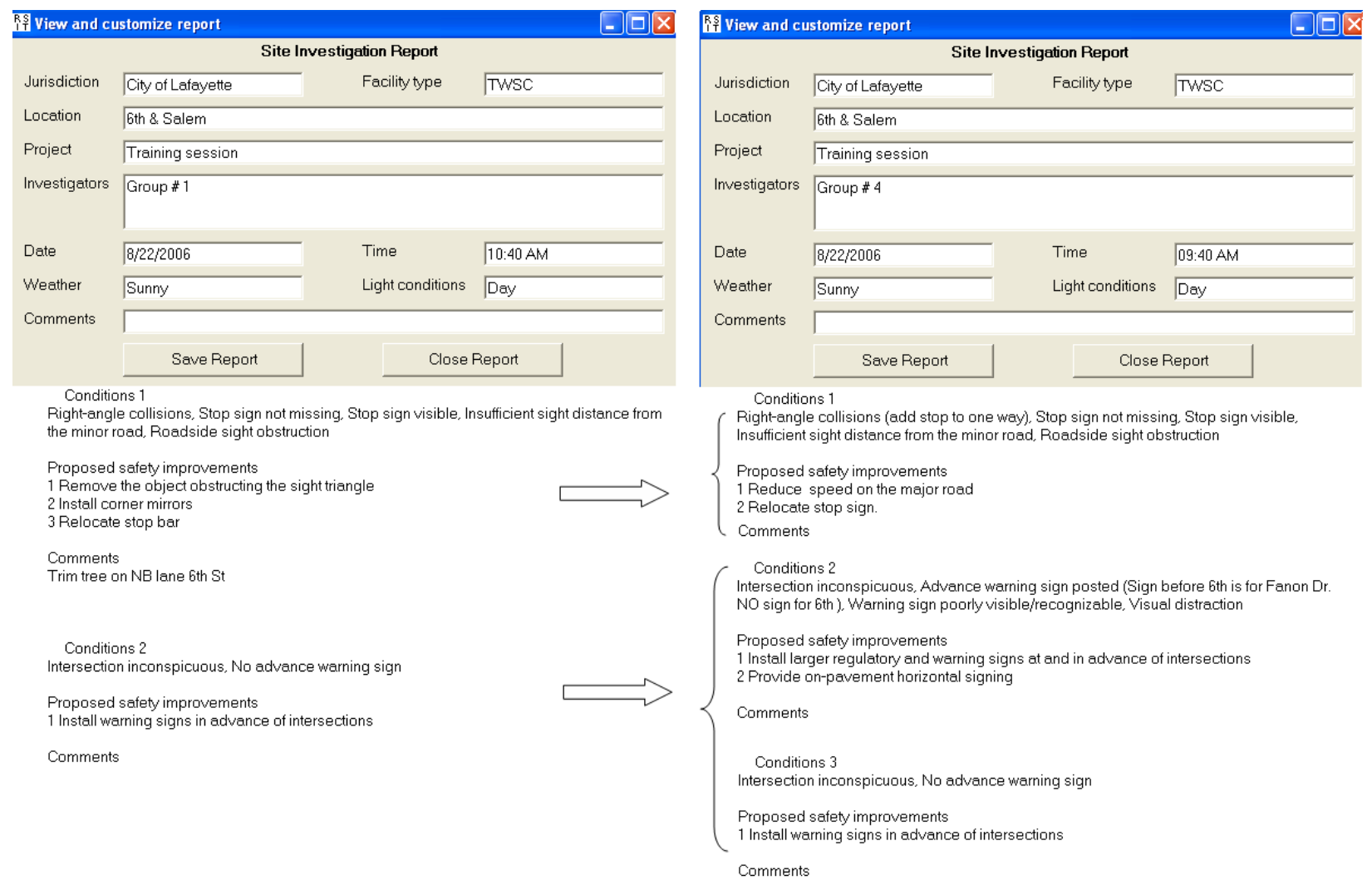

Figure 4-1 The prototype of RSIT - report

\subsection{Summary}

The proposed prototype with limited knowledge base guides an investigation team through a sequence of checks and determines the probable roadway factors contributing to crash occurrence. Even though the developed knowledge is complex, it has an easily understandable structure represented by a user-friendly interface. The knowledge structure and functionality of the GUI are easy to understand even for non-experts. The developed knowledge base provides a more detailed explanation for each individual roadway deficiency compared to the findings from the road safety report, which confirms the previous statement that even experienced investigators can overlook important factors. By using developed knowledge, the time spent on the investigation process should decrease. The implemented knowledge can decrease the size of the team, which 
usually should contain specialists from the different areas of geometry, safety, and human factors. In addition, by using the knowledge base in a user-friendly graphic interface, the automatically generated final report presents in an easy and understandable way the searching process including users comments and the proposed final solutions. Furthermore, the developed knowledge base can be used as a training tool to represent the logical connection between the time of the crash, the driver, and the roadway elements that contributed to roadway safety.

Although, the developed prototype of RSIT brought significant improvement over existing method, the evaluation part emphasized the need for modifications.

1) The knowledge developed for two-way stop-controlled intersection need to be revised and updated based on feedback from the evaluation part.

2) The investigation process should relate to the road components to allow tracking which parts of the road/intersection has been investigated and to allow documenting the findings in relation to these parts. For example, some safety checks apply to an entire intersection while some other to individual intersection approaches or exits.

3) The current knowledge must be expanded to include all-way stop-controlled intersection, road segment, rail road crossing, and signalized intersection.

4) The software should allow easier editing of the existing knowledge base by releasing the user from deciding how to "trim" or "expand" the knowledge base tree.

5) Uncertainty of some observations and conclusions together with their importance for a safety enhancement should be incorporated into the post-investigation process to help rank the identified multiple safety countermeasures for implementation. 


\section{RSIT2 CONCEPT}

The presented concept of RSIT2 is based on a novel idea of an optimal investigation based on an optimal checklist with its dynamic updating in the course of site investigation. This approach makes the investigation process the most effective (the least expensive). As the previous RSIT, RSIT2 uses rules as building blocks of the knowledge base. The knowledge updating can be executed by removing, adding, or modifying current rules.

First, we will introduce the concept of a rule-based checklist with estimating the investigation costs and some rule-of-thumb optimization strategies. Then, the knowledge base structure is explained, and finally, the RSIT2 software described in general terms.

\subsection{Checklist Optimization Problem}

Checklists are used to diagnose problems or/and to select relevant actions. In a general case, a checklist is a collection of if-then rules typically organized in two columns: the first column includes conditions and the second column includes relevant conclusions. Table 5-1 demonstrates this structure. Checklists are widely used because they are convenient, intuitive, and provide straightforward inventory of relevant knowledge. This inventory is easy to update by adding or removing rules.

Before we introduce an investigation cost function, let us propose a checklist structured differently than the one shown in Table 5-1. The modified checklist is easier to optimize than the conventional one.

Table 5-1 Conventional checklist

\begin{tabular}{|l|l|}
\hline IF & THEN \\
\hline$X_{1,1} \ldots$ and $X_{1, N 1}$ & $C_{1}$ \\
\hline$X_{2,1} \ldots$ and $X_{2, N 2}$ & $C_{2}$ \\
\hline$\ldots \ldots \ldots \ldots \ldots \ldots \ldots \ldots$ & $\cdots \cdots$ \\
\hline$X_{M, 1} \ldots$ and $X_{M, N M}$ & $C_{\mathrm{M}}$ \\
\hline
\end{tabular}


A conventional checklist shown in Table 5-1 is equivalent to the following set of rules:

\section{Equation 5-1}

Some of the atoms $X_{i . j}$ are the same. After removing the repetitions, renumbering the atoms from $1 \ldots N$, and reordering them in some way, the following sequence is obtained: $X_{n(1)}, X_{n(2)}, \ldots X_{n(N)}$, where $X_{n(i)}$ stands for an atom originally indexed between 1 and $N$, and being at position $i$ on the new checklist. These checks are performed in the order indicated by $i$. After the investigation is completed, the results include the checks with their logical values (true, false). These results and the set of rules (Equation 5-1) determine which conclusions $C_{j}$ are true (countermeasures to be selected). The proposed checklist structure including single conditions $X_{n(1)}, X_{n(2)}, \ldots X_{n(N)}$ is currently used by road safety engineers. The essential difference is the lack of formal rules that are the starting point for forming the checklist. Consideration of these rules in connection with the checklist allow three important operations lacking in the current road safety investigation: (1) Optimization of the checks order prior the investigation, (2) Updating the checklist in the course of the investigation, (3) Derivation of the relevant countermeasures after the investigation.

Let us now propose a method of evaluating the cost of an investigation. Let $X_{n(1)}$, $X_{n(2)}, \ldots X_{n(N)}$ be a list of checks. If performing check $n(i)$ costs and moving from check $n$ to check $m$ costs, then an investigation that follows a complete list of checks $X_{n(1)}, X_{n(2)}, \ldots X_{n(N)}$ costs

\section{Equation 5-2}


The investigation cost in Equation 5-2 assumes that all the checks are performed. Using the rules linking checks with countermeasures allows elimination of some checks during the investigation. Let the investigation be at line of the checklist. At this point of the investigation, a number of if-then rules are refuted because they include at least one of the false conditions among conditions checked. Other rules are still active. The check can be skipped if it does not support any of the remaining active rules. Let us denote the likelihood of skipping check $X_{n(i)}$ as $P_{n(i)}$. Now, the expected investigation cost is the sum of the expected cost of checks and the expected cost of transfers between checks:

\section{Equation 5-3}

In some cases, the transfer costs can be neglected and in some other cases the check costs can be neglected. Investigating a geographically large area that involves just visual inspection of spots takes a considerable time and resources to travel between the points while the cost of checks is small. This case is a traveling salesman problem and is well researched. In the case of investigating a geographically limited object such as a road intersection with variety of checks ranging from visual inspection to elaborated measurements, both the cost components are present and cannot be neglected.

There are three ways of improving a checklist and an efficiency of their use.

1) Checks that provide a great potential of eliminating other checks, particularly those expensive, should be placed close to the top of the list.

2) Some checks may be more expensive than other. These more expensive checks should appear close to the bottom of the list to give them a bigger chance to be refuted.

3) The pairs of checks with inexpensive transfer should be consecutive while the pairs with expensive transfer should be separated from each other. 


\subsection{RSIT2 Knowledge Representation}

For the purpose of the Road Safety Investigation Tool 2 (RSIT2), the knowledge representation has been changed. Instead of tree-like structure (Figure 5-1) implemented as embedded folders (Figure 5-2), the new knowledge takes a form of a collection of rules. To represent the new knowledge structure based on rules, two entities composing a rule are adopted: conditions $\left(X_{i}\right)$ and countermeasures $\left(C_{i}\right)$. Each rule can contains only one countermeasure and one or several conditions. For the development and implementation purpose, the rule is written in such a way that the countermeasure opens the rule and is followed by conditions as shown in Figure 5-3. This modification is syntactic only and it does not change the logical meaning of the rule.

The new structure adopted for RSIT 2 has a number of significant advantages.

a) The explicit use of rules tremendously helps the user update or modify the knowledge base by adding or removing rules that are logical and self explaining. A dedicated knowledge base editor (KB Editor) facilitates this important function.

b) Developing new knowledge bases conducted in this research is also easier because it does not require converting the rules into a tree - a non-trivial task for complex cases. The KB Editor can be used to enter a brand new knowledge base.

c) The rules can be easily generalized by adding variables that represent road components such as approaches, exits, etc. This modification allows developing a generic knowledge base that can be instantiated to a studied case by replacing the variables with the names of approaches, roads, exits, etc. This modification addresses the requirement that the tool better guides through the investigation by relating checks to specific road components and documents countermeasures more specifically. For example, the old condition: "Right-angle collisions", reads in the new version as: "Right-angle collisions between \#A1 and \#A2 vehicles". With additional conditions defining the two variables \#A1 and \#A2: "\#A1 major approach", “\#A2 minor approach”. 


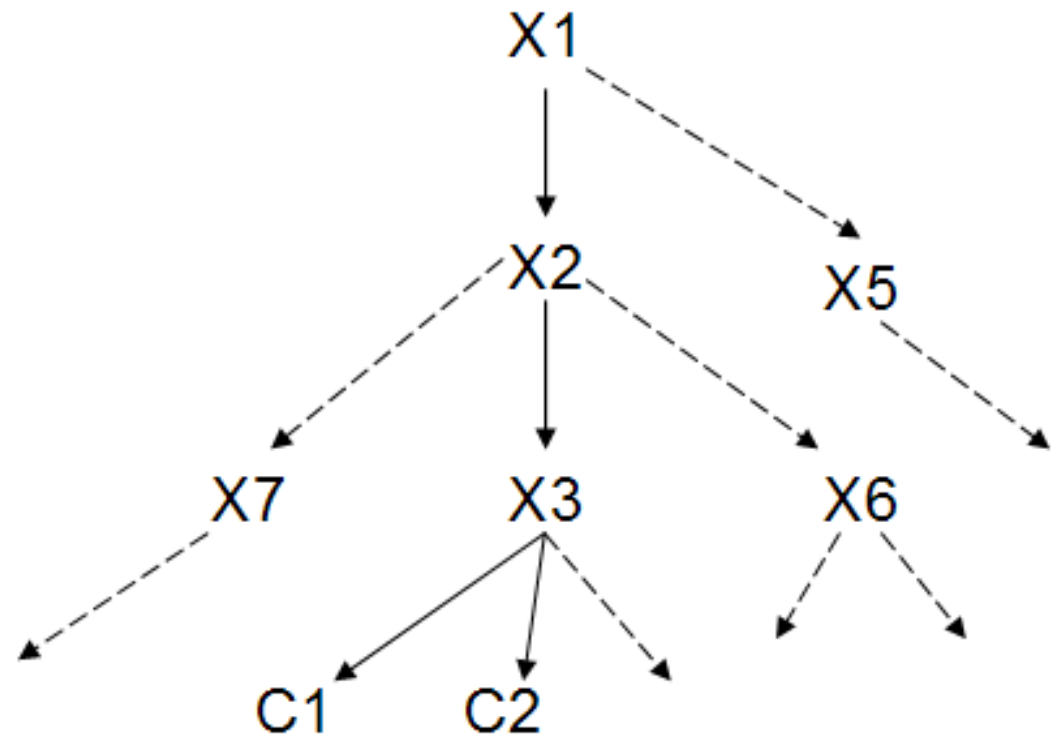

\section{Figure 5-1 RSIT Prototype Knowledge Structure}

TWSC Intersection

+ Driveways collisions

+1 Night time collisions

+9 Off-road collisions

- $\mathrm{V}$ Right-angle collisions

- Stop sign not missing

-1 Stop sign visible

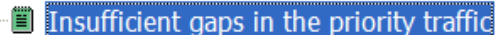

罯 Inadequate driver awareness on stop approach

+ Insufficient sight distance from the minor road

粗 Inadequate pavement marking

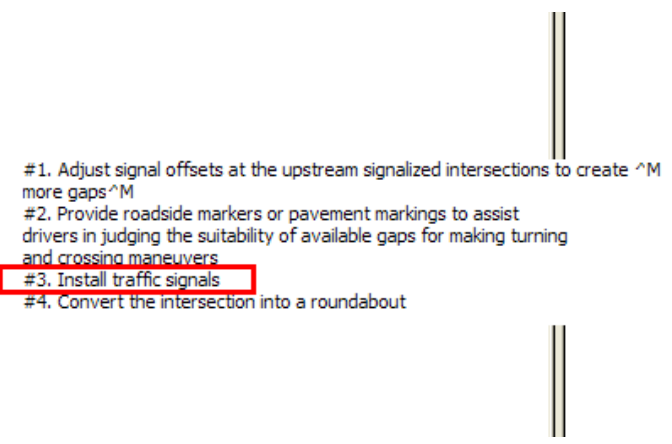

Figure 5-2 The Prototype of RSIT Knowledge Base

Install traffic signals at intersection TWSC Right-angle collisions between \#A1 and \#A2 vehicles \#A1 major approach \#A2 minor approach

- Stop sign visible on \#A2 approach Insufficient gaps between vehicle on \#A1 approach

Figure 5-3 RSIT 2 Rule Syntax 
d) The checklist composed of single checks-conditions can be optimized by ordering these checks in such a way that the expected cost of the investigation is the lowest. This cost includes the cost of checks and the cost of moving from one check to the other. The beforehand checklist optimization is possible thanks to a joint consideration of the checklist and the corresponding rules. This optimization task is equivalent to optimizing a tree structure - a task that does not have to be performed by the user.

e) The main sources of cost savings is skipping checks that do not have to be performed any longer because they do not affect the investigation results due to the outcome of the checks performed earlier. This real-time checklist updating is another unique feature of the developed method.

The two-way stop-controlled intersection knowledge base developed for the prototype of RSIT was updated. The new updated knowledge base includes over 200 rules. In addition, the knowledge bases for Road Segment, Signalized Intersection, Rail Road Crossing, and All-way Stop-Controlled intersection has been developed. They are presented in Appendix D.

Moreover, evaluation of identified countermeasures in order to help the user narrow the selection to a practical extent is introduced. The countermeasure evaluation is based on the expected safety reduction and cost level of the countermeasures.

To bring additional features including allow optimization of the checklist and ranking the identified countermeasures based on their expected safety effectiveness and cost, additional attributes checks and countermeasures are needed. Checks have associated the following attributes:

a) Probability of passing (being true)

b) Cost of the check

c) Off-Site (check performed before the investigation)

d) References (location where a file with additional explanatory material can be found)

The "Probability" attribute describes the assessed likelihood that a specific condition occurs (check passes). The "Cost" attribute tells how expensive the check is. Three cost levels 1-3 are used with level 3 being most expensive. The "Off-site" attribute 
defines that a check is performed prior the site investigation. The "References" provides a link to a relevant pdf document relevant to a given check.

Table 5-2 Example checks with corresponding attributes

\begin{tabular}{|l|c|c|c|c|}
\hline & Probability & Cost & $\begin{array}{c}\text { Off } \\
\text { Site }\end{array}$ & $\begin{array}{c}\text { Refere } \\
\text { nce }\end{array}$ \\
\hline Inadequate shoulders width on \#R1 road & 30 & 3 & $\mathrm{n}$ & 82302 \\
\hline Inadequate signing for multi-lane approach & 20 & 2 & $\mathrm{n}$ & $\mathrm{N}$ \\
\hline Too short acceleration lane for vehicles merging into \#E1 exit & 15 & 2 & $\mathrm{n}$ & $\mathrm{N}$ \\
\hline Inadequate visibility of pedestrians & 15 & 2 & $\mathrm{n}$ & $\mathrm{N}$ \\
\hline $\begin{array}{l}\text { Inadequate sight distance toward vehicles approaching } \\
\text { intersection on \#A1 approach }\end{array}$ & 40 & 2 & $\mathrm{n}$ & $422 \mathrm{a}$ \\
\hline Inadequate sight distance on horizontal curve & 20 & 2 & $\mathrm{n}$ & 4340 \\
\hline
\end{tabular}

Table 5-2 gives example checks with corresponding attributes in the format used only in the development phase. Check "Inadequate sight distance on horizontal curve" has the following parameters: a) Probability equal to 20 [\%], explains that this condition occurs in $20 \%$ cases; b) Cost level 2 indicates that this check is moderately expensive,; c) Offsite " $n$ " indicates that this check is NOT performed before the investigation but at the site, d) Reference “4340” provides a link to the Indiana Design Manual except (a pdf file) where relevant information can be found.

Also countermeasures have their own attributes needed for their ranking in the post-investigation phase:
a) $\mathrm{CRF}$
b) Cost
c) Road

The "CRF" (Crash Reduction Factor) describes the reduction in crashes possibly caused by a specific countermeasure. An extensive overview of available CRFs is presented in the last section of this chapter. The "Cost" attribute indicates the cost level of the countermeasure on the 1-5 scale with 5 indicating the highest cost level. The parameter "Road" tells to which types of roads this countermeasure can be applied. 
Table 5-3 Countermeasure Table

\begin{tabular}{|l|c|c|l|}
\hline & CRF & Cost & Road \\
\hline Eliminate shoulder drop-off on \#E1 exit & 28 & 3 & TWSC,AWSC \\
\hline Eliminate shoulder drop-off on \#R1 road & 28 & 3 & RS \\
\hline Employ emergency vehicle preemption & 70 & 3 & SIGN \\
\hline Enforce STOP sign compliance on \#A1 approach & 70 & 2 & TWSC,AWSC \\
\hline Enforce STOP sign compliance on \#A2 approach & 70 & 2 & TWSC \\
\hline Enhance delineation of sharp curves & 16 & 3 & RS \\
\hline Groove pavement surface on \#A1 approach & 27 & 3 & TWSC,AWSC \\
\hline Groove pavement surface on \#R1 road & 27 & 3 & RS \\
\hline
\end{tabular}

Example countermeasures with their attributes are presented in Table 5-3. For example, countermeasure "Groove pavement surface on \#R1 approach" has the following parameters: a) $\mathrm{CRF}$ equal to $27 \%$ indicates the percent of crash reduction expected to occur at locations where this countermeasure is needed and applied; b) Cost equal to 3 indicates that the countermeasures is moderately expensive, c) Road equal to "RS" tells that this countermeasure is applicable to road segments. The full list of conditions and countermeasures including associate parameters are attached in Appendix B and Appendix C.

\subsection{Summary of RSIT2 Components}

1) The user has access to the knowledge base in the form of conditions and conclusions through the KB Editor. The KB Editor allows viewing the KB, adding new rules, removing existing ones, or modifying them. The user is able to use existing conditions and countermeasures in new rules. Before adding new rules, the user checks if the needed conditions or countermeasures are already in the KB. The user has an option to add additional items if they are not included yet. The KB Editor includes a search tool for easy checking what is available before adding new items. All the existing attributes associated with the items in the $\mathrm{KB}$ are also displayed and subject to change, deletion, or addition.

2) Checklist Interface displays the current checklist and allows entering the results of check execution. It accepts the results at different levels of uncertainty. It allows 
the user full control over what checks are skipped and what cannot be skipped at all. The checklist are arranged in a specific order that promotes skipping redundant checks and reducing the time and cost of the investigation. Two important functions of the Checklist Interface are optimization of the checks order and updating the checklist.

3) Countermeasures Selector calculates the expected effectiveness of the countermeasures based on the investigation results and a rough estimation of costs level. It is integrated with the Project Evaluator which allows packaging the selected countermeasures into projects and estimating the project effectiveness.

4) Report Generator creates a report including the investigation results, selected countermeasures with their effectiveness and one or more proposed safety projects with their effectiveness. 


\section{RSIT2 KNOWLEDGE BASE}

The knowledge base development process contains three parts. First, the new concept of representing the knowledge base is introduced and the knowledge base for existing twoway stop-controlled intersection is revised and updated based on the feedback from the prototype of RSIT evaluation part and comments from SAC committee members. Next, the knowledge base for additional roadway entities including: all-way stop-controlled intersection, rail road crossing, road segment, and signalized intersection is developed. Finally, the existing crash reduction factors are reviewed and a final set of values developed.

\subsection{Sources of Knowledge}

The prototype RSIT includes the knowledge base for two-way stop-controlled intersections (TWSC). This knowledge base has been converted to rules as discussed in the previous chapter. This chapter described development of a new knowledge base for: road segments (RS), signalized intersections (SIG), rail road crossings (RRC), and allway stop-controlled intersections (AWSC). First, an overview of the development method is presented. Then, a detail review of the individual part of the new knowledge base is provided.

As the TWSC knowledge base, the new knowledge base was developed by extracting useful information from multiple sources:

a) Personal experience gained in past site investigations

b) Existing reports of site investigations by others

c) Short courses and workshops

d) Guidelines, manuals, textbooks, and research reports

The NCHRP 500-series reports (NCHRP 500, 2004) were the primary source of information. These reports are a well-organized compendium of strategies for improving safety at various roadway entities. Specific safety improvement strategies are recommended for many safety objectives included in the source. Furthermore, the 
NCHRP 500-series reports specify the confidence towards each of the proposed strategies represented by three levels: proven, tried, and experimental.

Other important sources included: NCHRP Report 383 (NCHRP 383, 1996), and NCHRP 457 (NCHRP 457, 2006). The NCHRP Report 383 provides a complete reference to an intersection sight distance analysis. It includes sight distance fundamentals that link the roadway geometry with the minimum sight-distance requirements for safe passing through intersection. The Intersection Diagnostics Review Model (Kindler et al. 2006), which uses the findings included in the NCHRP Report 383, provides a knowledge base relevant to sight conditions at intersections and the minimum stopping and decision sight distances drivers need. In addition, the NCHRP Report 457 provides additional information related to evaluation of intersection improvements.

Another source of information was the Traffic Engineering Handbook of the Institute of Transportation Engineers (Pline, 1999). This source gives a broad overview of various roadway elements as well as human factors that can affect safety. Traffic regulation study and guidance for using signs, traffic controls, and traffic calming devices are presented. The Traffic Engineering Handbook focuses on road users and addresses driver, pedestrian, and bicycle characteristics. Particularly useful for a safety investigation in this handbook are the safety countermeasures provided for specific accident patterns (pp. 206 - 209).

Human factors were taken into account during the process of developing the new knowledge base (Olson et al. 1996). After all, driver errors contribute in more than $90 \%$ of crashes. Driving involves many different tasks: "tracking, decision-making, navigation, adherence to regulations and warnings, tending environmental and mechanical system within the cab, communication, (...) watching for various events that may occur inside and outside the vehicle" (Proctor et al. 1994). Proctor at al. (Proctor et al. 1994) and Senders (Senders et al. 1991) distinguished three stages of human information processing: perception, cognition, and action. Olson (Olson et al. 1996) indicated that the perception stage is most important because how a driver perceives information determines the decision and action. Safety engineering can improve safety by applying various treatments that decrease the potential for driver perception errors. Improving the visibility of road signs and increasing the visibility of dangerous location 
are among low-cost improvements that may help enhance driver perception. Because of that, most of the human factors elements used to build the new knowledge base are related to improving driver awareness and visibility. Human factors consideration is also present in analyzing drivers' behavior on the investigated road and by proposing engineering solutions and police enforcement to prevent or at least reduce undesirable behaviors.

The next section discusses development of each part of the new knowledge based is presented.

\subsection{Knowledge Base Development}

The new knowledge base is organized as a set of rules. Each rule links safety, road geometry, traffic, and road user behavior with the relevant countermeasure.

The knowledge based was developed for five different roadway entities: two-way stop-controlled intersections (TWSC), all-way stop-controlled intersections (AWSC), railroad crossing (RRC), road segments (RS), and signalized intersections (SIGN). More than 360 countermeasures, and 240 conditions were developed and more than 1000 rules assembled using these elements. The following text characterizes in general terms the knowledge base developed for the five types of roads (segments and intersections).

More than 280 rules were developed for the RS knowledge base. When identifying various road and traffic deficiencies, several types of collisions frequently observed on road segments were considered. The most frequent are off-road collisions; therefore, developed knowledge base for road segment collisions focuses mostly on offroad collisions (more than 50\% rules). Moreover, the data analysis shows that off-road collisions are mostly related to horizontal curves, vertical curves, and to fixed object close to the travel way. The developed knowledge base addresses two general types of risk and corresponding remedies:

(1) Conditions upon which a driver leaves the travel way and adequate safety improvements to reduce this risk, and

(2) Conditions upon which the occupants of the out-of-control vehicle are exposed to severe consequences and countermeasures to reduce the risk of a severe outcome. 
In addition to off-road collisions other collisions were addressed in the knowledge base developed for road segment and included pedestrian/bicycle collisions, weather-related collisions, night-time collisions, driveway collisions, speed-related and head-on collisions.

The knowledge base developed for the four types of intersections contains: 170 rules for two-way stop-controlled intersections, 100 rules for all-way stop-controlled intersections, 220 rules for signalized intersections and 140 rules for railroad crossings. Because crash patterns at all types of intersections exhibit similarities, they are described without distinguishing the intersection types where possible.

a) Night-time collisions. This type of collisions was addressed in all established roadway entities. The deficiencies and countermeasures established for night-time collisions addresses mostly the visibility issue during night-time that affects perception stage of the driving task.

b) Speed-related collisions. This type of collisions was addressed in all established roadway entities. Speed-related crashes are organized in such a way that first check if adequate speed limit is posted, secondly, if the adequate speed limit is posted proposed different technique to address the excessive speed issue.

c) Adverse weather collisions. This type of collisions was addressed in all roadway entities. Adverse weather conditions are considered for winter separately from non-winter periods.

d) Driveway collisions. This type of collision was addressed for TWSC, AWSC, and RS. The driveway collisions rules address mostly the insufficient visibility and geometry design issues.

e) Right-angle collisions. This type of collisions was addressed for TWSC, SIGN intersections and RRC* . Right-angle collisions deserve a particularly strong focus at TWSC intersections where many rules address the geometry design and sight distance issues. The right-angle collision rules for SIGN intersections focus mostly on two cases where driver either does not notice the traffic lights or violate the traffic lights purposely. "The right-angle collisions at RRC are addressed through checking railroad crossing control devices (automatic gates vs. non- 
automatic gates). Moreover, the sight distance issues are pointed out as well as proposed safety improvements.

f) Rear-end collisions. This type of collisions is typical for SIGN intersections and to some extent for TWSC intersections. The knowledge base rules applied to these two types of intersections deal with the conditions and countermeasures addressing the rear-end collisions. The rear-end collision rules focus mainly on geometry design and, in case of SIGN intersections, on signal timing issues.

g) Head-on collisions. This type of collisions has a particularly considerable number of rules in the RS knowledge base. There are also rules addressing this type of collisions at TWSC and SIGN intersections. The head-on collision rules focus on deficiencies in geometry design and marking.

h) Pedestrian/bicycle collisions. This type of collision is possible on any road where pedestrians and bicycles are present. The rules addressing these collisions consider geometry design (crosswalk design, their placement, visibility, etc.) and, in the case of SIGN intersections, pedestrian/bicycle signals and their timing.

i) Left-turn and right-turn collisions. Both the types of collisions have been addressed by the knowledge base for SIGN intersections while left-turn collisions have been addressed by the knowledge base for TWSC intersections (right-turn collisions at TWSC have been already considered among right-angle collisions).

The above description is brief and does not cover all the types of crashes and circumstances. The full list of rules included in the developed knowledge base is presented in Appendix D.

\subsection{CRF Study}

The developed knowledge base includes 684 countermeasures. The next important and laborious step was to determine the effectiveness of these countermeasures measured with crash reduction factors (CRF). Twenty five sources were carefully reviewed and the found information compiled to obtain a CRF list. The list of these sources is shown at the end of this section. The CRF values obtained from this study were assigned to relevant countermeasures included in the developed RSIT2 knowledge base. The study has 
confirmed a significant discrepancy among reported values even for the same road improvements. In the case where several sources reported various CRF values for the same countermeasure, the average value was used as the final value. The reason for using this simple combining method was the lack of information about the reliability of individual CRF values.

An example result for the TWSC knowledge base is presented in Table 6-1. CRF values from different sources were placed in separate columns. Numbers in parenthesis refer to corresponding comments. In the presented example, the CRF value associated with the countermeasure: "Convert intersection into roundabout" has the following identified in our study values: $58,72,40$, and 38 . Two comments (5) and (6) were added to this result. The full list of the CRF values with corresponding comments is placed in Appendix E.

Out of 684 countermeasures included in the developed knowledge base, 424 have received corresponding CRF values and 260 had missing values. To overcome this gap, we matched the existing CRFs with countermeasures that were somehow similar to the CRF road improvements and used judgment to modify the CRF value. The values that were determined using this assessment are marked (italic format) as shown in Table 6-2.

The obtained CRFs values have been incorporated in the new RSIT2 knowledge base and used to rank countermeasures identified in the site investigation process. The ranking helps the safety analysts select the most promising countermeasures for implementation. The full list of CRF including list of comments is attached in Appendix E. 
Table 6-1 CRF Isolated Examples

\begin{tabular}{|c|c|c|c|c|c|c|c|c|c|c|c|c|c|c|c|c|c|c|c|c|}
\hline & \multicolumn{2}{|c|}{ S1 } & \multicolumn{2}{|c|}{ S2 } & \multicolumn{2}{|c|}{$\mathbf{S 3}$} & \multicolumn{2}{|c|}{ S4 } & \multicolumn{2}{|c|}{ S5 } & \multicolumn{2}{|c|}{ S6 } & \multicolumn{2}{|c|}{ S7 } & \multicolumn{2}{|c|}{ S8 } & \multicolumn{2}{|c|}{ S9,S10 } & \multicolumn{2}{|c|}{ S11-S21 } \\
\hline & $\mathbf{R}$ & $\mathbf{U}$ & $\mathbf{R}$ & $\mathbf{U}$ & $\mathbf{R}$ & $\mathbf{U}$ & $\mathbf{R}$ & $\mathbf{U}$ & $\mathbf{R}$ & $\mathbf{U}$ & $\mathbf{R}$ & $\mathbf{U}$ & $\mathbf{R}$ & $\mathbf{U}$ & $\mathbf{R}$ & $\mathbf{U}$ & $\mathbf{R}$ & $\mathbf{U}$ & $\mathbf{R}$ & $\mathbf{U}$ \\
\hline $\begin{array}{l}\text { Consider moving the right-turn lanes } \\
\text { on the major road laterally }\end{array}$ & & & & & & & & & & & & & & & & & & & & \\
\hline $\begin{array}{l}\text { Consider traffic signals with } \\
\text { protected left-turn phase }\end{array}$ & & & & & & & & & & & & & & & & & & & & \\
\hline $\begin{array}{l}\text { Construct Pedestrian Refuge Islands } \\
\text { and Raised Medians }\end{array}$ & & 46 & $\begin{array}{l}60 \\
(4)\end{array}$ & $\begin{array}{l}60 \\
(4)\end{array}$ & 32 & 23 & & & & & & & & & & 46 & & & & \\
\hline $\begin{array}{l}\text { Convert the intersection into a } \\
\text { roundabout }\end{array}$ & $\begin{array}{l}58 \\
(5)\end{array}$ & $\begin{array}{l}72 \\
(5)\end{array}$ & & & & & 40 & 40 & 40 & 40 & & & & & $\begin{array}{l}58 \\
(6)\end{array}$ & $\begin{array}{l}72 \\
(6)\end{array}$ & 58 & 72 & 38 & 38 \\
\hline $\begin{array}{l}\text { Eliminate screening by physical } \\
\text { objects }\end{array}$ & & & & & & & & & & & & & $\begin{array}{l}50 \\
(7)\end{array}$ & $\begin{array}{l}50 \\
(7)\end{array}$ & & & & & $\begin{array}{l}76 \\
(8) \\
\end{array}$ & $\begin{array}{l}76 \\
(8) \\
\end{array}$ \\
\hline Eliminate shoulder drop-off & & & & & & & & & & & 25 & 25 & & & & & & & $\begin{array}{l}30 \\
(9)\end{array}$ & $\begin{array}{l}30 \\
(9)\end{array}$ \\
\hline Enforce stop sign compliance & & & & & & & & & & & & & & & & & & & & \\
\hline Extend "no parking" zone. & & & & & 22 & 22 & & & & & $\begin{array}{l}35 \\
(10)\end{array}$ & $\begin{array}{l}35 \\
(10)\end{array}$ & & & & & & & 10 & 10 \\
\hline Groove pavement surface & & & 21 & 21 & & & 14 & 14 & & & & & & & & & & & 46 & 46 \\
\hline $\begin{array}{l}\text { Implement Road Narrowing } \\
\text { Measures }\end{array}$ & & & & & & & & & & & & & & & & & & & & \\
\hline Improve alignment/grade & & & & & & & & & & & & & & & & & & & & \\
\hline Improve channelization/delineation & & & & & & & & & & & $\begin{array}{l}30 \\
(11)\end{array}$ & $\begin{array}{l}30 \\
(11)\end{array}$ & 40 & 40 & & & & & 15 & \\
\hline Improve clear zone & & & & & $\begin{array}{l}25 \\
(12)\end{array}$ & $\begin{array}{l}25 \\
(12) \\
\end{array}$ & & & & & & & & & & & & & & \\
\hline Improve existing street lights & 30 & 30 & & & 50 & 50 & & & & & & & & & & & & & & \\
\hline Improve lanes width & $\begin{array}{l}8 \\
(13)\end{array}$ & & & & & & & & & & & & & & 8 & & & & & \\
\hline
\end{tabular}


Table 6-2 CRF Final Version (part of TWSC)

\begin{tabular}{|c|c|c|c|c|c|c|c|c|c|c|c|c|c|c|c|c|c|c|c|c|c|}
\hline & \multicolumn{2}{|c|}{ S1 } & \multicolumn{2}{|c|}{ S2 } & \multicolumn{2}{|c|}{ S3 } & \multicolumn{2}{|c|}{ S4 } & \multicolumn{2}{|c|}{ S5 } & \multicolumn{2}{|c|}{ S6 } & \multicolumn{2}{|c|}{ S7 } & \multicolumn{2}{|c|}{ S8 } & \multicolumn{2}{|c|}{ S9,S10 } & \multicolumn{2}{|c|}{$\begin{array}{l}\text { S11- } \\
\text { S21 } \\
\end{array}$} & \\
\hline & $\mathbf{R}$ & $\mathbf{U}$ & $\mathbf{R}$ & $\mathbf{U}$ & $\mathbf{R}$ & $\mathbf{U}$ & $\mathbf{R}$ & $\mathbf{U}$ & $\mathbf{R}$ & $\mathbf{U}$ & $\mathbf{R}$ & $\mathbf{U}$ & $\mathbf{R}$ & $\mathbf{U}$ & $\mathbf{R}$ & $\mathbf{U}$ & $\mathbf{R}$ & $\mathbf{U}$ & $\mathbf{R}$ & $\mathbf{U}$ & \\
\hline Install corner mirrors & & & & & & & & & & & & & & & & & & & & & 10 \\
\hline $\begin{array}{l}\text { Install curbing to define driveway } \\
\text { location }\end{array}$ & & & & & & & & & & & & & & & & & & & & & 20 \\
\hline Install curb-ramps & & & & & & & & & & & & & & & & & & & & & 15 \\
\hline Install flashing beacons & 30 & 30 & & & 50 & 50 & 30 & 30 & & & 30 & 30 & 24 & 24 & & & & & & & 33 \\
\hline $\begin{array}{l}\text { Install flashing beacons at stop- } \\
\text { controlled intersections }\end{array}$ & & & & & 50 & 50 & 30 & 30 & & & & & 24 & 24 & & & & & & & 35 \\
\hline Install fog - warning signs & & & & & & & 25 & 25 & & & & & & & & & & & & & 25 \\
\hline Install guardrails & & & & & 20 & 20 & 47 & 47 & & & 65 & 65 & & 4 & & & 44 & 44 & & & 40 \\
\hline Install indirect left-turn lanes & & & 34 & 34 & & & 35 & 35 & & & & & & & & & & & & & 35 \\
\hline $\begin{array}{l}\text { Install larger regulatory and warning } \\
\text { signs at and in advance of } \\
\text { intersections }\end{array}$ & & & & & & & & & & & 15 & 15 & 40 & 30 & & & & & & & 25 \\
\hline $\begin{array}{l}\text { Install larger regulatory and warning } \\
\text { signs at intersections }\end{array}$ & & & & & & & & & & & 15 & 15 & & & & & & & & & 15 \\
\hline Install left turn acceleration lane & & & 26 & 26 & & & & & & & 10 & 10 & & & & & & & & & 18 \\
\hline Install left turn bays & 28 & 27 & & & 20 & 20 & & & & & & & & & 28 & 27 & & & & & 25 \\
\hline Install lighting & 30 & 30 & 33 & 33 & 50 & 50 & & & & & 30 & 30 & 37 & 37 & & & & & 37 & 37 & 36 \\
\hline Install median divider & 15 & 15 & & & & & & & & & & & & & & & & & & & 15 \\
\hline $\begin{array}{l}\text { Install overhead flashing beacon } \\
\text { lights }\end{array}$ & 30 & 30 & & & 50 & 50 & 30 & 30 & & & 30 & 30 & 24 & 24 & & & & & & & 33 \\
\hline Install pavement markings & 10 & 10 & 21 & 21 & & & & & 21 & 21 & & & & & & & & & 10 & 10 & 16 \\
\hline Install pedestrian actuated signals & & & & & & & & & & & & & & & & & & & & & 35 \\
\hline
\end{tabular}




\section{RSIT2 USER MANUAL}

\subsection{RSIT2 CONCEPTS}

RSIT2 is based on a novel idea of an optimal checklist that is updated during site investigation. This approach makes the investigation process more time-effective and less expensive. RSIT2 uses rules as building blocks of the knowledge base which connects checks with countermeasures. The knowledge base can be updated by removing, adding, or modifying the rules.

The following part of the RSIT2 Concepts section introduces the background information which helps better understand the way RSIT2 works and may help read the next section describing the RSIT2 user interface.

\section{Conventional Checklist}

The traditional process of site investigation includes processing checks by following a list of checks, documenting the findings, and then identifying countermeasures that could be justified with the site investigation. The traditional checklist list checks sequentially: $\mathrm{X} 1$, $\mathrm{X} 2 \ldots \mathrm{XN}$, where $\mathrm{Xi}$ is a check such as Signalized intersection, Divided roadway, Poor drainage, Insufficient sight distance, etc. The identified countermeasures $\mathrm{C} 1, \mathrm{C} 2 \ldots \mathrm{CM}$ are such as: Remove sight distance obstruction, Increase yellow signal, etc.

This process has several drawbacks:

1. The check list has to be customized by the user to remove checks that are not applicable to the investigated site.

2. Checks on the customized list are considered at the site even when some of them could be skipped.

3. The order of checks may affect the investigation time but this is not considered when building the checklists. 
4. The checklist does not help keep track of the investigation progress when a check is applied multiple times to different road elements (approaches, exits, etc).

5. Checklists do not match the site investigation results with relevant countermeasures.

\section{Rule-based Knowledge Representation}

Rules are sometimes used to diagnose problems or/and to select relevant actions. An example rule may read:

IF

Signalized Intersection AND Rear-end collisions on approach

AND High speed on approach AND Short yellow signal on approach

THEN Increase yellow signal on approach.

In a general case of if-then rules, this knowledge base can be organized in a table with two columns: the first column includes conditions (or checks) and the second column includes relevant conclusions (or countermeasures). Table 7-1 demonstrates this structure where $\mathrm{X}_{\mathrm{i}}$ is a conditions or check such as: Signalized intersection, Divided roadway, Insufficient sight distance, etc. and $\mathrm{C}_{\mathrm{i}}$ is a countermeasure such as: Remove sight distance obstruction, Increase yellow signal, etc.

Table 7-1 Example of a rule-based checklist

\begin{tabular}{|c|c|}
\hline IF & THEN \\
\hline$X_{1}$ and $X_{2}$ & $C_{1}$ \\
\hline$X_{1}$ and $X_{3}$ and $X_{4}$ & $C_{2}$ \\
\hline$X_{2}$ and $X_{4}$ & $C_{2}$ \\
\hline$\ldots \ldots \ldots \cdots$ & $\cdots \cdots$ \\
\hline$X_{1}$ and $X_{23}$ and $X_{24}$ & $C_{\mathrm{M}}$ \\
\hline
\end{tabular}

The rule-based knowledge representation is intuitive and provides an inventory of relevant knowledge. This inventory of rules (called Knowledge Base) is easy to update by 
adding or removing rules. The rules explicitly link checks with relevant countermeasures which addresses the last drawback of conventional checklists. On the other hand, other drawbacks of conventional checklists used in safety investigation remain and some of them are even get worse due to multiple appearances of checks (for example, X1 in Table 7-1).

\section{RSIT2 Knowledge Base}

RST2 checks and countermeasures have added road component names to make them more specific. The example rule in the Rule-based Checklist section becomes:

IF Signalized Intersection AND Rear-end collisions on Main Street $N B$ approach AND High speed on Main Street NB approach

AND Short yellow signal on Main Street NB approach

THEN Increase yellow signal on Main Street NB approach.

The name of the approach added to the original rule is Main Street NB. To make the above rule applicable to any intersection, a variable \#A1 replaces the value Main Street $N B$ :

IF Signalized Intersection AND Rear-end collisions on \#A1 approach AND High speed on \#A1 approach

AND Short yellow signal on \#A1 approach

THEN Increase yellow signal on \#A1 approach.

This generic rule may be applied to each approach of any signalized intersection. It is multiplied the number of times equal the number of approaches, and then variable \#A1 is replaced with the names of these approaches.

The first step in building and using the RSIT2 checklist is developing a generic knowledge base (KB) that includes all known valid generic rules for various types of 
roads and intersections. RSIT2 offers a KB editor for that purpose which can be used to build a new KB or modify the one currently available.

\section{RSIT2 Checklist}

RSIT2 automatically converts the developed generic rule-based KB to a generic checklist by decomposing each rule into its elements, removing countermeasures, and removing multiple appearances of conditions.

RSIT2 customizes the generic checklist to a certain investigated site by multiplying checks to be used for multiple road components and replacing name variables with actual names of the road components. The second stage of the checklist customization is removing these conditions that can be claimed false based on the basic information entered by the user (traffic control, layout of the intersection legs, one-way streets, etc.). For example, the condition: Signalized intersection is removed from the checklist if the investigated intersection is not signalized. The next step is to update the checklist by removing these checks that become redundant after identifying false conditions. For example some checks are needed only at signalized intersections thus removing the Signalized intersection check entails removing all these other checks as well.

To reduce the investigation cost, RTSIT2 orders the obtained checks in a way that increases the investigation convenience (reduced walking at the site) and decreases the investigation effort. Easy and inexpensive checks that may eliminate the need for more labor demanding checks are performed first. In the current version, the checks are sorted according to the following rules:

1) Off-site checks are grouped together and precede on-site checks.

2) Checks within the off-site and on-site groups are further grouped if they applied to the same road component (approach, exit, road).

3) Within each group created with rule 2, the checks are sorted by cost with less expensive checks preceding the more expensive checks.

4) Within each group of same-cost checks formed with rule 3, the checks are sorted by the probability of passing with the checks least probable to pass preceding the 
checks more probable to pass (failing a check creates a chance for eliminating other checks).

\section{RSIT2 Files}

$\mathrm{KB}$ is stored in a file named KBname.rs2. The part KBname is provided by the user while the extension is fixed and cannot be changed. RSIT2 saves the KB file at a location selected by the user.

The user may also add reference files that include additional information helpful during a site investigation. These reference files are stored in a reference folder named KBname_files. Each KB file must have one reference folder. The KB file and the corresponding reference folder are associated by the common part KBname in their names and by saving the KBname.rs2 file and the KBname_files folder in the same directory.

A KB file can be converted to a text file to be seen by any text editor. The exported file has an extension KBname.csv.

During investigation, the user can save the current investigation log file to be able to stop the investigation and resume it later. The log file is named investigation.rsg where the name is provided by the user while the extension is fixed. This file is read by the RSIT2 to resume the investigation.

The investigation progress can be saved in the form of a checklist presented in the RSIT user interface with all the entries made by the user up to the time of saving the checklist. The name of the file is checklist.csv.

The investigation results including the identified countermeasures, the conditions supporting these countermeasures, and the assessed effectiveness of the countermeasures. This file is named report.txt and can be saved at any point of the investigation. 
The KB file and the reference files, which are input files used in multiple investigations. Any updated versions of the KB should be saved in a new folder and under a new name that reflects the version. Including dates in the KB names may be useful.

A single investigation may generate several output files. The output files generated by RSIT2 include the investigation log, progress, and results. Files generated in a single investigation should be saved in one folder with a name that helps identify the investigation.

\subsection{RSIT2 USER INTERFACE}

RSIT2 includes three components: Site Investigation tool, Knowledge Base editor, and Help module. These components can be run by clicking on a proper item listed in the RSIT2 starting window (Figure 7-1).

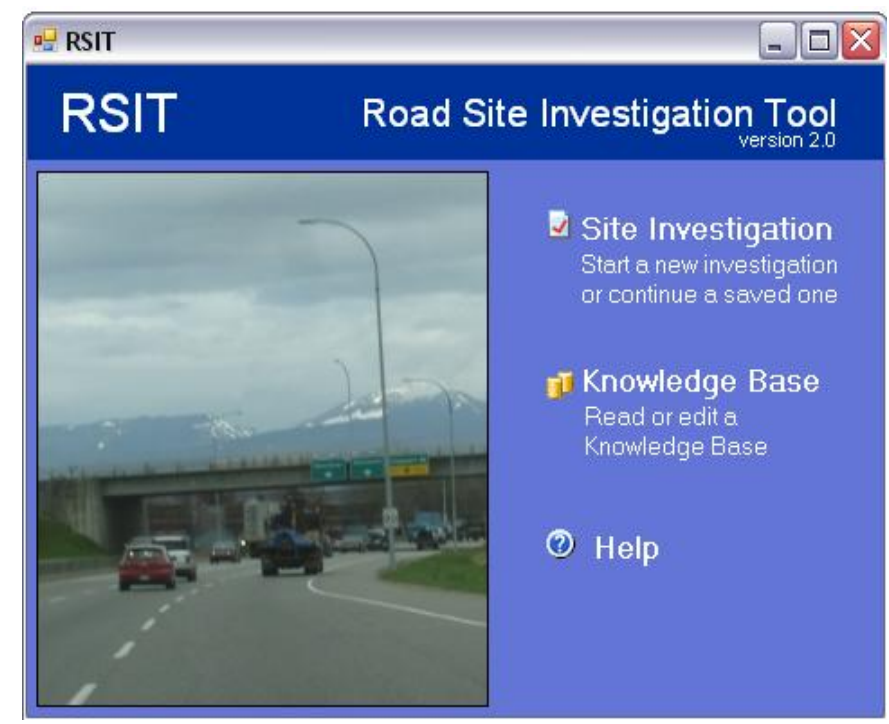

Figure 7-1 RSIT2 starting window

\section{Knowledge Base Editor}

The Knowledge Base editor interface is shown in Figure 7-2. The size of the interface can be adjusted with a mouse. The interface includes three parts: command line, display area, 
and editing area. These components are described in the following sections of the manual.

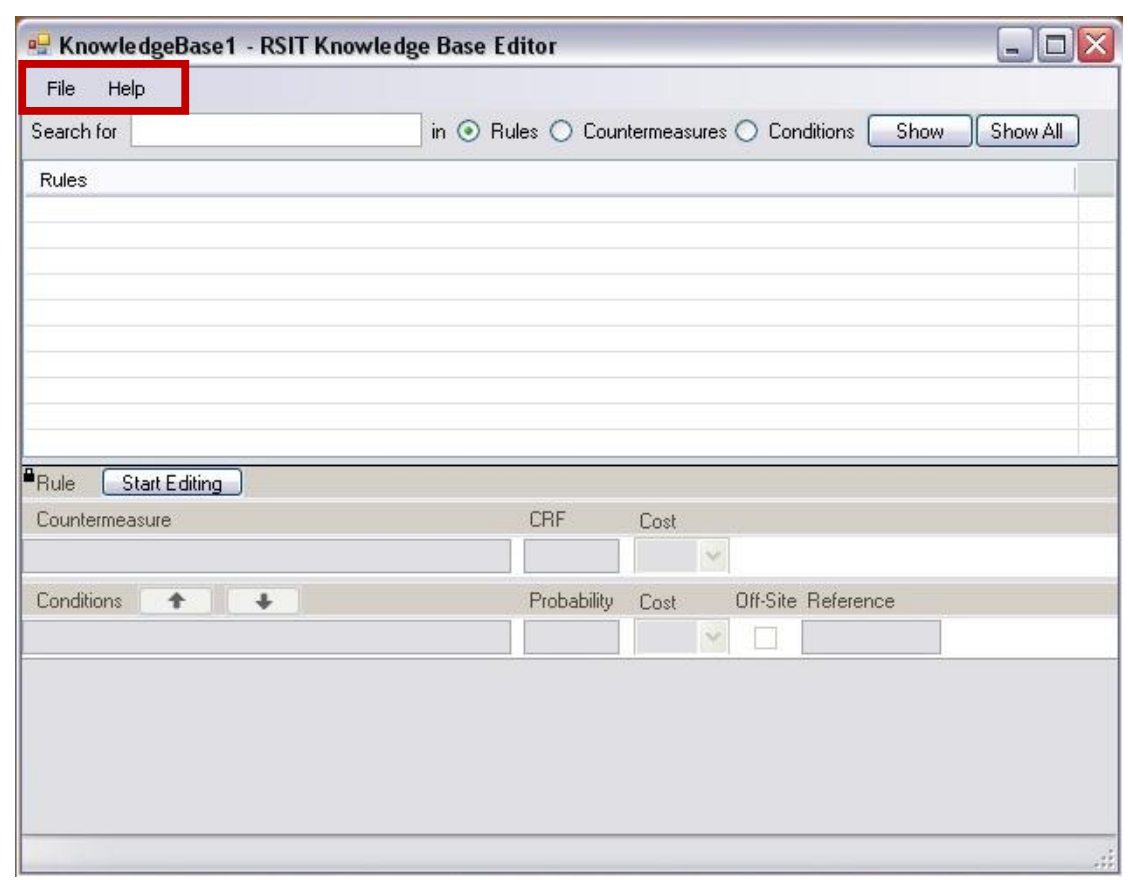

Figure 7-2 RSIT2 Editor

\section{Command Line}

The command line includes two options: File, and Help. The File pull-down list allows the following input-output file operations:

- New initiates development of a new knowledge base (KB)

- Open opens an existing KB file

- Save saves a currently opened KB file under the same name and in the same location. The extension of the KB file is .rs 2 .

- Save As saves a currently opened KB file under different name and/or in different location than the current

- Export converts a currently opened file into the text file format

- Exit ends the current KB editing session

The Help command opens the on-line user manual. 


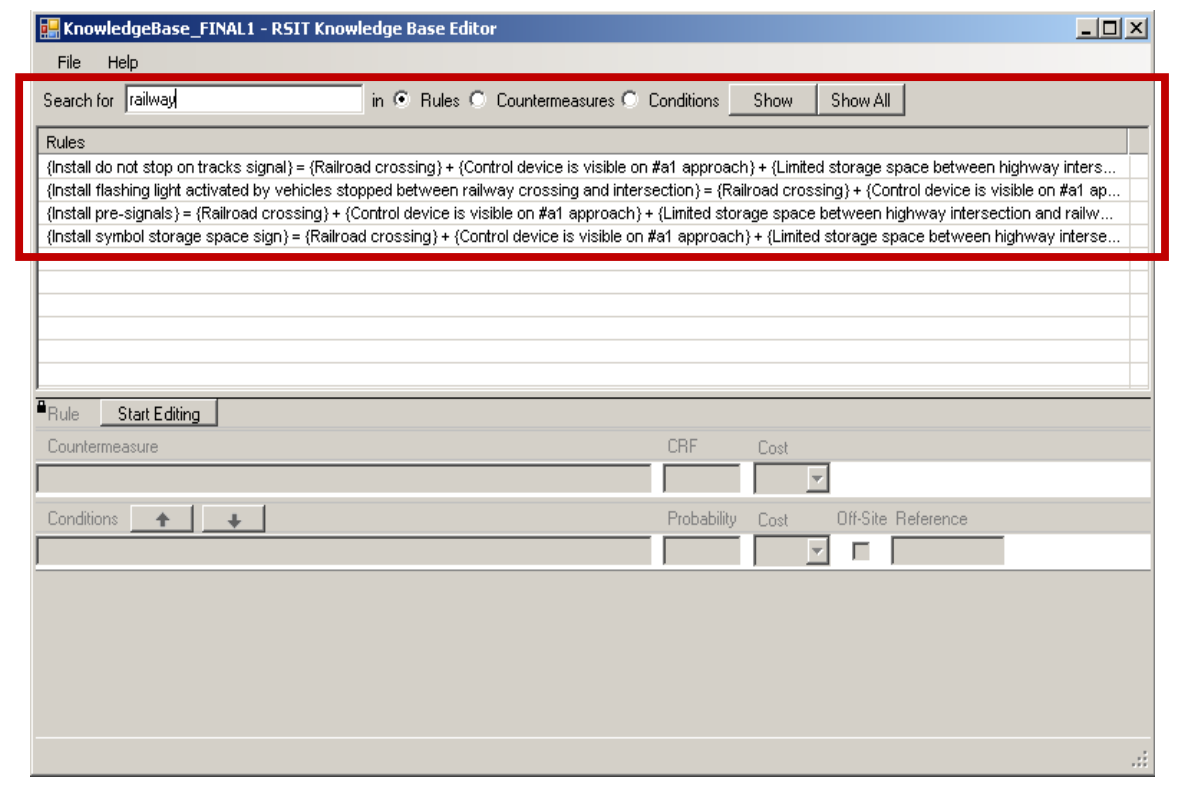

Figure 7-3 Display area

\section{Display Area}

The display area (Figure 7-3) includes a search tool that helps find in large knowledge bases conditions, countermeasures, and rules that include specific key words. Search can be performed on the entire knowledge base or on the results of the previous search. The key words are typed in the box after Search for. The user selects a radio to specify where the key words are to be looked for: in rules, in countermeasures, or in conditions. The Show button displays the results of the search while Show All resets the search. Figure 7-3 shows the result of searching the example KB for rules that include word railway. 


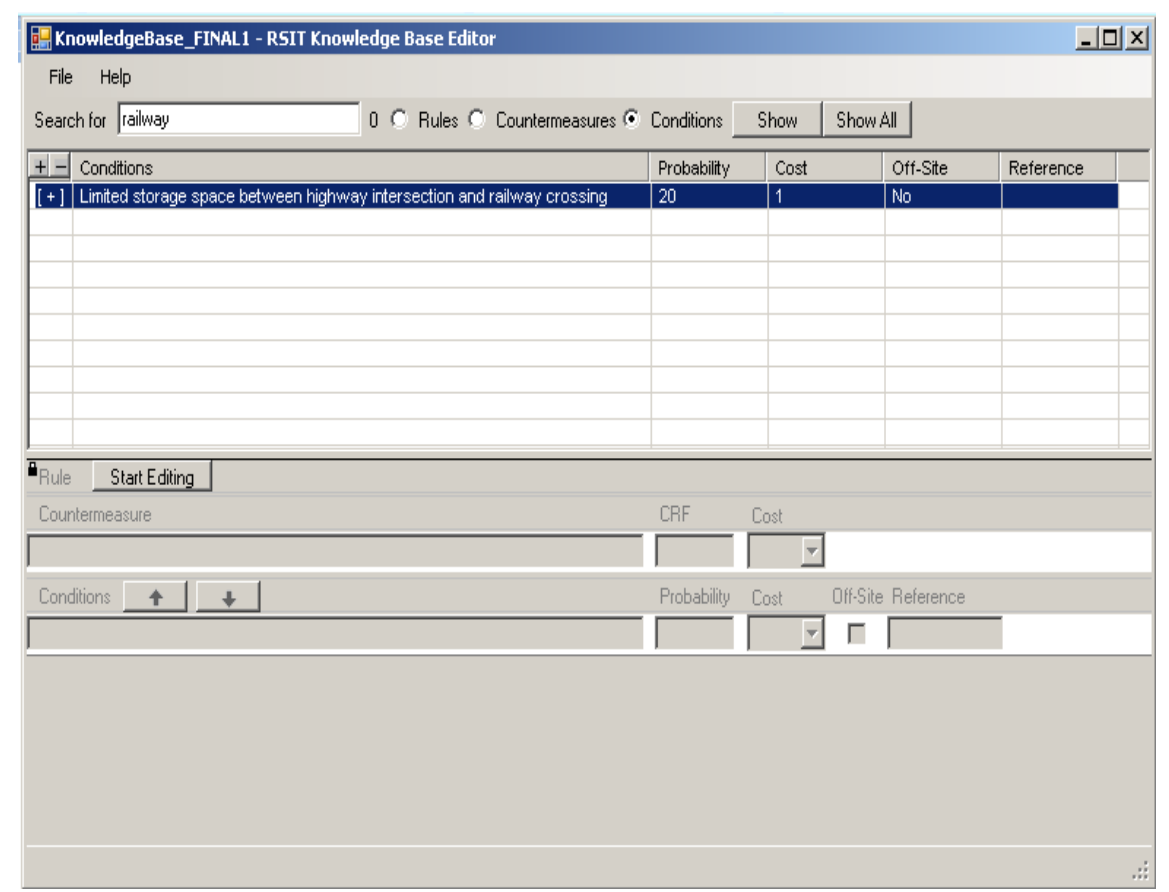

Figure 7-4 Display window with a condition by found by key word: railway

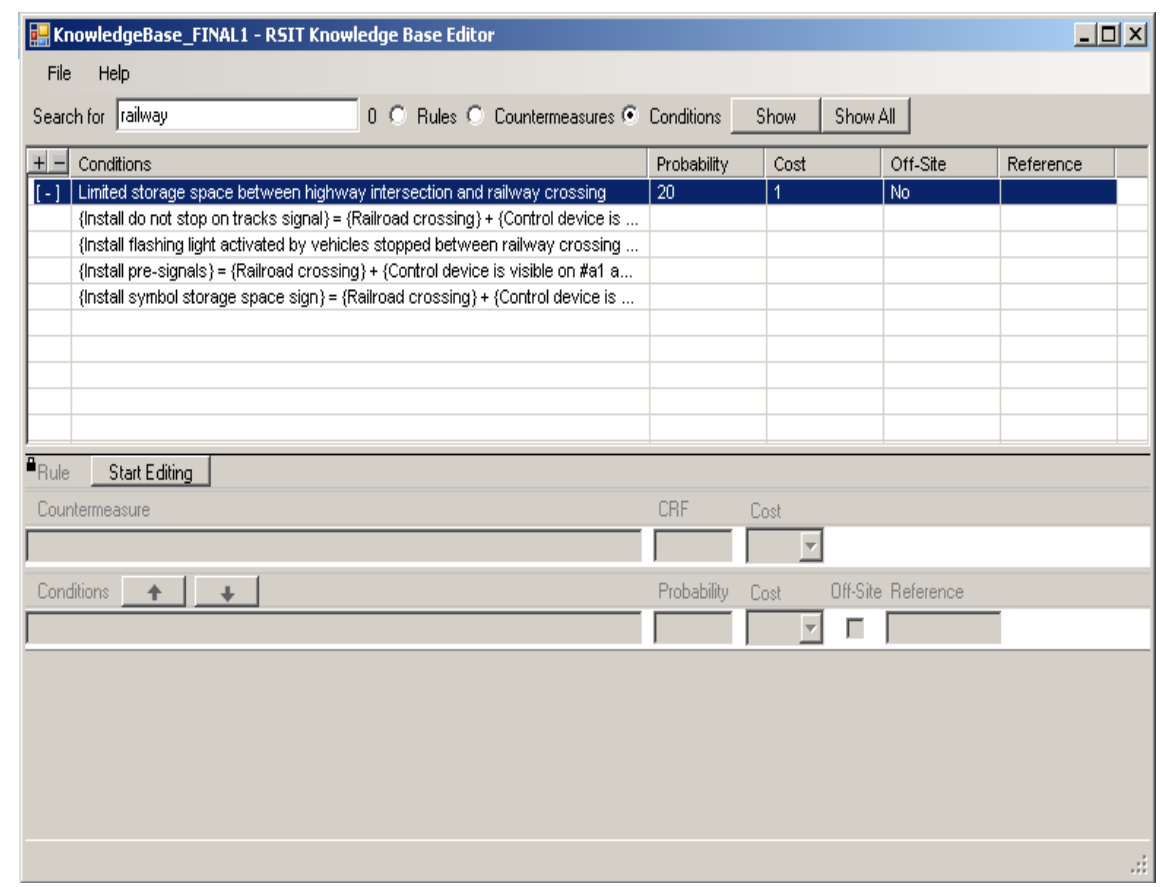

Figure 7-5 The found condition with an unfolded list of corresponding rules 
Figure 7-4 presents the results of a search for conditions that include word railway. The results are displayed in six columns. The third, fourth, fifth, and sixth columns contain the conditions' attributes.

1. [+] - clicking on this symbol shows the rules that include the found condition; the symbol changes to [-] if the rules are displayed (Figure 7-5).

2. Conditions - description of the condition.

3. Probability - chance in percent that the condition takes place at an investigated site (or that a corresponding check passes).

4. Cost - categorical assessment of the cost of the check measured on a scale from 1 to 5 with 1 being the least expensive check and 5 being the most expensive check

5. Off-Site -1 indicates that the condition is checked in the office and before the site investigation, 0 indicates otherwise.

6. Reference provides the name of the file that includes additional information relevant to the condition. This file is available for reading during the site investigation if needed. By convention, the reference files are stored in a folder named

A search results for countermeasures are displayed in four columns. The third and fourth columns include the countermeasures' attributes.

1. [+] - clicking on this symbol shows the rules that include the found countermeasure; the symbol changes to [-] if the rules are displayed.

2. Countermeasures - description of the countermeasure.

3. $\mathbf{C R F}$ - crash reduction factor in percent applicable to all crashes.

4. Cost - categorical assessment of the cost of the countermeasure measured on a scale from 1 to 5 with 1 being the least expensive countermeasure and 5 being the most expensive countermeasure.

\section{Editing Area}

The third component of the KB Editor interface is the editing area which provides the functionality required for entering and editing knowledge bases (Error! Reference 
source not found.). The editing in the current version of RSIT can be done only through rules. The user has to select Start Editing button (Figure 7-2) to activate the KB Editor.

When the KB Editor is active, the user can simply drag any rule displayed in the display area to the editing area or type a new rule directly in the lines of the editor. Another option is to drag some existing conditions from the list of currently displayed conditions above and type additional ones directly in the editor. The user has also an option of displaying countermeasures in the display window and drag a selected one to avoid typing of the countermeasure exists. This feature allows reusing conditions and countermeasures already presented in the current KB to avoid re-typing and associated risk of making mistakes.

Once the KB Editor detects non-empty countermeasure line and at least one non-empty condition line, the rule can be saved by pressing the enabled Update button. A rule dragged from the list of existing ones can be deleted right after dragging. Making any change to the rule disables the Delete button and enables the Update button. The updating operation replaces the old rule with the modified rule if some countermeasure attributes or condition attributes have changed while the text of countermeasure and conditions remained the same. The updating operation adds the new rule without deleting the old one if the countermeasure or condition text has changed. In this case, the new rule has to be saved and the End Editing button pressed. Then, the editing has to be again initiated by pressing the Start Editing button and the old rule dragged and deleted if not needed.

After the current KB is edited, it can be saved using the File operations. The window can be closed by pressing the cross button in the upper right corner. Selecting the Exit option in the File operations closes the window and shuts down the RSIT tool.

Pressing arrows in the editing area moves the current condition up or down the list of conditions. This feature is used to change the order of conditions without re-typing them. 


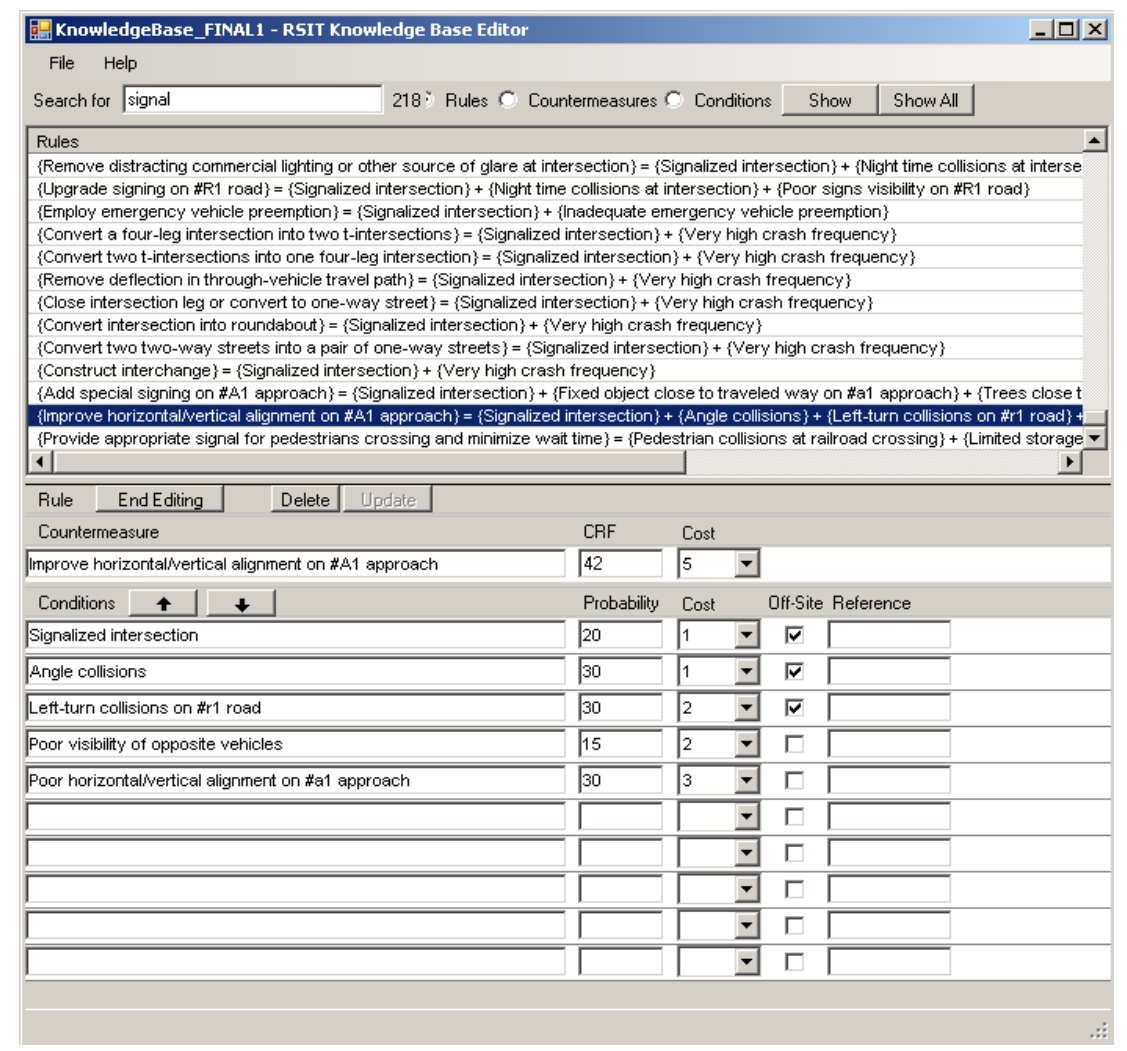

Figure 7-6 Editing area with a rule dragged from the display area

\section{Site Investigation Tool}

The Site Investigation tool facilitates functions needed to plan and execute a site investigation. The investigation tool has four windows:

(1) Project Information window,

(2) Intersection Diagram window,

(3) Checklist window, and

(4) Countermeasures window.

The top line in all the four windows has two buttons: Investigation and Help. The Investigation button allows two operations:

1. Initiation of a new investigation (option New) by loading a knowledge base, and

2. Resuming a past investigation (option Open) by loading a log file of a previous investigation. 
The currently conducted investigation can be saved in a log file by using options Save or Save As. The Exit option exits the RSIT. The Help button opens the on-line user help.

\section{Project Information}

The Project Information window allows entering all the major information about the investigation site: its name, jurisdiction, location, and the type of road (Figure 7-7). The names of the investigators, date, time, light conditions, and additional comments can be entered and saved as well.

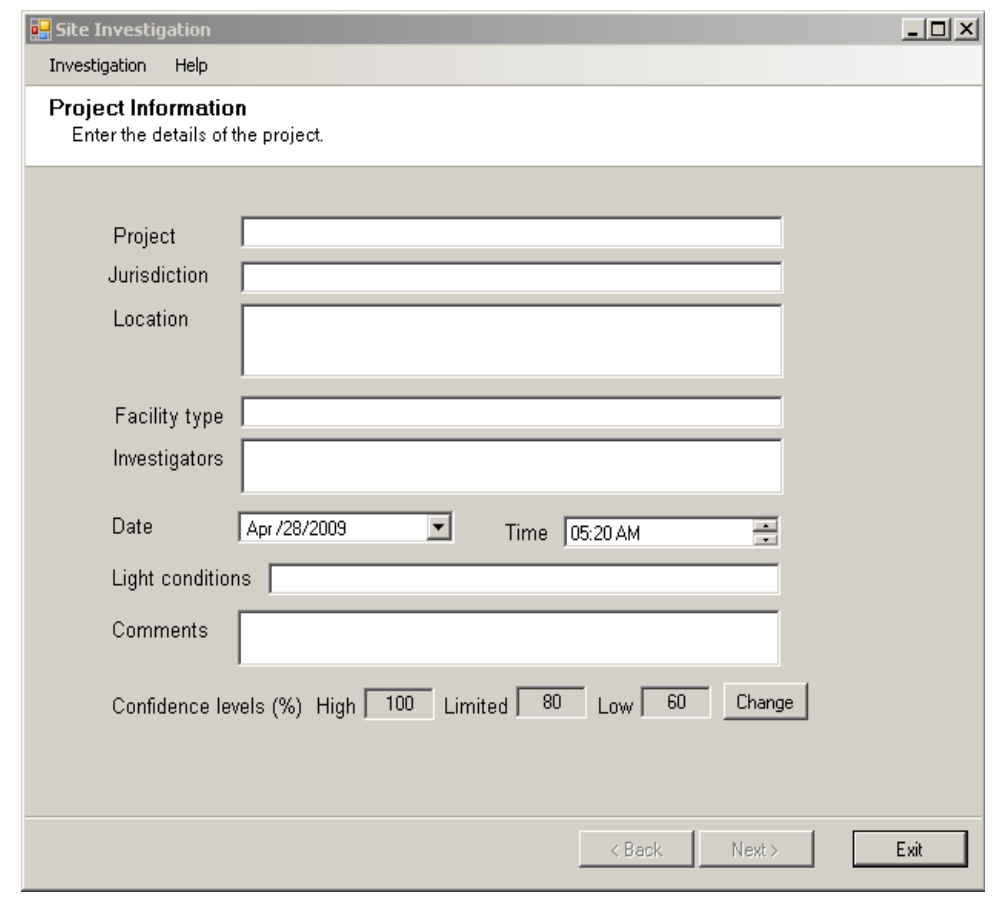

Figure 7-7 Project Information window 


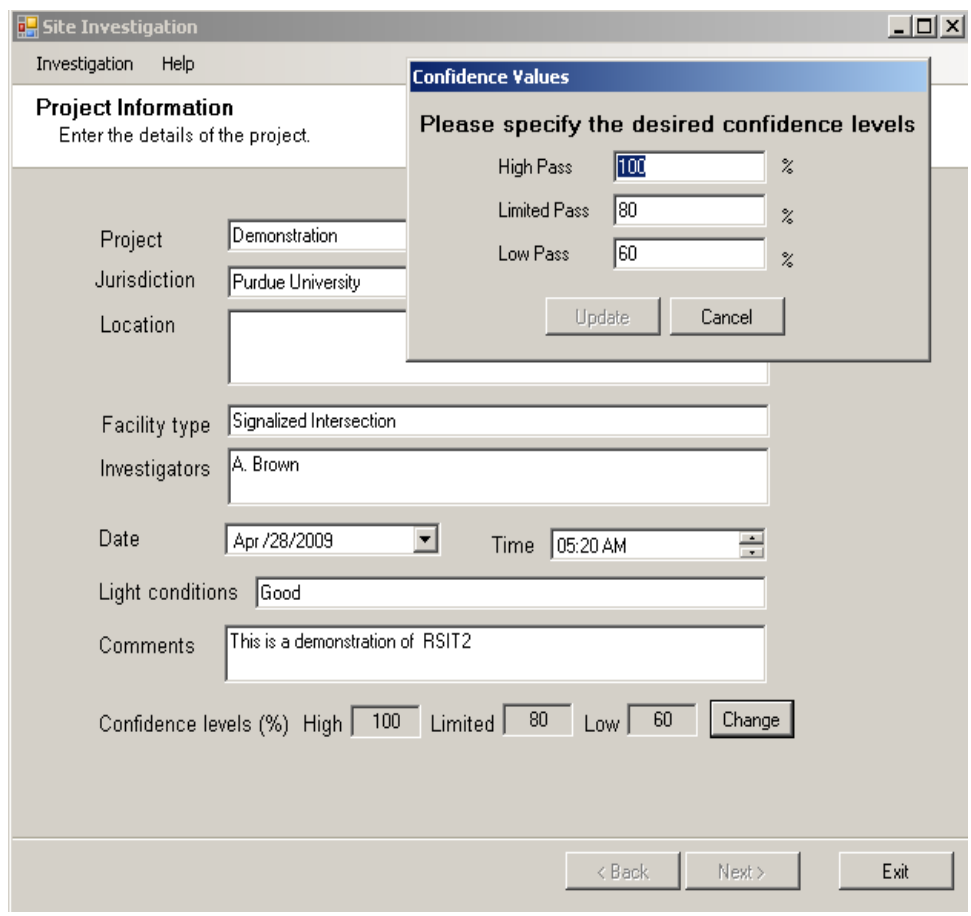

Figure 7-8 Changing the percent values associated with the three confidence levels

Figure 7-8 illustrates changing the percent values associated with the confidence levels at which checks can be passed. The 80 percent value assigned to the Limited Confidence Level means that when this passing level is selected, 80 percent of times the passing is correct and in $20 \%$ of cases the passing is not justified. Although this interpretation is based on frequency, the value reflects the user's preference. It is recommended, that the confidence levels are kept fixed across investigations to allow comparison of the results.

The Back and Next buttons facilitate navigation between the windows. Although most of the entries can be changed during the investigation and the tool will update the results, in some cases this updating is not possible and the current results may be erased. Proper warnings are issued in such cases.

\section{Intersection Diagram}

The Intersection Diagram window is used to specify major components of the investigated road and to name the road components (Figure 7-9). This information is used 


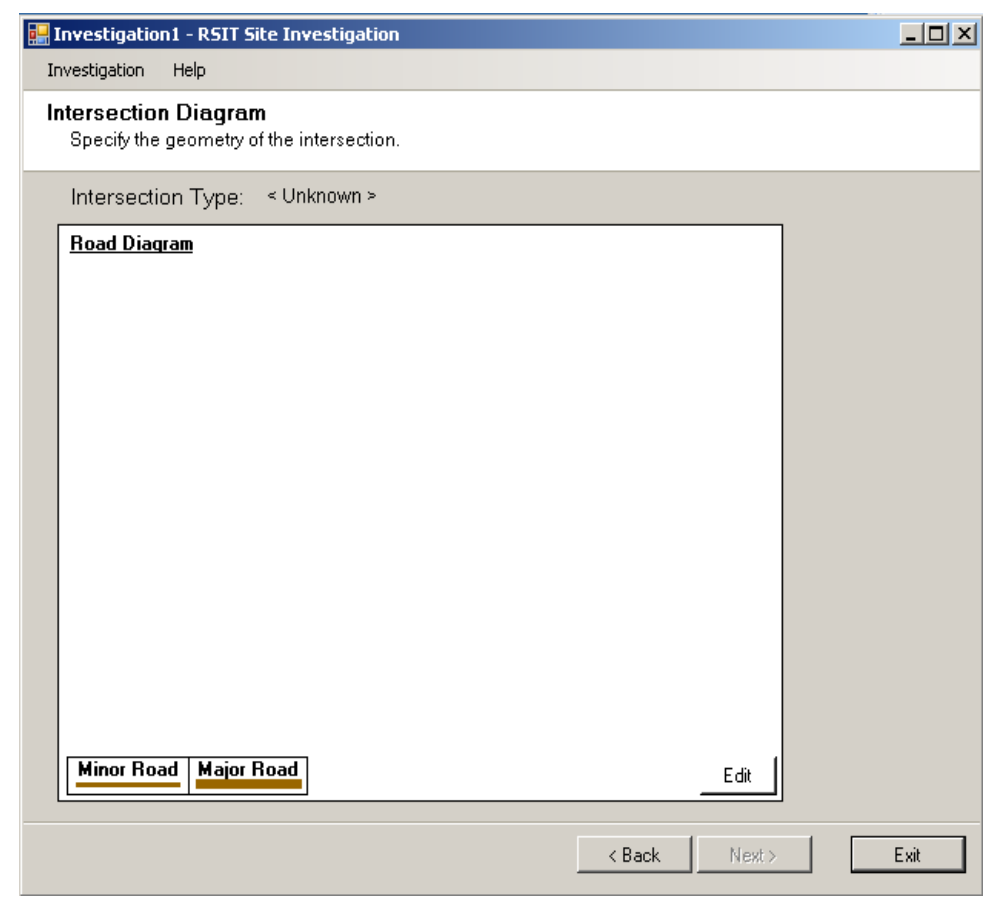

Figure 7-9 Intersection Diagram window

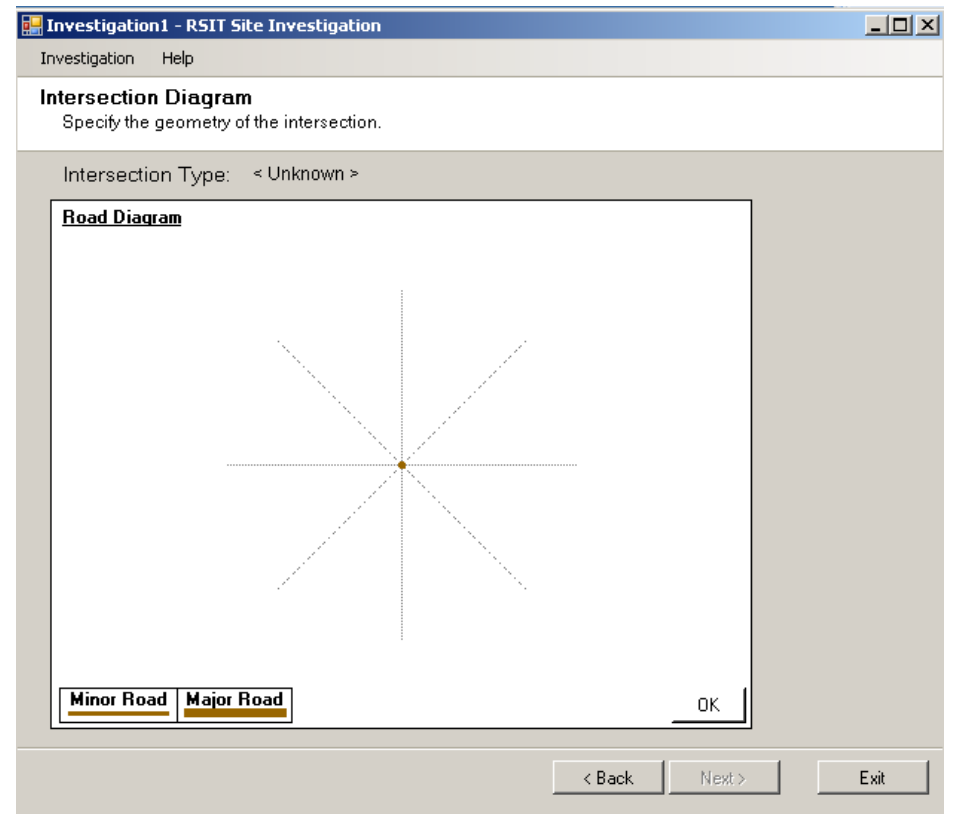

Figure 7-10 Activated editor of the Intersection Diagram

to customize the initial generic checklist by eliminating checks not applicable to the investigated road and by multiplying other checks applicable to multiple approaches, 
exits, crossing roads, etc. The names of the approaches and roads entered by the user in the Intersection Diagram are added to these checks to allow identification of the road components during the site investigation.

Pressing the Edit button in the bottom right corner activates the diagram editor. A simplified intersection diagram appears as the one shown in Figure 7-10. Defining the road components is done in several steps:

(1) Click with the mouse left button on any of the dashed lines to enter the intersection leg. Repeated clicking switches between two-way leg, one-way approach, one-way exit, and no-leg options.

(2) Click with the mouse left button on the name by any of the legs to modify the name. Press OK below the text box to accept the new name or press Cancel to bring to the previous name (Figure 7-11).

(3) Click with the mouse right button on the already entered leg to switch between minor and major roads. A major leg is coded with a thick line (Figure 7-12). A minor leg is coded with a thin line and a number between 1 and 4 . In some cases all legs may remain minor. If entering major legs is needed, then exactly two legs must be major.

(4) The two major legs form a road. A road passes an intersection on a straight or a sufficiently flat horizontal curve. A site investigation includes checks applied to such a pair of legs as a single road entity. The user has an option to define roads made of minor legs as well. This may be useful for signalized intersections. Two minor legs with the same number between 1 and 4 form a road. The numbers can be switched by clicking on them with the mouse left button.

(5) Click with the mouse left button on the traffic control sign by any of the approaches to switch between stop sign, yield sign, signal head, and no control (point).

(6) Click OK button in the bottom right corner of the diagram window to accept the current diagram and to exit the edit mode. 


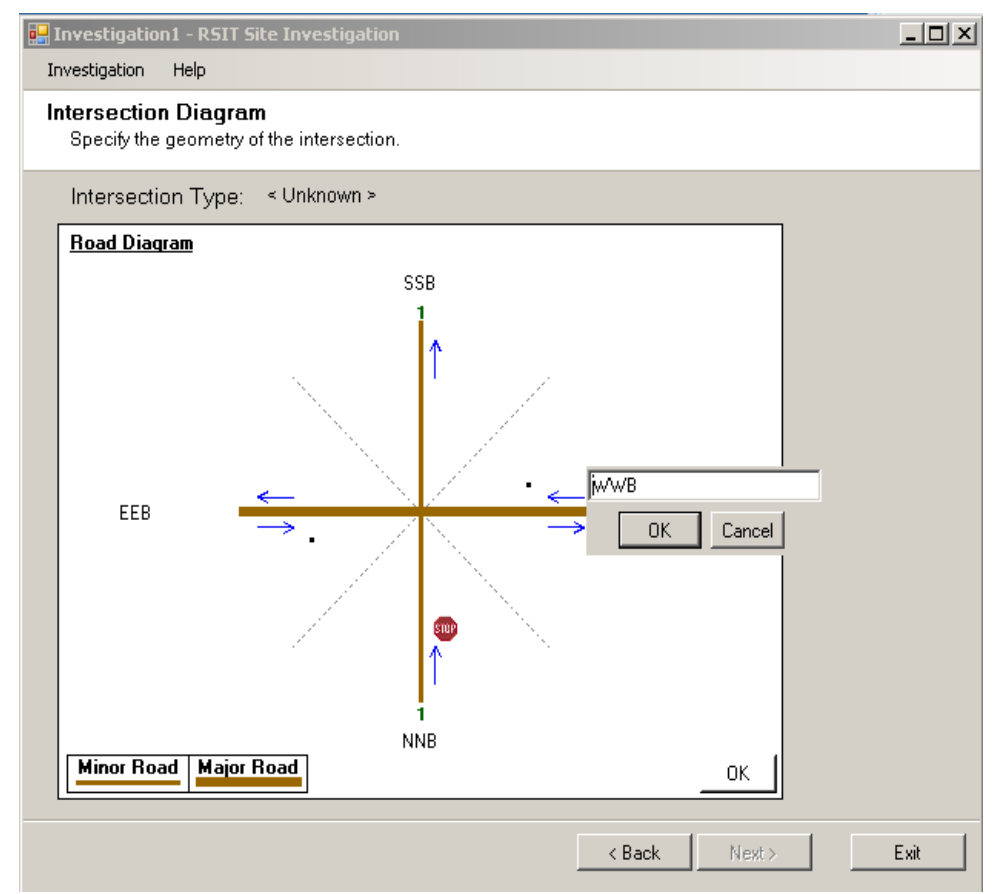

Figure 7-11 Opened text box to modify the road name in the Intersection Diagram window

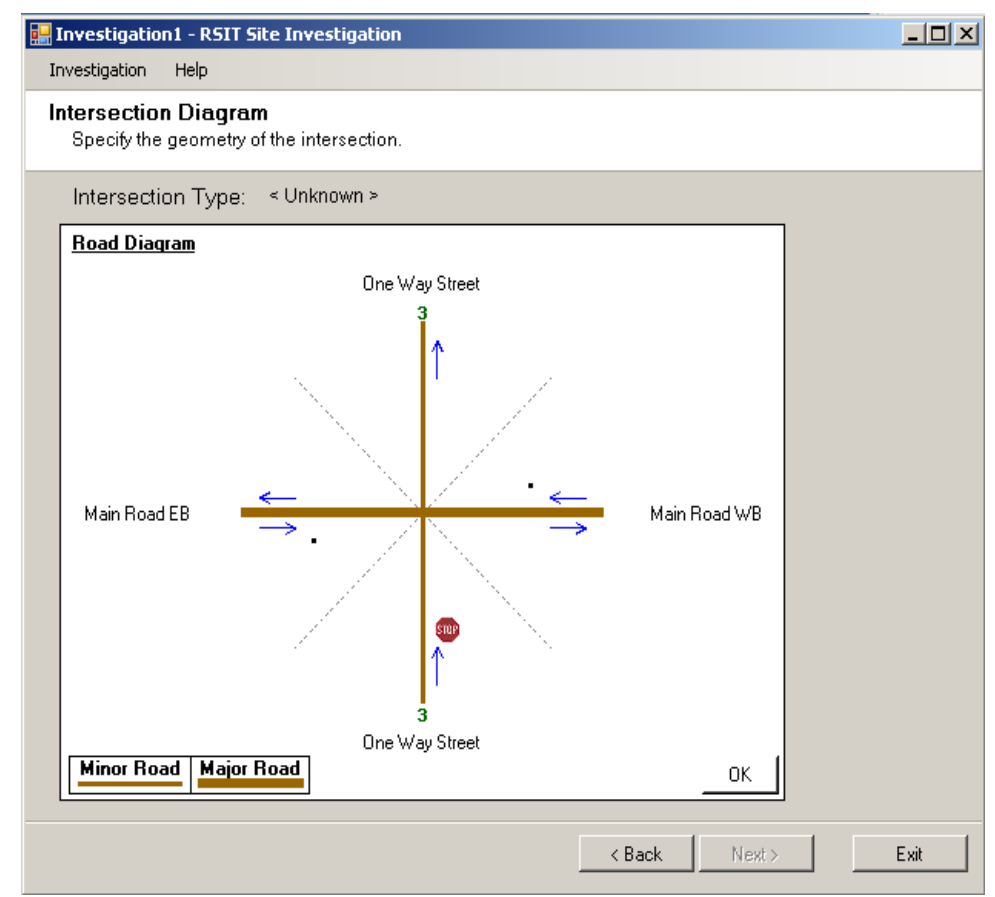

Figure 7-12 Intersection with a major and minor roads 


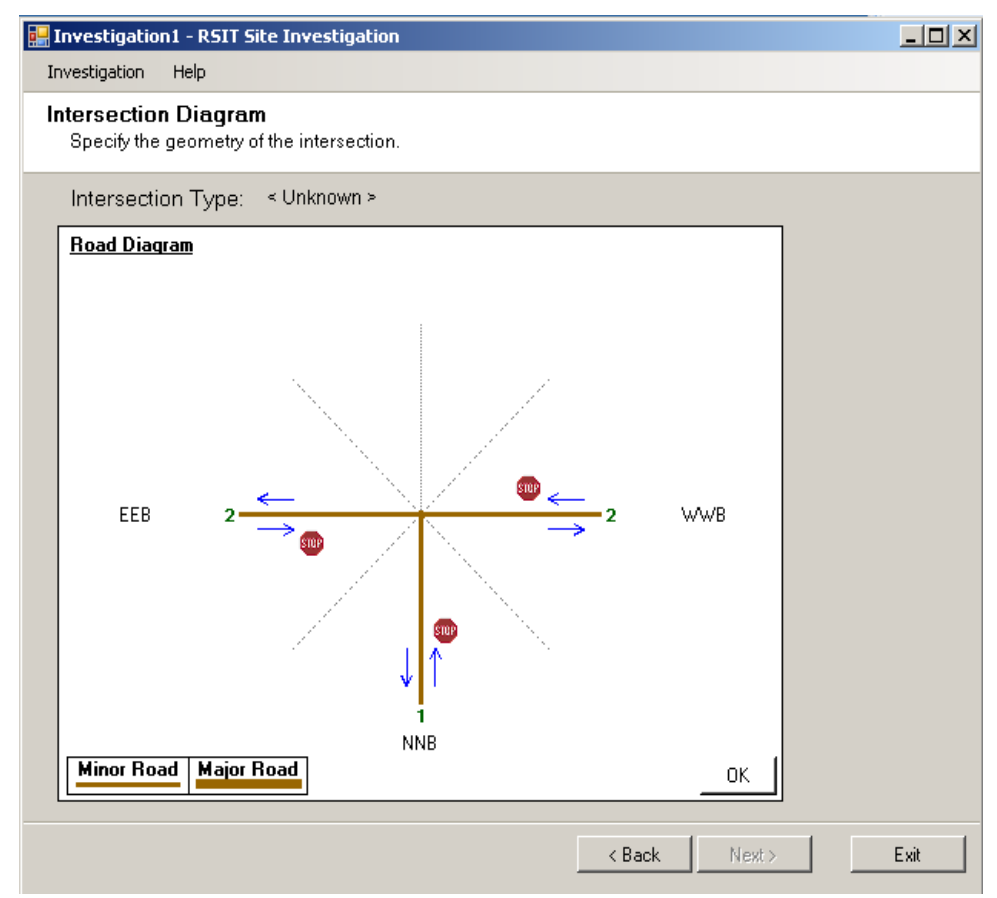

Figure 7-13 Intersection with a road that is not major

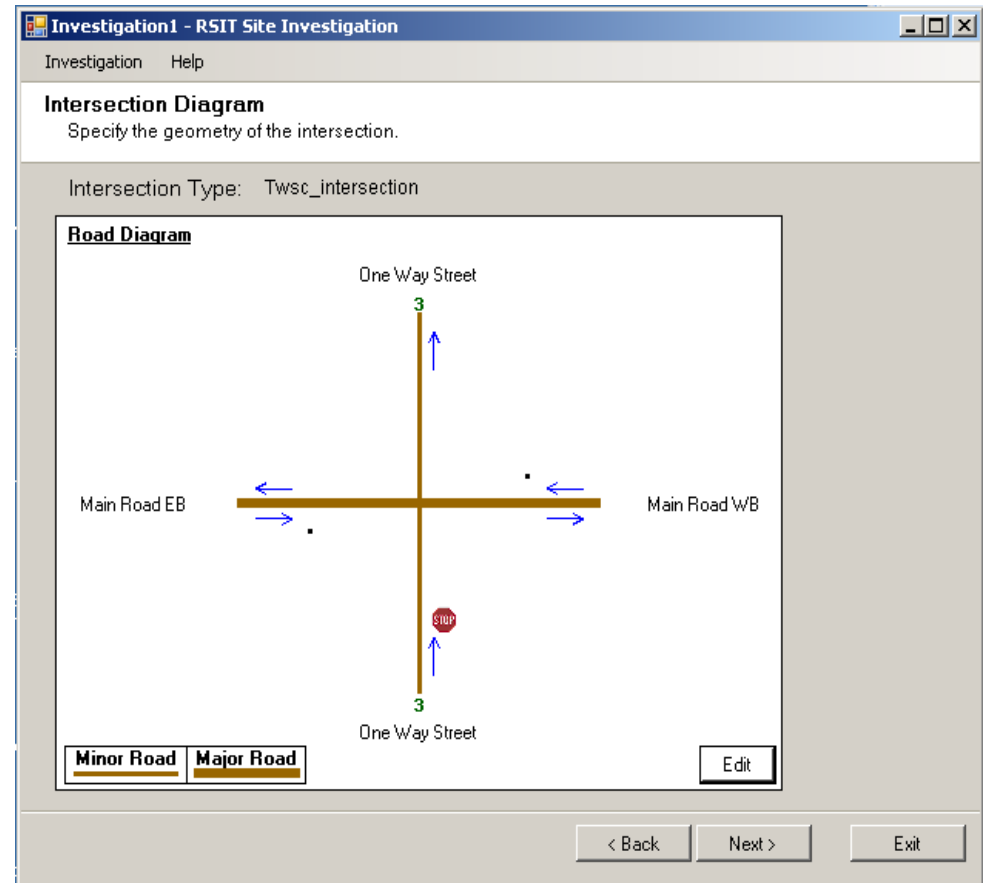

Figure 7-14 An example two-way stop-controlled intersection 


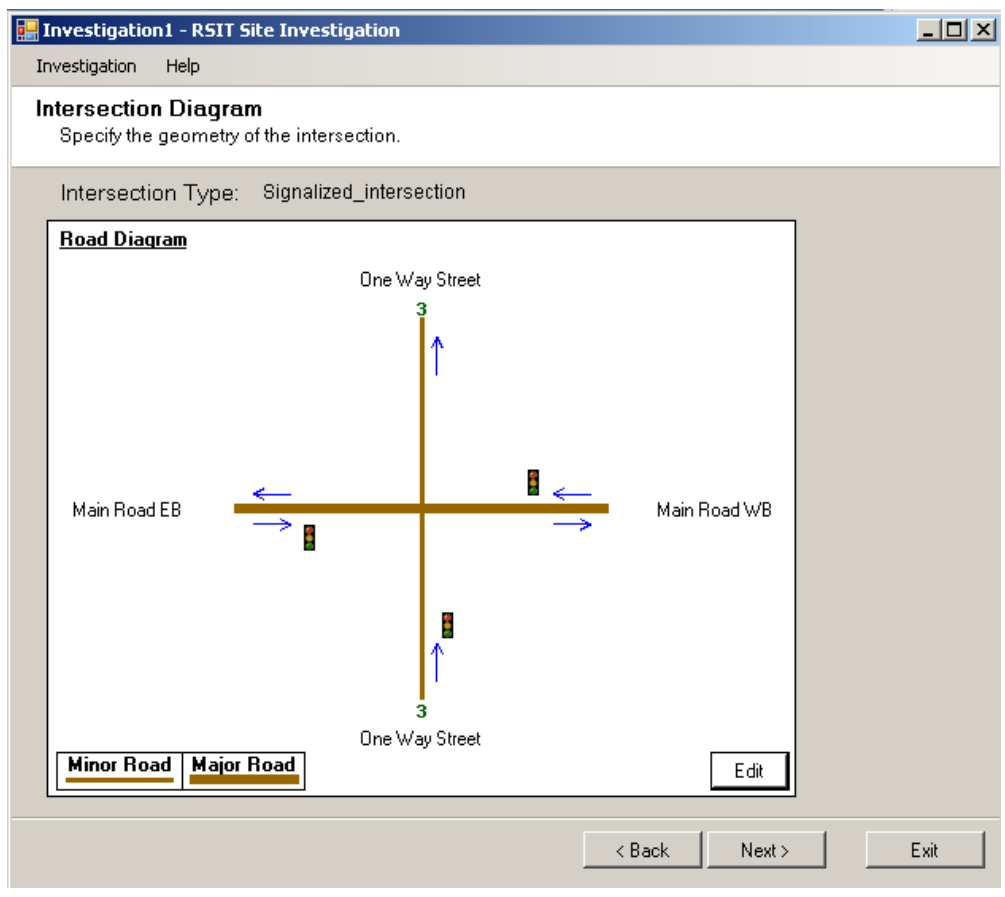

Figure 7-15 An example signalized intersection

RSIT2 checks the entered diagram to classify the type of road. The recognized type is displayed. In some cases, a message about an unrecognized type of road is displayed and the user must correct the entered diagram. Two examples of correct diagrams are shown in Figure 7-14 and Figure 7-15.

As mentioned earlier, correctly drawing a road/intersection diagram is important. It defines the type of the investigated road site, and the names the road components. These names are entered to the knowledge base rules to give them local/current meaning relevant for the site investigation. The knowledge base is then pre-processed based on the information entered in the road/intersection diagram and irrelevant checks removed from the generic knowledge base. The customized checklist is to be displayed in the checklist interface ready for use.

After successful entering the diagram, the user presses the Next button. 


\section{Checklist}

An example checklist displayed to the user after leaving the Intersection Diagram is shown in Figure 7-16. The user can perform checks in any order although the displayed order improves the investigation efficiency (reduces the number of checks required to complete the investigation). The savings are accomplished by eliminating checks that are not needed any longer because all the countermeasures supported by these checks have already been eliminated based on other checks performed earlier.

The checklist may be used in two modes: Hide mode (recommended) and Show All mode. In the Hide mode all processed and eliminated by RSIT checks are immediately removed from the display. The user sees only the checks that are currently remaining for processing.

The user can switch to the Show All mode at any time by pressing the Show All button. An example list in this mode is shown in Figure 7-17. The checks in shaded area are off-

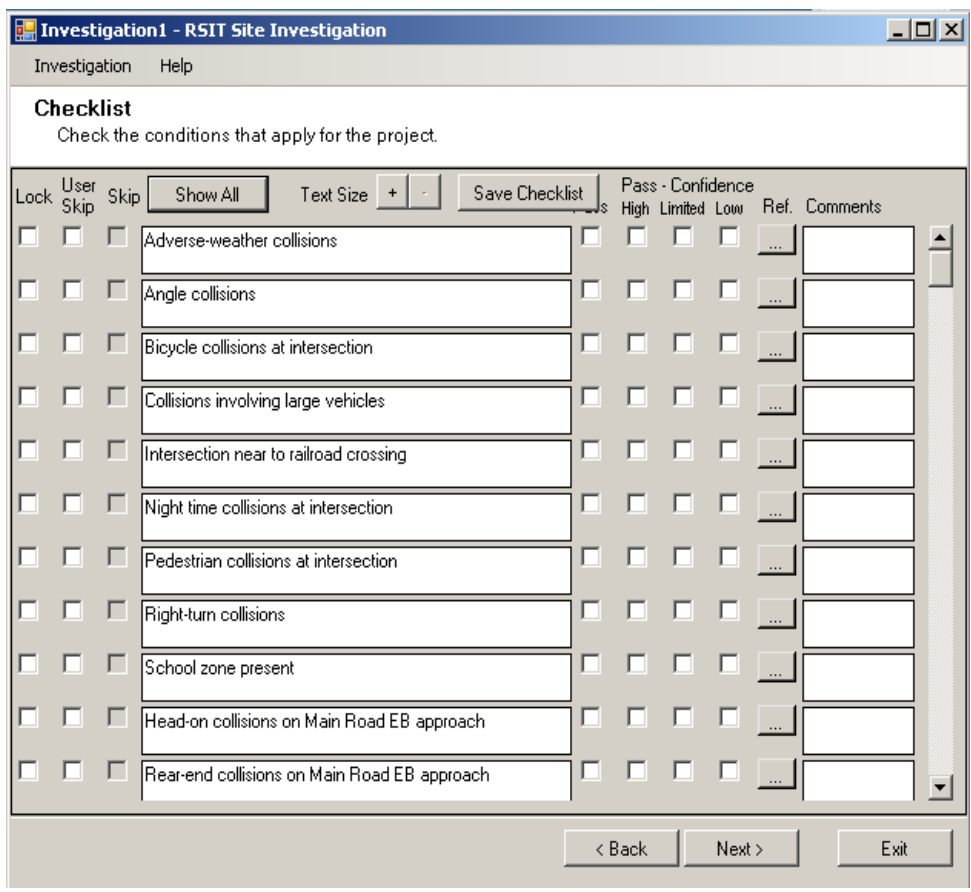

Figure 7-16 RSIT2 checklist interface with an example checklist 


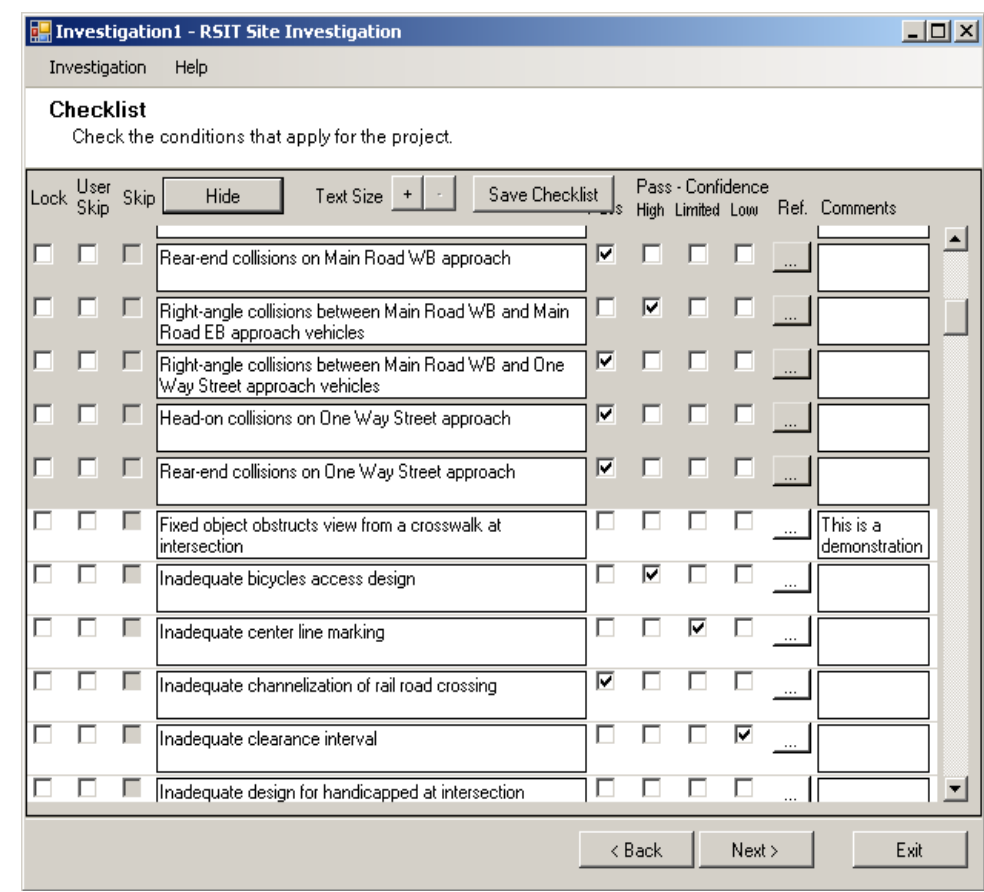

Figure 7-17 Checklist in the Show All mode

site checks that are typically processed before the investigation in the field. These checks appear on the list before other checks. All the checks are displayed together with user's passing/failing decision documented through marking proper boxes. These decisions can be changed by simply selecting different boxes. RSIT reprocesses the checklist immediately after each change is made to eliminate from processing or bring back checks for processing. Pressing the Hide button again hides both the already processed checks and the unneeded checks.

The Lock and User Skip columns of boxes in the front of the checklist give the user additional control over the checking process. A marked Lock box does not allow elimination of the marked check even if the check is not needed. A marked User Skip box eliminates the check regardless if it is needed or not. The third Skip column indicates if a given check has been skipped by RSIT2 based on checks already processed. Marks in this column are made by RSIT2 and not by the user. 
A check is performed if it has not been skipped by the time of its processing. The outcome is entered by selecting one of the four options following the check: No Pass, Pass - Confidence High, Pass - Confidence Limited, or Pass - Confidence Low. If the checked condition does not occur at the investigated site, then the No Pass option is selected, the other three options confirm the condition although at different levels of confidence. These values are set by the user in the Intersection Information window. These values are used to estimate the expected percent crash reduction as described in the following part of this manual.

If the user needs additional guidance before performing a check, he/she may call relevant references by selecting the button Ref (Reference) in the column next to the Pass boxes. A pop-up window will appear with available materials. No reference is available if the button is disabled.

The Comments column allows the user add any comments related to performed checks. All the user-entered comments are retained for future reference in a saved checklist. A

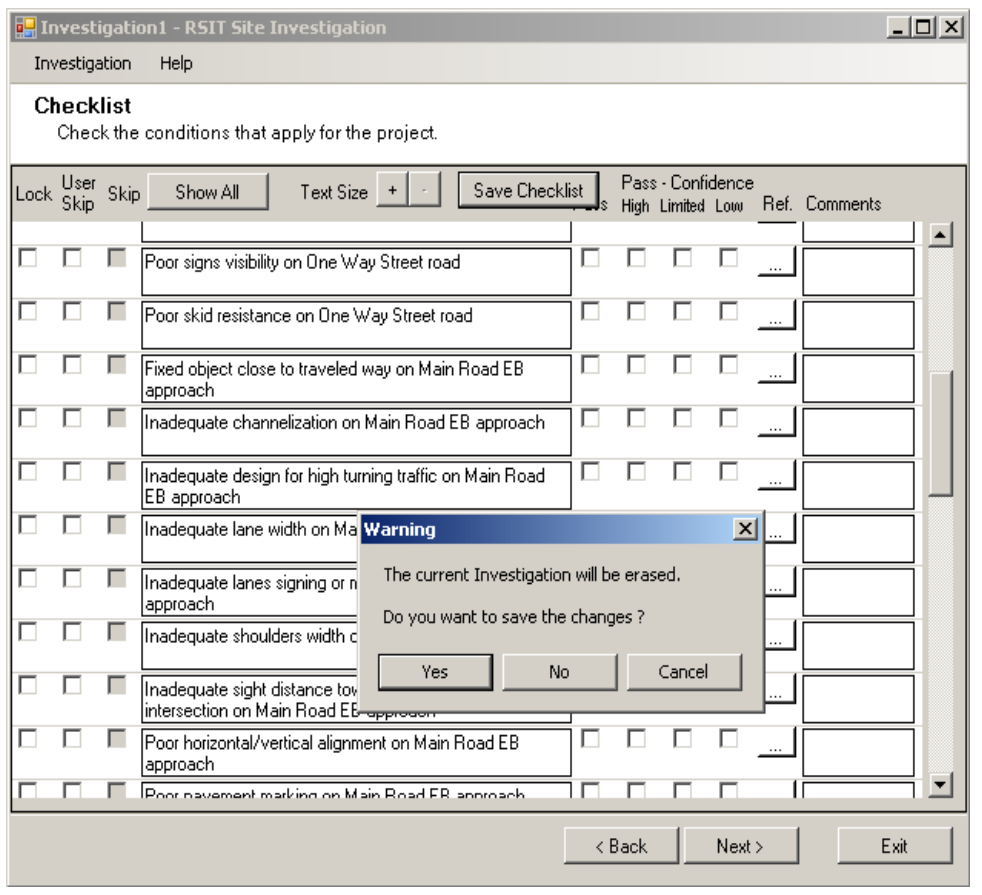

Figure 7-18 Warning triggered by the user's attempt to load another existing investigation log file 


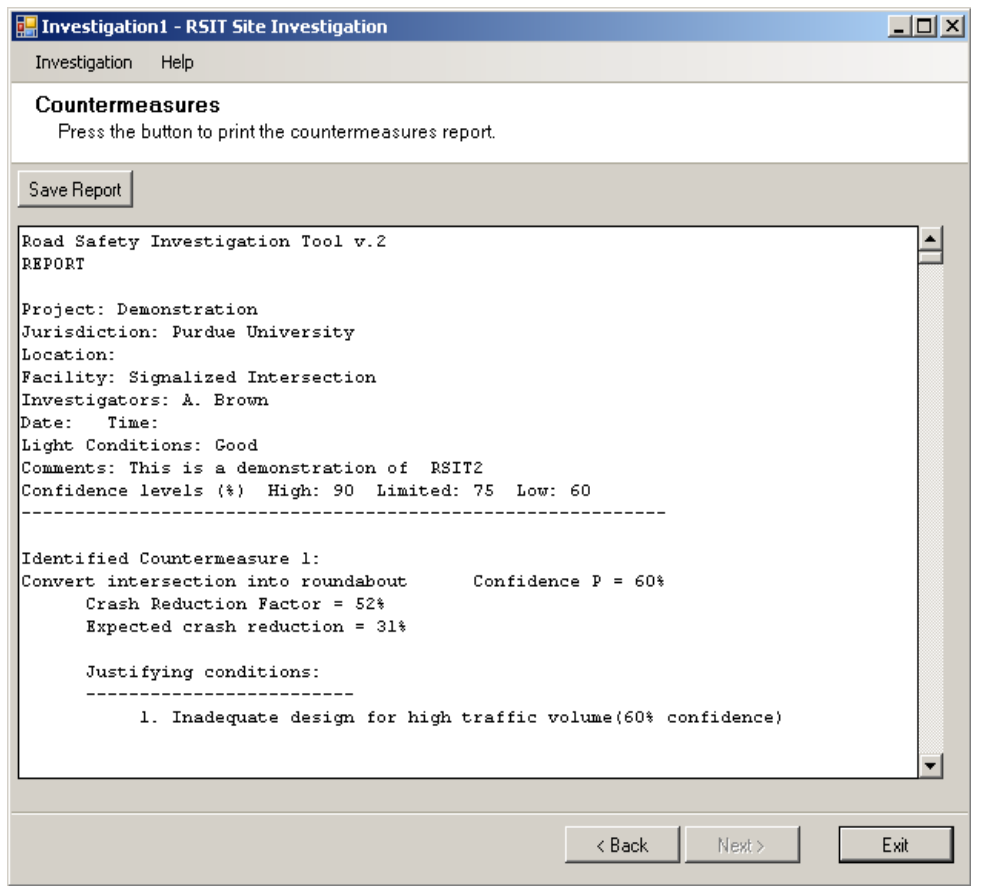

Figure 7-19 The top of the list of identified countermeasures

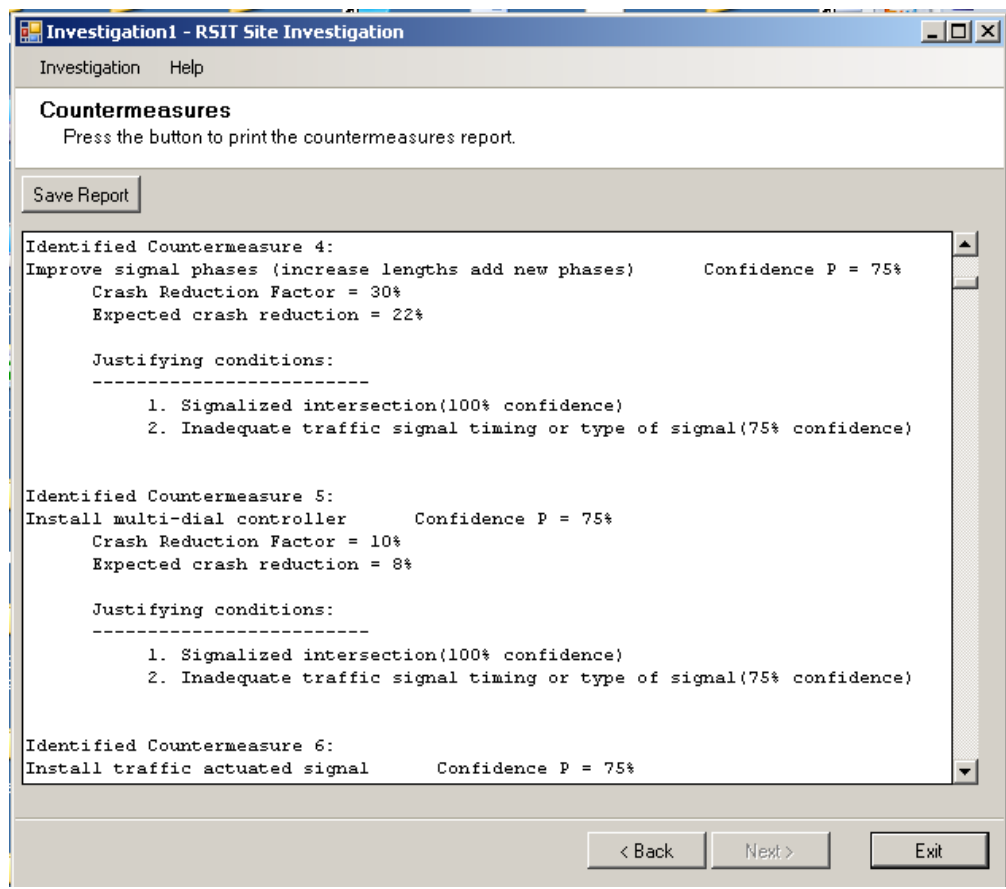

Figure 7-20 Example countermeasures identified by RSIT2 
comment appears also in the investigation report if the corresponding condition passes and the countermeasure supported by this condition is selected.

The user has an option to save the current investigation state for future continuation by pressing the Investigation option and use Save or Save As options. Any other actions such as initiating a new investigation or loading another existing one triggers a warning as the one shown in Figure 7-18.

The user has an option to save the current checklist with documented all operations on the checklist including the user comments in the last column by pressing the Save Checklist button. The saved file is the comma delimited text and can be opened with any text editor and by Excel.

The user can move to the previous window with the Intersection Diagram but any attempt to edit the diagram will cause loosing the current investigation if it is not saved because the checklist has to be recreated each time the diagram changes. The user can also move to the next window to learn what countermeasures have been identified so far (Figure 7-19). The format of the text in the Countermeasures window is exactly the same as the investigation report saved by the user.

\section{Site Investigation Report}

After the investigation is completed, the rules included in the knowledge base are used jointly with the results of the investigation (passed checks) to identify the countermeasures that are supported with the investigation results. A list of these countermeasures is displayed together with the successfully passed checks that support these countermeasures (Figure 7-19 and Figure 7-20). 
The user can save the investigation results in the format displayed in Figure 7-19 and Figure 7-20 by pressing the Save Report button. RSIT2-generated report includes four components:

1) The identification information including the location, investigators information, date, time of investigation, weather and other pertaining details.

2) The identified countermeasures with the local conditions that support these countermeasures. The confidence $P$ that the countermeasure is justified is the product of confidence levels of supporting conditions. A low value of $\mathrm{P}$ indicates that the local conditions weakly support the identified countermeasure because of the investigator's uncertainty about the conditions. If the investigator is fully convinced that all the supporting conditions appear at the investigated site then $\mathrm{P}$ is high and close to 100 percent.

3) The estimated effectiveness of the identified countermeasure expressed with the expected crash reduction. The expected crash reduction is the crash reduction factor times the confidence about the countermeasure $\mathrm{P}$ divided by 100. This expectancy reflects both the effectiveness of the countermeasure and the level of confidence that the countermeasure is justified. The assumption behind this calculation is that if the countermeasure is not justified then no crash reduction is expected.

The report is in the text format and is editable by any available text editor. 


\section{CONCLUSIONS}

A summary of the research findings, conclusion and recommendations for future research are presented in this chapter.

The analysis of the existing tools of high-crash site investigation revealed a need for a more advanced tool than checklists. Although checklists help investigate sites in a more organized manner than ad hoc investigation, they may lead to wasted time and incorrect findings if a considerable number of redundant checks are performed.

The proposed prototype of RSIT guides an investigation team through a sequence of checks and determines the probable roadway factors contributing to crash occurrence. Te limited to two-way stop-controlled intersection knowledge base was developed and evaluated. The knowledge structure and functionality of the GUI are easy to understand even for non-experts. The developed knowledge base provides a more detailed explanation for each individual roadway deficiency compared to the findings from the road safety report, which confirms the previous statement that even experienced investigators can overlook important factors. The prototype evaluation part confirmed usefulness of the proposed prototype of RSIT. Moreover, the feedback from evaluation part as well as SAC members' comments emphasized the need to create more flexible and improve version with extended knowledge base. As a result, the RSIT 2 was developed.

The RSIT 2 contains significant improvements over the prototype of RSIT.

First, the extended knowledge base for: all-way stop controlled intersection, signalized intersection, road segment and rail road crossing was developed. Secondly, a new structure of representing developed knowledge was established. The rule-base structure allows for more flexible and user friendly knowledge representation. Next, the editor was developed. This editor is a very useful tool and allows creating a new knowledge or easily updating existing knowledge. In addition, list of conditions and countermeasures used in the process of building the knowledge base is introduced. This list contains set of parameters associate with each condition and countermeasure. As a result, the efficiency 
evaluation under uncertain condition is introduced. This approach allows evaluating countermeasure safety by using reviewed CRFs values, and providing a safety ranking of proposed conditions. 


\section{List of references}

1. Albert Gan, at all. Update of Florida Crash Reduction Factors and Countermeasures to Improve the Development of District Safety Improvement Project. Miami, April 2005. (*)

2. American Traffic Safety Services Association Low Cost Local Road Safety Solutions Virginia, March 2006. (*)

3. Carrie E. Kindler, Nicholas D. Antonucci, Ingrid B. Potts, Timothy R. Neuman, Richard, M. Wood. IHSDM Intersection Diagnostic Review Model. http://www.tfhrc.gov/safety/ihsdm/pubs/02045/. (Accessed 06/09/2008)

4. Charles W. Niessner Crash Reduction Factors for Traffic Engineering and Intelligent Transportation Systems (ITS) Improvements: State-of-Knowledge Report Research Results Digest 299 National Cooperative Highway Research Program, November 2005. (*)

5. Christopher M. Monsere at all. Update and Enhancement of ODOT's Crash Reduction Factors. FHWA-OR-DR-06-11, Portland State University, June 2006. (*)

6. D.W. Harwood, J.M. Mason, R.E. Brydia, M.T. Pietrucha, G.L. Gittings. Intersection Sight Distance, NCHRP Report 383. Transportation Research Board, Washington, DC. 1996.

7. Evaluating Intersection Improvements: An Engineering Study Guide, NCHRP Report 457. http://onlinepubs.trb.org/onlinepubs/nchrp/esg/esg.pdf. Accessed July 1 2006. (Accessed 06/09/2008)

8. FHWA/USDOT "Toolbox of Countermeasures and Their Potential Effectiveness for Roadway Departure Crashes" Project number: FHWA-SA-07-013, September 2007. (*)

9. FHWA/USDOT "Desktop Reference for Crash Reduction Factors" Report number: FHWA-SA-07-015, September 2007. (*)

10. FHWA/USDOT "Toolbox of Countermeasures and Their Potential Effectiveness for Pedestrian Crashes" Project number: FHWA-SA-014, September 2007. (*)

11. FHWA/USDOT/ITE "Toolbox of Countermeasures and Their Potential Effectiveness for Intersection Crashes" September 2007. (*)

12. FHWA/USDOT "IHSDM" Washington D.C., 2006: http://www.tfhrc.gov/safety/ihsdm/pubs /06100/06100.pdf (Accessed 06/09/2008) 
13. http://www.beatthejam.org/planning/Safety/2005Data/Crash\%20Reduction $\% 20 \mathrm{Fa}$ ctors\%20Table\%202005.pdf (*) (Accessed 06/09/2008)

14. Illinois Department of Transportation Crash Reduction Factors for HSIP Program Projects. November 2006 (http://www.dot.state.il.us/safetyEng/Appendix\%20E.pdf). 06/09/2008)

15. Illinois Department of Transportation, Williamson County Road Safety Audits Report, Springfield, Illinois 2006

16. J. Treat et al. Tri-level Study of the Causes of Traffic Accidents: Final Report Volume 1. Technical Report, Federal Highway Administration, U.S. DOT, May 1979.

17. James L. Pline., Institute of Transportation Engineers. Traffic Engineering Handbook, 5th Edition, Washington, D.C. 1999.

18. Kenneth R. Agent at all. Development of Accident Reduction Factors. June 1996. $(*)$

19. Krammes, R.A., and Hayden, C. (2003) "Making Two-Lane Roads Safer" Public works January/February $2003 \cdot$ Vol. $66 \cdot$ No. 4.

20. LTAP, Hazard Elimination Study for $6^{\text {th }}$ Street and Salem Street, Lafayette, Indiana West Lafayette, 2006

21. LTAP, Hazard Elimination Study for Grant Street and Stadium Avenue West Lafayette, Indiana West Lafayette, 2006

22. LTAP, Hazard elimination study for Locust Rd. @ Ireland Rd. St. Joseph County, Indiana West Lafayette, 2006.

23. NCHRP Report 440 "Accident Mitigation Guild for Congested Rural Two-Lane Highways" Transportation Research Board (TRB), Washington, DC., 2000. (*)

24. NCHRP Report 500 Series, Volume 5. A Guide for Addressing Unsignalized Intersection Collisions. Transportation Research Board, Washington, D.C., 2003.

25. NCHRP Report 500 Series, Volume 5. A Guide for Addressing Unsignalized Intersection Collisions. Transportation Research Board, Washington, D.C., 2003. $(*)$

26. NCHRP Synthesis 336 Series. Road Safety Audits, A Synthesis of Highway Practice.” Transportation Research Board, Washington, D.C., 2004.

27. NCHRP Synthesis 383 Series. "Intersection Sight Distance" Transportation Research Board, Washington, D.C., 1996. 
28. NCHRP Report 457 "Evaluating Intersection Improvements: An Engineering Study Guide.” Transportation Research Board, Washington, D.C., 2006.

29. NCHRP Synthesis 500 Series, Volume 10 "A Guide for Reducing Collisions Involving Pedestrians" Transportation Research Board, Washington, D.C., 2004. $(*)$

30. NCHRP Synthesis 500 Series, Volume 12 "A Guide for Reducing Collisions at Signalized Intersection" Transportation Research Board, Washington, D.C., 2004. $(*)$

31. NCHRP Synthesis 500 Series, Volume 13 "A Guide for Reducing Collisions Involving Heavy Trucks" Transportation Research Board, Washington, D.C., 2004. (*)

32. NCHRP Synthesis 500 Series, Volume 14 : "A Guide for Reducing Crashes Involving Drowsy and Distracted Drivers" "Transportation Research Board, Washington, D.C., 2005. (*)

33. NCHRP Synthesis 500 Series, Volume 3: A Guide for Addressing Collisions with Trees in Hazardous Locations" Transportation Research Board, Washington, D.C., 2003. (*)

34. NCHRP Synthesis 500 Series, Volume 4:" Guide for Addressing Head-On Collisions" Transportation Research Board, Washington, D.C., 2003. (*)

35. NCHRP Synthesis 500 Series, Volume 6 "A Guide for Addressing Run-Off-Road Collisions” Transportation Research Board, Washington, D.C., 2003. (*)

36. NCHRP Synthesis 500 Series, Volume 7:" A Guide for Reducing Collisions on Horizontal Curves" Transportation Research Board, Washington, D.C., 2003. (*)

37. NCHRP Synthesis 500 Series, Volume 8 "A Guide for Reducing Collisions Involving Utility Poles" Transportation Research Board, Washington, D.C., 2004. (*)

38. North Caroline Department of Transportation North Carolina Project Development Crash Reduction Factor Information May 2007 (revised). (*)

39. Olson, Paul L. Forensic Aspects of Driver Perception and Response. Tucson, AZ: Lawyers \& Judges Pub. Co., c1996

40. Oregon Department of Transportation, Traffic Engineering and Operations Section Crash Reduction Factors. March 2006. (*) 
41. Primer A., Latham, F.E. and J.W. Trombly. Low Cost Traffic Engineering Improvements. FHWA-OP-03-078. Federal Highway Administration, Washington, D.C., April 2003.

42. Proctor, Robert W., Trisha Van Zandt. Human factors in simple and complex systems. Boston: Allyn and Bacon, c1994.

43. Road Safety Assessment Road safety audits for Williamson county, Illinois. Springfield 2006

44. Senders, John W., Neville P. Moray. Human error: cause, prediction, and reduction / analysis and synthesis. Hillsdale, N.J.: L. Erlbaum Associates, 1991.

45. Tarko, Andrew P., Mayank Kanodia. Hazard Elimination Program - Manual on Improving Safety of Indiana Road Intersection and Section. FHWA/IN/JTRP2003/19, Purdue University, West Lafayette, February 2004. (*)

46. U.S. Department of Transportation. Traffic Safety Facts 2004. National Center for Statistic and Analysis, Washington 2005.

47. Washington, S., and Oh, J., Bayesian methodology incorporating expert judgment for ranking countermeasure effectiveness under uncertainty: Example applied to at grade railroad crossings in Korea, Accident Analysis and Prevention 38 (2), 2006, pp. 234-247.(*)

48. Wiener E. L. Vigilance and inspection. In J.S. Warm (ed.) Sustained Attention in Human Performance. New York, 1984. 


\section{APPENDICES}


Appendix A

Indiana Safety Review Checklist 


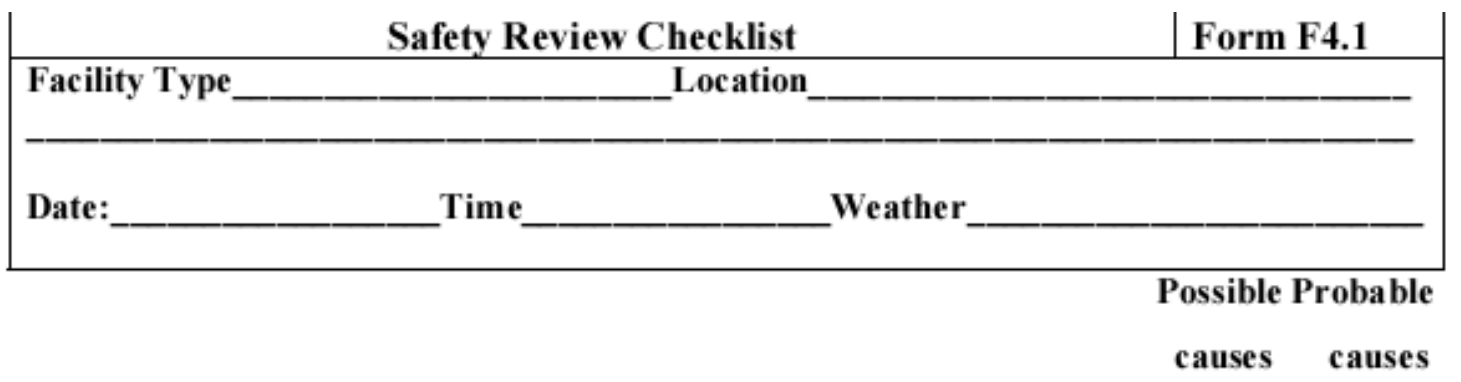

\section{Group A}

\section{Moving lanes}

Lane widths are inadequate for vehicle classes that are common to the location

Number of lanes inadequate for traffic

\section{Readability}

Lanes end abruptly without prior warning (lanes are not aligned)

\section{Auxiliary/Turning Lanes}

Inadequate advance warning of lane drops

\section{Driveways}

Improper location of driveways(e.g. driveways are too close to the intersection)

Driveways are closely spaced

Inadequate visibility of driveways

\section{Shoulders}

Shoulder width inadequate for vehicle classes that are common to the intersection

Inappropriate shoulder surfacing

Rumble strips not installed where warranted

Shoulders are poorly maintained

Insufficient contrast of shoulders

\section{Horizontal and vertical alignment}

Horizontal or vertical al ignment affect the visibility of the intersection

Abrupt changes in elevation

Inadequate visibility at sag and crest curves

Location at high side/low side of superelevation

Excessive curves that cause sliding in adverse weather condition

Excessive grades present, which could be unsafe in adverse weather conditions

\section{Pavement markings / Delineation}

Pavement markings (center lines, edge lines etc) are not clearly visible in day or night time conditions 


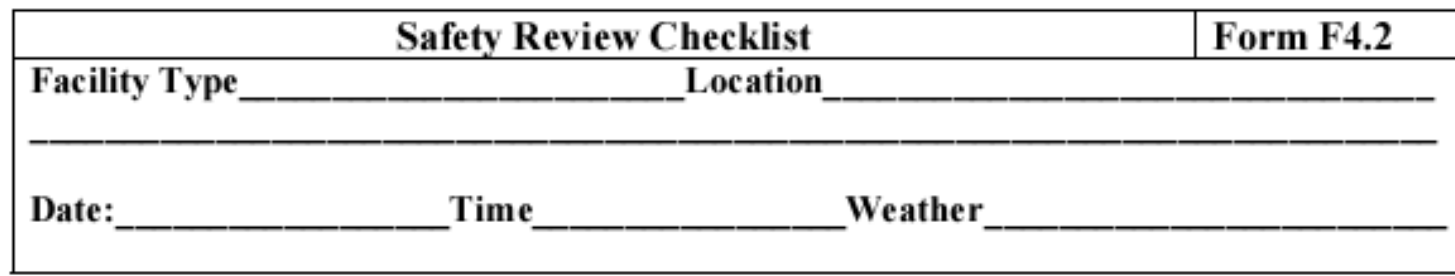

Possible Probable

causes causes

All necessary pavement markings not present

Presence of too many markings confusing the users

Pavement markings are inappropriate for the location

Old pavement markings have not been removed which may cause safety problems

Inadequate retroreflectivity of existing markings.

Road markings do not have sufficient contrast with the surfacing

\section{Light conditions}

Inadequate visibility during night time conditions

Severe headlight glares during night time operations

Luminaries create glare for road users on adjacent roads

Adjacent road lighting affects driver perception of road

Lighting interferes with traffic signs

Inadequate lighting for signs

\section{Signs}

Inadequate visibility of necessary, regulatory, warning and guide signs

in normal and adverse weather conditions

Incorrect location of regulatory, warning and directory signs (i.e., proper

height, offset, distance in advance of hazard)

Signs obstruct visibility

Signs are missing/redundant/broken

Signs are not maintained properly

Signs contradict each other

Any existing signs present those are no longer applicable

Signs are inconsistent with respect to standard fonts and phrases

Signs cannot be read from adequate safe distance

\section{Sight distance}

Inadequate sight distance, stopping sight distance or decision sight distance

Sight lines are obstructed by signs, buildings, landscaping, vegetation etc. 


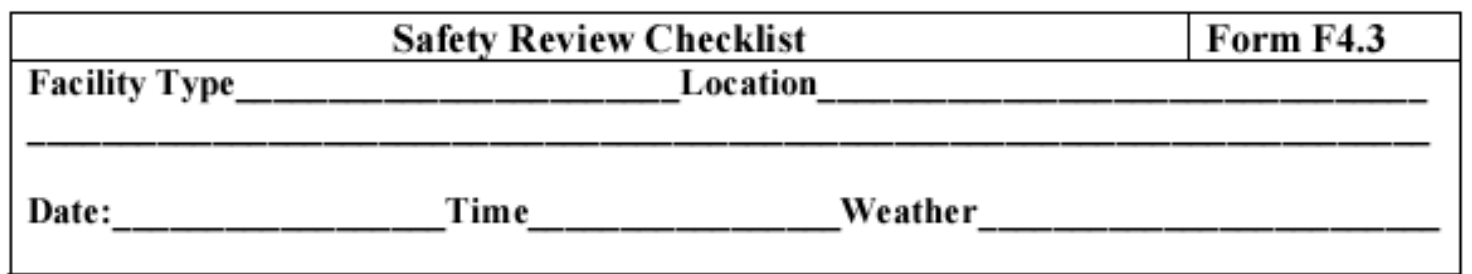

\section{Possible Probable \\ causes causes}

Sight lines are obstructed temporarily by parked vehicles, snow storage, seasonal foliage, etc.

Opportunity for passing is insufficient

\section{Pavement conditions}

Abrupt changes in pavement condition

\section{Skid resistance}

Presence of locations that have inadequate skid resistance

\section{Pavement defects}

Pavement has defects, which could result in safety problems(e.g. loss of steering control)

Pavement is not free of distresses (i.e. potholes, rutting, etc)

Changes in surface type (e.g. pavement ends) have drop offs /

poor transitions

Presence of loose aggregate/gravel in pavement

Presence of bleeding in pavement due to excess asphalt

\section{Drainage}

Presence of areas in pavement where ponding or sheet flow of water occurs resulting in safety problems

Drainage channel inappropriate for topography

Possibility of surface flooding or overflow from surrounding or intersecting drains and water courses

Presence of accumulated water during rainy conditions

Culverts are not protected

Embankments are too steep

\section{Barriers}

Clear zone is narrow

Guiderails are not designed properly

Inappropriate transition from one barrier to another 


Date:________ Time_________ Weather

Possible Probable

causes causes

Inadequate retro reflectivity of barriers

Inappropriate treatment of barrier ends

Median barriers sufficiently offset from roadway

\section{Medians}

Inappropriate spacing between median crossovers

Inadequate slopes of grass median

\section{Special Road Users}

Travel paths for pedestrians and cyclists are not properly signed and / or marked

Bus stops are not safely located with adequate clearance and visibility

from the traffic lane

Driver behavior indicating potential safety problems

Overrepresentation of a particular age group as users raising safety concerns

Too many drivers violating the speed limit

Skid marks

Frequent off tracking

Illegal parking

Pedestrians crossing illegally (jaywalking)

\section{Group B}

\section{Readability}

Confusing geometry which encourages wrong way entry

Layout is not consistent with adjacent interchanges

\section{Auxiliary/Turning Lanes}

Queues stretch beyond the auxiliary lanes

Deceleration length is short

Tapers are not marked properly

Tapers are not designed properly 


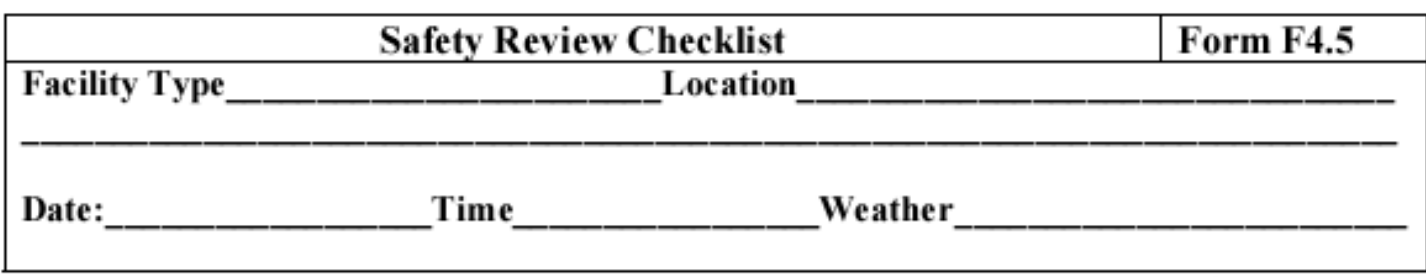

Possible Probable

causes causes

\section{Signs}

Inconsistency of signs and markings with adjacent interchanges

Driver behavior indicating potential safety problems

Presence of congestion (excessive queues and delays) leading to safety problems

Erratic maneuvers

Rapid breaking

Traffic conflicts

Wrong way entry

Violation of right of way

\section{Group C}

\section{Readability}

Intersection layout is complex (e.g. 5 leg intersection)

Layout is not consistent with adjacent intersections

\section{Channelization}

Presence of large unused area at the intersection

Island required to channel traffic at the intersection

Inadequate dimensions of the island

Inadequate visibility of the island

Confusing layout of islands

\section{Horizontal and vertical alignment}

Location of intersection (before, inside or after) a curve

Presence of sharp corners

\section{Curvature for turning movements}

Minimum design not provided for left and right turns(Insufficient widths and curves)

\section{Markings}

Stop bar not marked properly 


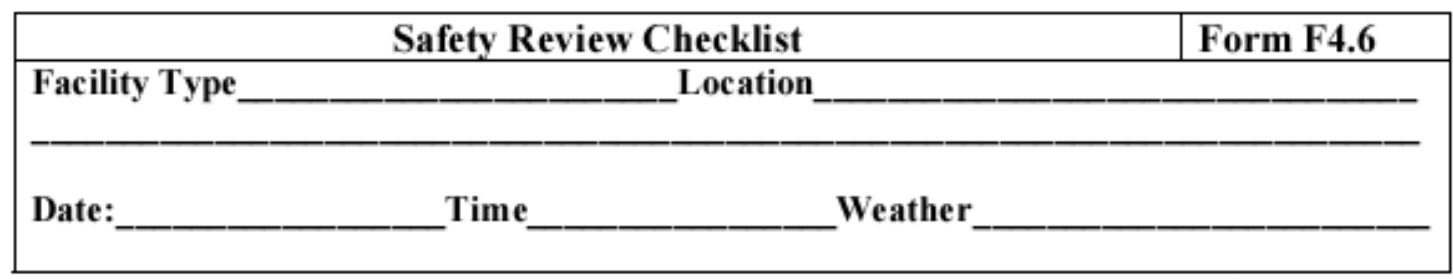

Possible Probable

causes causes

\section{Signs}

Inconsistency of signs and markings with adjacent intersections

\section{Sight distance}

Sight triangle is insufficient

\section{Median}

Excessively wide median

\section{Special Road Users}

Crossing points for pedestrians and cyclists not properly signed and / or marked

\section{Driver behavior indicating potential safety problems}

Violation of stop signs

Incorrect stopping position

Frequent blocking of continuous lanes by queues where auxiliary lanes do not exist

\section{Group D}

\section{Light conditions}

Lighting interferes with traffic signals

\section{Signals}

Inadequate warning for signals not visible from an appropriate sight distance?

(i.e., signs, flashing light, etc.)

High intensity signals/shields are not provided where sunset and sunrise glare may be a problem

Inadequate visibility of signals due to presence of billboards etc. (visual clutter) Traffic signals adjacent to roads affect driver perception of the intersection. Primary and secondary signal heads are not properly positioned Confusing signals for left turning vehicles (yellow trap, conjunction of permitted-protected phasing and lagging left-turns, lead lag phasing)

Auxiliary heads not provided where necessary

Bases not installed at the proper height 


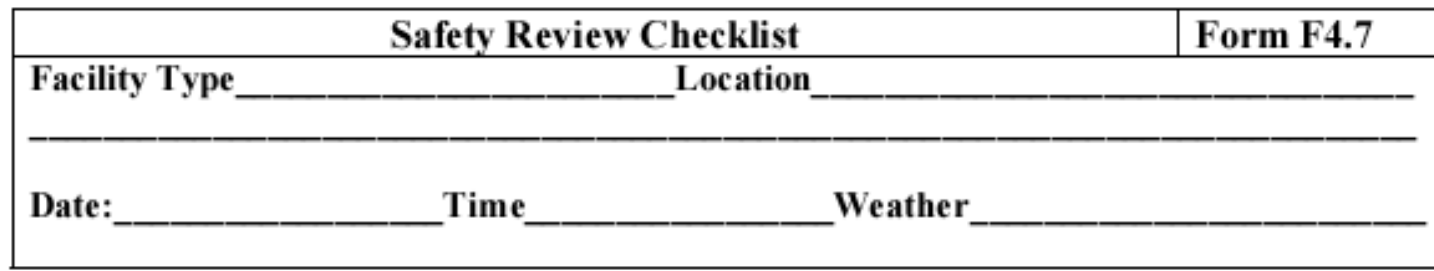

\section{Possible Probable \\ causes causes}

\section{Signal Phasing}

Minimal green and clearance phases are not provided

Signal phasing plan inconsistent with adjacent intersections

A dedicated left turn signal is required

\section{Light conditions}

Light interferes with traffic signals

Driver behavior indicating potential safety problems

Significant number of people running on red

\section{Group E}

\section{Railroad crossings}

Absence of railroad crossing signs on each approach to railroad crossings

Absence of advance warning signs at railroad crossing approaches

Presence of obstructions at rail road crossings which restrict sight distance

Grades of roadway approach to railroad crossings are not flat enough and encourage prevent vehicle snagging 


\section{Appendix B}

\section{Countermeasures List}

\begin{tabular}{|c|c|c|c|}
\hline Countermeasures & CRF & Cost & Road \\
\hline Install overhead STOP signs on \#A1 approach & 20 & 2 & RRC \\
\hline Install overhead STOP signs on \#A2 approach & 20 & 2 & TWSC,AWSC \\
\hline Add sign warning about off-road collisions on \#A1 approach & 10 & 1 & TWSC,AWSC \\
\hline Add sign warning about off-road collisions on \#E1 exit & 10 & 1 & TWSC,AWSC \\
\hline Add special signing & 10 & 1 & RS \\
\hline Add stop-lines in median opening on \#R1 road & 10 & 1 & TWSC \\
\hline Adjust minimum green or extension time & 10 & 2 & SIGN \\
\hline Adjust phase time and cycle time & 15 & 2 & SIGN \\
\hline Adjust yellow change interval & 8 & 2 & SIGN \\
\hline $\begin{array}{l}\text { Adjust signal offsets at upstream signalized intersection(s) on \#R1 road to } \\
\text { create more gaps }\end{array}$ & 20 & 3 & TWSC \\
\hline Channelize driveway(s) on \#A1 approach & 50 & 4 & TWSC,AWSC \\
\hline Channelize driveway(s) on \#E1 exit & 50 & 4 & TWSC,AWSC \\
\hline Channelize driveway(s) on \#R1 road & 50 & 4 & RS \\
\hline Change horizontal/vertical alignment on \#A1 approach & 42 & 5 & RRC,SIGN \\
\hline Change horizontal/vertical alignment on \#A2 approach & 42 & 5 & TWSC,AWSC \\
\hline Change horizontal/vertical alignment on \#R1 road & 42 & 5 & TWSC,AWSC \\
\hline Apply chip seal or slurry seal on \#A1 approach & 10 & 4 & TWSC,AWSC \\
\hline Apply ship seal or slurry seal on \#E1 exit & 10 & 4 & TWSC,AWSC \\
\hline Apply ship seal or slurry seal on \#R1 road & 10 & 4 & RS \\
\hline Close intersection leg or convert to one-way street & 40 & 4 & SIGN \\
\hline Close or relocate driveway(s) on \#A1 approach & 50 & 3 & TWSC,AWSC \\
\hline Close or relocate driveway(s) on \#R1 road & 50 & 3 & RS \\
\hline Close or relocate driveway(s) on \#E1 exit & 50 & 3 & TWSC,AWSC \\
\hline Close rail-road crossing point or install overpas & 90 & 5 & RRC \\
\hline Consider indirect left-turns on \#R1 road & 35 & 5 & TWSC \\
\hline
\end{tabular}




\begin{tabular}{|c|c|c|c|}
\hline Countermeasures & CRF & Cost & Road \\
\hline Consider a lateral relocation of right-turn lanes on \#A1 approach & 20 & 3 & TWSC \\
\hline Consider traffic signals with protected left-turn phase & 40 & 4 & TWSC \\
\hline Construct interchanges & 50 & 5 & SIGN \\
\hline Construct pedestrian refuge islands and raised medians & 45 & 3 & TWSC,AWSC \\
\hline Convert a four-leg intersection to two $\mathrm{T}$ intersection & 57 & 5 & SIGN \\
\hline Convert two-way streets to a one-way pair & 35 & 5 & SIGN \\
\hline Convert two $\mathrm{T}$ intersection to one four-leg intersection & 15 & 5 & SIGN \\
\hline Convert intersection into roundabout & 52 & 5 & TWSC,AWSC \\
\hline $\begin{array}{l}\text { Coordinate the railroad and traffic signal to provide preemption to the traffic } \\
\text { signal when trains are approaching the intersection }\end{array}$ & 28 & 3 & SIGN \\
\hline Create one way streets & 45 & 4 & SIGN \\
\hline Cut trees and other roadside vegetation obstruct the view & 25 & 3 & RS \\
\hline Create additional parking spaces & 10 & 3 & RS \\
\hline Eliminating compound curves & 25 & 4 & RS \\
\hline Eliminate screening of RRC by physical objects & 63 & 4 & $\mathrm{RRC}$ \\
\hline Eliminate screening by physical objects of crosswalk at intersection & 63 & 3 & $\mathrm{RRC}$ \\
\hline Eliminate screening by physical objects on \#R1 road & 62 & 3 & RS \\
\hline Eliminate shoulder drop-off on \#A1 approach & 28 & 3 & TWSC,AWSC \\
\hline Eliminate shoulder drop-off on \#E1 exit & 28 & 3 & TWSC,AWSC \\
\hline Eliminate shoulder drop-off on \#R1 road & 28 & 3 & RS \\
\hline Employ emergency vehicle preemption & 70 & 3 & SIGN \\
\hline Enforce STOP sign compliance on \#A1 approach & 70 & 2 & TWSC,AWSC \\
\hline Enforce STOP sign compliance on \#A2 approach & 70 & 2 & TWSC \\
\hline Enhance delineation of sharp curves & 16 & 3 & RS \\
\hline Groove pavement surface on \#A1 approach & 27 & 3 & TWSC,AWSC \\
\hline Groove pavement surface on \#R1 road & 27 & 3 & RS \\
\hline Consider traffic calming through narrowing \#R1 road & 30 & 3 & TWSC,AWSC \\
\hline Implement automated enforcement of red-light running (cameras) & 14 & 2 & SIGN \\
\hline Implement lighting / illumination measures on \#R1 road & 30 & 3 & RS \\
\hline Improve (add) coordinating signals & 25 & 3 & SIGN \\
\hline Improve (add) phases (length) & 30 & 3 & SIGN \\
\hline
\end{tabular}




\begin{tabular}{|c|c|c|c|}
\hline Countermeasures & CRF & Cost & Road \\
\hline Improve channelization/delineation on \#A1 approach & 31 & 3 & TWSC,AWSC \\
\hline Improve clear zone on \#R1 road & 25 & 3 & TWSC,AWSC,RS \\
\hline Improve existing street lights at the rail-road crossing points & 40 & 3 & RRC \\
\hline Improve gates visibility/reflectivity (flashing lights along the gate) & 75 & 2 & $\mathrm{RRC}$ \\
\hline Improve intersection skew angle & 40 & 3 & SIGN \\
\hline $\begin{array}{l}\text { Improve maintenance of rail infrastructure (Motion Sensitive Devices Constant } \\
\text { Warning Time) }\end{array}$ & 50 & 4 & $\mathrm{RRC}$ \\
\hline Improve maintenance of train detective devices & 10 & 3 & $\mathrm{RRC}$ \\
\hline Improve maintenance of visual/audio signals & 15 & 2 & $\mathrm{RRC}$ \\
\hline Improve maintenance/work of existing gates & 35 & 2 & $\mathrm{RRC}$ \\
\hline Improve or restore superelevation & 26 & 3 & RS \\
\hline Improve street lighting & 40 & 3 & TWSC,AWSC,SIGN \\
\hline Correct lane width on \#A1 approach & 8 & 3 & TWSC,AWSC \\
\hline Improve pavement marking in median opening on \#R1 road & 21 & 3 & TWSC \\
\hline Improve pavement markings along the curve on \#R1 road & 16 & 2 & RS \\
\hline Improve pavement markings and texturing & 16 & 3 & AWSC,TWSC,RRC \\
\hline Improve pavement marking on \#A1 approach & 21 & 3 & AWSC,TWSC,RRC,SIGN \\
\hline Provide photo video enforcement & 56 & 3 & AWSC,TWSC,RRC \\
\hline Improve signage and/or marking of crosswalk(s) & 25 & 2 & TWSC,AWSC \\
\hline Increase skid resistance on \#R1 road & 9 & 3 & TWSC,AWSC \\
\hline Improve traffic visibility from median opening toward \#A1 approach & 10 & 2 & TWSC \\
\hline Improve visibility of stop bar on \#A2 approach & 20 & 2 & TWSC \\
\hline Improve visibility of traffic signals & 30 & 2 & SIGN \\
\hline Improve visibility of the splittery-island & 25 & 2 & SIGN \\
\hline Improve visibility of intersection by providing enhanced signing and delineation & 30 & 3 & TWSC,AWSC,SIGN \\
\hline Install street lighting & 36 & 4 & TWSC,AWSC \\
\hline Improve winter maintenance & 25 & 3 & TWSC,AWSC \\
\hline Improve timeliness of winter maintenance & 20 & 3 & TWSC,AWSC \\
\hline Improve/install reflective signs on \#R1 road & 15 & 2 & TWSC,AWSC,SIGN \\
\hline Improve/install reflectorized pavement markers on \#R1 approach & 12 & 2 & TWSC \\
\hline Increase efficiency of use of existing parking spaces & 10 & 2 & RS \\
\hline
\end{tabular}




\begin{tabular}{|c|c|c|c|}
\hline Countermeasures & CRF & Cost & Road \\
\hline Increase radius of corner curves & 14 & 4 & TWSC \\
\hline Increase crosswalk setback on \#A1 approach & 15 & 3 & SIGN \\
\hline Increase crosswalk setback on \#E2 exit & 15 & 3 & TWSC \\
\hline Increase length of left-turn bay on \#A1 approach & 30 & 4 & TWSC \\
\hline Increase width of shoulders on \#A1 approach & 14 & 3 & TWSC,AWSC \\
\hline Increase width of shoulders on \#R1 road & 14 & 3 & TWSC,AWSC \\
\hline Install barrier curb or a guardrail on \#A1 approach & 33 & 3 & TWSC,AWSC \\
\hline Install barrier curb or a guardrail on \#E1 exit & 33 & 3 & TWSC,AWSC \\
\hline Install barrier curb or a guardrail on \#R1 road & 33 & 3 & $\mathrm{RS}$ \\
\hline Install a bicycles overpass or underpass & 90 & 5 & RRC \\
\hline Install pedestrian overpass or underpass & 90 & 5 & RRC \\
\hline Install triangular island on \#A2 approach & 25 & 3 & TWSC,AWSC \\
\hline Install acceleration lane on \#E1 exit sufficiently long for trucks & 26 & 5 & TWSC \\
\hline Install additional signal head on \#A1 approach & 41 & 2 & SIGN \\
\hline Install advance guide signs on \#A1 approach & 30 & 2 & SIGN \\
\hline Install advance warning sign on \#A1 approach & 26 & 2 & TWSC,AWSC,SIGN \\
\hline Install advance warning sign on \#A2 approach & 26 & 2 & TWSC \\
\hline Install advance warning sign on \#R1 road & 26 & 2 & $\mathrm{RS}$ \\
\hline Install advance warning sign of horizontal/vertical curve on \#R1 road & 26 & 2 & RS \\
\hline Install advisor speed sign & 26 & 1 & RS \\
\hline Install acceleration lane on \#E1 exit & 15 & 5 & TWSC \\
\hline Install active control devices & 50 & 4 & $\mathrm{RRC}$ \\
\hline Install utility polet marker & 16 & 1 & $\mathrm{RS}$ \\
\hline Install audible signals (warning bell) & 20 & 2 & $\mathrm{RRC}$ \\
\hline Install automated bicycles gates & 50 & 3 & $\mathrm{RRC}$ \\
\hline Install automated pedestrian gates & 30 & 3 & $\mathrm{RRC}$ \\
\hline Install automatic gates & 75 & 4 & $\mathrm{RRC}$ \\
\hline Install backplates & 15 & 2 & SIGN \\
\hline Install bicycles signals & 20 & 3 & RRC,SIGN \\
\hline Install brdige warning sign & 28 & 1 & $\mathrm{RS}$ \\
\hline Install cantilever flashing-light signal & 33 & 3 & $\mathrm{RRC}$ \\
\hline
\end{tabular}




\begin{tabular}{|c|c|c|c|}
\hline Countermeasures & CRF & Cost & Road \\
\hline Install DO NOT STOP ON TRACKS signal & 10 & 1 & $\mathrm{RRC}$ \\
\hline Install fence or chicane to prevent bicycles running across the track & 30 & 2 & $\mathrm{RRC}$ \\
\hline Install fence or chicane to prevent pedestrians running across the track & 30 & 2 & $\mathrm{RRC}$ \\
\hline Install fixed warning message signs & 10 & 1 & $\mathrm{RRC}$ \\
\hline Install object marker on \#A1 approach & 16 & 1 & TWSC,AWSC \\
\hline Install overhead signs & 10 & 2 & $\mathrm{RRC}, \mathrm{RS}$ \\
\hline Install object marker on \#E1 exit & 16 & 1 & TWSC,AWSC \\
\hline Install centerline rumble strips & 25 & 3 & $\mathrm{RS}$ \\
\hline Install corner mirrors (Local road/Low speed traffic) & 10 & 1 & TWSC \\
\hline Install "Cross only at Crosswalk" signs & 5 & 1 & RS \\
\hline Install curbing to define driveway(s) location on \#A1 approach & 20 & 3 & TWSC,AWSC \\
\hline Install curbing to define driveway(s) location on \#E1 exit & 20 & 3 & TWSC,AWSC \\
\hline Install curbing to define driveway(s) location on \#R1 road & 20 & 3 & RS \\
\hline Install curb-ramps & 15 & 3 & TWSC,AWSC \\
\hline Install flashing beacons on \#A1 approach & 35 & 2 & TWSC,AWSC,SIGN \\
\hline Install flashing beacons on \#R1 road & 35 & 2 & RS \\
\hline Install flashing beacons above STOP sign on \#A2 approach & 35 & 2 & TWSC,AWSC \\
\hline $\begin{array}{l}\text { Install flashing light activated by queuing traffic in the exit lane(s) from the } \\
\text { crossing }\end{array}$ & 25 & 2 & $\mathrm{RRC}$ \\
\hline Install fog-warning signs & 35 & 2 & TWSC \\
\hline Install four-quadrant gate arms & 25 & 2 & TWSC,AWSC \\
\hline Install gates with self deploying extension sections & 20 & 4 & $\mathrm{RRC}$ \\
\hline Install guardrails on \#A1 approach & 40 & 4 & TWSC,AWSC \\
\hline Install guardrails on \#E1 exit & 40 & 4 & TWSC,AWSC \\
\hline Install in-pavement luminaires & 20 & 2 & $\mathrm{RS}$ \\
\hline Install indirect left-turn lanes on \#A1 approach & 35 & 4 & TWSC \\
\hline Install indirect right-turn lanes on \#A1 approach & 30 & 4 & TWSC \\
\hline Install interactive truck rollover signing & 10 & 1 & $\mathrm{RS}$ \\
\hline Install larger regulatory signs & 25 & 2 & TWSC,AWSC \\
\hline Install larger signal lenses at the intersection & 11 & 2 & SIGN \\
\hline Install larger regulatory signs on both sides of road on \#A1 approach & 35 & 2 & TWSC,AWSC,SIGN \\
\hline
\end{tabular}




\begin{tabular}{|c|c|c|c|}
\hline Countermeasures & CRF & Cost & Road \\
\hline Install larger warning signs on \#A1 approach & 25 & 2 & TWSC,AWSC \\
\hline Install LED barrier-mounted guidance tubes & 20 & 2 & RS \\
\hline Install light-emitting diode (LED) & 20 & 2 & RS \\
\hline Install left-turn bay on \#A1 approach & 25 & 4 & TWSC \\
\hline Install left-turn lane on \#A1 approach & 27 & 4 & SIGN \\
\hline Install median barriers & 35 & 3 & RRC \\
\hline $\begin{array}{l}\text { Install median divider on \#A1 approach to prevent left-turns from/to } \\
\text { driveway(s) }\end{array}$ & 15 & 3 & TWSC \\
\hline Install median divider on \#E1 exit to prevent left-turns from/to driveway(s) & 15 & 3 & TWSC \\
\hline Install median divider on \#R1 road to prevent left-turns from/to driveway(s) & 15 & 3 & RS \\
\hline Install median retroreflective post delineators on the approach & 15 & 2 & $\mathrm{RRC}$ \\
\hline Install multi-dial controller & 10 & 2 & SIGN \\
\hline Install non-mountable curb islands & 20 & 2 & RRC \\
\hline Install overhead flashing beacon lights on \#A1 approach & 33 & 2 & TWSC,AWSC \\
\hline Install overhead flashing beacon lights on \#R1 road & 33 & 2 & RS \\
\hline Install pavement markings & 16 & 2 & TWSC,AWSC,RRC \\
\hline Install pedestrian actuated signals & 35 & 4 & TWSC,AWSC \\
\hline Install pedestrian barriers & 60 & 3 & TWSC,AWSC \\
\hline Install pedestrian crosswalks & 25 & 2 & TWSC,AWSC \\
\hline $\begin{array}{l}\text { Install physical barriers to restrict pedestrian crossing maneuvers at higher-risk } \\
\text { locations }\end{array}$ & 60 & 3 & SIGN \\
\hline Install post-mounted delineators & 22 & 2 & RS \\
\hline Install pre-signals & 50 & 4 & TWSC,AWSC \\
\hline Install raised crosswalks & 25 & 3 & TWSC,AWSC \\
\hline Install right right-turn lane on \#A1 approach & 13 & 4 & SIGN \\
\hline Install rumble strips on \#A1 approach & 32 & 2 & RRC,SIGN \\
\hline Install rumble strips on \#A2 approach & 32 & 2 & TWSC,AWSC \\
\hline Install rumble strips on \#R1 road & 32 & 2 & RS \\
\hline Install school crossing sign on \#R1 road & 16 & 1 & TWSC,AWSC \\
\hline Install school zone markings on \#R1 road & 20 & 1 & TWSC,AWSC \\
\hline Install school zone speed limit sign on \#R1 road & 25 & 1 & TWSC,AWSC \\
\hline Install shoulder rumble strips on \#A1 approach & 28 & 2 & TWSC,AWSC \\
\hline
\end{tabular}




\begin{tabular}{|c|c|c|c|}
\hline Countermeasures & CRF & Cost & Road \\
\hline Install sidewalks & 70 & 4 & RS \\
\hline Install sidewalk set-backs on \#R1 road & 20 & 2 & TWSC,AWSC \\
\hline Install speed humps & 40 & 2 & RRC \\
\hline $\begin{array}{l}\text { Install static or variable message signs displaying weather information on \#A1 } \\
\text { approach }\end{array}$ & 15 & 3 & $\mathrm{RRC}$ \\
\hline $\begin{array}{l}\text { Install static or variable message signs displaying weather information on \#R1 } \\
\text { road }\end{array}$ & 15 & 3 & RS \\
\hline Install stop sign on \#A1 approach & 40 & 1 & RRC \\
\hline Install street lights at intersection & 40 & 4 & TWSC,AWSC \\
\hline Install street lights at RRC & 40 & 4 & TWSC,AWSC \\
\hline Install street lights on \#R1 road & 40 & 4 & TWSC,AWSC \\
\hline Install street name signs at intersection & 25 & 2 & TWSC,AWSC,SIGN \\
\hline Install symbol storage space sign & 10 & 1 & $\mathrm{RRC}$ \\
\hline Install swing gates & 25 & 2 & RRC \\
\hline Install traffic actuated signal & 22 & 4 & SIGN \\
\hline Install traffic signals at intersection & 26 & 5 & TWSC,AWSC \\
\hline Install tree marker & 16 & 1 & $\mathrm{RS}$ \\
\hline Install utility poles marker & 5 & 1 & SIGN \\
\hline Install variable message signs and blank out signs & 20 & 2 & $\mathrm{RRC}$ \\
\hline Install vehicle-arresting barriers & 25 & 3 & $\mathrm{RRC}$ \\
\hline Install warning signs in advance of rail-road crossing & 45 & 1 & RRC \\
\hline Install warning signs on both side of road on \#A1 approach & 20 & 1 & AWSC,TWSC,RRC \\
\hline Install queue detection system & 22 & 3 & SIGN \\
\hline Install yield sign & 10 & 1 & RRC \\
\hline Install/improve center line markings & 34 & 2 & SIGN \\
\hline Install/improve signing or marking of pedestrian crosswalks at intersection & 16 & 2 & TWSC,AWSC \\
\hline Install/improve signing or marking of bycicles at intersection & 15 & 2 & SIGN \\
\hline Installing snow screens in areas exposed to snowdrifts on \#R1 road & 53 & 3 & TWSC,AWSC \\
\hline Lengthening clearance intervals & 12 & 2 & SIGN \\
\hline Optimize clearance interval & 9 & 2 & SIGN \\
\hline Overlay pavement on \#E1 exit & 21 & 4 & TWSC,AWSC \\
\hline Overlay pavement on \#R1 road & 21 & 4 & RS \\
\hline
\end{tabular}




\begin{tabular}{|c|c|c|c|}
\hline Countermeasures & CRF & Cost & Road \\
\hline Place utilities underground & 60 & 5 & RS \\
\hline Post adequate speed limit on \#R1 approach & 24 & 2 & TWSC,AWSC,RS \\
\hline Post adequate truck speed limit on \#R1 approach & 24 & 2 & TWSC,AWSC \\
\hline Post dynamic message sign to display speed of vehicles on \#A1 approach & 74 & 2 & TWSC,AWSC \\
\hline Post dynamic message sign to display speed of vehicles on \#R1 road & 74 & 2 & RS \\
\hline $\begin{array}{l}\text { Prohibit/Restrict trucks with very Long semitrailers on roads with horizontal } \\
\text { curves that cannot accommodate truck offtracking }\end{array}$ & 60 & 3 & RS \\
\hline Prohibit right turn on red & 33 & 1 & SIGN \\
\hline Properly maintain striping on median on \#R1 road & 10 & 2 & TWSC,AWSC \\
\hline Provide bypass lane on \#A1 approach with left-turn traffic & 10 & 3 & TWSC,AWSC \\
\hline $\begin{array}{l}\text { Provide double yellow centerline on median opening of divided highway at } \\
\text { intersections }\end{array}$ & 5 & 2 & TWSC \\
\hline Provide acceleration and deceleration lanes on \#A1 approach & 15 & 5 & TWSC \\
\hline Provide acceleration and deceleration lanes on \#E1 exit & 15 & 5 & TWSC \\
\hline Provide acceleration and deceleration lanes on \#R1 road & 15 & 5 & RS \\
\hline Provide adequate coordination between signals & 20 & 3 & SIGN \\
\hline $\begin{array}{l}\text { Provide adequate delineation and channelization for trucks left/right turn at } \\
\text { intersection }\end{array}$ & 30 & 3 & SIGN \\
\hline Provide adequate delineation for left-turns at intersection (markers or lines) & 30 & 3 & TWSC \\
\hline Provide adequate delineation for right-turns at intersection (markers or lines) & 30 & 3 & SIGN \\
\hline Provide adequate drainage on \#R1 road & 20 & 4 & TWSC,AWSC \\
\hline Provide adequate gates delay & 33 & 3 & $\mathrm{RRC}$ \\
\hline $\begin{array}{l}\text { Provide adequate information to train operator (for example: location of the } \\
\text { whistle post) }\end{array}$ & 15 & 2 & $\mathrm{RRC}$ \\
\hline $\begin{array}{l}\text { Provide appropriate signal intervals for pedestrians crossing and minimize wait } \\
\text { time }\end{array}$ & 35 & 2 & SIGN \\
\hline Provide adequate signals for handicapped & 25 & 2 & SIGN \\
\hline Provide adequate truck signing & 26 & 1 & RS \\
\hline Provide all-red clearance interval & 15 & 2 & SIGN \\
\hline Provide information of the crossing type & 10 & 1 & RRC \\
\hline Provide adequate lanes signing or marking on \#A1 approach & 21 & 3 & TWSC,AWSC \\
\hline Provide adequate turning markers or pavement markings for turning vehicle on & 14 & 2 & TWSC,AWSC \\
\hline
\end{tabular}




\begin{tabular}{|c|c|c|c|}
\hline Countermeasures & CRF & Cost & Road \\
\hline Provide breakaway and crashworthy mail box & 15 & 2 & RS \\
\hline Provide breakaway and crashworthy sign supports & 30 & 2 & RS \\
\hline $\begin{array}{l}\text { Provide dashed markings (extended left edgelines) for \#A1 approach } \\
\text { continuity across median opening at divided highway intersections }\end{array}$ & 22 & 2 & TWSC,AWSC \\
\hline Provide dynamic curve warning system on \#R1 road & 29 & 2 & RS \\
\hline Provide far-side left-turn signal on \#A1 approach & 28 & 3 & SIGN \\
\hline Provide/improve progression through a set of signalized intersections & 25 & 3 & SIGN \\
\hline Provide independent bicycle path on \#R1 road & 20 & 3 & TWSC,AWSC \\
\hline Provide louvers visors or special lenses on \#A1 approach & 10 & 2 & SIGN \\
\hline Provide median refuges at intersection & 46 & 3 & TWSC \\
\hline Provide median refuges on \#R1 road & 46 & 3 & $\mathrm{RS}$ \\
\hline Provide offset for left turn lanes on \#R1 road & 10 & 3 & TWSC \\
\hline Provide warning pavement marking on \#A1 approach & 10 & 2 & TWSC,AWSC \\
\hline Provide on-pavement horizontal signing on \#R1 road & 10 & 2 & RS \\
\hline Provide "Slippery when wet" signs on \#A1 approach & 10 & 1 & TWSC,AWSC \\
\hline Provide "Slippery when wet" signs on \#R1 road & 10 & 1 & TWSC,AWSC \\
\hline $\begin{array}{l}\text { Provide pedestrian-only phase or pedestrian-lead phase during signal } \\
\text { operation }\end{array}$ & 31 & 2 & SIGN \\
\hline Provide pavement marking (stop sign/chevron/etc.) on \#A2 approach & 30 & 2 & TWSC \\
\hline Provide pavement marking (stop sign/chevron/etc.) on \#R1 road & 30 & 2 & $\mathrm{RS}$ \\
\hline Provide protected left-turn phase for \#R1 road & 44 & 3 & SIGN \\
\hline Provide protected/permitted phase for \#R1 road & 10 & 3 & SIGN \\
\hline Provide split phases for \#R1 road & 25 & 3 & SIGN \\
\hline $\begin{array}{l}\text { Provide roadside markers or pavement markings on \#A1 approach to assist } \\
\text { drivers in judging suitability of available gaps for making turning and crossing } \\
\text { maneuvers }\end{array}$ & 10 & 2 & TWSC,AWSC \\
\hline Provide smooth paved shoulders on \#A1 approach & 24 & 3 & TWSC,AWSC \\
\hline Provide smooth paved shoulders on \#E1 exit & 24 & 3 & TWSC,AWSC \\
\hline Provide smooth paved shoulders on \#R1 road & 24 & 3 & RS \\
\hline Provide splitter islands on \#A2 approach & 35 & 3 & TWSC \\
\hline Provide spiral transition curves & 20 & 4 & RS \\
\hline Provide stop signs on \#A1 approach & 37 & 1 & TWSC,AWSC \\
\hline
\end{tabular}




\begin{tabular}{|c|c|c|c|}
\hline Countermeasures & CRF & Cost & Road \\
\hline Provide targeted speed enforcement on \#A1 approach & 70 & 3 & TWSC,AWSC \\
\hline Provide targeted speed enforcement on \#R1 road & 70 & 3 & RS \\
\hline $\begin{array}{l}\text { Provide/Improve pavement surface (improve transitions/drop offs/fixed } \\
\text { potholes/rutting/etc) on \#R1 road }\end{array}$ & 28 & 4 & TWSC,AWSC \\
\hline Provide visors to shade signal lenses from sunlight & 20 & 2 & SIGN \\
\hline Provide wider cross sections on two-lane roads & 20 & 4 & RS \\
\hline $\begin{array}{l}\text { Reallocate total two-lane roadway width (lane and shoulder) to include a } \\
\text { narrow "buffer median" }\end{array}$ & 50 & 4 & RS \\
\hline Reconstruct intersection & 45 & 4 & SIGN \\
\hline Redesign left-turning path from \#R1 road & 14 & 3 & TWSC,AWSC,SIGN \\
\hline Redesign right-turning path from \#R1 road & 14 & 3 & TWSC,AWSC,SIGN \\
\hline Reduce speed limit on \#A1 approach & 20 & 2 & TWSC,AWSC,SIGN \\
\hline Reduce speed limit on \#R1 road & 20 & 2 & RS \\
\hline Reduce number of lanes on \#A2 approach if allowed by capacity & 15 & 4 & TWSC \\
\hline Relocate control device away from the road & 20 & 2 & RRC \\
\hline Relocate crossing section & 30 & 5 & RRC \\
\hline Relocate driveway(s) from \#E1 exit & 33 & 4 & TWSC,AWSC \\
\hline Relocate driveway(s) from \#A1 approach & 33 & 4 & TWSC,AWSC \\
\hline Relocate driveway(s) from \#R1 road & 33 & 4 & RS \\
\hline Relocate or split stopping line on \#A2 approach & 10 & 1 & TWSC \\
\hline Relocate signal hardware out of clear zone & 62 & 3 & SIGN \\
\hline Relocate stop bar on \#A2 approach & 10 & 1 & TWSC,AWSC \\
\hline Relocate stop sign on \#A1 approach & 15 & 1 & TWSC,AWSC,RRC \\
\hline Relocate stop sign on \#A2 approach & 15 & 1 & TWSC,AWSC \\
\hline Relocate sign on \#A1 approach to make it visible & 20 & 1 & TWSC,AWSC \\
\hline Relocate sign on \#R1 road to make it visible & 20 & 1 & RS \\
\hline Relocate transit stop & 15 & 2 & SIGN \\
\hline Relocate traffic signals structure elements & 20 & 3 & SIGN \\
\hline Remove deflection in through-vehicle travel path & 20 & 2 & SIGN \\
\hline Remove distracting commercial lighting or other source of glare at intersection & 15 & 3 & TWSC,AWSC \\
\hline Remove distracting commercial lighting or other source of glare on \#R1 road & 15 & 3 & RS \\
\hline Remove late night/early morning flashing operation & 20 & 2 & SIGN \\
\hline
\end{tabular}




\begin{tabular}{|c|c|c|c|}
\hline Countermeasures & CRF & Cost & Road \\
\hline Remove or relocate object on \#A1 approach & 42 & 3 & TWSC,AWSC \\
\hline Remove or relocate object on \#E1 exit & 42 & 3 & TWSC,AWSC \\
\hline Remove or relocate sign & 29 & 2 & RS,SIGN \\
\hline Remove or relocate mail box on \#R1 road & 29 & 2 & RS \\
\hline Remove or relocate trees along \#R1 road & 46 & 3 & RS,SIGN \\
\hline Removed or relocate unnecessary signs "visual clutter" & 15 & 2 & SIGN \\
\hline Remove or relocate utility Pole along \#R1 road & 36 & 2 & RS,SIGN \\
\hline Remove sight obstruction on \#A1 approach & 13 & 2 & TWSC,AWSC \\
\hline Remove sight obstruction on \#E1 exit & 13 & 2 & TWSC,AWSC \\
\hline Remove sight obstruction on \#R1 road & 13 & 2 & RS \\
\hline Remove unwarranted signal & 56 & 3 & SIGN \\
\hline Remove object obstructing sight of control device & 20 & 3 & RRC \\
\hline Remove object obstructing sight of warning sign on \#A1 approach & 20 & 2 & TWSC,AWSC \\
\hline Remove object obstructing sight of warning sign on \#R1 road & 20 & 2 & RS \\
\hline Remove object obstructing sight of stop sign on \#A1 approach & 30 & 2 & TWSC,AWSC \\
\hline Remove object obstructing sight of stop sign on \#A2 approach & 30 & 2 & TWSC,AWSC \\
\hline Remove object obstructing sight triangle at RRC & 24 & 2 & RRC \\
\hline Remove object obstructing sight triangle at intersection & 16 & 2 & TWSC,AWSC,SIGN \\
\hline Replace poorly designed drain grates on \#R1 road with bicycle-safe types & 10 & 3 & TWSC,AWSC \\
\hline Replace / repair signals & 10 & 2 & RRC \\
\hline Replace, repair or clean control device & 20 & 2 & RRC \\
\hline Replace/Repair/Clean warning signs on \#A1 approach & 23 & 1 & TWSC,AWSC \\
\hline Reroute pedestrian paths at intersection & 30 & 3 & TWSC,AWSC \\
\hline Restrict left-turn maneuver on \#R1 road & 40 & 3 & TWSC \\
\hline Restrict left-turning at access point on \#A1 approach & 90 & 3 & TWSC \\
\hline Restrict left-turning at access point on \#E1 exit & 90 & 3 & TWSC \\
\hline Restrict left-turning at access point on \#R1 road & 90 & 3 & RS \\
\hline Restrict or eliminate turning maneuver on \#R1 road & 60 & 3 & SIGN \\
\hline Restrict parking close to intersection & 10 & 2 & TWSC,AWSC,SIGN \\
\hline Restrict parking close to RRC & 22 & 2 & RRC \\
\hline Restrict parking near driveway(s) on \#A1 approach & 19 & 2 & TWSC,AWSC \\
\hline
\end{tabular}




\begin{tabular}{|c|c|c|c|}
\hline Countermeasures & CRF & Cost & Road \\
\hline Restrict parking near driveway(s) on \#R1 road & 19 & 2 & RS \\
\hline Restrict parking near horizontal/vertical curves on \#R1 road & 18 & 2 & RS \\
\hline Signals to alert motorists that pedestrians are crossing at crossing point & 16 & 2 & TWSC,AWSC \\
\hline Signals to alert motorists that pedestrians are crossing at intersection & 16 & 2 & TWSC,AWSC \\
\hline Shield drivers from poles in hazardous locations & 20 & 2 & RS \\
\hline Trim vegetation at $R R C$ & 35 & 2 & $\mathrm{RRC}$ \\
\hline Trim vegetation at intersection & 35 & 2 & TWSC,AWSC,SIGN \\
\hline Upgrade signing on \#R1 road & 15 & 1 & TWSC,AWSC,RRC \\
\hline Use adequate methods to increase pavement friction on \#R1 road & 20 & 4 & TWSC,AWSC \\
\hline Use alternating passing lanes or four-lane sections at key locations & 29 & 3 & RS \\
\hline Use break-away devices on \#A1 approach & 30 & 3 & TWSC,AWSC \\
\hline Use break-away devices on \#R1 road & 30 & 3 & RS \\
\hline Use break-away devices on \#E1 exit & 30 & 3 & TWSC,AWSC \\
\hline $\begin{array}{l}\text { Use de-icing/anti-icing method to prevent snow or ice from forming or from } \\
\text { sticking to \#R1 road surface }\end{array}$ & 30 & 3 & TWSC,AWSC \\
\hline Use two red signal sections & 36 & 2 & SIGN \\
\hline Use school crossing guards at crossing point & 50 & 2 & TWSC,AWSC \\
\hline Use school crossing guards at intersection & 50 & 2 & TWSC,AWSC \\
\hline Widen lanes on \#R1 road to adequate width & 25 & 4 & RS \\
\hline $\begin{array}{l}\text { Widen median at intersection to provide sufficient protection to crossing } \\
\text { vehicles }\end{array}$ & 5 & 4 & TWSC,AWSC \\
\hline Widen median width on \#R1 road & 13 & 4 & TWSC,AWSC \\
\hline \multirow[t]{3}{*}{ Widen outside through lanes or add bike lanes on \#R1 road } & 15 & 3 & TWSC,AWSC \\
\hline & & $\begin{array}{l}\text { 1- LOW } \\
\text { COST }\end{array}$ & \\
\hline & & $\begin{array}{c}\text { 5- HIGH } \\
\text { COST }\end{array}$ & \\
\hline
\end{tabular}




\section{Appendix C}

\section{List of Conditions}

\begin{tabular}{|c|c|c|c|c|}
\hline $\begin{array}{ll} & \text { Conditions } \\
\end{array}$ & Probability & Cost & Off-Site & Reference \\
\hline \#A1 and \#A2 are right-angle approaches & 50 & 1 & $\mathrm{y}$ & $\mathrm{N}$ \\
\hline \#A1 major approach & 50 & 1 & $y$ & $\mathrm{~N}$ \\
\hline \#A2 minor approach & 50 & 1 & $\mathrm{y}$ & $\mathrm{N}$ \\
\hline \#E1 major exit & 50 & 1 & $\mathrm{y}$ & $\mathrm{N}$ \\
\hline \#E2 minor exit & 50 & 1 & $y$ & $\mathrm{~N}$ \\
\hline \#R1 major road & 50 & 1 & $y$ & $\mathrm{~N}$ \\
\hline \#R2 minor road & 50 & 1 & $\mathrm{y}$ & $\mathrm{N}$ \\
\hline Advance warning sign posted on \#A1 approach & 70 & 1 & $\mathrm{n}$ & $\mathrm{N}$ \\
\hline Advance warning sign posted on \#R1 road & 70 & 1 & $\mathrm{n}$ & $\mathrm{N}$ \\
\hline Adverse-weather collisions & 30 & 2 & $\mathrm{y}$ & $\mathrm{N}$ \\
\hline Angle collisions & 30 & 1 & $y$ & $\mathrm{~N}$ \\
\hline Automatic gates & 35 & 1 & $y$ & $\mathrm{~N}$ \\
\hline Automatic gates do not work properly & 10 & 2 & $\mathrm{n}$ & $\mathrm{N}$ \\
\hline AWSC & - & 1 & $y$ & $\mathrm{~N}$ \\
\hline Bicycle collisions at intersection & 20 & 2 & $\mathrm{y}$ & $\mathrm{N}$ \\
\hline Bicycle collisions at RRC & 20 & 2 & $\mathrm{y}$ & $\mathrm{N}$ \\
\hline Bicycle collisions on \#A1 approach & 20 & 2 & $\mathrm{y}$ & $\mathrm{N}$ \\
\hline Bicycle collisions on \#E1 exit & 20 & 2 & $\mathrm{y}$ & $\mathrm{N}$ \\
\hline Bicycle collisions on \#R1 road & 20 & 2 & $\mathrm{y}$ & $\mathrm{N}$ \\
\hline Bicycles crossing between already lowered gate arms & 30 & 2 & $\mathrm{n}$ & $\mathrm{N}$ \\
\hline Bicycles running across the track & 20 & 2 & $\mathrm{n}$ & $\mathrm{N}$ \\
\hline Collision on \#R1 road & 60 & 2 & $\mathrm{y}$ & $\mathrm{N}$ \\
\hline Control Device is visible on \#A1 approach & 70 & 2 & $\mathrm{n}$ & $\mathrm{N}$ \\
\hline Control Device is not visible on A1 approach & 30 & 2 & $\mathrm{n}$ & $\mathrm{N}$ \\
\hline Control device obstruction & 25 & 2 & $\mathrm{n}$ & $\mathrm{N}$ \\
\hline Crossing pedestrians at intersection are not adequate recognizable & 10 & 3 & $\mathrm{n}$ & $\mathrm{N}$ \\
\hline
\end{tabular}




\begin{tabular}{|c|c|c|c|c|}
\hline $\begin{aligned} \text { Conditions } \\
\end{aligned}$ & Probability & Cost & Off-Site & Reference \\
\hline Crosswalk not recognizable by drivers on \#R1 road & 30 & 3 & $\mathrm{n}$ & $\mathrm{N}$ \\
\hline Divided \#R1 road & 40 & 1 & $\mathrm{n}$ & $\mathrm{N}$ \\
\hline Driver not aware of encroach into opposing lane & 30 & 1 & $\mathrm{n}$ & $\mathrm{N}$ \\
\hline Drivers crossing between already lowered gate arms & 50 & 1 & $\mathrm{n}$ & $\mathrm{N}$ \\
\hline Driveway(s) collisions on \#A1 approach & 20 & 2 & $\mathrm{y}$ & $\mathrm{N}$ \\
\hline Driveway(s) collisions on \#E1 exit & 20 & 2 & $\mathrm{y}$ & $\mathrm{N}$ \\
\hline Driveway(s) collisions on \#R1 road & 20 & 2 & $\mathrm{y}$ & $\mathrm{N}$ \\
\hline Driveway(s) design inadequate for high traffic on \#A1 approach & 20 & 4 & $\mathrm{n}$ & $\mathrm{N}$ \\
\hline Driveway(s) design inadequate for high traffic on \#E1 exit & 20 & 4 & $\mathrm{n}$ & $\mathrm{N}$ \\
\hline Driveway(s) design inadequate for high traffic on \#R1 road & 20 & 4 & $\mathrm{n}$ & $\mathrm{N}$ \\
\hline Driveway(s) on \#A1 approach close to intersection & 40 & 2 & $\mathrm{y}$ & $\mathrm{N}$ \\
\hline Driveway(s) on \#E1 exit close to intersection & 40 & 2 & $\mathrm{y}$ & $\mathrm{N}$ \\
\hline Edgedrops present on \#A1 approach & 40 & 2 & $\mathrm{n}$ & $\mathrm{N}$ \\
\hline Edgedrops present on \#E1 approach & 30 & 2 & $\mathrm{n}$ & $\mathrm{N}$ \\
\hline Excessive speed on \#R1 road & 30 & 3 & $\mathrm{n}$ & $\mathrm{N}$ \\
\hline Exclusive lanes for left turns on \#A1 approach & 30 & 2 & $\mathrm{n}$ & $\mathrm{N}$ \\
\hline Fix object close to traveled way on \#A1 approach & 40 & 2 & $\mathrm{n}$ & 49301 \\
\hline Fix object close to traveled way on \#R1 road & 40 & 2 & $\mathrm{n}$ & 49301 \\
\hline Fix object close to traveled way on \#E1 exit & 40 & 2 & $\mathrm{n}$ & 49301 \\
\hline Frequent stop sign violations on \#A1 approach & 40 & 3 & $\mathrm{n}$ & $\mathrm{N}$ \\
\hline Frequent stop sign violations on \#A2 approach & 40 & 3 & $\mathrm{n}$ & $\mathrm{N}$ \\
\hline Frequently speed violation on \#R1 road & 40 & 3 & $\mathrm{n}$ & 403 \\
\hline High turning volume on \#R1 road & 50 & 3 & $\mathrm{n}$ & $\mathrm{N}$ \\
\hline High traffic volume on \#R1 road & 50 & 3 & $\mathrm{n}$ & $\mathrm{N}$ \\
\hline Head-on collisions on \#R1 road & 20 & 2 & $\mathrm{y}$ & $\mathrm{N}$ \\
\hline Head-on collisions on \#A1 approach & 20 & 2 & $\mathrm{y}$ & $\mathrm{N}$ \\
\hline Horizontal curve & 35 & 1 & $\mathrm{n}$ & $\mathrm{N}$ \\
\hline Improperly control device maintained & 35 & 1 & $\mathrm{n}$ & $\mathrm{N}$ \\
\hline Improperly stop sign maintained on \#A1 approach & 20 & 2 & $\mathrm{n}$ & $\mathrm{N}$ \\
\hline Improperly stop sign maintained on \#A2 approach & 20 & 2 & $\mathrm{n}$ & $\mathrm{N}$ \\
\hline Inadequate advance warning of $\mathrm{RRC}$ & 15 & 1 & $\mathrm{n}$ & $\mathrm{N}$ \\
\hline
\end{tabular}




\begin{tabular}{|c|c|c|c|c|}
\hline Conditions & Probability & Cost & Off-Site & Reference \\
\hline Inadequate bicycles access design & 25 & 3 & $\mathrm{n}$ & 517 \\
\hline Inadequate channelization of rail road crossing & 30 & 2 & $\mathrm{n}$ & $\mathrm{N}$ \\
\hline Inadequate channelization on \#A1 approach & 25 & 3 & $\mathrm{n}$ & $\mathrm{N}$ \\
\hline Inadequate center line markings & 15 & 1 & $\mathrm{n}$ & $\mathrm{N}$ \\
\hline Inadequate clearance interval & 10 & 2 & $\mathrm{n}$ & $\mathrm{N}$ \\
\hline Inadequate control device & 10 & 1 & $\mathrm{n}$ & $\mathrm{N}$ \\
\hline Inadequate coordination between signals & 10 & 2 & $\mathrm{n}$ & $\mathrm{N}$ \\
\hline Too short pedestrian walk signal & 10 & 2 & $\mathrm{n}$ & $\mathrm{N}$ \\
\hline Inadequate crosswalk placement at intersection & 15 & 3 & $\mathrm{n}$ & $\mathrm{N}$ \\
\hline Inadequate design for handicap at intersection & 20 & 2 & $\mathrm{n}$ & 51108 \\
\hline Inadequate design for high traffic volume & 15 & 3 & $\mathrm{n}$ & $\mathrm{N}$ \\
\hline $\begin{array}{l}\text { Inadequate design for considerable number of trucks entering \#E1 exit from \#A2 } \\
\text { approach }\end{array}$ & 20 & 3 & $\mathrm{n}$ & $\mathrm{N}$ \\
\hline $\begin{array}{l}\text { Inadequate design for considerable turning volume on \#A2 approach entering on \#E1 } \\
\text { exit }\end{array}$ & 20 & 3 & $\mathrm{n}$ & $\mathrm{N}$ \\
\hline Inadequate design for high turning traffic on \#A1 approach & 20 & 4 & $\mathrm{n}$ & $462 \mathrm{D}$ \\
\hline Inadequate design of existing crosswalk & 15 & 3 & $\mathrm{n}$ & $\mathrm{N}$ \\
\hline Inadequate design of horizontal curve & 20 & 3 & $\mathrm{n}$ & $432 a-432 b$ \\
\hline Inadequate design of vertical curve & 20 & 3 & $\mathrm{n}$ & $432 a-432 b$ \\
\hline Inadequate gates visibility & 25 & 2 & $\mathrm{n}$ & $\mathrm{N}$ \\
\hline Inadequate intersection skew anlge & 10 & 2 & $\mathrm{n}$ & $\mathrm{N}$ \\
\hline Inadequate lanes signing or marking on \#A1 approach & 25 & 2 & $\mathrm{n}$ & 76203 \\
\hline Inadequate lanes width on \#A1 approach & 25 & 2 & $\mathrm{n}$ & 5540501 \\
\hline Inadequate lanes width on \#R1 road & 25 & 2 & $\mathrm{n}$ & 5540501 \\
\hline Inadequate lighting of crosswalk at intersection & 30 & 3 & $\mathrm{n}$ & 7830 \\
\hline Inadequate left-turning path from \#R1 road & 10 & 3 & $\mathrm{n}$ & 4640 \\
\hline Inadequate left turn channelization & 10 & 2 & $\mathrm{n}$ & $\mathrm{N}$ \\
\hline Inadequate left turn channelization/delineation for turning trucks & 10 & 2 & $\mathrm{n}$ & $\mathrm{N}$ \\
\hline Inadequate location of transit stop & 5 & 1 & $\mathrm{n}$ & $\mathrm{N}$ \\
\hline Inadequate maintenance of striping on median on \#R1 road & 15 & 2 & $\mathrm{n}$ & $\mathrm{N}$ \\
\hline Inadequate pavement marking at intersection & 15 & 2 & $\mathrm{n}$ & 76203 \\
\hline Inadequate pavement marking for turning vehicle on \#R1 road & 15 & 2 & $\mathrm{n}$ & $\mathrm{N}$ \\
\hline
\end{tabular}




\begin{tabular}{|c|c|c|c|c|}
\hline Conditions & Probability & Cost & Off-Site & Reference \\
\hline Inadequate pavement width to accommodate turning trucks & 10 & 2 & $\mathrm{n}$ & $\mathrm{N}$ \\
\hline Inadequate phases design & 15 & 3 & $\mathrm{n}$ & $\mathrm{N}$ \\
\hline Inadequate protection for pedestrians at intersection & 30 & 3 & $\mathrm{n}$ & $\mathrm{N}$ \\
\hline Inadequate protection for pedestrians on crosswalk at intersection & 30 & 3 & $\mathrm{n}$ & $\mathrm{N}$ \\
\hline Inadequate protection of pedestrians on \#R1 road & 30 & 3 & $\mathrm{n}$ & $\mathrm{N}$ \\
\hline Inadequate rail infrastructure & 15 & 2 & $\mathrm{n}$ & $\mathrm{N}$ \\
\hline Inadequate right turn channelization & 15 & 2 & $\mathrm{n}$ & $\mathrm{N}$ \\
\hline Inadequate median width at intersection & 20 & 2 & $\mathrm{n}$ & 45201 \\
\hline Inadequate road alignment on \#R1 road & 30 & 4 & $\mathrm{n}$ & 46102 \\
\hline Mail Boxes close to traveled way & 35 & 1 & $\mathrm{n}$ & 511101 \\
\hline Missing double yellow centerline in the meadin opening on \#R1 road & 15 & 1 & $\mathrm{n}$ & $\mathrm{N}$ \\
\hline Missing stoplines in the median opening on \#R1 road & 15 & 1 & $\mathrm{n}$ & $\mathrm{N}$ \\
\hline Inadequate road marking on crossing median on \#R1 road & 25 & 2 & $\mathrm{n}$ & $\mathrm{N}$ \\
\hline Inadequate shoulders width on \#A1 approach & 30 & 3 & $\mathrm{n}$ & 82302 \\
\hline Inadequate shoulders width on \#R1 road & 30 & 3 & $\mathrm{n}$ & 82302 \\
\hline Inadequate signing for multi-lane approach & 20 & 2 & $\mathrm{n}$ & $\mathrm{N}$ \\
\hline Inadequate space for merging vehicles into \#A1 approach & 15 & 2 & $\mathrm{n}$ & $\mathrm{N}$ \\
\hline Inadequate visibility of pedestrians & 15 & 2 & $\mathrm{n}$ & $\mathrm{N}$ \\
\hline Inadequate sight distance toward approaching vehicle on \#A1 approach & 40 & 2 & $\mathrm{n}$ & $422 a$ \\
\hline Inadequate sight distance on horizontal curve & 20 & 2 & $\mathrm{n}$ & 4340 \\
\hline Inadequate shoulder design on \#A1 approach & 20 & 3 & $\mathrm{n}$ & 5290206 \\
\hline Inadequate shoulder design on \#E1 exit & 20 & 3 & $\mathrm{n}$ & 5290206 \\
\hline Inadequate Snow/Slush/Ice maintenance & 25 & 3 & $\mathrm{n}$ & $\mathrm{N}$ \\
\hline Inadequate Snow/Slush/Ice maintenance on \#A1 approach & 25 & 3 & $\mathrm{n}$ & $\mathrm{N}$ \\
\hline Inadequate Snow/Slush/lce maintenance on \#R1 road & 25 & 3 & $\mathrm{n}$ & $\mathrm{N}$ \\
\hline Inadequate street lights at intersection & 30 & 3 & $\mathrm{n}$ & 7830 \\
\hline Inadequate street lights at rail road crossing & 30 & 3 & $\mathrm{n}$ & 7830 \\
\hline Inadequate street lights on \#R1 road & 30 & 3 & $\mathrm{n}$ & 7830 \\
\hline Inadequate traffic signal timing or type of signal & 10 & 2 & $\mathrm{n}$ & 77507 \\
\hline Inadequate emergency vehicle preemption & 5 & 2 & $\mathrm{n}$ & $\mathrm{N}$ \\
\hline Inadequate visibility on approaching train & 25 & 2 & $\mathrm{n}$ & $\mathrm{N}$ \\
\hline
\end{tabular}




\begin{tabular}{|c|c|c|c|c|}
\hline $\begin{array}{r}\text { Conditions } \\
\end{array}$ & Probability & Cost & Off-Site & Reference \\
\hline Inadequate visibility of driveway(s) on \#E1 exit & 35 & 3 & $\mathrm{n}$ & $\mathrm{N}$ \\
\hline Inadequate visibility of driveway(s) on \#R1 road & 35 & 3 & $\mathrm{n}$ & $\mathrm{N}$ \\
\hline Inadequate visibility of crosswalk at intersection & 30 & 3 & $\mathrm{n}$ & 7620302 \\
\hline Inadequate visibility of traffic signals & 20 & 2 & $\mathrm{n}$ & 77501 \\
\hline Inadequate warning on approaching train & 15 & 2 & $\mathrm{n}$ & $\mathrm{N}$ \\
\hline Insufficient bridge visibility & 10 & 2 & $\mathrm{n}$ & $\mathrm{N}$ \\
\hline Insufficient delineation along the curve & 15 & 2 & $\mathrm{n}$ & $\mathrm{N}$ \\
\hline Insufficient gaps between vehicle on \#A1 approach & 50 & 3 & $\mathrm{n}$ & $4610 h 1$ \\
\hline Insufficient gaps between vehicles on \#R1 road & 50 & 3 & $\mathrm{n}$ & 4610h1 \\
\hline Insufficient fog warning & 20 & 2 & $\mathrm{n}$ & $\mathrm{N}$ \\
\hline Insufficient median width on \#R1 road & 25 & 2 & $\mathrm{n}$ & 4520 \\
\hline Insufficient school zone warning on \#R1 road & 15 & 2 & $\mathrm{n}$ & $\mathrm{N}$ \\
\hline Insufficient sight distance from \#A1 approach toward \#A2 approach & 35 & 3 & $\mathrm{n}$ & $422 a$ \\
\hline Insufficient sight distance from \#A2 approach & 35 & 3 & $\mathrm{n}$ & $422 a$ \\
\hline Insufficient sight distance to the crossing & 35 & 3 & $\mathrm{n}$ & $422 a$ \\
\hline Insufficient warning & 20 & 2 & $\mathrm{n}$ & $\mathrm{N}$ \\
\hline Intersection near to railroad crossing & 5 & 1 & $\mathrm{n}$ & $\mathrm{N}$ \\
\hline Intersection difficult to recognize for driver on \#A1 approach & 30 & 2 & $\mathrm{n}$ & $\mathrm{N}$ \\
\hline Intersection difficult to recognize for driver on \#A2 approach & 30 & 2 & $\mathrm{n}$ & $\mathrm{N}$ \\
\hline Intersection exhibit very high crash frequency & 15 & 2 & $\mathrm{n}$ & $\mathrm{N}$ \\
\hline Intersection inconspicuous & 45 & 2 & $\mathrm{n}$ & $\mathrm{N}$ \\
\hline Intersection inconspicuous from \#A1 approach & 45 & 2 & $\mathrm{n}$ & $\mathrm{N}$ \\
\hline Intersection inconspicuous from \#A2 approach & 45 & 2 & $\mathrm{n}$ & $\mathrm{N}$ \\
\hline Intersections too small to allow simultaneous left turns & 10 & 2 & $\mathrm{n}$ & $\mathrm{N}$ \\
\hline Intersection type "T" & 15 & 1 & $\mathrm{y}$ & $\mathrm{N}$ \\
\hline Intersections with significantly unbalanced opposing left-turn volumes & 15 & 2 & $\mathrm{n}$ & $\mathrm{N}$ \\
\hline Large vehicle collision & 5 & 1 & $\mathrm{y}$ & $\mathrm{N}$ \\
\hline Left-turn bay not present on \#A1 approach & 30 & 1 & $\mathrm{n}$ & $\mathrm{N}$ \\
\hline Left-turn bay present on \#A1 approach & 70 & 1 & $\mathrm{n}$ & $\mathrm{N}$ \\
\hline Left-turn collisions at intersection & 30 & 2 & $\mathrm{y}$ & $\mathrm{N}$ \\
\hline Left-turn collisions on \#E2 exit & 30 & 2 & $\mathrm{y}$ & $\mathrm{N}$ \\
\hline
\end{tabular}




\begin{tabular}{|c|c|c|c|c|}
\hline Conditions & Probability & Cost & Off-Site & Reference \\
\hline Limited storage space between a nearby highway intersection and the tracks & 20 & 1 & $\mathrm{n}$ & $\mathrm{N}$ \\
\hline Long delay on \#R2 road & 20 & 2 & $\mathrm{n}$ & $\mathrm{N}$ \\
\hline Long distance between crosswalks on \#R1 road & 15 & 2 & $\mathrm{n}$ & $\mathrm{N}$ \\
\hline Long queues of left-turn vehicles at intersection & 15 & 2 & $\mathrm{n}$ & $\mathrm{N}$ \\
\hline Median object obstructs sight distance on \#R1 road & 20 & 3 & $\mathrm{n}$ & $\mathrm{N}$ \\
\hline Multilane on \#R1 road make difficult for bicycles to cross on \#R1 road & 15 & 3 & $\mathrm{n}$ & $\mathrm{N}$ \\
\hline Multilane on \#R1 road make difficult for pedestrian to cross on \#R1 road & 15 & 3 & $\mathrm{n}$ & $\mathrm{N}$ \\
\hline Narrow clear zone on \#R1 road & 25 & 2 & $\mathrm{n}$ & $492 \mathrm{~A}$ \\
\hline Night time collisions at intersection & 30 & 2 & $\mathrm{y}$ & $\mathrm{N}$ \\
\hline Night time collisions at rail road crossing & 30 & 1 & $\mathrm{y}$ & $\mathrm{N}$ \\
\hline Night time collisions for drivers on \#R1 road & 30 & 2 & $\mathrm{y}$ & $\mathrm{N}$ \\
\hline No advance warning sign on \#A1 approach & 20 & 1 & $\mathrm{n}$ & $\mathrm{N}$ \\
\hline No advance warning sign on \#R1 road & 20 & 1 & $\mathrm{n}$ & $\mathrm{N}$ \\
\hline No baypass for left turn traffic on \#A1 approach & 30 & 1 & $\mathrm{n}$ & $\mathrm{N}$ \\
\hline No posted speed limit on \#A1 approach & 10 & 1 & $\mathrm{n}$ & $\mathrm{N}$ \\
\hline No posted speed limit on \#R1 road & 10 & 1 & $\mathrm{n}$ & $\mathrm{N}$ \\
\hline No sidewalk present & 10 & 1 & $\mathrm{n}$ & $\mathrm{N}$ \\
\hline No school zone speed limit posted on \#R1 road & 5 & 1 & $\mathrm{n}$ & $\mathrm{N}$ \\
\hline No street name signs present at intersection & 5 & 1 & $\mathrm{n}$ & 75505 \\
\hline Not Automatic gates & 35 & 1 & $\mathrm{y}$ & $\mathrm{N}$ \\
\hline One left turn lane & 5 & 1 & $\mathrm{n}$ & $\mathrm{N}$ \\
\hline Parking cars close to intersection & 5 & 1 & $\mathrm{n}$ & $\mathrm{N}$ \\
\hline Pavement defects on \#R1 road & 45 & 2 & $\mathrm{n}$ & 5260 \\
\hline Pedestrian collisions at intersection & 30 & 2 & $\mathrm{n}$ & $\mathrm{N}$ \\
\hline Pedestrian collisions at RRC & 20 & 2 & $\mathrm{n}$ & $\mathrm{N}$ \\
\hline Pedestrian collisions on \#R1 road & 30 & 2 & $\mathrm{n}$ & $\mathrm{N}$ \\
\hline Pedestrians may block \#E2 exits & 20 & 2 & $\mathrm{n}$ & $\mathrm{N}$ \\
\hline Pedestrians running across the track & 40 & 2 & $\mathrm{n}$ & $\mathrm{N}$ \\
\hline Pedestrians walking along road & 20 & 2 & $\mathrm{n}$ & $\mathrm{N}$ \\
\hline Physical object obstruct view from a crosswalk at intersection & 30 & 2 & $\mathrm{n}$ & $\mathrm{N}$ \\
\hline Percent of tall vehicles considerable on approach \#A2 & 10 & 2 & $\mathrm{n}$ & $\mathrm{N}$ \\
\hline
\end{tabular}




\begin{tabular}{|c|c|c|c|c|}
\hline $\begin{array}{r}\text { Conditions } \\
\end{array}$ & Probability & Cost & Off-Site & Reference \\
\hline Poor horizontal/vertical alignment on \#A1 approach & 30 & 3 & $\mathrm{n}$ & $432 a-432 b$ \\
\hline Poor horizontal/vertical alignment on \#A2 approach & 30 & 3 & $\mathrm{n}$ & $432 a-432 b$ \\
\hline Poor horizontal/vertical alignment on \#R1 road & 30 & 3 & $\mathrm{n}$ & $432 a-432 b$ \\
\hline Poor pavement marking on \#A1 approach & 25 & 2 & $\mathrm{n}$ & 76203 \\
\hline Poor signs reflectivity for drivers on \#R1 road & 15 & 2 & $\mathrm{n}$ & 75203 \\
\hline Poor signs visibility on \#R1 road & 20 & 2 & $\mathrm{n}$ & $\mathrm{N}$ \\
\hline Poor skid resistance on \#A1 approach & 30 & 3 & $\mathrm{n}$ & $\mathrm{N}$ \\
\hline Poor skid resistance on \#R1 road & 30 & 3 & $\mathrm{n}$ & $\mathrm{N}$ \\
\hline Poor visibility of opposite vehicles & 15 & 2 & $\mathrm{n}$ & $\mathrm{N}$ \\
\hline Poorly designed drain grates on $\mathrm{R} 1$ road & 15 & 4 & $\mathrm{n}$ & 5550302 \\
\hline Posted speed limit on \#R1 road & 80 & 1 & $\mathrm{n}$ & $\mathrm{N}$ \\
\hline Off-road collisions on \#A1 approach & 40 & 2 & $\mathrm{y}$ & $\mathrm{N}$ \\
\hline Off-road collisions on \#E1 exit & 40 & 2 & $\mathrm{y}$ & $\mathrm{N}$ \\
\hline Off-road collisions on \#R1 road & 40 & 2 & $\mathrm{y}$ & $\mathrm{N}$ \\
\hline Opposite left turn vehicles on \#R1 road obstruct each other sight distance & 20 & 2 & $\mathrm{n}$ & $\mathrm{N}$ \\
\hline $\mathrm{RRC}$ & - & 1 & $\mathrm{y}$ & $\mathrm{N}$ \\
\hline Rear-end collisions on \#A1 approach & 25 & 2 & $\mathrm{y}$ & $\mathrm{N}$ \\
\hline Rear-end collisions on \#E1 exit & 20 & 2 & $\mathrm{y}$ & $\mathrm{N}$ \\
\hline Red-light running & 10 & 2 & $\mathrm{n}$ & $\mathrm{N}$ \\
\hline Red-light violation & 10 & 2 & $\mathrm{n}$ & $\mathrm{N}$ \\
\hline Regulatory sign poorly visible/recognizable at intersection & 15 & 1 & $\mathrm{n}$ & $\mathrm{N}$ \\
\hline Regulatory sign posted at intersection & 70 & 1 & $\mathrm{n}$ & $\mathrm{N}$ \\
\hline Right-angle collisions between \#A1 and \#A2 vehicles & 40 & 2 & $\mathrm{y}$ & $\mathrm{N}$ \\
\hline Right-angle collisions between \#R1 and \#R2 vehicles & 40 & 2 & $\mathrm{y}$ & $\mathrm{N}$ \\
\hline Right turn collision & 15 & 2 & $\mathrm{y}$ & $\mathrm{N}$ \\
\hline Right-turn lanes not present on \#A1 approach & 50 & 1 & $\mathrm{n}$ & $\mathrm{N}$ \\
\hline Right-turn lanes present on \#A1 approach & 50 & 1 & $\mathrm{n}$ & $\mathrm{N}$ \\
\hline Right-turning vehicles on \#A1 approach obstruct sight of vehicles on \#A2 approach & 25 & 2 & $\mathrm{n}$ & $\mathrm{N}$ \\
\hline Road inadequate design for trucks & 10 & 2 & $\mathrm{n}$ & $\mathrm{N}$ \\
\hline Roadside sight obstruction at intersection & 30 & 3 & $\mathrm{n}$ & $422 a$ \\
\hline Roadside sight obstruction at RRC & 30 & 3 & $\mathrm{n}$ & $422 a$ \\
\hline
\end{tabular}




\begin{tabular}{|c|c|c|c|c|}
\hline Conditions & Probability & Cost & Off-Site & Reference \\
\hline Roadway inconspicuous & 45 & 2 & $\mathrm{n}$ & $\mathrm{N}$ \\
\hline RS & - & 1 & $y$ & $\mathrm{~N}$ \\
\hline School zone present & 40 & 1 & $\mathrm{n}$ & $\mathrm{N}$ \\
\hline Sight distance obstructed by other vehicles on \#A2 approach & 20 & 2 & $\mathrm{n}$ & $\mathrm{N}$ \\
\hline Sight distance obstructed by other vehicles parked close to intersection & 20 & 2 & $\mathrm{n}$ & $\mathrm{N}$ \\
\hline SIGN & - & 1 & y & $\mathrm{N}$ \\
\hline Sign Supports close to traveled way & 30 & 1 & $\mathrm{n}$ & $\mathrm{N}$ \\
\hline Signalized intersection(s) on \#R1 coordinated with the investigated intersection & 10 & 1 & $\mathrm{n}$ & $\mathrm{N}$ \\
\hline Significant high traffic volume turning from \#A2 approach into \#A1 approach & 15 & 3 & $\mathrm{n}$ & $\mathrm{N}$ \\
\hline Signifficant number of pedestrian crossing at intersection & 15 & 3 & $\mathrm{n}$ & $\mathrm{N}$ \\
\hline Signifficant number of bicycles on \#R1 road & 10 & 3 & $\mathrm{n}$ & $\mathrm{N}$ \\
\hline Signifficant number of pedestrian crossing intersection & 15 & 3 & $\mathrm{n}$ & $\mathrm{N}$ \\
\hline Slippery pavement (no winter) on \#A1 approach & 20 & 3 & $\mathrm{n}$ & ch52 \\
\hline Slippery pavement (no winter) on \#E1 exit & 20 & 3 & $\mathrm{n}$ & ch52 \\
\hline Slippery pavement (winter) on \#A1 approach & 10 & 3 & $\mathrm{n}$ & $\operatorname{ch} 52$ \\
\hline Slippery pavement (winter) on \#R1 road & 10 & 3 & $\mathrm{n}$ & ch52 \\
\hline Speed violation on \#R1 road & 35 & 2 & $\mathrm{n}$ & $\mathrm{N}$ \\
\hline Stop sign not visible on \#A2 approach & 30 & 2 & $\mathrm{n}$ & $\mathrm{N}$ \\
\hline Stop sign not visible on \#R2 road & 30 & 2 & $\mathrm{n}$ & $\mathrm{N}$ \\
\hline Stop sign obstruction on \#A1 approach & 15 & 1 & $\mathrm{n}$ & $\mathrm{N}$ \\
\hline Stop sign obstruction on \#A2 approach & 20 & 2 & $\mathrm{n}$ & $\mathrm{N}$ \\
\hline Stop sign visible on \#A2 approach & 70 & 2 & $\mathrm{n}$ & $\mathrm{N}$ \\
\hline Stop sign(s) visible on \#R2 road & 70 & 2 & $\mathrm{n}$ & $\mathrm{N}$ \\
\hline Two or more left turn lanes & 10 & 1 & $\mathrm{n}$ & $\mathrm{N}$ \\
\hline Trees close to traveled way & 25 & 2 & $\mathrm{n}$ & $\mathrm{N}$ \\
\hline Trucks collisions & 10 & 2 & $y$ & $\mathrm{~N}$ \\
\hline TWSC & - & 1 & $n$ & $\mathrm{~N}$ \\
\hline Undivided \#R1 road & 60 & 1 & $\mathrm{n}$ & $\mathrm{N}$ \\
\hline Unwarranted traffic control & 5 & 3 & $\mathrm{n}$ & $\mathrm{N}$ \\
\hline Utility Poles close to traveled way & 20 & 2 & $\mathrm{n}$ & $\mathrm{N}$ \\
\hline Vehicles queues forming across the railroad tracks & 5 & 1 & $\mathrm{n}$ & $\mathrm{N}$ \\
\hline
\end{tabular}




\begin{tabular}{|c|c|c|c|c|}
\hline $\begin{array}{c}\text { Conditions } \\
\end{array}$ & Probability & Cost & Off-Site & Reference \\
\hline Vehicle queues extend beyond taper on \#A1 approach & 20 & 2 & $\mathrm{n}$ & $\mathrm{N}$ \\
\hline Vehicle queues extend beyond turning bay on \#A1 approach & 20 & 2 & $\mathrm{n}$ & $\mathrm{N}$ \\
\hline Vehicles slow down before entering turning bay on \#A1 approach & 60 & 2 & $\mathrm{n}$ & $\mathrm{N}$ \\
\hline Vertical curve & 40 & 2 & $\mathrm{n}$ & $\mathrm{N}$ \\
\hline Visual/Audio warning signal does not work correctly & 15 & 1 & $\mathrm{n}$ & $\mathrm{N}$ \\
\hline Visual distraction at intersection & 60 & 2 & $\mathrm{n}$ & $\mathrm{N}$ \\
\hline Visual distraction on \#A1 approach & 60 & 2 & $\mathrm{n}$ & $\mathrm{N}$ \\
\hline Visual distraction on \#R1 road & 60 & 2 & $\mathrm{n}$ & $\mathrm{N}$ \\
\hline Warning sign obstruction on \#A1 approach & 10 & 1 & $\mathrm{n}$ & $\mathrm{N}$ \\
\hline Warning sign obstruction on \#R1 road & 10 & 1 & $\mathrm{n}$ & $\mathrm{N}$ \\
\hline Warning sign poorly visible/recognizable on \#A1 approach & 10 & 2 & $\mathrm{n}$ & $754 a$ \\
\hline Warning sign poorly visible/recognizable on \#R1 road & 10 & 2 & $\mathrm{n}$ & $754 a$ \\
\hline \multirow[t]{3}{*}{ Wide median on \#R1 road } & 10 & 2 & $\mathrm{n}$ & $\mathrm{N}$ \\
\hline & & $\begin{array}{l}\text { 1-LOW } \\
\text { COST }\end{array}$ & y-yes & $\begin{array}{l}\text { N- NO } \\
\text { DATA }\end{array}$ \\
\hline & & $\begin{array}{l}\text { 5-HIGH } \\
\text { COST }\end{array}$ & n-no & \\
\hline
\end{tabular}




\section{Appendix D}

\section{RSIT 2 Knowledge Base}

\begin{tabular}{|c|c|c|c|c|c|c|c|}
\hline $\begin{array}{l}\text { Close or relocate } \\
\text { driveway(s) on \#A1 } \\
\text { approach }\end{array}$ & TWSC & $\begin{array}{l}\text { Driveway(s) } \\
\text { collisions on } \\
\text { \#A1 } \\
\text { approach }\end{array}$ & $\begin{array}{l}\text { Driveway(s) on } \\
\text { \#A1 approach } \\
\text { close to } \\
\text { intersection }\end{array}$ & $\begin{array}{l}\text { Inadequate } \\
\text { visibility of } \\
\text { driveway(s) on } \\
\text { \#A1 approach }\end{array}$ & $\begin{array}{l}\text { Driveway(s) design } \\
\text { inadequate for high } \\
\text { traffic on \#A1 approach }\end{array}$ & & \\
\hline $\begin{array}{l}\text { Close or relocate } \\
\text { driveway(s) on \#E1 exit }\end{array}$ & TWSC & $\begin{array}{l}\text { Driveway(s) } \\
\text { collisions on } \\
\text { \#E1 exit }\end{array}$ & $\begin{array}{l}\text { Driveway(s) on } \\
\text { \#E1 exit close } \\
\text { to intersection }\end{array}$ & $\begin{array}{l}\text { Inadequate } \\
\text { visibility of } \\
\text { driveway(s) on } \\
\text { \#E1 exit }\end{array}$ & $\begin{array}{l}\text { Driveway(s) design } \\
\text { inadequate for high } \\
\text { traffic on \#E1 exit }\end{array}$ & & \\
\hline $\begin{array}{l}\text { Relocate driveway(s) } \\
\text { from \#E1 exit }\end{array}$ & TWSC & $\begin{array}{l}\text { Driveway(s) } \\
\text { collisions on } \\
\text { \#1 exit }\end{array}$ & $\begin{array}{l}\text { Driveway(s) on } \\
\text { \#E1 exit close } \\
\text { to intersection }\end{array}$ & $\begin{array}{l}\text { Inadequate } \\
\text { visibility of } \\
\text { driveway(s) on } \\
\text { \#E1 exit }\end{array}$ & $\begin{array}{l}\text { Driveway(s) design } \\
\text { inadequate for high } \\
\text { traffic on \#E1 exit }\end{array}$ & & \\
\hline $\begin{array}{l}\text { Channelize driveway(s) } \\
\text { on \#A1 approach }\end{array}$ & TWSC & $\begin{array}{l}\text { Driveway(s) } \\
\text { collisions on } \\
\text { \#A1 } \\
\text { approach }\end{array}$ & $\begin{array}{l}\text { Driveway(s) on } \\
\text { \#A1 approach } \\
\text { close to } \\
\text { intersection }\end{array}$ & $\begin{array}{l}\text { Inadequate } \\
\text { visibility of } \\
\text { driveway(s) on } \\
\text { \#A1 approach }\end{array}$ & $\begin{array}{l}\text { Driveway(s) design } \\
\text { inadequate for high } \\
\text { traffic on \#A1 approach }\end{array}$ & & \\
\hline $\begin{array}{l}\text { Channelize driveway(s) } \\
\text { on \#E1 exit }\end{array}$ & TWSC & $\begin{array}{l}\text { Driveway(s) } \\
\text { collisions on } \\
\text { \#E1 exit }\end{array}$ & $\begin{array}{l}\text { Driveway(s) on } \\
\text { \#E1 exit close } \\
\text { to intersection }\end{array}$ & $\begin{array}{l}\text { Inadequate } \\
\text { visibility of } \\
\text { driveway(s) on } \\
\text { \#E1 exit }\end{array}$ & $\begin{array}{l}\text { Driveway(s) design } \\
\text { inadequate for high } \\
\text { traffic on \#E1 exit }\end{array}$ & & \\
\hline $\begin{array}{l}\text { Install curbing to define } \\
\text { driveway(s) location on } \\
\text { \#A1 approach }\end{array}$ & TWSC & $\begin{array}{l}\text { Driveway(s) } \\
\text { collisions on } \\
\text { \#A1 } \\
\text { approach }\end{array}$ & $\begin{array}{l}\text { Driveway(s) on } \\
\# \text { A1 approach } \\
\text { close to } \\
\text { intersection }\end{array}$ & $\begin{array}{l}\text { Inadequate } \\
\text { visibility of } \\
\text { driveway(s) on } \\
\text { \#A1 approach }\end{array}$ & $\begin{array}{l}\text { Driveway(s) design } \\
\text { inadequate for high } \\
\text { traffic on \#A1 approach }\end{array}$ & & \\
\hline $\begin{array}{l}\text { Install curbing to define } \\
\text { driveway(s) location on } \\
\text { \#E1 exit }\end{array}$ & TWSC & $\begin{array}{l}\text { Driveway(s) } \\
\text { collisions on } \\
\text { \#E1 exit }\end{array}$ & $\begin{array}{l}\text { Driveway(s) on } \\
\text { \#E1 exit close } \\
\text { to intersection }\end{array}$ & $\begin{array}{l}\text { Inadequate } \\
\text { visibility of } \\
\text { driveway(s) on } \\
\text { \#E1 exit }\end{array}$ & $\begin{array}{l}\text { Driveway(s) design } \\
\text { inadequate for high } \\
\text { traffic on \#E1 exit }\end{array}$ & & \\
\hline $\begin{array}{l}\text { Install median divider } \\
\text { on \#A1 approach to } \\
\text { prevent left-turns } \\
\text { from/to driveway(s) }\end{array}$ & TWSC & $\begin{array}{l}\text { Driveway(s) } \\
\text { collisions on } \\
\text { \#A1 } \\
\text { approach }\end{array}$ & $\begin{array}{l}\text { Driveway(s) on } \\
\text { \#A1 approach } \\
\text { close to } \\
\text { intersection }\end{array}$ & $\begin{array}{l}\text { Inadequate } \\
\text { visibility of } \\
\text { driveway(s) on } \\
\text { \#A1 approach }\end{array}$ & $\begin{array}{l}\text { Driveway(s) design } \\
\text { inadequate for high } \\
\text { traffic on \#A1 approach }\end{array}$ & & \\
\hline
\end{tabular}




\begin{tabular}{|c|c|c|c|c|c|c|c|}
\hline $\begin{array}{l}\text { Install median divider } \\
\text { on \#E1 exit to prevent } \\
\text { left-turns from/to } \\
\text { driveway(s) }\end{array}$ & TWSC & $\begin{array}{l}\text { Driveway(s) } \\
\text { collisions on } \\
\text { \#E1 exit }\end{array}$ & $\begin{array}{l}\text { Driveway(s) on } \\
\text { \#E1 exit close } \\
\text { to intersection }\end{array}$ & $\begin{array}{l}\text { Inadequate } \\
\text { visibility of } \\
\text { driveway(s) on } \\
\text { \#E1 exit }\end{array}$ & $\begin{array}{l}\text { Driveway(s) design } \\
\text { inadequate for high } \\
\text { traffic on \#E1 exit }\end{array}$ & & \\
\hline $\begin{array}{l}\text { Provide deceleration } \\
\text { lane on \#A1 approach }\end{array}$ & TWSC & $\begin{array}{l}\text { Driveway(s) } \\
\text { collisions on } \\
\text { \#A1 } \\
\text { approach }\end{array}$ & $\begin{array}{l}\text { Driveway(s) on } \\
\text { \#A1 approach } \\
\text { close to } \\
\text { intersection }\end{array}$ & $\begin{array}{l}\text { Inadequate } \\
\text { visibility of } \\
\text { driveway(s) on } \\
\text { \#A1 approach }\end{array}$ & $\begin{array}{l}\text { Driveway(s) design } \\
\text { inadequate for high } \\
\text { traffic on \#A1 approach }\end{array}$ & & \\
\hline $\begin{array}{l}\text { Provide acceleration } \\
\text { lane on \#E1 exit }\end{array}$ & TWSC & $\begin{array}{l}\text { Driveway(s) } \\
\text { collisions on } \\
\text { \#E1 exit }\end{array}$ & $\begin{array}{l}\text { Driveway(s) on } \\
\text { \#E1 exit close } \\
\text { to intersection }\end{array}$ & $\begin{array}{l}\text { Inadequate } \\
\text { visibility of } \\
\text { driveway(s) on } \\
\text { \#E1 exit }\end{array}$ & $\begin{array}{l}\text { Driveway(s) design } \\
\text { inadequate for high } \\
\text { traffic on \#E1 exit }\end{array}$ & & \\
\hline $\begin{array}{l}\text { Relocate driveway(s) } \\
\text { from \#A1 approach }\end{array}$ & TWSC & $\begin{array}{l}\text { Driveway(s) } \\
\text { collisions on } \\
\text { \#A1 } \\
\text { approach }\end{array}$ & $\begin{array}{l}\text { Driveway(s) on } \\
\text { \#A1 approach } \\
\text { close to } \\
\text { intersection }\end{array}$ & $\begin{array}{l}\text { Inadequate } \\
\text { visibility of } \\
\text { driveway(s) on } \\
\text { \#A1 approach }\end{array}$ & $\begin{array}{l}\text { Driveway(s) design } \\
\text { inadequate for high } \\
\text { traffic on \#A1 approach }\end{array}$ & & \\
\hline $\begin{array}{l}\text { Remove sight } \\
\text { obstruction on \#A1 } \\
\text { approach }\end{array}$ & TWSC & $\begin{array}{l}\text { Driveway(s) } \\
\text { collisions on } \\
\text { \#A1 } \\
\text { approach }\end{array}$ & $\begin{array}{l}\text { Driveway(s) on } \\
\text { \#A1 approach } \\
\text { close to } \\
\text { intersection }\end{array}$ & $\begin{array}{l}\text { Inadequate } \\
\text { visibility of } \\
\text { driveway(s) on } \\
\text { \#A1 approach }\end{array}$ & & & \\
\hline $\begin{array}{l}\text { Remove sight } \\
\text { obstruction on \#E1 exit }\end{array}$ & TWSC & $\begin{array}{l}\text { Driveway(s) } \\
\text { collisions on } \\
\text { \#E1 exit }\end{array}$ & $\begin{array}{l}\text { Driveway(s) on } \\
\text { \#E1 exit close } \\
\text { to intersection }\end{array}$ & $\begin{array}{l}\text { Inadequate } \\
\text { visibility of } \\
\text { driveway(s) on } \\
\text { \#E1 exit }\end{array}$ & & & \\
\hline $\begin{array}{l}\text { Restrict left-turning at } \\
\text { access point on \#A1 } \\
\text { approach }\end{array}$ & TWSC & $\begin{array}{l}\text { Driveway(s) } \\
\text { collisions on } \\
\text { \#A1 } \\
\text { approach }\end{array}$ & $\begin{array}{l}\text { Driveway(s) on } \\
\text { \#A1 approach } \\
\text { close to } \\
\text { intersection }\end{array}$ & $\begin{array}{l}\text { Inadequate } \\
\text { visibility of } \\
\text { driveway(s) on } \\
\text { \#A1 approach }\end{array}$ & $\begin{array}{l}\text { Driveway(s) design } \\
\text { inadequate for high } \\
\text { traffic on \#A1 approach }\end{array}$ & & \\
\hline $\begin{array}{l}\text { Restrict left-turning at } \\
\text { access point on \#E1 } \\
\text { exit }\end{array}$ & TWSC & $\begin{array}{l}\text { Driveway(s) } \\
\text { collisions on } \\
\text { \#E1 exit }\end{array}$ & $\begin{array}{l}\text { Driveway(s) on } \\
\text { \#E1 exit close } \\
\text { to intersection }\end{array}$ & $\begin{array}{l}\text { Inadequate } \\
\text { visibility of } \\
\text { driveway(s) on } \\
\text { \#E1 exit }\end{array}$ & $\begin{array}{l}\text { Driveway(s) design } \\
\text { inadequate for high } \\
\text { traffic on \#E1 exit }\end{array}$ & & \\
\hline $\begin{array}{l}\text { Restrict parking near } \\
\text { driveway(s) on \#A1 } \\
\text { approach }\end{array}$ & TWSC & $\begin{array}{l}\text { Driveway(s) } \\
\text { collisions on } \\
\text { \#A1 } \\
\text { approach }\end{array}$ & $\begin{array}{l}\text { Driveway(s) on } \\
\text { \#A1 approach } \\
\text { close to } \\
\text { intersection }\end{array}$ & $\begin{array}{l}\text { Inadequate } \\
\text { visibility of } \\
\text { driveway(s) on } \\
\text { \#A1 approach }\end{array}$ & & & \\
\hline
\end{tabular}




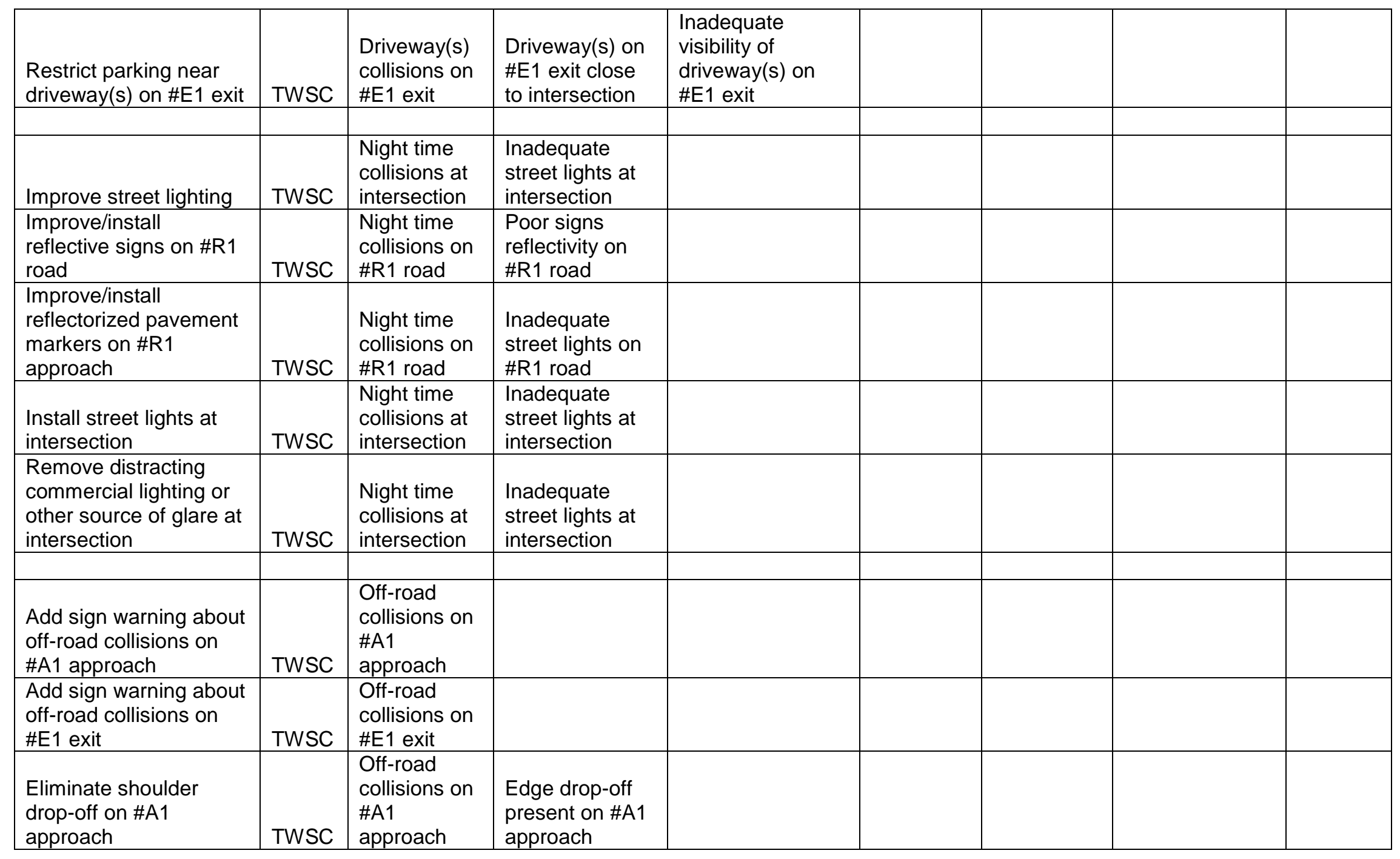




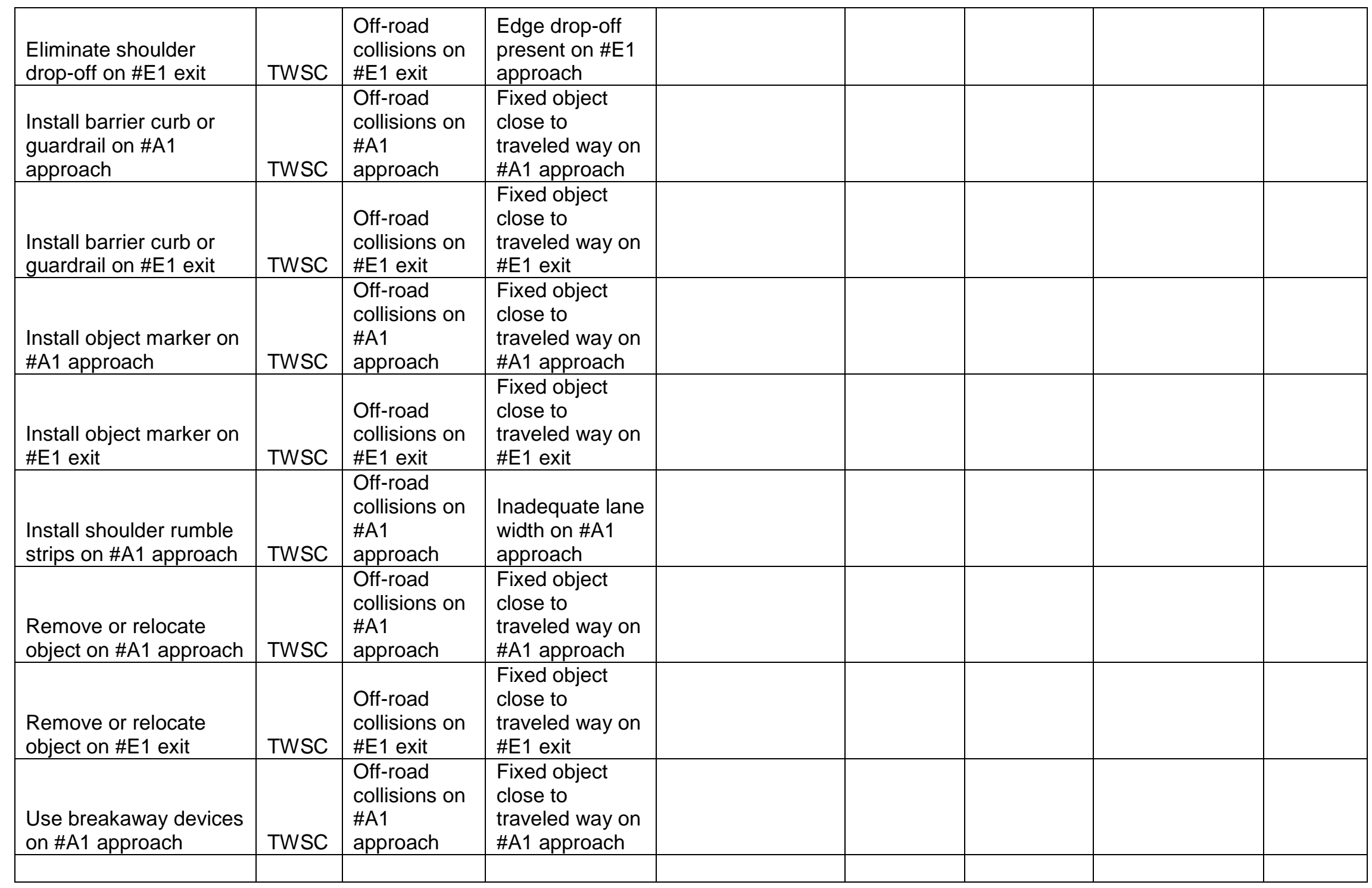




\begin{tabular}{|c|c|c|c|c|c|c|c|c|}
\hline $\begin{array}{l}\text { Use breakaway devices } \\
\text { on \#E1 exit }\end{array}$ & TWSC & $\begin{array}{l}\text { Off-road } \\
\text { collisions on } \\
\text { \#E1 exit }\end{array}$ & $\begin{array}{l}\text { Fixed object } \\
\text { close to } \\
\text { traveled way on } \\
\text { \#E1 exit }\end{array}$ & & & & & \\
\hline $\begin{array}{l}\text { Install overhead STOP } \\
\text { sign(s) on \#A2 } \\
\text { approach }\end{array}$ & TWSC & $\begin{array}{l}\text { Right-angle } \\
\text { collisions } \\
\text { between \#A1 } \\
\text { and \#A2 } \\
\text { vehicles }\end{array}$ & $\begin{array}{l}\text { \#A1 major } \\
\text { approach }\end{array}$ & $\begin{array}{l}\text { \#A2 minor } \\
\text { approach }\end{array}$ & \multicolumn{2}{|c|}{$\begin{array}{l}\text { Percent of tall vehicles } \\
\text { considerable on } \\
\text { approach \#A2 }\end{array}$} & & \\
\hline $\begin{array}{l}\text { Add stoplines in } \\
\text { median opening on } \\
\text { \#R1 road }\end{array}$ & TWSC & $\begin{array}{l}\text { Right-angle } \\
\text { collisions } \\
\text { between \#A1 } \\
\text { and \#A2 } \\
\text { vehicles }\end{array}$ & $\begin{array}{l}\text { \#A1 major } \\
\text { approach }\end{array}$ & $\begin{array}{l}\text { \#A2 minor } \\
\text { approach }\end{array}$ & $\begin{array}{l}\text { Stop sign } \\
\text { visible on } \\
\text { \#A2 } \\
\text { approach }\end{array}$ & $\begin{array}{l}\text { Divided } \\
\text { \#R1 road }\end{array}$ & $\begin{array}{l}\text { Missing } \\
\text { stoplines in the } \\
\text { median opening } \\
\text { on \#R1 road }\end{array}$ & $\begin{array}{l}\text { Wide } \\
\text { median } \\
\text { on \#R1 } \\
\text { road }\end{array}$ \\
\hline $\begin{array}{l}\text { Adjust signal offsets } \\
\text { along \#R1 road to } \\
\text { create longer inter- } \\
\text { vehicle gaps }\end{array}$ & TWSC & $\begin{array}{l}\text { Right-angle } \\
\text { collisions } \\
\text { between } \\
\text { \#R1 and } \\
\text { \#R2 vehicles }\end{array}$ & \#R1 major road & \#R2 minor road & $\begin{array}{l}\text { Stop } \\
\text { sign(s) } \\
\text { visible on } \\
\text { \#R2 road }\end{array}$ & $\begin{array}{l}\text { Insufficient } \\
\text { gaps } \\
\text { between } \\
\text { vehicles on } \\
\text { \#R1 road }\end{array}$ & \multicolumn{2}{|c|}{$\begin{array}{l}\text { Signalized intersection(s) } \\
\text { on \#R1 coordinated with } \\
\text { the investigated } \\
\text { intersection }\end{array}$} \\
\hline $\begin{array}{l}\text { Consider intersection } \\
\text { with indirect left-turns }\end{array}$ & TWSC & $\begin{array}{l}\text { Right-angle } \\
\text { collisions } \\
\text { between \#A1 } \\
\text { and \#A2 } \\
\text { vehicles }\end{array}$ & $\begin{array}{l}\text { \#A1 major } \\
\text { approach }\end{array}$ & $\begin{array}{l}\text { \#A2 minor } \\
\text { approach }\end{array}$ & $\begin{array}{l}\text { Stop sign } \\
\text { visible on } \\
\text { \#A2 } \\
\text { approach }\end{array}$ & $\begin{array}{l}\text { Divided } \\
\text { \#R1 road }\end{array}$ & $\begin{array}{l}\text { Significant high } \\
\text { traffic volume } \\
\text { turning from } \\
\text { \#A2 approach } \\
\text { into \#E1 exit }\end{array}$ & \\
\hline $\begin{array}{l}\text { Consider a lateral } \\
\text { relocation of right-turn } \\
\text { lanes on \#A1 approach }\end{array}$ & TWSC & $\begin{array}{l}\text { Right-angle } \\
\text { collisions } \\
\text { between \#A1 } \\
\text { and \#A2 } \\
\text { vehicles }\end{array}$ & $\begin{array}{l}\text { \#A1 major } \\
\text { approach }\end{array}$ & $\begin{array}{l}\text { \#A2 minor } \\
\text { approach }\end{array}$ & $\begin{array}{l}\text { Stop sign } \\
\text { visible on } \\
\text { \#A2 } \\
\text { approach }\end{array}$ & $\begin{array}{l}\text { Insufficient } \\
\text { sight } \\
\text { distance } \\
\text { from \#A2 } \\
\text { approach }\end{array}$ & \multicolumn{2}{|c|}{$\begin{array}{l}\text { Right-turning vehicles on } \\
\# A 1 \text { approach obstruct } \\
\text { sight of vehicles on \#A2 } \\
\text { approach }\end{array}$} \\
\hline $\begin{array}{l}\text { Convert intersection } \\
\text { into roundabout }\end{array}$ & TWSC & $\begin{array}{l}\text { Right-angle } \\
\text { collisions } \\
\text { between \#A1 } \\
\text { and \#A2 } \\
\text { vehicles }\end{array}$ & $\begin{array}{l}\text { \#A1 major } \\
\text { approach }\end{array}$ & $\begin{array}{l}\text { \#A2 minor } \\
\text { approach }\end{array}$ & $\begin{array}{l}\text { Stop sign } \\
\text { visible on } \\
\text { \#A2 } \\
\text { approach }\end{array}$ & $\begin{array}{l}\text { Insufficient } \\
\text { gaps } \\
\text { between } \\
\text { vehicle on } \\
\text { \#A1 } \\
\text { approach }\end{array}$ & & \\
\hline
\end{tabular}




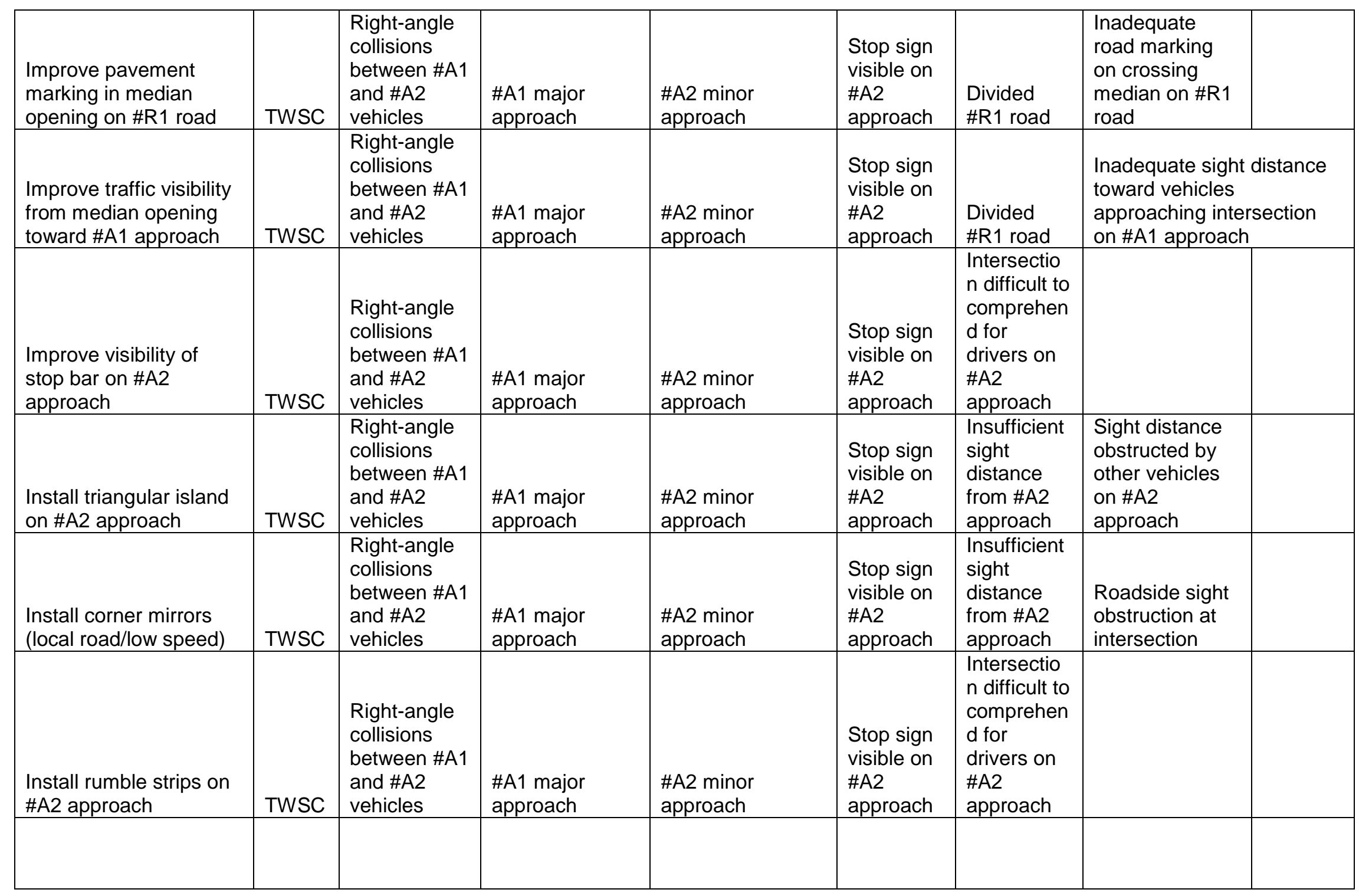




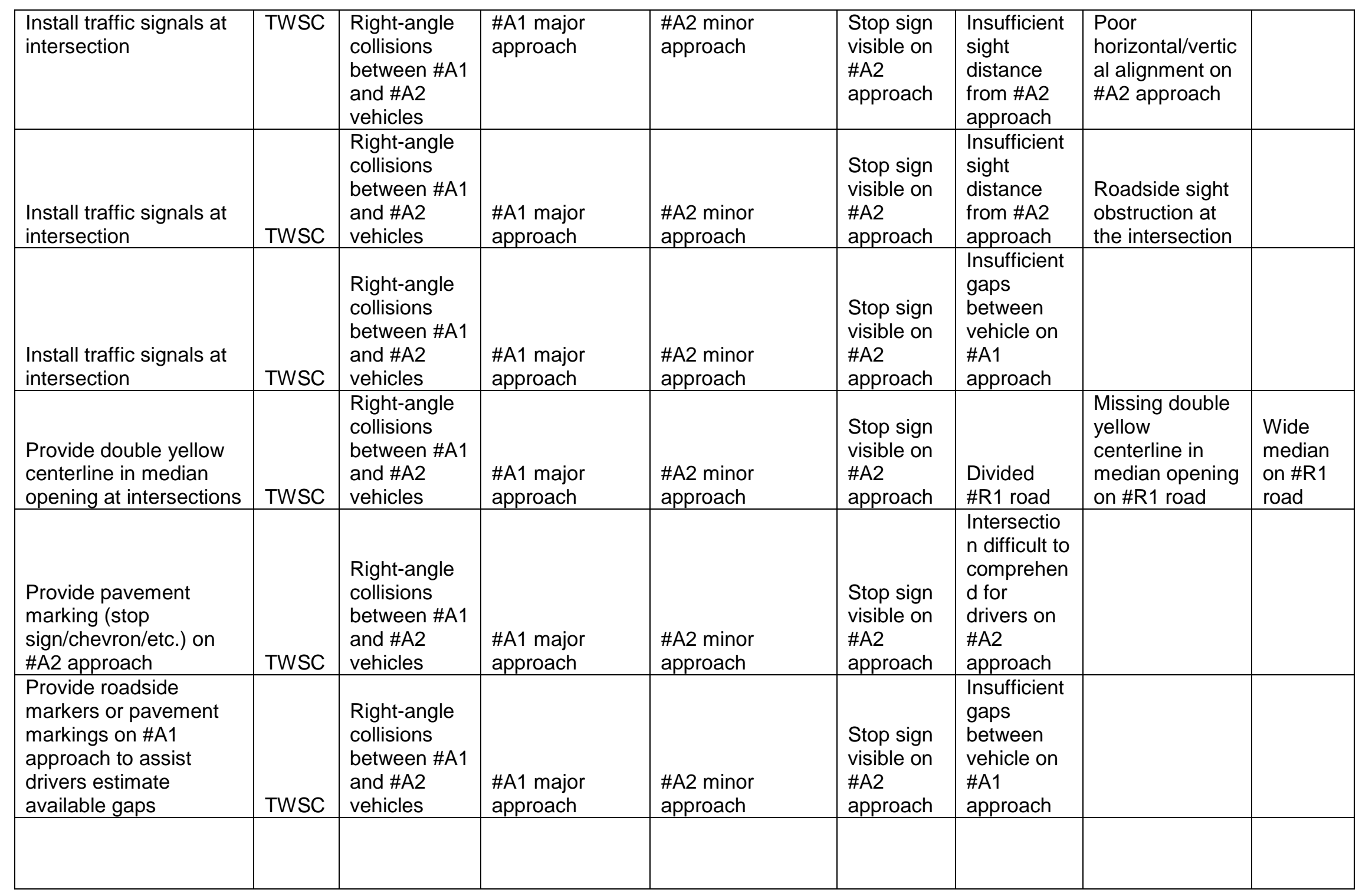




\begin{tabular}{|c|c|c|c|c|c|c|c|}
\hline $\begin{array}{l}\text { Provide splitter islands } \\
\text { on \#A2 approach }\end{array}$ & TWSC & $\begin{array}{l}\text { Right-angle } \\
\text { collisions } \\
\text { between \#A1 } \\
\text { and \#A2 } \\
\text { vehicles }\end{array}$ & $\begin{array}{l}\text { \#A1 major } \\
\text { approach }\end{array}$ & $\begin{array}{l}\text { \#A2 minor } \\
\text { approach }\end{array}$ & $\begin{array}{l}\text { Stop sign } \\
\text { visible on } \\
\text { \#A2 } \\
\text { approach }\end{array}$ & $\begin{array}{l}\text { Intersectio } \\
\text { n difficult to } \\
\text { comprehen } \\
d \text { for } \\
\text { drivers on } \\
\text { \#A2 } \\
\text { approach }\end{array}$ & \\
\hline $\begin{array}{l}\text { Reduce speed limit on } \\
\text { \#A1 approach }\end{array}$ & TWSC & $\begin{array}{l}\text { Right-angle } \\
\text { collisions } \\
\text { between \#A1 } \\
\text { and \#A2 } \\
\text { vehicles }\end{array}$ & $\begin{array}{l}\text { \#A1 major } \\
\text { approach }\end{array}$ & $\begin{array}{l}\text { \#A2 minor } \\
\text { approach }\end{array}$ & $\begin{array}{l}\text { Stop sign } \\
\text { visible on } \\
\text { \#A2 } \\
\text { approach }\end{array}$ & $\begin{array}{l}\text { Insufficient } \\
\text { sight } \\
\text { distance } \\
\text { from \#A1 } \\
\text { approach } \\
\text { toward \#A2 } \\
\text { approach }\end{array}$ & $\begin{array}{l}\text { Roadside sight } \\
\text { obstruction at } \\
\text { intersection }\end{array}$ \\
\hline $\begin{array}{l}\text { Reduce number of } \\
\text { lanes on \#A2 approach } \\
\text { if allowed by capacity }\end{array}$ & TWSC & $\begin{array}{l}\text { Right-angle } \\
\text { collisions } \\
\text { between \#A1 } \\
\text { and \#A2 } \\
\text { vehicles }\end{array}$ & $\begin{array}{l}\text { \#A1 major } \\
\text { approach }\end{array}$ & $\begin{array}{l}\text { \#A2 minor } \\
\text { approach }\end{array}$ & $\begin{array}{l}\text { Stop sign } \\
\text { visible on } \\
\text { \#A2 } \\
\text { approach }\end{array}$ & $\begin{array}{l}\text { Insufficient } \\
\text { sight } \\
\text { distance } \\
\text { from \#A2 } \\
\text { approach }\end{array}$ & $\begin{array}{l}\text { Sight distance } \\
\text { obstructed by } \\
\text { other vehicles } \\
\text { on \#A2 } \\
\text { approach }\end{array}$ \\
\hline $\begin{array}{l}\text { Relocate or split } \\
\text { stopping line on \#A2 } \\
\text { approach }\end{array}$ & TWSC & $\begin{array}{l}\text { Right-angle } \\
\text { collisions } \\
\text { between \#A1 } \\
\text { and \#A2 } \\
\text { vehicles }\end{array}$ & $\begin{array}{l}\text { \#A1 major } \\
\text { approach }\end{array}$ & $\begin{array}{l}\text { \#A2 minor } \\
\text { approach }\end{array}$ & $\begin{array}{l}\text { Stop sign } \\
\text { visible on } \\
\text { \#A2 } \\
\text { approach }\end{array}$ & $\begin{array}{l}\text { Insufficient } \\
\text { sight } \\
\text { distance } \\
\text { from \#A2 } \\
\text { approach }\end{array}$ & $\begin{array}{l}\text { Sight distance } \\
\text { obstructed by } \\
\text { other vehicles } \\
\text { on \#A2 } \\
\text { approach }\end{array}$ \\
\hline $\begin{array}{l}\text { Relocate stop bar on } \\
\text { \#A2 approach }\end{array}$ & TWSC & $\begin{array}{l}\text { Right-angle } \\
\text { collisions } \\
\text { between \#A1 } \\
\text { and \#A2 } \\
\text { vehicles }\end{array}$ & $\begin{array}{l}\text { \#A1 major } \\
\text { approach }\end{array}$ & $\begin{array}{l}\text { \#A2 minor } \\
\text { approach }\end{array}$ & $\begin{array}{l}\text { Stop sign } \\
\text { visible on } \\
\text { \#A2 } \\
\text { approach }\end{array}$ & $\begin{array}{l}\text { Insufficient } \\
\text { sight } \\
\text { distance } \\
\text { from \#A2 } \\
\text { approach }\end{array}$ & $\begin{array}{l}\text { Roadside sight } \\
\text { obstruction at } \\
\text { intersection }\end{array}$ \\
\hline $\begin{array}{l}\text { Relocate stop sign on } \\
\text { \#A2 approach }\end{array}$ & TWSC & $\begin{array}{l}\text { Right-angle } \\
\text { collisions } \\
\text { between \#A1 } \\
\text { and \#A2 } \\
\text { vehicles }\end{array}$ & $\begin{array}{l}\text { \#A1 major } \\
\text { approach }\end{array}$ & $\begin{array}{l}\text { \#A2 minor } \\
\text { approach }\end{array}$ & $\begin{array}{l}\text { Stop sign } \\
\text { NOT } \\
\text { visible on } \\
\text { \#A2 } \\
\text { approach }\end{array}$ & $\begin{array}{l}\text { Stop sign } \\
\text { obstruction } \\
\text { on \#A2 } \\
\text { approach }\end{array}$ & \\
\hline & & & & & & & \\
\hline
\end{tabular}




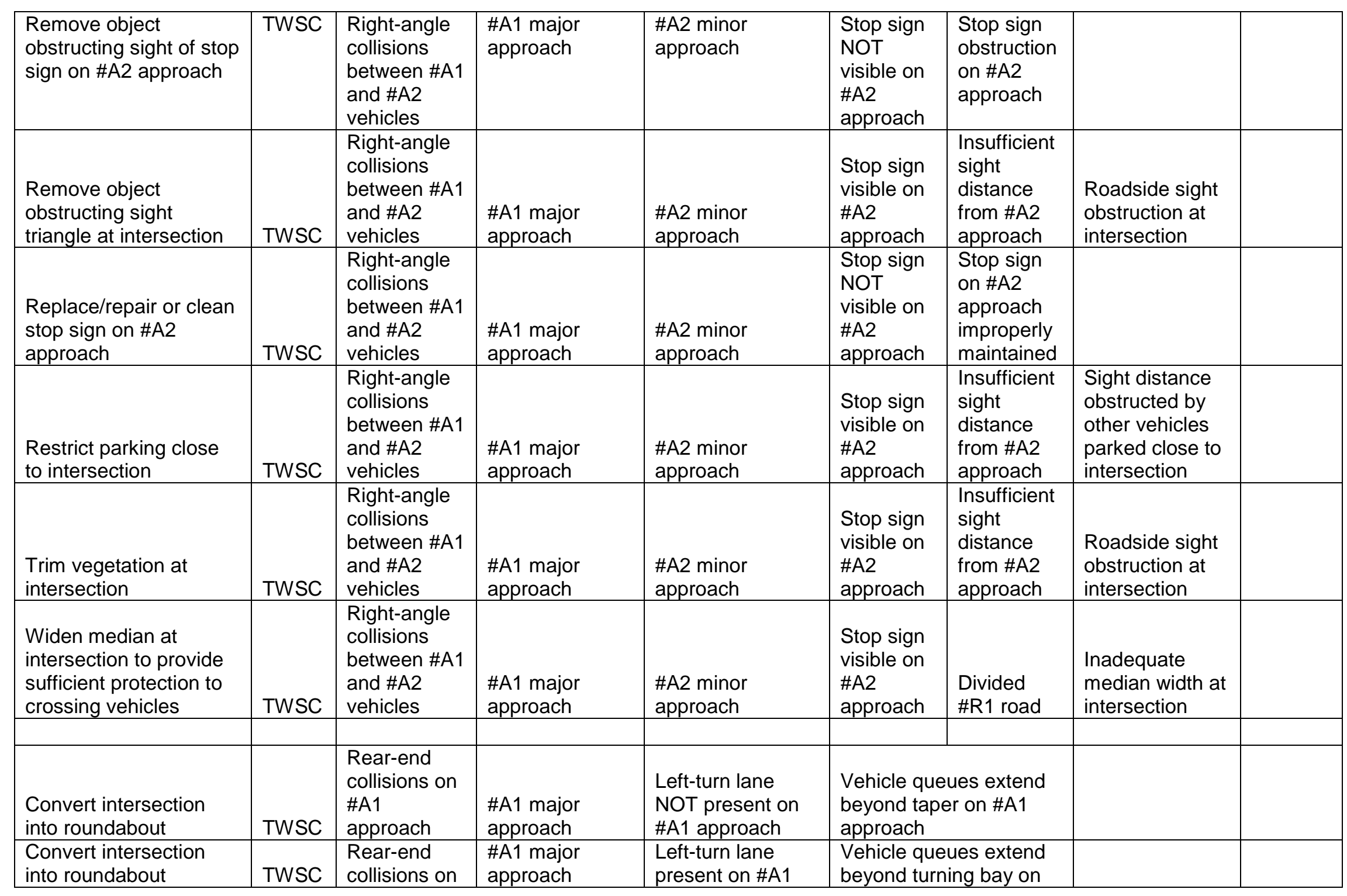




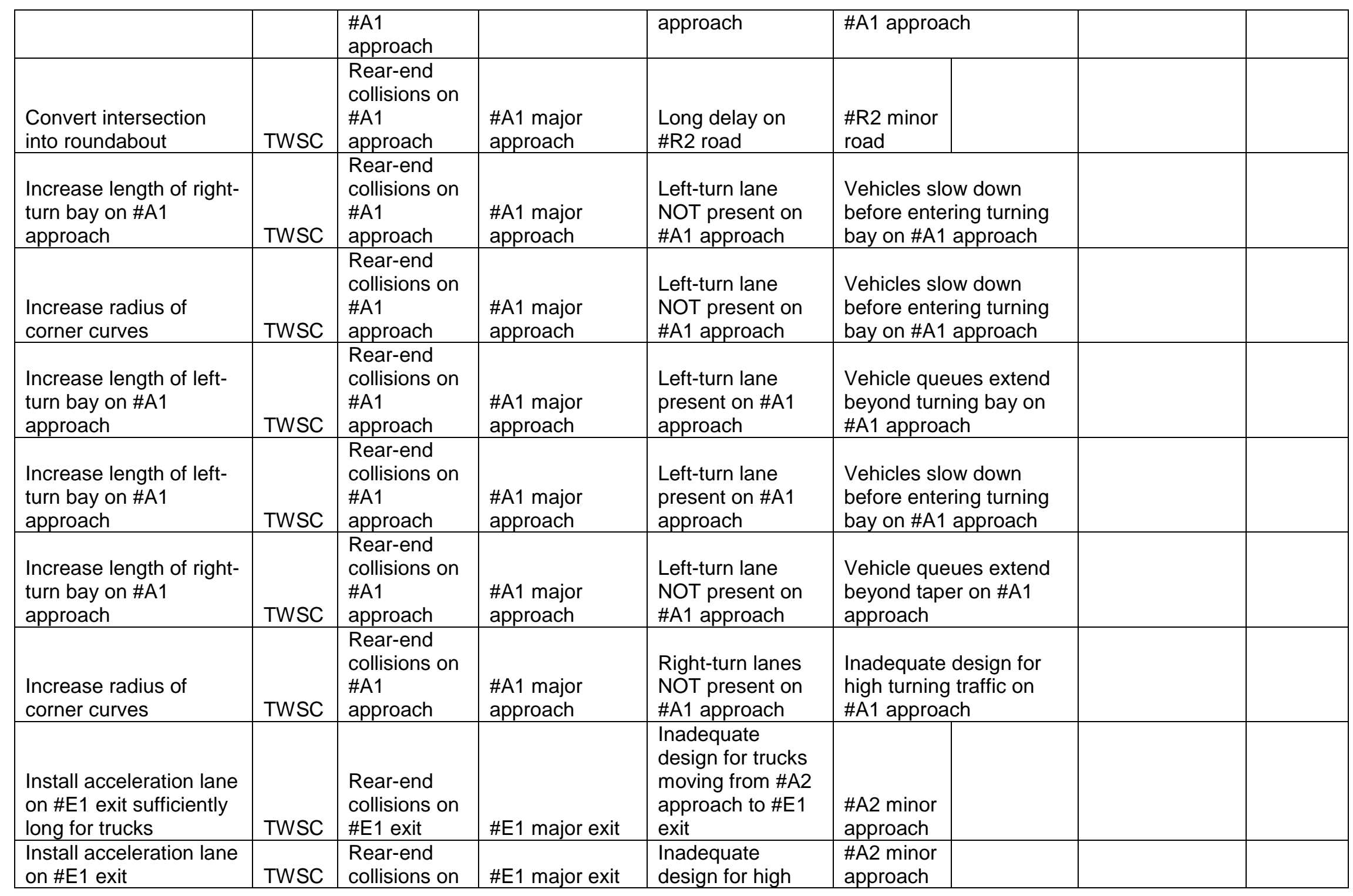




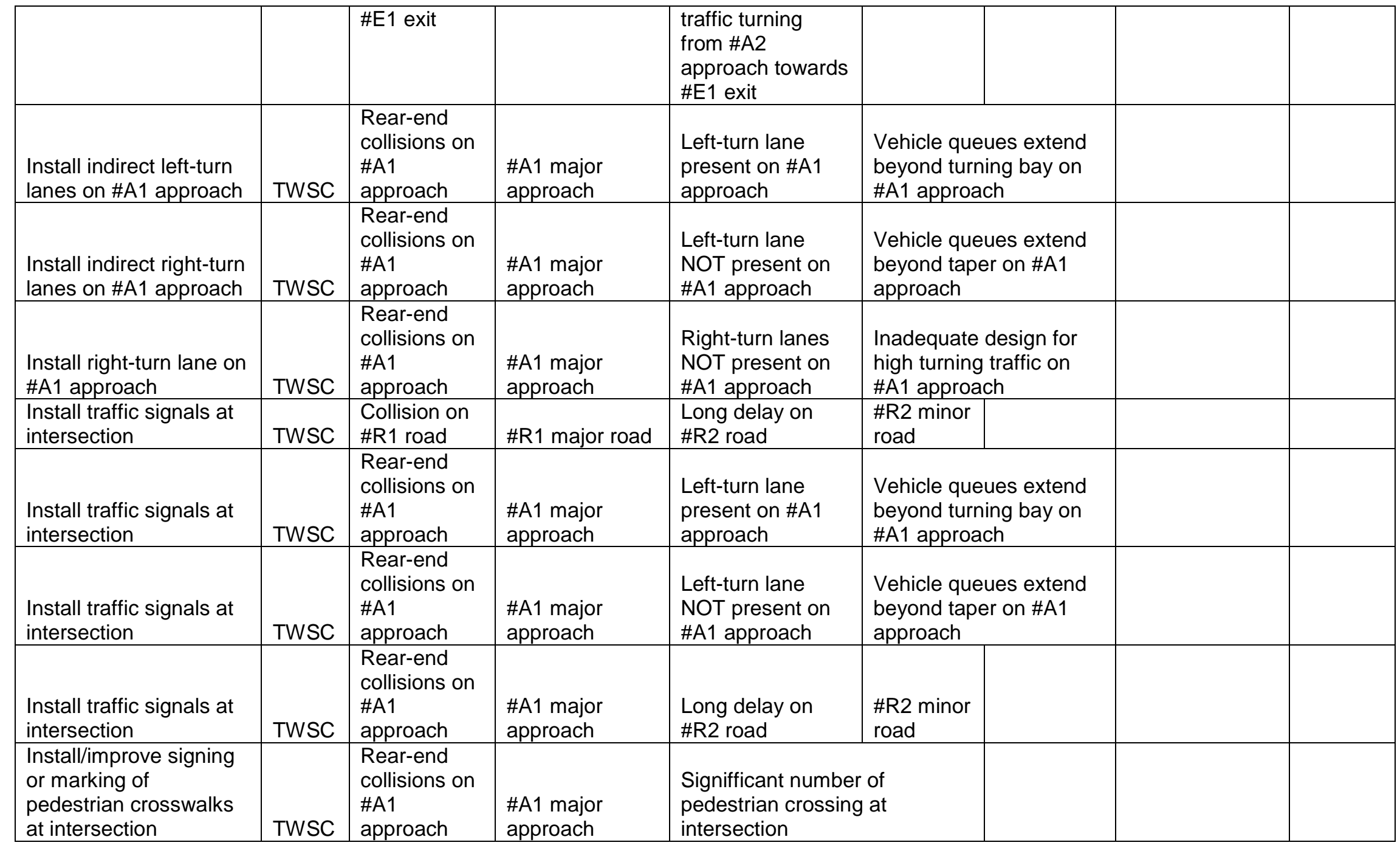




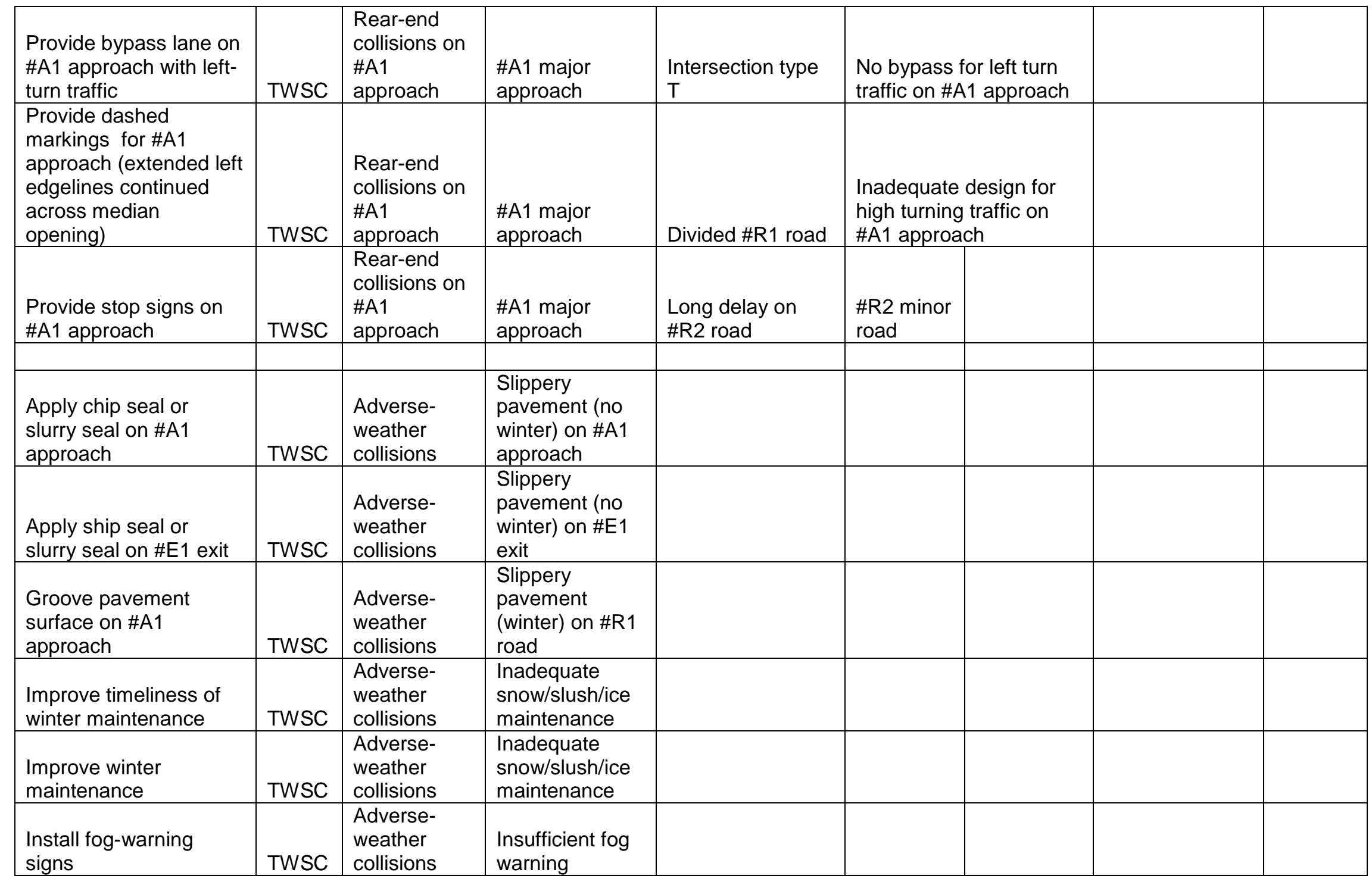




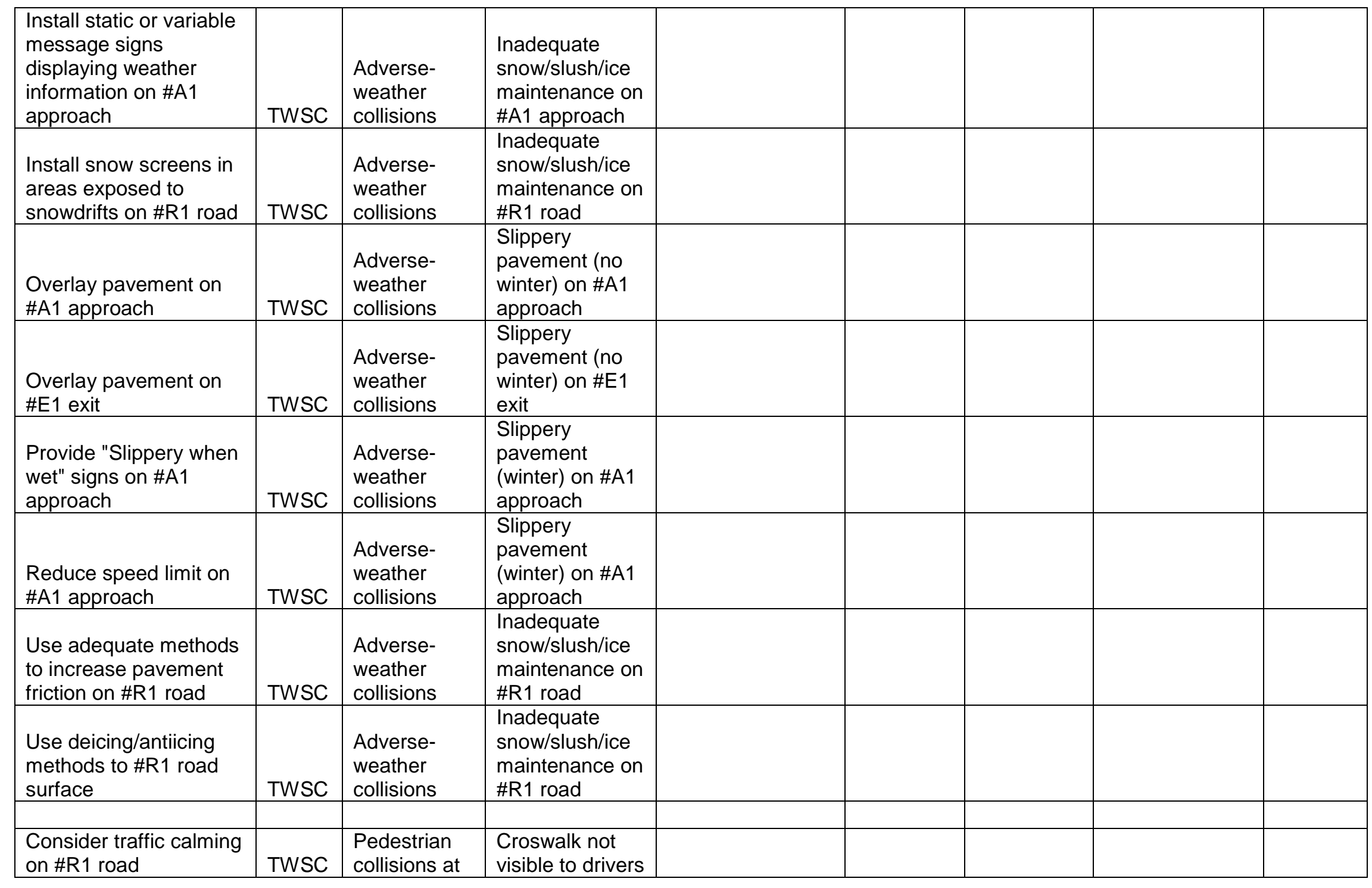




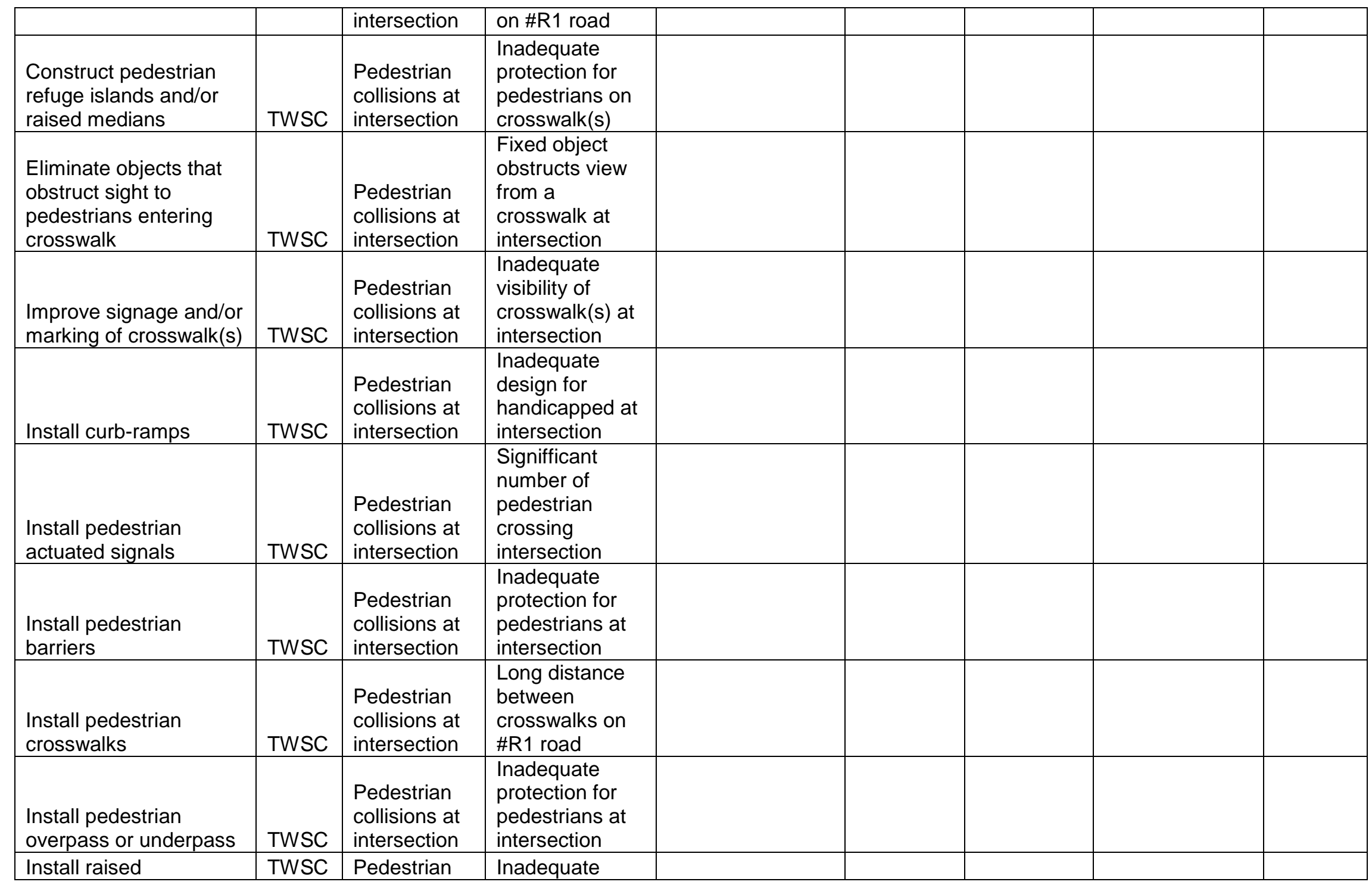




\begin{tabular}{|c|c|c|c|c|c|c|c|c|}
\hline crosswalks & & $\begin{array}{l}\text { collisions at } \\
\text { intersection }\end{array}$ & $\begin{array}{l}\text { visibility of } \\
\text { crosswalk(s) at } \\
\text { intersection }\end{array}$ & & & & & \\
\hline $\begin{array}{l}\text { Install school crossing } \\
\text { sign on \#R1 road }\end{array}$ & TWSC & $\begin{array}{l}\text { Pedestrian } \\
\text { collisions at } \\
\text { intersection }\end{array}$ & $\begin{array}{l}\text { School zone } \\
\text { present }\end{array}$ & $\begin{array}{l}\text { Insufficient school } \\
\text { zone warning on } \\
\text { \#R1 road }\end{array}$ & & & & \\
\hline $\begin{array}{l}\text { Install school zone } \\
\text { markings on \#R1 road }\end{array}$ & TWSC & $\begin{array}{l}\text { Pedestrian } \\
\text { collisions at } \\
\text { intersection }\end{array}$ & $\begin{array}{l}\text { School zone } \\
\text { present }\end{array}$ & $\begin{array}{l}\text { Insufficient school } \\
\text { zone warning on } \\
\text { \#R1 road }\end{array}$ & & & & \\
\hline $\begin{array}{l}\text { Install school zone } \\
\text { speed limit sign on \#R1 } \\
\text { road }\end{array}$ & TWSC & $\begin{array}{l}\text { Pedestrian } \\
\text { collisions at } \\
\text { intersection }\end{array}$ & $\begin{array}{l}\text { School zone } \\
\text { present }\end{array}$ & $\begin{array}{l}\text { No school zone } \\
\text { speed limit } \\
\text { posted on \#R1 } \\
\text { road }\end{array}$ & & & & \\
\hline $\begin{array}{l}\text { Increase sidewalk } \\
\text { setback on \#R1 road }\end{array}$ & TWSC & $\begin{array}{l}\text { Pedestrian } \\
\text { collisions on } \\
\text { \#R1 road }\end{array}$ & $\begin{array}{l}\text { Inadequate } \\
\text { protection of } \\
\text { pedestrians on } \\
\text { \#R1 road }\end{array}$ & & & & & \\
\hline Install street lighting & TWSC & $\begin{array}{l}\text { Pedestrian } \\
\text { collisions at } \\
\text { intersection }\end{array}$ & $\begin{array}{l}\text { Inadequate } \\
\text { lighting of } \\
\text { crosswalk at } \\
\text { intersection }\end{array}$ & & & & & \\
\hline $\begin{array}{l}\text { Provide separate } \\
\text { bicycle path on \#R1 } \\
\text { road }\end{array}$ & TWSC & $\begin{array}{l}\text { Bicycle } \\
\text { collisions on } \\
\text { \#R1 road } \\
\end{array}$ & $\begin{array}{l}\text { Inadequate } \\
\text { bicycles space } \\
\text { on \#R1 road } \\
\end{array}$ & & & & & \\
\hline $\begin{array}{l}\text { Provide separate } \\
\text { bicycle path on \#R1 } \\
\text { road }\end{array}$ & TWSC & $\begin{array}{l}\text { Bicycle } \\
\text { collisions on } \\
\text { \#R1 road }\end{array}$ & $\begin{array}{l}\text { Signifficant } \\
\text { number of } \\
\text { bicycles on \#R1 } \\
\text { road }\end{array}$ & & & & & \\
\hline $\begin{array}{l}\text { Provide median refuges } \\
\text { at intersection }\end{array}$ & TWSC & $\begin{array}{l}\text { Bicycle } \\
\text { collisions at } \\
\text { intersection }\end{array}$ & $\begin{array}{l}\text { High traffic } \\
\text { volume on \#R1 } \\
\text { road }\end{array}$ & & & & & \\
\hline $\begin{array}{l}\text { Provide median refuges } \\
\text { at intersection }\end{array}$ & TWSC & $\begin{array}{l}\text { Bicycle } \\
\text { collisions at } \\
\text { intersection }\end{array}$ & \multicolumn{2}{|c|}{$\begin{array}{l}\text { Multiple lanes on \#R1 road make } \\
\text { difficult for bicycles to cross \#R1 } \\
\text { road }\end{array}$} & & & & \\
\hline $\begin{array}{l}\text { Provide smooth paved } \\
\text { shoulders on \#A1 } \\
\text { approach }\end{array}$ & TWSC & $\begin{array}{l}\text { Bicycle } \\
\text { collisions on } \\
\text { \#A1 }\end{array}$ & $\begin{array}{l}\text { Inadequate } \\
\text { shoulder design } \\
\text { on \#A1 }\end{array}$ & & & & & \\
\hline
\end{tabular}




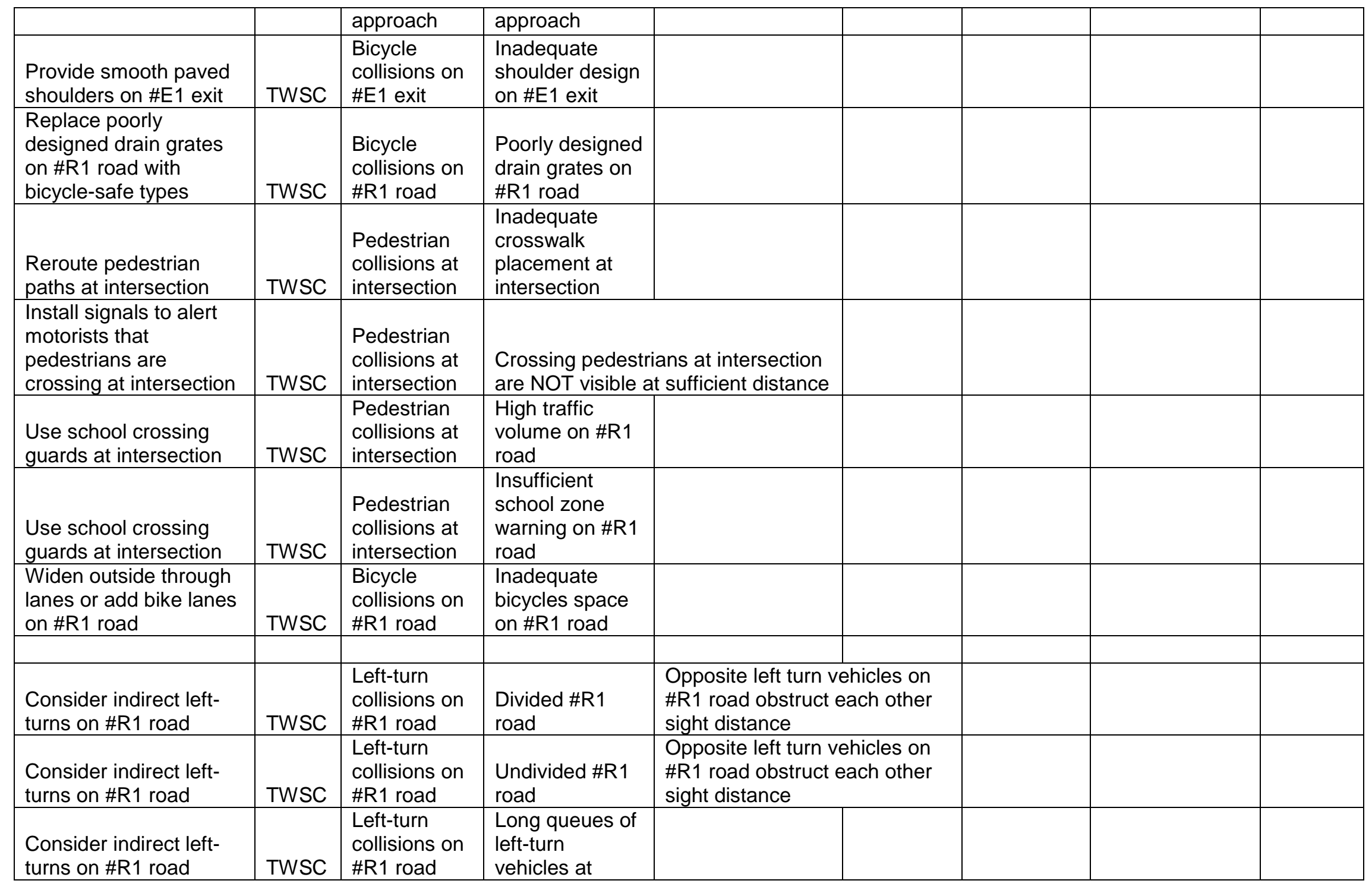




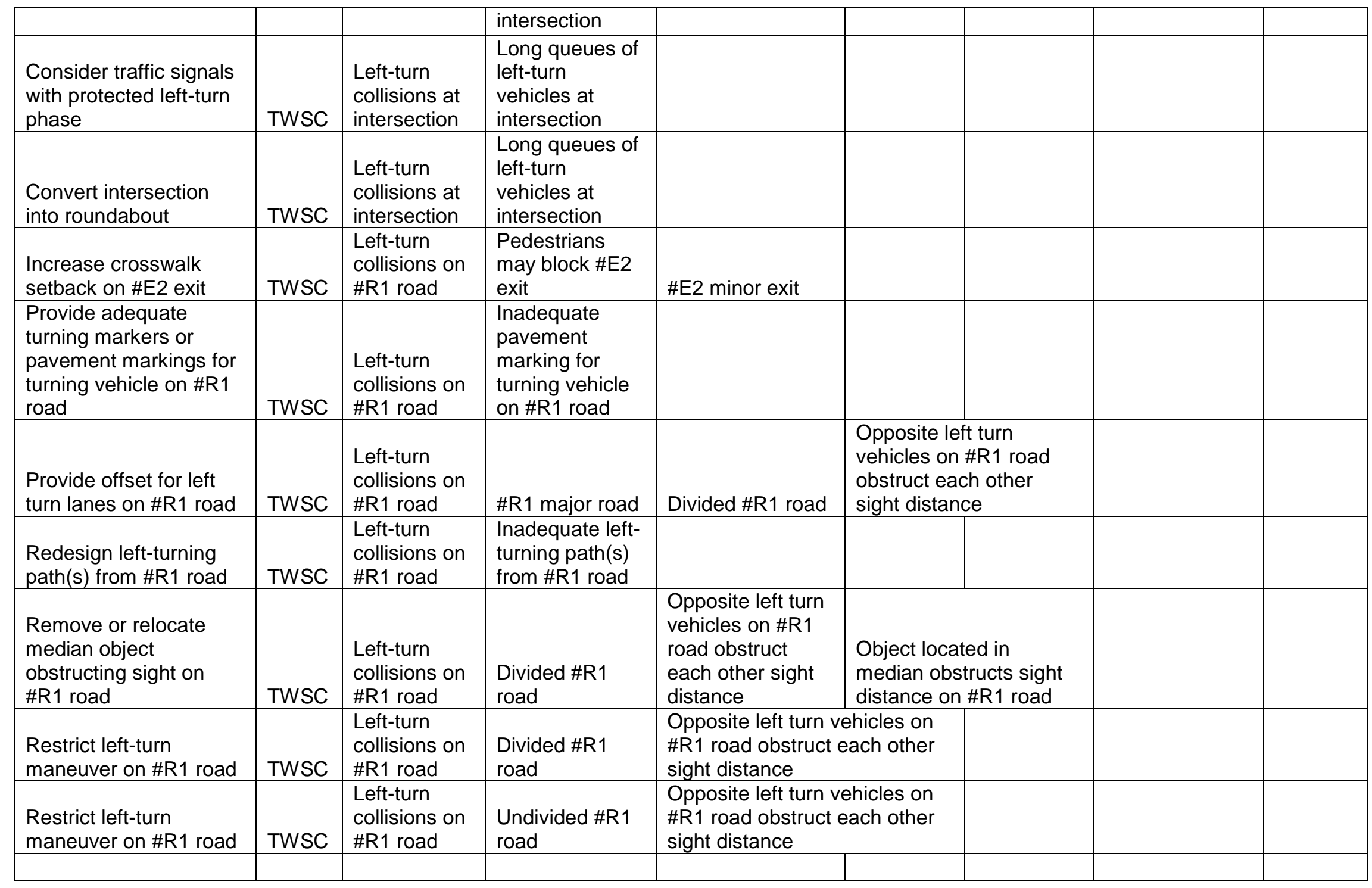




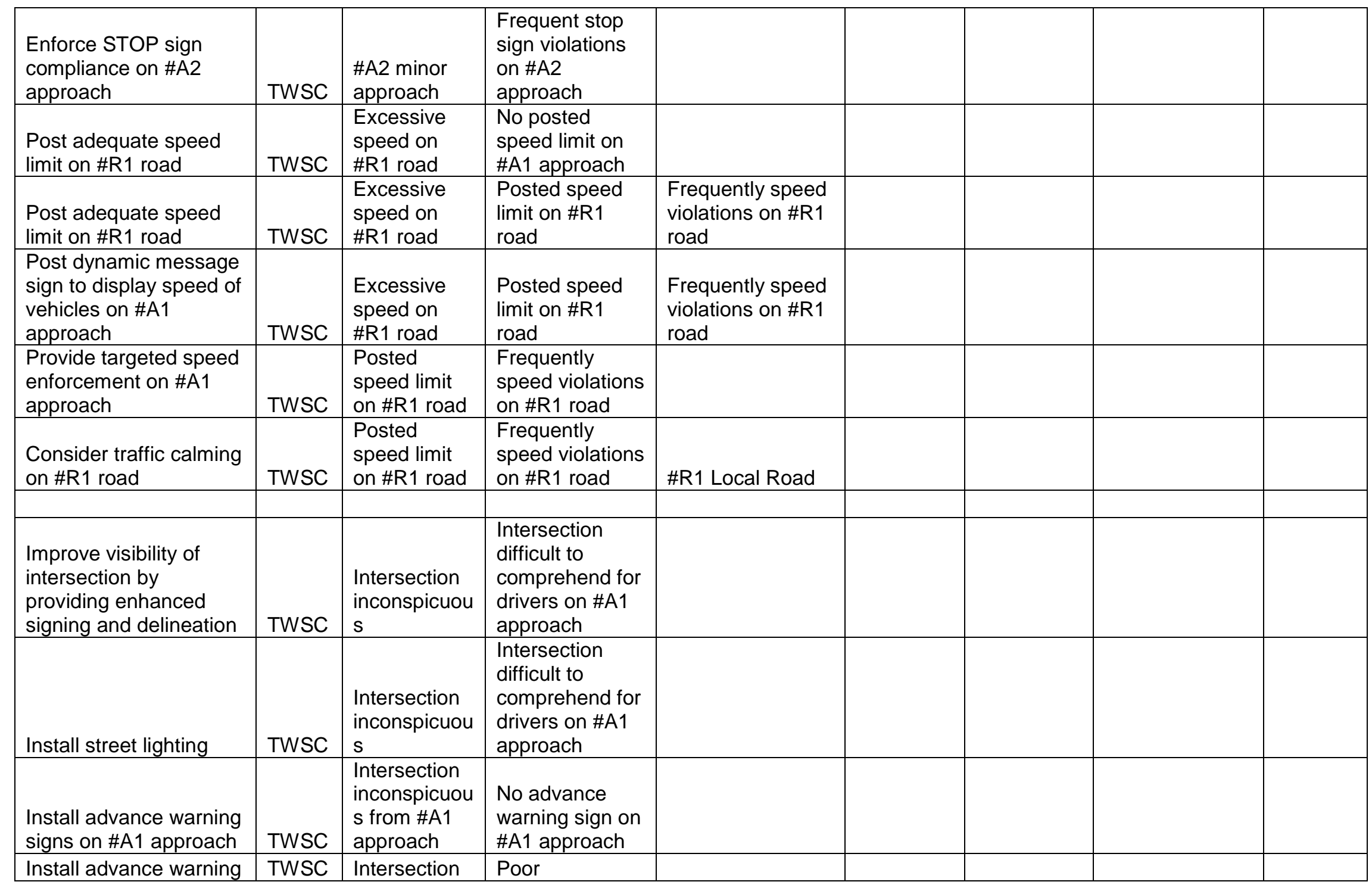




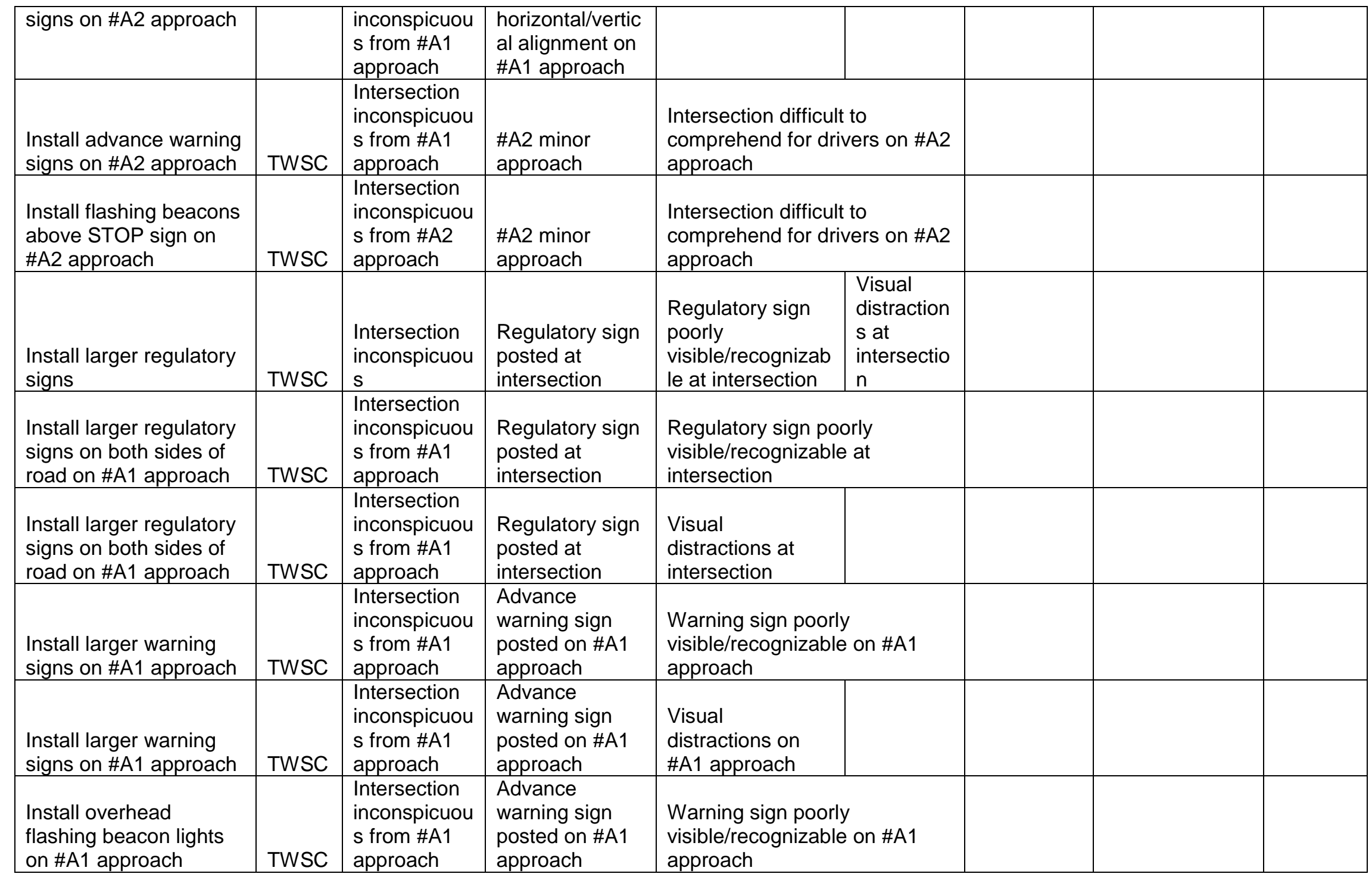




\begin{tabular}{|c|c|c|c|c|c|c|c|}
\hline $\begin{array}{l}\text { Install street name } \\
\text { signs at intersection }\end{array}$ & TWSC & $\begin{array}{l}\text { Intersection } \\
\text { inconspicuou } \\
\text { s }\end{array}$ & $\begin{array}{l}\text { No street name } \\
\text { signs present at } \\
\text { intersection }\end{array}$ & & & & \\
\hline $\begin{array}{l}\text { Install warning signs on } \\
\text { both sides of road on } \\
\# A 1 \text { approach }\end{array}$ & TWSC & $\begin{array}{l}\text { Intersection } \\
\text { inconspicuou } \\
\text { s from \#A1 } \\
\text { approach }\end{array}$ & $\begin{array}{l}\text { Advance } \\
\text { warning sign } \\
\text { posted on \#A1 } \\
\text { approach }\end{array}$ & $\begin{array}{l}\text { Warning sign } \\
\text { poorly } \\
\text { visible/recognizab } \\
\text { le on \#A1 } \\
\text { approach }\end{array}$ & $\begin{array}{l}\text { Visual } \\
\text { distraction } \\
\text { s on \#A1 } \\
\text { approach }\end{array}$ & & \\
\hline $\begin{array}{l}\text { Provide warning } \\
\text { pavement marking on } \\
\text { \#A1 approach }\end{array}$ & TWSC & $\begin{array}{l}\text { Intersection } \\
\text { inconspicuou } \\
\text { s from \#A1 } \\
\text { approach }\end{array}$ & $\begin{array}{l}\text { Advance } \\
\text { warning sign } \\
\text { posted on \#A1 } \\
\text { approach }\end{array}$ & $\begin{array}{l}\text { Warning sign } \\
\text { poorly } \\
\text { visible/recognizab } \\
\text { le on \#A1 } \\
\text { approach }\end{array}$ & $\begin{array}{l}\text { Visual } \\
\text { distraction } \\
\text { s on \#A1 } \\
\text { approach }\end{array}$ & & \\
\hline $\begin{array}{l}\text { Relocate sign on \#A1 } \\
\text { approach to make it } \\
\text { visible }\end{array}$ & TWSC & $\begin{array}{l}\text { Intersection } \\
\text { inconspicuou } \\
\text { s from \#A1 } \\
\text { approach }\end{array}$ & $\begin{array}{l}\text { Advance } \\
\text { warning sign } \\
\text { posted on \#A1 } \\
\text { approach }\end{array}$ & $\begin{array}{l}\text { Warning sign } \\
\text { poorly } \\
\text { visible/recognizab } \\
\text { le on \#A1 } \\
\text { approach }\end{array}$ & \multicolumn{2}{|c|}{$\begin{array}{l}\text { Warning sign obstructed } \\
\text { on \#A1 approach }\end{array}$} & \\
\hline $\begin{array}{l}\text { Remove object } \\
\text { obstructing sight of } \\
\text { warning sign on \#A1 } \\
\text { approach }\end{array}$ & TWSC & $\begin{array}{l}\text { Intersection } \\
\text { inconspicuou } \\
\text { s from \#A1 } \\
\text { approach }\end{array}$ & $\begin{array}{l}\text { Advance } \\
\text { warning sign } \\
\text { posted on \#A1 } \\
\text { approach }\end{array}$ & $\begin{array}{l}\text { Warning sign } \\
\text { poorly } \\
\text { visible/recognizab } \\
\text { le on \#A1 } \\
\text { approach }\end{array}$ & \multicolumn{2}{|c|}{$\begin{array}{l}\text { Warning sign obstructed } \\
\text { on \#A1 approach }\end{array}$} & \\
\hline $\begin{array}{l}\text { Replace/repair/Clean } \\
\text { warning signs on \#A1 } \\
\text { approach }\end{array}$ & TWSC & $\begin{array}{l}\text { Intersection } \\
\text { inconspicuou } \\
\text { s from \#A1 } \\
\text { approach }\end{array}$ & $\begin{array}{l}\text { Advance } \\
\text { warning sign } \\
\text { posted on \#A1 } \\
\text { approach }\end{array}$ & \multicolumn{2}{|c|}{$\begin{array}{l}\text { Warning sign poorly } \\
\text { visible/recognizable on \#A1 } \\
\text { approach }\end{array}$} & & \\
\hline $\begin{array}{l}\text { Improve } \\
\text { horizontal/vertical } \\
\text { alignment on \#A2 } \\
\text { approach }\end{array}$ & \multicolumn{2}{|c|}{$\begin{array}{l}\text { Poor } \\
\text { horizontal/vertical } \\
\text { alignment on \#A2 } \\
\text { approach }\end{array}$} & & & & & \\
\hline $\begin{array}{l}\text { Change } \\
\text { horizontal/vertical } \\
\text { alignment on \#R1 road }\end{array}$ & \multicolumn{2}{|c|}{$\begin{array}{l}\text { Poor } \\
\text { horizontal/vertical } \\
\text { alignment on \#R1 } \\
\text { road }\end{array}$} & & & & & \\
\hline
\end{tabular}




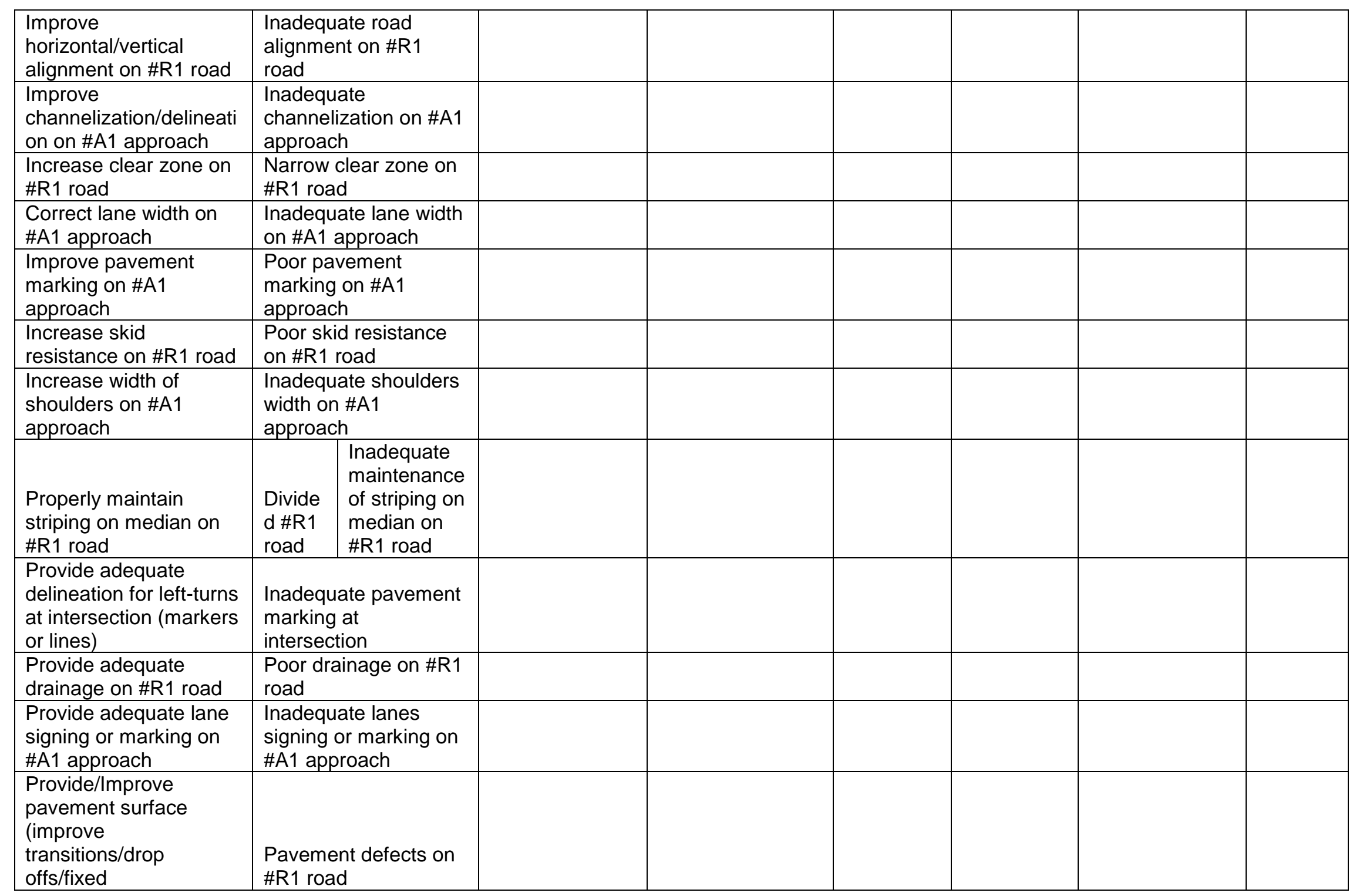




\begin{tabular}{|l|l|l|l|l|l|}
\hline $\begin{array}{l}\text { potholes/rutting/etc) on } \\
\text { \#R1 road }\end{array}$ & & & & & \\
\hline $\begin{array}{l}\text { Widen lanes on \#A1 } \\
\text { approach to adequate } \\
\text { width }\end{array}$ & $\begin{array}{l}\text { Inadequate lane width } \\
\text { on \#A1 approach }\end{array}$ & & & & \\
\hline $\begin{array}{l}\text { Widen median width on } \\
\text { \#R1 road }\end{array}$ & $\begin{array}{l}\text { Insufficient median } \\
\text { width on \#R1 road }\end{array}$ & & & & \\
\hline
\end{tabular}

\section{All-way stop-controlled intersection}

\begin{tabular}{|c|c|c|c|c|c|}
\hline $\begin{array}{l}\text { Close or relocate driveway(s) on \#A1 } \\
\text { approach }\end{array}$ & AWSC & $\begin{array}{l}\text { Driveway(s) } \\
\text { collisions on \#A1 } \\
\text { approach }\end{array}$ & $\begin{array}{l}\text { Driveway(s) on \#A1 } \\
\text { approach close to } \\
\text { intersection }\end{array}$ & $\begin{array}{l}\text { Inadequate visibility of } \\
\text { driveway(s) on \#A1 } \\
\text { approach }\end{array}$ & $\begin{array}{l}\text { Driveway(s) design } \\
\text { inadequate for high } \\
\text { traffic on \#A1 approach }\end{array}$ \\
\hline $\begin{array}{l}\text { Close or relocate driveway(s) on \#E1 } \\
\text { exit }\end{array}$ & AWSC & $\begin{array}{l}\text { Driveway(s) } \\
\text { collisions on \#E1 } \\
\text { exit }\end{array}$ & $\begin{array}{l}\text { Driveway(s) on \#E1 } \\
\text { exit close to } \\
\text { intersection }\end{array}$ & $\begin{array}{l}\text { Inadequate visibility of } \\
\text { driveway(s) on \#E1 } \\
\text { exit }\end{array}$ & $\begin{array}{l}\text { Driveway(s) design } \\
\text { inadequate for high } \\
\text { traffic on \#E1 exit }\end{array}$ \\
\hline Relocate driveway(s) from \#E1 exit & AWSC & $\begin{array}{l}\text { Driveway(s) } \\
\text { collisions on \#E1 } \\
\text { exit }\end{array}$ & $\begin{array}{l}\text { Driveway(s) on \#E1 } \\
\text { exit close to } \\
\text { intersection }\end{array}$ & $\begin{array}{l}\text { Inadequate visibility of } \\
\text { driveway(s) on \#E1 } \\
\text { exit }\end{array}$ & $\begin{array}{l}\text { Driveway(s) design } \\
\text { inadequate for high } \\
\text { traffic on \#E1 exit }\end{array}$ \\
\hline $\begin{array}{l}\text { Channelize driveway(s) on \#A1 } \\
\text { approach }\end{array}$ & AWSC & $\begin{array}{l}\text { Driveway(s) } \\
\text { collisions on \#A1 } \\
\text { approach }\end{array}$ & $\begin{array}{l}\text { Driveway(s) on \#A1 } \\
\text { approach close to } \\
\text { intersection }\end{array}$ & $\begin{array}{l}\text { Inadequate visibility of } \\
\text { driveway(s) on \#A1 } \\
\text { approach }\end{array}$ & $\begin{array}{l}\text { Driveway(s) design } \\
\text { inadequate for high } \\
\text { traffic on \#A1 approach }\end{array}$ \\
\hline Channelize driveway(s) on \#E1 exit & AWSC & $\begin{array}{l}\text { Driveway(s) } \\
\text { collisions on \#E1 } \\
\text { exit }\end{array}$ & $\begin{array}{l}\text { Driveway(s) on \#E1 } \\
\text { exit close to } \\
\text { intersection }\end{array}$ & $\begin{array}{l}\text { Inadequate visibility of } \\
\text { driveway(s) on \#E1 } \\
\text { exit }\end{array}$ & $\begin{array}{l}\text { Driveway(s) design } \\
\text { inadequate for high } \\
\text { traffic on \#E1 exit }\end{array}$ \\
\hline $\begin{array}{l}\text { Install curbing to define driveway(s) } \\
\text { location on \#A1 approach }\end{array}$ & AWSC & $\begin{array}{l}\text { Driveway(s) } \\
\text { collisions on \#A1 } \\
\text { approach }\end{array}$ & $\begin{array}{l}\text { Driveway(s) on \#A1 } \\
\text { approach close to } \\
\text { intersection }\end{array}$ & $\begin{array}{l}\text { Inadequate visibility of } \\
\text { driveway(s) on \#A1 } \\
\text { approach }\end{array}$ & $\begin{array}{l}\text { Driveway(s) design } \\
\text { inadequate for high } \\
\text { traffic on \#A1 approach }\end{array}$ \\
\hline $\begin{array}{l}\text { Install curbing to define driveway(s) } \\
\text { location on \#E1 exit }\end{array}$ & AWSC & $\begin{array}{l}\text { Driveway(s) } \\
\text { collisions on \#E1 } \\
\text { exit }\end{array}$ & $\begin{array}{l}\text { Driveway(s) on \#E1 } \\
\text { exit close to } \\
\text { intersection }\end{array}$ & $\begin{array}{l}\text { Inadequate visibility of } \\
\text { driveway(s) on \#E1 } \\
\text { exit }\end{array}$ & $\begin{array}{l}\text { Driveway(s) design } \\
\text { inadequate for high } \\
\text { traffic on \#E1 exit }\end{array}$ \\
\hline $\begin{array}{l}\text { Install median divider on \#A1 } \\
\text { approach to prevent left-turns from/to }\end{array}$ & AWSC & $\begin{array}{l}\text { Driveway(s) } \\
\text { collisions on \#A1 }\end{array}$ & $\begin{array}{l}\text { Driveway(s) on \#A1 } \\
\text { approach close to }\end{array}$ & $\begin{array}{l}\text { Inadequate visibility of } \\
\text { driveway(s) on \#A1 }\end{array}$ & $\begin{array}{l}\text { Driveway(s) design } \\
\text { inadequate for high }\end{array}$ \\
\hline
\end{tabular}




\begin{tabular}{|c|c|c|c|c|c|}
\hline driveway(s) & & approach & intersection & approach & traffic on \#A1 approach \\
\hline $\begin{array}{l}\text { Install median divider on \#E1 exit to } \\
\text { prevent left-turns from/to driveway(s) }\end{array}$ & AWSC & $\begin{array}{l}\text { Driveway(s) } \\
\text { collisions on \#E1 } \\
\text { exit }\end{array}$ & $\begin{array}{l}\text { Driveway(s) on \#E1 } \\
\text { exit close to } \\
\text { intersection }\end{array}$ & $\begin{array}{l}\text { Inadequate visibility of } \\
\text { driveway(s) on \#E1 } \\
\text { exit }\end{array}$ & $\begin{array}{l}\text { Driveway(s) design } \\
\text { inadequate for high } \\
\text { traffic on \#E1 exit }\end{array}$ \\
\hline $\begin{array}{l}\text { Provide deceleration lane on \#A1 } \\
\text { approach }\end{array}$ & AWSC & $\begin{array}{l}\text { Driveway(s) } \\
\text { collisions on \#A1 } \\
\text { approach }\end{array}$ & $\begin{array}{l}\text { Driveway(s) on \#A1 } \\
\text { approach close to } \\
\text { intersection }\end{array}$ & $\begin{array}{l}\text { Inadequate visibility of } \\
\text { driveway(s) on \#A1 } \\
\text { approach }\end{array}$ & $\begin{array}{l}\text { Driveway(s) design } \\
\text { inadequate for high } \\
\text { traffic on \#A1 approach }\end{array}$ \\
\hline Provide acceleration lane on \#E1 exit & AWSC & $\begin{array}{l}\text { Driveway(s) } \\
\text { collisions on \#E1 } \\
\text { exit }\end{array}$ & $\begin{array}{l}\text { Driveway(s) on \#E1 } \\
\text { exit close to } \\
\text { intersection }\end{array}$ & $\begin{array}{l}\text { Inadequate visibility of } \\
\text { driveway(s) on \#E1 } \\
\text { exit }\end{array}$ & $\begin{array}{l}\text { Driveway(s) design } \\
\text { inadequate for high } \\
\text { traffic on \#E1 exit }\end{array}$ \\
\hline $\begin{array}{l}\text { Relocate driveway(s) from \#A1 } \\
\text { approach }\end{array}$ & AWSC & $\begin{array}{l}\text { Driveway(s) } \\
\text { collisions on \#A1 } \\
\text { approach }\end{array}$ & $\begin{array}{l}\text { Driveway(s) on \#A1 } \\
\text { approach close to } \\
\text { intersection }\end{array}$ & $\begin{array}{l}\text { Inadequate visibility of } \\
\text { driveway(s) on \#A1 } \\
\text { approach }\end{array}$ & $\begin{array}{l}\text { Driveway(s) design } \\
\text { inadequate for high } \\
\text { traffic on \#A1 approach }\end{array}$ \\
\hline $\begin{array}{l}\text { Remove sight obstruction on \#A1 } \\
\text { approach }\end{array}$ & AWSC & $\begin{array}{l}\text { Driveway(s) } \\
\text { collisions on \#A1 } \\
\text { approach }\end{array}$ & $\begin{array}{l}\text { Driveway(s) on \#A1 } \\
\text { approach close to } \\
\text { intersection }\end{array}$ & \multicolumn{2}{|c|}{$\begin{array}{l}\text { Inadequate visibility of driveway(s) on \#A1 } \\
\text { approach }\end{array}$} \\
\hline Remove sight obstruction on \#E1 exit & AWSC & $\begin{array}{l}\text { Driveway(s) } \\
\text { collisions on \#E1 } \\
\text { exit }\end{array}$ & $\begin{array}{l}\text { Driveway(s) on \#E1 } \\
\text { exit close to } \\
\text { intersection }\end{array}$ & \multicolumn{2}{|c|}{ Inadequate visibility of driveway(s) on \#E1 exit } \\
\hline $\begin{array}{l}\text { Restrict left-turning at access point on } \\
\text { \#A1 approach }\end{array}$ & AWSC & $\begin{array}{l}\text { Driveway(s) } \\
\text { collisions on \#A1 } \\
\text { approach }\end{array}$ & $\begin{array}{l}\text { Driveway(s) on \#A1 } \\
\text { approach close to } \\
\text { intersection }\end{array}$ & $\begin{array}{l}\text { Inadequate visibility of } \\
\text { driveway(s) on \#A1 } \\
\text { approach }\end{array}$ & $\begin{array}{l}\text { Driveway(s) design } \\
\text { inadequate for high } \\
\text { traffic on \#A1 approach }\end{array}$ \\
\hline $\begin{array}{l}\text { Restrict left-turning at access point on } \\
\text { \#E1 exit }\end{array}$ & AWSC & $\begin{array}{l}\text { Driveway(s) } \\
\text { collisions on \#E1 } \\
\text { exit }\end{array}$ & $\begin{array}{l}\text { Driveway(s) on \#E1 } \\
\text { exit close to } \\
\text { intersection }\end{array}$ & $\begin{array}{l}\text { Inadequate visibility of } \\
\text { driveway(s) on \#E1 } \\
\text { exit }\end{array}$ & $\begin{array}{l}\text { Driveway(s) design } \\
\text { inadequate for high } \\
\text { traffic on \#E1 exit }\end{array}$ \\
\hline $\begin{array}{l}\text { Restrict parking near driveway(s) on } \\
\text { \#A1 approach }\end{array}$ & AWSC & $\begin{array}{l}\text { Driveway(s) } \\
\text { collisions on \#A1 } \\
\text { approach }\end{array}$ & $\begin{array}{l}\text { Driveway(s) on \#A1 } \\
\text { approach close to } \\
\text { intersection }\end{array}$ & \multicolumn{2}{|c|}{$\begin{array}{l}\text { Inadequate visibility of driveway(s) on \#A1 } \\
\text { approach }\end{array}$} \\
\hline $\begin{array}{l}\text { Restrict parking near driveway(s) on } \\
\text { \#E1 exit }\end{array}$ & AWSC & $\begin{array}{l}\text { Driveway(s) } \\
\text { collisions on \#E1 } \\
\text { exit }\end{array}$ & $\begin{array}{l}\text { Driveway(s) on \#E1 } \\
\text { exit close to } \\
\text { intersection }\end{array}$ & \multicolumn{2}{|c|}{ Inadequate visibility of driveway(s) on \#E1 exit } \\
\hline Improve street lighting & AWSC & $\begin{array}{l}\text { Night time collisions } \\
\text { at intersection }\end{array}$ & $\begin{array}{l}\text { Inadequate street } \\
\text { lights at intersection }\end{array}$ & & \\
\hline $\begin{array}{l}\text { Improve/install reflective signs on \#R1 } \\
\text { road }\end{array}$ & AWSC & $\begin{array}{l}\text { Night time collisions } \\
\text { on \#R1 road }\end{array}$ & $\begin{array}{l}\text { Poor signs } \\
\text { reflectivity on \#R1 }\end{array}$ & & \\
\hline
\end{tabular}




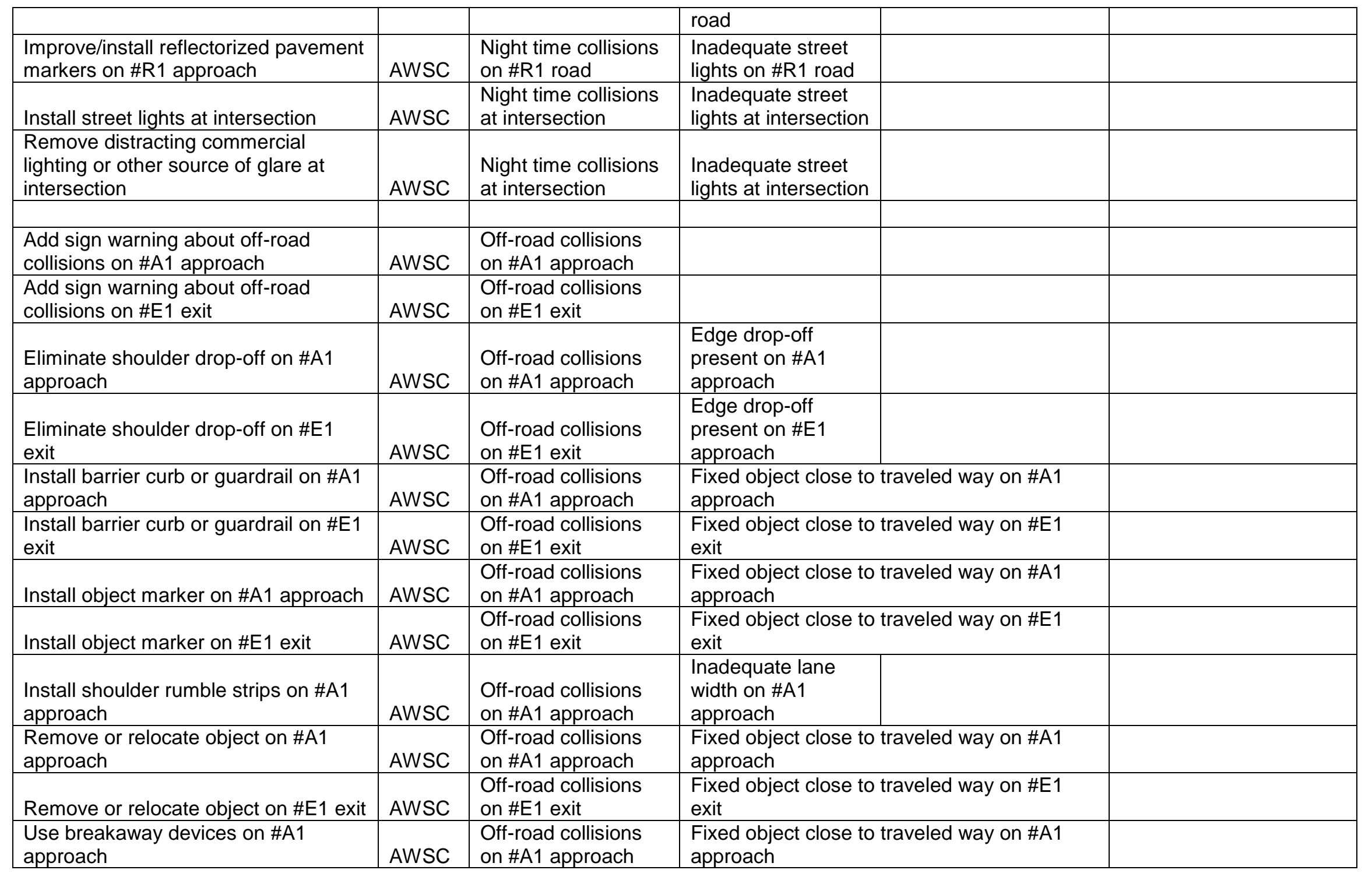




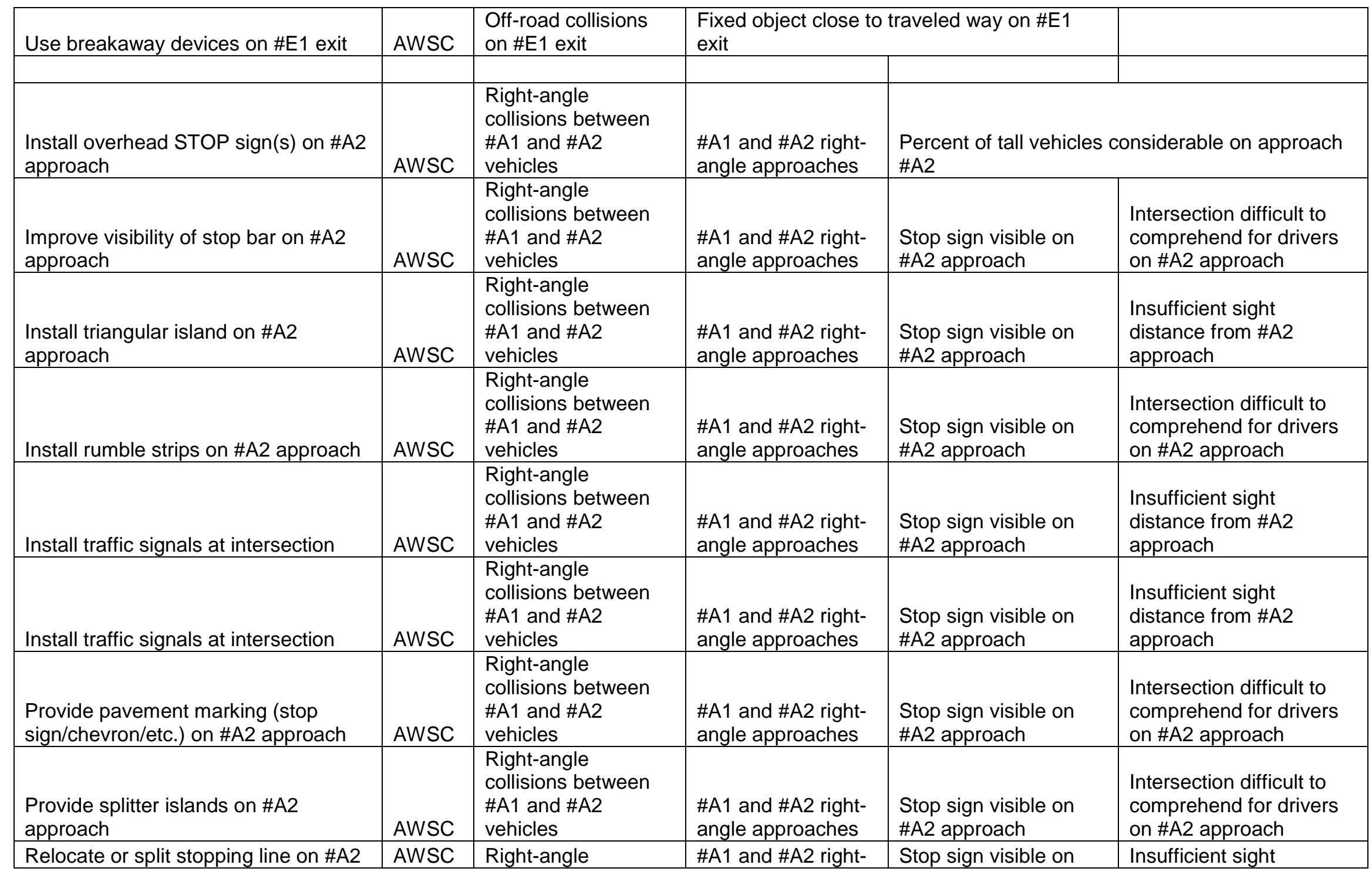




\begin{tabular}{|c|c|c|c|c|c|}
\hline approach & & $\begin{array}{l}\text { collisions between } \\
\text { \#A1 and \#A2 } \\
\text { vehicles }\end{array}$ & angle approaches & \#A2 approach & $\begin{array}{l}\text { distance from \#A2 } \\
\text { approach }\end{array}$ \\
\hline Relocate stop bar on \#A2 approach & AWSC & $\begin{array}{l}\text { Right-angle } \\
\text { collisions between } \\
\text { \#A1 and \#A2 } \\
\text { vehicles }\end{array}$ & $\begin{array}{l}\text { \#A1 and \#A2 right- } \\
\text { angle approaches }\end{array}$ & $\begin{array}{l}\text { Stop sign visible on } \\
\text { \#A2 approach }\end{array}$ & $\begin{array}{l}\text { Insufficient sight } \\
\text { distance from \#A2 } \\
\text { approach }\end{array}$ \\
\hline Relocate stop sign on \#A2 approach & AWSC & $\begin{array}{l}\text { Right-angle } \\
\text { collisions between } \\
\text { \#A1 and \#A2 } \\
\text { vehicles }\end{array}$ & $\begin{array}{l}\text { \#A1 and \#A2 right- } \\
\text { angle approaches }\end{array}$ & $\begin{array}{l}\text { Stop sign NOT visible } \\
\text { on \#A2 approach }\end{array}$ & $\begin{array}{l}\text { Stop sign obstruction on } \\
\text { \#A2 approach }\end{array}$ \\
\hline $\begin{array}{l}\text { Remove object obstructing sight of } \\
\text { stop sign on \#A2 approach }\end{array}$ & AWSC & $\begin{array}{l}\text { Right-angle } \\
\text { collisions between } \\
\text { \#A1 and \#A2 } \\
\text { vehicles }\end{array}$ & $\begin{array}{l}\text { \#A1 and \#A2 right- } \\
\text { angle approaches }\end{array}$ & $\begin{array}{l}\text { Stop sign NOT visible } \\
\text { on \#A2 approach }\end{array}$ & $\begin{array}{l}\text { Stop sign obstruction on } \\
\text { \#A2 approach }\end{array}$ \\
\hline $\begin{array}{l}\text { Remove object obstructing sight } \\
\text { triangle at intersection }\end{array}$ & AWSC & $\begin{array}{l}\text { Right-angle } \\
\text { collisions between } \\
\text { \#A1 and \#A2 } \\
\text { vehicles }\end{array}$ & $\begin{array}{l}\text { \#A1 and \#A2 right- } \\
\text { angle approaches }\end{array}$ & $\begin{array}{l}\text { Stop sign visible on } \\
\text { \#A2 approach }\end{array}$ & $\begin{array}{l}\text { Insufficient sight } \\
\text { distance from \#A2 } \\
\text { approach }\end{array}$ \\
\hline $\begin{array}{l}\text { Replace/repair or clean stop sign on } \\
\text { \#A2 approach }\end{array}$ & AWSC & $\begin{array}{l}\text { Right-angle } \\
\text { collisions between } \\
\text { \#A1 and \#A2 } \\
\text { vehicles }\end{array}$ & $\begin{array}{l}\text { \#A1 and \#A2 right- } \\
\text { angle approaches }\end{array}$ & $\begin{array}{l}\text { Stop sign NOT visible } \\
\text { on \#A2 approach }\end{array}$ & $\begin{array}{l}\text { Stop sign on \#A2 } \\
\text { approach improperly } \\
\text { maintained }\end{array}$ \\
\hline Restrict parking close to intersection & AWSC & $\begin{array}{l}\text { Right-angle } \\
\text { collisions between } \\
\text { \#A1 and \#A2 } \\
\text { vehicles }\end{array}$ & $\begin{array}{l}\text { \#A1 and \#A2 right- } \\
\text { angle approaches }\end{array}$ & $\begin{array}{l}\text { Stop sign visible on } \\
\text { \#A2 approach }\end{array}$ & $\begin{array}{l}\text { Insufficient sight } \\
\text { distance from \#A2 } \\
\text { approach }\end{array}$ \\
\hline Trim vegetation at intersection & AWSC & $\begin{array}{l}\text { Right-angle } \\
\text { collisions between } \\
\text { \#A1 and \#A2 } \\
\text { vehicles }\end{array}$ & $\begin{array}{l}\text { \#A1 and \#A2 right- } \\
\text { angle approaches }\end{array}$ & $\begin{array}{l}\text { Stop sign visible on } \\
\text { \#A2 approach }\end{array}$ & $\begin{array}{l}\text { Insufficient sight } \\
\text { distance from \#A2 } \\
\text { approach }\end{array}$ \\
\hline $\begin{array}{l}\text { Apply chip seal or slurry seal on \#A1 } \\
\text { approach }\end{array}$ & AWSC & $\begin{array}{l}\text { Adverse-weather } \\
\text { collisions }\end{array}$ & \multicolumn{2}{|c|}{$\begin{array}{l}\text { Slippery pavement (no winter) on \#A1 } \\
\text { approach }\end{array}$} & \\
\hline $\begin{array}{l}\text { Apply ship seal or slurry seal on \#E1 } \\
\text { exit }\end{array}$ & AWSC & $\begin{array}{l}\text { Adverse-weather } \\
\text { collisions }\end{array}$ & $\begin{array}{l}\text { Slippery pavement } \\
\text { (no winter) on \#E1 }\end{array}$ & & \\
\hline
\end{tabular}




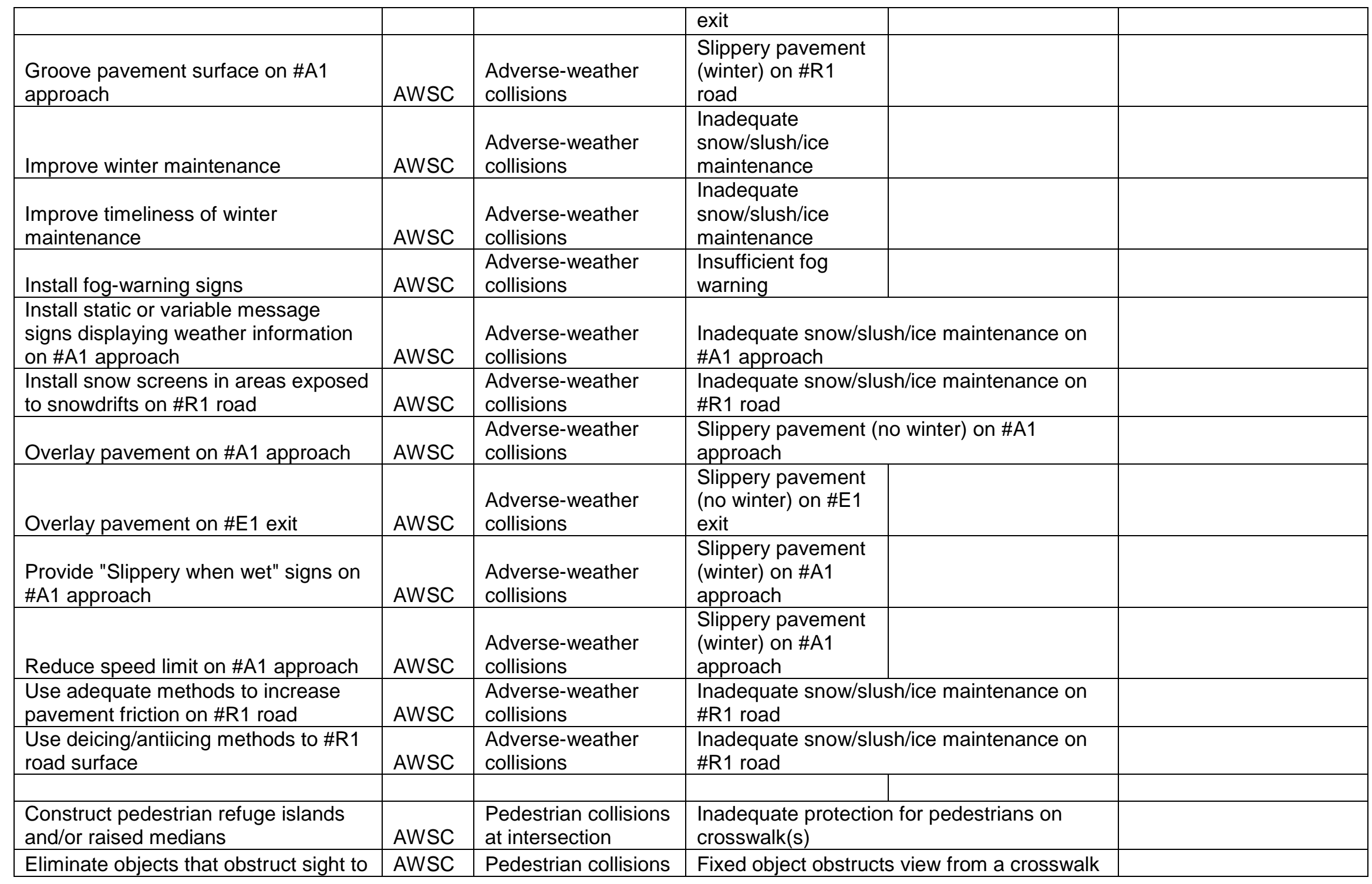




\begin{tabular}{|c|c|c|c|c|c|}
\hline pedestrians entering crosswalk & & at intersection & \multicolumn{2}{|l|}{ at intersection } & \\
\hline Consider traffic calming on \#R1 road & AWSC & $\begin{array}{l}\text { Pedestrian collisions } \\
\text { at intersection }\end{array}$ & $\begin{array}{l}\text { Croswalk not visible } \\
\text { to drivers on \#R1 } \\
\text { road }\end{array}$ & & \\
\hline 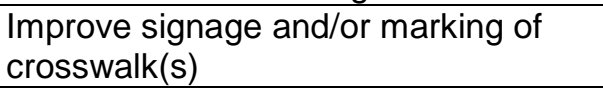 & AWSC & $\begin{array}{l}\text { Pedestrian collisions } \\
\text { at intersection }\end{array}$ & \multicolumn{2}{|c|}{$\begin{array}{l}\text { Inadequate visibility of crosswalk(s) at } \\
\text { intersection }\end{array}$} & \\
\hline $\begin{array}{l}\text { Install pedestrian overpass or } \\
\text { underpass }\end{array}$ & AWSC & $\begin{array}{l}\text { Pedestrian collisions } \\
\text { at intersection }\end{array}$ & \multicolumn{2}{|c|}{$\begin{array}{l}\text { Inadequate protection for pedestrians at } \\
\text { intersection }\end{array}$} & \\
\hline Install curb-ramps & AWSC & $\begin{array}{l}\text { Pedestrian collisions } \\
\text { at intersection }\end{array}$ & \multicolumn{2}{|c|}{$\begin{array}{l}\text { Inadequate design for handicapped at } \\
\text { intersection }\end{array}$} & \\
\hline Install street lighting & AWSC & $\begin{array}{l}\text { Pedestrian collisions } \\
\text { at intersection }\end{array}$ & \multicolumn{2}{|c|}{$\begin{array}{l}\text { Inadequate lighting of crosswalk at } \\
\text { intersection }\end{array}$} & \\
\hline Install pedestrian actuated signals & AWSC & $\begin{array}{l}\text { Pedestrian collisions } \\
\text { at intersection }\end{array}$ & \multicolumn{2}{|c|}{$\begin{array}{l}\text { Signifficant number of pedestrian crossing } \\
\text { intersection }\end{array}$} & \\
\hline Install pedestrian barriers & AWSC & $\begin{array}{l}\text { Pedestrian collisions } \\
\text { at intersection }\end{array}$ & \multicolumn{2}{|c|}{$\begin{array}{l}\text { Inadequate protection for pedestrians at } \\
\text { intersection }\end{array}$} & \\
\hline Install pedestrian crosswalks & AWSC & $\begin{array}{l}\text { Pedestrian collisions } \\
\text { at intersection }\end{array}$ & \multicolumn{2}{|c|}{$\begin{array}{l}\text { Long distance between crosswalks on \#R1 } \\
\text { road }\end{array}$} & \\
\hline Install raised crosswalks & AWSC & $\begin{array}{l}\text { Pedestrian collisions } \\
\text { at intersection }\end{array}$ & \multicolumn{2}{|c|}{$\begin{array}{l}\text { Inadequate visibility of crosswalk(s) at } \\
\text { intersection }\end{array}$} & \\
\hline $\begin{array}{l}\text { Install school crossing sign on \#R1 } \\
\text { road }\end{array}$ & AWSC & $\begin{array}{l}\text { Pedestrian collisions } \\
\text { at intersection }\end{array}$ & $\begin{array}{l}\text { Insufficient school } \\
\text { zone warning on } \\
\text { \#R1 road }\end{array}$ & & \\
\hline $\begin{array}{l}\text { Install school zone markings on \#R1 } \\
\text { road }\end{array}$ & AWSC & $\begin{array}{l}\text { Pedestrian collisions } \\
\text { at intersection }\end{array}$ & $\begin{array}{l}\text { School zone } \\
\text { present }\end{array}$ & $\begin{array}{l}\text { Insufficient school } \\
\text { zone warning on \#R1 } \\
\text { road }\end{array}$ & \\
\hline $\begin{array}{l}\text { Install school zone speed limit sign on } \\
\text { \#R1 road }\end{array}$ & AWSC & $\begin{array}{l}\text { Pedestrian collisions } \\
\text { at intersection }\end{array}$ & $\begin{array}{l}\text { School zone } \\
\text { present }\end{array}$ & No school zone speed I & mit posted on \#R1 road \\
\hline $\begin{array}{l}\text { Increase sidewalk setback on \#R1 } \\
\text { road }\end{array}$ & AWSC & $\begin{array}{l}\text { Pedestrian collisions } \\
\text { on \#R1 road }\end{array}$ & \multicolumn{2}{|c|}{$\begin{array}{l}\text { Inadequate protection of pedestrians on \#R1 } \\
\text { road }\end{array}$} & \\
\hline $\begin{array}{l}\text { Provide separate bicycle path on \#R1 } \\
\text { road }\end{array}$ & AWSC & $\begin{array}{l}\text { Bicycle collisions on } \\
\text { \#R1 road }\end{array}$ & $\begin{array}{l}\text { Inadequate bicycles } \\
\text { space on \#R1 road }\end{array}$ & & \\
\hline $\begin{array}{l}\text { Provide separate bicycle path on \#R1 } \\
\text { road }\end{array}$ & AWSC & $\begin{array}{l}\text { Bicycle collisions on } \\
\text { \#R1 road }\end{array}$ & $\begin{array}{l}\text { Signifficant number } \\
\text { of bicycles on \#R1 } \\
\text { road }\end{array}$ & & \\
\hline Provide median refuges at & AWSC & Bicycle collisions at & High traffic volume & & \\
\hline
\end{tabular}




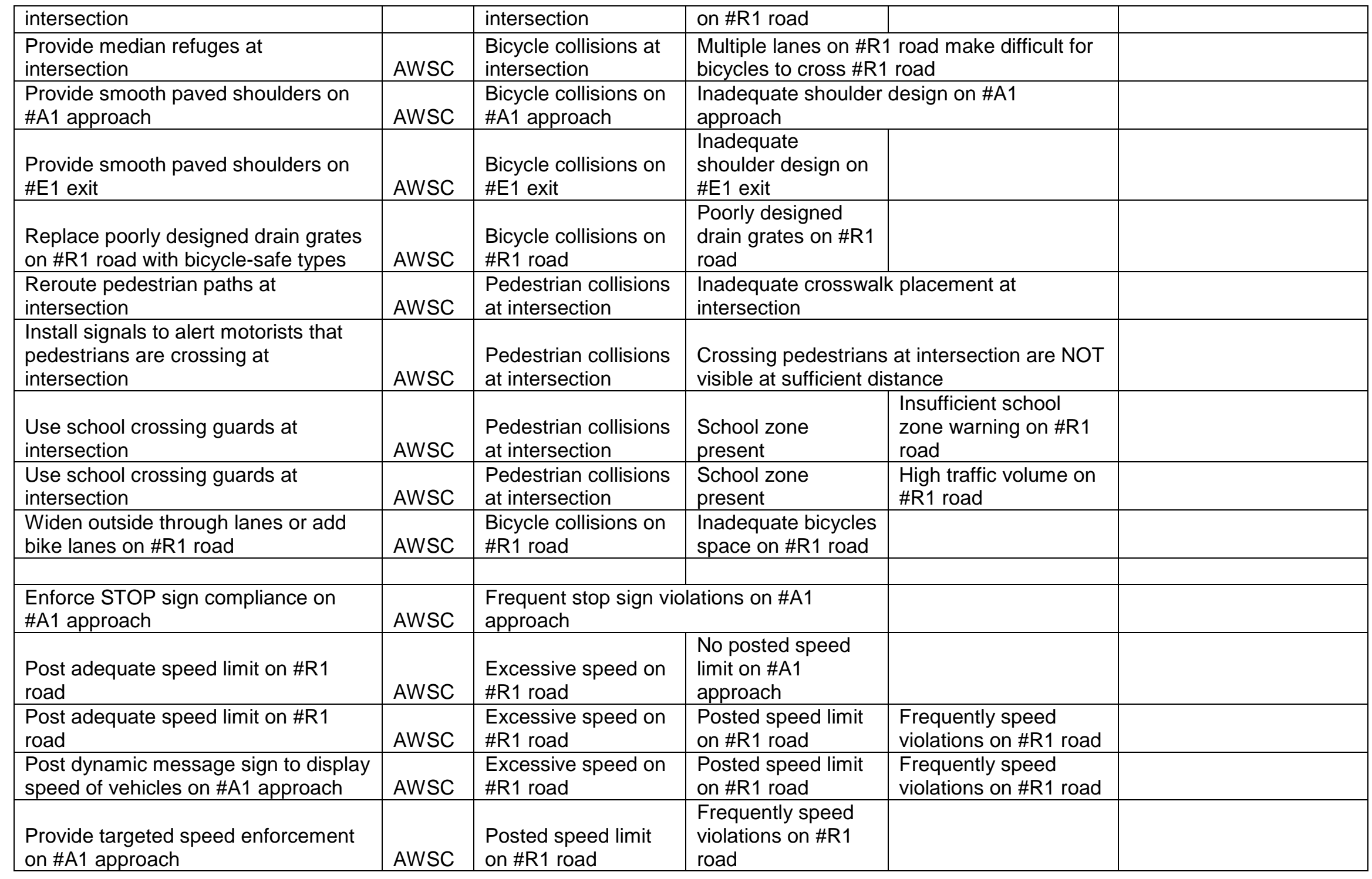




\begin{tabular}{|c|c|c|c|c|c|}
\hline Consider traffic calming on \#R1 road & AWSC & $\begin{array}{l}\text { Posted speed limit } \\
\text { on \#R1 road }\end{array}$ & $\begin{array}{l}\text { Frequently speed } \\
\text { violations on \#R1 } \\
\text { road }\end{array}$ & & \\
\hline $\begin{array}{l}\text { Improve visibility of intersection by } \\
\text { providing enhanced signing and } \\
\text { delineation }\end{array}$ & AWSC & $\begin{array}{l}\text { Intersection } \\
\text { inconspicuous }\end{array}$ & \multirow{2}{*}{\multicolumn{2}{|c|}{$\begin{array}{l}\text { Intersection difficult to comprehend for } \\
\text { drivers on \#A1 approach } \\
\text { Intersection difficult to comprehend for } \\
\text { drivers on \#A1 approach }\end{array}$}} & \\
\hline Install street lighting & AWSC & $\begin{array}{l}\text { Intersection } \\
\text { inconspicuous }\end{array}$ & & & \\
\hline $\begin{array}{l}\text { Install advance warning signs on \#A1 } \\
\text { approach }\end{array}$ & AWSC & $\begin{array}{l}\text { Intersection } \\
\text { inconspicuous from } \\
\text { \#A1 approach }\end{array}$ & $\begin{array}{l}\text { No advance } \\
\text { warning sign on } \\
\text { \#A1 approach }\end{array}$ & & \\
\hline $\begin{array}{l}\text { Install flashing beacons on \#A1 } \\
\text { approach }\end{array}$ & AWSC & $\begin{array}{l}\text { Intersection } \\
\text { inconspicuous from } \\
\text { \#A1 approach }\end{array}$ & \multicolumn{2}{|c|}{$\begin{array}{l}\text { Intersection difficult to comprehend for } \\
\text { drivers on \#A1 approach }\end{array}$} & \\
\hline Install larger regulatory signs & AWSC & $\begin{array}{l}\text { Intersection } \\
\text { inconspicuous }\end{array}$ & $\begin{array}{l}\text { Regulatory sign } \\
\text { posted at } \\
\text { intersection }\end{array}$ & $\begin{array}{l}\text { Regulatory sign poorly } \\
\text { visible/recognizable at } \\
\text { intersection }\end{array}$ & $\begin{array}{l}\text { Visual distractions at } \\
\text { intersection }\end{array}$ \\
\hline $\begin{array}{l}\text { Install larger regulatory signs on both } \\
\text { sides of road on \#A1 approach }\end{array}$ & AWSC & $\begin{array}{l}\text { Intersection } \\
\text { inconspicuous from } \\
\text { \#A1 approach }\end{array}$ & $\begin{array}{l}\text { Regulatory sign } \\
\text { posted at } \\
\text { intersection }\end{array}$ & \multicolumn{2}{|c|}{$\begin{array}{l}\text { Regulatory sign poorly visible/recognizable at } \\
\text { intersection }\end{array}$} \\
\hline $\begin{array}{l}\text { Install larger regulatory signs on both } \\
\text { sides of road on \#A1 approach }\end{array}$ & AWSC & $\begin{array}{l}\text { Intersection } \\
\text { inconspicuous from } \\
\text { \#A1 approach }\end{array}$ & $\begin{array}{l}\text { Regulatory sign } \\
\text { posted at } \\
\text { intersection }\end{array}$ & $\begin{array}{l}\text { Visual distractions at } \\
\text { intersection }\end{array}$ & \\
\hline $\begin{array}{l}\text { Install larger warning signs on \#A1 } \\
\text { approach }\end{array}$ & AWSC & $\begin{array}{l}\text { Intersection } \\
\text { inconspicuous from } \\
\text { \#A1 approach }\end{array}$ & $\begin{array}{l}\text { Advance warning } \\
\text { sign posted on \#A1 } \\
\text { approach }\end{array}$ & \multicolumn{2}{|c|}{$\begin{array}{l}\text { Warning sign poorly visible/recognizable on \#A1 } \\
\text { approach }\end{array}$} \\
\hline $\begin{array}{l}\text { Install larger warning signs on \#A1 } \\
\text { approach }\end{array}$ & AWSC & $\begin{array}{l}\text { Intersection } \\
\text { inconspicuous from } \\
\text { \#A1 approach }\end{array}$ & $\begin{array}{l}\text { Advance warning } \\
\text { sign posted on \#A1 } \\
\text { approach }\end{array}$ & $\begin{array}{l}\text { Visual distractions on } \\
\text { \#A1 approach }\end{array}$ & \\
\hline $\begin{array}{l}\text { Install overhead flashing beacon lights } \\
\text { on \#A1 approach }\end{array}$ & AWSC & $\begin{array}{l}\text { Intersection } \\
\text { inconspicuous from } \\
\text { \#A1 approach }\end{array}$ & $\begin{array}{l}\text { Advance warning } \\
\text { sign posted on \#A1 } \\
\text { approach }\end{array}$ & \multicolumn{2}{|c|}{$\begin{array}{l}\text { Warning sign poorly visible/recognizable on \#A1 } \\
\text { approach }\end{array}$} \\
\hline $\begin{array}{l}\text { Install street name signs at } \\
\text { intersection }\end{array}$ & AWSC & $\begin{array}{l}\text { Intersection } \\
\text { inconspicuous }\end{array}$ & $\begin{array}{l}\text { No street name } \\
\text { signs present at } \\
\text { intersection }\end{array}$ & & \\
\hline
\end{tabular}




\begin{tabular}{|c|c|c|c|c|c|}
\hline $\begin{array}{l}\text { Install warning signs on both sides of } \\
\text { road on \#A1 approach }\end{array}$ & AWSC & $\begin{array}{l}\text { Intersection } \\
\text { inconspicuous from } \\
\text { \#A1 approach }\end{array}$ & $\begin{array}{l}\text { Advance warning } \\
\text { sign posted on \#A1 } \\
\text { approach }\end{array}$ & $\begin{array}{l}\text { Warning sign poorly } \\
\text { visible/recognizable on } \\
\text { \#A1 approach }\end{array}$ & $\begin{array}{l}\text { Visual distractions on } \\
\text { \#A1 approach }\end{array}$ \\
\hline $\begin{array}{l}\text { Provide warning pavement marking } \\
\text { on \#A1 approach }\end{array}$ & AWSC & $\begin{array}{l}\text { Intersection } \\
\text { inconspicuous from } \\
\text { \#A1 approach }\end{array}$ & $\begin{array}{l}\text { Advance warning } \\
\text { sign posted on \#A1 } \\
\text { approach }\end{array}$ & $\begin{array}{l}\text { Warning sign poorly } \\
\text { visible/recognizable on } \\
\text { \#A1 approach }\end{array}$ & $\begin{array}{l}\text { Visual distractions on } \\
\text { \#A1 approach }\end{array}$ \\
\hline $\begin{array}{l}\text { Relocate sign on \#A1 approach to } \\
\text { make it visible }\end{array}$ & AWSC & $\begin{array}{l}\text { Intersection } \\
\text { inconspicuous from } \\
\text { \#A1 approach }\end{array}$ & $\begin{array}{l}\text { Advance warning } \\
\text { sign posted on \#A1 } \\
\text { approach }\end{array}$ & $\begin{array}{l}\text { Warning sign poorly } \\
\text { visible/recognizable on } \\
\text { \#A1 approach }\end{array}$ & $\begin{array}{l}\text { Warning sign obstructed } \\
\text { on \#A1 approach }\end{array}$ \\
\hline $\begin{array}{l}\text { Remove object obstructing sight of } \\
\text { warning sign on \#A1 approach }\end{array}$ & AWSC & $\begin{array}{l}\text { Intersection } \\
\text { inconspicuous from } \\
\text { \#A1 approach }\end{array}$ & $\begin{array}{l}\text { Advance warning } \\
\text { sign posted on \#A1 } \\
\text { approach }\end{array}$ & $\begin{array}{l}\text { Warning sign poorly } \\
\text { visible/recognizable on } \\
\text { \#A1 approach }\end{array}$ & $\begin{array}{l}\text { Warning sign obstructed } \\
\text { on \#A1 approach }\end{array}$ \\
\hline $\begin{array}{l}\text { Replace/repair/Clean warning signs } \\
\text { on \#A1 approach }\end{array}$ & AWSC & $\begin{array}{l}\text { Intersection } \\
\text { inconspicuous from } \\
\text { \#A1 approach }\end{array}$ & $\begin{array}{l}\text { Advance warning } \\
\text { sign posted on \#A1 } \\
\text { approach }\end{array}$ & \multicolumn{2}{|c|}{$\begin{array}{l}\text { Warning sign poorly visible/recognizable on \#A1 } \\
\text { approach }\end{array}$} \\
\hline $\begin{array}{l}\text { Improve horizontal/vertical alignment } \\
\text { on \#A2 approach }\end{array}$ & \multicolumn{2}{|c|}{$\begin{array}{l}\text { Poor horizontal/vertical } \\
\text { alignment on \#A2 approach }\end{array}$} & & & \\
\hline $\begin{array}{l}\text { Change horizontal/vertical alignment } \\
\text { on \#R1 road }\end{array}$ & \multicolumn{2}{|c|}{$\begin{array}{l}\text { Poor horizontal/vertical } \\
\text { alignment on \#R1 road }\end{array}$} & & & \\
\hline $\begin{array}{l}\text { Improve horizontal/vertical alignment } \\
\text { on \#R1 road }\end{array}$ & \multicolumn{2}{|c|}{$\begin{array}{l}\text { Inadequate road alignment on } \\
\text { \#R1 road }\end{array}$} & & & \\
\hline Increase clear zone on \#R1 road & \multicolumn{2}{|c|}{$\begin{array}{l}\text { Narrow clear zone on \#R1 } \\
\text { road }\end{array}$} & & & \\
\hline Correct lane width on \#A1 approach & \multicolumn{2}{|c|}{$\begin{array}{l}\text { Inadequate lane width on \#A1 } \\
\text { approach }\end{array}$} & & & \\
\hline $\begin{array}{l}\text { Improve pavement marking on \#A1 } \\
\text { approach }\end{array}$ & \multicolumn{2}{|c|}{$\begin{array}{l}\text { Poor pavement marking on } \\
\text { \#A1 approach }\end{array}$} & & & \\
\hline Increase skid resistance on \#R1 road & \multicolumn{2}{|c|}{$\begin{array}{l}\text { Poor skid resistance on \#R1 } \\
\text { road }\end{array}$} & & & \\
\hline $\begin{array}{l}\text { Increase width of shoulders on \#A1 } \\
\text { approach }\end{array}$ & \multicolumn{2}{|c|}{$\begin{array}{l}\text { Inadequate shoulders width on } \\
\text { \#A1 approach }\end{array}$} & & & \\
\hline $\begin{array}{l}\text { Provide adequate drainage on \#R1 } \\
\text { road }\end{array}$ & \multicolumn{2}{|c|}{ Poor drainage on \#R1 road } & & & \\
\hline $\begin{array}{l}\text { Provide adequate lane signing or } \\
\text { marking on \#A1 approach }\end{array}$ & \multicolumn{2}{|c|}{$\begin{array}{l}\text { Inadequate lanes signing or } \\
\text { marking on \#A1 approach }\end{array}$} & & & \\
\hline
\end{tabular}




\begin{tabular}{|l|l|l|l|}
\hline $\begin{array}{l}\text { Provide/Improve pavement surface } \\
\text { (improve transitions/drop offs/fixed } \\
\text { potholes/rutting/etc) on \#R1 road }\end{array}$ & Pavement defects on \#R1 road & & \\
\hline $\begin{array}{l}\text { Widen lanes on \#A1 approach to } \\
\text { adequate width }\end{array}$ & $\begin{array}{l}\text { Inadequate lane width on \#A1 } \\
\text { approach }\end{array}$ & & \\
\hline Convert intersection into roundabout & $\begin{array}{l}\text { Inadequate design for high } \\
\text { traffic volume }\end{array}$ & & \\
\hline
\end{tabular}

\section{Rail Road Crossing}

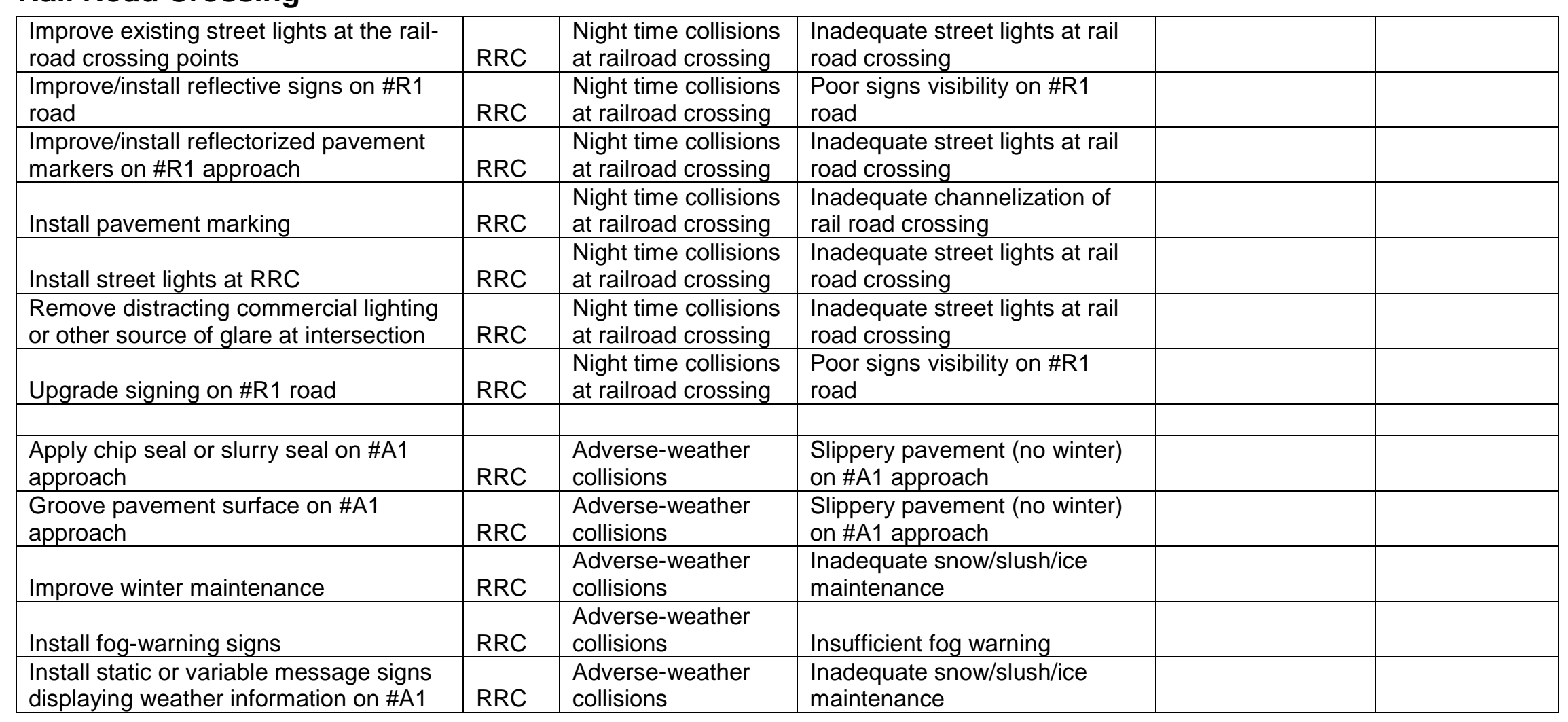




\begin{tabular}{|c|c|c|c|c|c|}
\hline approach & & & & & \\
\hline $\begin{array}{l}\text { Install snow screens in areas exposed } \\
\text { to snowdrifts on \#R1 road }\end{array}$ & $\mathrm{RRC}$ & $\begin{array}{l}\text { Adverse-weather } \\
\text { collisions }\end{array}$ & $\begin{array}{l}\text { Inadequate snow/slush/ice } \\
\text { maintenance }\end{array}$ & & \\
\hline Overlay pavement on \#A1 approach & $\mathrm{RRC}$ & $\begin{array}{l}\text { Adverse-weather } \\
\text { collisions }\end{array}$ & $\begin{array}{l}\text { Slippery pavement (no winter) } \\
\text { on \#A1 approach }\end{array}$ & & \\
\hline Post adequate speed limit on \#R1 road & $\mathrm{RRC}$ & $\begin{array}{l}\text { Adverse-weather } \\
\text { collisions }\end{array}$ & $\begin{array}{l}\text { Slippery pavement (no winter) } \\
\text { on \#A1 approach }\end{array}$ & & \\
\hline $\begin{array}{l}\text { Provide "Slippery when wet" signs on } \\
\text { \#A1 approach }\end{array}$ & $\mathrm{RRC}$ & $\begin{array}{l}\text { Adverse-weather } \\
\text { collisions }\end{array}$ & $\begin{array}{l}\text { Slippery pavement (no winter) } \\
\text { on \#A1 approach }\end{array}$ & & \\
\hline $\begin{array}{l}\text { Use deicing/antiicing methods to \#R1 } \\
\text { road surface }\end{array}$ & $\mathrm{RRC}$ & $\begin{array}{l}\text { Adverse-weather } \\
\text { collisions }\end{array}$ & $\begin{array}{l}\text { Inadequate snow/slush/ice } \\
\text { maintenance }\end{array}$ & & \\
\hline Post adequate speed limit on \#R1 road & $\mathrm{RRC}$ & $\begin{array}{l}\text { Excessive speed on } \\
\text { \#R1 road }\end{array}$ & $\begin{array}{l}\text { No posted speed limit on \#A1 } \\
\text { approach }\end{array}$ & & \\
\hline $\begin{array}{l}\text { Provide targeted speed enforcement on } \\
\text { \#A1 approach }\end{array}$ & $\mathrm{RRC}$ & $\begin{array}{l}\text { Excessive speed on } \\
\text { \#R1 road }\end{array}$ & Posted speed limit on \#R1 road & $\begin{array}{l}\text { Speed violations } \\
\text { on \#R1 road }\end{array}$ & \\
\hline $\begin{array}{l}\text { Post dynamic message sign to display } \\
\text { speed of vehicles on \#A1 approach }\end{array}$ & $\mathrm{RRC}$ & $\begin{array}{l}\text { Excessive speed on } \\
\text { \#R1 road }\end{array}$ & Posted speed limit on \#R1 road & $\begin{array}{l}\text { Speed violations } \\
\text { on \#R1 road }\end{array}$ & \\
\hline Post adequate speed limit on \#R1 road & $\mathrm{RRC}$ & $\begin{array}{l}\text { Excessive speed on } \\
\text { \#R1 road }\end{array}$ & Posted speed limit on \#R1 road & $\begin{array}{l}\text { Speed violations } \\
\text { on \#R1 road }\end{array}$ & \\
\hline $\begin{array}{l}\text { Eliminate screening of RRC by physical } \\
\text { objects }\end{array}$ & $\mathrm{RRC}$ & $\begin{array}{l}\text { Bicycle collisions at } \\
\text { RRC }\end{array}$ & $\begin{array}{l}\text { Inadequate visibility on } \\
\text { approaching train }\end{array}$ & & \\
\hline $\begin{array}{l}\text { Improve pavement marking and } \\
\text { texturing }\end{array}$ & $\mathrm{RRC}$ & $\begin{array}{l}\text { Bicycle collisions at } \\
\text { RRC }\end{array}$ & $\begin{array}{l}\text { Inadequate bicycles access } \\
\text { design }\end{array}$ & & \\
\hline $\begin{array}{l}\text { Improve signage and/or marking of } \\
\text { crosswalk(s) }\end{array}$ & $\mathrm{RRC}$ & $\begin{array}{l}\text { Bicycle collisions at } \\
\text { RRC }\end{array}$ & $\begin{array}{l}\text { Inadequate bicycles access } \\
\text { design }\end{array}$ & & \\
\hline Install bicycles overpass or underpass & $\mathrm{RRC}$ & $\begin{array}{l}\text { Bicycle collisions at } \\
\text { RRC }\end{array}$ & $\begin{array}{l}\text { Inadequate bicycles access } \\
\text { design }\end{array}$ & & \\
\hline $\begin{array}{l}\text { Install fence or chicane to prevent } \\
\text { bicycles riding across track }\end{array}$ & $\mathrm{RRC}$ & $\begin{array}{l}\text { Bicycle collisions at } \\
\text { RRC }\end{array}$ & $\begin{array}{l}\text { Bicycles running across the } \\
\text { track }\end{array}$ & & \\
\hline Install active control devices & $\mathrm{RRC}$ & $\begin{array}{l}\text { Bicycle collisions at } \\
\text { RRC }\end{array}$ & $\begin{array}{l}\text { Inadequate bicycles access } \\
\text { design }\end{array}$ & & \\
\hline Install audible signals (warning bell) & $\mathrm{RRC}$ & $\begin{array}{l}\text { Bicycle collisions at } \\
\text { RRC }\end{array}$ & $\begin{array}{l}\text { Inadequate warning about } \\
\text { approaching train }\end{array}$ & & \\
\hline Install automated bicycles gates & $\mathrm{RRC}$ & $\begin{array}{l}\text { Bicycle collisions at } \\
\text { RRC }\end{array}$ & $\begin{array}{l}\text { Inadequate bicycles access } \\
\text { design }\end{array}$ & & \\
\hline
\end{tabular}




\begin{tabular}{|c|c|c|c|c|}
\hline Install bicycle signals & $\mathrm{RRC}$ & $\begin{array}{l}\text { Bicycle collisions at } \\
\text { RRC }\end{array}$ & $\begin{array}{l}\text { Inadequate bicycles access } \\
\text { design }\end{array}$ & \\
\hline Install fixed warning message signs & $\mathrm{RRC}$ & $\begin{array}{l}\text { Bicycle collisions at } \\
\text { RRC }\end{array}$ & $\begin{array}{l}\text { Inadequate bicycles access } \\
\text { design }\end{array}$ & \\
\hline $\begin{array}{l}\text { Install flashing beacons on \#A1 } \\
\text { approach }\end{array}$ & $\mathrm{RRC}$ & $\begin{array}{l}\text { Bicycle collisions at } \\
\text { RRC }\end{array}$ & $\begin{array}{l}\text { Inadequate bicycles access } \\
\text { design }\end{array}$ & \\
\hline Install four-quadrant gate arms & $\mathrm{RRC}$ & $\begin{array}{l}\text { Bicycle collisions at } \\
\text { RRC }\end{array}$ & Automatic gates & $\begin{array}{l}\text { Bicycles crossing between already } \\
\text { lowered gate arms }\end{array}$ \\
\hline $\begin{array}{l}\text { Install gates with self deploying } \\
\text { extension sections }\end{array}$ & $\mathrm{RRC}$ & $\begin{array}{l}\text { Bicycle collisions at } \\
\text { RRC }\end{array}$ & Automatic gates & $\begin{array}{l}\text { Bicycles crossing between already } \\
\text { lowered gate arms }\end{array}$ \\
\hline Install street lighting & $\mathrm{RRC}$ & $\begin{array}{l}\text { Bicycle collisions at } \\
\text { RRC }\end{array}$ & $\begin{array}{l}\text { Inadequate warning about } \\
\text { approaching train }\end{array}$ & \\
\hline Install median barriers & $\mathrm{RRC}$ & $\begin{array}{l}\text { Bicycle collisions at } \\
\text { RRC }\end{array}$ & Automatic gates & $\begin{array}{l}\text { Bicycles crossing between already } \\
\text { lowered gate arms }\end{array}$ \\
\hline Install non-mountable curb islands & $\mathrm{RRC}$ & $\begin{array}{l}\text { Bicycle collisions at } \\
\text { RRC }\end{array}$ & Automatic gates & $\begin{array}{l}\text { Bicycles crossing between already } \\
\text { lowered gate arms }\end{array}$ \\
\hline Install swing gates & $\mathrm{RRC}$ & $\begin{array}{l}\text { Bicycle collisions at } \\
\text { RRC }\end{array}$ & $\begin{array}{l}\text { Inadequate bicycles access } \\
\text { design }\end{array}$ & \\
\hline $\begin{array}{l}\text { Install variable message signs and } \\
\text { blank out signs }\end{array}$ & $\mathrm{RRC}$ & $\begin{array}{l}\text { Bicycle collisions at } \\
\text { RRC }\end{array}$ & $\begin{array}{l}\text { Inadequate bicycles access } \\
\text { design }\end{array}$ & \\
\hline Install vehicle-arresting barriers & $\mathrm{RRC}$ & $\begin{array}{l}\text { Bicycle collisions at } \\
\text { RRC }\end{array}$ & Automatic gates & $\begin{array}{l}\text { Bicycles crossing between already } \\
\text { lowered gate arms }\end{array}$ \\
\hline $\begin{array}{l}\text { Eliminate screening of RRC by physical } \\
\text { objects }\end{array}$ & $\mathrm{RRC}$ & $\begin{array}{l}\text { Pedestrian } \\
\text { collisions at RRC }\end{array}$ & $\begin{array}{l}\text { Inadequate visibility on } \\
\text { approaching train }\end{array}$ & \\
\hline $\begin{array}{l}\text { Improve pavement marking and } \\
\text { texturing }\end{array}$ & $\mathrm{RRC}$ & $\begin{array}{l}\text { Pedestrian } \\
\text { collisions at RRC }\end{array}$ & $\begin{array}{l}\text { Inadequate design of existing } \\
\text { crosswalk }\end{array}$ & \\
\hline $\begin{array}{l}\text { Improve signage and/or marking of } \\
\operatorname{crosswalk(s)}\end{array}$ & $\mathrm{RRC}$ & $\begin{array}{l}\text { Pedestrian } \\
\text { collisions at } \mathrm{RRC}\end{array}$ & $\begin{array}{l}\text { Inadequate design of existing } \\
\text { crosswalk }\end{array}$ & \\
\hline $\begin{array}{l}\text { Install fence or chicane to prevent } \\
\text { pedestrians running across track }\end{array}$ & $\mathrm{RRC}$ & $\begin{array}{l}\text { Pedestrian } \\
\text { collisions at } \mathrm{RRC}\end{array}$ & $\begin{array}{l}\text { Pedestrians running across the } \\
\text { track }\end{array}$ & \\
\hline $\begin{array}{l}\text { Install pedestrian overpass or } \\
\text { underpass }\end{array}$ & $\mathrm{RRC}$ & $\begin{array}{l}\text { Pedestrian } \\
\text { collisions at RRC }\end{array}$ & $\begin{array}{l}\text { Inadequate design of existing } \\
\text { crosswalk }\end{array}$ & \\
\hline Install active control devices & $\mathrm{RRC}$ & $\begin{array}{l}\text { Pedestrian } \\
\text { collisions at } \mathrm{RRC}\end{array}$ & $\begin{array}{l}\text { Inadequate design of existing } \\
\text { crosswalk }\end{array}$ & \\
\hline Install audible signals (warning bell) & $\mathrm{RRC}$ & $\begin{array}{l}\text { Pedestrian } \\
\text { collisions at RRC }\end{array}$ & $\begin{array}{l}\text { Inadequate warning about } \\
\text { approaching train }\end{array}$ & \\
\hline
\end{tabular}




\begin{tabular}{|c|c|c|c|c|c|}
\hline Install automated pedestrian gates & $\mathrm{RRC}$ & $\begin{array}{l}\text { Pedestrian } \\
\text { collisions at RRC }\end{array}$ & $\begin{array}{l}\text { Inadequate design of existing } \\
\text { crosswalk }\end{array}$ & & \\
\hline Install fixed warning message signs & $\mathrm{RRC}$ & $\begin{array}{l}\text { Pedestrian } \\
\text { collisions at RRC }\end{array}$ & $\begin{array}{l}\text { Inadequate design of existing } \\
\text { crosswalk }\end{array}$ & & \\
\hline $\begin{array}{l}\text { Install flashing beacons on \#A1 } \\
\text { approach }\end{array}$ & $\mathrm{RRC}$ & $\begin{array}{l}\text { Pedestrian } \\
\text { collisions at RRC }\end{array}$ & $\begin{array}{l}\text { Inadequate design of existing } \\
\text { crosswalk }\end{array}$ & & \\
\hline Install street lighting & $\mathrm{RRC}$ & $\begin{array}{l}\text { Pedestrian } \\
\text { collisions at RRC }\end{array}$ & $\begin{array}{l}\text { Inadequate warning about } \\
\text { approaching train }\end{array}$ & & \\
\hline Install pedestrian barriers & $\mathrm{RRC}$ & $\begin{array}{l}\text { Pedestrian } \\
\text { collisions at RRC }\end{array}$ & $\begin{array}{l}\text { Inadequate design of existing } \\
\text { crosswalk }\end{array}$ & & \\
\hline Install pedestrian barriers & $\mathrm{RRC}$ & $\begin{array}{l}\text { Pedestrian } \\
\text { collisions at RRC }\end{array}$ & $\begin{array}{l}\text { Inadequate design of existing } \\
\text { crosswalk }\end{array}$ & & \\
\hline Install swing gates & $\mathrm{RRC}$ & $\begin{array}{l}\text { Pedestrian } \\
\text { collisions at RRC }\end{array}$ & $\begin{array}{l}\text { Inadequate design of existing } \\
\text { crosswalk }\end{array}$ & & \\
\hline $\begin{array}{l}\text { Install variable message signs and } \\
\text { blank out signs }\end{array}$ & $\mathrm{RRC}$ & $\begin{array}{l}\text { Pedestrian } \\
\text { collisions at RRC }\end{array}$ & $\begin{array}{l}\text { Inadequate design of existing } \\
\text { crosswalk }\end{array}$ & & \\
\hline & & & & & \\
\hline & & & & & \\
\hline $\begin{array}{l}\text { Install overhead STOP sign(s) on \#A1 } \\
\text { approach }\end{array}$ & $\mathrm{RRC}$ & $\begin{array}{l}\text { Control device is } \\
\text { visible on \#A1 } \\
\text { approach }\end{array}$ & No automatic gates & $\begin{array}{l}\text { Stop sign } \\
\text { obstruction on } \\
\# A 1 \text { approach }\end{array}$ & \\
\hline $\begin{array}{l}\text { Install overhead STOP sign(s) on \#A1 } \\
\text { approach }\end{array}$ & $\mathrm{RRC}$ & $\begin{array}{l}\text { Control device is } \\
\text { NOT visible on \#A1 } \\
\text { approach }\end{array}$ & Control device obstruction & & \\
\hline $\begin{array}{l}\text { Improve horizontal/vertical alignment on } \\
\text { \#A1 approach }\end{array}$ & $\mathrm{RRC}$ & $\begin{array}{l}\text { Control device is } \\
\text { visible on \#A1 } \\
\text { approach }\end{array}$ & $\begin{array}{l}\text { Insufficient sight distance to the } \\
\text { crossing }\end{array}$ & \multicolumn{2}{|c|}{$\begin{array}{l}\text { Poor horizontal/vertical alignment } \\
\text { on \#A1 approach }\end{array}$} \\
\hline $\begin{array}{l}\text { Improve horizontal/vertical alignment on } \\
\text { \#A1 approach }\end{array}$ & $\mathrm{RRC}$ & $\begin{array}{l}\text { Control device is } \\
\text { visible on \#A1 } \\
\text { approach }\end{array}$ & $\begin{array}{l}\text { Insufficient sight distance to the } \\
\text { crossing }\end{array}$ & \multicolumn{2}{|c|}{$\begin{array}{l}\text { Poor horizontal/vertical alignment } \\
\text { on \#A1 approach }\end{array}$} \\
\hline $\begin{array}{l}\text { Improve horizontal/vertical alignment on } \\
\text { \#A1 approach }\end{array}$ & $\mathrm{RRC}$ & $\begin{array}{l}\text { Control device is } \\
\text { NOT visible on \#A1 } \\
\text { approach }\end{array}$ & $\begin{array}{l}\text { Poor horizontal/vertical } \\
\text { alignment on \#A1 approach }\end{array}$ & & \\
\hline $\begin{array}{l}\text { Close at-grade rail-road crossing or } \\
\text { install overpass }\end{array}$ & $\mathrm{RRC}$ & $\begin{array}{l}\text { Control device is } \\
\text { visible on \#A1 }\end{array}$ & No automatic gates & $\begin{array}{l}\text { Inadequate design } \\
\text { for high traffic }\end{array}$ & \\
\hline
\end{tabular}




\begin{tabular}{|c|c|c|c|c|c|}
\hline & & approach & & volume & \\
\hline $\begin{array}{l}\text { Close at-grade rail-road crossing or } \\
\text { install overpass }\end{array}$ & $\mathrm{RRC}$ & $\begin{array}{l}\text { Control device is } \\
\text { visible on \#A1 } \\
\text { approach }\end{array}$ & Automatic gates & $\begin{array}{l}\text { Inadequate design } \\
\text { for high traffic } \\
\text { volume }\end{array}$ & \\
\hline Restrict parking close to RRC & $\mathrm{RRC}$ & $\begin{array}{l}\text { Control device is } \\
\text { visible on \#A1 } \\
\text { approach }\end{array}$ & $\begin{array}{l}\text { Insufficient sight distance to the } \\
\text { crossing }\end{array}$ & Automatic gates & $\begin{array}{l}\text { Roadside sight } \\
\text { obstruction at } \\
\text { RRC }\end{array}$ \\
\hline Restrict parking close to RRC & $\mathrm{RRC}$ & $\begin{array}{l}\text { Control device is } \\
\text { visible on \#A1 } \\
\text { approach }\end{array}$ & $\begin{array}{l}\text { Insufficient sight distance to the } \\
\text { crossing }\end{array}$ & $\begin{array}{l}\text { No automatic } \\
\text { gates }\end{array}$ & $\begin{array}{l}\text { Roadside sight } \\
\text { obstruction at } \\
\text { RRC }\end{array}$ \\
\hline $\begin{array}{l}\text { Improve gates visibility/reflectivity } \\
\text { (flashing lights along the gate) }\end{array}$ & $\mathrm{RRC}$ & $\begin{array}{l}\text { Control device is } \\
\text { visible on \#A1 } \\
\text { approach }\end{array}$ & Automatic gates & $\begin{array}{l}\text { Inadequate gates } \\
\text { visibility }\end{array}$ & \\
\hline $\begin{array}{l}\text { Install advanced warning devices on rail } \\
\text { track sensitive to train motion }\end{array}$ & $\mathrm{RRC}$ & $\begin{array}{l}\text { Control device is } \\
\text { visible on \#A1 } \\
\text { approach }\end{array}$ & Inadequate rail infrastructure & & \\
\hline $\begin{array}{l}\text { Improve maintenance of train detective } \\
\text { devices }\end{array}$ & $\mathrm{RRC}$ & $\begin{array}{l}\text { Control device is } \\
\text { visible on \#A1 } \\
\text { approach }\end{array}$ & Automatic gates & $\begin{array}{l}\text { Automatic gates } \\
\text { do not work } \\
\text { properly }\end{array}$ & \\
\hline $\begin{array}{l}\text { Improve maintenance of visual/audio } \\
\text { signals }\end{array}$ & $\mathrm{RRC}$ & $\begin{array}{l}\text { Control device is } \\
\text { visible on \#A1 } \\
\text { approach }\end{array}$ & $\begin{array}{l}\text { Visual/audio warning signal } \\
\text { does NOT work correctly }\end{array}$ & & \\
\hline $\begin{array}{l}\text { Improve maintenance/work of existing } \\
\text { gates }\end{array}$ & $\mathrm{RRC}$ & $\begin{array}{l}\text { Control device is } \\
\text { visible on \#A1 } \\
\text { approach }\end{array}$ & Automatic gates & $\begin{array}{l}\text { Automatic gates } \\
\text { do not work } \\
\text { properly }\end{array}$ & \\
\hline $\begin{array}{l}\text { Improve/install reflective signs on \#R1 } \\
\text { road }\end{array}$ & $\mathrm{RRC}$ & $\begin{array}{l}\text { Control device is } \\
\text { visible on \#A1 } \\
\text { approach }\end{array}$ & No automatic gates & $\begin{array}{l}\text { Inadequate } \\
\text { control device }\end{array}$ & \\
\hline $\begin{array}{l}\text { Install advance warning signs on \#A1 } \\
\text { approach }\end{array}$ & $\mathrm{RRC}$ & $\begin{array}{l}\text { Control device is } \\
\text { NOT visible on \#A1 } \\
\text { approach }\end{array}$ & $\begin{array}{l}\text { Poor horizontal/vertical } \\
\text { alignment on \#A1 approach }\end{array}$ & & \\
\hline Install audible signals (warning bell) & $\mathrm{RRC}$ & $\begin{array}{l}\text { Control device is } \\
\text { visible on \#A1 } \\
\text { approach }\end{array}$ & No automatic gates & $\begin{array}{l}\text { Inadequate } \\
\text { control device }\end{array}$ & \\
\hline Install automatic gates & $\mathrm{RRC}$ & $\begin{array}{l}\text { Control device is } \\
\text { visible on \#A1 }\end{array}$ & No automatic gates & $\begin{array}{l}\text { Inadequate } \\
\text { control device }\end{array}$ & \\
\hline
\end{tabular}




\begin{tabular}{|c|c|c|c|c|c|}
\hline & & approach & & & \\
\hline Install automatic gates & $\mathrm{RRC}$ & $\begin{array}{l}\text { Control device is } \\
\text { visible on \#A1 } \\
\text { approach }\end{array}$ & No automatic gates & \multicolumn{2}{|c|}{$\begin{array}{l}\text { Frequent stop sign violations on } \\
\text { \#A1 approach }\end{array}$} \\
\hline Install cantilever flashing-light signal & RRC & $\begin{array}{l}\text { Control device is } \\
\text { visible on \#A1 } \\
\text { approach }\end{array}$ & No automatic gates & $\begin{array}{l}\text { Inadequate } \\
\text { control device }\end{array}$ & \\
\hline Install cantilever flashing-light signal & $\mathrm{RRC}$ & $\begin{array}{l}\text { Control device is } \\
\text { visible on \#A1 } \\
\text { approach }\end{array}$ & No automatic gates & $\begin{array}{l}\text { Inadequate } \\
\text { signing for multi- } \\
\text { lane approach }\end{array}$ & \\
\hline $\begin{array}{l}\text { Install corner mirrors (local road/low } \\
\text { speed) }\end{array}$ & $\mathrm{RRC}$ & $\begin{array}{l}\text { Control device is } \\
\text { visible on \#A1 } \\
\text { approach }\end{array}$ & $\begin{array}{l}\text { Insufficient sight distance to the } \\
\text { crossing }\end{array}$ & $\begin{array}{l}\text { No automatic } \\
\text { gates }\end{array}$ & $\begin{array}{l}\text { Roadside sight } \\
\text { obstruction at } \\
\text { RRC }\end{array}$ \\
\hline Install crossbuck & $\mathrm{RRC}$ & $\begin{array}{l}\text { Control device is } \\
\text { visible on \#A1 } \\
\text { approach }\end{array}$ & No automatic gates & $\begin{array}{l}\text { Inadequate } \\
\text { control device }\end{array}$ & \\
\hline $\begin{array}{l}\text { Install DO NOT STOP ON TRACKS } \\
\text { signal }\end{array}$ & $\mathrm{RRC}$ & $\begin{array}{l}\text { Control device is } \\
\text { visible on \#A1 } \\
\text { approach }\end{array}$ & $\begin{array}{l}\text { Limited storage space between } \\
\text { highway intersection and } \\
\text { railway crossing }\end{array}$ & & \\
\hline $\begin{array}{l}\text { Install flashing beacons on \#A1 } \\
\text { approach }\end{array}$ & $\mathrm{RRC}$ & $\begin{array}{l}\text { Control device is } \\
\text { visible on \#A1 } \\
\text { approach }\end{array}$ & $\begin{array}{l}\text { Inadequate advance warning of } \\
\mathrm{RRC}\end{array}$ & & \\
\hline $\begin{array}{l}\text { Install flashing beacons on \#A1 } \\
\text { approach }\end{array}$ & $\mathrm{RRC}$ & $\begin{array}{l}\text { Control device is } \\
\text { visible on \#A1 } \\
\text { approach }\end{array}$ & Automatic gates & $\begin{array}{l}\text { Inadequate gates } \\
\text { visibility }\end{array}$ & \\
\hline $\begin{array}{l}\text { Install flashing beacons on \#A1 } \\
\text { approach }\end{array}$ & $\mathrm{RRC}$ & $\begin{array}{l}\text { Control device is } \\
\text { visible on \#A1 } \\
\text { approach }\end{array}$ & No automatic gates & $\begin{array}{l}\text { Inadequate } \\
\text { control device }\end{array}$ & \\
\hline $\begin{array}{l}\text { Install flashing light activated by } \\
\text { vehicles stopped between railway } \\
\text { crossing and intersection }\end{array}$ & $\mathrm{RRC}$ & $\begin{array}{l}\text { Control device is } \\
\text { visible on \#A1 } \\
\text { approach }\end{array}$ & $\begin{array}{l}\text { Limited storage space between } \\
\text { highway intersection and } \\
\text { railway crossing }\end{array}$ & & \\
\hline Install four-quadrant gate arms & $\mathrm{RRC}$ & $\begin{array}{l}\text { Control device is } \\
\text { visible on \#A1 } \\
\text { approach }\end{array}$ & Automatic gates & $\begin{array}{l}\text { Drivers crossing bet } \\
\text { lowered gate arms }\end{array}$ & ween already \\
\hline Install automatic gates & $\mathrm{RRC}$ & $\begin{array}{l}\text { Control device is } \\
\text { visible on \#A1 }\end{array}$ & No automatic gates & $\begin{array}{l}\text { Inadequate design } \\
\text { for high traffic }\end{array}$ & \\
\hline
\end{tabular}




\begin{tabular}{|c|c|c|c|c|c|}
\hline & & approach & & volume & \\
\hline $\begin{array}{l}\text { Install gates with self deploying } \\
\text { extension sections }\end{array}$ & $\mathrm{RRC}$ & $\begin{array}{l}\text { Control device is } \\
\text { visible on \#A1 } \\
\text { approach }\end{array}$ & Automatic gates & \multicolumn{2}{|c|}{$\begin{array}{l}\text { Drivers crossing between already } \\
\text { lowered gate arms }\end{array}$} \\
\hline Install median barriers & RRC & $\begin{array}{l}\text { Control device is } \\
\text { visible on \#A1 } \\
\text { approach }\end{array}$ & Automatic gates & \multicolumn{2}{|c|}{$\begin{array}{l}\text { Drivers crossing between already } \\
\text { lowered gate arms }\end{array}$} \\
\hline $\begin{array}{l}\text { Install median retroreflective post } \\
\text { delineators on the approach }\end{array}$ & $\mathrm{RRC}$ & $\begin{array}{l}\text { Control device is } \\
\text { visible on \#A1 } \\
\text { approach }\end{array}$ & Automatic gates & \multicolumn{2}{|c|}{$\begin{array}{l}\text { Drivers crossing between already } \\
\text { lowered gate arms }\end{array}$} \\
\hline Install non-mountable curb islands & $\mathrm{RRC}$ & $\begin{array}{l}\text { Control device is } \\
\text { visible on \#A1 } \\
\text { approach }\end{array}$ & Automatic gates & \multicolumn{2}{|c|}{$\begin{array}{l}\text { Drivers crossing between already } \\
\text { lowered gate arms }\end{array}$} \\
\hline Install overhead signs & $\mathrm{RRC}$ & $\begin{array}{l}\text { Control device is } \\
\text { visible on \#A1 } \\
\text { approach }\end{array}$ & No automatic gates & $\begin{array}{l}\text { Inadequate } \\
\text { signing for multi- } \\
\text { lane approach }\end{array}$ & \\
\hline Install pre-signals & $\mathrm{RRC}$ & $\begin{array}{l}\text { Control device is } \\
\text { visible on \#A1 } \\
\text { approach }\end{array}$ & $\begin{array}{l}\text { Limited storage space between } \\
\text { highway intersection and } \\
\text { railway crossing }\end{array}$ & & \\
\hline Install rumble strips on \#A1 approach & $\mathrm{RRC}$ & $\begin{array}{l}\text { Control device is } \\
\text { visible on \#A1 } \\
\text { approach }\end{array}$ & $\begin{array}{l}\text { Inadequate advance warning of } \\
\mathrm{RRC}\end{array}$ & & \\
\hline $\begin{array}{l}\text { Install warning signs on both sides of } \\
\text { road on \#A1 approach }\end{array}$ & RRC & $\begin{array}{l}\text { Control device is } \\
\text { visible on \#A1 } \\
\text { approach }\end{array}$ & $\begin{array}{l}\text { Inadequate advance warning of } \\
\mathrm{RRC}\end{array}$ & & \\
\hline Install speed humps & $\mathrm{RRC}$ & $\begin{array}{l}\text { Control device is } \\
\text { visible on \#A1 } \\
\text { approach }\end{array}$ & $\begin{array}{l}\text { Insufficient sight distance to the } \\
\text { crossing }\end{array}$ & Automatic gates & $\begin{array}{l}\text { Roadside sight } \\
\text { obstruction at } \\
\text { RRC }\end{array}$ \\
\hline Install speed humps & $\mathrm{RRC}$ & $\begin{array}{l}\text { Control device is } \\
\text { visible on \#A1 } \\
\text { approach }\end{array}$ & $\begin{array}{l}\text { Insufficient sight distance to the } \\
\text { crossing }\end{array}$ & $\begin{array}{l}\text { No automatic } \\
\text { gates }\end{array}$ & $\begin{array}{l}\text { Roadside sight } \\
\text { obstruction at } \\
\text { RRC }\end{array}$ \\
\hline Install stop sign on \#A1 approach & $\mathrm{RRC}$ & $\begin{array}{l}\text { Control device is } \\
\text { visible on \#A1 } \\
\text { approach }\end{array}$ & $\begin{array}{l}\text { Insufficient sight distance to the } \\
\text { crossing }\end{array}$ & $\begin{array}{l}\text { No automatic } \\
\text { gates }\end{array}$ & $\begin{array}{l}\text { Roadside sight } \\
\text { obstruction at } \\
\text { RRC }\end{array}$ \\
\hline Install stop sign on \#A1 approach & $\mathrm{RRC}$ & $\begin{array}{l}\text { Control device is } \\
\text { visible on \#A1 }\end{array}$ & No automatic gates & $\begin{array}{l}\text { Inadequate } \\
\text { control device }\end{array}$ & \\
\hline
\end{tabular}




\begin{tabular}{|c|c|c|c|c|c|}
\hline & & approach & & & \\
\hline Install symbol storage space sign & $\mathrm{RRC}$ & $\begin{array}{l}\text { Control device is } \\
\text { visible on \#A1 } \\
\text { approach }\end{array}$ & $\begin{array}{l}\text { Limited storage space between } \\
\text { highway intersection and } \\
\text { railway crossing }\end{array}$ & & \\
\hline Install vehicle-arresting barriers & $\mathrm{RRC}$ & $\begin{array}{l}\text { Control device is } \\
\text { visible on \#A1 } \\
\text { approach }\end{array}$ & Automatic gates & \multicolumn{2}{|c|}{$\begin{array}{l}\text { Drivers crossing between already } \\
\text { lowered gate arms }\end{array}$} \\
\hline $\begin{array}{l}\text { Install warning signs in advance of rail- } \\
\text { road crossing }\end{array}$ & $\mathrm{RRC}$ & $\begin{array}{l}\text { Control device is } \\
\text { visible on \#A1 } \\
\text { approach }\end{array}$ & $\begin{array}{l}\text { Inadequate advance warning of } \\
\mathrm{RRC}\end{array}$ & & \\
\hline Install yield sign & $\mathrm{RRC}$ & $\begin{array}{l}\text { Control device is } \\
\text { visible on \#A1 } \\
\text { approach }\end{array}$ & No automatic gates & $\begin{array}{l}\text { Inadequate } \\
\text { control device }\end{array}$ & \\
\hline Post adequate speed limit on \#R1 road & $\mathrm{RRC}$ & $\begin{array}{l}\text { Control device is } \\
\text { visible on \#A1 } \\
\text { approach }\end{array}$ & $\begin{array}{l}\text { Insufficient sight distance to the } \\
\text { crossing }\end{array}$ & $\begin{array}{l}\text { Roadside sight } \\
\text { obstruction at } \\
\text { RRC }\end{array}$ & \\
\hline Provide adequate gates delay & $\mathrm{RRC}$ & $\begin{array}{l}\text { Control device is } \\
\text { visible on \#A1 } \\
\text { approach }\end{array}$ & Automatic gates & $\begin{array}{l}\text { Automatic gates } \\
\text { do not work } \\
\text { properly }\end{array}$ & \\
\hline $\begin{array}{l}\text { Provide adequate information to train } \\
\text { operator (for example: location of the } \\
\text { whistle post) }\end{array}$ & $\mathrm{RRC}$ & $\begin{array}{l}\text { Control device is } \\
\text { visible on \#A1 } \\
\text { approach }\end{array}$ & Inadequate rail infrastructure & & \\
\hline Provide information of crossing type & $\mathrm{RRC}$ & $\begin{array}{l}\text { Control device is } \\
\text { visible on \#A1 } \\
\text { approach }\end{array}$ & $\begin{array}{l}\text { Inadequate advance warning of } \\
\mathrm{RRC}\end{array}$ & & \\
\hline $\begin{array}{l}\text { Improve pavement marking on \#A1 } \\
\text { approach }\end{array}$ & $\mathrm{RRC}$ & $\begin{array}{l}\text { Control device is } \\
\text { visible on \#A1 } \\
\text { approach }\end{array}$ & $\begin{array}{l}\text { Inadequate advance warning of } \\
\mathrm{RRC}\end{array}$ & & \\
\hline Provide photo/video enforcement & $\mathrm{RRC}$ & $\begin{array}{l}\text { Control device is } \\
\text { visible on \#A1 } \\
\text { approach }\end{array}$ & Automatic gates & $\begin{array}{l}\text { Drivers crossing } \mathrm{b} \\
\text { lowered gate arm }\end{array}$ & veen already \\
\hline Reduce speed limit on \#A1 approach & $\mathrm{RRC}$ & $\begin{array}{l}\text { Control device is } \\
\text { visible on \#A1 } \\
\text { approach }\end{array}$ & $\begin{array}{l}\text { Insufficient sight distance to the } \\
\text { crossing }\end{array}$ & Automatic gates & $\begin{array}{l}\text { Roadside sight } \\
\text { obstruction at } \\
\text { RRC }\end{array}$ \\
\hline Reduce speed limit on \#A1 approach & $\mathrm{RRC}$ & $\begin{array}{l}\text { Control device is } \\
\text { visible on \#A1 }\end{array}$ & $\begin{array}{l}\text { Insufficient sight distance to the } \\
\text { crossing }\end{array}$ & $\begin{array}{l}\text { No automatic } \\
\text { gates }\end{array}$ & $\begin{array}{l}\text { Roadside sight } \\
\text { obstruction at }\end{array}$ \\
\hline
\end{tabular}




\begin{tabular}{|c|c|c|c|c|c|}
\hline & & approach & & & $\mathrm{RRC}$ \\
\hline $\begin{array}{l}\text { Relocate control device away from the } \\
\text { road }\end{array}$ & $\mathrm{RRC}$ & $\begin{array}{l}\text { Control device is } \\
\text { NOT visible on \#A1 } \\
\text { approach }\end{array}$ & Control device obstruction & & \\
\hline Relocate stop bar on \#A1 approach & $\mathrm{RRC}$ & $\begin{array}{l}\text { Control device is } \\
\text { visible on \#A1 } \\
\text { approach }\end{array}$ & $\begin{array}{l}\text { Insufficient sight distance to the } \\
\text { crossing }\end{array}$ & $\begin{array}{l}\text { No automatic } \\
\text { gates }\end{array}$ & $\begin{array}{l}\text { Roadside sight } \\
\text { obstruction at } \\
\text { RRC }\end{array}$ \\
\hline Relocate stop sign on \#A1 approach & $\mathrm{RRC}$ & $\begin{array}{l}\text { Control device is } \\
\text { visible on \#A1 } \\
\text { approach }\end{array}$ & $\begin{array}{l}\text { Insufficient sight distance to the } \\
\text { crossing }\end{array}$ & $\begin{array}{l}\text { No automatic } \\
\text { gates }\end{array}$ & $\begin{array}{l}\text { Roadside sight } \\
\text { obstruction at } \\
\text { RRC }\end{array}$ \\
\hline Relocate stop sign on \#A1 approach & $\mathrm{RRC}$ & $\begin{array}{l}\text { Control device is } \\
\text { visible on \#A1 } \\
\text { approach }\end{array}$ & No automatic gates & $\begin{array}{l}\text { Stop sign } \\
\text { obstruction on } \\
\text { \#A1 approach }\end{array}$ & \\
\hline Relocate crossing section & $\mathrm{RRC}$ & $\begin{array}{l}\text { Control device is } \\
\text { visible on \#A1 } \\
\text { approach }\end{array}$ & No automatic gates & $\begin{array}{l}\text { Inadequate design } \\
\text { for high traffic } \\
\text { volume }\end{array}$ & \\
\hline $\begin{array}{l}\text { Remove object obstructing sight of } \\
\text { control device }\end{array}$ & $\mathrm{RRC}$ & $\begin{array}{l}\text { Control device is } \\
\text { NOT visible on \#A1 } \\
\text { approach }\end{array}$ & Control device obstruction & & \\
\hline $\begin{array}{l}\text { Remove object obstructing sight of stop } \\
\text { sign on \#A1 approach }\end{array}$ & $\mathrm{RRC}$ & $\begin{array}{l}\text { Control device is } \\
\text { visible on \#A1 } \\
\text { approach }\end{array}$ & No automatic gates & $\begin{array}{l}\text { Stop sign } \\
\text { obstruction on } \\
\text { \#A1 approach }\end{array}$ & \\
\hline $\begin{array}{l}\text { Remove object obstructing sight triangle } \\
\text { at RRC }\end{array}$ & RRC & $\begin{array}{l}\text { Control device is } \\
\text { visible on \#A1 } \\
\text { approach }\end{array}$ & $\begin{array}{l}\text { Insufficient sight distance to the } \\
\text { crossing }\end{array}$ & Automatic gates & $\begin{array}{l}\text { Roadside sight } \\
\text { obstruction at } \\
\text { RRC }\end{array}$ \\
\hline $\begin{array}{l}\text { Remove object obstructing sight triangle } \\
\text { at RRC }\end{array}$ & $\mathrm{RRC}$ & $\begin{array}{l}\text { Control device is } \\
\text { visible on \#A1 } \\
\text { approach }\end{array}$ & $\begin{array}{l}\text { Insufficient sight distance to the } \\
\text { crossing }\end{array}$ & $\begin{array}{l}\text { No automatic } \\
\text { gates }\end{array}$ & $\begin{array}{l}\text { Roadside sight } \\
\text { obstruction at } \\
\text { RRC }\end{array}$ \\
\hline Replace/repair signals & $\mathrm{RRC}$ & $\begin{array}{l}\text { Control device is } \\
\text { visible on \#A1 } \\
\text { approach }\end{array}$ & $\begin{array}{l}\text { Visual/audio warning signal } \\
\text { does NOT work correctly }\end{array}$ & & \\
\hline Replace, repair or clean control device & $\mathrm{RRC}$ & $\begin{array}{l}\text { Control device is } \\
\text { NOT visible on \#A1 } \\
\text { approach }\end{array}$ & $\begin{array}{l}\text { Control device improperly } \\
\text { maintained }\end{array}$ & & \\
\hline Replace/repair signals & $\mathrm{RRC}$ & $\begin{array}{l}\text { Control device is } \\
\text { visible on \#A1 }\end{array}$ & No automatic gates & \multicolumn{2}{|c|}{$\begin{array}{l}\text { Stop sign on \#A1 approach } \\
\text { improperly maintained }\end{array}$} \\
\hline
\end{tabular}




\begin{tabular}{|c|c|c|c|c|c|}
\hline & & approach & & & \\
\hline Trim vegetation at $R R C$ & $\mathrm{RRC}$ & $\begin{array}{l}\text { Control device is } \\
\text { visible on \#A1 } \\
\text { approach }\end{array}$ & $\begin{array}{l}\text { Insufficient sight distance to the } \\
\text { crossing }\end{array}$ & $\begin{array}{l}\text { Roadside sight } \\
\text { obstruction at } \\
\text { RRC }\end{array}$ & \\
\hline Trim vegetation at $R R C$ & $\mathrm{RRC}$ & $\begin{array}{l}\text { Control device is } \\
\text { visible on \#A1 } \\
\text { approach }\end{array}$ & $\begin{array}{l}\text { Insufficient sight distance to the } \\
\text { crossing }\end{array}$ & Automatic gates & $\begin{array}{l}\text { Roadside sight } \\
\text { obstruction at } \\
\text { RRC }\end{array}$ \\
\hline Trim vegetation at $R R C$ & $\mathrm{RRC}$ & $\begin{array}{l}\text { Control device is } \\
\text { visible on \#A1 } \\
\text { approach }\end{array}$ & $\begin{array}{l}\text { Insufficient sight distance to the } \\
\text { crossing }\end{array}$ & $\begin{array}{l}\text { No automatic } \\
\text { gates }\end{array}$ & $\begin{array}{l}\text { Roadside sight } \\
\text { obstruction at } \\
\text { RRC }\end{array}$ \\
\hline Provide photo/video enforcement & $\mathrm{RRC}$ & $\begin{array}{l}\text { Control device is } \\
\text { visible on \#A1 } \\
\text { approach }\end{array}$ & No automatic gates & \multicolumn{2}{|c|}{$\begin{array}{l}\text { Frequent stop sign violations on } \\
\# A 1 \text { approach }\end{array}$} \\
\hline Increase skid resistance on \#R1 road & \multicolumn{2}{|c|}{$\begin{array}{l}\text { Poor skid resistance on \#R1 } \\
\text { road }\end{array}$} & & & \\
\hline Provide adequate drainage on \#R1 road & \multicolumn{2}{|c|}{ Poor drainage on \#R1 road } & & & \\
\hline $\begin{array}{l}\text { Provide/Improve pavement surface } \\
\text { (improve transitions/drop offs/fixed } \\
\text { potholes/rutting/etc) on \#R1 road }\end{array}$ & \multicolumn{2}{|c|}{$\begin{array}{l}\text { Pavement defects on \#R1 } \\
\text { road }\end{array}$} & & & \\
\hline $\begin{array}{l}\text { Improve channelization/delineation on } \\
\text { \#A1 approach }\end{array}$ & \multicolumn{2}{|c|}{$\begin{array}{l}\text { Inadequate channelization of } \\
\text { rail road crossing }\end{array}$} & & & \\
\hline $\begin{array}{l}\text { Improve horizontal/vertical alignment on } \\
\text { \#A1 approach }\end{array}$ & \multicolumn{2}{|c|}{$\begin{array}{l}\text { Poor horizontal/vertical } \\
\text { alignment on \#A1 approach }\end{array}$} & & & \\
\hline $\begin{array}{l}\text { Improve horizontal/vertical alignment on } \\
\text { \#R1 road }\end{array}$ & \multicolumn{2}{|c|}{$\begin{array}{l}\text { Inadequate road alignment } \\
\text { on \#R1 road }\end{array}$} & & & \\
\hline $\begin{array}{l}\text { Improve pavement marking on \#A1 } \\
\text { approach }\end{array}$ & \multicolumn{2}{|c|}{$\begin{array}{l}\text { Poor pavement marking on } \\
\text { \#A1 approach }\end{array}$} & & & \\
\hline Provide adequate drainage on \#R1 road & \multicolumn{2}{|c|}{ Poor drainage on \#R1 road } & & & \\
\hline
\end{tabular}




\section{Signalized Intersection}

\begin{tabular}{|c|c|c|c|c|}
\hline $\begin{array}{l}\text { Improve visibility of intersection by } \\
\text { providing enhanced signing and } \\
\text { delineation }\end{array}$ & SIGN & $\begin{array}{l}\text { Inadequate lanes } \\
\text { signing or marking on } \\
\text { \#A1 approach }\end{array}$ & & \\
\hline $\begin{array}{l}\text { Install advance guide signs on \#A1 } \\
\text { approach }\end{array}$ & SIGN & $\begin{array}{l}\text { Inadequate lanes } \\
\text { signing or marking on } \\
\text { \#A1 approach }\end{array}$ & & \\
\hline Install street name signs at intersection & SIGN & $\begin{array}{l}\text { Inadequate lanes } \\
\text { signing or marking on } \\
\text { \#A1 approach }\end{array}$ & & \\
\hline $\begin{array}{l}\text { Install advance warning signs on \#A1 } \\
\text { approach }\end{array}$ & SIGN & $\begin{array}{l}\text { Inadequate lanes } \\
\text { signing or marking on } \\
\# A 1 \text { approach }\end{array}$ & & \\
\hline $\begin{array}{l}\text { Improve pavement marking on \#A1 } \\
\text { approach }\end{array}$ & SIGN & $\begin{array}{l}\text { Inadequate lanes } \\
\text { signing or marking on } \\
\text { \#A1 approach }\end{array}$ & & \\
\hline Install overhead signs & SIGN & $\begin{array}{l}\text { Inadequate lanes } \\
\text { signing or marking on } \\
\# A 1 \text { approach }\end{array}$ & & \\
\hline Install post-mounted delineators & SIGN & $\begin{array}{l}\text { Inadequate lanes } \\
\text { signing or marking on } \\
\text { \#A1 approach }\end{array}$ & & \\
\hline $\begin{array}{l}\text { Install flashing beacons on \#A1 } \\
\text { approach }\end{array}$ & SIGN & $\begin{array}{l}\text { Inadequate lanes } \\
\text { signing or marking on } \\
\text { \#A1 approach }\end{array}$ & & \\
\hline $\begin{array}{l}\text { Install larger regulatory signs on both } \\
\text { sides of road on \#A1 approach }\end{array}$ & SIGN & $\begin{array}{l}\text { Inadequate lanes } \\
\text { signing or marking on } \\
\text { \#A1 approach }\end{array}$ & & \\
\hline $\begin{array}{l}\text { Improve/install reflective signs on \#R1 } \\
\text { road }\end{array}$ & SIGN & \begin{tabular}{l|l} 
Inadequate lanes & \\
signing or marking on & \\
\#A1 approach & \\
\end{tabular} & & \\
\hline $\begin{array}{l}\text { Remove object obstructing sight } \\
\text { triangle at intersection }\end{array}$ & SIGN & $\begin{array}{l}\text { Inadequate sight distance toward vehicles } \\
\text { approaching intersection on \#A1 approach }\end{array}$ & & \\
\hline Trim vegetation at intersection & SIGN & $\begin{array}{l}\text { Inadequate sight distance toward vehicles } \\
\text { approaching intersection on \#A1 approach }\end{array}$ & & \\
\hline
\end{tabular}




\begin{tabular}{|c|c|c|c|c|c|}
\hline Post adequate speed limit on \#R1 road & SIGN & \multicolumn{2}{|c|}{$\begin{array}{l}\text { Inadequate sight distance toward vehicles } \\
\text { approaching intersection on \#A1 approach }\end{array}$} & & \\
\hline Restrict parking close to intersection & SIGN & \multicolumn{2}{|c|}{$\begin{array}{l}\text { Inadequate sight distance toward vehicles } \\
\text { approaching intersection on \#A1 approach }\end{array}$} & & \\
\hline Improve street lighting & SIGN & \multicolumn{2}{|c|}{$\begin{array}{l}\text { Inadequate sight distance toward vehicles } \\
\text { approaching intersection on \#A1 approach }\end{array}$} & & \\
\hline Install rumble strips on \#A1 approach & SIGN & \multicolumn{2}{|c|}{$\begin{array}{l}\text { Inadequate sight distance toward vehicles } \\
\text { approaching intersection on \#A1 approach }\end{array}$} & & \\
\hline Install queue detection system & SIGN & \multicolumn{2}{|c|}{$\begin{array}{l}\text { Inadequate sight distance toward vehicles } \\
\text { approaching intersection on \#A1 approach }\end{array}$} & & \\
\hline $\begin{array}{l}\text { Improve horizontal/vertical alignment } \\
\text { on \#A1 approach }\end{array}$ & SIGN & $\begin{array}{l}\text { Poor } \\
\text { horizontal/vertical } \\
\text { alignment on \#A1 } \\
\text { approach }\end{array}$ & & & \\
\hline Improve intersection skew angle & SIGN & $\begin{array}{l}\text { Poor } \\
\text { horizontal/vertical } \\
\text { alignment on \#A1 } \\
\text { approach }\end{array}$ & & & \\
\hline Add special signing & SIGN & $\begin{array}{l}\text { Fixed object close to } \\
\text { traveled way on \#A1 } \\
\text { approach }\end{array}$ & $\begin{array}{l}\text { Trees close to } \\
\text { traveled way }\end{array}$ & & \\
\hline Add special signing & SIGN & $\begin{array}{l}\text { Fixed object close to } \\
\text { traveled way on \#A1 } \\
\text { approach }\end{array}$ & $\begin{array}{l}\text { Utility poles close } \\
\text { to traveled way }\end{array}$ & & \\
\hline $\begin{array}{l}\text { Install barrier curb or guardrail on \#A1 } \\
\text { approach }\end{array}$ & SIGN & $\begin{array}{l}\text { Fixed object close to } \\
\text { traveled way on \#A1 } \\
\text { approach }\end{array}$ & $\begin{array}{l}\text { Trees close to } \\
\text { traveled way }\end{array}$ & & \\
\hline $\begin{array}{l}\text { Install barrier curb or guardrail on \#A1 } \\
\text { approach }\end{array}$ & SIGN & $\begin{array}{l}\text { Fixed object close to } \\
\text { traveled way on \#A1 } \\
\text { approach }\end{array}$ & $\begin{array}{l}\text { Utility poles close } \\
\text { to traveled way }\end{array}$ & & \\
\hline Install tree marker & SIGN & $\begin{array}{l}\text { Fixed object close to } \\
\text { traveled way on \#A1 } \\
\text { approach }\end{array}$ & $\begin{array}{l}\text { Trees close to } \\
\text { traveled way }\end{array}$ & & \\
\hline Install utility poles marker & SIGN & $\begin{array}{l}\text { Fixed object close to } \\
\text { traveled way on \#A1 }\end{array}$ & $\begin{array}{l}\text { Utility poles close } \\
\text { to traveled way }\end{array}$ & & \\
\hline
\end{tabular}




\begin{tabular}{|c|c|c|c|c|c|}
\hline & & approach & & & \\
\hline Increase clear zone on \#R1 road & SIGN & $\begin{array}{l}\text { Fixed object close to } \\
\text { traveled way on \#A1 } \\
\text { approach }\end{array}$ & $\begin{array}{l}\text { Trees close to } \\
\text { traveled way }\end{array}$ & & \\
\hline Place utilities underground & SIGN & $\begin{array}{l}\text { Fixed object close to } \\
\text { traveled way on \#A1 } \\
\text { approach }\end{array}$ & $\begin{array}{l}\text { Utility poles close } \\
\text { to traveled way }\end{array}$ & & \\
\hline $\begin{array}{l}\text { Provide breakaway and crashworthy } \\
\text { sign supports }\end{array}$ & SIGN & $\begin{array}{l}\text { Fixed object close to } \\
\text { traveled way on \#A1 } \\
\text { approach }\end{array}$ & $\begin{array}{l}\text { Sign supports close } \\
\text { to traveled way }\end{array}$ & & \\
\hline $\begin{array}{l}\text { Relocate signal hardware out of clear } \\
\text { zone }\end{array}$ & SIGN & $\begin{array}{l}\text { Fixed object close to } \\
\text { traveled way on \#A1 } \\
\text { approach }\end{array}$ & $\begin{array}{l}\text { Signal hardware } \\
\text { close to traveled } \\
\text { way }\end{array}$ & & \\
\hline Remove or relocate sign & SIGN & $\begin{array}{l}\text { Fixed object close to } \\
\text { traveled way on \#A1 } \\
\text { approach }\end{array}$ & $\begin{array}{l}\text { Sign supports close } \\
\text { to traveled way }\end{array}$ & & \\
\hline $\begin{array}{l}\text { Remove or relocate trees along \#R1 } \\
\text { road }\end{array}$ & SIGN & $\begin{array}{l}\text { Fixed object close to } \\
\text { traveled way on \#A1 } \\
\text { approach }\end{array}$ & $\begin{array}{l}\text { Trees close to } \\
\text { traveled way }\end{array}$ & & \\
\hline $\begin{array}{l}\text { Remove or relocate utility pole along } \\
\text { \#R1 road }\end{array}$ & SIGN & $\begin{array}{l}\text { Fixed object close to } \\
\text { traveled way on \#A1 } \\
\text { approach }\end{array}$ & $\begin{array}{l}\text { Utility poles close } \\
\text { to traveled way }\end{array}$ & & \\
\hline $\begin{array}{l}\text { Shield drivers from poles in hazardous } \\
\text { locations }\end{array}$ & SIGN & $\begin{array}{l}\text { Fixed object close to } \\
\text { traveled way on \#A1 } \\
\text { approach }\end{array}$ & $\begin{array}{l}\text { Utility poles close } \\
\text { to traveled way }\end{array}$ & & \\
\hline $\begin{array}{l}\text { Use breakaway devices on \#A1 } \\
\text { approach }\end{array}$ & SIGN & $\begin{array}{l}\text { Fixed object close to } \\
\text { traveled way on \#A1 } \\
\text { approach }\end{array}$ & $\begin{array}{l}\text { Utility poles close } \\
\text { to traveled way }\end{array}$ & & \\
\hline $\begin{array}{l}\text { Widen lanes on \#A1 approach to } \\
\text { adequate width }\end{array}$ & SIGN & $\begin{array}{l}\text { Inadequate lane width } \\
\text { on \#A1 approach }\end{array}$ & & & \\
\hline Increase skid resistance on \#R1 road & SIGN & $\begin{array}{l}\text { Poor skid resistance } \\
\text { on \#R1 road }\end{array}$ & & & \\
\hline $\begin{array}{l}\text { Groove pavement surface on \#A1 } \\
\text { approach }\end{array}$ & SIGN & $\begin{array}{l}\text { Poor skid resistance } \\
\text { on \#A1 approach }\end{array}$ & & & \\
\hline $\begin{array}{l}\text { Provide/Improve pavement surface } \\
\text { (improve transitions/drop offs/fixed }\end{array}$ & SIGN & $\begin{array}{l}\text { Pavement defects on } \\
\text { \#R1 road }\end{array}$ & & & \\
\hline
\end{tabular}




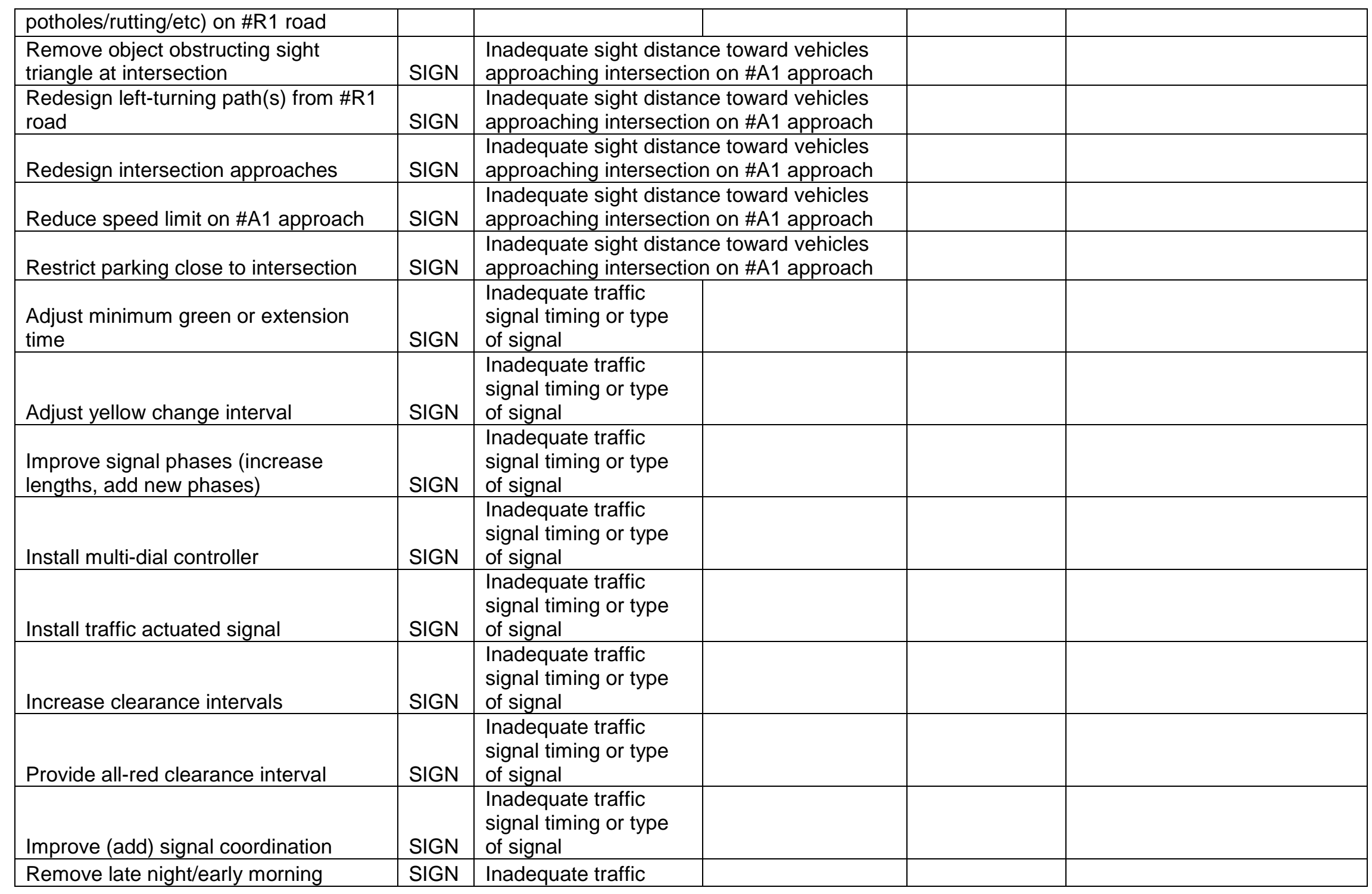




\begin{tabular}{|c|c|c|c|c|c|}
\hline flashing operation & & $\begin{array}{l}\text { signal timing or type } \\
\text { of signal }\end{array}$ & & & \\
\hline Improve (add) signal coordination & SIGN & $\begin{array}{l}\text { Inadequate traffic } \\
\text { signal timing or type } \\
\text { of signal }\end{array}$ & & & \\
\hline $\begin{array}{l}\text { Install additional signal head on \#A1 } \\
\text { approach }\end{array}$ & SIGN & $\begin{array}{l}\text { Inadequate visibility of } \\
\text { traffic signals }\end{array}$ & & & \\
\hline Install backplates & SIGN & $\begin{array}{l}\text { Inadequate visibility of } \\
\text { traffic signals }\end{array}$ & & & \\
\hline Install larger signal lenses & SIGN & $\begin{array}{l}\text { Inadequate visibility of } \\
\text { traffic signals }\end{array}$ & & & \\
\hline $\begin{array}{l}\text { Provide far-side left-turn signal on \#A1 } \\
\text { approach }\end{array}$ & SIGN & $\begin{array}{l}\text { Inadequate visibility of } \\
\text { traffic signals }\end{array}$ & & & \\
\hline $\begin{array}{l}\text { Provide louvers visors or special } \\
\text { lenses on \#A1 approach }\end{array}$ & SIGN & $\begin{array}{l}\text { Inadequate visibility of } \\
\text { traffic signals }\end{array}$ & & & \\
\hline Reduce speed limit on \#A1 approach & SIGN & $\begin{array}{l}\text { Inadequate visibility of } \\
\text { traffic signals }\end{array}$ & & & \\
\hline $\begin{array}{l}\text { Relocate traffic signals structure } \\
\text { elements }\end{array}$ & SIGN & $\begin{array}{l}\text { Inadequate visibility of } \\
\text { traffic signals }\end{array}$ & & & \\
\hline $\begin{array}{l}\text { Removed or relocate unnecessary } \\
\text { signs "visual clutter" }\end{array}$ & SIGN & $\begin{array}{l}\text { Inadequate visibility of } \\
\text { traffic signals }\end{array}$ & & & \\
\hline Use two red signal sections & SIGN & $\begin{array}{l}\text { Inadequate visibility of } \\
\text { traffic signals }\end{array}$ & & & \\
\hline $\begin{array}{l}\text { Provide visors to shade signal lenses } \\
\text { from sunlight }\end{array}$ & SIGN & $\begin{array}{l}\text { Inadequate visibility of } \\
\text { traffic signals }\end{array}$ & & & \\
\hline $\begin{array}{l}\text { Provide adequate drainage on \#R1 } \\
\text { road }\end{array}$ & SIGN & $\begin{array}{l}\text { Poor drainage on \#R1 } \\
\text { road }\end{array}$ & & & \\
\hline Remove unwarranted signal & SIGN & $\begin{array}{l}\text { Unwarranted traffic } \\
\text { control }\end{array}$ & & & \\
\hline $\begin{array}{l}\text { Improve horizontal/vertical alignment } \\
\text { on \#A1 approach }\end{array}$ & SIGN & Angle collisions & $\begin{array}{l}\text { Left-turn collisions } \\
\text { on \#R1 road }\end{array}$ & $\begin{array}{l}\text { Poor visibility } \\
\text { of opposite } \\
\text { vehicles }\end{array}$ & $\begin{array}{l}\text { Poor horizontal/vertical } \\
\text { alignment on \#A1 approach }\end{array}$ \\
\hline $\begin{array}{l}\text { Consider indirect left-turns on \#R1 } \\
\text { road }\end{array}$ & SIGN & Angle collisions & $\begin{array}{l}\text { Left-turn collisions } \\
\text { on \#R1 road }\end{array}$ & $\begin{array}{l}\text { High turning } \\
\text { volume on } \\
\text { \#R1 road }\end{array}$ & \\
\hline
\end{tabular}




\begin{tabular}{|c|c|c|c|c|c|}
\hline $\begin{array}{l}\text { Consider indirect left-turns on \#R1 } \\
\text { road }\end{array}$ & SIGN & Angle collisions & $\begin{array}{l}\text { Left-turn collisions } \\
\text { on \#R1 road }\end{array}$ & $\begin{array}{l}\text { Poor visibility } \\
\text { of opposite } \\
\text { vehicles }\end{array}$ & $\begin{array}{l}\text { Opposite left turn vehicles on } \\
\text { \#R1 road obstruct each other } \\
\text { sight distance }\end{array}$ \\
\hline $\begin{array}{l}\text { Increase length of left-turn bay on \#A1 } \\
\text { approach }\end{array}$ & SIGN & Angle collisions & $\begin{array}{l}\text { Left-turn collisions } \\
\text { on \#R1 road }\end{array}$ & $\begin{array}{l}\text { Inadequate left } \\
\text { turn } \\
\text { channelization }\end{array}$ & \\
\hline $\begin{array}{l}\text { Increase length of right-turn bay on } \\
\text { \#A1 approach }\end{array}$ & SIGN & Angle collisions & Right-turn collisions & $\begin{array}{l}\text { Inadequate } \\
\text { right turn } \\
\text { channelization }\end{array}$ & \\
\hline $\begin{array}{l}\text { Install advance warning signs on \#A1 } \\
\text { approach }\end{array}$ & SIGN & Angle collisions & Right-turn collisions & $\begin{array}{l}\text { Inadequate sigh } \\
\text { approaching inte }\end{array}$ & $\begin{array}{l}\text { distance toward vehicles } \\
\text { rsection on \#A1 approach }\end{array}$ \\
\hline Install left-turn lane on \#A1 approach & SIGN & Angle collisions & $\begin{array}{l}\text { Left-turn collisions } \\
\text { on \#R1 road }\end{array}$ & $\begin{array}{l}\text { Inadequate left } \\
\text { turn } \\
\text { channelization }\end{array}$ & \\
\hline Install right-turn lane on \#A1 approach & SIGN & Angle collisions & Right-turn collisions & $\begin{array}{l}\text { Inadequate } \\
\text { right turn } \\
\text { channelization }\end{array}$ & \\
\hline Install right-turn lane on \#A1 approach & SIGN & Angle collisions & Right-turn collisions & $\begin{array}{l}\text { High turning } \\
\text { volume on } \\
\text { \#R1 road }\end{array}$ & \\
\hline $\begin{array}{l}\text { Provide offset for left turn lanes on \#R1 } \\
\text { road }\end{array}$ & SIGN & Angle collisions & $\begin{array}{l}\text { Left-turn collisions } \\
\text { on \#R1 road }\end{array}$ & $\begin{array}{l}\text { Inadequate left } \\
\text { turn } \\
\text { channelization }\end{array}$ & \\
\hline $\begin{array}{l}\text { Provide offset for left turn lanes on \#R1 } \\
\text { road }\end{array}$ & SIGN & Angle collisions & $\begin{array}{l}\text { Left-turn collisions } \\
\text { on \#R1 road }\end{array}$ & $\begin{array}{l}\text { Poor visibility } \\
\text { of opposite } \\
\text { vehicles }\end{array}$ & $\begin{array}{l}\text { Opposite left turn vehicles on } \\
\text { \#R1 road obstruct each other } \\
\text { sight distance }\end{array}$ \\
\hline $\begin{array}{l}\text { Provide protected left-turn phase for } \\
\text { \#R1 road }\end{array}$ & SIGN & Angle collisions & $\begin{array}{l}\text { Left-turn collisions } \\
\text { on \#R1 road }\end{array}$ & $\begin{array}{l}\text { Single left-turn } \\
\text { lane }\end{array}$ & Inadequate phase design \\
\hline $\begin{array}{l}\text { Provide protected left-turn phase for } \\
\text { \#R1 road }\end{array}$ & SIGN & Angle collisions & $\begin{array}{l}\text { Left-turn collisions } \\
\text { on \#R1 road }\end{array}$ & $\begin{array}{l}\text { Two or more } \\
\text { left-turn lanes }\end{array}$ & \\
\hline $\begin{array}{l}\text { Provide protected left-turn phase for } \\
\text { \#R1 road }\end{array}$ & SIGN & Angle collisions & $\begin{array}{l}\text { Left-turn collisions } \\
\text { on \#R1 road }\end{array}$ & $\begin{array}{l}\text { Two or more } \\
\text { left-turn lanes }\end{array}$ & Inadequate phase design \\
\hline $\begin{array}{l}\text { Provide protected/permitted phase for } \\
\text { \#R1 road }\end{array}$ & SIGN & Angle collisions & $\begin{array}{l}\text { Left-turn collisions } \\
\text { on \#R1 road }\end{array}$ & $\begin{array}{l}\text { Single left-turn } \\
\text { lane }\end{array}$ & Inadequate phase design \\
\hline Provide split phases for \#R1 road & SIGN & Angle collisions & $\begin{array}{l}\text { Left-turn collisions } \\
\text { on \#R1 road }\end{array}$ & \multicolumn{2}{|c|}{$\begin{array}{l}\text { Intersection area too small to allow } \\
\text { simultaneous left turns }\end{array}$} \\
\hline
\end{tabular}




\begin{tabular}{|c|c|c|c|c|c|}
\hline Provide split phases for \#R1 road & SIGN & Angle collisions & $\begin{array}{l}\text { Left-turn collisions } \\
\text { on \#R1 road }\end{array}$ & \multicolumn{2}{|c|}{$\begin{array}{l}\text { Intersection with unbalanced left-turn volumes } \\
\text { on opposite approaches }\end{array}$} \\
\hline Provide split phases for \#R1 road & SIGN & Angle collisions & $\begin{array}{l}\text { Left-turn collisions } \\
\text { on \#R1 road }\end{array}$ & $\begin{array}{l}\text { Poor visibility } \\
\text { of opposite } \\
\text { vehicles }\end{array}$ & $\begin{array}{l}\text { Opposite left turn vehicles on } \\
\text { \#R1 road obstruct each other } \\
\text { sight distance }\end{array}$ \\
\hline $\begin{array}{l}\text { Redesign left-turning path(s) from \#R1 } \\
\text { road }\end{array}$ & SIGN & Angle collisions & $\begin{array}{l}\text { Left-turn collisions } \\
\text { on \#R1 road }\end{array}$ & $\begin{array}{l}\text { Inadequate left } \\
\text { turn } \\
\text { channelization }\end{array}$ & \\
\hline $\begin{array}{l}\text { Redesign right-turning path(s) from } \\
\text { \#R1 road }\end{array}$ & SIGN & Angle collisions & Right-turn collisions & $\begin{array}{l}\text { Inadequate } \\
\text { right turn } \\
\text { channelization }\end{array}$ & \\
\hline Reduce speed limit on \#A1 approach & SIGN & Angle collisions & Right-turn collisions & \multicolumn{2}{|c|}{$\begin{array}{l}\text { Inadequate sight distance toward vehicles } \\
\text { approaching intersection on \#A1 approach }\end{array}$} \\
\hline $\begin{array}{l}\text { Remove or relocate median object } \\
\text { obstructing sight on \#R1 road }\end{array}$ & SIGN & Angle collisions & $\begin{array}{l}\text { Left-turn collisions } \\
\text { on \#R1 road }\end{array}$ & $\begin{array}{l}\text { Poor visibility } \\
\text { of opposite } \\
\text { vehicles }\end{array}$ & $\begin{array}{l}\text { Object located in median } \\
\text { obstructs sight distance on } \\
\text { \#R1 road }\end{array}$ \\
\hline $\begin{array}{l}\text { Remove sight obstruction on \#A1 } \\
\text { approach }\end{array}$ & SIGN & Angle collisions & Right-turn collisions & \multicolumn{2}{|c|}{$\begin{array}{l}\text { Inadequate sight distance toward vehicles } \\
\text { approaching intersection on \#A1 approach }\end{array}$} \\
\hline $\begin{array}{l}\text { Restrict left-turn maneuver on \#R1 } \\
\text { road }\end{array}$ & SIGN & Angle collisions & $\begin{array}{l}\text { Left-turn collisions } \\
\text { on \#R1 road }\end{array}$ & $\begin{array}{l}\text { High turning } \\
\text { volume on } \\
\text { \#R1 road }\end{array}$ & \\
\hline $\begin{array}{l}\text { Restrict left-turn maneuver on \#R1 } \\
\text { road }\end{array}$ & SIGN & Angle collisions & $\begin{array}{l}\text { Left-turn collisions } \\
\text { on \#R1 road }\end{array}$ & $\begin{array}{l}\text { Poor visibility } \\
\text { of opposite } \\
\text { vehicles }\end{array}$ & $\begin{array}{l}\text { Opposite left turn vehicles on } \\
\text { \#R1 road obstruct each other } \\
\text { sight distance }\end{array}$ \\
\hline $\begin{array}{l}\text { Restrict or eliminate turning maneuver } \\
\text { on \#R1 road }\end{array}$ & SIGN & Angle collisions & Right-turn collisions & $\begin{array}{l}\text { High turning } \\
\text { volume on } \\
\text { \#R1 road }\end{array}$ & \\
\hline $\begin{array}{l}\text { Restrict or eliminate turning maneuver } \\
\text { on \#R1 road }\end{array}$ & SIGN & Angle collisions & $\begin{array}{l}\text { Left-turn collisions } \\
\text { on \#R1 road }\end{array}$ & $\begin{array}{l}\text { High turning } \\
\text { volume on } \\
\text { \#R1 road }\end{array}$ & \\
\hline Restrict parking close to intersection & SIGN & Angle collisions & Right-turn collisions & $\begin{array}{l}\text { Parking cars } \\
\text { close to } \\
\text { intersection }\end{array}$ & \\
\hline Restrict parking close to intersection & SIGN & Angle collisions & Right-turn collisions & \multicolumn{2}{|c|}{$\begin{array}{l}\text { Inadequate sight distance toward vehicles } \\
\text { approaching intersection on \#A1 approach }\end{array}$} \\
\hline
\end{tabular}




\begin{tabular}{|c|c|c|c|c|c|}
\hline Improve (add) signal coordination & SIGN & $\begin{array}{l}\text { Rear-end collisions on } \\
\text { \#A1 approach }\end{array}$ & $\begin{array}{l}\text { Inadequate signal } \\
\text { coordination }\end{array}$ & & \\
\hline $\begin{array}{l}\text { Provide adequate turning markers or } \\
\text { pavement markings for turning vehicle } \\
\text { on \#R1 road }\end{array}$ & SIGN & $\begin{array}{l}\text { Rear-end collisions on } \\
\text { \#A1 approach }\end{array}$ & $\begin{array}{l}\text { Inadequate left turn } \\
\text { channelization }\end{array}$ & & \\
\hline $\begin{array}{l}\text { Redesign left-turning path(s) from \#R1 } \\
\text { road }\end{array}$ & SIGN & $\begin{array}{l}\text { Rear-end collisions on } \\
\text { \#A1 approach }\end{array}$ & $\begin{array}{l}\text { Inadequate left turn } \\
\text { channelization }\end{array}$ & & \\
\hline Install left-turn lane on \#A1 approach & SIGN & $\begin{array}{l}\text { Rear-end collisions on } \\
\text { \#A1 approach }\end{array}$ & $\begin{array}{l}\text { Inadequate left turn } \\
\text { channelization }\end{array}$ & & \\
\hline $\begin{array}{l}\text { Increase length of left-turn bay on \#A1 } \\
\text { approach }\end{array}$ & SIGN & $\begin{array}{l}\text { Rear-end collisions on } \\
\text { \#A1 approach }\end{array}$ & $\begin{array}{l}\text { Inadequate left turn } \\
\text { channelization }\end{array}$ & & \\
\hline $\begin{array}{l}\text { Increase length of left-turn bay on \#A1 } \\
\text { approach }\end{array}$ & SIGN & $\begin{array}{l}\text { Rear-end collisions on } \\
\text { \#A1 approach }\end{array}$ & $\begin{array}{l}\text { Inadequate left turn } \\
\text { channelization }\end{array}$ & & \\
\hline $\begin{array}{l}\text { Provide offset for left turn lanes on \#R1 } \\
\text { road }\end{array}$ & SIGN & $\begin{array}{l}\text { Rear-end collisions on } \\
\text { \#A1 approach }\end{array}$ & $\begin{array}{l}\text { Inadequate left turn } \\
\text { channelization }\end{array}$ & & \\
\hline Improve visibility of the splitter-island & SIGN & $\begin{array}{l}\text { Rear-end collisions on } \\
\text { \#A1 approach }\end{array}$ & $\begin{array}{l}\text { Inadequate left turn } \\
\text { channelization }\end{array}$ & & \\
\hline $\begin{array}{l}\text { Increase length of right-turn bay on } \\
\text { \#A1 approach }\end{array}$ & SIGN & $\begin{array}{l}\text { Rear-end collisions on } \\
\text { \#A1 approach }\end{array}$ & $\begin{array}{l}\text { Inadequate left turn } \\
\text { channelization }\end{array}$ & & \\
\hline $\begin{array}{l}\text { Increase length of right-turn bay on } \\
\text { \#A1 approach }\end{array}$ & SIGN & $\begin{array}{l}\text { Rear-end collisions on } \\
\text { \#A1 approach }\end{array}$ & $\begin{array}{l}\text { Inadequate left turn } \\
\text { channelization }\end{array}$ & & \\
\hline $\begin{array}{l}\text { Provide adequate delineation for right- } \\
\text { turns at intersection (markers or lines) }\end{array}$ & SIGN & $\begin{array}{l}\text { Rear-end collisions on } \\
\text { \#A1 approach }\end{array}$ & $\begin{array}{l}\text { Inadequate left turn } \\
\text { channelization }\end{array}$ & & \\
\hline Install right-turn lane on \#A1 approach & SIGN & $\begin{array}{l}\text { Rear-end collisions on } \\
\text { \#A1 approach }\end{array}$ & $\begin{array}{l}\text { Inadequate left turn } \\
\text { channelization }\end{array}$ & & \\
\hline $\begin{array}{l}\text { Adjust minimum green or extension } \\
\text { time }\end{array}$ & SIGN & $\begin{array}{l}\text { Rear-end collisions on } \\
\text { \#A1 approach }\end{array}$ & $\begin{array}{l}\text { Inadequate traffic } \\
\text { signal timing or } \\
\text { type of signal }\end{array}$ & & \\
\hline Adjust phase time and cycle time & SIGN & $\begin{array}{l}\text { Rear-end collisions on } \\
\text { \#A1 approach }\end{array}$ & $\begin{array}{l}\text { Inadequate traffic } \\
\text { signal timing or } \\
\text { type of signal }\end{array}$ & & \\
\hline Adjust yellow change interval & SIGN & $\begin{array}{l}\text { Rear-end collisions on } \\
\text { \#A1 approach }\end{array}$ & $\begin{array}{l}\text { Inadequate traffic } \\
\text { signal timing or } \\
\text { type of signal }\end{array}$ & & \\
\hline Install multi-dial controller & SIGN & $\begin{array}{l}\text { Rear-end collisions on } \\
\text { \#A1 approach }\end{array}$ & $\begin{array}{l}\text { Inadequate traffic } \\
\text { signal timing or }\end{array}$ & & \\
\hline
\end{tabular}




\begin{tabular}{|c|c|c|c|c|c|}
\hline & & & type of signal & & \\
\hline Provide all-red clearance interval & SIGN & $\begin{array}{l}\text { Rear-end collisions on } \\
\text { \#A1 approach }\end{array}$ & $\begin{array}{l}\text { Inadequate traffic } \\
\text { signal timing or } \\
\text { type of signal }\end{array}$ & & \\
\hline Improve (add) signal coordination & SIGN & $\begin{array}{l}\text { Rear-end collisions on } \\
\text { \#A1 approach }\end{array}$ & $\begin{array}{l}\text { Inadequate traffic } \\
\text { signal timing or } \\
\text { type of signal }\end{array}$ & & \\
\hline $\begin{array}{l}\text { Remove late night/early morning } \\
\text { flashing operation }\end{array}$ & SIGN & $\begin{array}{l}\text { Rear-end collisions on } \\
\text { \#A1 approach }\end{array}$ & $\begin{array}{l}\text { Inadequate traffic } \\
\text { signal timing or } \\
\text { type of signal }\end{array}$ & & \\
\hline $\begin{array}{l}\text { Increase crosswalk setback on \#A1 } \\
\text { approach }\end{array}$ & SIGN & $\begin{array}{l}\text { Rear-end collisions on } \\
\text { \#A1 approach }\end{array}$ & $\begin{array}{l}\text { Pedestrians may } \\
\text { block \#E2 exit }\end{array}$ & & \\
\hline Prohibit right turn on red & SIGN & $\begin{array}{l}\text { Rear-end collisions on } \\
\text { \#A1 approach }\end{array}$ & $\begin{array}{l}\text { Pedestrians may } \\
\text { block \#E2 exit }\end{array}$ & & \\
\hline Relocate transit stop & SIGN & $\begin{array}{l}\text { Rear-end collisions on } \\
\text { \#A1 approach }\end{array}$ & $\begin{array}{l}\text { Inadequate location } \\
\text { of transit stop }\end{array}$ & & \\
\hline $\begin{array}{l}\text { Improve channelization/delineation on } \\
\text { \#A1 approach }\end{array}$ & SIGN & $\begin{array}{l}\text { Head-on collisions on } \\
\text { \#A1 approach }\end{array}$ & $\begin{array}{l}\text { Inadequate } \\
\text { channelization on } \\
\text { \#A1 approach }\end{array}$ & & \\
\hline Create one-way streets & SIGN & $\begin{array}{l}\text { Head-on collisions on } \\
\text { \#A1 approach }\end{array}$ & $\begin{array}{l}\text { Roadway design } \mathrm{NO} \\
\text { traffic conditions }\end{array}$ & adequate for & \\
\hline $\begin{array}{l}\text { Improve horizontal/vertical alignment } \\
\text { on \#R1 road }\end{array}$ & SIGN & $\begin{array}{l}\text { Head-on collisions on } \\
\text { \#A1 approach }\end{array}$ & $\begin{array}{l}\text { Poor } \\
\text { horizontal/vertical } \\
\text { alignment on \#R1 } \\
\text { road }\end{array}$ & & \\
\hline Install/improve center line marking & SIGN & $\begin{array}{l}\text { Head-on collisions on } \\
\text { \#A1 approach }\end{array}$ & $\begin{array}{l}\text { Inadequate center } \\
\text { line marking }\end{array}$ & & \\
\hline Restrict parking close to intersection & SIGN & $\begin{array}{l}\text { Head-on collisions on } \\
\text { \#A1 approach }\end{array}$ & $\begin{array}{l}\text { Parking cars close } \\
\text { to intersection }\end{array}$ & & \\
\hline Optimize clearance intervals & SIGN & $\begin{array}{l}\text { Right-angle collisions } \\
\text { between \#A1 and \#A2 } \\
\text { vehicles }\end{array}$ & Red-light running & $\begin{array}{l}\text { Inadequate } \\
\text { clearance } \\
\text { interval }\end{array}$ & \\
\hline $\begin{array}{l}\text { Implement automated enforcement of } \\
\text { red-light running (cameras) }\end{array}$ & SIGN & $\begin{array}{l}\text { Right-angle collisions } \\
\text { between \#A1 and \#A2 }\end{array}$ & Red-light running & & \\
\hline
\end{tabular}




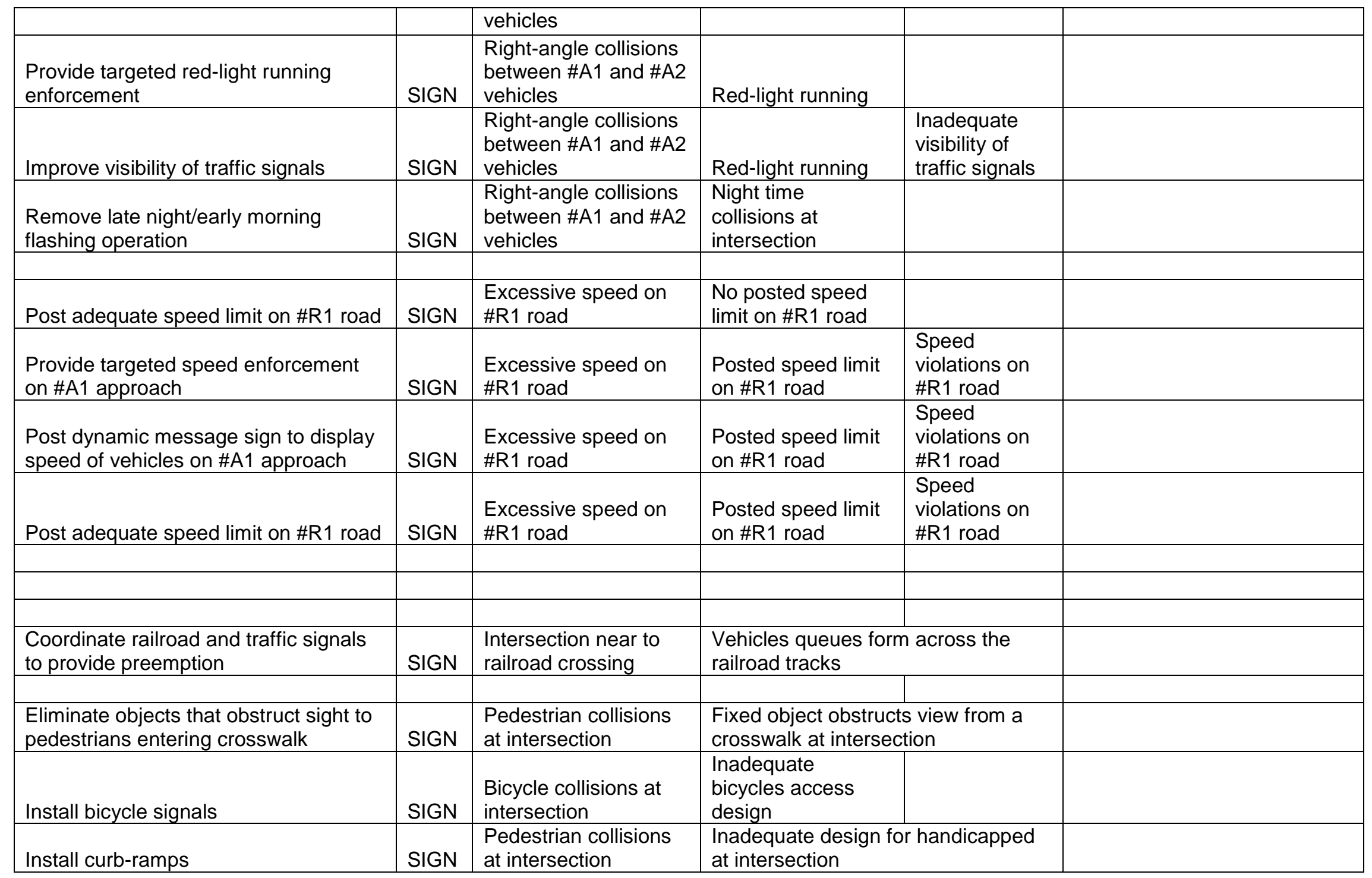




\begin{tabular}{|c|c|c|c|c|c|}
\hline $\begin{array}{l}\text { Install flashing beacons on \#A1 } \\
\text { approach }\end{array}$ & SIGN & $\begin{array}{l}\text { Pedestrian collisions } \\
\text { at intersection }\end{array}$ & $\begin{array}{l}\text { School zone } \\
\text { present }\end{array}$ & \multicolumn{2}{|c|}{ Insufficient school zone warning on \#R1 road } \\
\hline Install pedestrian actuated signals & SIGN & $\begin{array}{l}\text { Pedestrian collisions } \\
\text { at intersection }\end{array}$ & \multicolumn{2}{|c|}{$\begin{array}{l}\text { Signifficant number of pedestrian } \\
\text { crossing intersection }\end{array}$} & \\
\hline $\begin{array}{l}\text { Install pedestrian overpass or } \\
\text { underpass }\end{array}$ & SIGN & $\begin{array}{l}\text { Pedestrian collisions } \\
\text { at intersection }\end{array}$ & \multicolumn{2}{|c|}{$\begin{array}{l}\text { Inadequate protection for } \\
\text { pedestrians at intersection }\end{array}$} & \\
\hline $\begin{array}{l}\text { Install physical barriers to restrict } \\
\text { pedestrian crossing maneuvers at } \\
\text { higher-risk locations }\end{array}$ & SIGN & $\begin{array}{l}\text { Pedestrian collisions } \\
\text { at intersection }\end{array}$ & \multicolumn{2}{|c|}{$\begin{array}{l}\text { Inadequate protection for } \\
\text { pedestrians at intersection }\end{array}$} & \\
\hline Install raised crosswalks & SIGN & $\begin{array}{l}\text { Pedestrian collisions } \\
\text { at intersection }\end{array}$ & \multicolumn{2}{|c|}{$\begin{array}{l}\text { Inadequate visibility of crosswalk(s) } \\
\text { at intersection }\end{array}$} & \\
\hline $\begin{array}{l}\text { Install school crossing sign on \#R1 } \\
\text { road }\end{array}$ & SIGN & $\begin{array}{l}\text { Pedestrian collisions } \\
\text { at intersection }\end{array}$ & $\begin{array}{l}\text { School zone } \\
\text { present }\end{array}$ & \multirow{2}{*}{$\begin{array}{l}\text { Insufficient sch } \\
\text { of pedestrians }\end{array}$} & ol zone warning on \#R1 road \\
\hline $\begin{array}{l}\text { Increase sidewalk setback on \#R1 } \\
\text { road }\end{array}$ & SIGN & $\begin{array}{l}\text { Pedestrian collisions } \\
\text { at intersection }\end{array}$ & $\begin{array}{l}\text { Inadequate protection of pedestrians } \\
\text { on \#R1 road }\end{array}$ & & \\
\hline Install sidewalks & SIGN & $\begin{array}{l}\text { Pedestrian collisions } \\
\text { at intersection }\end{array}$ & $\begin{array}{l}\text { No sidewalk } \\
\text { present }\end{array}$ & & \\
\hline Install street lighting & SIGN & $\begin{array}{l}\text { Pedestrian collisions } \\
\text { at intersection }\end{array}$ & $\begin{array}{l}\text { Inadequate lighting } \\
\text { of crosswalk at } \\
\text { intersection }\end{array}$ & & \\
\hline Install street lighting & SIGN & $\begin{array}{l}\text { Pedestrian collisions } \\
\text { at intersection }\end{array}$ & $\begin{array}{l}\text { School zone } \\
\text { present }\end{array}$ & Inadequate ligh & ing of crosswalk at intersection \\
\hline $\begin{array}{l}\text { Install/improve signing or marking of } \\
\text { bicycles at intersection }\end{array}$ & SIGN & $\begin{array}{l}\text { Bicycle collisions at } \\
\text { intersection }\end{array}$ & \multicolumn{2}{|c|}{$\begin{array}{l}\text { Inadequate design for high turning } \\
\text { traffic on \#A1 approach }\end{array}$} & \\
\hline $\begin{array}{l}\text { Install/improve signing or marking of } \\
\text { pedestrian crosswalks at intersection }\end{array}$ & SIGN & $\begin{array}{l}\text { Pedestrian collisions } \\
\text { at intersection }\end{array}$ & \multicolumn{2}{|c|}{$\begin{array}{l}\text { Inadequate visibility of crosswalk(s) } \\
\text { at intersection }\end{array}$} & \\
\hline Optimize clearance intervals & SIGN & $\begin{array}{l}\text { Pedestrian collisions } \\
\text { at intersection }\end{array}$ & $\begin{array}{l}\text { Inadequate } \\
\text { clearance interval }\end{array}$ & & \\
\hline Prohibit right turn on red & SIGN & $\begin{array}{l}\text { Pedestrian collisions } \\
\text { at intersection }\end{array}$ & \multicolumn{2}{|c|}{$\begin{array}{l}\text { Signifficant number of pedestrian } \\
\text { crossing intersection }\end{array}$} & \\
\hline $\begin{array}{l}\text { Provide adequate drainage on \#R1 } \\
\text { road }\end{array}$ & SIGN & $\begin{array}{l}\text { Bicycle collisions at } \\
\text { intersection }\end{array}$ & $\begin{array}{l}\text { Inadequate gates } \\
\text { visibility }\end{array}$ & & \\
\hline $\begin{array}{l}\text { Provide adequate drainage on \#R1 } \\
\text { road }\end{array}$ & SIGN & $\begin{array}{l}\text { Pedestrian collisions } \\
\text { at intersection }\end{array}$ & $\begin{array}{l}\text { Poor drainage on } \\
\text { \#R1 road }\end{array}$ & & \\
\hline $\begin{array}{l}\text { Provide adequate signals for } \\
\text { handicapped }\end{array}$ & SIGN & $\begin{array}{l}\text { Pedestrian collisions } \\
\text { at intersection }\end{array}$ & \multicolumn{2}{|c|}{$\begin{array}{l}\text { Inadequate design for handicapped } \\
\text { at intersection }\end{array}$} & \\
\hline
\end{tabular}




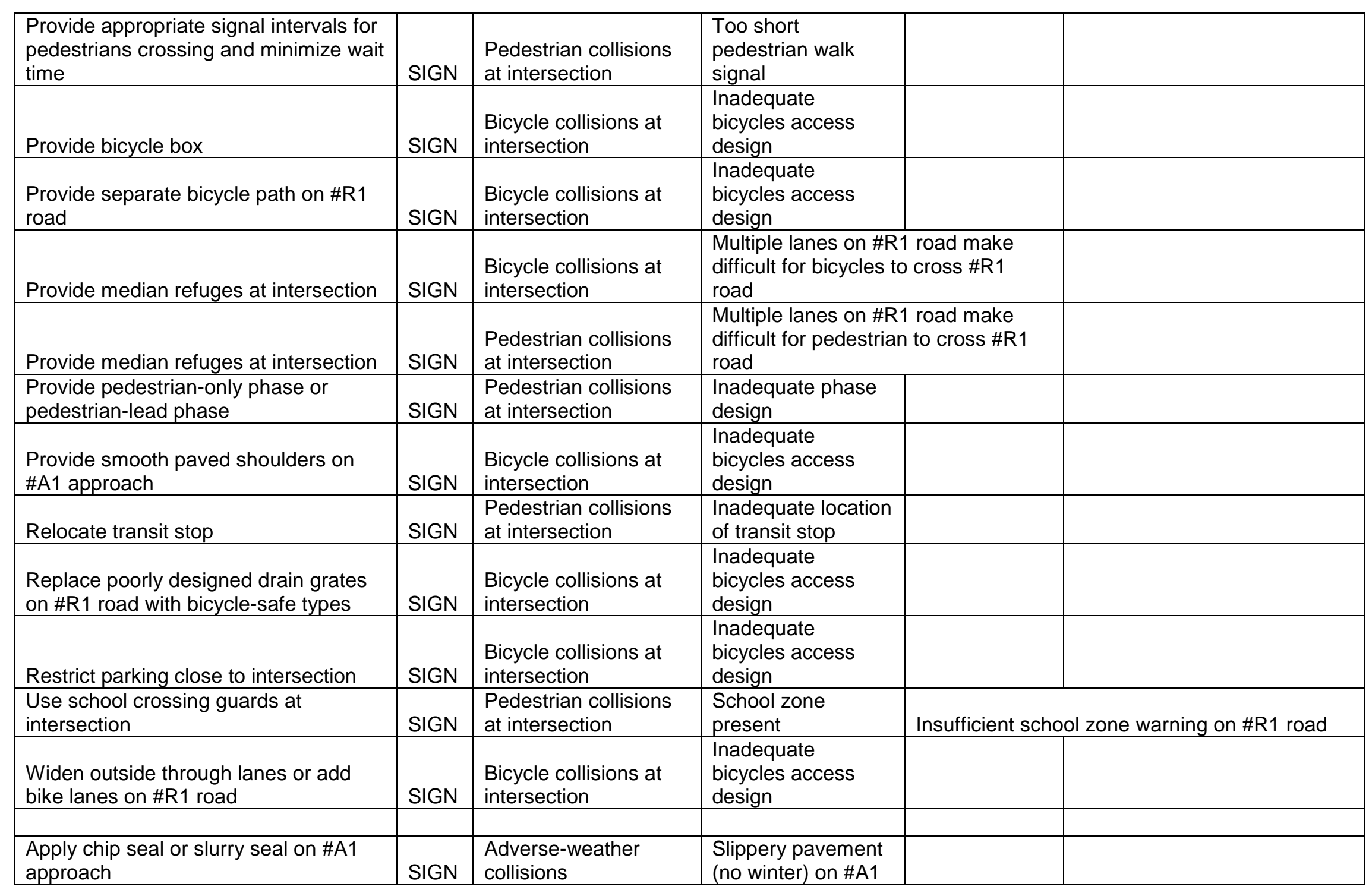




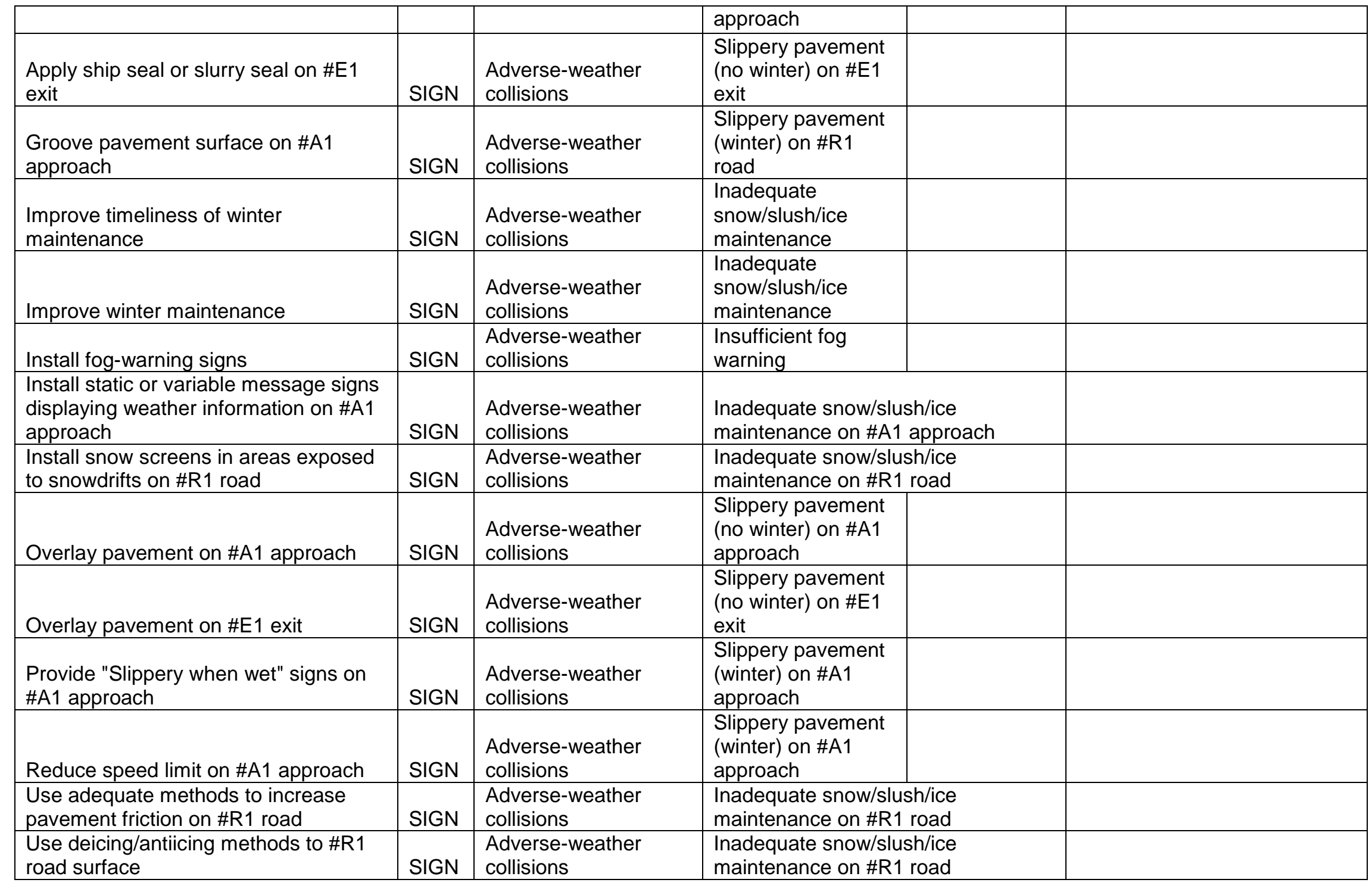




\begin{tabular}{|c|c|c|c|c|c|}
\hline $\begin{array}{l}\text { Provide adequate delineation and } \\
\text { channelization at intersection for } \\
\text { left/right-turning trucks }\end{array}$ & SIGN & $\begin{array}{l}\text { Collisions involving } \\
\text { large vehicles }\end{array}$ & $\begin{array}{l}\text { Inadequate left turn } \\
\text { channelization/deline } \\
\text { trucks }\end{array}$ & tion for turning & \\
\hline Increase clearance intervals & SIGN & $\begin{array}{l}\text { Collisions involving } \\
\text { large vehicles }\end{array}$ & $\begin{array}{l}\text { Inadequate } \\
\text { clearance interval }\end{array}$ & & \\
\hline $\begin{array}{l}\text { Provide additional pavement to } \\
\text { accommodate turning trucks }\end{array}$ & SIGN & $\begin{array}{l}\text { Collisions involving } \\
\text { large vehicles }\end{array}$ & $\begin{array}{l}\text { Pavement width } \\
\text { inadequate for } \\
\text { turning trucks }\end{array}$ & & \\
\hline Improve intersection skew angle & SIGN & $\begin{array}{l}\text { Collisions involving } \\
\text { large vehicles }\end{array}$ & $\begin{array}{l}\text { Inadequate } \\
\text { intersection skew } \\
\text { angle }\end{array}$ & & \\
\hline Improve street lighting & SIGN & $\begin{array}{l}\text { Night time collisions at } \\
\text { intersection }\end{array}$ & $\begin{array}{l}\text { Inadequate street } \\
\text { lights at } \\
\text { intersection }\end{array}$ & & \\
\hline $\begin{array}{l}\text { Improve/install reflective signs on \#R1 } \\
\text { road }\end{array}$ & SIGN & $\begin{array}{l}\text { Night time collisions at } \\
\text { intersection }\end{array}$ & $\begin{array}{l}\text { Poor signs } \\
\text { reflectivity on \#R1 } \\
\text { road }\end{array}$ & & \\
\hline $\begin{array}{l}\text { Improve/install reflectorized pavement } \\
\text { markers on \#R1 approach }\end{array}$ & SIGN & $\begin{array}{l}\text { Night time collisions at } \\
\text { intersection }\end{array}$ & $\begin{array}{l}\text { Inadequate street } \\
\text { lights on \#R1 road }\end{array}$ & & \\
\hline Install street lights at intersection & SIGN & $\begin{array}{l}\text { Night time collisions at } \\
\text { intersection }\end{array}$ & $\begin{array}{l}\text { Inadequate street } \\
\text { lights at } \\
\text { intersection }\end{array}$ & & \\
\hline $\begin{array}{l}\text { Remove distracting commercial lighting } \\
\text { or other source of glare at intersection }\end{array}$ & SIGN & $\begin{array}{l}\text { Night time collisions at } \\
\text { intersection }\end{array}$ & $\begin{array}{l}\text { Inadequate street } \\
\text { lights at } \\
\text { intersection }\end{array}$ & & \\
\hline Upgrade signing on \#R1 road & SIGN & $\begin{array}{l}\text { Night time collisions at } \\
\text { intersection }\end{array}$ & $\begin{array}{l}\text { Poor signs visibility } \\
\text { on \#R1 road }\end{array}$ & & \\
\hline Employ emergency vehicle preemption & SIGN & $\begin{array}{l}\text { Inadequate } \\
\text { emergency vehicle } \\
\text { preemption }\end{array}$ & & & \\
\hline $\begin{array}{l}\text { Convert a four-leg intersection into two } \\
\text { T-intersections }\end{array}$ & SIGN & $\begin{array}{l}\text { Very high crash } \\
\text { frequency }\end{array}$ & & & \\
\hline
\end{tabular}




\begin{tabular}{|l|l|l|l|l|l|}
\hline $\begin{array}{l}\text { Convert two T-intersections into one } \\
\text { four-leg intersection }\end{array}$ & SIGN & $\begin{array}{l}\text { Very high crash } \\
\text { frequency }\end{array}$ & & \\
\hline $\begin{array}{l}\text { Remove deflection in through-vehicle } \\
\text { travel path }\end{array}$ & SIGN & $\begin{array}{l}\text { Very high crash } \\
\text { frequency }\end{array}$ & & & \\
\hline $\begin{array}{l}\text { Close intersection leg or convert to } \\
\text { one-way street }\end{array}$ & SIGN & $\begin{array}{l}\text { Very high crash } \\
\text { frequency }\end{array}$ & & & \\
\hline Convert intersection into roundabout & SIGN & $\begin{array}{l}\text { Very high crash } \\
\text { frequency }\end{array}$ & & & \\
\hline $\begin{array}{l}\text { Convert two two-way streets into a pair } \\
\text { of one-way streets }\end{array}$ & SIGN & $\begin{array}{l}\text { Very high crash } \\
\text { frequency }\end{array}$ & & & \\
\hline Construct interchange & SIGN & $\begin{array}{l}\text { Very high crash } \\
\text { frequency }\end{array}$ & & & \\
\hline
\end{tabular}




\section{Road Segment}

\begin{tabular}{|c|c|c|c|c|c|}
\hline Close or relocate driveway(s) on \#R1 road & RS & $\begin{array}{l}\text { Driveway(s) } \\
\text { collisions on } \\
\text { \#R1 road }\end{array}$ & $\begin{array}{l}\text { Inadequate visibility } \\
\text { of driveway(s) on \#R1 } \\
\text { road }\end{array}$ & \multicolumn{2}{|c|}{$\begin{array}{l}\text { Driveway(s) design inadequate for high traffic } \\
\text { on \#R1 road }\end{array}$} \\
\hline Channelize driveway(s) on \#R1 road & RS & $\begin{array}{l}\text { Driveway(s) } \\
\text { collisions on } \\
\text { \#R1 road }\end{array}$ & $\begin{array}{l}\text { Inadequate visibility } \\
\text { of driveway(s) on \#R1 } \\
\text { road }\end{array}$ & \multicolumn{2}{|c|}{$\begin{array}{l}\text { Driveway(s) design inadequate for high traffic } \\
\text { on \#R1 road }\end{array}$} \\
\hline $\begin{array}{l}\text { Install curbing to define driveway(s) location } \\
\text { on \#R1 road }\end{array}$ & RS & $\begin{array}{l}\text { Driveway(s) } \\
\text { collisions on } \\
\text { \#R1 road }\end{array}$ & $\begin{array}{l}\text { Inadequate visibility } \\
\text { of driveway(s) on \#R1 } \\
\text { road }\end{array}$ & \multicolumn{2}{|c|}{$\begin{array}{l}\text { Driveway(s) design inadequate for high traffic } \\
\text { on \#R1 road }\end{array}$} \\
\hline $\begin{array}{l}\text { Install median divider on \#R1 road to prevent } \\
\text { left-turns from/to driveway(s) }\end{array}$ & RS & $\begin{array}{l}\text { Driveway(s) } \\
\text { collisions on } \\
\text { \#R1 road }\end{array}$ & $\begin{array}{l}\text { Inadequate visibility } \\
\text { of driveway(s) on \#R1 } \\
\text { road }\end{array}$ & \multicolumn{2}{|c|}{$\begin{array}{l}\text { Driveway(s) design inadequate for high traffic } \\
\text { on \#R1 road }\end{array}$} \\
\hline $\begin{array}{l}\text { Provide acceleration/deceleration lanes on } \\
\text { \#R1 road }\end{array}$ & RS & $\begin{array}{l}\text { Driveway(s) } \\
\text { collisions on } \\
\text { \#R1 road }\end{array}$ & $\begin{array}{l}\text { Inadequate visibility } \\
\text { of driveway(s) on \#R1 } \\
\text { road }\end{array}$ & \multicolumn{2}{|c|}{$\begin{array}{l}\text { Driveway(s) design inadequate for high traffic } \\
\text { on \#R1 road }\end{array}$} \\
\hline Relocate driveway(s) from \#R1 road & RS & $\begin{array}{l}\text { Driveway(s) } \\
\text { collisions on } \\
\text { \#R1 road }\end{array}$ & $\begin{array}{l}\text { Inadequate visibility } \\
\text { of driveway(s) on \#R1 } \\
\text { road }\end{array}$ & \multicolumn{2}{|c|}{$\begin{array}{l}\text { Driveway(s) design inadequate for high traffic } \\
\text { on \#R1 road }\end{array}$} \\
\hline Remove sight obstruction on \#R1 road & RS & $\begin{array}{l}\text { Driveway(s) } \\
\text { collisions on } \\
\text { \#R1 road }\end{array}$ & \multicolumn{2}{|c|}{ Inadequate visibility of driveway(s) on \#R1 road } & \\
\hline $\begin{array}{l}\text { Restrict left-turning at access point on \#R1 } \\
\text { road }\end{array}$ & RS & $\begin{array}{l}\text { Driveway(s) } \\
\text { collisions on } \\
\text { \#R1 road }\end{array}$ & $\begin{array}{l}\text { Inadequate visibility } \\
\text { of driveway(s) on \#R1 } \\
\text { road }\end{array}$ & \multicolumn{2}{|c|}{$\begin{array}{l}\text { Driveway(s) design inadequate for high traffic } \\
\text { on \#R1 road }\end{array}$} \\
\hline $\begin{array}{l}\text { Restrict parking near driveway(s) on \#R1 } \\
\text { road }\end{array}$ & RS & $\begin{array}{l}\text { Driveway(s) } \\
\text { collisions on } \\
\text { \#R1 road } \\
\end{array}$ & \multicolumn{2}{|c|}{ Inadequate visibility of driveway(s) on \#R1 road } & \\
\hline Improve street lighting & RS & $\begin{array}{l}\text { Night time } \\
\text { collisions on } \\
\text { \#R1 road }\end{array}$ & $\begin{array}{l}\text { Inadequate street } \\
\text { lights on \#R1 road }\end{array}$ & & \\
\hline Improve/install reflective signs on \#R1 road & RS & $\begin{array}{l}\text { Night time } \\
\text { collisions on } \\
\text { \#R1 road }\end{array}$ & $\begin{array}{l}\text { Poor signs reflectivity } \\
\text { on \#R1 road }\end{array}$ & & \\
\hline
\end{tabular}




\begin{tabular}{|c|c|c|c|c|}
\hline $\begin{array}{l}\text { Improve/install reflectorized pavement } \\
\text { markers on \#R1 approach }\end{array}$ & RS & $\begin{array}{l}\text { Night time } \\
\text { collisions on } \\
\text { \#R1 road }\end{array}$ & $\begin{array}{l}\text { Inadequate street } \\
\text { lights on \#R1 road }\end{array}$ & \\
\hline Install street lights on \#R1 road & RS & $\begin{array}{l}\text { Night time } \\
\text { collisions on } \\
\text { \#R1 road }\end{array}$ & $\begin{array}{l}\text { Inadequate street } \\
\text { lights on \#R1 road }\end{array}$ & \\
\hline $\begin{array}{l}\text { Remove distracting commercial lighting or } \\
\text { other source of glare on \#R1 road }\end{array}$ & RS & $\begin{array}{l}\text { Night time } \\
\text { collisions on } \\
\text { \#R1 road }\end{array}$ & $\begin{array}{l}\text { Inadequate street } \\
\text { lights on \#R1 road }\end{array}$ & \\
\hline $\begin{array}{l}\text { Construct pedestrian refuge islands and/or } \\
\text { raised medians }\end{array}$ & RS & $\begin{array}{l}\text { Pedestrian } \\
\text { collisions on } \\
\text { \#R1 road }\end{array}$ & Crossing point & Inadequate design of existing crosswalk \\
\hline $\begin{array}{l}\text { Eliminate objects that obstruct sight distance } \\
\text { on \#R1 road }\end{array}$ & RS & $\begin{array}{l}\text { Pedestrian } \\
\text { collisions on } \\
\text { \#R1 road }\end{array}$ & Crossing point & Inadequate design of existing crosswalk \\
\hline $\begin{array}{l}\text { Eliminate objects that obstruct sight distance } \\
\text { on \#R1 road }\end{array}$ & RS & $\begin{array}{l}\text { Pedestrian } \\
\text { collisions on } \\
\text { \#R1 road }\end{array}$ & $\begin{array}{l}\text { Inadequate visibility } \\
\text { of pedestrians }\end{array}$ & \\
\hline $\begin{array}{l}\text { Implement lighting and/or illumination } \\
\text { measures on \#R1 road }\end{array}$ & RS & $\begin{array}{l}\text { Pedestrian } \\
\text { collisions on } \\
\text { \#R1 road }\end{array}$ & $\begin{array}{l}\text { Inadequate visibility } \\
\text { of pedestrians }\end{array}$ & \\
\hline Consider traffic calming on \#R1 road & RS & $\begin{array}{l}\text { Pedestrian } \\
\text { collisions on } \\
\text { \#R1 road }\end{array}$ & Crossing point & Inadequate design of existing crosswalk \\
\hline 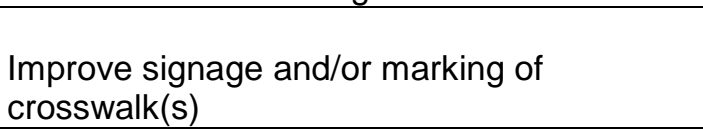 & RS & $\begin{array}{l}\text { Pedestrian } \\
\text { collisions on } \\
\text { \#R1 road }\end{array}$ & Crossing point & Inadequate design of existing crosswalk \\
\hline Install "Cross only at Crosswalk" signs & RS & $\begin{array}{l}\text { Pedestrian } \\
\text { collisions on } \\
\text { \#R1 road }\end{array}$ & $\begin{array}{l}\text { Pedestrians walking } \\
\text { along road }\end{array}$ & \\
\hline Install pedestrian overpass or underpass & RS & $\begin{array}{l}\text { Pedestrian } \\
\text { collisions on } \\
\text { \#R1 road }\end{array}$ & Crossing point & Inadequate design of existing crosswalk \\
\hline Install curb-ramps & RS & $\begin{array}{l}\text { Pedestrian } \\
\text { collisions on }\end{array}$ & Crossing point & Inadequate design of existing crosswalk \\
\hline
\end{tabular}




\begin{tabular}{|c|c|c|c|c|c|}
\hline & & \#R1 road & & & \\
\hline Install street lighting & RS & $\begin{array}{l}\text { Pedestrian } \\
\text { collisions on } \\
\text { \#R1 road } \\
\end{array}$ & Crossing point & \multicolumn{2}{|c|}{ Inadequate design of existing crosswalk } \\
\hline Install pedestrian overpass or underpass & RS & $\begin{array}{l}\text { Pedestrian } \\
\text { collisions on } \\
\text { \#R1 road }\end{array}$ & $\begin{array}{l}\text { Pedestrians walking } \\
\text { along road }\end{array}$ & & \\
\hline Install pedestrian actuated signals & RS & $\begin{array}{l}\text { Pedestrian } \\
\text { collisions on } \\
\text { \#R1 road } \\
\end{array}$ & Crossing point & \multicolumn{2}{|c|}{ Long distance between crosswalks on \#R1 road } \\
\hline Install pedestrian barriers & RS & $\begin{array}{l}\text { Pedestrian } \\
\text { collisions on } \\
\text { \#R1 road }\end{array}$ & $\begin{array}{l}\text { Pedestrians walking } \\
\text { along road }\end{array}$ & & \\
\hline Install pedestrian crosswalks & RS & $\begin{array}{l}\text { Pedestrian } \\
\text { collisions on } \\
\text { \#R1 road } \\
\end{array}$ & Crossing point & \multicolumn{2}{|c|}{ Long distance between crosswalks on \#R1 road } \\
\hline Install pedestrian crosswalks & RS & $\begin{array}{l}\text { Pedestrian } \\
\text { collisions on } \\
\text { \#R1 road } \\
\end{array}$ & \multicolumn{2}{|c|}{ Long distance between crosswalks on \#R1 road } & \\
\hline Install raised crosswalks & RS & $\begin{array}{l}\text { Pedestrian } \\
\text { collisions on } \\
\text { \#R1 road }\end{array}$ & Crossing point & \multicolumn{2}{|c|}{ Inadequate design of existing crosswalk } \\
\hline Install school crossing sign on \#R1 road & RS & $\begin{array}{l}\text { Pedestrian } \\
\text { collisions on } \\
\text { \#R1 road } \\
\end{array}$ & Crossing point & School zone present & $\begin{array}{l}\text { Insufficient school } \\
\text { zone warning on } \\
\text { \#R1 road }\end{array}$ \\
\hline Install school zone markings on \#R1 road & RS & $\begin{array}{l}\text { Pedestrian } \\
\text { collisions on } \\
\text { \#R1 road } \\
\end{array}$ & Crossing point & School zone present & $\begin{array}{l}\text { Insufficient school } \\
\text { zone warning on } \\
\text { \#R1 road }\end{array}$ \\
\hline Increase sidewalk setback on \#R1 road & RS & $\begin{array}{l}\text { Pedestrian } \\
\text { collisions on } \\
\text { \#R1 road } \\
\end{array}$ & Crossing point & \multicolumn{2}{|c|}{ Inadequate design of existing crosswalk } \\
\hline Install sidewalks & RS & $\begin{array}{l}\text { Pedestrian } \\
\text { collisions on } \\
\text { \#R1 road } \\
\end{array}$ & $\begin{array}{l}\text { Pedestrians walking } \\
\text { along road }\end{array}$ & & \\
\hline Post adequate speed limit on \#R1 road & RS & $\begin{array}{l}\text { Pedestrian } \\
\text { collisions on }\end{array}$ & Crossing point & School zone present & $\begin{array}{l}\text { Insufficient school } \\
\text { zone warning on }\end{array}$ \\
\hline
\end{tabular}




\begin{tabular}{|c|c|c|c|c|c|}
\hline & & \#R1 road & & & \#R1 road \\
\hline Provide separate bicycle path on \#R1 road & RS & $\begin{array}{l}\text { Bicycle } \\
\text { collisions on } \\
\text { \#R1 road }\end{array}$ & $\begin{array}{l}\text { Inadequate bicycles } \\
\text { access design }\end{array}$ & & \\
\hline Provide median refuges on \#R1 road & RS & $\begin{array}{l}\text { Bicycle } \\
\text { collisions on } \\
\text { \#R1 road }\end{array}$ & $\begin{array}{l}\text { Inadequate bicycles } \\
\text { access design }\end{array}$ & & \\
\hline $\begin{array}{l}\text { Provide smooth paved shoulders on \#R1 } \\
\text { road }\end{array}$ & RS & $\begin{array}{l}\text { Bicycle } \\
\text { collisions on } \\
\text { \#R1 road }\end{array}$ & $\begin{array}{l}\text { Inadequate bicycles } \\
\text { access design }\end{array}$ & & \\
\hline $\begin{array}{l}\text { Replace poorly designed drain grates on \#R1 } \\
\text { road with bicycle-safe types }\end{array}$ & RS & $\begin{array}{l}\text { Bicycle } \\
\text { collisions on } \\
\text { \#R1 road }\end{array}$ & $\begin{array}{l}\text { Inadequate bicycles } \\
\text { access design }\end{array}$ & & \\
\hline $\begin{array}{l}\text { Install signals to alert motorists that } \\
\text { pedestrians are crossing at crossing point }\end{array}$ & RS & $\begin{array}{l}\text { Pedestrian } \\
\text { collisions on } \\
\text { \#R1 road }\end{array}$ & Crossing point & Inadequate design of exi & ing crosswalk \\
\hline Use school crossing guards at crossing point & RS & $\begin{array}{l}\text { Pedestrian } \\
\text { collisions on } \\
\text { \#R1 road }\end{array}$ & Crossing point & School zone present & $\begin{array}{l}\text { Insufficient school } \\
\text { zone warning on } \\
\text { \#R1 road }\end{array}$ \\
\hline $\begin{array}{l}\text { Widen outside through lanes or add bike } \\
\text { lanes on \#R1 road }\end{array}$ & RS & $\begin{array}{l}\text { Bicycle } \\
\text { collisions on } \\
\text { \#R1 road }\end{array}$ & $\begin{array}{l}\text { Inadequate bicycles } \\
\text { access design }\end{array}$ & & \\
\hline Apply ship seal or slurry seal on \#R1 road & RS & $\begin{array}{l}\text { Adverse- } \\
\text { weather } \\
\text { collisions }\end{array}$ & $\begin{array}{l}\text { Slippery pavement } \\
\text { (winter) on \#R1 road }\end{array}$ & & \\
\hline Groove pavement surface on \#R1 road & RS & $\begin{array}{l}\text { Adverse- } \\
\text { weather } \\
\text { collisions }\end{array}$ & $\begin{array}{l}\text { Slippery pavement } \\
\text { (winter) on \#R1 road }\end{array}$ & & \\
\hline Improve winter maintenance & RS & $\begin{array}{l}\text { Adverse- } \\
\text { weather } \\
\text { collisions }\end{array}$ & \multicolumn{2}{|c|}{$\begin{array}{l}\text { Inadequate snow/slush/ice maintenance on \#R1 } \\
\text { road }\end{array}$} & \\
\hline Improve timeliness of winter maintenance & RS & $\begin{array}{l}\text { Adverse- } \\
\text { weather } \\
\text { collisions }\end{array}$ & \multicolumn{2}{|c|}{$\begin{array}{l}\text { Inadequate snow/slush/ice maintenance on \#R1 } \\
\text { road }\end{array}$} & \\
\hline Install fog-warning signs & RS & Adverse- & Insufficient fog & & \\
\hline
\end{tabular}




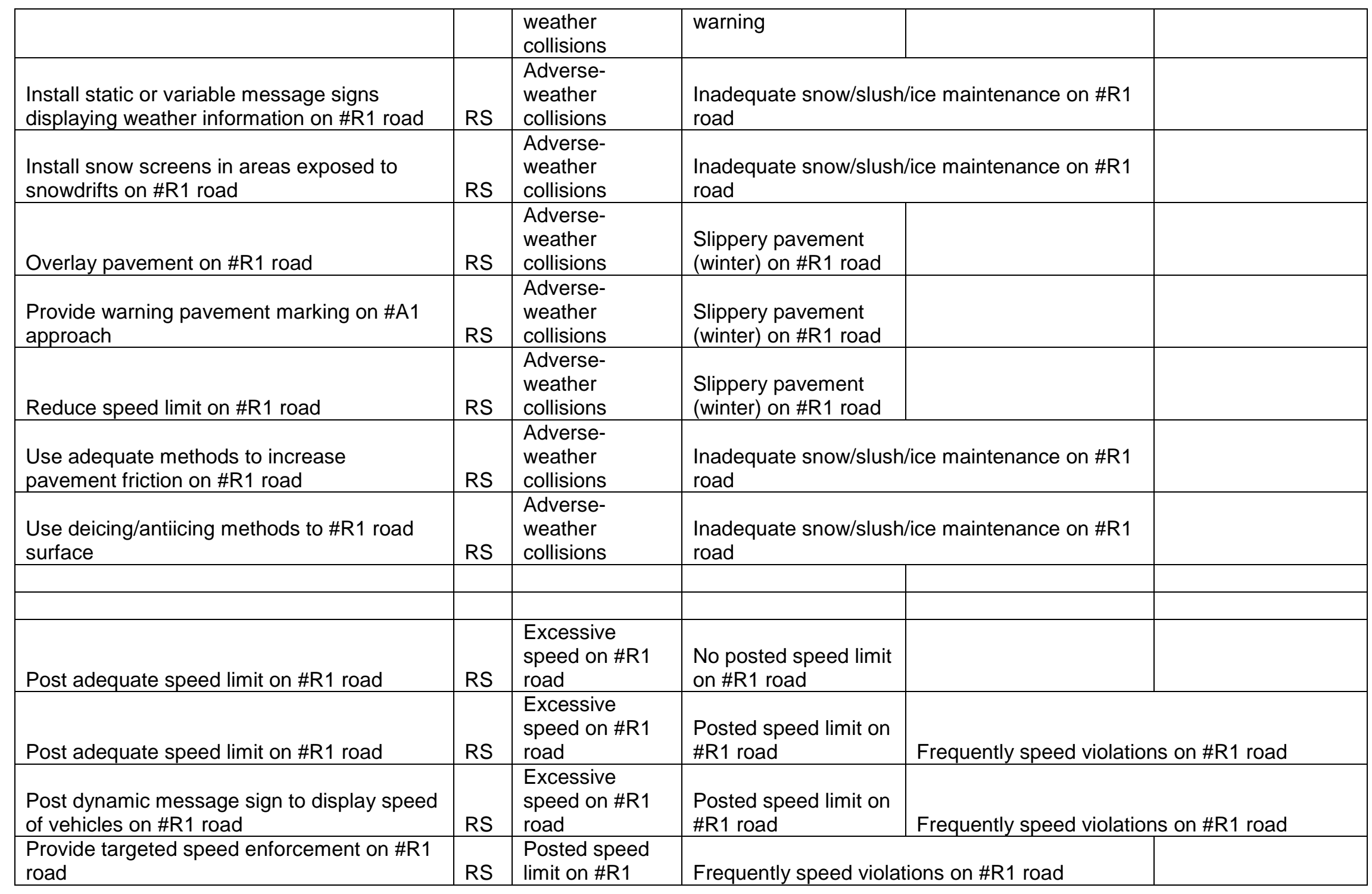




\begin{tabular}{|c|c|c|c|c|c|}
\hline & & road & & & \\
\hline Add special signing & RS & $\begin{array}{l}\text { Off-road } \\
\text { collisions on } \\
\text { \#R1 road } \\
\end{array}$ & Horizontal curve & $\begin{array}{l}\text { Fixed object close to } \\
\text { traveled way on \#R1 } \\
\text { road }\end{array}$ & $\begin{array}{l}\text { Insufficient bridge } \\
\text { visibility }\end{array}$ \\
\hline Install barrier curb or guardrail on \#R1 road & RS & $\begin{array}{l}\text { Off-road } \\
\text { collisions on } \\
\text { \#R1 road }\end{array}$ & Horizontal curve & $\begin{array}{l}\text { Fixed object close to } \\
\text { traveled way on \#R1 } \\
\text { road }\end{array}$ & $\begin{array}{l}\text { Insufficient bridge } \\
\text { visibility }\end{array}$ \\
\hline Increase clear zone on \#R1 road & RS & $\begin{array}{l}\text { Off-road } \\
\text { collisions on } \\
\text { \#R1 road } \\
\end{array}$ & Horizontal curve & $\begin{array}{l}\text { Fixed object close to } \\
\text { traveled way on \#R1 } \\
\text { road }\end{array}$ & $\begin{array}{l}\text { Insufficient bridge } \\
\text { visibility }\end{array}$ \\
\hline Add special signing & RS & $\begin{array}{l}\text { Off-road } \\
\text { collisions on } \\
\text { \#R1 road } \\
\end{array}$ & Vertical curve & $\begin{array}{l}\text { Fixed object close to } \\
\text { traveled way on \#R1 } \\
\text { road }\end{array}$ & $\begin{array}{l}\text { Insufficient bridge } \\
\text { visibility }\end{array}$ \\
\hline Install barrier curb or guardrail on \#R1 road & RS & $\begin{array}{l}\text { Off-road } \\
\text { collisions on } \\
\text { \#R1 road } \\
\end{array}$ & Vertical curve & $\begin{array}{l}\text { Fixed object close to } \\
\text { traveled way on \#R1 } \\
\text { road }\end{array}$ & $\begin{array}{l}\text { Insufficient bridge } \\
\text { visibility }\end{array}$ \\
\hline Increase clear zone on \#R1 road & RS & $\begin{array}{l}\text { Off-road } \\
\text { collisions on } \\
\text { \#R1 road } \\
\end{array}$ & Vertical curve & $\begin{array}{l}\text { Fixed object close to } \\
\text { traveled way on \#R1 } \\
\text { road }\end{array}$ & $\begin{array}{l}\text { Insufficient bridge } \\
\text { visibility }\end{array}$ \\
\hline $\begin{array}{l}\text { Provide breakaway and crashworthy mail } \\
\text { boxes }\end{array}$ & RS & $\begin{array}{l}\text { Off-road } \\
\text { collisions on } \\
\text { \#R1 road } \\
\end{array}$ & Horizontal curve & $\begin{array}{l}\text { Fixed object close to } \\
\text { traveled way on \#R1 } \\
\text { road }\end{array}$ & $\begin{array}{l}\text { Mail boxes close to } \\
\text { traveled way }\end{array}$ \\
\hline Remove or relocate mail box on \#R1 road & RS & $\begin{array}{l}\text { Off-road } \\
\text { collisions on } \\
\text { \#R1 road } \\
\end{array}$ & Horizontal curve & $\begin{array}{l}\text { Fixed object close to } \\
\text { traveled way on \#R1 } \\
\text { road }\end{array}$ & $\begin{array}{l}\text { Mail boxes close to } \\
\text { traveled way }\end{array}$ \\
\hline $\begin{array}{l}\text { Provide breakaway and crashworthy mail } \\
\text { boxes }\end{array}$ & RS & $\begin{array}{l}\text { Off-road } \\
\text { collisions on } \\
\text { \#R1 road } \\
\end{array}$ & Vertical curve & $\begin{array}{l}\text { Fixed object close to } \\
\text { traveled way on \#R1 } \\
\text { road }\end{array}$ & $\begin{array}{l}\text { Mail boxes close to } \\
\text { traveled way }\end{array}$ \\
\hline Remove or relocate mail box on \#R1 road & RS & $\begin{array}{l}\text { Off-road } \\
\text { collisions on } \\
\text { \#R1 road } \\
\end{array}$ & Vertical curve & $\begin{array}{l}\text { Fixed object close to } \\
\text { traveled way on \#R1 } \\
\text { road }\end{array}$ & $\begin{array}{l}\text { Mail boxes close to } \\
\text { traveled way }\end{array}$ \\
\hline $\begin{array}{l}\text { Provide breakaway and crashworthy sign } \\
\text { supports }\end{array}$ & RS & $\begin{array}{l}\text { Off-road } \\
\text { collisions on } \\
\text { \#R1 road }\end{array}$ & Horizontal curve & $\begin{array}{l}\text { Fixed object close to } \\
\text { traveled way on \#R1 } \\
\text { road }\end{array}$ & $\begin{array}{l}\text { Sign supports close } \\
\text { to traveled way }\end{array}$ \\
\hline Remove or relocate sign & RS & Off-road & Horizontal curve & Fixed object close to & Sign supports close \\
\hline
\end{tabular}




\begin{tabular}{|c|c|c|c|c|c|}
\hline & & $\begin{array}{l}\text { collisions on } \\
\text { \#R1 road }\end{array}$ & & $\begin{array}{l}\text { traveled way on \#R1 } \\
\text { road }\end{array}$ & to traveled way \\
\hline $\begin{array}{l}\text { Provide breakaway and crashworthy sign } \\
\text { supports }\end{array}$ & RS & $\begin{array}{l}\text { Off-road } \\
\text { collisions on } \\
\text { \#R1 road }\end{array}$ & Vertical curve & $\begin{array}{l}\text { Fixed object close to } \\
\text { traveled way on \#R1 } \\
\text { road }\end{array}$ & $\begin{array}{l}\text { Sign supports close } \\
\text { to traveled way }\end{array}$ \\
\hline Remove or relocate sign & RS & $\begin{array}{l}\text { Off-road } \\
\text { collisions on } \\
\text { \#R1 road } \\
\end{array}$ & Vertical curve & $\begin{array}{l}\text { Fixed object close to } \\
\text { traveled way on \#R1 } \\
\text { road }\end{array}$ & $\begin{array}{l}\text { Sign supports close } \\
\text { to traveled way }\end{array}$ \\
\hline Add special signing & RS & $\begin{array}{l}\text { Off-road } \\
\text { collisions on } \\
\text { \#R1 road }\end{array}$ & Horizontal curve & $\begin{array}{l}\text { Fixed object close to } \\
\text { traveled way on \#R1 } \\
\text { road }\end{array}$ & $\begin{array}{l}\text { Trees close to } \\
\text { traveled way }\end{array}$ \\
\hline Install barrier curb or guardrail on \#R1 road & RS & $\begin{array}{l}\text { Off-road } \\
\text { collisions on } \\
\text { \#R1 road }\end{array}$ & Horizontal curve & $\begin{array}{l}\text { Fixed object close to } \\
\text { traveled way on \#R1 } \\
\text { road }\end{array}$ & $\begin{array}{l}\text { Trees close to } \\
\text { traveled way }\end{array}$ \\
\hline Install tree marker & RS & $\begin{array}{l}\text { Off-road } \\
\text { collisions on } \\
\text { \#R1 road } \\
\end{array}$ & Horizontal curve & $\begin{array}{l}\text { Fixed object close to } \\
\text { traveled way on \#R1 } \\
\text { road }\end{array}$ & $\begin{array}{l}\text { Trees close to } \\
\text { traveled way }\end{array}$ \\
\hline Increase clear zone on \#R1 road & RS & $\begin{array}{l}\text { Off-road } \\
\text { collisions on } \\
\text { \#R1 road }\end{array}$ & Horizontal curve & $\begin{array}{l}\text { Fixed object close to } \\
\text { traveled way on \#R1 } \\
\text { road }\end{array}$ & $\begin{array}{l}\text { Trees close to } \\
\text { traveled way }\end{array}$ \\
\hline Remove or relocate trees along \#R1 road & RS & $\begin{array}{l}\text { Off-road } \\
\text { collisions on } \\
\text { \#R1 road }\end{array}$ & Horizontal curve & $\begin{array}{l}\text { Fixed object close to } \\
\text { traveled way on \#R1 } \\
\text { road }\end{array}$ & $\begin{array}{l}\text { Trees close to } \\
\text { traveled way }\end{array}$ \\
\hline Add special signing & RS & $\begin{array}{l}\text { Off-road } \\
\text { collisions on } \\
\text { \#R1 road }\end{array}$ & Vertical curve & $\begin{array}{l}\text { Fixed object close to } \\
\text { traveled way on \#R1 } \\
\text { road }\end{array}$ & $\begin{array}{l}\text { Trees close to } \\
\text { traveled way }\end{array}$ \\
\hline Install barrier curb or guardrail on \#R1 road & RS & $\begin{array}{l}\text { Off-road } \\
\text { collisions on } \\
\text { \#R1 road } \\
\end{array}$ & Vertical curve & $\begin{array}{l}\text { Fixed object close to } \\
\text { traveled way on \#R1 } \\
\text { road }\end{array}$ & $\begin{array}{l}\text { Trees close to } \\
\text { traveled way }\end{array}$ \\
\hline Install tree marker & RS & $\begin{array}{l}\text { Off-road } \\
\text { collisions on } \\
\text { \#R1 road }\end{array}$ & Vertical curve & $\begin{array}{l}\text { Fixed object close to } \\
\text { traveled way on \#R1 } \\
\text { road }\end{array}$ & $\begin{array}{l}\text { Trees close to } \\
\text { traveled way }\end{array}$ \\
\hline Increase clear zone on \#R1 road & RS & $\begin{array}{l}\text { Off-road } \\
\text { collisions on } \\
\text { \#R1 road }\end{array}$ & Vertical curve & $\begin{array}{l}\text { Fixed object close to } \\
\text { traveled way on \#R1 } \\
\text { road }\end{array}$ & $\begin{array}{l}\text { Trees close to } \\
\text { traveled way }\end{array}$ \\
\hline Remove or relocate trees along \#R1 road & RS & Off-road & Vertical curve & Fixed object close to & Trees close to \\
\hline
\end{tabular}




\begin{tabular}{|c|c|c|c|c|c|}
\hline & & $\begin{array}{l}\text { collisions on } \\
\text { \#R1 road }\end{array}$ & & $\begin{array}{l}\text { traveled way on \#R1 } \\
\text { road }\end{array}$ & traveled way \\
\hline Add special signing & RS & $\begin{array}{l}\text { Off-road } \\
\text { collisions on } \\
\text { \#R1 road }\end{array}$ & Horizontal curve & $\begin{array}{l}\text { Fixed object close to } \\
\text { traveled way on \#R1 } \\
\text { road }\end{array}$ & $\begin{array}{l}\text { Utility poles close to } \\
\text { traveled way }\end{array}$ \\
\hline Install barrier curb or guardrail on \#R1 road & RS & $\begin{array}{l}\text { Off-road } \\
\text { collisions on } \\
\text { \#R1 road } \\
\end{array}$ & Horizontal curve & $\begin{array}{l}\text { Fixed object close to } \\
\text { traveled way on \#R1 } \\
\text { road }\end{array}$ & $\begin{array}{l}\text { Utility poles close to } \\
\text { traveled way }\end{array}$ \\
\hline Install utility polet marker & RS & $\begin{array}{l}\text { Off-road } \\
\text { collisions on } \\
\text { \#R1 road }\end{array}$ & Horizontal curve & $\begin{array}{l}\text { Fixed object close to } \\
\text { traveled way on \#R1 } \\
\text { road }\end{array}$ & $\begin{array}{l}\text { Utility poles close to } \\
\text { traveled way }\end{array}$ \\
\hline Place utilities underground & RS & $\begin{array}{l}\text { Off-road } \\
\text { collisions on } \\
\text { \#R1 road } \\
\end{array}$ & Horizontal curve & $\begin{array}{l}\text { Fixed object close to } \\
\text { traveled way on \#R1 } \\
\text { road }\end{array}$ & $\begin{array}{l}\text { Utility poles close to } \\
\text { traveled way }\end{array}$ \\
\hline $\begin{array}{l}\text { Remove or relocate utility pole along \#R1 } \\
\text { road }\end{array}$ & RS & $\begin{array}{l}\text { Off-road } \\
\text { collisions on } \\
\text { \#R1 road } \\
\end{array}$ & Horizontal curve & $\begin{array}{l}\text { Fixed object close to } \\
\text { traveled way on \#R1 } \\
\text { road }\end{array}$ & $\begin{array}{l}\text { Utility poles close to } \\
\text { traveled way }\end{array}$ \\
\hline Use breakaway devices on \#R1 road & RS & $\begin{array}{l}\text { Off-road } \\
\text { collisions on } \\
\text { \#R1 road }\end{array}$ & Horizontal curve & $\begin{array}{l}\text { Fixed object close to } \\
\text { traveled way on \#R1 } \\
\text { road }\end{array}$ & $\begin{array}{l}\text { Utility poles close to } \\
\text { traveled way }\end{array}$ \\
\hline Add special signing & RS & $\begin{array}{l}\text { Off-road } \\
\text { collisions on } \\
\text { \#R1 road }\end{array}$ & Vertical curve & $\begin{array}{l}\text { Fixed object close to } \\
\text { traveled way on \#R1 } \\
\text { road }\end{array}$ & $\begin{array}{l}\text { Utility poles close to } \\
\text { traveled way }\end{array}$ \\
\hline Install barrier curb or guardrail on \#R1 road & RS & $\begin{array}{l}\text { Off-road } \\
\text { collisions on } \\
\text { \#R1 road } \\
\end{array}$ & Vertical curve & $\begin{array}{l}\text { Fixed object close to } \\
\text { traveled way on \#R1 } \\
\text { road }\end{array}$ & $\begin{array}{l}\text { Utility poles close to } \\
\text { traveled way }\end{array}$ \\
\hline Install utility polet marker & RS & $\begin{array}{l}\text { Off-road } \\
\text { collisions on } \\
\text { \#R1 road } \\
\end{array}$ & Vertical curve & $\begin{array}{l}\text { Fixed object close to } \\
\text { traveled way on \#R1 } \\
\text { road }\end{array}$ & $\begin{array}{l}\text { Utility poles close to } \\
\text { traveled way }\end{array}$ \\
\hline Place utilities underground & RS & $\begin{array}{l}\text { Off-road } \\
\text { collisions on } \\
\text { \#R1 road }\end{array}$ & Vertical curve & $\begin{array}{l}\text { Fixed object close to } \\
\text { traveled way on \#R1 } \\
\text { road }\end{array}$ & $\begin{array}{l}\text { Utility poles close to } \\
\text { traveled way }\end{array}$ \\
\hline $\begin{array}{l}\text { Remove or relocate utility pole along \#R1 } \\
\text { road }\end{array}$ & RS & $\begin{array}{l}\text { Off-road } \\
\text { collisions on } \\
\text { \#R1 road }\end{array}$ & Vertical curve & $\begin{array}{l}\text { Fixed object close to } \\
\text { traveled way on \#R1 } \\
\text { road }\end{array}$ & $\begin{array}{l}\text { Utility poles close to } \\
\text { traveled way }\end{array}$ \\
\hline Use breakaway devices on \#R1 road & RS & Off-road & Vertical curve & Fixed object close to & Utility poles close to \\
\hline
\end{tabular}




\begin{tabular}{|c|c|c|c|c|c|}
\hline & & $\begin{array}{l}\text { collisions on } \\
\text { \#R1 road }\end{array}$ & & $\begin{array}{l}\text { traveled way on \#R1 } \\
\text { road }\end{array}$ & traveled way \\
\hline $\begin{array}{l}\text { Change horizontal/vertical alignment on \#R1 } \\
\text { road }\end{array}$ & RS & $\begin{array}{l}\text { Off-road } \\
\text { collisions on } \\
\text { \#R1 road }\end{array}$ & Vertical curve & \multicolumn{2}{|c|}{ Inadequate design of vertical curve } \\
\hline Eliminate compound curves & RS & $\begin{array}{l}\text { Off-road } \\
\text { collisions on } \\
\text { \#R1 road }\end{array}$ & Horizontal curve & \multicolumn{2}{|c|}{ Inadequate design of horizontal curve } \\
\hline Improve or restore superelevation & RS & $\begin{array}{l}\text { Off-road } \\
\text { collisions on } \\
\text { \#R1 road }\end{array}$ & Horizontal curve & \multicolumn{2}{|c|}{ Inadequate design of horizontal curve } \\
\hline $\begin{array}{l}\text { Improve horizontal/vertical alignment on \#R1 } \\
\text { road }\end{array}$ & RS & $\begin{array}{l}\text { Off-road } \\
\text { collisions on } \\
\text { \#R1 road }\end{array}$ & Horizontal curve & \multicolumn{2}{|c|}{ Inadequate design of horizontal curve } \\
\hline Provide spiral transition curves & RS & $\begin{array}{l}\text { Off-road } \\
\text { collisions on } \\
\text { \#R1 road }\end{array}$ & Horizontal curve & \multicolumn{2}{|c|}{ Inadequate design of horizontal curve } \\
\hline Widen lanes on \#R1 road to adequate width & RS & $\begin{array}{l}\text { Off-road } \\
\text { collisions on } \\
\text { \#R1 road }\end{array}$ & Horizontal curve & \multicolumn{2}{|c|}{ Inadequate lane width on \#R1 road } \\
\hline Install centerline rumble strips & RS & $\begin{array}{l}\text { Off-road } \\
\text { collisions on } \\
\text { \#R1 road }\end{array}$ & Horizontal curve & \multicolumn{2}{|c|}{ Inadequate lane width on \#R1 road } \\
\hline $\begin{array}{l}\text { Install shoulder rumble strips on \#A1 } \\
\text { approach }\end{array}$ & RS & $\begin{array}{l}\text { Off-road } \\
\text { collisions on } \\
\text { \#R1 road }\end{array}$ & Horizontal curve & \multicolumn{2}{|c|}{ Inadequate lane width on \#R1 road } \\
\hline Widen lanes on \#R1 road to adequate width & RS & $\begin{array}{l}\text { Off-road } \\
\text { collisions on } \\
\text { \#R1 road }\end{array}$ & Vertical curve & \multicolumn{2}{|c|}{ Inadequate lane width on \#R1 road } \\
\hline Install centerline rumble strips & RS & $\begin{array}{l}\text { Off-road } \\
\text { collisions on } \\
\text { \#R1 road }\end{array}$ & Vertical curve & \multicolumn{2}{|c|}{ Inadequate lane width on \#R1 road } \\
\hline $\begin{array}{l}\text { Install shoulder rumble strips on \#A1 } \\
\text { approach }\end{array}$ & RS & $\begin{array}{l}\text { Off-road } \\
\text { collisions on } \\
\text { \#R1 road }\end{array}$ & Vertical curve & \multicolumn{2}{|c|}{ Inadequate lane width on \#R1 road } \\
\hline Cut or trim trees and other roadside & RS & Off-road & Horizontal curve & \multicolumn{2}{|c|}{ Inadequate sight distance on horizontal curve } \\
\hline
\end{tabular}




\begin{tabular}{|c|c|c|c|c|}
\hline vegetation that obstruct sight distance & & $\begin{array}{l}\text { collisions on } \\
\text { \#R1 road }\end{array}$ & & \\
\hline Remove sight obstruction on \#R1 road & RS & $\begin{array}{l}\text { Off-road } \\
\text { collisions on } \\
\text { \#R1 road }\end{array}$ & Horizontal curve & Inadequate sight distance on horizontal curve \\
\hline Restrict parking & RS & $\begin{array}{l}\text { Off-road } \\
\text { collisions on } \\
\text { \#R1 road } \\
\end{array}$ & Horizontal curve & Inadequate sight distance on horizontal curve \\
\hline Add special signing & RS & $\begin{array}{l}\text { Off-road } \\
\text { collisions on } \\
\text { \#R1 road }\end{array}$ & $\begin{array}{l}\text { Fixed object close to } \\
\text { traveled way on \#R1 } \\
\text { road }\end{array}$ & Insufficient bridge visibility \\
\hline Install barrier curb or guardrail on \#R1 road & RS & $\begin{array}{l}\text { Off-road } \\
\text { collisions on } \\
\text { \#R1 road }\end{array}$ & $\begin{array}{l}\text { Fixed object close to } \\
\text { traveled way on \#R1 } \\
\text { road }\end{array}$ & Insufficient bridge visibility \\
\hline Increase clear zone on \#R1 road & RS & $\begin{array}{l}\text { Off-road } \\
\text { collisions on } \\
\text { \#R1 road } \\
\end{array}$ & $\begin{array}{l}\text { Fixed object close to } \\
\text { traveled way on \#R1 } \\
\text { road }\end{array}$ & Insufficient bridge visibility \\
\hline $\begin{array}{l}\text { Improve pavement marking along the curve } \\
\text { on \#R1 road }\end{array}$ & RS & $\begin{array}{l}\text { Off-road } \\
\text { collisions on } \\
\text { \#R1 road }\end{array}$ & Horizontal curve & Insufficient delineation along the curve \\
\hline Install in-pavement luminaires & RS & $\begin{array}{l}\text { Off-road } \\
\text { collisions on } \\
\text { \#R1 road }\end{array}$ & Horizontal curve & Insufficient delineation along the curve \\
\hline Install LED barrier-mounted guidance tubes & RS & $\begin{array}{l}\text { Off-road } \\
\text { collisions on } \\
\text { \#R1 road }\end{array}$ & Horizontal curve & Insufficient delineation along the curve \\
\hline Install light-emitting diode (LED) & $\mathrm{RS}$ & $\begin{array}{l}\text { Off-road } \\
\text { collisions on } \\
\text { \#R1 road }\end{array}$ & Horizontal curve & Insufficient delineation along the curve \\
\hline $\begin{array}{l}\text { Delineate guardrail located outside the } \\
\text { roadway curve }\end{array}$ & RS & $\begin{array}{l}\text { Off-road } \\
\text { collisions on } \\
\text { \#R1 road }\end{array}$ & Horizontal curve & Insufficient delineation along the curve \\
\hline $\begin{array}{l}\text { Provide pavement marking (stop } \\
\text { sign/chevron/etc.) on \#R1 road }\end{array}$ & RS & $\begin{array}{l}\text { Off-road } \\
\text { collisions on } \\
\text { \#R1 road } \\
\end{array}$ & Horizontal curve & Insufficient delineation along the curve \\
\hline Install post-mounted delineators & RS & Off-road & Horizontal curve & Insufficient delineation along the curve \\
\hline
\end{tabular}




\begin{tabular}{|c|c|c|c|c|}
\hline & & $\begin{array}{l}\text { collisions on } \\
\text { \#R1 road }\end{array}$ & & \\
\hline $\begin{array}{l}\text { Improve pavement marking along the curve } \\
\text { on \#R1 road }\end{array}$ & RS & $\begin{array}{l}\text { Off-road } \\
\text { collisions on } \\
\text { \#R1 road }\end{array}$ & Horizontal curve & Insufficient delineation along the curve \\
\hline Install advisor speed sign & RS & $\begin{array}{l}\text { Off-road } \\
\text { collisions on } \\
\text { \#R1 road }\end{array}$ & Horizontal curve & Insufficient warning \\
\hline Install centerline rumble strips & RS & $\begin{array}{l}\text { Off-road } \\
\text { collisions on } \\
\text { \#R1 road }\end{array}$ & Horizontal curve & Insufficient warning \\
\hline Install flashing beacons on \#R1 road & RS & $\begin{array}{l}\text { Off-road } \\
\text { collisions on } \\
\text { \#R1 road }\end{array}$ & Horizontal curve & Insufficient warning \\
\hline $\begin{array}{l}\text { Install advance warning signs of } \\
\text { horizontal/vertical curve on \#R1 road }\end{array}$ & RS & $\begin{array}{l}\text { Off-road } \\
\text { collisions on } \\
\text { \#R1 road }\end{array}$ & Horizontal curve & Insufficient warning \\
\hline Install street lighting & RS & $\begin{array}{l}\text { Off-road } \\
\text { collisions on } \\
\text { \#R1 road }\end{array}$ & Horizontal curve & Insufficient warning \\
\hline $\begin{array}{l}\text { Install shoulder rumble strips on \#A1 } \\
\text { approach }\end{array}$ & RS & $\begin{array}{l}\text { Off-road } \\
\text { collisions on } \\
\text { \#R1 road }\end{array}$ & Horizontal curve & Insufficient warning \\
\hline Install advance warning signs on \#R1 road & RS & $\begin{array}{l}\text { Off-road } \\
\text { collisions on } \\
\text { \#R1 road }\end{array}$ & Horizontal curve & Insufficient warning \\
\hline $\begin{array}{l}\text { Provide curve dynamic warning system on } \\
\text { \#R1 road }\end{array}$ & RS & $\begin{array}{l}\text { Off-road } \\
\text { collisions on } \\
\text { \#R1 road } \\
\end{array}$ & Horizontal curve & Insufficient warning \\
\hline Install rumble strips on \#A1 approach & RS & $\begin{array}{l}\text { Off-road } \\
\text { collisions on } \\
\text { \#R1 road }\end{array}$ & Horizontal curve & Insufficient warning \\
\hline Install advisor speed sign & RS & $\begin{array}{l}\text { Off-road } \\
\text { collisions on } \\
\text { \#R1 road }\end{array}$ & Vertical curve & Insufficient warning \\
\hline Install flashing beacons on \#R1 road & RS & Off-road & Vertical curve & Insufficient warning \\
\hline
\end{tabular}




\begin{tabular}{|c|c|c|c|c|}
\hline & & $\begin{array}{l}\text { collisions on } \\
\text { \#R1 road }\end{array}$ & & \\
\hline Install street lighting & RS & $\begin{array}{l}\text { Off-road } \\
\text { collisions on } \\
\text { \#R1 road } \\
\end{array}$ & Vertical curve & Insufficient warning \\
\hline Install advance warning signs on \#R1 road & RS & $\begin{array}{l}\text { Off-road } \\
\text { collisions on } \\
\text { \#R1 road }\end{array}$ & Vertical curve & Insufficient warning \\
\hline $\begin{array}{l}\text { Install advance warning signs of } \\
\text { horizontal/vertical curve on \#R1 road }\end{array}$ & RS & $\begin{array}{l}\text { Off-road } \\
\text { collisions on } \\
\text { \#R1 road } \\
\end{array}$ & Vertical curve & Insufficient warning \\
\hline Install rumble strips on \#A1 approach & RS & $\begin{array}{l}\text { Off-road } \\
\text { collisions on } \\
\text { \#R1 road } \\
\end{array}$ & Vertical curve & Insufficient warning \\
\hline $\begin{array}{l}\text { Provide advance warning of unexpected } \\
\text { changes in horizontal alignment }\end{array}$ & RS & $\begin{array}{l}\text { Off-road } \\
\text { collisions on } \\
\text { \#R1 road }\end{array}$ & Horizontal curve & Insufficient warning \\
\hline $\begin{array}{l}\text { Provide breakaway and crashworthy mail } \\
\text { boxes }\end{array}$ & RS & $\begin{array}{l}\text { Off-road } \\
\text { collisions on } \\
\text { \#R1 road }\end{array}$ & $\begin{array}{l}\text { Fixed object close to } \\
\text { traveled way on \#R1 } \\
\text { road }\end{array}$ & Mail boxes close to traveled way \\
\hline Remove or relocate mail box on \#R1 road & RS & $\begin{array}{l}\text { Off-road } \\
\text { collisions on } \\
\text { \#R1 road }\end{array}$ & $\begin{array}{l}\text { Fixed object close to } \\
\text { traveled way on \#R1 } \\
\text { road }\end{array}$ & Mail boxes close to traveled way \\
\hline $\begin{array}{l}\text { Provide/Improve pavement surface (improve } \\
\text { transitions/drop offs/fixed } \\
\text { potholes/rutting/etc) on \#R1 road }\end{array}$ & RS & $\begin{array}{l}\text { Off-road } \\
\text { collisions on } \\
\text { \#R1 road } \\
\end{array}$ & Horizontal curve & Pavement defects on \#R1 road \\
\hline $\begin{array}{l}\text { Provide/Improve pavement surface (improve } \\
\text { transitions/drop offs/fixed } \\
\text { potholes/rutting/etc) on \#R1 road }\end{array}$ & RS & $\begin{array}{l}\text { Off-road } \\
\text { collisions on } \\
\text { \#R1 road } \\
\end{array}$ & Vertical curve & Pavement defects on \#R1 road \\
\hline Provide adequate drainage on \#R1 road & RS & $\begin{array}{l}\text { Off-road } \\
\text { collisions on } \\
\text { \#R1 road }\end{array}$ & Horizontal curve & Poorly designed drain grates on \#R1 road \\
\hline Provide adequate drainage on \#R1 road & RS & $\begin{array}{l}\text { Off-road } \\
\text { collisions on } \\
\text { \#R1 road }\end{array}$ & Vertical curve & Poorly designed drain grates on \#R1 road \\
\hline Increase skid resistance on \#R1 road & RS & Off-road & Horizontal curve & Poor skid resistance on \#R1 road \\
\hline
\end{tabular}




\begin{tabular}{|c|c|c|c|c|}
\hline & & $\begin{array}{l}\text { collisions on } \\
\text { \#R1 road }\end{array}$ & & \\
\hline Groove pavement surface on \#R1 road & RS & $\begin{array}{l}\text { Off-road } \\
\text { collisions on } \\
\text { \#R1 road } \\
\end{array}$ & Horizontal curve & Poor skid resistance on \#R1 road \\
\hline Increase skid resistance on \#R1 road & RS & $\begin{array}{l}\text { Off-road } \\
\text { collisions on } \\
\text { \#R1 road }\end{array}$ & Vertical curve & Poor skid resistance on \#R1 road \\
\hline Groove pavement surface on \#R1 road & RS & $\begin{array}{l}\text { Off-road } \\
\text { collisions on } \\
\text { \#R1 road }\end{array}$ & Vertical curve & Poor skid resistance on \#R1 road \\
\hline $\begin{array}{l}\text { Provide breakaway and crashworthy sign } \\
\text { supports }\end{array}$ & RS & $\begin{array}{l}\text { Off-road } \\
\text { collisions on } \\
\text { \#R1 road }\end{array}$ & $\begin{array}{l}\text { Fixed object close to } \\
\text { traveled way on \#R1 } \\
\text { road }\end{array}$ & Sign supports close to traveled way \\
\hline Remove or relocate sign & RS & $\begin{array}{l}\text { Off-road } \\
\text { collisions on } \\
\text { \#R1 road } \\
\end{array}$ & $\begin{array}{l}\text { Fixed object close to } \\
\text { traveled way on \#R1 } \\
\text { road }\end{array}$ & Sign supports close to traveled way \\
\hline Add special signing & RS & $\begin{array}{l}\text { Off-road } \\
\text { collisions on } \\
\text { \#R1 road }\end{array}$ & $\begin{array}{l}\text { Fixed object close to } \\
\text { traveled way on \#R1 } \\
\text { road }\end{array}$ & Trees close to traveled way \\
\hline Install barrier curb or guardrail on \#R1 road & RS & $\begin{array}{l}\text { Off-road } \\
\text { collisions on } \\
\text { \#R1 road }\end{array}$ & $\begin{array}{l}\text { Fixed object close to } \\
\text { traveled way on \#R1 } \\
\text { road }\end{array}$ & Trees close to traveled way \\
\hline Install tree marker & RS & $\begin{array}{l}\text { Off-road } \\
\text { collisions on } \\
\text { \#R1 road }\end{array}$ & $\begin{array}{l}\text { Fixed object close to } \\
\text { traveled way on \#R1 } \\
\text { road }\end{array}$ & Trees close to traveled way \\
\hline Increase clear zone on \#R1 road & RS & $\begin{array}{l}\text { Off-road } \\
\text { collisions on } \\
\text { \#R1 road }\end{array}$ & $\begin{array}{l}\text { Fixed object close to } \\
\text { traveled way on \#R1 } \\
\text { road }\end{array}$ & Trees close to traveled way \\
\hline Remove or relocate trees along \#R1 road & RS & $\begin{array}{l}\text { Off-road } \\
\text { collisions on } \\
\text { \#R1 road }\end{array}$ & $\begin{array}{l}\text { Fixed object close to } \\
\text { traveled way on \#R1 } \\
\text { road }\end{array}$ & Trees close to traveled way \\
\hline Add special signing & RS & $\begin{array}{l}\text { Off-road } \\
\text { collisions on } \\
\text { \#R1 road } \\
\end{array}$ & $\begin{array}{l}\text { Fixed object close to } \\
\text { traveled way on \#R1 } \\
\text { road }\end{array}$ & Utility poles close to traveled way \\
\hline Install barrier curb or guardrail on \#R1 road & $\mathrm{RS}$ & Off-road & Fixed object close to & Utility poles close to traveled way \\
\hline
\end{tabular}




\begin{tabular}{|c|c|c|c|c|c|}
\hline & & $\begin{array}{l}\text { collisions on } \\
\text { \#R1 road }\end{array}$ & $\begin{array}{l}\text { traveled way on \#R1 } \\
\text { road }\end{array}$ & & \\
\hline Install utility polet marker & RS & $\begin{array}{l}\text { Off-road } \\
\text { collisions on } \\
\text { \#R1 road } \\
\end{array}$ & $\begin{array}{l}\text { Fixed object close to } \\
\text { traveled way on \#R1 } \\
\text { road }\end{array}$ & \multicolumn{2}{|c|}{ Utility poles close to traveled way } \\
\hline Place utilities underground & RS & $\begin{array}{l}\text { Off-road } \\
\text { collisions on } \\
\text { \#R1 road } \\
\end{array}$ & $\begin{array}{l}\text { Fixed object close to } \\
\text { traveled way on \#R1 } \\
\text { road }\end{array}$ & \multicolumn{2}{|c|}{ Utility poles close to traveled way } \\
\hline $\begin{array}{l}\text { Remove or relocate utility pole along \#R1 } \\
\text { road }\end{array}$ & RS & $\begin{array}{l}\text { Off-road } \\
\text { collisions on } \\
\text { \#R1 road } \\
\end{array}$ & $\begin{array}{l}\text { Fixed object close to } \\
\text { traveled way on \#R1 } \\
\text { road }\end{array}$ & \multicolumn{2}{|c|}{ Utility poles close to traveled way } \\
\hline $\begin{array}{l}\text { Shield drivers from poles in hazardous } \\
\text { locations }\end{array}$ & RS & $\begin{array}{l}\text { Off-road } \\
\text { collisions on } \\
\text { \#R1 road }\end{array}$ & $\begin{array}{l}\text { Fixed object close to } \\
\text { traveled way on \#R1 } \\
\text { road }\end{array}$ & \multicolumn{2}{|c|}{ Utility poles close to traveled way } \\
\hline Use breakaway devices on \#R1 road & RS & $\begin{array}{l}\text { Off-road } \\
\text { collisions on } \\
\text { \#R1 road } \\
\end{array}$ & $\begin{array}{l}\text { Fixed object close to } \\
\text { traveled way on \#R1 } \\
\text { road }\end{array}$ & \multicolumn{2}{|c|}{ Utility poles close to traveled way } \\
\hline Widen lanes on \#R1 road to adequate width & RS & $\begin{array}{l}\text { Off-road } \\
\text { collisions on } \\
\text { \#R1 road }\end{array}$ & $\begin{array}{l}\text { Inadequate lane } \\
\text { width on \#R1 road }\end{array}$ & & \\
\hline Install centerline rumble strips & RS & $\begin{array}{l}\text { Off-road } \\
\text { collisions on } \\
\text { \#R1 road }\end{array}$ & $\begin{array}{l}\text { Inadequate lane } \\
\text { width on \#R1 road }\end{array}$ & & \\
\hline $\begin{array}{l}\text { Install shoulder rumble strips on \#A1 } \\
\text { approach }\end{array}$ & RS & $\begin{array}{l}\text { Off-road } \\
\text { collisions on } \\
\text { \#R1 road }\end{array}$ & $\begin{array}{l}\text { Inadequate lane } \\
\text { width on \#R1 road }\end{array}$ & & \\
\hline Install pavement marking & RS & $\begin{array}{l}\text { Off-road } \\
\text { collisions on } \\
\text { \#R1 road } \\
\end{array}$ & \multicolumn{3}{|c|}{ Inadequate pavement marking on \#R1 road } \\
\hline Improve pavement marking and texturing & RS & $\begin{array}{l}\text { Off-road } \\
\text { collisions on } \\
\text { \#R1 road }\end{array}$ & \multirow{2}{*}{\multicolumn{3}{|c|}{$\begin{array}{l}\text { Inadequate pavement marking on \#R1 road } \\
\text { Inadequate road } \\
\text { alignment on \#R1 } \\
\text { road }\end{array}$}} \\
\hline $\begin{array}{l}\text { Provide safer slopes and ditches to prevent } \\
\text { rollovers }\end{array}$ & RS & $\begin{array}{l}\text { Off-road } \\
\text { collisions on } \\
\text { \#R1 road }\end{array}$ & & & \\
\hline Enhance delineation of sharp curves & RS & Off-road & Inadequate road & & \\
\hline
\end{tabular}




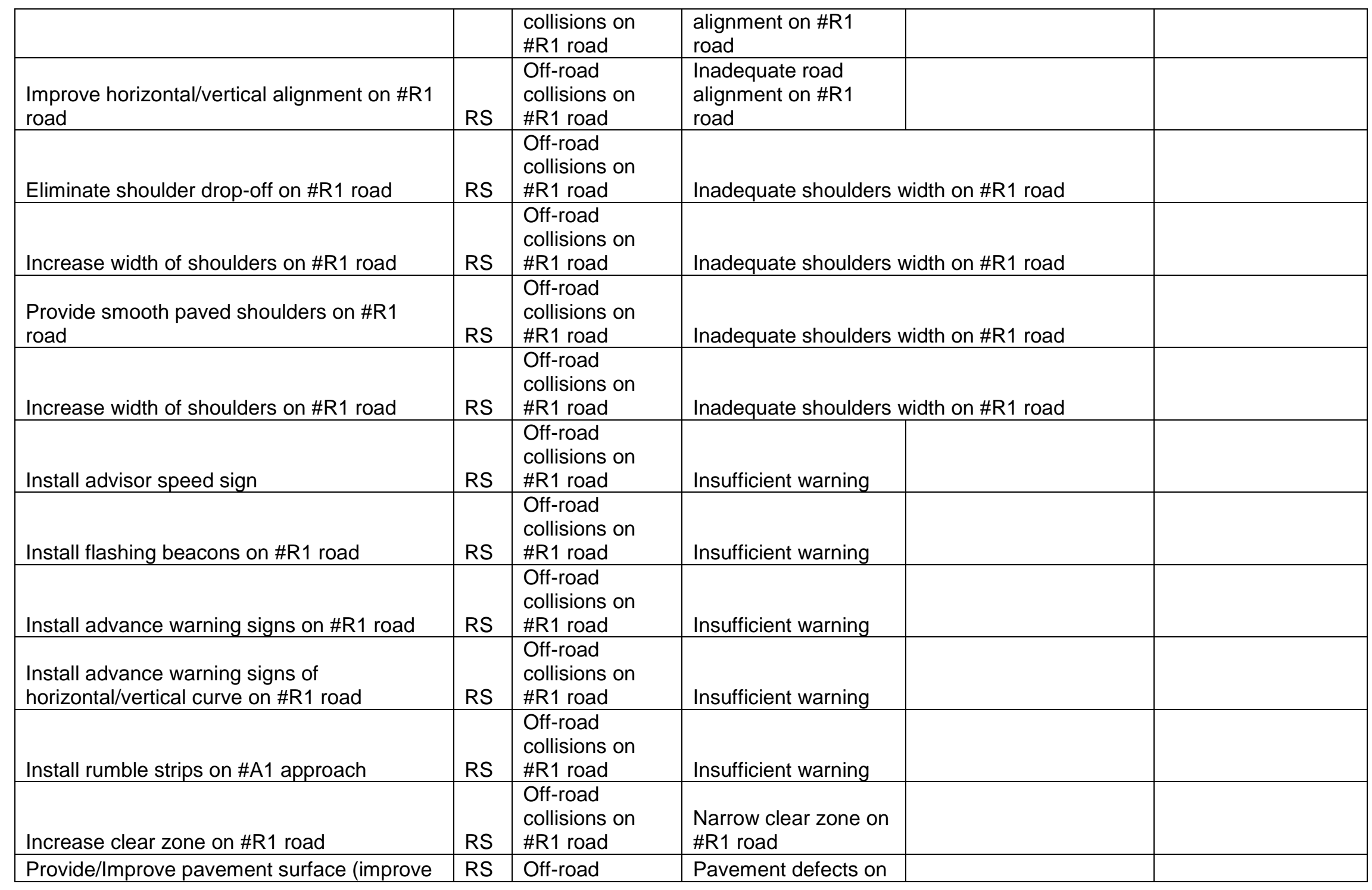




\begin{tabular}{|c|c|c|c|c|}
\hline $\begin{array}{l}\text { transitions/drop offs/fixed } \\
\text { potholes/rutting/etc) on \#R1 road }\end{array}$ & & $\begin{array}{l}\text { Collisions on } \\
\text { \#R1 road }\end{array}$ & \#R1 road & \\
\hline Provide adequate drainage on \#R1 road & RS & $\begin{array}{l}\text { Off-road } \\
\text { collisions on } \\
\text { \#R1 road } \\
\end{array}$ & $\begin{array}{l}\text { Poor drainage on } \\
\text { \#R1 road }\end{array}$ & \\
\hline Increase skid resistance on \#R1 road & RS & $\begin{array}{l}\text { Off-road } \\
\text { collisions on } \\
\text { \#R1 road }\end{array}$ & $\begin{array}{l}\text { Poor skid resistance } \\
\text { on \#R1 road }\end{array}$ & \\
\hline Groove pavement surface on \#R1 road & RS & $\begin{array}{l}\text { Off-road } \\
\text { collisions on } \\
\text { \#R1 road }\end{array}$ & $\begin{array}{l}\text { Poor skid resistance } \\
\text { on \#R1 road }\end{array}$ & \\
\hline Create additional parking spaces & RS & $\begin{array}{l}\text { Off-road } \\
\text { collisions on } \\
\text { \#R1 road }\end{array}$ & Truck collisions & \\
\hline Provide adequate truck signing & RS & $\begin{array}{l}\text { Off-road } \\
\text { collisions on } \\
\text { \#R1 road }\end{array}$ & Truck collisions & \\
\hline Install rumble strips on \#A1 approach & RS & $\begin{array}{l}\text { Off-road } \\
\text { collisions on } \\
\text { \#R1 road }\end{array}$ & Truck collisions & \\
\hline $\begin{array}{l}\text { Increase efficiency of use of existing parking } \\
\text { spaces }\end{array}$ & RS & $\begin{array}{l}\text { Off-road } \\
\text { collisions on } \\
\text { \#R1 road }\end{array}$ & Truck collisions & \\
\hline Install interactive truck rollover signing & RS & $\begin{array}{l}\text { Off-road } \\
\text { collisions on } \\
\text { \#R1 road }\end{array}$ & Truck collisions & \\
\hline Post adequate speed limit on \#R1 road & RS & $\begin{array}{l}\text { Off-road } \\
\text { collisions on } \\
\text { \#R1 road } \\
\end{array}$ & Truck collisions & \\
\hline $\begin{array}{l}\text { Provide targeted speed enforcement on \#R1 } \\
\text { road }\end{array}$ & RS & $\begin{array}{l}\text { Off-road } \\
\text { collisions on } \\
\text { \#R1 road }\end{array}$ & Truck collisions & \\
\hline $\begin{array}{l}\text { Change horizontal/vertical alignment on \#R1 } \\
\text { road }\end{array}$ & RS & $\begin{array}{l}\text { Head-on } \\
\text { collisions on } \\
\text { \#R1 road }\end{array}$ & Vertical curve & Inadequate design of vertical curve \\
\hline
\end{tabular}




\begin{tabular}{|c|c|c|c|c|}
\hline Widen lanes on \#R1 road to adequate width & RS & $\begin{array}{l}\text { Head-on } \\
\text { collisions on } \\
\text { \#R1 road }\end{array}$ & Horizontal curve & Inadequate lane width on \#R1 road \\
\hline Install centerline rumble strips & RS & $\begin{array}{l}\text { Head-on } \\
\text { collisions on } \\
\text { \#R1 road }\end{array}$ & Horizontal curve & Inadequate lane width on \#R1 road \\
\hline Widen lanes on \#R1 road to adequate width & RS & $\begin{array}{l}\text { Head-on } \\
\text { collisions on } \\
\text { \#R1 road }\end{array}$ & Vertical curve & Inadequate lane width on \#R1 road \\
\hline Install rumble strips on \#A1 approach & RS & $\begin{array}{l}\text { Head-on } \\
\text { collisions on } \\
\text { \#R1 road }\end{array}$ & Vertical curve & Inadequate lane width on \#R1 road \\
\hline Install interactive truck rollover signing & RS & $\begin{array}{l}\text { Head-on } \\
\text { collisions on } \\
\text { \#R1 road }\end{array}$ & Horizontal curve & Inadequate road design for trucks \\
\hline $\begin{array}{l}\text { Prohibit long semitrailers if horizontal } \\
\text { curve(s) cannot accommodate truck } \\
\text { offtracking }\end{array}$ & RS & $\begin{array}{l}\text { Head-on } \\
\text { collisions on } \\
\text { \#R1 road }\end{array}$ & Horizontal curve & Inadequate road design for trucks \\
\hline Post adequate truck speed limit on \#R1 road & RS & $\begin{array}{l}\text { Head-on } \\
\text { collisions on } \\
\text { \#R1 road }\end{array}$ & Horizontal curve & Inadequate road design for trucks \\
\hline $\begin{array}{l}\text { Cut or trim trees and other roadside } \\
\text { vegetation that obstruct sight distance }\end{array}$ & RS & $\begin{array}{l}\text { Head-on } \\
\text { collisions on } \\
\text { \#R1 road }\end{array}$ & Horizontal curve & Inadequate sight distance on horizontal curve \\
\hline Remove other obstructions & RS & $\begin{array}{l}\text { Head-on } \\
\text { collisions on } \\
\text { \#R1 road }\end{array}$ & Horizontal curve & Inadequate sight distance on horizontal curve \\
\hline $\begin{array}{l}\text { Restrict parking near horizontal/vertical } \\
\text { curves on \#R1 road }\end{array}$ & RS & $\begin{array}{l}\text { Head-on } \\
\text { collisions on } \\
\text { \#R1 road }\end{array}$ & Horizontal curve & Inadequate sight distance on horizontal curve \\
\hline Improve pavement marking and texturing & RS & $\begin{array}{l}\text { Head-on } \\
\text { collisions on } \\
\text { \#R1 road }\end{array}$ & Horizontal curve & Insufficient delineation along the curve \\
\hline Install in-pavement luminaires & RS & $\begin{array}{l}\text { Head-on } \\
\text { collisions on } \\
\text { \#R1 road }\end{array}$ & Horizontal curve & Insufficient delineation along the curve \\
\hline
\end{tabular}




\begin{tabular}{|c|c|c|c|c|}
\hline Install LED barrier-mounted guidance tubes & RS & $\begin{array}{l}\text { Head-on } \\
\text { collisions on } \\
\text { \#R1 road }\end{array}$ & Horizontal curve & Insufficient delineation along the curve \\
\hline Install light-emitting diode (LED) & RS & $\begin{array}{l}\text { Head-on } \\
\text { collisions on } \\
\text { \#1 road }\end{array}$ & Horizontal curve & Insufficient delineation along the curve \\
\hline $\begin{array}{l}\text { Delineate guardrail located outside the } \\
\text { roadway curve }\end{array}$ & RS & $\begin{array}{l}\text { Head-on } \\
\text { collisions on } \\
\text { \#R1 road }\end{array}$ & Horizontal curve & Insufficient delineation along the curve \\
\hline $\begin{array}{l}\text { Provide pavement marking (stop } \\
\text { sign/chevron/etc.) on \#R1 road }\end{array}$ & RS & $\begin{array}{l}\text { Head-on } \\
\text { collisions on } \\
\text { \#R1 road }\end{array}$ & Horizontal curve & Insufficient delineation along the curve \\
\hline Install post-mounted delineators & RS & $\begin{array}{l}\text { Head-on } \\
\text { collisions on } \\
\text { \#R1 road }\end{array}$ & Horizontal curve & Insufficient delineation along the curve \\
\hline $\begin{array}{l}\text { Improve pavement marking along the curve } \\
\text { on \#R1 road }\end{array}$ & RS & $\begin{array}{l}\text { Head-on } \\
\text { collisions on } \\
\text { \#R1 road }\end{array}$ & Horizontal curve & Insufficient delineation along the curve \\
\hline Install advisor speed sign & RS & $\begin{array}{l}\text { Head-on } \\
\text { collisions on } \\
\text { \#R1 road }\end{array}$ & Horizontal curve & Insufficient warning \\
\hline Install flashing beacons on \#R1 road & RS & $\begin{array}{l}\text { Head-on } \\
\text { collisions on } \\
\text { \#R1 road }\end{array}$ & Horizontal curve & Insufficient warning \\
\hline $\begin{array}{l}\text { Install advance warning signs of } \\
\text { horizontal/vertical curve on \#R1 road }\end{array}$ & RS & $\begin{array}{l}\text { Head-on } \\
\text { collisions on } \\
\text { \#R1 road }\end{array}$ & Horizontal curve & Insufficient warning \\
\hline Install rumble strips on \#A1 approach & RS & $\begin{array}{l}\text { Head-on } \\
\text { collisions on } \\
\text { \#R1 road }\end{array}$ & Horizontal curve & Insufficient warning \\
\hline Install advance warning signs on \#R1 road & RS & $\begin{array}{l}\text { Head-on } \\
\text { collisions on } \\
\text { \#R1 road }\end{array}$ & Horizontal curve & Insufficient warning \\
\hline $\begin{array}{l}\text { Provide curve dynamic warning system on } \\
\text { \#R1 road }\end{array}$ & RS & $\begin{array}{l}\text { Head-on } \\
\text { collisions on } \\
\text { \#R1 road }\end{array}$ & Horizontal curve & Insufficient warning \\
\hline
\end{tabular}




\begin{tabular}{|c|c|c|c|c|}
\hline Install rumble strips on \#A1 approach & RS & $\begin{array}{l}\text { Head-on } \\
\text { collisions on } \\
\text { \#R1 road }\end{array}$ & Horizontal curve & Insufficient warning \\
\hline Install advisor speed sign & RS & $\begin{array}{l}\text { Head-on } \\
\text { collisions on } \\
\text { \#R1 road }\end{array}$ & Vertical curve & Insufficient warning \\
\hline Install flashing beacons on \#R1 road & RS & $\begin{array}{l}\text { Head-on } \\
\text { collisions on } \\
\text { \#R1 road }\end{array}$ & Vertical curve & Insufficient warning \\
\hline Install street lighting & RS & $\begin{array}{l}\text { Head-on } \\
\text { collisions on } \\
\text { \#R1 road }\end{array}$ & Vertical curve & Insufficient warning \\
\hline Install advance warning signs on \#R1 road & RS & $\begin{array}{l}\text { Head-on } \\
\text { collisions on } \\
\text { \#R1 road }\end{array}$ & Vertical curve & Insufficient warning \\
\hline Install rumble strips on \#A1 approach & RS & $\begin{array}{l}\text { Head-on } \\
\text { collisions on } \\
\text { \#R1 road }\end{array}$ & Vertical curve & Insufficient warning \\
\hline $\begin{array}{l}\text { Install advance warning signs of } \\
\text { horizontal/vertical curve on \#R1 road }\end{array}$ & RS & $\begin{array}{l}\text { Head-on } \\
\text { collisions on } \\
\text { \#R1 road }\end{array}$ & Horizontal curve & Insufficient warning \\
\hline $\begin{array}{l}\text { Install advance warning signs of } \\
\text { horizontal/vertical curve on \#R1 road }\end{array}$ & RS & $\begin{array}{l}\text { Head-on } \\
\text { collisions on } \\
\text { \#R1 road }\end{array}$ & Vertical curve & Insufficient warning \\
\hline $\begin{array}{l}\text { Provide/Improve pavement surface (improve } \\
\text { transitions/drop offs/fixed } \\
\text { potholes/rutting/etc) on \#R1 road }\end{array}$ & RS & $\begin{array}{l}\text { Head-on } \\
\text { collisions on } \\
\text { \#R1 road }\end{array}$ & Horizontal curve & Pavement defects on \#R1 road \\
\hline $\begin{array}{l}\text { Provide/Improve pavement surface (improve } \\
\text { transitions/drop offs/fixed } \\
\text { potholes/rutting/etc) on \#R1 road }\end{array}$ & RS & $\begin{array}{l}\text { Head-on } \\
\text { collisions on } \\
\text { \#R1 road }\end{array}$ & Vertical curve & Pavement defects on \#R1 road \\
\hline Provide adequate drainage on \#R1 road & RS & $\begin{array}{l}\text { Head-on } \\
\text { collisions on } \\
\text { \#R1 road }\end{array}$ & Horizontal curve & Poor drainage on \#R1 road \\
\hline Provide adequate drainage on \#R1 road & RS & $\begin{array}{l}\text { Head-on } \\
\text { collisions on } \\
\text { \#R1 road }\end{array}$ & Vertical curve & Poor drainage on \#R1 road \\
\hline
\end{tabular}




\begin{tabular}{|c|c|c|c|c|c|}
\hline Increase skid resistance on \#R1 road & RS & $\begin{array}{l}\text { Head-on } \\
\text { collisions on } \\
\text { \#R1 road }\end{array}$ & Horizontal curve & \multicolumn{2}{|c|}{ Poor skid resistance on \#R1 road } \\
\hline Increase skid resistance on \#R1 road & RS & $\begin{array}{l}\text { Head-on } \\
\text { collisions on } \\
\text { \#R1 road }\end{array}$ & Vertical curve & \multicolumn{2}{|c|}{ Poor skid resistance on \#R1 road } \\
\hline Groove pavement surface on \#R1 road & RS & $\begin{array}{l}\text { Head-on } \\
\text { collisions on } \\
\text { \#R1 road }\end{array}$ & Vertical curve & Poor skid resistance & on \#R1 road \\
\hline Install centerline rumble strips & RS & $\begin{array}{l}\text { Head-on } \\
\text { collisions on } \\
\text { \#R1 road }\end{array}$ & \multicolumn{3}{|c|}{ Frequent encroachments into opposing lane } \\
\hline Install median barriers & RS & $\begin{array}{l}\text { Head-on } \\
\text { collisions on } \\
\text { \#R1 road }\end{array}$ & \multicolumn{3}{|c|}{ Frequent encroachments into opposing lane } \\
\hline Install centerline rumble strips & RS & $\begin{array}{l}\text { Head-on } \\
\text { collisions on } \\
\text { \#R1 road }\end{array}$ & \multicolumn{3}{|c|}{ Frequent encroachments into opposing lane } \\
\hline $\begin{array}{l}\text { Provide wider cross sections on two-lane } \\
\text { roads }\end{array}$ & RS & $\begin{array}{l}\text { Head-on } \\
\text { collisions on } \\
\text { \#R1 road }\end{array}$ & \multicolumn{3}{|c|}{ Frequent encroachments into opposing lane } \\
\hline $\begin{array}{l}\text { Reallocate total two-lane roadway width (lane } \\
\text { and shoulder) to include a narrow "buffer } \\
\text { median" }\end{array}$ & RS & $\begin{array}{l}\text { Head-on } \\
\text { collisions on } \\
\text { \#R1 road }\end{array}$ & \multicolumn{3}{|c|}{ Frequent encroachments into opposing lane } \\
\hline Install median barriers & RS & $\begin{array}{l}\text { Head-on } \\
\text { collisions on } \\
\text { \#R1 road }\end{array}$ & \multicolumn{3}{|c|}{ Inadequate design for high traffic volume } \\
\hline $\begin{array}{l}\text { Use alternating passing lanes or four-lane } \\
\text { sections at key locations }\end{array}$ & RS & $\begin{array}{l}\text { Head-on } \\
\text { collisions on } \\
\text { \#R1 road }\end{array}$ & \multicolumn{3}{|c|}{ Inadequate design for high traffic volume } \\
\hline Widen lanes on \#R1 road to adequate width & RS & $\begin{array}{l}\text { Head-on } \\
\text { collisions on } \\
\text { \#R1 road }\end{array}$ & $\begin{array}{l}\text { Inadequate lane } \\
\text { width on \#R1 road }\end{array}$ & & \\
\hline Install centerline rumble strips & RS & $\begin{array}{l}\text { Head-on } \\
\text { collisions on } \\
\text { \#R1 road }\end{array}$ & $\begin{array}{l}\text { Inadequate lane } \\
\text { width on \#R1 road }\end{array}$ & & \\
\hline
\end{tabular}




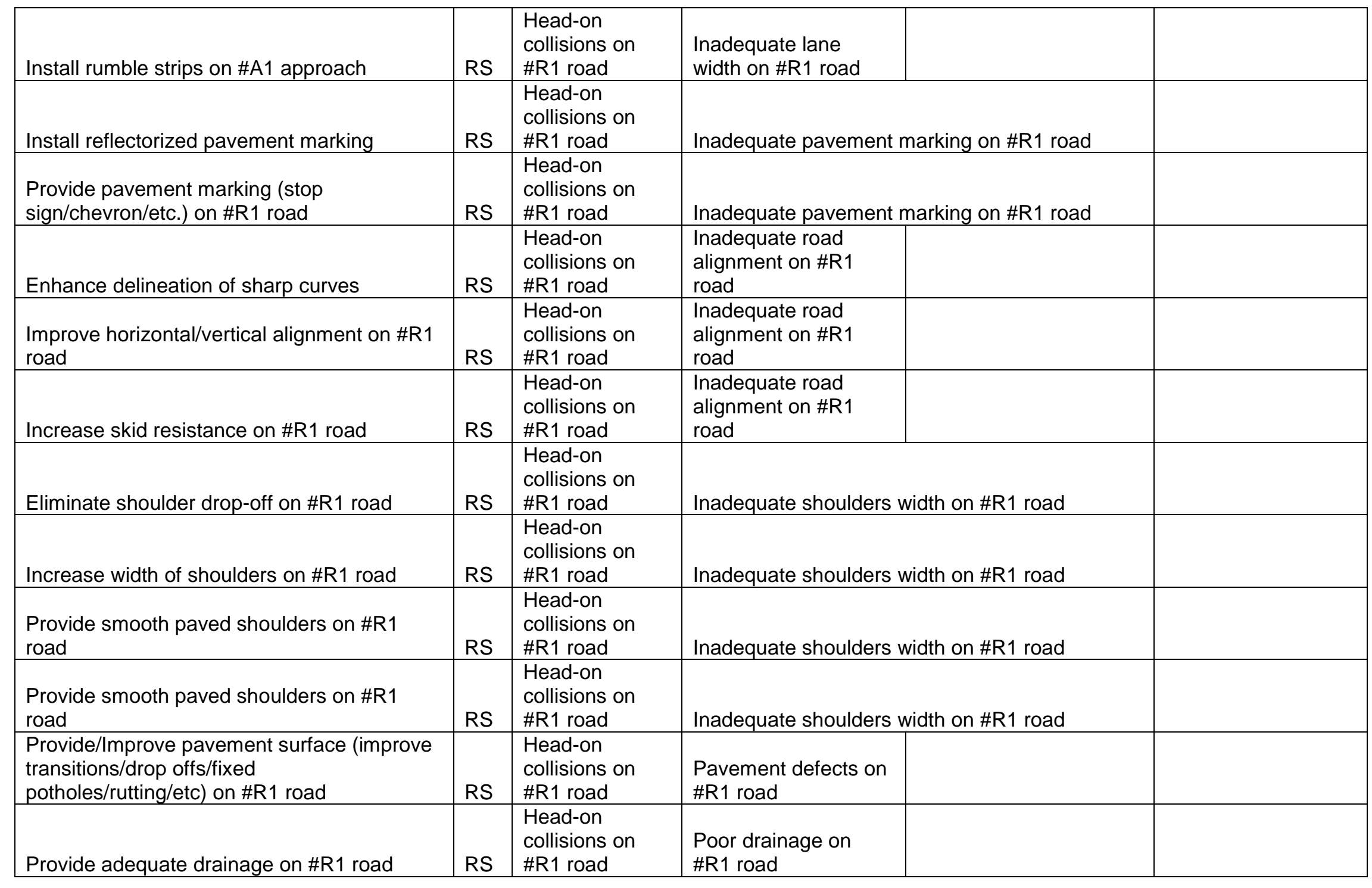




\begin{tabular}{|c|c|c|c|c|c|}
\hline Increase skid resistance on \#R1 road & RS & $\begin{array}{l}\text { Head-on } \\
\text { collisions on } \\
\text { \#R1 road } \\
\end{array}$ & $\begin{array}{l}\text { Poor skid resistance } \\
\text { on \#R1 road }\end{array}$ & & \\
\hline Groove pavement surface on \#R1 road & RS & $\begin{array}{l}\text { Head-on } \\
\text { collisions on } \\
\text { \#R1 road }\end{array}$ & $\begin{array}{l}\text { Poor skid resistance } \\
\text { on \#R1 road }\end{array}$ & & \\
\hline Create additional parking spaces & RS & $\begin{array}{l}\text { Head-on } \\
\text { collisions on } \\
\text { \#R1 road } \\
\end{array}$ & Truck collisions & & \\
\hline Provide adequate truck signing & RS & $\begin{array}{l}\text { Head-on } \\
\text { collisions on } \\
\text { \#R1 road }\end{array}$ & Truck collisions & & \\
\hline Install rumble strips on \#A1 approach & RS & $\begin{array}{l}\text { Head-on } \\
\text { collisions on } \\
\text { \#R1 road } \\
\end{array}$ & Truck collisions & & \\
\hline $\begin{array}{l}\text { Increase efficiency of use of existing parking } \\
\text { spaces }\end{array}$ & RS & $\begin{array}{l}\text { Head-on } \\
\text { collisions on } \\
\text { \#R1 road }\end{array}$ & Truck collisions & & \\
\hline Install interactive truck rollover signing & RS & $\begin{array}{l}\text { Head-on } \\
\text { collisions on } \\
\text { \#R1 road }\end{array}$ & Truck collisions & & \\
\hline Post adequate speed limit on \#R1 road & RS & $\begin{array}{l}\text { Head-on } \\
\text { collisions on } \\
\text { \#R1 road }\end{array}$ & Truck collisions & & \\
\hline $\begin{array}{l}\text { Provide targeted speed enforcement on \#R1 } \\
\text { road }\end{array}$ & RS & $\begin{array}{l}\text { Head-on } \\
\text { collisions on } \\
\text { \#R1 road } \\
\end{array}$ & Truck collisions & & \\
\hline Install advance warning signs on \#R1 road & RS & $\begin{array}{l}\text { Roadway } \\
\text { inconspicuous }\end{array}$ & $\begin{array}{l}\text { No advance warning } \\
\text { sign on \#R1 road }\end{array}$ & & \\
\hline $\begin{array}{l}\text { Install advance warning signs of } \\
\text { horizontal/vertical curve on \#R } 1 \text { road }\end{array}$ & RS & $\begin{array}{l}\text { Roadway } \\
\text { inconspicuous }\end{array}$ & $\begin{array}{l}\text { Advance warning } \\
\text { sign posted on \#R1 } \\
\text { road }\end{array}$ & $\begin{array}{l}\text { Warning sign poorly } \\
\text { visible/recognizable on } \\
\text { \#R1 road }\end{array}$ & $\begin{array}{l}\text { Visual distractions } \\
\text { on \#R1 road }\end{array}$ \\
\hline $\begin{array}{l}\text { Install overhead flashing beacon lights on } \\
\text { \#R1 road }\end{array}$ & RS & $\begin{array}{l}\text { Roadway } \\
\text { inconspicuous }\end{array}$ & $\begin{array}{l}\text { Advance warning } \\
\text { sign posted on \#R1 } \\
\text { road }\end{array}$ & $\begin{array}{l}\text { Warning sign poorly } \\
\text { visible/recognizable on } \\
\text { \#R1 road }\end{array}$ & $\begin{array}{l}\text { Visual distractions } \\
\text { on \#R1 road }\end{array}$ \\
\hline
\end{tabular}




\begin{tabular}{|l|l|l|l|l|l|}
\hline $\begin{array}{l}\text { Install larger regulatory signs on both sides of } \\
\text { road on \#R1 road }\end{array}$ & $R S$ & $\begin{array}{l}\text { Roadway } \\
\text { inconspicuous }\end{array}$ & $\begin{array}{l}\text { Advance warning } \\
\text { sign posted on \#R1 } \\
\text { road }\end{array}$ & $\begin{array}{l}\text { Warning sign poorly } \\
\text { visible/recognizable on } \\
\text { \#R1 road }\end{array}$ & $\begin{array}{l}\text { Visual distractions } \\
\text { on \#R1 road }\end{array}$ \\
\hline $\begin{array}{l}\text { Provide warning pavement marking on \#A1 } \\
\text { approach }\end{array}$ & $R S$ & $\begin{array}{l}\text { Roadway } \\
\text { inconspicuous }\end{array}$ & $\begin{array}{l}\text { Advance warning } \\
\text { sign posted on \#R1 } \\
\text { road }\end{array}$ & $\begin{array}{l}\text { Warning sign poorly } \\
\text { visible/recognizable on } \\
\text { \#R1 road }\end{array}$ & $\begin{array}{l}\text { Visual distractions } \\
\text { on \#R1 road }\end{array}$ \\
\hline Relocate sign on \#R1 road to make it visible & $\mathrm{RS}$ & $\begin{array}{l}\text { Roadway } \\
\text { inconspicuous }\end{array}$ & $\begin{array}{l}\text { Advance warning } \\
\text { sign posted on \#R1 } \\
\text { road }\end{array}$ & $\begin{array}{l}\text { Warning sign poorly } \\
\text { visible/recognizable on } \\
\text { \#R1 road }\end{array}$ & $\begin{array}{l}\text { Warning sign } \\
\text { obstructed on \#R1 } \\
\text { road }\end{array}$ \\
\hline $\begin{array}{l}\text { Remove object obstructing sight of warning } \\
\text { sign on \#R1 road }\end{array}$ & $\mathrm{RS}$ & $\begin{array}{l}\text { Roadway } \\
\text { inconspicuous }\end{array}$ & $\begin{array}{l}\text { Advance warning } \\
\text { sign posted on \#R1 } \\
\text { road }\end{array}$ & $\begin{array}{l}\text { Warning sign poorly } \\
\text { visible/recognizable on } \\
\text { \#R1 road }\end{array}$ & $\begin{array}{l}\text { Warning sign } \\
\text { obstructed on \#R1 } \\
\text { road }\end{array}$ \\
\hline $\begin{array}{l}\text { Remove object obstructing sight of warning } \\
\text { sign on \#R1 road }\end{array}$ & $\mathrm{RS}$ & $\begin{array}{l}\text { Roadway } \\
\text { inconspicuous }\end{array}$ & $\begin{array}{l}\text { Advance warning } \\
\text { sign posted on \#R1 } \\
\text { road }\end{array}$ & $\begin{array}{l}\text { Warning sign poorly visible/recognizable on } \\
\text { \#R1 road }\end{array}$ \\
\hline
\end{tabular}




\section{Appendix E}

\section{CRF study}

\section{Two-Way Stop-Controlled Intersection}

\begin{tabular}{|c|c|c|c|c|c|c|c|c|c|c|c|c|c|c|c|c|c|c|c|c|}
\hline & \multicolumn{2}{|c|}{ S1 } & \multicolumn{2}{|c|}{ S2 } & \multicolumn{2}{|c|}{ S3 } & \multicolumn{2}{|c|}{ S4 } & \multicolumn{2}{|c|}{ S5 } & \multicolumn{2}{|c|}{ S6 } & \multicolumn{2}{|c|}{ S7 } & \multicolumn{2}{|c|}{ S8 } & \multicolumn{2}{|c|}{ S9,S10 } & \multicolumn{2}{|c|}{ S11-S21 } \\
\hline & $\mathbf{R}$ & $\mathbf{U}$ & $\mathbf{R}$ & $\mathbf{U}$ & $\mathbf{R}$ & $\mathbf{U}$ & $\mathbf{R}$ & $\mathbf{U}$ & $\mathbf{R}$ & $\mathbf{U}$ & $\mathbf{R}$ & $\mathbf{U}$ & $\mathbf{R}$ & $\mathbf{U}$ & $\mathbf{R}$ & $\mathbf{U}$ & $\mathbf{R}$ & $\mathbf{U}$ & $\mathbf{R}$ & $\mathbf{U}$ \\
\hline Install raised crosswalk & & & 25 & 25 & & & & & 25 & 25 & & & & & & & 46 & & & \\
\hline $\begin{array}{l}\text { Installing snow screens } \\
\text { in areas exposed to } \\
\text { snowdrifts }\end{array}$ & & & & & 53 & 53 & & & & & & & & & & & & & & \\
\hline $\begin{array}{l}\text { Properly maintain the } \\
\text { striping }\end{array}$ & & & & & & & & & & & & & & & & & & & & \\
\hline $\begin{array}{l}\text { Use school crossing } \\
\text { guards }\end{array}$ & & & & & & & & & & & & & & & & & & & & \\
\hline $\begin{array}{l}\text { Add overhead stop- } \\
\text { signs if the percent of } \\
\text { tall vehciles is } \\
\text { considerable }\end{array}$ & & & & & & & & & & & & & & & & & & & & \\
\hline Add special signing & & & & & & & & & & & & & & & & & & & & \\
\hline $\begin{array}{l}\text { Add stop-lines in the } \\
\text { median opening }\end{array}$ & & & & & & & & & & & & & & & & & & & & \\
\hline $\begin{array}{l}\text { Adjust signal offsets at } \\
\text { the upstream } \\
\text { signalized intersections } \\
\text { to create more gaps }\end{array}$ & & & & & & & & & & & & & & & & & & & & \\
\hline $\begin{array}{l}\text { Change } \\
\text { horizontal/vertical } \\
\text { alignment }\end{array}$ & & & 58 & 58 & & & & & & & 50 & 50 & 50 & 50 & & & & & 10 & 10 \\
\hline
\end{tabular}




\begin{tabular}{|c|c|c|c|c|c|c|c|c|c|c|c|c|c|c|c|c|c|c|c|c|}
\hline & \multicolumn{2}{|c|}{ S1 } & \multicolumn{2}{|c|}{ S2 } & \multicolumn{2}{|c|}{ S3 } & \multicolumn{2}{|c|}{ S4 } & \multicolumn{2}{|c|}{ S5 } & \multicolumn{2}{|c|}{ S6 } & \multicolumn{2}{|c|}{ S7 } & \multicolumn{2}{|c|}{ S8 } & \multicolumn{2}{|c|}{ S9,S10 } & \multicolumn{2}{|c|}{ S11-S21 } \\
\hline & $\mathbf{R}$ & $\mathbf{U}$ & $\mathbf{R}$ & $\mathbf{U}$ & $\mathbf{R}$ & $\mathbf{U}$ & $\mathbf{R}$ & $\mathbf{U}$ & $\mathbf{R}$ & $\mathbf{U}$ & $\mathbf{R}$ & $\mathbf{U}$ & $\mathbf{R}$ & $\mathbf{U}$ & $\mathbf{R}$ & $\mathbf{U}$ & $\mathbf{R}$ & $\mathbf{U}$ & $\mathbf{R}$ & $\mathbf{U}$ \\
\hline $\begin{array}{l}\text { Chip and seal or slurry } \\
\text { seal approaches }\end{array}$ & & & & & & & & & & & & & & & & & & & & \\
\hline Close curb lanes & & & & & & & & & & & & & & & & & & & & \\
\hline $\begin{array}{l}\text { Close or relocate the } \\
\text { driveways }\end{array}$ & & & 50 & 50 & & & & & & & & & & & & & & & & \\
\hline $\begin{array}{l}\text { Consider indirect left } \\
\text { turns }\end{array}$ & & & 34 & 34 & & & 35 & 35 & 25 & 25 & 41 & 41 & $\begin{array}{l}32 \\
(1) \\
\end{array}$ & $\begin{array}{l}32 \\
(1) \\
\end{array}$ & & & $\begin{array}{l}48 \\
(2) \\
\end{array}$ & $\begin{array}{l}47 \\
(2)\end{array}$ & $\begin{array}{l}24 \\
(3) \\
\end{array}$ & \\
\hline $\begin{array}{l}\text { Consider intersection } \\
\text { type "J" (no directly left } \\
\text { turn) }\end{array}$ & & & & & & & & & & & & & & & & & & & & \\
\hline $\begin{array}{l}\text { Consider moving the } \\
\text { right-turn lanes on the } \\
\text { major road laterally }\end{array}$ & & & & & & & & & & & & & & & & & & & & \\
\hline $\begin{array}{l}\text { Consider traffic signals } \\
\text { with protected left-turn } \\
\text { phase }\end{array}$ & & & & & & & & & & & & & & & & & & & & \\
\hline $\begin{array}{l}\text { Construct Pedestrian } \\
\text { Refuge Islands and } \\
\text { Raised Medians }\end{array}$ & & 46 & $\begin{array}{l}60 \\
(4)\end{array}$ & $\begin{array}{l}60 \\
(4)\end{array}$ & 32 & 23 & & & & & & & & & & 46 & & & & \\
\hline $\begin{array}{l}\text { Convert the } \\
\text { intersection into a } \\
\text { roundabout }\end{array}$ & $\begin{array}{l}58 \\
(5)\end{array}$ & $\begin{array}{l}72 \\
(5)\end{array}$ & & & & & 40 & 40 & 40 & 40 & & & & & $\begin{array}{l}58 \\
(6)\end{array}$ & $\begin{array}{l}72 \\
(6)\end{array}$ & 58 & 72 & 38 & 38 \\
\hline $\begin{array}{l}\text { Eliminate screening by } \\
\text { physical objects }\end{array}$ & & & & & & & & & & & & & $\begin{array}{l}50 \\
(7)\end{array}$ & $\begin{array}{l}50 \\
(7)\end{array}$ & & & & & $\begin{array}{l}76 \\
(8)\end{array}$ & $\begin{array}{l}76 \\
(8)\end{array}$ \\
\hline $\begin{array}{l}\text { Eliminate shoulder } \\
\text { drop-off }\end{array}$ & & & & & & & & & & & 25 & 25 & & & & & & & $\begin{array}{l}30 \\
(9)\end{array}$ & $\begin{array}{l}30 \\
(9)\end{array}$ \\
\hline $\begin{array}{l}\text { Enforce stop sign } \\
\text { compliance }\end{array}$ & & & & & & & & & & & & & & & & & & & & \\
\hline $\begin{array}{l}\text { Extend "no parking" } \\
\text { zone. }\end{array}$ & & & & & 22 & 22 & & & & & $\begin{array}{l}35 \\
(1 \\
0)\end{array}$ & $\begin{array}{l}35 \\
(1 \\
0)\end{array}$ & & & & & & & 10 & 10 \\
\hline Groove pavement & & & 21 & 21 & & & 14 & 14 & & & & & & & & & & & 46 & 46 \\
\hline
\end{tabular}




\begin{tabular}{|c|c|c|c|c|c|c|c|c|c|c|c|c|c|c|c|c|c|c|c|c|}
\hline & \multicolumn{2}{|c|}{ S1 } & \multicolumn{2}{|c|}{ S2 } & \multicolumn{2}{|c|}{ S3 } & \multicolumn{2}{|c|}{ S4 } & \multicolumn{2}{|c|}{ S5 } & \multicolumn{2}{|c|}{ S6 } & \multicolumn{2}{|c|}{ S7 } & \multicolumn{2}{|c|}{ S8 } & \multicolumn{2}{|c|}{ S9,S10 } & \multicolumn{2}{|c|}{ S11-S21 } \\
\hline & $\mathbf{R}$ & $\mathbf{U}$ & $\mathbf{R}$ & $\mathbf{U}$ & $\mathbf{R}$ & $\mathbf{U}$ & $\mathbf{R}$ & $\mathbf{U}$ & $\mathbf{R}$ & $\mathbf{U}$ & $\mathbf{R}$ & $\mathbf{U}$ & $\mathbf{R}$ & $\mathbf{U}$ & $\mathbf{R}$ & $\mathbf{U}$ & $\mathbf{R}$ & $\mathbf{U}$ & $\mathbf{R}$ & $\mathbf{U}$ \\
\hline surface & & & & & & & & & & & & & & & & & & & & \\
\hline $\begin{array}{l}\text { Implement Road } \\
\text { Narrowing Measures }\end{array}$ & & & & & & & & & & & & & & & & & & & & \\
\hline $\begin{array}{l}\text { Improve } \\
\text { alignment/grade }\end{array}$ & & & & & & & & & & & & & & & & & & & & \\
\hline $\begin{array}{l}\text { Improve } \\
\text { channelization/delineati } \\
\text { on }\end{array}$ & & & & & & & & & & & $\begin{array}{l}30 \\
(1 \\
1)\end{array}$ & $\begin{array}{l}30 \\
(1 \\
1)\end{array}$ & 40 & 40 & & & & & 15 & \\
\hline Improve clear zone & & & & & $\begin{array}{l}25 \\
(1 \\
2)\end{array}$ & $\begin{array}{l}25 \\
(1 \\
2) \\
\end{array}$ & & & & & & & & & & & & & & \\
\hline $\begin{array}{l}\text { Improve existing street } \\
\text { lights }\end{array}$ & 30 & 30 & & & 50 & 50 & & & & & & & & & & & & & & \\
\hline Improve lanes width & $\begin{array}{l}8 \\
(1 \\
3)\end{array}$ & & & & & & & & & & & & & & 8 & & & & & \\
\hline $\begin{array}{l}\text { Improve pavement } \\
\text { marking }\end{array}$ & & & 21 & 21 & & & & & & & & & & & & & & & & \\
\hline $\begin{array}{l}\text { Improve pavement } \\
\text { marking in the median } \\
\text { opening }\end{array}$ & & & 21 & 21 & & & & & & & & & & & & & & & & \\
\hline $\begin{array}{l}\text { Improve signage } \\
\text { and/or marking of the } \\
\text { crosswalk }\end{array}$ & & & & & & & & & & & 25 & 25 & & & & & & & & \\
\hline $\begin{array}{l}\text { Improve skid } \\
\text { resistance }\end{array}$ & & & 9 & 9 & & & & & & & & & & & & & & & & \\
\hline $\begin{array}{l}\text { Improve visibility from } \\
\text { median toward major } \\
\text { approach }\end{array}$ & & & & & & & & & & & & & & & & & & & 10 & 10 \\
\hline $\begin{array}{l}\text { Improve visibility of } \\
\text { intersections by } \\
\text { providing enhanced } \\
\text { signing and delineation }\end{array}$ & & & & & & & & & & & $\begin{array}{l}30 \\
(1 \\
1) \\
\end{array}$ & $\begin{array}{l}30 \\
(1 \\
1) \\
\end{array}$ & & & & & & & & \\
\hline Improve visibility of the & 30 & 30 & 33 & 33 & 50 & 50 & & & & & 30 & 30 & 37 & 37 & & & & & 37 & 37 \\
\hline
\end{tabular}




\begin{tabular}{|c|c|c|c|c|c|c|c|c|c|c|c|c|c|c|c|c|c|c|c|c|}
\hline & \multicolumn{2}{|c|}{ S1 } & \multicolumn{2}{|c|}{ S2 } & \multicolumn{2}{|c|}{ S3 } & \multicolumn{2}{|c|}{ S4 } & \multicolumn{2}{|c|}{ S5 } & \multicolumn{2}{|c|}{ S6 } & \multicolumn{2}{|c|}{ S7 } & \multicolumn{2}{|c|}{ S8 } & \multicolumn{2}{|c|}{ S9,S10 } & \multicolumn{2}{|c|}{ S11-S21 } \\
\hline & $\mathbf{R}$ & $\mathbf{U}$ & $\mathbf{R}$ & $\mathbf{U}$ & $\mathbf{R}$ & $\mathbf{U}$ & $\mathbf{R}$ & $\mathbf{U}$ & $\mathbf{R}$ & $\mathbf{U}$ & $\mathbf{R}$ & $\mathbf{U}$ & $\mathbf{R}$ & $\mathbf{U}$ & $\mathbf{R}$ & $\mathbf{U}$ & $\mathbf{R}$ & $\mathbf{U}$ & $\mathbf{R}$ & $\mathbf{U}$ \\
\hline $\begin{array}{l}\text { intersection by } \\
\text { providing lighting }\end{array}$ & & & & & $\begin{array}{l}(1 \\
4)\end{array}$ & $\begin{array}{l}(1 \\
4) \\
\end{array}$ & & & & & & & & & & & & & & \\
\hline $\begin{array}{l}\text { Improve winter } \\
\text { maintenance methods }\end{array}$ & & & & & & & & & & & & & & & & & & & & \\
\hline $\begin{array}{l}\text { Improve winter } \\
\text { maintenance } \\
\text { preparedness (shorter } \\
\text { response time) }\end{array}$ & & & & & & & & & & & & & & & & & & & & \\
\hline $\begin{array}{l}\text { Improve/install } \\
\text { reflective signs }\end{array}$ & & & 15 & 15 & & & & & & & & & & & & & & & & \\
\hline $\begin{array}{l}\text { Improve/install } \\
\text { reflectorized pavement } \\
\text { markers }\end{array}$ & & & & & & & & & & & & & & & & & & & & \\
\hline $\begin{array}{l}\text { Increase length of the } \\
\text { right turn lanes }\end{array}$ & & & 28 & 28 & & & $\begin{array}{l}15 \\
(1 \\
5) \\
\end{array}$ & $\begin{array}{l}15 \\
(1 \\
5)\end{array}$ & & & 28 & 28 & & & & & & & & \\
\hline $\begin{array}{l}\text { Increase radius of the } \\
\text { corner curve }\end{array}$ & & & 13 & 13 & & & & & & & 15 & 15 & & & & & & & & \\
\hline $\begin{array}{l}\text { Increase the crosswalk } \\
\text { setback }\end{array}$ & & & & & & & & & & & & & & & & & & & & \\
\hline $\begin{array}{l}\text { Increase the length of } \\
\text { the left turn lanes }\end{array}$ & & & 28 & 28 & & & 15 & 15 & & & 28 & 28 & & & & & & & & \\
\hline $\begin{array}{l}\text { Increase the turning } \\
\text { path radius }\end{array}$ & & & 13 & 13 & & & & & & & 15 & 15 & & & & & & & & \\
\hline $\begin{array}{l}\text { Increase width of the } \\
\text { shoulders }\end{array}$ & $\begin{array}{l}9 \\
(1 \\
6) \\
\end{array}$ & & 14 & 14 & & & & & & & 20 & 20 & & & 9 & & & & 14 & \\
\hline $\begin{array}{l}\text { Install a barrier curb or } \\
\text { a guardrail }\end{array}$ & $\begin{array}{l}45 \\
(1 \\
7)\end{array}$ & $\begin{array}{l}45 \\
(1 \\
7)\end{array}$ & 11 & 11 & & & $\begin{array}{l}47 \\
(1 \\
8)\end{array}$ & $\begin{array}{l}47 \\
(1 \\
8)\end{array}$ & & & & & & 4 & & & $\begin{array}{l}44 \\
(7)\end{array}$ & $\begin{array}{l}44 \\
(7)\end{array}$ & & \\
\hline $\begin{array}{l}\text { Install a pedestrian } \\
\text { overpass or underpass }\end{array}$ & & & 86 & 86 & & & & & & & & & & & & & & & 91 & 91 \\
\hline Install a stop sign & & & 35 & 35 & & & & & & & 39 & 39 & & & & & & & & \\
\hline Install a triangular & & & & & & & & & & & & & & & & & & & & \\
\hline
\end{tabular}




\begin{tabular}{|c|c|c|c|c|c|c|c|c|c|c|c|c|c|c|c|c|c|c|c|c|}
\hline & \multicolumn{2}{|c|}{ S1 } & \multicolumn{2}{|c|}{ S2 } & \multicolumn{2}{|c|}{ S3 } & \multicolumn{2}{|c|}{ S4 } & \multicolumn{2}{|c|}{ S5 } & \multicolumn{2}{|c|}{ S6 } & \multicolumn{2}{|c|}{ S7 } & \multicolumn{2}{|c|}{ S8 } & \multicolumn{2}{|c|}{$\mathrm{S} 9, \mathrm{S10}$} & \multicolumn{2}{|c|}{ S11-S21 } \\
\hline & $\mathbf{R}$ & $\mathbf{U}$ & $\mathbf{R}$ & $\mathbf{U}$ & $\mathbf{R}$ & $\mathbf{U}$ & $\mathbf{R}$ & $\mathbf{U}$ & $\mathbf{R}$ & $\mathbf{U}$ & $\mathbf{R}$ & $\mathbf{U}$ & $\mathbf{R}$ & $\mathbf{U}$ & $\mathbf{R}$ & $\mathbf{U}$ & $\mathbf{R}$ & $\mathbf{U}$ & $\mathbf{R}$ & $\mathbf{U}$ \\
\hline $\begin{array}{l}\text { island to relocate the } \\
\text { right-turning movement } \\
\text { away from other } \\
\text { movements }\end{array}$ & & & & & & & & & & & & & & & & & & & & \\
\hline $\begin{array}{l}\text { Install acceleration } \\
\text { lanes sufficiently long } \\
\text { for trucks }\end{array}$ & & & 26 & 26 & & & & & & & & & & & & & & & & \\
\hline $\begin{array}{l}\text { Install advance } \\
\text { warning sign }\end{array}$ & 20 & 20 & 25 & 25 & & & $\begin{array}{l}25 \\
(1 \\
9) \\
\end{array}$ & $\begin{array}{l}25 \\
(1 \\
9) \\
\end{array}$ & & & & & 30 & 40 & & & & & & \\
\hline $\begin{array}{l}\text { Install an acceleration } \\
\text { lane }\end{array}$ & & & 26 & 26 & & & & & & & 10 & 10 & 10 & 10 & & & & & & \\
\hline Install an object marker & & & & & & & 16 & 16 & & & & & & & & & & & & \\
\hline $\begin{array}{l}\text { Install channelization of } \\
\text { the driveways }\end{array}$ & & & & & & & & & $\begin{array}{l}50 \\
(2 \\
0)\end{array}$ & $\begin{array}{l}50 \\
(2 \\
0)\end{array}$ & & & & & & & & & & \\
\hline Install corner mirrors & & & & & & & & & & & & & & & & & & & & \\
\hline $\begin{array}{l}\text { Install curbing to define } \\
\text { driveway location }\end{array}$ & & & & & & & & & & & & & & & & & & & & \\
\hline Install curb-ramps & & & & & & & & & & & & & & & & & & & & \\
\hline Install flashing beacons & 30 & 30 & & & 50 & 50 & $\begin{array}{l}30 \\
(2 \\
1) \\
\end{array}$ & $\begin{array}{l}30 \\
(2 \\
1) \\
\end{array}$ & & & 30 & 30 & 24 & 24 & & & & & & \\
\hline $\begin{array}{l}\text { Install flashing beacons } \\
\text { at stop-controlled } \\
\text { intersections }\end{array}$ & & & & & 50 & 50 & $\begin{array}{l}30 \\
(2 \\
1) \\
\end{array}$ & $\begin{array}{l}30 \\
(2 \\
1)\end{array}$ & & & & & 24 & 24 & & & & & & \\
\hline $\begin{array}{l}\text { Install fog - warning } \\
\text { signs }\end{array}$ & & & & & & & $\begin{array}{l}25 \\
(1 \\
9)\end{array}$ & $\begin{array}{l}25 \\
(1 \\
9)\end{array}$ & & & & & & & & & & & & \\
\hline Install guardrails & & & & & 20 & 20 & 47 & 47 & & & $\begin{array}{l}65 \\
(4)\end{array}$ & $\begin{array}{l}65 \\
(4)\end{array}$ & & 4 & & & $\begin{array}{l}44 \\
(4)\end{array}$ & $\begin{array}{l}44 \\
(4)\end{array}$ & & \\
\hline $\begin{array}{l}\text { Install indirect left-turn } \\
\text { lanes }\end{array}$ & & & $\begin{array}{l}34 \\
(2 \\
2)\end{array}$ & $\begin{array}{l}34 \\
(2 \\
2)\end{array}$ & & & 35 & 35 & & & & & & & & & & & & \\
\hline
\end{tabular}




\begin{tabular}{|c|c|c|c|c|c|c|c|c|c|c|c|c|c|c|c|c|c|c|c|c|}
\hline & \multicolumn{2}{|c|}{ S1 } & \multicolumn{2}{|c|}{ S2 } & \multicolumn{2}{|c|}{ S3 } & \multicolumn{2}{|c|}{ S4 } & \multicolumn{2}{|c|}{ S5 } & \multicolumn{2}{|c|}{ S6 } & \multicolumn{2}{|c|}{ S7 } & \multicolumn{2}{|c|}{ S8 } & \multicolumn{2}{|c|}{ S9,S10 } & \multicolumn{2}{|c|}{ S11-S21 } \\
\hline & $\mathbf{R}$ & $\mathbf{U}$ & $\mathbf{R}$ & $\mathbf{U}$ & $\mathbf{R}$ & $\mathbf{U}$ & $\mathbf{R}$ & $\mathbf{U}$ & $\mathbf{R}$ & $\mathbf{U}$ & $\mathbf{R}$ & $\mathbf{U}$ & $\mathbf{R}$ & $\mathbf{U}$ & $\mathbf{R}$ & $\mathbf{U}$ & $\mathbf{R}$ & $\mathbf{U}$ & $\mathbf{R}$ & $\mathbf{U}$ \\
\hline $\begin{array}{l}\text { Install larger regulatory } \\
\text { and warning signs at } \\
\text { and in advance of } \\
\text { intersections }\end{array}$ & & & & & & & & & & & $\begin{array}{l}15 \\
(2 \\
3)\end{array}$ & $\begin{array}{l}15 \\
(2 \\
3)\end{array}$ & 40 & 30 & & & & & & \\
\hline $\begin{array}{l}\text { Install larger regulatory } \\
\text { and warning signs at } \\
\text { intersections }\end{array}$ & & & & & & & & & & & $\begin{array}{l}15 \\
(2 \\
3)\end{array}$ & $\begin{array}{l}15 \\
(2 \\
3)\end{array}$ & & & & & & & & \\
\hline $\begin{array}{l}\text { Install left turn } \\
\text { acceleration lane }\end{array}$ & & & 26 & 26 & & & & & & & 10 & 10 & & & & & & & & \\
\hline Install left turn bays & 28 & 27 & & & 20 & 20 & & & & & & & & & $\begin{array}{l}28 \\
(2 \\
4)\end{array}$ & $\begin{array}{l}27 \\
(2 \\
4) \\
\end{array}$ & & & & \\
\hline Install lighting & 30 & 30 & 33 & 33 & $\begin{array}{l}50 \\
(2 \\
5)\end{array}$ & $\begin{array}{l}50 \\
(2 \\
5)\end{array}$ & & & & & 30 & 30 & 37 & 37 & & & & & $\begin{array}{l}37 \\
(2 \\
6)\end{array}$ & $\begin{array}{l}37 \\
(2 \\
6)\end{array}$ \\
\hline Install median divider & $\begin{array}{l}15 \\
(2 \\
7)\end{array}$ & $\begin{array}{l}15 \\
(2 \\
7)\end{array}$ & & & & & & & & & & & & & & & & & & \\
\hline $\begin{array}{l}\text { Install overhead } \\
\text { flashing beacon lights }\end{array}$ & 30 & 30 & & & 50 & 50 & $\begin{array}{l}30 \\
(2 \\
1)\end{array}$ & $\begin{array}{l}30 \\
(2 \\
1)\end{array}$ & & & 30 & 30 & 24 & 24 & & & & & & \\
\hline $\begin{array}{l}\text { Install pavement } \\
\text { markings }\end{array}$ & 10 & 10 & 21 & 21 & & & & & 21 & 21 & & & & & & & & & 10 & 10 \\
\hline $\begin{array}{l}\text { Install pedestrian } \\
\text { actuated signals }\end{array}$ & & & & & & & & & & & & & & & & & & & & \\
\hline $\begin{array}{l}\text { Install pedestrian } \\
\text { barriers }\end{array}$ & & & & & & & & & & & & & & & & & & & & \\
\hline $\begin{array}{l}\text { Install pedestrian } \\
\text { crosswalk }\end{array}$ & & & 25 & 25 & & & & & 25 & 25 & & & & & & & & & & \\
\hline $\begin{array}{l}\text { Install reflectorized } \\
\text { pavement marking }\end{array}$ & & & & & & & & & & & & & & & & & & & & \\
\hline $\begin{array}{l}\text { Install regulatory signs } \\
\text { on the both side of the } \\
\text { road }\end{array}$ & & & & & & & & & & & & & & & & & & & & \\
\hline
\end{tabular}




\begin{tabular}{|c|c|c|c|c|c|c|c|c|c|c|c|c|c|c|c|c|c|c|c|c|}
\hline & \multicolumn{2}{|c|}{ S1 } & \multicolumn{2}{|c|}{ S2 } & \multicolumn{2}{|c|}{ S3 } & \multicolumn{2}{|c|}{ S4 } & \multicolumn{2}{|c|}{ S5 } & \multicolumn{2}{|c|}{ S6 } & \multicolumn{2}{|c|}{ S7 } & \multicolumn{2}{|c|}{ S8 } & \multicolumn{2}{|c|}{ S9,S10 } & \multicolumn{2}{|c|}{ S11-S21 } \\
\hline & $\mathbf{R}$ & $\mathbf{U}$ & $\mathbf{R}$ & $\mathbf{U}$ & $\mathbf{R}$ & $\mathbf{U}$ & $\mathbf{R}$ & $\mathbf{U}$ & $\mathbf{R}$ & $\mathbf{U}$ & $\mathbf{R}$ & $\mathbf{U}$ & $\mathbf{R}$ & $\mathbf{U}$ & $\mathbf{R}$ & $\mathbf{U}$ & $\mathbf{R}$ & $\mathbf{U}$ & $\mathbf{R}$ & $\mathbf{U}$ \\
\hline $\begin{array}{l}\text { Install right turn bays if } \\
\text { the number of vehicle } \\
\text { turning right is } \\
\text { considerable }\end{array}$ & & & & & & & & & & & & & & & & & & & & \\
\hline Install right turn lane & & 14 & $\begin{array}{l}25 \\
(2 \\
8) \\
\end{array}$ & $\begin{array}{l}25 \\
(2 \\
8) \\
\end{array}$ & & & 25 & 25 & & & 24 & 24 & & & 14 & & 26 & & 10 & \\
\hline Install rumble strips & & & 32 & 32 & 28 & 28 & & & & & $\begin{array}{l}25 \\
(2 \\
3)\end{array}$ & $\begin{array}{l}25 \\
(2 \\
3)\end{array}$ & & & 21 & & & & 50 & 50 \\
\hline $\begin{array}{l}\text { Install school crossing } \\
\text { sign }\end{array}$ & & & 18 & 18 & & & & & & & 14 & 14 & & & & & & & & \\
\hline $\begin{array}{l}\text { Install school zone } \\
\text { markings }\end{array}$ & & & & & & & & & & & & & & & & & & & & \\
\hline $\begin{array}{l}\text { Install sidewalk set- } \\
\text { backs }\end{array}$ & & & & & & & & & & & & & & & & & & & & \\
\hline Install speed limit sign & & & & & & & & & & & & & & & & & & & & \\
\hline $\begin{array}{l}\text { Install static or variable } \\
\text { message signs } \\
\text { displaying weather } \\
\text { information }\end{array}$ & & & 15 & 15 & & & & & & & $\begin{array}{l}15 \\
(2 \\
3)\end{array}$ & $\begin{array}{l}15 \\
(2 \\
3)\end{array}$ & & & & & & & & \\
\hline Install street lights & 30 & 30 & & & $\begin{array}{l}50 \\
(2 \\
5)\end{array}$ & $\begin{array}{l}50 \\
(2 \\
5) \\
\end{array}$ & & & & & & & & & & & & & & \\
\hline $\begin{array}{l}\text { Install streets name } \\
\text { signs }\end{array}$ & & & & & & & & & & & 25 & 25 & & & & & & & & \\
\hline Install traffic signals & & 14 & $\begin{array}{l}30 \\
(2 \\
9)\end{array}$ & $\begin{array}{l}30 \\
(2 \\
9)\end{array}$ & & & & & & & 28 & 28 & 28 & 28 & & 23 & $\begin{array}{l}23 \\
(3 \\
0) \\
\end{array}$ & $\begin{array}{l}23 \\
(3 \\
0) \\
\end{array}$ & & \\
\hline $\begin{array}{l}\text { Install/Improve lighting } \\
\text { at the access points }\end{array}$ & & & $\begin{array}{l}33 \\
(3 \\
1) \\
\end{array}$ & $\begin{array}{l}33 \\
(3 \\
1) \\
\end{array}$ & & & & & & & 30 & 30 & 37 & 37 & & & & & $\begin{array}{l}37 \\
(2 \\
6)\end{array}$ & $\begin{array}{l}37 \\
(2 \\
6)\end{array}$ \\
\hline $\begin{array}{l}\text { Install/improve signing } \\
\text { or marking of }\end{array}$ & & & & & & & & & & & 15 & 15 & & & & & 16 & 16 & & \\
\hline
\end{tabular}




\begin{tabular}{|c|c|c|c|c|c|c|c|c|c|c|c|c|c|c|c|c|c|c|c|c|}
\hline & \multicolumn{2}{|c|}{ S1 } & \multicolumn{2}{|c|}{ S2 } & \multicolumn{2}{|c|}{ S3 } & \multicolumn{2}{|c|}{ S4 } & \multicolumn{2}{|c|}{ S5 } & \multicolumn{2}{|c|}{ S6 } & \multicolumn{2}{|c|}{ S7 } & \multicolumn{2}{|c|}{ S8 } & \multicolumn{2}{|c|}{ S9,S10 } & \multicolumn{2}{|c|}{ S11-S21 } \\
\hline & $\mathbf{R}$ & $\mathbf{U}$ & $\mathbf{R}$ & $\mathbf{U}$ & $\mathbf{R}$ & $\mathbf{U}$ & $\mathbf{R}$ & $\mathbf{U}$ & $\mathbf{R}$ & $\mathbf{U}$ & $\mathbf{R}$ & $\mathbf{U}$ & $\mathbf{R}$ & $\mathbf{U}$ & $\mathbf{R}$ & $\mathbf{U}$ & $\mathbf{R}$ & $\mathbf{U}$ & $\mathbf{R}$ & $\mathbf{U}$ \\
\hline pedestrian crosswalks & & & & & & & & & & & & & & & & & & & & \\
\hline $\begin{array}{l}\text { Install/improve warning } \\
\text { signs }\end{array}$ & & & 25 & 25 & 20 & 20 & $\begin{array}{l}25 \\
(2 \\
3)\end{array}$ & $\begin{array}{l}25 \\
(2 \\
3)\end{array}$ & & & 25 & 25 & & & & & & & & \\
\hline $\begin{array}{l}\text { Move driveway to side } \\
\text { street }\end{array}$ & & & $\begin{array}{l}50 \\
(3 \\
2)\end{array}$ & $\begin{array}{l}50 \\
(3 \\
2)\end{array}$ & 15 & 15 & & & & & & & & & & & & & & \\
\hline Overlay pavement & & & 17 & 17 & 18 & 18 & 27 & 27 & & & & & & & & & & & & \\
\hline $\begin{array}{l}\text { Post an adequate } \\
\text { speed limit }\end{array}$ & & & & & 28 & 28 & & & & & & & 20 & 20 & & & & & & \\
\hline $\begin{array}{l}\text { Post dynamic message } \\
\text { sign to display the } \\
\text { speed of approaching } \\
\text { vehicles. }\end{array}$ & & & & & & & & & & & & & & & & & $\begin{array}{l}74 \\
(3 \\
3) \\
\end{array}$ & $\begin{array}{l}74 \\
(3 \\
3)\end{array}$ & & \\
\hline $\begin{array}{l}\text { Provide "Slippery when } \\
\text { wet" signs }\end{array}$ & & & & & & & & & & & & & & & & & & & & \\
\hline $\begin{array}{l}\text { Provide a bypass lane } \\
\text { on the approach with } \\
\text { left-turn traffic }\end{array}$ & & & & & & & & & & & & & & & & & & & & \\
\hline $\begin{array}{l}\text { Provide a double } \\
\text { yellow centerline on } \\
\text { the median opening of } \\
\text { a divided highway at } \\
\text { intersections }\end{array}$ & & & & & & & & & & & & & & & & & & & & \\
\hline $\begin{array}{l}\text { Provide acceleration } \\
\text { and deceleration lanes }\end{array}$ & & & 26 & 26 & & & & & & & $\begin{array}{l}10 \\
(1 \\
1)\end{array}$ & $\begin{array}{l}10 \\
(1 \\
1)\end{array}$ & 10 & 10 & & & & & & \\
\hline $\begin{array}{l}\text { Provide adequate } \\
\text { delineation for left- } \\
\text { turns at the intersection } \\
\text { (markers or lines) }\end{array}$ & & & & & & & & & & & 30 & 30 & & & & & & & & \\
\hline $\begin{array}{l}\text { Provide adequate } \\
\text { drainage }\end{array}$ & & & 20 & 20 & & & & & & & & & & & & & & & & \\
\hline Provide adequate & & & & & 21 & 21 & & & & & & & & & & & & & & \\
\hline
\end{tabular}




\begin{tabular}{|c|c|c|c|c|c|c|c|c|c|c|c|c|c|c|c|c|c|c|c|c|}
\hline & \multicolumn{2}{|c|}{ S1 } & \multicolumn{2}{|c|}{ S2 } & \multicolumn{2}{|c|}{ S3 } & \multicolumn{2}{|c|}{ S4 } & \multicolumn{2}{|c|}{ S5 } & \multicolumn{2}{|c|}{ S6 } & \multicolumn{2}{|c|}{ S7 } & \multicolumn{2}{|c|}{ S8 } & \multicolumn{2}{|c|}{ S9,S10 } & \multicolumn{2}{|c|}{ S11-S21 } \\
\hline & $\mathbf{R}$ & $\mathbf{U}$ & $\mathbf{R}$ & $\mathbf{U}$ & $\mathbf{R}$ & $\mathbf{U}$ & $\mathbf{R}$ & $\mathbf{U}$ & $\mathbf{R}$ & $\mathbf{U}$ & $\mathbf{R}$ & $\mathbf{U}$ & $\mathbf{R}$ & $\mathbf{U}$ & $\mathbf{R}$ & $\mathbf{U}$ & $\mathbf{R}$ & $\mathbf{U}$ & $\mathbf{R}$ & $\mathbf{U}$ \\
\hline $\begin{array}{l}\text { lanes signing or } \\
\text { marking }\end{array}$ & & & & & $\begin{array}{l}3 \\
4) \\
\end{array}$ & $\begin{array}{l}3 \\
4) \\
\end{array}$ & & & & & & & & & & & & & & \\
\hline $\begin{array}{l}\text { Provide adequate } \\
\text { turning markers or } \\
\text { pavement markings }\end{array}$ & 10 & 10 & 21 & 21 & & & & & & & & & & & & & & & 10 & 10 \\
\hline $\begin{array}{l}\text { Provide dashed } \\
\text { markings (extended left } \\
\text { edgelines) for major- } \\
\text { road continuity across } \\
\text { the median opening at } \\
\text { divided highway } \\
\text { intersections }\end{array}$ & & & & & $\begin{array}{l}24 \\
(3 \\
5)\end{array}$ & $\begin{array}{l}24 \\
(3 \\
5)\end{array}$ & & & & & 30 & 30 & $\begin{array}{l}11 \\
(3 \\
6) \\
\end{array}$ & $\begin{array}{l}11 \\
(3 \\
6) \\
\end{array}$ & & & & & & \\
\hline $\begin{array}{l}\text { Provide independent } \\
\text { bicycle path where } \\
\text { necessary }\end{array}$ & & & & & & & & & & & & & & & & & & & & \\
\hline $\begin{array}{l}\text { Provide median } \\
\text { refuges }\end{array}$ & & 46 & & & & & & & & & & & & & & & & & & \\
\hline $\begin{array}{l}\text { Provide offset for left } \\
\text { turn lanes on the } \\
\text { opposite approaches }\end{array}$ & & & & & & & 10 & 10 & & & & & & & & & & & & \\
\hline $\begin{array}{l}\text { Provide on-pavement } \\
\text { horizontal signing }\end{array}$ & & & & & & & & & & & & & & & & & & & & \\
\hline $\begin{array}{l}\text { Provide pavement } \\
\text { marking (stop sign, } \\
\text { chevron, etc.) }\end{array}$ & & & 21 & 21 & $\begin{array}{l}35 \\
(3 \\
7)\end{array}$ & $\begin{array}{l}35 \\
(3 \\
7)\end{array}$ & & & & & & & 35 & 35 & & & $\begin{array}{l}25 \\
(3 \\
7)\end{array}$ & & & \\
\hline $\begin{array}{l}\text { Provide roadside } \\
\text { markers or pavement } \\
\text { markings to assist } \\
\text { drivers in judging the } \\
\text { suitability of available } \\
\text { gaps for making } \\
\text { turning and crossing } \\
\text { maneuvers }\end{array}$ & & & & & & & & & & & & & & & & & & & & \\
\hline Provide smooth paved & & & 15 & 15 & & & & & & & & & & 53 & & & & & 14 & \\
\hline
\end{tabular}




\begin{tabular}{|c|c|c|c|c|c|c|c|c|c|c|c|c|c|c|c|c|c|c|c|c|}
\hline & \multicolumn{2}{|c|}{ S1 } & \multicolumn{2}{|c|}{ S2 } & \multicolumn{2}{|c|}{ S3 } & \multicolumn{2}{|c|}{ S4 } & \multicolumn{2}{|c|}{ S5 } & \multicolumn{2}{|c|}{ S6 } & \multicolumn{2}{|c|}{ S7 } & \multicolumn{2}{|c|}{ S8 } & \multicolumn{2}{|c|}{ S9,S10 } & \multicolumn{2}{|c|}{ S11-S21 } \\
\hline & $\mathbf{R}$ & $\mathbf{U}$ & $\mathbf{R}$ & $\mathbf{U}$ & $\mathbf{R}$ & $\mathbf{U}$ & $\mathbf{R}$ & $\mathbf{U}$ & $\mathbf{R}$ & U & $\mathbf{R}$ & $\mathbf{U}$ & $\mathbf{R}$ & $\mathbf{U}$ & $\mathbf{R}$ & $\mathbf{U}$ & $\mathbf{R}$ & $\mathbf{U}$ & $\mathbf{R}$ & $\mathbf{U}$ \\
\hline shoulders & & & & & & & & & & & & & & $\begin{array}{l}3 \\
8)\end{array}$ & & & & & & \\
\hline Provide splitter islands & & & & & & & & & & & & & & & & & & & & \\
\hline Provide stop signs & & & 35 & 35 & & & & & & & 39 & 39 & & & & & & & & \\
\hline $\begin{array}{l}\text { Provide targeted speed } \\
\text { enforcement }\end{array}$ & & & & & & & & & & & & & & & & & & & & \\
\hline $\begin{array}{l}\text { Provide traffic calming } \\
\text { on intersection } \\
\text { approaches through a } \\
\text { combination of } \\
\text { geometric and traffic } \\
\text { control devices }\end{array}$ & & & & & & & & & & & & & & & & & & & & \\
\hline $\begin{array}{l}\text { Provide traffic signal if } \\
\text { warranted }\end{array}$ & & & & & & & & & & & 28 & 28 & & & & $\begin{array}{l}23 \\
(3 \\
9)\end{array}$ & $\begin{array}{l}23 \\
(4 \\
0)\end{array}$ & $\begin{array}{l}23 \\
(4 \\
0)\end{array}$ & & \\
\hline $\begin{array}{l}\text { Provide visible stop } \\
\text { bars }\end{array}$ & & & & & & & & & & & & & & & & & & & & \\
\hline $\begin{array}{l}\text { Provide/Improve } \\
\text { pavement surface } \\
\text { (improve transitions, } \\
\text { drop offs, fixed pothols, } \\
\text { rutting, etc) }\end{array}$ & & & & & & & & & & & & & & & & & & & & \\
\hline Reconstruct the radius & & & 13 & 13 & & & & & & & 15 & 15 & & & & & & & & \\
\hline $\begin{array}{l}\text { Reduce speed on the } \\
\text { major road }\end{array}$ & & & & & & & & & & & 20 & 20 & $\begin{array}{l}20 \\
(4 \\
1)\end{array}$ & $\begin{array}{l}20 \\
(4 \\
1)\end{array}$ & & & & & & \\
\hline Reduce speed limit & & & & & & & & & & & & & 20 & 20 & & & & & & \\
\hline $\begin{array}{l}\text { Reduce the number of } \\
\text { lanes on the minor } \\
\text { approach if allowed by } \\
\text { capacity }\end{array}$ & & & & & & & & & & & & & & & & & & & & \\
\hline Relocate crosswalk & & & & & & & & & & & & & & & & & & & & \\
\hline Relocate islands & & & & & & & & & & & & & & & & & & & & \\
\hline
\end{tabular}




\begin{tabular}{|c|c|c|c|c|c|c|c|c|c|c|c|c|c|c|c|c|c|c|c|c|}
\hline & \multicolumn{2}{|c|}{ S1 } & \multicolumn{2}{|c|}{ S2 } & \multicolumn{2}{|c|}{ S3 } & \multicolumn{2}{|c|}{ S4 } & \multicolumn{2}{|c|}{ S5 } & \multicolumn{2}{|c|}{ S6 } & \multicolumn{2}{|c|}{ S7 } & \multicolumn{2}{|c|}{ S8 } & \multicolumn{2}{|c|}{ S9,S10 } & \multicolumn{2}{|c|}{ S11-S21 } \\
\hline & $\mathbf{R}$ & $\mathbf{U}$ & $\mathbf{R}$ & $\mathbf{U}$ & $\mathbf{R}$ & $\mathbf{U}$ & $\mathbf{R}$ & $\mathbf{U}$ & $\mathbf{R}$ & $\mathbf{U}$ & $\mathbf{R}$ & $\mathbf{U}$ & $\mathbf{R}$ & $\mathbf{U}$ & $\mathbf{R}$ & $\mathbf{U}$ & $\mathbf{R}$ & $\mathbf{U}$ & $\mathbf{R}$ & $\mathbf{U}$ \\
\hline $\begin{array}{l}\text { Relocate or split the } \\
\text { stopping line }\end{array}$ & & & & & & & & & & & & & & & & & & & & \\
\hline Relocate stop bar & & & & & & & & & & & & & & & & & & & & \\
\hline Relocate stop sign & & & & & & & & & & & & & & & & & & & & \\
\hline $\begin{array}{l}\text { Relocate the sign to } \\
\text { make it visible }\end{array}$ & & & & & & & & & & & & & & & & & & & & \\
\hline $\begin{array}{l}\text { Remove distracting } \\
\text { commercial lighting or } \\
\text { other source of glare }\end{array}$ & & & & & & & & & & & 15 & 15 & & & & & & & & \\
\hline $\begin{array}{l}\text { Remove or re-locate } \\
\text { the median object } \\
\text { obstructing sight of } \\
\text { opposite traffic }\end{array}$ & $\begin{array}{l}12 \\
(4 \\
2)\end{array}$ & & & & & & & & & & & & & & $\begin{array}{l}13 \\
(4 \\
3)\end{array}$ & & & & 10 & 10 \\
\hline $\begin{array}{l}\text { Remove or relocate the } \\
\text { object }\end{array}$ & & & & & 60 & 60 & $\begin{array}{l}29 \\
(4 \\
4)\end{array}$ & $\begin{array}{l}29 \\
(4 \\
4)\end{array}$ & & & 30 & 30 & $\begin{array}{l}50 \\
(7)\end{array}$ & $\begin{array}{l}50 \\
(7)\end{array}$ & & & & & & \\
\hline $\begin{array}{l}\text { Remove sight } \\
\text { obstructions }\end{array}$ & $\begin{array}{l}12 \\
(4 \\
2)\end{array}$ & & & & & & & & & & & & & & $\begin{array}{l}13 \\
(4 \\
3) \\
\end{array}$ & & & & & \\
\hline $\begin{array}{l}\text { Remove the object } \\
\text { obstructing sight of the } \\
\text { warning sign }\end{array}$ & & & & & & & & & & & & & & & & & & & & \\
\hline $\begin{array}{l}\text { Remove the object } \\
\text { obstructing the sight of } \\
\text { the stop sign }\end{array}$ & & & & & & & & & & & & & & & & & & & & \\
\hline $\begin{array}{l}\text { Remove the object } \\
\text { obstructing the sight } \\
\text { triangle }\end{array}$ & $\begin{array}{l}12 \\
(4 \\
2)\end{array}$ & & & & & & 25 & 25 & & & & & & & $\begin{array}{l}13 \\
(4 \\
3)\end{array}$ & & & & 10 & 10 \\
\hline $\begin{array}{l}\text { Replace poorly } \\
\text { designed drain grates } \\
\text { with bicycle-safe types }\end{array}$ & & & & & & & & & & & & & & & & & & & & \\
\hline $\begin{array}{l}\text { Replace, repair, or } \\
\text { clean the sign }\end{array}$ & & & & & $\begin{array}{l}5 \\
(4 \\
5) \\
\end{array}$ & $\begin{array}{l}5 \\
(4 \\
5) \\
\end{array}$ & & & & & & & & & & & & & & \\
\hline
\end{tabular}




\begin{tabular}{|c|c|c|c|c|c|c|c|c|c|c|c|c|c|c|c|c|c|c|c|c|}
\hline & \multicolumn{2}{|c|}{ S1 } & \multicolumn{2}{|c|}{ S2 } & \multicolumn{2}{|c|}{ S3 } & \multicolumn{2}{|c|}{ S4 } & \multicolumn{2}{|c|}{ S5 } & \multicolumn{2}{|c|}{ S6 } & \multicolumn{2}{|c|}{ S7 } & \multicolumn{2}{|c|}{$\mathbf{S 8}$} & \multicolumn{2}{|c|}{ S9,S10 } & \multicolumn{2}{|c|}{ S11-S21 } \\
\hline & $\mathbf{R}$ & $\mathbf{U}$ & $\mathbf{R}$ & $\mathbf{U}$ & $\mathbf{R}$ & $\mathbf{U}$ & $\mathbf{R}$ & $\mathbf{U}$ & $\mathbf{R}$ & $\mathbf{U}$ & $\mathbf{R}$ & $\mathbf{U}$ & $\mathbf{R}$ & $\mathbf{U}$ & $\mathbf{R}$ & $\mathbf{U}$ & $\mathbf{R}$ & $\mathbf{U}$ & $\mathbf{R}$ & $\mathbf{U}$ \\
\hline $\begin{array}{l}\text { Replace, repair, or } \\
\text { clean the warning } \\
\text { signs }\end{array}$ & & & & & 20 & 20 & 25 & 25 & & & & & & & & & & & & \\
\hline $\begin{array}{l}\text { Reroute pedestrian } \\
\text { paths }\end{array}$ & & & & & & & & & & & & & & & & & & & & \\
\hline $\begin{array}{l}\text { Restrict left-turn } \\
\text { maneuver }\end{array}$ & & & & & $\begin{array}{l}40 \\
(4 \\
6)\end{array}$ & & & & & & & & & & & & & & & \\
\hline $\begin{array}{l}\text { Restrict left-turning at } \\
\text { the access points }\end{array}$ & & & & & & & & & & & & & & & & & & & $\begin{array}{l}10 \\
0 \\
(4 \\
7) \\
\end{array}$ & \\
\hline $\begin{array}{l}\text { Restrict parking near } \\
\text { the driveway }\end{array}$ & & & & & $\begin{array}{l}22 \\
(4 \\
8)\end{array}$ & $\begin{array}{l}22 \\
(4 \\
8)\end{array}$ & & & & & 35 & 35 & 8 & 8 & & & & & 10 & 10 \\
\hline $\begin{array}{l}\text { Restrict parking on the } \\
\text { approach }\end{array}$ & & & & & & & & & & & & & & & & & & & 10 & 10 \\
\hline $\begin{array}{l}\text { Signals to alert } \\
\text { motorists that } \\
\text { pedestrians are } \\
\text { crossing }\end{array}$ & & & & & & & & & & & & & & & & & 16 & 16 & & \\
\hline Trim vegetation & & & & & & & & & & & & & & & & & & & & \\
\hline Upgrade signing & & & & & $\begin{array}{l}5 \\
(4 \\
5)\end{array}$ & $\begin{array}{l}5 \\
(4 \\
5)\end{array}$ & & & & & 24 & 24 & & & & & & & & \\
\hline $\begin{array}{l}\text { Use "Slippery when } \\
\text { wet" signs }\end{array}$ & & & & & & & & & & & & & & & & & & & & \\
\hline $\begin{array}{l}\text { Use break-away } \\
\text { devices }\end{array}$ & & & & & 30 & 30 & & & & & & & & & & & & & & \\
\hline $\begin{array}{l}\text { Use salt to prevent } \\
\text { snow or ice from } \\
\text { forming or from sticking } \\
\text { to the road surface }\end{array}$ & & & & & & & & & & & & & & & & & & & & \\
\hline Use sand to increase & & & & & & & & & & & & & & & & & & & & \\
\hline
\end{tabular}




\begin{tabular}{|c|c|c|c|c|c|c|c|c|c|c|c|c|c|c|c|c|c|c|c|c|}
\hline & \multicolumn{2}{|c|}{ S1 } & \multicolumn{2}{|c|}{ S2 } & \multicolumn{2}{|c|}{ S3 } & \multicolumn{2}{|c|}{ S4 } & \multicolumn{2}{|c|}{ S5 } & \multicolumn{2}{|c|}{ S6 } & \multicolumn{2}{|c|}{ S7 } & \multicolumn{2}{|c|}{ S8 } & \multicolumn{2}{|c|}{ S9,S10 } & \multicolumn{2}{|c|}{ S11-S21 } \\
\hline & $\mathbf{R}$ & $\mathbf{U}$ & $\mathbf{R}$ & $\mathbf{U}$ & $\mathbf{R}$ & $\mathbf{U}$ & $\mathbf{R}$ & $\mathbf{U}$ & $\mathbf{R}$ & $\mathbf{U}$ & $\mathbf{R}$ & $\mathbf{U}$ & $\mathbf{R}$ & $\mathbf{U}$ & $\mathbf{R}$ & $\mathbf{U}$ & $\mathbf{R}$ & $\mathbf{U}$ & $\mathbf{R}$ & $\mathbf{U}$ \\
\hline pavement friction & & & & & & & & & & & & & & & & & & & & \\
\hline $\begin{array}{l}\text { Vehicles in the right- } \\
\text { turn lane on the majo } \\
\text { road block the minor- } \\
\text { road drivers' view of } \\
\text { traffic approaching on } \\
\text { the major road }\end{array}$ & & & & & & & & & & & & & & & & & & & & \\
\hline $\begin{array}{l}\text { Widen lanes to } \\
\text { adequate width }\end{array}$ & & & 40 & 40 & 38 & & $\begin{array}{l}22 \\
14 \\
9(\end{array}$ & $\begin{array}{l}22 \\
(4 \\
9)\end{array}$ & $\begin{array}{l}30 \\
(5 \\
0)\end{array}$ & $\begin{array}{l}30 \\
(5 \\
0)\end{array}$ & 25 & 25 & $\begin{array}{l}23 \\
(5 \\
1)\end{array}$ & $\begin{array}{l}23 \\
(5 \\
1)\end{array}$ & $\begin{array}{l}8 \\
(5 \\
2)\end{array}$ & & & & 13 & 13 \\
\hline $\begin{array}{l}\text { Widen the median to } \\
\text { provide sufficient } \\
\text { protection to crossing } \\
\text { vehicles }\end{array}$ & & & & & & & & & & & & & & & & & & & & \\
\hline $\begin{array}{l}\text { Widen the median } \\
\text { width }\end{array}$ & & & & & & & & & & & & & 13 & 13 & & & & & & \\
\hline $\begin{array}{l}\text { Widen the outside } \\
\text { through lanes or add } \\
\text { bike lanes }\end{array}$ & & & & & & & & & & & & & & & & & & & & \\
\hline
\end{tabular}




\section{COMMENTS}

(1) - added painted lane

(2) - rural both approaches, 4 legs

(3) - four legs intersection

(4) - reduction in pedestrians fatal only

(5) - for single lane approach; multiple lane approach $\mathrm{CRF}=5 \%$ only urban

(6) - single lane

(7) - fatal only

(8) - conflict elimination

(9) - shoulder treatment

(10) - eliminate parking

(11) - post delineation

(12) - widen by 5,8,10,15,20 [ft] CRF is respectively: $13 \%, 21 \%, 25 \%, 35 \%, 44 \%$

(13) - widen lanes: 9ft-12ft $\mathrm{CRF}=12 \% ; 10 \mathrm{ft}-12 \mathrm{ft} \mathrm{CRF}=8 \% ; 11 \mathrm{ft}-12 \mathrm{ft} \mathrm{CRF}=2 \%$

(14) - provide illumination for intersection

(15) - general any turn lane

(16) - for width: $2 \mathrm{ft}-8 \mathrm{ft}: 12 \%, 4 \mathrm{ft}-8 \mathrm{ft}: 9 \%, 6 \mathrm{ft}-8 \mathrm{ft}: 5 \%$ (only for paved shoulder)

(17) - fatal $\mathrm{CRF}=44 \%$; injury $\mathrm{CRF}=47 \%$

(18)- total injury crashes (incl. fatal)

(19) - warning general

(20) - channelization - CRF $=50 \%$ for Rear End, Head On, Sideswipe Same Direction, Sideswipe Opposite Direction, Left Turn, and Run-off-the-Road crashes.

(21) - for intersection only

(22) - provide left turn lane without signal

(23) - general warning and large sign

(24) - 4 leg intersection

(25) - provide illumination for intersection

(26) - CRF between $25 \%-50 \%$

(27) - add mountable median

(28) - turning, angle, rear-end, sideswipe - over 
(29) - 3 leg: all types $\mathrm{CRF}=14 \%$; angle $\mathrm{CRF}=34 \%$, rear-end $\mathrm{CRF}=(-50 \%), 4$ leg: all types $\mathrm{CRF}=23 \%$; angle $\mathrm{CRF}=67 \%$, rear-end $\mathrm{CRF}=(-38 \%)$

(30) - 4 leg all crashes

(31) - treat as intersection

(32) - relocate driveway entrance: left, right, angle $=10 \%$; rear, head $=20 \%$

(33) - speed reduction (not crashes)

(34) - pavement markings

(35) - edge line markings

(36) - edge lines

(37) - chevron

(38) - urban multilane

(39) - 4 leg intersection

(40)- 4 leg both approaches

(41) - modify speed limit

(42) - the following reduction applies: in 1 quadrant - 5\%, in 2 quadrant - 9\%, in 3 quadrant $13 \%$, in 4 quadrant $-17 \%$

(43) - 3 quadrant

(44) - removed fixed object (relocate $=35 \%$ )

(45) - general upgrade signing

(46) - prohibit turning

(47) - only turning crashes

(48) - prohibit on street parking

(49) - pavement widening

(50) - widening and resurfacing or widening alone

(51) - average from $1 \mathrm{ft}$ and $2 \mathrm{ft}$

(52) $-10 \mathrm{ft}-12 \mathrm{ft}$

(53) - average from width: $4 \mathrm{ft}, 10 \mathrm{ft}, 20 \mathrm{ft}$ 


\section{Rail Road Crossing}

\begin{tabular}{|c|c|c|c|c|c|c|c|c|c|c|c|c|c|c|c|c|c|c|c|c|c|c|}
\hline & \multicolumn{2}{|c|}{ S1 } & \multicolumn{2}{|c|}{ S2 } & \multicolumn{2}{|c|}{ S3 } & \multicolumn{2}{|c|}{ S4 } & \multicolumn{2}{|c|}{ S5 } & \multicolumn{2}{|c|}{ S6 } & \multicolumn{2}{|c|}{ S7 } & \multicolumn{2}{|c|}{ S8 } & \multicolumn{2}{|c|}{ S9-S10 } & \multicolumn{2}{|c|}{ S11-S21 } & \multicolumn{2}{|c|}{$\mathrm{S} 22$} \\
\hline & $\mathbf{R}$ & $\mathbf{U}$ & $\mathbf{R}$ & $\mathbf{U}$ & $\mathbf{R}$ & $\mathbf{U}$ & $\mathbf{R}$ & $\mathbf{U}$ & $\mathbf{R}$ & $\mathbf{U}$ & $\mathbf{R}$ & $\mathbf{U}$ & $\mathbf{R}$ & $\mathbf{U}$ & $\mathbf{R}$ & $\mathbf{U}$ & $\mathbf{R}$ & $\mathbf{U}$ & $\mathbf{R}$ & $\mathbf{U}$ & $\mathbf{R}$ & $\mathbf{U}$ \\
\hline $\begin{array}{l}\text { Add overhead stop- } \\
\text { signs if the percent of } \\
\text { tall vehicles is } \\
\text { considerable }\end{array}$ & & & & & & & & & & & & & & & & & & & & & & \\
\hline $\begin{array}{l}\text { Change } \\
\text { horizontal/vertical } \\
\text { alignment (skewed- } \\
\text { angle crossing) }\end{array}$ & & & & & & & & & & & & & & & & & & & & & $\begin{array}{l}45 \\
(1)\end{array}$ & $\begin{array}{l}45 \\
(1)\end{array}$ \\
\hline $\begin{array}{l}\text { Chip and seal or slurry } \\
\text { seal approaches }\end{array}$ & & & & & & & & & & & & & & & & & & & & & & \\
\hline $\begin{array}{l}\text { Close or install } \\
\text { overpass }\end{array}$ & & & & & & & & & & & & & & & & & & & & & & \\
\hline $\begin{array}{l}\text { Close rail-road } \\
\text { crossing point }\end{array}$ & & & & & & & & & & & & & & & & & & & & & & \\
\hline $\begin{array}{l}\text { Eliminate screening by } \\
\text { physical objects }\end{array}$ & & & & & & & & & & & & & & & & & & & & & & \\
\hline $\begin{array}{l}\text { Extend "no parking" } \\
\text { zone. }\end{array}$ & & & & & & & & & & & & & & & & & & & & & & \\
\hline $\begin{array}{l}\text { Groove pavement } \\
\text { surface }\end{array}$ & & & & & & & & & & & & & & & & & & & & & & \\
\hline $\begin{array}{l}\text { Improve } \\
\text { channelization/delineati } \\
\text { on }\end{array}$ & & & & & & & & & & & $\begin{array}{l}30 \\
(2)\end{array}$ & $\begin{array}{l}30 \\
(2)\end{array}$ & 40 & 40 & & & 75 & 75 & 15 & & & \\
\hline $\begin{array}{l}\text { Improve existing street } \\
\text { lights at the rail-road } \\
\text { crossing points }\end{array}$ & 30 & 30 & & & 50 & 50 & & & & & & & & & & & & & & & & \\
\hline $\begin{array}{l}\text { Improve gates } \\
\text { visibility/reflectivity } \\
\text { (flashing lights along }\end{array}$ & & & $\begin{array}{l}75 \\
(3)\end{array}$ & $\begin{array}{l}75 \\
(3)\end{array}$ & & & & & & & & & & & & & & & & & & \\
\hline
\end{tabular}




\begin{tabular}{|c|c|c|c|c|c|c|c|c|c|c|c|c|c|c|c|c|c|c|c|c|c|c|}
\hline \multirow[b]{3}{*}{ the gate) } & \multicolumn{2}{|c|}{ S1 } & \multicolumn{2}{|c|}{ S2 } & \multicolumn{2}{|c|}{ S3 } & \multicolumn{2}{|c|}{ S4 } & \multicolumn{2}{|c|}{ S5 } & \multicolumn{2}{|c|}{ S6 } & \multicolumn{2}{|c|}{ S7 } & \multicolumn{2}{|c|}{ S8 } & \multicolumn{2}{|c|}{ S9-S10 } & \multicolumn{2}{|c|}{ S11-S21 } & \multicolumn{2}{|c|}{ S22 } \\
\hline & $\mathbf{R}$ & $\mathbf{U}$ & $\mathbf{R}$ & $\mathbf{U}$ & $\mathbf{R}$ & $\mathbf{U}$ & $\mathbf{R}$ & $\mathbf{U}$ & $\mathbf{R}$ & $\mathbf{U}$ & $\mathbf{R}$ & $\mathbf{U}$ & $\mathbf{R}$ & $\mathbf{U}$ & $\mathbf{R}$ & $\mathbf{U}$ & $\mathbf{R}$ & $\mathbf{U}$ & $\mathbf{R}$ & $\mathbf{U}$ & $\mathbf{R}$ & $\mathbf{U}$ \\
\hline & & & & & & & & & & & & & & & & & & & & & & \\
\hline $\begin{array}{l}\text { Improve maintenance } \\
\text { of rail infrastructure } \\
\text { (Motion Sensitive } \\
\text { Devices, Constant } \\
\text { Warning Time) }\end{array}$ & & & & & & & & & & & & & & & & & & & & & $\begin{array}{l}50 \\
(4)\end{array}$ & $\begin{array}{l}50 \\
(4)\end{array}$ \\
\hline $\begin{array}{l}\text { Improve maintenance } \\
\text { of train detective } \\
\text { devices }\end{array}$ & & & & & & & & & & & & & & & & & & & & & & \\
\hline $\begin{array}{l}\text { Improve maintenance } \\
\text { of visual/audio signals }\end{array}$ & & & & & & & & & & & & & & & & & & & & & & \\
\hline $\begin{array}{l}\text { Improve } \\
\text { maintenance/work of } \\
\text { existing gates }\end{array}$ & & & & & & & & & & & & & & & & & & & & & & \\
\hline $\begin{array}{l}\text { Improve pavement } \\
\text { markings and texturing }\end{array}$ & & & & & & & & & & & & & & & & & & & & & & \\
\hline $\begin{array}{l}\text { Improve signage of the } \\
\text { crosswalk }\end{array}$ & & & & & & & & & & & 25 & 25 & & & & & & & & & & \\
\hline $\begin{array}{l}\text { Improve skid } \\
\text { resistance }\end{array}$ & & & 9 & 9 & & & & & & & & & & & & & & & & & & \\
\hline $\begin{array}{l}\text { Improve winter } \\
\text { maintenance methods }\end{array}$ & & & & & & & & & & & & & & & & & & & & & & \\
\hline $\begin{array}{l}\text { Improve winter } \\
\text { maintenance } \\
\text { preparedness (shorter } \\
\text { response time) }\end{array}$ & & & & & & & & & & & & & & & & & & & & & & \\
\hline $\begin{array}{l}\text { Improve/install } \\
\text { reflective signs }\end{array}$ & & & 15 & 15 & & & & & & & & & & & & & & & & & & \\
\hline $\begin{array}{l}\text { Improve/install } \\
\text { reflectorized pavement } \\
\text { markers }\end{array}$ & & & & & & & & & & & & & & & & & & & & & & \\
\hline $\begin{array}{l}\text { Install a bicycles } \\
\text { overpass or underpass }\end{array}$ & & & & & & & & & & & & & & & & & & & & & & \\
\hline Install a fence or & & & & & & & & & & & & & & & & & & & & & & \\
\hline
\end{tabular}




\begin{tabular}{|c|c|c|c|c|c|c|c|c|c|c|c|c|c|c|c|c|c|c|c|c|c|c|}
\hline \multirow{3}{*}{$\begin{array}{l}\text { chicane to prevent } \\
\text { bicycles running across } \\
\text { the track }\end{array}$} & \multicolumn{2}{|c|}{ S1 } & \multicolumn{2}{|c|}{ S2 } & \multicolumn{2}{|c|}{ S3 } & \multicolumn{2}{|c|}{ S4 } & \multicolumn{2}{|c|}{ S5 } & \multicolumn{2}{|c|}{ S6 } & \multicolumn{2}{|c|}{ S7 } & \multicolumn{2}{|c|}{ S8 } & \multicolumn{2}{|c|}{ S9-S10 } & \multicolumn{2}{|c|}{ S11-S21 } & \multicolumn{2}{|c|}{$\mathrm{S} 22$} \\
\hline & $\mathbf{R}$ & $\mathbf{U}$ & $\mathbf{R}$ & $\mathbf{U}$ & $\mathbf{R}$ & $\mathbf{U}$ & $\mathbf{R}$ & $\mathbf{U}$ & $\mathbf{R}$ & $\mathbf{U}$ & $\mathbf{R}$ & $\mathbf{U}$ & $\mathbf{R}$ & $\mathbf{U}$ & $\mathbf{R}$ & $\mathbf{U}$ & $\mathbf{R}$ & $\mathbf{U}$ & $\mathbf{R}$ & $\mathbf{U}$ & $\mathbf{R}$ & $\mathbf{U}$ \\
\hline & & & & & & & & & & & & & & & & & & & & & & \\
\hline \multicolumn{23}{|l|}{$\begin{array}{l}\text { Install a fence or } \\
\text { chicane to prevent } \\
\text { pedestrians running } \\
\text { across the track }\end{array}$} \\
\hline $\begin{array}{l}\text { Install a pedestrian } \\
\text { overpass or underpass }\end{array}$ & & & 86 & 86 & & & & & & & & & & & & & & & & & & \\
\hline $\begin{array}{l}\text { Install active control } \\
\text { devices }\end{array}$ & 50 & 50 & & & & & & & & & & & & & & & & & & & & \\
\hline \multicolumn{23}{|l|}{$\begin{array}{l}\text { Install adequate control } \\
\text { device }\end{array}$} \\
\hline $\begin{array}{l}\text { Install advance } \\
\text { warning sign }\end{array}$ & 20 & 20 & 25 & 25 & & & $\begin{array}{l}30 \\
(5)\end{array}$ & $\begin{array}{l}30 \\
(5) \\
\end{array}$ & 40 & 40 & & & 30 & 40 & & & & & & & 50 & 50 \\
\hline $\begin{array}{l}\text { Install audible signals } \\
\text { (warning bell) }\end{array}$ & & & & & & & & & 50 & 50 & & & & & & & & & & & & \\
\hline $\begin{array}{l}\text { Install automated } \\
\text { bicycles/pedestrians } \\
\text { gates }\end{array}$ & & & & & & & & & & & & & & & & & & & & & 50 & 50 \\
\hline \multicolumn{23}{|l|}{$\begin{array}{l}\text { Install automated } \\
\text { pedestrian gates }\end{array}$} \\
\hline Install automatic gates & & & 75 & 75 & & & & & & & & & & & & & & & & & & \\
\hline \multicolumn{23}{|l|}{ Install bicycles signals } \\
\hline \multicolumn{23}{|l|}{$\begin{array}{l}\text { Install cantilever } \\
\text { flashing-light signal }\end{array}$} \\
\hline \multicolumn{23}{|l|}{ Install corner mirrors } \\
\hline Install crossbuck & & & & & & & & & 60 & 60 & & & & & & & & & & & & \\
\hline \multicolumn{23}{|l|}{$\begin{array}{l}\text { Install DO NOT STOP } \\
\text { ON TRACKS signal }\end{array}$} \\
\hline \multicolumn{23}{|l|}{$\begin{array}{l}\text { Install fixed message } \\
\text { signs }\end{array}$} \\
\hline Install flashers & & & 75 & 75 & & & & & & & & & & & & & & & & & & \\
\hline
\end{tabular}




\begin{tabular}{|c|c|c|c|c|c|c|c|c|c|c|c|c|c|c|c|c|c|c|c|c|c|c|}
\hline \multirow{3}{*}{ Install flashing beacons } & \multicolumn{2}{|c|}{ S1 } & \multicolumn{2}{|c|}{ S2 } & \multicolumn{2}{|c|}{ S3 } & \multicolumn{2}{|c|}{ S4 } & \multicolumn{2}{|c|}{ S5 } & \multicolumn{2}{|c|}{ S6 } & \multicolumn{2}{|c|}{ S7 } & \multicolumn{2}{|c|}{ S8 } & \multicolumn{2}{|c|}{ S9-S10 } & \multicolumn{2}{|c|}{ S11-S21 } & \multicolumn{2}{|c|}{ S22 } \\
\hline & \multirow[b]{2}{*}{30} & \multirow{2}{*}{$\mathbf{U}$} & \multirow{2}{*}{$\begin{array}{l}\mathbf{R} \\
65 \\
\end{array}$} & \multirow{2}{*}{$\begin{array}{l}\mathbf{U} \\
65\end{array}$} & \multirow{2}{*}{$\begin{array}{l}\mathbf{R} \\
50 \\
\end{array}$} & \multirow{2}{*}{$\begin{array}{l}\mathbf{U} \\
50 \\
\end{array}$} & \multirow{2}{*}{\begin{tabular}{r|}
$\mathbf{R}$ \\
30 \\
$(5)$ \\
\end{tabular}} & \multirow{2}{*}{$\begin{array}{r}\mathbf{U} \\
30 \\
(5) \\
\end{array}$} & \multirow{2}{*}{$\begin{array}{l}\mathbf{R} \\
60 \\
\end{array}$} & \multirow{2}{*}{$\begin{array}{l}\mathbf{U} \\
60 \\
\end{array}$} & \multirow{2}{*}{$\begin{array}{l}\mathbf{R} \\
58 \\
\end{array}$} & \multirow{2}{*}{$\mathbf{U}$} & \multirow{2}{*}{$\begin{array}{l}\mathbf{R} \\
24 \\
\end{array}$} & \multirow{2}{*}{$\begin{array}{l}\mathbf{U} \\
24 \\
\end{array}$} & \multirow[t]{2}{*}{$\mathbf{R}$} & \multirow[t]{2}{*}{$\mathbf{U}$} & $\mathbf{R}$ & $\mathbf{U}$ & $\mathbf{R}$ & $\mathbf{U}$ & $\mathbf{R}$ & $\mathbf{U}$ \\
\hline & & & & & & & & & & & & & & & & & & & & & & \\
\hline $\begin{array}{l}\text { Install flashing light } \\
\text { activated by queuing } \\
\text { traffic in the exit lane(s) } \\
\text { from the crossing }\end{array}$ & & & & & & & & & & & & & & & & & & & & & & \\
\hline $\begin{array}{l}\text { Install fog - warning } \\
\text { signs }\end{array}$ & & & & & & & 25 & 25 & & & & & & & & & & & & & & \\
\hline $\begin{array}{l}\text { Install four-quadrant } \\
\text { gate arms }\end{array}$ & & & & & & & & & & & & & & & & & & & & & 55 & 55 \\
\hline Install gates & & & $\begin{array}{l}65 \\
(6)\end{array}$ & $\begin{array}{l}65 \\
(6)\end{array}$ & & & & & & & & & & & & & & & & & $\begin{array}{l}46 \\
(7)\end{array}$ & $\begin{array}{l}46 \\
(7)\end{array}$ \\
\hline $\begin{array}{l}\text { Install gates with self } \\
\text { deploying extension } \\
\text { sections }\end{array}$ & & & & & & & & & & & & & & & & & & & & & & \\
\hline Install lighting & 30 & 30 & 33 & 33 & $\begin{array}{l}50 \\
(8)\end{array}$ & $\begin{array}{l}50 \\
(8) \\
\end{array}$ & & & & & 30 & 30 & 37 & 37 & & & & & $\begin{array}{l}37 \\
(9)\end{array}$ & $\begin{array}{l}37 \\
(9)\end{array}$ & 45 & 45 \\
\hline Install median barriers & & & & & & & & & & & & & & & & & & & & & & \\
\hline $\begin{array}{l}\text { Install median } \\
\text { retroreflective post } \\
\text { delineators on the } \\
\text { approach }\end{array}$ & & & & & & & & & & & & & & & & & & & & & & \\
\hline $\begin{array}{l}\text { Install non-mountable } \\
\text { curb islands }\end{array}$ & & & & & & & & & & & & & & & & & & & & & & \\
\hline Install overhead signs & & & & & & & & & & & & & & & & & & & & & & \\
\hline $\begin{array}{l}\text { Install pavement } \\
\text { markings }\end{array}$ & 10 & 10 & 21 & 21 & & & & & 21 & 21 & & & & & 10 & 10 & & & 10 & 10 & & \\
\hline $\begin{array}{l}\text { Install pedestrian } \\
\text { barriers }\end{array}$ & & & & & & & & & & & & & & & & & & & & & & \\
\hline $\begin{array}{l}\text { Install pedestrian } \\
\text { signals }\end{array}$ & & & & & & & & & & & & & & & & & & & & & & \\
\hline Install pre-signals & & & & & & & & & & & & & & & & & & & & & 50 & 50 \\
\hline Install rumble strips & & & 32 & 32 & 28 & 28 & & & & & $\begin{array}{l}25 \\
(1\end{array}$ & $\begin{array}{l}25 \\
(1\end{array}$ & & & 21 & & & & 50 & 50 & & \\
\hline
\end{tabular}




\begin{tabular}{|c|c|c|c|c|c|c|c|c|c|c|c|c|c|c|c|c|c|c|c|c|c|c|}
\hline & \multicolumn{2}{|c|}{ S1 } & \multicolumn{2}{|c|}{ S2 } & \multicolumn{2}{|c|}{ S3 } & \multicolumn{2}{|c|}{ S4 } & \multicolumn{2}{|c|}{ S5 } & \multicolumn{2}{|c|}{ S6 } & \multicolumn{2}{|c|}{ S7 } & \multicolumn{2}{|c|}{ S8 } & \multicolumn{2}{|c|}{ S9-S10 } & \multicolumn{2}{|c|}{ S11-S21 } & \multicolumn{2}{|c|}{ S22 } \\
\hline & $\mathbf{R}$ & $\mathbf{U}$ & $\mathbf{R}$ & $\mathbf{U}$ & $\mathbf{R}$ & $\mathbf{U}$ & $\mathbf{R}$ & $\mathbf{U}$ & $\mathbf{R}$ & $\mathbf{U}$ & $\mathbf{R}$ & $\mathbf{U}$ & $\mathbf{R}$ & $\mathbf{U}$ & $\mathbf{R}$ & $\mathbf{U}$ & $\mathbf{R}$ & $\mathbf{U}$ & $\mathbf{R}$ & $\mathbf{U}$ & $\mathbf{R}$ & $\mathbf{U}$ \\
\hline & & & & & & & & & & & $0)$ & $0)$ & & & & & & & & & & \\
\hline $\begin{array}{l}\text { Install signs on both } \\
\text { sides of highway }\end{array}$ & & & & & & & & & & & & & & & & & & & & & & \\
\hline Install speed humps & & & & & & & & & & & & & & & & & & & & & 40 & 40 \\
\hline $\begin{array}{l}\text { Install static or variable } \\
\text { message signs } \\
\text { displaying weather } \\
\text { information }\end{array}$ & & & $\begin{array}{l}15 \\
(1 \\
1) \\
\end{array}$ & $\begin{array}{l}15 \\
(1 \\
1) \\
\end{array}$ & & & & & & & 15 & 15 & & & & & & & & & & \\
\hline Install stop sign & & & 35 & 35 & & & & & & & 39 & 39 & & & & & & & & & 46 & 46 \\
\hline Install street lights & 30 & 30 & & & $\begin{array}{l}50 \\
(8)\end{array}$ & $\begin{array}{l}50 \\
(8)\end{array}$ & & & & & & & & & & & & & & & & \\
\hline Install swing gates & & & & & & & & & & & & & & & & & & & & & & \\
\hline $\begin{array}{l}\text { Install symbol storage } \\
\text { space sign }\end{array}$ & & & & & & & & & & & & & & & & & & & & & & \\
\hline $\begin{array}{l}\text { Install variable } \\
\text { message signs and } \\
\text { blank out signs }\end{array}$ & & & & & & & & & & & & & & & & & & & & & & \\
\hline $\begin{array}{l}\text { Install vehicle-arresting } \\
\text { barriers }\end{array}$ & & & & & & & & & & & & & & & & & & & & & & \\
\hline $\begin{array}{l}\text { Install warning signs in } \\
\text { advance of rail-road } \\
\text { crossing }\end{array}$ & & & & & & & & & 40 & 40 & & & & & & & & & & & 50 & 50 \\
\hline Install yield sign & & & & & & & & & & & & & & & & & & & & & & \\
\hline $\begin{array}{l}\text { Installing snow screens } \\
\text { in areas exposed to } \\
\text { snowdrifts }\end{array}$ & & & & & & & & & & & & & & & & & & & & & & \\
\hline $\begin{array}{l}\text { Overlay pavement } \\
\text { (friction course) }\end{array}$ & & & 17 & 17 & 18 & 18 & 27 & 27 & & & & & & & & & & & & & & \\
\hline $\begin{array}{l}\text { Post a lower speed } \\
\text { limit for cars and/or } \\
\text { trains }\end{array}$ & & & & & & & & & & & & & & & & & & & & & & \\
\hline $\begin{array}{l}\text { Post an adequate } \\
\text { speed limit }\end{array}$ & & & & & 28 & 28 & & & & & & & 20 & 20 & & & & & & & & \\
\hline
\end{tabular}




\begin{tabular}{|c|c|c|c|c|c|c|c|c|c|c|c|c|c|c|c|c|c|c|c|c|c|c|}
\hline \multirow[b]{3}{*}{$\begin{array}{l}\text { Post dynamic message } \\
\text { sign to display the } \\
\text { speed of approaching } \\
\text { vehicles. }\end{array}$} & \multicolumn{2}{|c|}{ S1 } & \multicolumn{2}{|c|}{ S2 } & \multicolumn{2}{|c|}{ S3 } & \multicolumn{2}{|c|}{ S4 } & \multicolumn{2}{|c|}{ S5 } & \multicolumn{2}{|c|}{ S6 } & \multicolumn{2}{|c|}{ S7 } & \multicolumn{2}{|c|}{ S8 } & \multicolumn{2}{|c|}{ S9-S10 } & \multicolumn{2}{|c|}{$\mathrm{S} 11-\mathrm{S} 21$} & \multicolumn{2}{|c|}{ S22 } \\
\hline & $\mathbf{R}$ & $\mathbf{U}$ & $\mathbf{R}$ & $\mathbf{U}$ & $\mathbf{R}$ & $\mathbf{U}$ & $\mathbf{R}$ & $\mathbf{U}$ & $\mathbf{R}$ & $\mathbf{U}$ & $\mathbf{R}$ & $\mathbf{U}$ & $\mathbf{R}$ & $\mathbf{U}$ & $\mathbf{R}$ & $\mathbf{U}$ & $\mathbf{R}$ & $\mathbf{U}$ & $\mathbf{R}$ & $\mathbf{U}$ & $\mathbf{R}$ & $\mathbf{U}$ \\
\hline & & & & & & & & & & & & & & & & & $\begin{array}{l}74 \\
(1 \\
2)\end{array}$ & $\begin{array}{l}74 \\
(1 \\
2)\end{array}$ & & & & \\
\hline $\begin{array}{l}\text { Provide adequate } \\
\text { delay }\end{array}$ & & & & & & & & & & & & & & & & & & & & & $\begin{array}{l}33 \\
(1 \\
3)\end{array}$ & $\begin{array}{l}33 \\
(1 \\
3)\end{array}$ \\
\hline $\begin{array}{l}\text { Provide adequate } \\
\text { drainage }\end{array}$ & & & 32 & 32 & & & & & & & & & & & & & & & & & & \\
\hline $\begin{array}{l}\text { Provide adequate gate } \\
\text { interval }\end{array}$ & & & & & & & & & & & & & & & & & & & & & $\begin{array}{l}33 \\
(1 \\
3)\end{array}$ & $\begin{array}{l}33 \\
(1 \\
3)\end{array}$ \\
\hline $\begin{array}{l}\text { Provide adequate } \\
\text { information to train } \\
\text { operator (for example: } \\
\text { location of the whistle } \\
\text { post) }\end{array}$ & & & & & & & & & & & & & & & & & & & & & & \\
\hline $\begin{array}{l}\text { Provide information of } \\
\text { the crossing type }\end{array}$ & & & & & & & & & & & & & & & & & & & & & & \\
\hline $\begin{array}{l}\text { Provide pavement } \\
\text { marking }\end{array}$ & & & & & 35 & 35 & & & & & 10 & 10 & 35 & 35 & & & & & & & & \\
\hline $\begin{array}{l}\text { Provide photo video } \\
\text { enforcement }\end{array}$ & & & & & & & & & & & & & & & & & & & & & 56 & 56 \\
\hline $\begin{array}{l}\text { Provide targeted speed } \\
\text { enforcement }\end{array}$ & & & & & & & & & & & & & & & & & & & & & & \\
\hline $\begin{array}{l}\text { Provide traffic calming } \\
\text { on approaches through } \\
\text { a combination of } \\
\text { geometric and traffic } \\
\text { control devices }\end{array}$ & & & & & & & & & & & & & & & & & & & & & & \\
\hline $\begin{array}{l}\text { Provide/Improve } \\
\text { pavement surface } \\
\text { (improve transitions, } \\
\text { drop offs, fixed }\end{array}$ & & & & & & & & & & & 34 & 34 & & & & & & & & & & \\
\hline
\end{tabular}




\begin{tabular}{|c|c|c|c|c|c|c|c|c|c|c|c|c|c|c|c|c|c|c|c|c|c|c|}
\hline \multirow[b]{3}{*}{ potholes, rutting, etc) } & \multicolumn{2}{|c|}{ S1 } & \multicolumn{2}{|c|}{ S2 } & \multicolumn{2}{|c|}{ S3 } & \multicolumn{2}{|c|}{ S4 } & \multicolumn{2}{|c|}{ S5 } & \multicolumn{2}{|c|}{ S6 } & \multicolumn{2}{|c|}{ S7 } & \multicolumn{2}{|c|}{ S8 } & \multicolumn{2}{|c|}{ S9-S10 } & \multicolumn{2}{|c|}{ S11-S21 } & \multicolumn{2}{|c|}{ S22 } \\
\hline & $\mathbf{R}$ & $\mathbf{U}$ & $\mathbf{R}$ & $\mathbf{U}$ & $\mathbf{R}$ & $\mathbf{U}$ & $\mathbf{R}$ & $\mathbf{U}$ & $\mathbf{R}$ & $\mathbf{U}$ & $\mathbf{R}$ & $\mathbf{U}$ & $\mathbf{R}$ & $\mathbf{U}$ & $\mathbf{R}$ & $\mathbf{U}$ & $\mathbf{R}$ & $\mathbf{U}$ & $\mathbf{R}$ & $\mathbf{U}$ & $\mathbf{R}$ & $\mathbf{U}$ \\
\hline & & & & & & & & & & & & & & & & & & & & & & \\
\hline Relocate control devic & & & & & & & & & & & & & & & & & & & & & & \\
\hline $\begin{array}{l}\text { Relocate stop bar (If } \\
\text { stop sign present) }\end{array}$ & & & & & & & & & & & & & & & & & & & & & & \\
\hline Relocate stop sign & & & & & & & & & & & & & & & & & & & & & & \\
\hline $\begin{array}{l}\text { Relocate the crossing } \\
\text { section }\end{array}$ & & & & & & & & & & & & & & & & & & & & & & \\
\hline $\begin{array}{l}\text { Remove distracting } \\
\text { commercial lights }\end{array}$ & & & & & & & & & & & $\begin{array}{l}15 \\
(1 \\
4)\end{array}$ & $\begin{array}{l}15 \\
(1 \\
4) \\
\end{array}$ & & & & & & & & & & \\
\hline $\begin{array}{l}\text { Remove the object } \\
\text { obstructing the sight of } \\
\text { the control device }\end{array}$ & & & & & & & & & & & & & & & & & & & & & & \\
\hline $\begin{array}{l}\text { Remove the object } \\
\text { obstructing the sight of } \\
\text { the stop sign }\end{array}$ & & & & & & & & & & & & & & & & & & & & & 47 & 47 \\
\hline $\begin{array}{l}\text { Remove the object } \\
\text { obstructing the sight } \\
\text { triangle }\end{array}$ & $\begin{array}{l}12 \\
(1 \\
5) \\
\end{array}$ & & & & & & $\begin{array}{l}25 \\
(1 \\
6) \\
\end{array}$ & $\begin{array}{l}25 \\
(1 \\
6) \\
\end{array}$ & & & & & & & $\begin{array}{l}13 \\
(1 \\
7) \\
\end{array}$ & & & & 10 & 10 & 50 & 50 \\
\hline $\begin{array}{l}\text { Remove the object } \\
\text { obstructing view from } \\
\text { the approach }\end{array}$ & & & & & & & & & & & & & & & & & & & & & 50 & 50 \\
\hline $\begin{array}{l}\text { Replace / repair } \\
\text { signals }\end{array}$ & & & & & & & & & & & & & & & & & & & & & & \\
\hline $\begin{array}{l}\text { Replace, repair, or } \\
\text { clean the control } \\
\text { device }\end{array}$ & & & & & & & & & & & & & & & & & & & & & & \\
\hline $\begin{array}{l}\text { Replace, repair, or } \\
\text { clean the sign }\end{array}$ & & & & & $\begin{array}{l}5 \\
(1 \\
8)\end{array}$ & $\begin{array}{l}5 \\
(1 \\
8)\end{array}$ & & & & & & & & & & & & & & & & \\
\hline Trim vegetation & & & & & & & & & & & & & & & & & & & & & & \\
\hline Upgrade signing & & & & & $\begin{array}{l}5 \\
(1 \\
\end{array}$ & $\begin{array}{l}5 \\
(1 \\
\end{array}$ & & & & & 24 & 24 & & & & & & & & & & \\
\hline
\end{tabular}




\begin{tabular}{|c|c|c|c|c|c|c|c|c|c|c|c|c|c|c|c|c|c|c|c|c|c|c|}
\hline & \multicolumn{2}{|c|}{ S1 } & \multicolumn{2}{|c|}{ S2 } & \multicolumn{2}{|c|}{ S3 } & \multicolumn{2}{|c|}{ S4 } & \multicolumn{2}{|c|}{ S5 } & \multicolumn{2}{|c|}{ S6 } & \multicolumn{2}{|c|}{ S7 } & \multicolumn{2}{|c|}{ S8 } & \multicolumn{2}{|c|}{ S9-S10 } & \multicolumn{2}{|c|}{ S11-S21 } & \multicolumn{2}{|c|}{ S22 } \\
\hline & $\mathbf{R}$ & $\mathbf{U}$ & $\mathbf{R}$ & $\mathbf{U}$ & $\mathbf{R}$ & $\mathbf{U}$ & $\mathbf{R}$ & $\mathbf{U}$ & $\mathbf{R}$ & $\mathbf{U}$ & $\mathbf{R}$ & $\mathbf{U}$ & $\mathbf{R}$ & $\mathbf{U}$ & $\mathbf{R}$ & $\mathbf{U}$ & $\mathbf{R}$ & $\mathbf{U}$ & $\mathbf{R}$ & $\mathbf{U}$ & $\mathbf{R}$ & $\mathbf{U}$ \\
\hline & & & & & 8) & 8) & & & & & & & & & & & & & & & & \\
\hline $\begin{array}{l}\text { Use "Slippery when } \\
\text { wet" sign (temporary) }\end{array}$ & & & & & & & & & & & & & & & & & & & & & & \\
\hline $\begin{array}{l}\text { Use photo video } \\
\text { enforcement }\end{array}$ & & & & & & & & & & & & & & & & & & & & & 56 & 56 \\
\hline $\begin{array}{l}\text { Use salt to prevent } \\
\text { snow or ice from } \\
\text { forming or from sticking } \\
\text { to the road surface }\end{array}$ & & & & & & & & & & & & & & & & & & & & & & \\
\hline $\begin{array}{l}\text { Use sand to increase } \\
\text { pavement friction }\end{array}$ & & & & & & & & & & & & & & & & & & & & & & \\
\hline
\end{tabular}




\section{COMMENTS:}

(1) Crossing angle

(2) Post delineation

(3) Install flasher

(4) Constant warning

(5) Warning general

(6) Automated gates

(7) 2Q gates

(8) Provide illumination for intersection

(9) CRF between 25\%-50\% (average reported)

(10)Rumble strips general

(11)General variable message sign

(12)Reported speed reduction (not crashes reduction)

(13)Gate delay

(14)Glare screen

(15) The following reduction applies: in 1 quadrant - 5\%, in 2 quadrant - 9\%, in 3 quadrant 13\%, in 4 quadrant $-17 \%$

(16) Clear sight triangle

(17) 3 quadrant

(18) General upgrade signing 


\section{Road Segment}

\begin{tabular}{|c|c|c|c|c|c|c|c|c|c|c|c|c|c|c|c|c|c|c|c|c|}
\hline & \multicolumn{2}{|c|}{ S1 } & \multicolumn{2}{|c|}{ S2 } & \multicolumn{2}{|c|}{ S3 } & \multicolumn{2}{|c|}{ S4 } & \multicolumn{2}{|c|}{ S5 } & \multicolumn{2}{|c|}{ S6 } & \multicolumn{2}{|c|}{ S7 } & \multicolumn{2}{|c|}{ S8 } & \multicolumn{2}{|c|}{ S9,S10 } & \multicolumn{2}{|c|}{ S11-S21 } \\
\hline & $\mathbf{R}$ & $\mathbf{U}$ & $\mathbf{R}$ & $\mathbf{U}$ & $\mathbf{R}$ & $\mathbf{U}$ & $\mathbf{R}$ & $\mathbf{U}$ & $\mathbf{R}$ & $\mathbf{U}$ & $\mathbf{R}$ & $\mathbf{U}$ & $\mathbf{R}$ & $\mathbf{U}$ & $\mathbf{R}$ & $\mathbf{U}$ & $\mathbf{R}$ & $\mathbf{U}$ & $\mathbf{R}$ & $\mathbf{U}$ \\
\hline Add special signing & & & & & & & & & & & & & & & & & & & & \\
\hline $\begin{array}{l}\text { Centerline rumble } \\
\text { strips for two-lane } \\
\text { roads }\end{array}$ & & & $\begin{array}{l}32 \\
(1)\end{array}$ & $\begin{array}{l}32 \\
(1) \\
\end{array}$ & $\begin{array}{l}32 \\
(1) \\
\end{array}$ & $\begin{array}{l}32 \\
(1) \\
\end{array}$ & & & & & $\begin{array}{l}25 \\
(1) \\
\end{array}$ & $\begin{array}{l}25 \\
(1) \\
\end{array}$ & & & 12 & & 22 & 22 & & \\
\hline $\begin{array}{l}\text { Chip and seal or slurry } \\
\text { seal approaches }\end{array}$ & & & & & & & & & & & & & & & & & & & & \\
\hline $\begin{array}{l}\text { Close or relocate the } \\
\text { driveways }\end{array}$ & & & 50 & 50 & $\begin{array}{l}15 \\
(2) \\
\end{array}$ & $\begin{array}{l}15 \\
(2) \\
\end{array}$ & & & & & & & & & & & & & & \\
\hline $\begin{array}{l}\text { Construct pedestrian } \\
\text { refuge islands and } \\
\text { raised medians }\end{array}$ & & 46 & & & & & & & & & & & & & & & & & & \\
\hline $\begin{array}{l}\text { Create additional } \\
\text { parking spaces }\end{array}$ & & & & & & & & & & & & & & & & & & & & \\
\hline $\begin{array}{l}\text { Cut trees and other } \\
\text { roadside vegetation } \\
\text { obstruct the view }\end{array}$ & & & & & 25 & 25 & & & & & & & & & & & & & & \\
\hline $\begin{array}{l}\text { Design safer slopes } \\
\text { and ditches to prevent } \\
\text { rollovers }\end{array}$ & & & & & $\begin{array}{l}35 \\
(3)\end{array}$ & $\begin{array}{l}35 \\
(3)\end{array}$ & & & & & & & & & & & & & & \\
\hline $\begin{array}{l}\text { Eliminate screening by } \\
\text { physical objects }\end{array}$ & & & & & $\begin{array}{l}60 \\
(4) \\
\end{array}$ & $\begin{array}{l}60 \\
(4) \\
\end{array}$ & & & & & & & $\begin{array}{l}50 \\
(5) \\
\end{array}$ & $\begin{array}{l}50 \\
(5)\end{array}$ & & & & & $\begin{array}{l}76 \\
(6) \\
\end{array}$ & $\begin{array}{l}76 \\
(6)\end{array}$ \\
\hline $\begin{array}{l}\text { Eliminate shoulder } \\
\text { drop-offs }\end{array}$ & & & & & & & & & & & 25 & 25 & & & & & & & $\begin{array}{l}30 \\
(7)\end{array}$ & $\begin{array}{l}30 \\
(7)\end{array}$ \\
\hline $\begin{array}{l}\text { Eliminating compound } \\
\text { curves }\end{array}$ & & & & & & & & & & & & & & & & & & & & \\
\hline $\begin{array}{l}\text { Enhance delineation of } \\
\text { sharp curves }\end{array}$ & & & & & 16 & 16 & & & & & & & 17 & 17 & & & 15 & & 15 & \\
\hline $\begin{array}{l}\text { Enhance delineation of } \\
\text { sharp curves segment }\end{array}$ & & & & & 16 & 16 & & & & & & & & & & & 15 & & 15 & \\
\hline $\begin{array}{l}\text { Groove pavement } \\
\text { surface }\end{array}$ & & & 21 & 21 & & & 14 & 14 & & & & & & & & & & & 46 & 46 \\
\hline $\begin{array}{l}\text { Identify and treat truck } \\
\text { crash roadway }\end{array}$ & & & & & & & & & & & & & & & & & & & & \\
\hline
\end{tabular}




\begin{tabular}{|c|c|c|c|c|c|c|c|c|c|c|c|c|c|c|c|c|c|c|c|c|}
\hline & \multicolumn{2}{|c|}{ S1 } & \multicolumn{2}{|c|}{ S2 } & \multicolumn{2}{|c|}{ S3 } & \multicolumn{2}{|c|}{ S4 } & \multicolumn{2}{|c|}{ S5 } & \multicolumn{2}{|c|}{ S6 } & \multicolumn{2}{|c|}{ S7 } & \multicolumn{2}{|c|}{ S8 } & \multicolumn{2}{|c|}{ S9,S10 } & \multicolumn{2}{|c|}{ S11-S21 } \\
\hline & $\mathbf{R}$ & $\mathbf{U}$ & $\mathbf{R}$ & $\mathbf{U}$ & $\mathbf{R}$ & $\mathbf{U}$ & $\mathbf{R}$ & $\mathbf{U}$ & $\mathbf{R}$ & $\mathbf{U}$ & $\mathbf{R}$ & $\mathbf{U}$ & $\mathbf{R}$ & $\mathbf{U}$ & $\mathbf{R}$ & $\mathbf{U}$ & $\mathbf{R}$ & $\mathbf{U}$ & $\mathbf{R}$ & $\mathbf{U}$ \\
\hline segments-signing & & & & & & & & & & & & & & & & & & & & \\
\hline $\begin{array}{l}\text { Implement lighting / } \\
\text { illumination measures }\end{array}$ & 23 & 23 & 28 & 28 & & & & & & & 25 & 25 & 37 & 37 & & & & & $\begin{array}{l}37 \\
(8)\end{array}$ & $\begin{array}{l}37 \\
(8)\end{array}$ \\
\hline $\begin{array}{l}\text { Implement road } \\
\text { narrowing measures }\end{array}$ & & & & & & & & & & & & & & & & & & & & \\
\hline Improve alignment & & & & & & & & & & & & & & & & & & & & \\
\hline $\begin{array}{l}\text { Improve } \\
\text { channelization/delineati } \\
\text { on }\end{array}$ & & & & & & & & & & & $\begin{array}{l}30 \\
(9)\end{array}$ & $\begin{array}{l}30 \\
(9)\end{array}$ & 40 & 40 & & & & & & \\
\hline Improve clear zone & & & & & $\begin{array}{l}25 \\
(1 \\
0) \\
\end{array}$ & $\begin{array}{l}25 \\
(1 \\
0)\end{array}$ & & & & & & & & & & & & & & \\
\hline $\begin{array}{l}\text { Improve existing street } \\
\text { lights }\end{array}$ & 30 & 30 & & & $\begin{array}{l}50 \\
(1 \\
1) \\
\end{array}$ & $\begin{array}{l}50 \\
(1 \\
1) \\
\end{array}$ & & & & & & & & & & & & & & \\
\hline Improve lanes width & $\begin{array}{l}9 \\
(1 \\
2) \\
\end{array}$ & & & & & & & & & & $\begin{array}{l}25 \\
(1 \\
3)\end{array}$ & $\begin{array}{l}25 \\
(1 \\
3)\end{array}$ & $\begin{array}{l}23 \\
(1 \\
4)\end{array}$ & $\begin{array}{l}23 \\
(1 \\
4)\end{array}$ & $\begin{array}{l}8 \\
(1 \\
5)\end{array}$ & & & & & \\
\hline $\begin{array}{l}\text { Improve or restore } \\
\text { superelevation }\end{array}$ & $\begin{array}{l}11 \\
(1 \\
6)\end{array}$ & & 28 & 28 & & & & & & & $\begin{array}{l}40 \\
(1 \\
7)\end{array}$ & $\begin{array}{l}40 \\
(1 \\
7)\end{array}$ & & & $\begin{array}{l}11 \\
(1 \\
8) \\
\end{array}$ & & & & & \\
\hline $\begin{array}{l}\text { Improve pavement } \\
\text { markings }\end{array}$ & & & 21 & 21 & 10 & 10 & & & 21 & 21 & & & & & & & & & 10 & 10 \\
\hline $\begin{array}{l}\text { Improve pavements } \\
\text { resistant }\end{array}$ & & & & & & & & & & & & & & & & & & & & \\
\hline $\begin{array}{l}\text { Improve } \\
\text { reflectorization/conspic } \\
\text { uity of pedestrians }\end{array}$ & & & & & & & & & & & & & & & & & & & & \\
\hline $\begin{array}{l}\text { Improve signage } \\
\text { and/or marking of the } \\
\text { crosswalk }\end{array}$ & & & & & 5 & 5 & & & & & $\begin{array}{l}25 \\
(1 \\
9)\end{array}$ & $\begin{array}{l}25 \\
(1 \\
9) \\
\end{array}$ & & & & & & & & \\
\hline $\begin{array}{l}\text { Improve skid } \\
\text { resistance }\end{array}$ & & & 9 & 9 & & & & & & & & & & & & & & & 20 & 20 \\
\hline $\begin{array}{l}\text { Improve skid } \\
\text { resistance along the } \\
\text { segment }\end{array}$ & & & 9 & 9 & & & & & & & & & & & & & & & & \\
\hline
\end{tabular}




\begin{tabular}{|c|c|c|c|c|c|c|c|c|c|c|c|c|c|c|c|c|c|c|c|c|}
\hline & \multicolumn{2}{|c|}{ S1 } & \multicolumn{2}{|c|}{ S2 } & \multicolumn{2}{|c|}{ S3 } & \multicolumn{2}{|c|}{ S4 } & \multicolumn{2}{|c|}{ S5 } & \multicolumn{2}{|c|}{ S6 } & \multicolumn{2}{|c|}{ S7 } & \multicolumn{2}{|c|}{ S8 } & \multicolumn{2}{|c|}{ S9,S10 } & \multicolumn{2}{|c|}{ S11-S21 } \\
\hline & $\mathbf{R}$ & $\mathbf{U}$ & $\mathbf{R}$ & $\mathbf{U}$ & $\mathbf{R}$ & $\mathbf{U}$ & $\mathbf{R}$ & $\mathbf{U}$ & $\mathbf{R}$ & $\mathbf{U}$ & $\mathbf{R}$ & $\mathbf{U}$ & $\mathbf{R}$ & $\mathbf{U}$ & $\mathbf{R}$ & $\mathbf{U}$ & $\mathbf{R}$ & $\mathbf{U}$ & $\mathbf{R}$ & $\mathbf{U}$ \\
\hline $\begin{array}{l}\text { Improve winter } \\
\text { maintenance methods }\end{array}$ & & & & & & & & & & & & & & & & & & & & \\
\hline $\begin{array}{l}\text { Improve winter } \\
\text { maintenance } \\
\text { preparedness (shorter } \\
\text { response time) }\end{array}$ & & & & & & & & & & & & & & & & & & & & \\
\hline $\begin{array}{l}\text { Improve/install } \\
\text { reflective signs }\end{array}$ & & & 15 & 15 & & & & & & & & & & & & & & & & \\
\hline $\begin{array}{l}\text { Improve/install } \\
\text { reflectorized pavement } \\
\text { markers }\end{array}$ & & & & & & & & & & & & & & & & & & & & \\
\hline $\begin{array}{l}\text { Incorporate rumble } \\
\text { strips into new and } \\
\text { existing roadways }\end{array}$ & & & 32 & 32 & $\begin{array}{l}32 \\
(1)\end{array}$ & $\begin{array}{l}32 \\
(1)\end{array}$ & & & & & 25 & 25 & & & & & & & & \\
\hline $\begin{array}{l}\text { Increase efficiency of } \\
\text { use of existing parking } \\
\text { spaces }\end{array}$ & & & & & & & & & & & & & & & & & & & & \\
\hline $\begin{array}{l}\text { Increase width of the } \\
\text { shoulders }\end{array}$ & & & 14 & 14 & & & 8 & 8 & & & 20 & 20 & & & $\begin{array}{l}9 \\
(2 \\
0)\end{array}$ & & & & & \\
\hline $\begin{array}{l}\text { Install "Cross only at } \\
\text { Crosswalk" signs }\end{array}$ & & & & & & & & & & & & & & & & & & & & \\
\hline $\begin{array}{l}\text { Install a barrier curb or } \\
\text { a guardrail }\end{array}$ & & & 11 & 11 & 20 & 20 & 47 & 47 & & & & & & 4 & & & $\begin{array}{l}44 \\
(5) \\
\end{array}$ & $\begin{array}{l}44 \\
(5) \\
\end{array}$ & & \\
\hline $\begin{array}{l}\text { Install a bridge warning } \\
\text { sign }\end{array}$ & & & 25 & 25 & & & $\begin{array}{l}25 \\
(2 \\
1) \\
\end{array}$ & $\begin{array}{l}25 \\
(2 \\
1) \\
\end{array}$ & & & 34 & 34 & & & & & & & & \\
\hline $\begin{array}{l}\text { Install a pedestrian } \\
\text { overpass or underpass }\end{array}$ & & & 86 & 86 & & & & & & & & & & & & & & & 91 & 91 \\
\hline Install a tree marker & & & & & $\begin{array}{l}16 \\
(2 \\
2)\end{array}$ & $\begin{array}{l}16 \\
(2 \\
2)\end{array}$ & & & & & & & & & & & & & & \\
\hline $\begin{array}{l}\text { Install advance } \\
\text { warning signs }\end{array}$ & & & 25 & 25 & $\begin{array}{l}36 \\
(2 \\
3) \\
\end{array}$ & & $\begin{array}{l}25 \\
(2 \\
1) \\
\end{array}$ & $\begin{array}{l}25 \\
(2 \\
1) \\
\end{array}$ & & & & & & & & & & & & \\
\hline Install advisor speed & & & & & & & & & & & 26 & 26 & & & & & & & & \\
\hline
\end{tabular}




\begin{tabular}{|c|c|c|c|c|c|c|c|c|c|c|c|c|c|c|c|c|c|c|c|c|}
\hline & \multicolumn{2}{|c|}{ S1 } & \multicolumn{2}{|c|}{ S2 } & \multicolumn{2}{|c|}{ S3 } & \multicolumn{2}{|c|}{ S4 } & \multicolumn{2}{|c|}{ S5 } & \multicolumn{2}{|c|}{ S6 } & \multicolumn{2}{|c|}{ S7 } & \multicolumn{2}{|c|}{ S8 } & \multicolumn{2}{|c|}{ S9,S10 } & \multicolumn{2}{|c|}{ S11-S21 } \\
\hline & $\mathbf{R}$ & $\mathbf{U}$ & $\mathbf{R}$ & $\mathbf{U}$ & $\mathbf{R}$ & $\mathbf{U}$ & $\mathbf{R}$ & $\mathbf{U}$ & $\mathbf{R}$ & $\mathbf{U}$ & $\mathbf{R}$ & $\mathbf{U}$ & $\mathbf{R}$ & $\mathbf{U}$ & $\mathbf{R}$ & $\mathbf{U}$ & $\mathbf{R}$ & $\mathbf{U}$ & $\mathbf{R}$ & $\mathbf{U}$ \\
\hline sign & & & & & & & & & & & & & & & & & & & & \\
\hline $\begin{array}{l}\text { Install anutility polet } \\
\text { marker }\end{array}$ & & & & & $\begin{array}{l}16 \\
(2 \\
4)\end{array}$ & $\begin{array}{l}16 \\
(2 \\
4)\end{array}$ & & & & & & & & & & & & & & \\
\hline $\begin{array}{l}\text { Install central rumble } \\
\text { strips }\end{array}$ & $\begin{array}{l}12 \\
(2 \\
5) \\
\end{array}$ & & & & & & 14 & 14 & & & $\begin{array}{l}25 \\
(2 \\
6)\end{array}$ & $\begin{array}{l}25 \\
(2 \\
6)\end{array}$ & & & 12 & & 22 & 22 & 54 & 54 \\
\hline $\begin{array}{l}\text { Install channelization of } \\
\text { the driveways }\end{array}$ & & & & & & & & & $\begin{array}{l}50 \\
(2 \\
7)\end{array}$ & $\begin{array}{l}50 \\
(2 \\
7)\end{array}$ & & & & & & & & & & \\
\hline $\begin{array}{l}\text { Install curbing to define } \\
\text { driveway location }\end{array}$ & & & & & & & & & & & & & & & & & & & & \\
\hline Install curb-ramps & & & & & & & & & & & & & & & & & & & & \\
\hline Install flashing beacons & 30 & 30 & & & 50 & 50 & & & & & 30 & 30 & 54 & 54 & & & & & & \\
\hline $\begin{array}{l}\text { Install fog - warning } \\
\text { signs }\end{array}$ & & & & & & & $\begin{array}{l}25 \\
(2 \\
1) \\
\end{array}$ & $\begin{array}{l}25 \\
(2 \\
1) \\
\end{array}$ & & & & & & & & & & & & \\
\hline $\begin{array}{l}\text { Install Horizontal } \\
\text { alignment signs (Turn, } \\
\text { Curve, Reverse Turn, } \\
\text { Winding Road) }\end{array}$ & 30 & 30 & & & & & & & & & & & & & & & & & & \\
\hline $\begin{array}{l}\text { Install in-pavement } \\
\text { luminaires }\end{array}$ & & & & & & & & & & & & & & & & & & & & \\
\hline $\begin{array}{l}\text { Install interactive truck } \\
\text { rollover signing }\end{array}$ & & & & & & & & & & & & & & & & & & & & \\
\hline $\begin{array}{l}\text { Install larger regulatory } \\
\text { and warning signs at } \\
\text { and in advance of } \\
\text { hazardous location }\end{array}$ & & & & & & & & & & & $\begin{array}{l}32 \\
(2 \\
8)\end{array}$ & $\begin{array}{l}32 \\
(2 \\
8)\end{array}$ & & & & & & & & \\
\hline $\begin{array}{l}\text { Install LED barrier- } \\
\text { mounted guidance } \\
\text { tubes }\end{array}$ & & & & & & & & & & & & & & & & & & & & \\
\hline $\begin{array}{l}\text { Install light-emitting } \\
\text { diode (LED) }\end{array}$ & & & & & & & & & & & & & & & & & & & & \\
\hline Install lighting & 30 & 30 & 23 & 23 & $\begin{array}{l}50 \\
(1\end{array}$ & $\begin{array}{l}50 \\
(1\end{array}$ & & & & & 25 & 25 & 37 & 37 & & & & & $\begin{array}{l}37 \\
(8)\end{array}$ & $\begin{array}{l}37 \\
(8)\end{array}$ \\
\hline
\end{tabular}




\begin{tabular}{|c|c|c|c|c|c|c|c|c|c|c|c|c|c|c|c|c|c|c|c|c|}
\hline & \multicolumn{2}{|c|}{ S1 } & \multicolumn{2}{|c|}{ S2 } & \multicolumn{2}{|c|}{ S3 } & \multicolumn{2}{|c|}{ S4 } & \multicolumn{2}{|c|}{ S5 } & \multicolumn{2}{|c|}{ S6 } & \multicolumn{2}{|c|}{ S7 } & \multicolumn{2}{|c|}{ S8 } & \multicolumn{2}{|c|}{ S9,S10 } & \multicolumn{2}{|c|}{ S11-S21 } \\
\hline & $\mathbf{R}$ & $\mathbf{U}$ & $\mathbf{R}$ & $\mathbf{U}$ & $\mathbf{R}$ & $\mathbf{U}$ & $\mathbf{R}$ & $\mathbf{U}$ & $\mathbf{R}$ & $\mathbf{U}$ & $\mathbf{R}$ & $\mathbf{U}$ & $\mathbf{R}$ & $\mathbf{U}$ & $\mathbf{R}$ & $\mathbf{U}$ & $\mathbf{R}$ & $\mathbf{U}$ & $\mathbf{R}$ & $\mathbf{U}$ \\
\hline & & & & & 1) & 1) & & & & & & & & & & & & & & \\
\hline Install median barriers & $\begin{array}{l}60 \\
(5)\end{array}$ & & & & 61 & 61 & & & $\begin{array}{l}63 \\
(5)\end{array}$ & $\begin{array}{l}63 \\
(5) \\
\end{array}$ & & & $\begin{array}{l}90 \\
(5)\end{array}$ & $\begin{array}{l}90 \\
(5)\end{array}$ & $\begin{array}{l}90 \\
(5)\end{array}$ & & & & & \\
\hline Install median divider & & & 15 & 15 & & & & & & & & & & & & & & & & \\
\hline $\begin{array}{l}\text { Install midlane rumble } \\
\text { strips }\end{array}$ & & & $\begin{array}{l}32 \\
(2 \\
6) \\
\end{array}$ & $\begin{array}{l}32 \\
(2 \\
6) \\
\end{array}$ & & & 14 & 14 & & & $\begin{array}{l}25 \\
(2 \\
6) \\
\end{array}$ & $\begin{array}{l}25 \\
(2 \\
6) \\
\end{array}$ & & & 12 & & 22 & 22 & $\begin{array}{l}54 \\
(3 \\
0) \\
\end{array}$ & $\begin{array}{l}54 \\
(3 \\
0) \\
\end{array}$ \\
\hline $\begin{array}{l}\text { Install overhead } \\
\text { flashing beacon lights }\end{array}$ & 30 & 30 & & & $\begin{array}{l}50 \\
(1 \\
1) \\
\end{array}$ & $\begin{array}{l}50 \\
(1 \\
1) \\
\end{array}$ & & & & & 30 & 30 & $\begin{array}{l}54 \\
(3 \\
1) \\
\end{array}$ & $\begin{array}{l}54 \\
(3 \\
1) \\
\end{array}$ & & & & & & \\
\hline $\begin{array}{l}\text { Install } \\
\text { Overpasses/Underpas } \\
\text { ses }\end{array}$ & & & 86 & 86 & & & & & & & & & & & & & & & & \\
\hline $\begin{array}{l}\text { Install pavement } \\
\text { markings }\end{array}$ & & & 21 & 21 & 10 & 10 & & & 21 & 21 & & & & & & & & & 10 & 10 \\
\hline $\begin{array}{l}\text { Install pedestrian } \\
\text { actuated signals }\end{array}$ & & & & & & & & & & & & & & & & & & & & \\
\hline $\begin{array}{l}\text { Install pedestrian } \\
\text { barriers }\end{array}$ & & & & & & & & & & & & & & & & & & & & \\
\hline $\begin{array}{l}\text { Install pedestrian } \\
\text { crosswalk }\end{array}$ & & & 25 & 25 & & & & & 25 & 25 & & & & & & & & & & \\
\hline Install raised crosswalk & & & & & & & & & & & & & & & & & 46 & & & \\
\hline $\begin{array}{l}\text { Install reflectorized } \\
\text { pavement markings }\end{array}$ & 10 & 10 & 10 & 10 & & & & & & & & & & & & & & & & \\
\hline $\begin{array}{l}\text { Install regulatory signs } \\
\text { on the both side of the } \\
\text { road }\end{array}$ & & & & & & & & & & & & & & & & & & & & \\
\hline Install rumble strips & & & 32 & 32 & & & & & & & $\begin{array}{l}25 \\
(2 \\
6) \\
\end{array}$ & $\begin{array}{l}25 \\
(2 \\
6) \\
\end{array}$ & & & 21 & & & & & \\
\hline $\begin{array}{l}\text { Install school crossing } \\
\text { sign }\end{array}$ & & & 18 & 18 & & & & & & & 14 & 14 & & & & & & & & \\
\hline $\begin{array}{l}\text { Install school zone } \\
\text { markings }\end{array}$ & & & & & & & & & & & & & & & & & & & & \\
\hline $\begin{array}{l}\text { Install shoulder rumble } \\
\text { strips }\end{array}$ & 21 & & & & & & 29 & 29 & 30 & 30 & $\begin{array}{l}25 \\
(2\end{array}$ & $\begin{array}{l}25 \\
(2\end{array}$ & & & 21 & & 25 & 25 & 35 & 35 \\
\hline
\end{tabular}




\begin{tabular}{|c|c|c|c|c|c|c|c|c|c|c|c|c|c|c|c|c|c|c|c|c|}
\hline & \multicolumn{2}{|c|}{ S1 } & \multicolumn{2}{|c|}{ S2 } & \multicolumn{2}{|c|}{ S3 } & \multicolumn{2}{|c|}{ S4 } & \multicolumn{2}{|c|}{ S5 } & \multicolumn{2}{|c|}{ S6 } & \multicolumn{2}{|c|}{ S7 } & \multicolumn{2}{|c|}{ S8 } & \multicolumn{2}{|c|}{ S9,S10 } & \multicolumn{2}{|c|}{ S11-S21 } \\
\hline & $\mathbf{R}$ & $\mathbf{U}$ & $\mathbf{R}$ & $\mathbf{U}$ & $\mathbf{R}$ & $\mathbf{U}$ & $\mathbf{R}$ & $\mathbf{U}$ & $\mathbf{R}$ & $\mathbf{U}$ & $\mathbf{R}$ & $\mathbf{U}$ & $\mathbf{R}$ & $\mathbf{U}$ & $\mathbf{R}$ & $\mathbf{U}$ & $\mathbf{R}$ & $\mathbf{U}$ & $\mathbf{R}$ & $\mathbf{U}$ \\
\hline & & & & & & & & & & & 6) & 6) & & & & & & & & \\
\hline $\begin{array}{l}\text { Install sidewalk set- } \\
\text { backs }\end{array}$ & & & & & & & & & & & & & & & & & & & & \\
\hline Install sidewalks & & & 74 & 74 & & & & & & & 65 & 65 & & & & & & & $\begin{array}{l}70 \\
(3 \\
1) \\
\end{array}$ & $\begin{array}{l}70 \\
(3 \\
1) \\
\end{array}$ \\
\hline Install speed limit sign & & & & & & & & & & & & & & & & & & & & \\
\hline $\begin{array}{l}\text { Install static or variable } \\
\text { message signs } \\
\text { displaying weather } \\
\text { information }\end{array}$ & & & $\begin{array}{l}15 \\
(3 \\
2) \\
\end{array}$ & $\begin{array}{l}15 \\
(3 \\
2) \\
\end{array}$ & & & & & & & 15 & 15 & & & & & & & & \\
\hline Install street lights & 30 & 30 & & & $\begin{array}{l}50 \\
(1 \\
1) \\
\end{array}$ & $\begin{array}{l}50 \\
(1 \\
1) \\
\end{array}$ & & & & & & & & & & & & & & \\
\hline $\begin{array}{l}\text { Install warning signs } \\
\text { (can be install on both } \\
\text { side of the road) }\end{array}$ & & & 25 & 25 & $\begin{array}{l}36 \\
(3 \\
2) \\
\end{array}$ & & 25 & 25 & & & & & & & & & & & & \\
\hline $\begin{array}{l}\text { Install/Improve lighting } \\
\text { at the access points }\end{array}$ & & & 33 & 33 & & & & & & & 25 & 25 & 37 & 37 & & & & & $\begin{array}{l}37 \\
(3 \\
3) \\
37 \\
(8)\end{array}$ & $\begin{array}{l}37 \\
(8) \\
\end{array}$ \\
\hline $\begin{array}{l}\text { Installing snow screens } \\
\text { in areas exposed to } \\
\text { snowdrifts }\end{array}$ & & & & & 53 & 53 & & & & & & & & & & & & & & \\
\hline $\begin{array}{l}\text { Modify horizontal } \\
\text { alignment }\end{array}$ & & & 45 & 45 & 40 & & $\begin{array}{l}66 \\
(5) \\
\end{array}$ & $\begin{array}{l}66 \\
(5) \\
\end{array}$ & & & 40 & 40 & & & & & & & 64 & 64 \\
\hline $\begin{array}{l}\text { Modify roadside clear } \\
\text { zone }\end{array}$ & & & & & $\begin{array}{l}25 \\
(1 \\
0) \\
\end{array}$ & $\begin{array}{l}25 \\
(1 \\
0) \\
\end{array}$ & & & & & & & & & & & & & & \\
\hline $\begin{array}{l}\text { Modify speed limits } \\
\text { and increase } \\
\text { enforcement to reduce } \\
\text { truck and other vehicle } \\
\text { speeds }\end{array}$ & & & & & & & & & & & 20 & 20 & & & & & & & & \\
\hline Move driveway to side & & & 50 & 50 & 15 & 15 & & & & & & & & & & & & & & \\
\hline
\end{tabular}




\begin{tabular}{|c|c|c|c|c|c|c|c|c|c|c|c|c|c|c|c|c|c|c|c|c|}
\hline & \multicolumn{2}{|c|}{ S1 } & \multicolumn{2}{|c|}{ S2 } & \multicolumn{2}{|c|}{ S3 } & \multicolumn{2}{|c|}{ S4 } & \multicolumn{2}{|c|}{ S5 } & \multicolumn{2}{|c|}{ S6 } & \multicolumn{2}{|c|}{ S7 } & \multicolumn{2}{|c|}{ S8 } & \multicolumn{2}{|c|}{ S9,S10 } & \multicolumn{2}{|c|}{ S11-S21 } \\
\hline & $\mathbf{R}$ & $\mathbf{U}$ & $\mathbf{R}$ & $\mathbf{U}$ & $\mathbf{R}$ & $\mathbf{U}$ & $\mathbf{R}$ & $\mathbf{U}$ & $\mathbf{R}$ & $\mathbf{U}$ & $\mathbf{R}$ & $\mathbf{U}$ & $\mathbf{R}$ & $\mathbf{U}$ & $\mathbf{R}$ & $\mathbf{U}$ & $\mathbf{R}$ & $\mathbf{U}$ & $\mathbf{R}$ & $\mathbf{U}$ \\
\hline street & & & (2) & (2) & & & & & & & & & & & & & & & & \\
\hline $\begin{array}{l}\text { Overlay pavement } \\
\text { (friction course) }\end{array}$ & & & 17 & 17 & 18 & 18 & 27 & 27 & & & & & 13 & 34 & & & & & & \\
\hline Pave shoulders & & & 15 & 15 & & & & & & & 15 & 15 & & $\begin{array}{l}53 \\
(3 \\
3) \\
\end{array}$ & & & & & & \\
\hline $\begin{array}{l}\text { Place utilities } \\
\text { underground }\end{array}$ & & & & & & & & & & & & & & & & & & & & \\
\hline $\begin{array}{l}\text { Placed delineation on } \\
\text { guardrail located } \\
\text { outside the roadway } \\
\text { shoulder, along the } \\
\text { curve }\end{array}$ & & & & & 16 & 16 & & & & & $\begin{array}{l}30 \\
(9)\end{array}$ & $\begin{array}{l}30 \\
(9) \\
\end{array}$ & 17 & 17 & & & 15 & & 15 & \\
\hline $\begin{array}{l}\text { Post an adequate } \\
\text { speed limit }\end{array}$ & & & & & 28 & 28 & & & & & & & 20 & 20 & & & & & & \\
\hline $\begin{array}{l}\text { Post dynamic message } \\
\text { sign to display the } \\
\text { speed of approaching } \\
\text { vehicles. }\end{array}$ & & & & & & & & & & & & & & & & & $\begin{array}{l}74 \\
(3 \\
4) \\
\end{array}$ & $\begin{array}{l}74 \\
(3 \\
4)\end{array}$ & & \\
\hline $\begin{array}{l}\text { Profiled thermoplastic } \\
\text { stripes for centerline }\end{array}$ & & & & & & & & & & & & & & & & & & & & \\
\hline $\begin{array}{l}\text { Prohibit/Restrict trucks } \\
\text { with very Long } \\
\text { semitrailers on roads } \\
\text { with horizontal curves } \\
\text { that cannot } \\
\text { accommodate truck } \\
\text { offtracking }\end{array}$ & & & & & & & & & & & & & & & & & & & & \\
\hline $\begin{array}{l}\text { Provide acceleration } \\
\text { and deceleration lanes }\end{array}$ & & & 26 & 26 & & & & & & & 10 & 10 & & & & & & & & \\
\hline $\begin{array}{l}\text { Provide adequate } \\
\text { drainage }\end{array}$ & & & 20 & 20 & & & & & & & & & & & & & & & & \\
\hline $\begin{array}{l}\text { Provide adequate } \\
\text { speed limit for trucks }\end{array}$ & & & & & & & & & & & $\begin{array}{l}20 \\
(3 \\
5) \\
\end{array}$ & $\begin{array}{l}20 \\
(3 \\
5) \\
\end{array}$ & & & & & & & & \\
\hline Provide adequate & & & 87 & 87 & 88 & & 40 & 40 & 40 & 40 & 40 & 40 & 45 & 45 & & & & & & \\
\hline
\end{tabular}




\begin{tabular}{|c|c|c|c|c|c|c|c|c|c|c|c|c|c|c|c|c|c|c|c|c|}
\hline & \multicolumn{2}{|c|}{ S1 } & \multicolumn{2}{|c|}{ S2 } & \multicolumn{2}{|c|}{ S3 } & \multicolumn{2}{|c|}{ S4 } & \multicolumn{2}{|c|}{ S5 } & \multicolumn{2}{|c|}{ S6 } & \multicolumn{2}{|c|}{ S7 } & \multicolumn{2}{|c|}{ S8 } & \multicolumn{2}{|c|}{ S9,S10 } & \multicolumn{2}{|c|}{ S11-S21 } \\
\hline & $\mathbf{R}$ & $\mathbf{U}$ & $\mathbf{R}$ & $\mathbf{U}$ & $\mathbf{R}$ & $\mathbf{U}$ & $\mathbf{R}$ & $\mathbf{U}$ & $\mathbf{R}$ & $\mathbf{U}$ & $\mathbf{R}$ & $\mathbf{U}$ & $\mathbf{R}$ & $\mathbf{U}$ & $\mathbf{R}$ & $\mathbf{U}$ & $\mathbf{R}$ & $\mathbf{U}$ & $\mathbf{R}$ & $\mathbf{U}$ \\
\hline $\begin{array}{l}\text { vertical curve design } \\
\text { (crest/redesign) }\end{array}$ & & & & & & & & & & & & & & & & & & & & \\
\hline $\begin{array}{l}\text { Provide advance } \\
\text { warning of unexpected } \\
\text { changes in horizontal } \\
\text { alignment }\end{array}$ & & & & & & & $\begin{array}{l}25 \\
(2 \\
1) \\
\end{array}$ & $\begin{array}{l}25 \\
(2 \\
1) \\
\end{array}$ & & & & & 30 & 30 & & & & & 18 & 18 \\
\hline $\begin{array}{l}\text { Provide advance } \\
\text { warning of unexpected } \\
\text { changes in } \\
\text { horizontal/vertical } \\
\text { alignment }\end{array}$ & & & & & & & $\begin{array}{l}25 \\
(2 \\
1) \\
\end{array}$ & $\begin{array}{l}25 \\
(2 \\
1) \\
\end{array}$ & & & $\begin{array}{l}32 \\
(2 \\
8) \\
\end{array}$ & $\begin{array}{l}32 \\
(2 \\
8) \\
\end{array}$ & 30 & 30 & & & & & & \\
\hline $\begin{array}{l}\text { Provide advance } \\
\text { warning of unexpected } \\
\text { changes in vertical } \\
\text { alignment }\end{array}$ & & & & & & & $\begin{array}{l}25 \\
(2 \\
1)\end{array}$ & $\begin{array}{l}25 \\
(2 \\
1)\end{array}$ & & & $\begin{array}{l}32 \\
(2 \\
8)\end{array}$ & $\begin{array}{l}32 \\
(2 \\
8)\end{array}$ & 30 & 30 & & & & & & \\
\hline $\begin{array}{l}\text { Provide breakaway } \\
\text { and crashworthy mail } \\
\text { box }\end{array}$ & & & & & & & & & & & & & & & & & & & & \\
\hline $\begin{array}{l}\text { Provide breakaway } \\
\text { and crashworthy sign } \\
\text { supports }\end{array}$ & & & & & 30 & 30 & & & & & & & & & & & & & & \\
\hline Provide chevrons & & & & & 35 & 35 & & & & & 30 & 30 & 35 & 35 & & & 25 & & & \\
\hline $\begin{array}{l}\text { Provide dynamic curve } \\
\text { warning system }\end{array}$ & & & & & & & & & & & & & & & & & & & $\begin{array}{l}10 \\
0 \\
(3 \\
6)\end{array}$ & \\
\hline $\begin{array}{l}\text { Provide enhanced } \\
\text { pavement markings }\end{array}$ & & & 10 & 10 & 10 & 10 & & & & & & & & & & & & & 10 & 10 \\
\hline $\begin{array}{l}\text { Provide grooved } \\
\text { pavement }\end{array}$ & & & 21 & 21 & & & 14 & 14 & & & & & & & & & & & 46 & 46 \\
\hline $\begin{array}{l}\text { Provide independent } \\
\text { bicycle path where } \\
\text { necessary }\end{array}$ & & & & & & & & & & & & & & & & & & & & \\
\hline $\begin{array}{l}\text { Provide median } \\
\text { refuges }\end{array}$ & & $\begin{array}{l}46 \\
(3 \\
7) \\
\end{array}$ & & & & & & & & & & & & & & & & & & \\
\hline
\end{tabular}




\begin{tabular}{|c|c|c|c|c|c|c|c|c|c|c|c|c|c|c|c|c|c|c|c|c|}
\hline & \multicolumn{2}{|c|}{ S1 } & \multicolumn{2}{|c|}{ S2 } & \multicolumn{2}{|c|}{ S3 } & \multicolumn{2}{|c|}{ S4 } & \multicolumn{2}{|c|}{ S5 } & \multicolumn{2}{|c|}{ S6 } & \multicolumn{2}{|c|}{ S7 } & \multicolumn{2}{|c|}{ S8 } & \multicolumn{2}{|c|}{ S9,S10 } & \multicolumn{2}{|c|}{ S11-S21 } \\
\hline & $\mathbf{R}$ & $\mathbf{U}$ & $\mathbf{R}$ & $\mathbf{U}$ & $\mathbf{R}$ & $\mathbf{U}$ & $\mathbf{R}$ & $\mathbf{U}$ & $\mathbf{R}$ & $\mathbf{U}$ & $\mathbf{R}$ & $\mathbf{U}$ & $\mathbf{R}$ & $\mathbf{U}$ & $\mathbf{R}$ & $\mathbf{U}$ & $\mathbf{R}$ & $\mathbf{U}$ & $\mathbf{R}$ & $\mathbf{U}$ \\
\hline $\begin{array}{l}\text { Provide on-pavement } \\
\text { horizontal signing }\end{array}$ & & & & & & & & & & & & & & & & & $\begin{array}{l}10 \\
(3 \\
4)\end{array}$ & & & \\
\hline $\begin{array}{l}\text { Provide post-mounted } \\
\text { delineators }\end{array}$ & & & & & 16 & 16 & & & & & 30 & 30 & 25 & 25 & & & 15 & & 15 & \\
\hline $\begin{array}{l}\text { Provide smooth paved } \\
\text { shoulders }\end{array}$ & & & 15 & 15 & & & $\begin{array}{l}8 \\
(3 \\
8) \\
\end{array}$ & $\begin{array}{l}8 \\
(3 \\
8) \\
\end{array}$ & & & & & & $\begin{array}{l}53 \\
(3 \\
9) \\
\end{array}$ & & & & & & \\
\hline $\begin{array}{l}\text { Provide targeted speed } \\
\text { enforcement }\end{array}$ & & & & & & & & & & & & & & & & & & & & \\
\hline $\begin{array}{l}\text { Provide traffic calming } \\
\text { through a combination } \\
\text { of geometric and traffic } \\
\text { control devices }\end{array}$ & & & & & & & & & & & & & & & & & & & & \\
\hline $\begin{array}{l}\text { Provide wider cross } \\
\text { sections on two-lane } \\
\text { roads }\end{array}$ & & & & & & & & & & & & & & & & & & & & \\
\hline $\begin{array}{l}\text { Provide/Improve } \\
\text { pavement surface } \\
\text { (improve transitions, } \\
\text { drop offs, fixed } \\
\text { potholes, rutting, etc) }\end{array}$ & & & & & & & & & & & & & & & & & & & & \\
\hline $\begin{array}{l}\text { Provide/Improve } \\
\text { pavement surface } \\
\text { along the segment } \\
\text { (improve transitions, } \\
\text { drop offs, fixed } \\
\text { potholes, rutting, etc) }\end{array}$ & & & & & & & & & & & & & & & & & & & & \\
\hline $\begin{array}{l}\text { Providing spiral } \\
\text { transition curves }\end{array}$ & & & & & & & & & & & & & & & & & & & & \\
\hline $\begin{array}{l}\text { Raised pavement } \\
\text { markers }\end{array}$ & & & & & 9 & 9 & & & & & 10 & 10 & 4 & 4 & & & 9 & 9 & & \\
\hline $\begin{array}{l}\text { Reallocate total two- } \\
\text { lane roadway width } \\
\text { (lane and shoulder) to } \\
\text { include a narrow } \\
\text { "buffer median" }\end{array}$ & & & & & & & & & & & & & & & & & & & $\begin{array}{l}50 \\
(4 \\
0)\end{array}$ & $\begin{array}{l}50 \\
(4 \\
0)\end{array}$ \\
\hline
\end{tabular}




\begin{tabular}{|c|c|c|c|c|c|c|c|c|c|c|c|c|c|c|c|c|c|c|c|c|}
\hline & \multicolumn{2}{|c|}{ S1 } & \multicolumn{2}{|c|}{ S2 } & \multicolumn{2}{|c|}{ S3 } & \multicolumn{2}{|c|}{ S4 } & \multicolumn{2}{|c|}{ S5 } & \multicolumn{2}{|c|}{ S6 } & \multicolumn{2}{|c|}{ S7 } & \multicolumn{2}{|c|}{ S8 } & \multicolumn{2}{|c|}{ S9,S10 } & \multicolumn{2}{|c|}{ S11-S21 } \\
\hline & $\mathbf{R}$ & $\mathbf{U}$ & $\mathbf{R}$ & $\mathbf{U}$ & $\mathbf{R}$ & $\mathbf{U}$ & $\mathbf{R}$ & $\mathbf{U}$ & $\mathbf{R}$ & $\mathbf{U}$ & $\mathbf{R}$ & $\mathbf{U}$ & $\mathbf{R}$ & $\mathbf{U}$ & $\mathbf{R}$ & $\mathbf{U}$ & $\mathbf{R}$ & $\mathbf{U}$ & $\mathbf{R}$ & $\mathbf{U}$ \\
\hline $\begin{array}{l}\text { Relocate the sign to } \\
\text { make it visible }\end{array}$ & & & & & & & & & & & & & & & & & & & & \\
\hline $\begin{array}{l}\text { Remove distracting } \\
\text { commercial lights }\end{array}$ & & & & & & & & & & & & & & & & & & & & \\
\hline $\begin{array}{l}\text { Remove or relocate } \\
\text { mail box }\end{array}$ & & & & & $\begin{array}{l}29 \\
(4 \\
1) \\
\end{array}$ & $\begin{array}{l}29 \\
(4 \\
1) \\
\end{array}$ & & & & & $\begin{array}{l}30 \\
(4 \\
2) \\
\end{array}$ & $\begin{array}{l}30 \\
(4 \\
2) \\
\end{array}$ & & & & & & & $\begin{array}{l}27 \\
(4 \\
3) \\
\end{array}$ & $\begin{array}{l}27 \\
(4 \\
3) \\
\end{array}$ \\
\hline $\begin{array}{l}\text { Remove or relocate } \\
\text { sign }\end{array}$ & & & & & $\begin{array}{l}29 \\
(4 \\
1)\end{array}$ & $\begin{array}{l}29 \\
(4 \\
1)\end{array}$ & & & & & & & & & & & & & & \\
\hline $\begin{array}{l}\text { Remove or relocate } \\
\text { trees }\end{array}$ & & & & & 25 & 25 & & & & & & & & & & & & & $\begin{array}{l}66 \\
(5)\end{array}$ & $\begin{array}{l}66 \\
(5)\end{array}$ \\
\hline $\begin{array}{l}\text { Remove or relocate } \\
\text { utility Poles }\end{array}$ & & & & & 38 & 38 & & & & & & & & & & & & & 32 & \\
\hline $\begin{array}{l}\text { Remove sight } \\
\text { obstructions }\end{array}$ & & & & & & & & & & & & & & & & & & & & \\
\hline $\begin{array}{l}\text { Remove the object } \\
\text { obstructing sight of the } \\
\text { warning sign }\end{array}$ & & & & & & & & & & & & & & & & & & & & \\
\hline $\begin{array}{l}\text { Removed other } \\
\text { obstructions }\end{array}$ & & & & & 29 & 29 & & & & & & & & & & & & & & \\
\hline $\begin{array}{l}\text { Replace poorly } \\
\text { designed drain grates } \\
\text { with bicycle-safe types }\end{array}$ & & & & & & & & & & & & & & & & & & & & \\
\hline $\begin{array}{l}\text { Replace, repair, or } \\
\text { clean the warning } \\
\text { signs }\end{array}$ & & & & & & & $\begin{array}{l}25 \\
(2 \\
1)\end{array}$ & $\begin{array}{l}25 \\
(2 \\
1) \\
\end{array}$ & & & 24 & 24 & 30 & 40 & & & & & & \\
\hline $\begin{array}{l}\text { Restrict left-turning at } \\
\text { the access points }\end{array}$ & & & & & $\begin{array}{l}45 \\
(4 \\
4) \\
\end{array}$ & $\begin{array}{l}45 \\
(4 \\
4) \\
\end{array}$ & & & & & & & & & & & & & & \\
\hline Restrict parking & & & & & & & & & & & $\begin{array}{l}35 \\
(4 \\
5) \\
\end{array}$ & $\begin{array}{l}35 \\
(4 \\
5) \\
\end{array}$ & 8 & 8 & & & & & 10 & 10 \\
\hline $\begin{array}{l}\text { Restrict parking near } \\
\text { the driveway }\end{array}$ & & & & & & & & & & & & & & & & & & & & \\
\hline Shield drivers from & & & & & & & & & & & & & & & & & & & & \\
\hline
\end{tabular}




\begin{tabular}{|c|c|c|c|c|c|c|c|c|c|c|c|c|c|c|c|c|c|c|c|c|}
\hline & \multicolumn{2}{|c|}{ S1 } & \multicolumn{2}{|c|}{ S2 } & \multicolumn{2}{|c|}{ S3 } & \multicolumn{2}{|c|}{ S4 } & \multicolumn{2}{|c|}{ S5 } & \multicolumn{2}{|c|}{ S6 } & \multicolumn{2}{|c|}{ S7 } & \multicolumn{2}{|c|}{ S8 } & \multicolumn{2}{|c|}{ S9,S10 } & \multicolumn{2}{|c|}{ S11-S21 } \\
\hline & $\mathbf{R}$ & $\mathbf{U}$ & $\mathbf{R}$ & $\mathbf{U}$ & $\mathbf{R}$ & $\mathbf{U}$ & $\mathbf{R}$ & $\mathbf{U}$ & $\mathbf{R}$ & $\mathbf{U}$ & $\mathbf{R}$ & $\mathbf{U}$ & $\mathbf{R}$ & $\mathbf{U}$ & $\mathbf{R}$ & $\mathbf{U}$ & $\mathbf{R}$ & $\mathbf{U}$ & $\mathbf{R}$ & $\mathbf{U}$ \\
\hline $\begin{array}{l}\text { poles in hazardous } \\
\text { locations }\end{array}$ & & & & & & & & & & & & & & & & & & & & \\
\hline $\begin{array}{l}\text { Signals to alert } \\
\text { motorists that } \\
\text { pedestrians are } \\
\text { crossing }\end{array}$ & & & & & & & & & & & & & & & & & & & & \\
\hline Upgrade signing & & & & & 5 & 5 & & & & & 24 & 24 & & & & & & & & \\
\hline $\begin{array}{l}\text { Use "Slippery when } \\
\text { wet" sign (temporary) }\end{array}$ & & & & & & & & & & & & & & & & & & & & \\
\hline $\begin{array}{l}\text { Use alternating } \\
\text { passing lanes or four- } \\
\text { lane sections at key } \\
\text { locations }\end{array}$ & & & & & 30 & 30 & $\begin{array}{l}25 \\
(4 \\
6) \\
\end{array}$ & $\begin{array}{l}25 \\
(4 \\
6) \\
\end{array}$ & & & & & & & & & 35 & 35 & 25 & 25 \\
\hline $\begin{array}{l}\text { Use break-away } \\
\text { devices }\end{array}$ & & & & & 30 & 30 & & & & & & & & & & & & & & \\
\hline $\begin{array}{l}\text { Use salt to prevent } \\
\text { snow or ice from } \\
\text { forming or from sticking } \\
\text { to the road surface }\end{array}$ & & & & & & & & & & & & & & & & & & & & \\
\hline $\begin{array}{l}\text { Use sand to increase } \\
\text { pavement friction }\end{array}$ & & & & & & & & & & & & & & & & & & & & \\
\hline $\begin{array}{l}\text { Use school crossing } \\
\text { guards }\end{array}$ & & & & & & & & & & & & & & & & & & & & \\
\hline $\begin{array}{l}\text { Use transverse striping } \\
\text { treatments }\end{array}$ & & & & & & & & & & & & & & & & & & & & \\
\hline $\begin{array}{l}\text { Widen and/or pave } \\
\text { shoulders }\end{array}$ & $\begin{array}{l}9 \\
(4 \\
7) \\
\end{array}$ & & 14 & 14 & & & 8 & 8 & & & 20 & 20 & & $\begin{array}{l}53 \\
(4 \\
8) \\
\end{array}$ & & & & & 19 & 19 \\
\hline $\begin{array}{l}\text { Widen the outside } \\
\text { through lanes or add } \\
\text { bike lanes }\end{array}$ & & & & & & & & & & & & & & & & & & & & \\
\hline
\end{tabular}




\section{COMMENTS:}

(1) - install rumble strips (general)

(2) - relocate driveway entrance: left, right angle $\mathrm{CRF}=10 \%$; rear, head $\mathrm{CRF}=20 \%$

(3) - flatted side slope

(4) - relocate object (general) fatal only $\mathrm{CRF}=60 \%$

(5) - fatal only

(6) - conflict elimination

(7) - shoulder treatment

(8) - CRF for lighting between 25\%-50\% (average)

(9) - post delineation

(10) - widen by 5,8,10,15,20 [ft] CRF is respectively: $13 \%, 21 \%, 25 \%, 35 \%, 44 \%$

(11) - provide illumination for Intersection

(12) - for width: $2 \mathrm{ft}-8 \mathrm{ft}: 12 \%, 4 \mathrm{ft}-8 \mathrm{ft}: 9 \%, 6 \mathrm{ft}-8 \mathrm{ft}: 5 \%$ (only for paved shoulder)

(13) - widen pavement

(14) - average from $1 \mathrm{ft}$ and $2 \mathrm{ft}$

(15) - lane width: between $10 \mathrm{ft}-12 \mathrm{ft}$

(16) - CRF: $15 \%$ for $0.02-0.04 ; 11 \%$ for $0.04-0.06$ and $5 \%$ for $0.06-0.08$

(17) - modify superelevation

(18) - range 0.04-0.08

(19) - mark crosswalk

(20) - shoulder width: 4ft-8ft

(21) - warning general

(22) - object marker install

(23) $-\mathrm{CRF}=36 \%$ for rural 2 lane; more than 2 lane: $\mathrm{CRF}=18 \%$

(24) - object marker install

(25) $-\mathrm{CRF}=21 \%$ for head-on and sideswipe

(26) - rumble strips general

(27) - Channelization - CRF=50\% for Rear End, Head On, Sideswipe Same Direction, Sideswipe Opposite Direction, Left Turn, and Run-off-the-Road crashes.

(28) - curve warning sign

(29) - install mountable median

(30) - head-on only 
(31) - CRF between $50 \%-90 \%$

(32) $-\mathrm{CRF}=36 \%$ for rural 2 lane

(33) - paved multilane urban

(34) - speed reduction (not crashes)

(35) - modify speed limit

(36) - from 10 crashes to 0 during 3 years

(37) - pedestrians

(38) - improve/ widen shoulder

(39) - paved shoulder multilane urban

(40) - head-on collision only

(41) - removed fixed object (relocate $\mathrm{CRF}=35 \%$ )

(42) - remove fixed object

(43) - CRF between $14 \%-40 \%$

(44) - prohibit let-turn maneuver

(45) - eliminate parking

(46) - upgrade no passing zone

(47) - for width: 2-8:12\%, 4-8:9\%, 6-8:5\% (only for paved shoulder)

(48) - paved multilane urban area 


\section{Signalized Intersection}

\begin{tabular}{|c|c|c|c|c|c|c|c|c|c|c|c|c|c|c|c|c|c|c|c|c|}
\hline & \multicolumn{2}{|c|}{ S1 } & \multicolumn{2}{|c|}{ S2 } & \multicolumn{2}{|c|}{ S3 } & \multicolumn{2}{|c|}{ S4 } & \multicolumn{2}{|c|}{ S5 } & \multicolumn{2}{|c|}{ S6 } & \multicolumn{2}{|c|}{ S7 } & \multicolumn{2}{|c|}{ S8 } & \multicolumn{2}{|c|}{ S9,S10 } & \multicolumn{2}{|c|}{ S11-S21 } \\
\hline & $\mathbf{R}$ & $\mathbf{U}$ & $\mathbf{R}$ & $\mathbf{U}$ & $\mathbf{R}$ & $\mathbf{U}$ & $\mathbf{R}$ & $\mathbf{U}$ & $\mathbf{R}$ & $\mathbf{U}$ & $\mathbf{R}$ & $\mathbf{U}$ & $\mathbf{R}$ & $\mathbf{U}$ & $\mathbf{R}$ & $\mathbf{U}$ & $\mathbf{R}$ & $\mathbf{U}$ & $\mathbf{R}$ & $\mathbf{U}$ \\
\hline $\begin{array}{l}\text { Add } \\
\text { illuminated/reflectoriz } \\
\text { ed name signs }\end{array}$ & 23 & 23 & 28 & 28 & & & & & & & & & & & & & & & & \\
\hline Add special signing & & & & & & & & & & & & & & & & & & & & \\
\hline $\begin{array}{l}\text { Adjust minimum } \\
\text { green or extension } \\
\text { time }\end{array}$ & & & & & & & & & & & & & & & & & & & & \\
\hline $\begin{array}{l}\text { Adjust phase time } \\
\text { and cycle time }\end{array}$ & & & & & & & & & & & & & & & & & & & & \\
\hline $\begin{array}{l}\text { Adjust yellow change } \\
\text { interval }\end{array}$ & & & & & & & & & & & & & & & & $\begin{array}{l}8 \\
(1)\end{array}$ & & 8 & & \\
\hline $\begin{array}{l}\text { Change } \\
\text { horizontal/vertical } \\
\text { alignment }\end{array}$ & & & 58 & 58 & & & & & & & 50 & 50 & 50 & 50 & & & & & & \\
\hline $\begin{array}{l}\text { Channelize } \\
\text { intersections }\end{array}$ & & & & & & & & & $\begin{array}{l}50 \\
(2)\end{array}$ & $\begin{array}{l}50 \\
(2)\end{array}$ & & & 70 & 70 & & & & & & \\
\hline $\begin{array}{l}\text { Chip and seal or } \\
\text { slurry seal } \\
\text { approaches }\end{array}$ & & & & & & & & & & & & & & & & & & & & \\
\hline Clear sight triangles & $\begin{array}{l}12 \\
(3) \\
\end{array}$ & & & & & & 25 & 25 & & & & & & & & & & & & \\
\hline $\begin{array}{l}\text { Close intersection leg } \\
\text { or convert to one-way } \\
\text { street }\end{array}$ & & & & & & & & & & & & & & & & & & & & \\
\hline $\begin{array}{l}\text { Consider indirect left } \\
\text { turns }\end{array}$ & & & $\begin{array}{l}28 \\
(4) \\
\end{array}$ & $\begin{array}{l}28 \\
(4)\end{array}$ & & & 25 & 25 & 25 & 25 & 22 & 22 & & & & & & 37 & & \\
\hline $\begin{array}{l}\text { Construct } \\
\text { interchanges }\end{array}$ & & & & & & & & & & & & & & & & & & & & \\
\hline $\begin{array}{l}\text { Convert a four-leg } \\
\text { intersection to two } \mathrm{T} \\
\text { intersection }\end{array}$ & & & & & & & & & & & & & & & & & & & & \\
\hline $\begin{array}{l}\text { Convert intersection } \\
\text { to roundabouts }\end{array}$ & $\begin{array}{l}58 \\
(5)\end{array}$ & $\begin{array}{l}72 \\
(5)\end{array}$ & & & & & 40 & 40 & 40 & 40 & & & & & 58 & 72 & 58 & 72 & 38 & 38 \\
\hline
\end{tabular}




\begin{tabular}{|c|c|c|c|c|c|c|c|c|c|c|c|c|c|c|c|c|c|c|c|c|}
\hline & \multicolumn{2}{|c|}{ S1 } & \multicolumn{2}{|c|}{$\mathbf{S 2}$} & \multicolumn{2}{|c|}{ S3 } & \multicolumn{2}{|c|}{$\mathbf{S 4}$} & \multicolumn{2}{|c|}{ S5 } & \multicolumn{2}{|c|}{ S6 } & \multicolumn{2}{|c|}{ S7 } & \multicolumn{2}{|c|}{ S8 } & \multicolumn{2}{|c|}{ S9,S10 } & \multicolumn{2}{|c|}{ S11-S21 } \\
\hline & $\mathbf{R}$ & $\mathbf{U}$ & $\mathbf{R}$ & $\mathbf{U}$ & $\mathbf{R}$ & $\mathbf{U}$ & $\mathbf{R}$ & $\mathbf{U}$ & $\mathbf{R}$ & $\mathbf{U}$ & $\mathbf{R}$ & $\mathbf{U}$ & $\mathbf{R}$ & $\mathbf{U}$ & $\mathbf{R}$ & $\mathbf{U}$ & $\mathbf{R}$ & $\mathbf{U}$ & $\mathbf{R}$ & $\mathbf{U}$ \\
\hline $\begin{array}{l}\text { Convert two T } \\
\text { intersection to one } \\
\text { four-leg intersection }\end{array}$ & & & & & & & & & & & & & & & & & & & & \\
\hline $\begin{array}{l}\text { Convert two-way } \\
\text { streets to a one-way } \\
\text { pair }\end{array}$ & & & & & & & & & & & & & & & & & & & & \\
\hline $\begin{array}{l}\text { Coordinate the } \\
\text { railroad and traffic } \\
\text { signal to provide } \\
\text { preemption to the } \\
\text { traffic signal when } \\
\text { trains are } \\
\text { approaching the } \\
\text { intersection }\end{array}$ & & & & & & & $\begin{array}{l}30 \\
(6)\end{array}$ & $\begin{array}{l}30 \\
(6)\end{array}$ & & & & & & & & & & & 25 & 25 \\
\hline $\begin{array}{l}\text { Create one way } \\
\text { streets }\end{array}$ & & & & & & & & & & & & & & & & & & & & \\
\hline Delineate turn path & & & & & & & & & & & 30 & 30 & & & & & & & & \\
\hline $\begin{array}{l}\text { Eliminate screening } \\
\text { by physical objects }\end{array}$ & & & & & $\begin{array}{l}60 \\
(7)\end{array}$ & $\begin{array}{l}60 \\
(7)\end{array}$ & & & & & & & 50 & 50 & & & & & $\begin{array}{l}76 \\
(8)\end{array}$ & 76 \\
\hline $\begin{array}{l}\text { Employ emergency } \\
\text { vehicle preemption }\end{array}$ & & & & & & & & & & & & & & & & & & & $\begin{array}{l}70 \\
(9) \\
\end{array}$ & $\begin{array}{l}70 \\
(9) \\
\end{array}$ \\
\hline $\begin{array}{l}\text { Encourage safe } \\
\text { speeds through } \\
\text { design }\end{array}$ & & & & & & & & & & & & & & & & & & & & \\
\hline $\begin{array}{l}\text { Extend "no parking" } \\
\text { zone. }\end{array}$ & & & & & 22 & 22 & & & & & $\begin{array}{l}35 \\
(10 \\
) \\
\end{array}$ & $\begin{array}{l}35 \\
(10 \\
) \\
\end{array}$ & 8 & 8 & & & & & 10 & 10 \\
\hline $\begin{array}{l}\text { Groove pavement } \\
\text { surface }\end{array}$ & & & 21 & 21 & & & 14 & 14 & & & & & & & & & & & 46 & 46 \\
\hline $\begin{array}{l}\text { Implement automated } \\
\text { enforcement of red- } \\
\text { light running } \\
\text { (cameras) }\end{array}$ & & 9 & & & & & & & & & & & & & & 9 & & $\begin{array}{l}25 \\
(11 \\
)\end{array}$ & & \\
\hline $\begin{array}{l}\text { Improve (add) } \\
\text { coordinating signals }\end{array}$ & & & & & & & & & & & & & & & & & & & 25 & 25 \\
\hline Improve (add) & & & & & 30 & 30 & & & & & & & & & & & & & & \\
\hline
\end{tabular}




\begin{tabular}{|c|c|c|c|c|c|c|c|c|c|c|c|c|c|c|c|c|c|c|c|c|}
\hline & \multicolumn{2}{|c|}{ S1 } & \multicolumn{2}{|c|}{ S2 } & \multicolumn{2}{|c|}{ S3 } & \multicolumn{2}{|c|}{$\mathbf{S 4}$} & \multicolumn{2}{|c|}{ S5 } & \multicolumn{2}{|c|}{ S6 } & \multicolumn{2}{|c|}{ S7 } & \multicolumn{2}{|c|}{ S8 } & \multicolumn{2}{|c|}{ S9,S10 } & \multicolumn{2}{|c|}{ S11-S21 } \\
\hline & $\mathbf{R}$ & $\mathbf{U}$ & $\mathbf{R}$ & $\mathbf{U}$ & $\mathbf{R}$ & $\mathbf{U}$ & $\mathbf{R}$ & $\mathbf{U}$ & $\mathbf{R}$ & $\mathbf{U}$ & $\mathbf{R}$ & $\mathbf{U}$ & $\mathbf{R}$ & $\mathbf{U}$ & $\mathbf{R}$ & $\mathbf{U}$ & $\mathbf{R}$ & $\mathbf{U}$ & $\mathbf{R}$ & $\mathbf{U}$ \\
\hline phases (length) & & & & & )$^{(12}$ & )$^{(12}$ & & & & & & & & & & & & & & \\
\hline $\begin{array}{l}\text { Improve } \\
\text { alignment/grade }\end{array}$ & & & & & & & & & & & & & & & & & & & & \\
\hline $\begin{array}{l}\text { Improve existing } \\
\text { street lights }\end{array}$ & 30 & 30 & & & $\begin{array}{l}50 \\
(13 \\
) \\
\end{array}$ & $\begin{array}{l}50 \\
(13 \\
) \\
\end{array}$ & & & & & & & & & & & & & & \\
\hline $\begin{array}{l}\text { Improve intersection } \\
\text { angle }\end{array}$ & & & & & & & & & & & & & & & & & & & & \\
\hline $\begin{array}{l}\text { Improve intersection } \\
\text { skew angle }\end{array}$ & & & & & & & 40 & 40 & & & & & & & & & & & & \\
\hline $\begin{array}{l}\text { Improve left-turn lane } \\
\text { geometry }\end{array}$ & & & & & & & & & & & & & & & & & & & & \\
\hline $\begin{array}{l}\text { Improve right-turn } \\
\text { lane geometry }\end{array}$ & & & & & & & & & & & & & & & & & & & & \\
\hline $\begin{array}{l}\text { Improve signing and } \\
\text { delineation }\end{array}$ & & & & & & & & & & & $\begin{array}{l}30 \\
(14 \\
) \\
\end{array}$ & $\begin{array}{l}30 \\
(14 \\
)\end{array}$ & 17 & 17 & & & & & 15 & \\
\hline $\begin{array}{l}\text { Improve skid } \\
\text { resistance }\end{array}$ & & & 9 & 9 & & & & & & & & & & & & & & & & \\
\hline $\begin{array}{l}\text { Improve winter } \\
\text { maintenance } \\
\text { methods }\end{array}$ & & & & & & & & & & & & & & & & & & & & \\
\hline $\begin{array}{l}\text { Improve winter } \\
\text { maintenance } \\
\text { preparedness } \\
\text { (shorter response } \\
\text { time) }\end{array}$ & & & & & & & & & & & & & & & & & & & & \\
\hline $\begin{array}{l}\text { Improve/install } \\
\text { reflective signs }\end{array}$ & & & 15 & 15 & & & & & & & & & & & & & & & & \\
\hline $\begin{array}{l}\text { Improve/install } \\
\text { reflectorized } \\
\text { pavement markers }\end{array}$ & & & & & & & & & & & & & & & & & & & & \\
\hline $\begin{array}{l}\text { Increase the } \\
\text { crosswalk setback }\end{array}$ & & & & & & & & & & & & & & & & & & & & \\
\hline Install a barrier curb & & & 11 & 11 & 20 & 20 & 47 & 47 & & & & & & 4 & & & & 44 & & \\
\hline
\end{tabular}




\begin{tabular}{|c|c|c|c|c|c|c|c|c|c|c|c|c|c|c|c|c|c|c|c|c|}
\hline & \multicolumn{2}{|c|}{ S1 } & \multicolumn{2}{|c|}{ S2 } & \multicolumn{2}{|c|}{ S3 } & \multicolumn{2}{|c|}{ S4 } & \multicolumn{2}{|c|}{ S5 } & \multicolumn{2}{|c|}{ S6 } & \multicolumn{2}{|c|}{ S7 } & \multicolumn{2}{|c|}{ S8 } & \multicolumn{2}{|c|}{ S9,S10 } & \multicolumn{2}{|c|}{ S11-S21 } \\
\hline & $\mathbf{R}$ & $\mathbf{U}$ & $\mathbf{R}$ & $\mathbf{U}$ & $\mathbf{R}$ & $\mathbf{U}$ & $\mathbf{R}$ & $\mathbf{U}$ & $\mathbf{R}$ & $\mathbf{U}$ & $\mathbf{R}$ & $\mathbf{U}$ & $\mathbf{R}$ & $\mathbf{U}$ & $\mathbf{R}$ & $\mathbf{U}$ & $\mathbf{R}$ & $\mathbf{U}$ & $\mathbf{R}$ & $\mathbf{U}$ \\
\hline or a guardrail & & & & & & & )$^{(15}$ & )$^{15}$ & & & & & & & & & & )$^{(16}$ & & \\
\hline Install a tree marker & & & & & $\begin{array}{l}16 \\
(17 \\
)\end{array}$ & $\begin{array}{l}16 \\
(17 \\
)^{1}\end{array}$ & & & & & & & & & & & & & & \\
\hline $\begin{array}{l}\text { Install additional } \\
\text { signal head }\end{array}$ & & & & & $\begin{array}{l}50 \\
(18 \\
)^{5}\end{array}$ & $\begin{array}{l}50 \\
(18 \\
)^{2}\end{array}$ & & & & & & & )$^{31(19}$ & $\begin{array}{l}31 \\
(19 \\
) \\
\end{array}$ & & & & & & \\
\hline $\begin{array}{l}\text { Install adequate } \\
\text { signings }\end{array}$ & & & 30 & 30 & & & & & & & & & & & & & & & & \\
\hline $\begin{array}{l}\text { Install an utility poles } \\
\text { marker }\end{array}$ & & & & & $\begin{array}{l}16 \\
(17 \\
)\end{array}$ & $\begin{array}{l}16 \\
(17 \\
)^{17}\end{array}$ & & & & & & & & & & & & & & \\
\hline Install backplates & & & & & & & $\begin{array}{l}5 \\
(20 \\
) \\
\end{array}$ & $\begin{array}{l}5 \\
(20 \\
)\end{array}$ & & & 24 & 24 & & & & & & & & \\
\hline $\begin{array}{l}\text { Install bicycles traffic } \\
\text { lights }\end{array}$ & & & & & & & & & & & & & & & & & & & & \\
\hline Install curb-ramps & & & & & & & & & & & & & & & & & & & & \\
\hline $\begin{array}{l}\text { Install flashing } \\
\text { beacons }\end{array}$ & 30 & 30 & & & $\begin{array}{l}50 \\
(21 \\
)\end{array}$ & $\begin{array}{l}50 \\
(21 \\
)^{2}\end{array}$ & $\begin{array}{l}30 \\
(22 \\
)\end{array}$ & $\begin{array}{l}30 \\
(22 \\
)\end{array}$ & & & 30 & 30 & 24 & 24 & & & & & & \\
\hline $\begin{array}{l}\text { Install fog - warning } \\
\text { signs }\end{array}$ & & & $\begin{array}{l}25 \\
(23 \\
) \\
\end{array}$ & $\begin{array}{l}25 \\
(23 \\
) \\
\end{array}$ & & & $\begin{array}{l}25 \\
(24 \\
) \\
\end{array}$ & $\begin{array}{l}25 \\
(24 \\
) \\
\end{array}$ & & & & & 24 & 24 & & & & & & \\
\hline $\begin{array}{l}\text { Install larger signal } \\
\text { lenses }\end{array}$ & & & $\begin{array}{l}10 \\
(25 \\
)\end{array}$ & $\begin{array}{l}10 \\
(25 \\
)\end{array}$ & & & & & & & $\begin{array}{l}12 \\
(25 \\
)\end{array}$ & $\begin{array}{l}12 \\
(25 \\
)\end{array}$ & 10 & 10 & & & & & & \\
\hline Install larger signs & & & & & & & & & & & & & & & & & & & & \\
\hline Install left-turn lane & & & 28 & 28 & & & & & 25 & 25 & & & & & & & & & & \\
\hline Install median driver & & & & & & & & & & & & & & & & & & & & \\
\hline $\begin{array}{l}\text { Install multi-dialer } \\
\text { controller }\end{array}$ & & & & & & & & & & & & & & & & & & & & \\
\hline $\begin{array}{l}\text { Install overhead } \\
\text { street signing }\end{array}$ & & & & & & & & & & & & & & & & & & & & \\
\hline Install painted/raised & & & 23 & 23 & & & & & & & 10 & 10 & 10 & 10 & & & & & & \\
\hline
\end{tabular}




\begin{tabular}{|c|c|c|c|c|c|c|c|c|c|c|c|c|c|c|c|c|c|c|c|c|}
\hline & \multicolumn{2}{|c|}{ S1 } & \multicolumn{2}{|c|}{ S2 } & \multicolumn{2}{|c|}{ S3 } & \multicolumn{2}{|c|}{$\mathbf{S 4}$} & \multicolumn{2}{|c|}{ S5 } & \multicolumn{2}{|c|}{ S6 } & \multicolumn{2}{|c|}{ S7 } & \multicolumn{2}{|c|}{ S8 } & \multicolumn{2}{|c|}{ S9,S10 } & \multicolumn{2}{|c|}{ S11-S21 } \\
\hline & $\mathbf{R}$ & $\mathbf{U}$ & $\mathbf{R}$ & $\mathbf{U}$ & $\mathbf{R}$ & $\mathbf{U}$ & $\mathbf{R}$ & $\mathbf{U}$ & $\mathbf{R}$ & $\mathbf{U}$ & $\mathbf{R}$ & $\mathbf{U}$ & $\mathbf{R}$ & $\mathbf{U}$ & $\mathbf{R}$ & $\mathbf{U}$ & $\mathbf{R}$ & $\mathbf{U}$ & $\mathbf{R}$ & $\mathbf{U}$ \\
\hline $\begin{array}{l}\text { islands (provide } \\
\text { adequate visibility of } \\
\text { the island) }\end{array}$ & & & & & & & & & & & & & & & & & & & & \\
\hline $\begin{array}{l}\text { Install pedestrian } \\
\text { overpasses }\end{array}$ & & & 86 & 86 & & & & & & & & & & & & & & & 91 & 91 \\
\hline $\begin{array}{l}\text { Install physical } \\
\text { barriers to restrict } \\
\text { pedestrian crossing } \\
\text { maneuvers at higher- } \\
\text { risk locations }\end{array}$ & & & & & & & & & & & & & & & & & & & & \\
\hline $\begin{array}{l}\text { Install queue } \\
\text { detection system }\end{array}$ & & & & & & & & & & & & & & & & & & & & \\
\hline $\begin{array}{l}\text { Install raised } \\
\text { crosswalk }\end{array}$ & & & 25 & 25 & & & & & 25 & 25 & & & & & & & & 46 & & \\
\hline Install right-turn lane & & 4 & $\begin{array}{l}17 \\
(26 \\
\end{array}$ & $\begin{array}{l}17 \\
(26 \\
)\end{array}$ & & & & & & & & & & & & & & & & \\
\hline $\begin{array}{l}\text { Install rumble strips } \\
\text { on approach }\end{array}$ & & & $\begin{array}{l}32 \\
(27 \\
) \\
\end{array}$ & $\begin{array}{l}32 \\
(27 \\
) \\
\end{array}$ & & & & & & & $\begin{array}{l}25 \\
(28 \\
)\end{array}$ & $\begin{array}{l}25 \\
(28 \\
) \\
\end{array}$ & & & & & & & 50 & 50 \\
\hline $\begin{array}{l}\text { Install static or } \\
\text { variable message } \\
\text { signs displaying } \\
\text { weather information }\end{array}$ & & & $\begin{array}{l}15 \\
(29 \\
) \\
\end{array}$ & $\begin{array}{l}15 \\
(29 \\
) \\
\end{array}$ & & & & & & & $\begin{array}{l}15 \\
(30 \\
) \\
\end{array}$ & $\begin{array}{l}15 \\
(30 \\
) \\
\end{array}$ & & & & & & & & \\
\hline Install street lights & 30 & 30 & & & $\begin{array}{l}50 \\
(13 \\
) \\
\end{array}$ & $\begin{array}{l}50 \\
(13 \\
\end{array}$ & & & & & & & & & & & & & & \\
\hline $\begin{array}{l}\text { Install traffic actuated } \\
\text { signal }\end{array}$ & & & & & & & & & & & 22 & 22 & & & & & & & & \\
\hline Install warning sign & & & 25 & 25 & 20 & 20 & 25 & 25 & & & & & 20 & 15 & & & & & & \\
\hline $\begin{array}{l}\text { Install/improve center } \\
\text { line markings }\end{array}$ & & & 33 & 33 & 33 & 33 & & & & & 35 & 35 & & & & & & & & \\
\hline $\begin{array}{l}\text { Install/improve } \\
\text { Intersection lighting }\end{array}$ & 30 & 30 & 33 & 33 & $\begin{array}{l}50 \\
(31 \\
) \\
\end{array}$ & $\begin{array}{l}50 \\
(31 \\
) \\
\end{array}$ & & & & & 30 & 30 & 37 & 37 & & & & & 37 & 37 \\
\hline Installing snow & & & & & 53 & 53 & & & & & & & & & & & & & & \\
\hline
\end{tabular}




\begin{tabular}{|c|c|c|c|c|c|c|c|c|c|c|c|c|c|c|c|c|c|c|c|c|}
\hline & \multicolumn{2}{|c|}{ S1 } & \multicolumn{2}{|c|}{$\mathbf{S 2}$} & \multicolumn{2}{|c|}{ S3 } & \multicolumn{2}{|c|}{$\mathbf{S 4}$} & \multicolumn{2}{|c|}{ S5 } & \multicolumn{2}{|c|}{ S6 } & \multicolumn{2}{|c|}{ S7 } & \multicolumn{2}{|c|}{ S8 } & \multicolumn{2}{|c|}{ S9,S10 } & \multicolumn{2}{|c|}{ S11-S21 } \\
\hline & $\mathbf{R}$ & $\mathbf{U}$ & $\mathbf{R}$ & $\mathbf{U}$ & $\mathbf{R}$ & $\mathbf{U}$ & $\mathbf{R}$ & $\mathbf{U}$ & $\mathbf{R}$ & $\mathbf{U}$ & $\mathbf{R}$ & $\mathbf{U}$ & $\mathbf{R}$ & $\mathbf{U}$ & $\mathbf{R}$ & $\mathbf{U}$ & $\mathbf{R}$ & $\mathbf{U}$ & $\mathbf{R}$ & $\mathbf{U}$ \\
\hline $\begin{array}{l}\text { screens in areas } \\
\text { exposed to snowdrifts }\end{array}$ & & & & & & & & & & & & & & & & & & & & \\
\hline $\begin{array}{l}\text { Lengthen left-turn } \\
\text { lane }\end{array}$ & $\begin{array}{l}9 \\
(32 \\
) \\
\end{array}$ & & 28 & 28 & & & $\begin{array}{l}15 \\
(33 \\
) \\
\end{array}$ & $\begin{array}{l}15 \\
(33 \\
)\end{array}$ & & & & & & & & & & & & \\
\hline $\begin{array}{l}\text { Lengthen right-turn } \\
\text { lane }\end{array}$ & $\begin{array}{l}9 \\
(32 \\
)\end{array}$ & & 28 & 28 & & & $\begin{array}{l}15 \\
(33 \\
)\end{array}$ & $\begin{array}{l}15 \\
(33 \\
)\end{array}$ & & & & & & & & & & & & \\
\hline $\begin{array}{l}\text { Lengthening } \\
\text { clearance intervals }\end{array}$ & & $\begin{array}{l}8 \\
(34 \\
) \\
\end{array}$ & 15 & 15 & & & & & & & & & & & & & & 8 & & \\
\hline $\begin{array}{l}\text { Modify roadside clear } \\
\text { zone }\end{array}$ & & & & & $\begin{array}{l}25 \\
(35 \\
)\end{array}$ & $\begin{array}{l}25 \\
(35 \\
)\end{array}$ & & & & & & & & & & & & & 9 & 9 \\
\hline $\begin{array}{l}\text { Optimize clearance } \\
\text { interval }\end{array}$ & & & & & & & & & & & & & & & & & & & 9 & 9 \\
\hline $\begin{array}{l}\text { Overlay pavement } \\
\text { (friction course) }\end{array}$ & & & 17 & 17 & 18 & 18 & 27 & 27 & & & & & 13 & 34 & & & & & & \\
\hline $\begin{array}{l}\text { Place utilities } \\
\text { underground }\end{array}$ & & & & & & & & & & & & & & & & & & & & \\
\hline $\begin{array}{l}\text { Post an adequate } \\
\text { speed limit }\end{array}$ & & & & & 28 & 28 & & & & & & & 20 & 20 & & & & & & \\
\hline $\begin{array}{l}\text { Post dynamic } \\
\text { message sign to } \\
\text { display the speed of } \\
\text { approaching vehicles. }\end{array}$ & & & & & & & & & & & & & & & & & $\begin{array}{l}74 \\
(36 \\
)\end{array}$ & $\begin{array}{l}74 \\
(36 \\
) \\
\end{array}$ & & \\
\hline $\begin{array}{l}\text { Prohibit right turn on } \\
\text { red }\end{array}$ & & & 20 & 20 & 45 & 45 & & & & & & & & & & & & & & \\
\hline $\begin{array}{l}\text { Prohibit turning } \\
\text { movements }\end{array}$ & & & & & 40 & & & & & & 45 & 45 & & & & & & & & \\
\hline $\begin{array}{l}\text { Provide additional } \\
\text { pavement }\end{array}$ & & & & & & & & & & & & & & & & & & & & \\
\hline $\begin{array}{l}\text { Provide adequate } \\
\text { channelization and } \\
\text { dealination }\end{array}$ & & & 22 & 22 & & & & & $\begin{array}{l}50 \\
(2) \\
\end{array}$ & $\begin{array}{l}50 \\
(2) \\
\end{array}$ & & & & & & & & & & \\
\hline Provide adequate & & & 20 & 20 & & & & & & & & & & & & & & & & \\
\hline
\end{tabular}




\begin{tabular}{|c|c|c|c|c|c|c|c|c|c|c|c|c|c|c|c|c|c|c|c|c|}
\hline & \multicolumn{2}{|c|}{ S1 } & \multicolumn{2}{|c|}{ S2 } & \multicolumn{2}{|c|}{ S3 } & \multicolumn{2}{|c|}{$\mathbf{S 4}$} & \multicolumn{2}{|c|}{ S5 } & \multicolumn{2}{|c|}{ S6 } & \multicolumn{2}{|c|}{ S7 } & \multicolumn{2}{|c|}{ S8 } & \multicolumn{2}{|c|}{ S9,S10 } & \multicolumn{2}{|c|}{ S11-S21 } \\
\hline & $\mathbf{R}$ & $\mathbf{U}$ & $\mathbf{R}$ & $\mathbf{U}$ & $\mathbf{R}$ & $\mathbf{U}$ & $\mathbf{R}$ & $\mathbf{U}$ & $\mathbf{R}$ & $\mathbf{U}$ & $\mathbf{R}$ & $\mathbf{U}$ & $\mathbf{R}$ & $\mathbf{U}$ & $\mathbf{R}$ & $\mathbf{U}$ & $\mathbf{R}$ & $\mathbf{U}$ & $\mathbf{R}$ & $\mathbf{U}$ \\
\hline drainage & & & & & & & & & & & & & & & & & & & & \\
\hline $\begin{array}{l}\text { Provide adequate } \\
\text { lane width }\end{array}$ & $\begin{array}{l}9 \\
(32 \\
) \\
\end{array}$ & & & & & & & & & & $\begin{array}{l}25 \\
(37 \\
)\end{array}$ & $\begin{array}{l}25 \\
(37 \\
) \\
\end{array}$ & & & & $\begin{array}{l}8 \\
(38 \\
1\end{array}$ & & & & \\
\hline $\begin{array}{l}\text { Provide adequate } \\
\text { left-turn lane width }\end{array}$ & $\begin{array}{l}9 \\
(32 \\
) \\
\end{array}$ & & & & & & & & & & & & & & & $\begin{array}{l}8 \\
(38 \\
1 \\
\end{array}$ & & & & \\
\hline $\begin{array}{l}\text { Provide adequate } \\
\text { right-turn lane width }\end{array}$ & $\begin{array}{l}9 \\
(32 \\
)\end{array}$ & & & & & & & & & & & & & & & $\begin{array}{l}8 \\
(38 \\
)\end{array}$ & & & & \\
\hline $\begin{array}{l}\text { Provide adequate } \\
\text { signals for } \\
\text { handicapped }\end{array}$ & & & & & & & & & & & & & & & & & & & & \\
\hline $\begin{array}{l}\text { Provide adequate } \\
\text { signing }\end{array}$ & & & 30 & 30 & & & & & & & & & & & & & & & & \\
\hline $\begin{array}{l}\text { Provide adequate } \\
\text { signs and markings }\end{array}$ & & & 30 & 30 & & & & & & & & & & & & & & & & \\
\hline $\begin{array}{l}\text { Provide all-red } \\
\text { clearance interval }\end{array}$ & & & 15 & 15 & & & & & & & & & & & & & & & & \\
\hline $\begin{array}{l}\text { Provide appropriate } \\
\text { intervals for crossings } \\
\text { and minimize wait } \\
\text { time }\end{array}$ & & & & & & & & & & & & & & & & & & & & \\
\hline Provide bicycle box & & & & & & & & & & & & & & & & & & & & \\
\hline $\begin{array}{l}\text { Provide breakaway } \\
\text { and crashworthy sign } \\
\text { supports }\end{array}$ & & & & & 30 & 30 & & & & & & & & & & & & & & \\
\hline $\begin{array}{l}\text { Provide } \\
\text { channelization }\end{array}$ & & & 22 & 22 & & & & & $\begin{array}{l}50 \\
(2)\end{array}$ & $\begin{array}{l}50 \\
(2)\end{array}$ & & & & & & & & & & \\
\hline $\begin{array}{l}\text { Provide continuous } \\
\text { sidewalks }\end{array}$ & & & 74 & 74 & & & & & & & 65 & 65 & & & & & & & & \\
\hline $\begin{array}{l}\text { Provide crossing } \\
\text { guards for school } \\
\text { children }\end{array}$ & & & & & & & & & & & & & & & & & & & & \\
\hline $\begin{array}{l}\text { Provide far-side left- } \\
\text { turn signal }\end{array}$ & & & & & & & & & & & & & & & & & & & & \\
\hline
\end{tabular}




\begin{tabular}{|c|c|c|c|c|c|c|c|c|c|c|c|c|c|c|c|c|c|c|c|c|}
\hline & \multicolumn{2}{|c|}{ S1 } & \multicolumn{2}{|c|}{ S2 } & \multicolumn{2}{|c|}{ S3 } & \multicolumn{2}{|c|}{ S4 } & \multicolumn{2}{|c|}{ S5 } & \multicolumn{2}{|c|}{ S6 } & \multicolumn{2}{|c|}{ S7 } & \multicolumn{2}{|c|}{ S8 } & \multicolumn{2}{|c|}{ s9,S10 } & \multicolumn{2}{|c|}{ S11-S21 } \\
\hline & $\mathbf{R}$ & $\mathbf{U}$ & $\mathbf{R}$ & $\mathbf{U}$ & $\mathbf{R}$ & $\mathbf{U}$ & $\mathbf{R}$ & $\mathbf{U}$ & $\mathbf{R}$ & $\mathbf{U}$ & $\mathbf{R}$ & $\mathbf{U}$ & $\mathbf{R}$ & $\mathbf{U}$ & $\mathbf{R}$ & $\mathbf{U}$ & $\mathbf{R}$ & $\mathbf{U}$ & $\mathbf{R}$ & $\mathbf{U}$ \\
\hline $\begin{array}{l}\text { Provide grooved } \\
\text { pavement }\end{array}$ & & & 21 & 21 & & & 14 & 14 & & & & & & & & & & & 46 & 46 \\
\hline $\begin{array}{l}\text { Provide independent } \\
\text { bicycle path where } \\
\text { necessary }\end{array}$ & & & & & & & & & & & & & & & & & & & & \\
\hline $\begin{array}{l}\text { Provide indirect left } \\
\text { turn (can be for high } \\
\text { left turn volume) }\end{array}$ & & & $\begin{array}{l}28 \\
(4) \\
\end{array}$ & $\begin{array}{l}28 \\
(4) \\
\end{array}$ & & & 25 & 25 & 25 & 25 & 22 & 22 & & & & & & & & \\
\hline $\begin{array}{l}\text { Provide intersection } \\
\text { lighting }\end{array}$ & 30 & 30 & 33 & 33 & 50 & 50 & & & & & 30 & 30 & 37 & 37 & & & & 37 & 37 & 37 \\
\hline $\begin{array}{l}\text { Provide lights in } \\
\text { crosswalks in schools } \\
\text { zones }\end{array}$ & & & & & & & & & & & & & & & & & & & $\begin{array}{l}43 \\
(39 \\
1\end{array}$ & $\begin{array}{l}43 \\
(39 \\
) \\
\end{array}$ \\
\hline $\begin{array}{l}\text { Provide longer } \\
\text { clearance intervals }\end{array}$ & & & $\begin{array}{l}15 \\
(40 \\
) \\
\end{array}$ & $\begin{array}{l}15 \\
(40 \\
) \\
\end{array}$ & & & & & & & & & & & & & & & & \\
\hline $\begin{array}{l}\text { Provide louvers, } \\
\text { visors, or special } \\
\text { lenses so drivers are } \\
\text { able to view signals } \\
\text { only for their } \\
\text { approach }\end{array}$ & & & & & & & & & & & & & & & & & & & & \\
\hline $\begin{array}{l}\text { Provide median } \\
\text { refuges }\end{array}$ & & 46 & & & & & & & & & & & & & & & & & & \\
\hline $\begin{array}{l}\text { Provide offset for left } \\
\text { turn lanes on the } \\
\text { opposite approaches }\end{array}$ & & & & & & & $\begin{array}{l}37 \\
(41 \\
) \\
\end{array}$ & $\begin{array}{l}37 \\
(41 \\
) \\
\end{array}$ & & & & & & & & & & & & \\
\hline $\begin{array}{l}\text { Provide pavement } \\
\text { markings }\end{array}$ & & & 21 & 21 & 10 & 10 & & & 21 & 21 & & & & & & & & & 10 & 10 \\
\hline $\begin{array}{l}\text { Provide pedestrian } \\
\text { push buttons }\end{array}$ & & & & & & & & & & & & & & & & & & & & \\
\hline $\begin{array}{l}\text { Provide pedestrian- } \\
\text { only phase or } \\
\text { pedestrian-lead } \\
\text { phase during signal } \\
\text { operation }\end{array}$ & & & 20 & 20 & & & & & & & 23 & 23 & & & & & & & $\begin{array}{l}50 \\
(42 \\
)\end{array}$ & $\begin{array}{l}50 \\
(42 \\
)\end{array}$ \\
\hline
\end{tabular}




\begin{tabular}{|c|c|c|c|c|c|c|c|c|c|c|c|c|c|c|c|c|c|c|c|c|}
\hline & \multicolumn{2}{|c|}{ S1 } & \multicolumn{2}{|c|}{ S2 } & \multicolumn{2}{|c|}{ S3 } & \multicolumn{2}{|c|}{ S4 } & \multicolumn{2}{|c|}{ S5 } & \multicolumn{2}{|c|}{ S6 } & \multicolumn{2}{|c|}{ S7 } & \multicolumn{2}{|c|}{ S8 } & \multicolumn{2}{|c|}{ s9,S10 } & \multicolumn{2}{|c|}{ S11-S21 } \\
\hline & $\mathbf{R}$ & $\mathbf{U}$ & $\mathbf{R}$ & $\mathbf{U}$ & $\mathbf{R}$ & $\mathbf{U}$ & $\mathbf{R}$ & $\mathbf{U}$ & $\mathbf{R}$ & $\mathbf{U}$ & $\mathbf{R}$ & $\mathbf{U}$ & $\mathbf{R}$ & $\mathbf{U}$ & $\mathbf{R}$ & $\mathbf{U}$ & $\mathbf{R}$ & $\mathbf{U}$ & $\mathbf{R}$ & $\mathbf{U}$ \\
\hline $\begin{array}{l}\text { Provide positive } \\
\text { offset for left-turn } \\
\text { lanes }\end{array}$ & & & & & & & $\begin{array}{l}37 \\
(41 \\
) \\
\end{array}$ & $\begin{array}{l}37 \\
(41 \\
) \\
\end{array}$ & & & & & & & & & & & & \\
\hline $\begin{array}{l}\text { Provide right turn } \\
\text { lane }\end{array}$ & & & 25 & 25 & & & 25 & 25 & & & 24 & 24 & & & & 4 & & $\begin{array}{l}8 \\
(43 \\
1 \\
\end{array}$ & & \\
\hline $\begin{array}{l}\text { Provide roadside } \\
\text { delineators }\end{array}$ & & & & & & & & & & & 30 & 30 & & & & & & & 15 & \\
\hline $\begin{array}{l}\text { Provide sidewalk set- } \\
\text { backs }\end{array}$ & & & & & & & & & & & & & & & & & & & & \\
\hline $\begin{array}{l}\text { Provide smooth } \\
\text { paved shoulders }\end{array}$ & & & 15 & 15 & & & & & & & 15 & 15 & & $\begin{array}{l}53 \\
(44 \\
) \\
\end{array}$ & & & & & & \\
\hline $\begin{array}{l}\text { Provide target } \\
\text { conventional } \\
\text { enforcement of traffic } \\
\text { laws }\end{array}$ & & & & & & & & & & & & & & & & & & & & \\
\hline $\begin{array}{l}\text { Provide target } \\
\text { enforcement }\end{array}$ & & & & & & & & & & & & & & & & & & & & \\
\hline $\begin{array}{l}\text { Provide targeted } \\
\text { speed enforcement }\end{array}$ & & & & & & & & & & & & & & & & & & & & \\
\hline $\begin{array}{l}\text { Provide traffic } \\
\text { calming on } \\
\text { intersection } \\
\text { approaches through } \\
\text { a combination of } \\
\text { geometric and traffic } \\
\text { control devices }\end{array}$ & & & & & & & & & & & & & & & & & & & & \\
\hline $\begin{array}{l}\text { Provide visors to } \\
\text { shade signal lenses } \\
\text { from sunlight }\end{array}$ & & & & & & & & & & & & & & & & & & & & \\
\hline $\begin{array}{l}\text { Provide/Improve } \\
\text { pavement surface } \\
\text { (improve transitions, } \\
\text { drop offs, fixed } \\
\text { potholes, rutting, etc) }\end{array}$ & & & & & & & & & & & & & & & & & & & & \\
\hline
\end{tabular}




\begin{tabular}{|c|c|c|c|c|c|c|c|c|c|c|c|c|c|c|c|c|c|c|c|c|}
\hline & \multicolumn{2}{|c|}{ S1 } & \multicolumn{2}{|c|}{$\mathrm{S} 2$} & \multicolumn{2}{|c|}{ S3 } & \multicolumn{2}{|c|}{ S4 } & \multicolumn{2}{|c|}{ S5 } & \multicolumn{2}{|c|}{ S6 } & \multicolumn{2}{|c|}{ S7 } & \multicolumn{2}{|c|}{ S8 } & \multicolumn{2}{|c|}{ S9,S10 } & \multicolumn{2}{|c|}{ S11-S21 } \\
\hline & $\mathbf{R}$ & $\mathbf{U}$ & $\mathbf{R}$ & $\mathbf{U}$ & $\mathbf{R}$ & $\mathbf{U}$ & $\mathbf{R}$ & $\mathbf{U}$ & $\mathbf{R}$ & $\mathbf{U}$ & $\mathbf{R}$ & $\mathbf{U}$ & $\mathbf{R}$ & $\mathbf{U}$ & $\mathbf{R}$ & $\mathbf{U}$ & $\mathbf{R}$ & $\mathbf{U}$ & $\mathbf{R}$ & $\mathbf{U}$ \\
\hline $\begin{array}{l}\text { Provide/improve } \\
\text { progression through } \\
\text { a set of signalized } \\
\text { intersections }\end{array}$ & & & & & & & & & & & & & & & & & & & & \\
\hline $\begin{array}{l}\text { Provide/improve } \\
\text { signal progression }\end{array}$ & & & & & & & & & & & & & & & & & & & & \\
\hline $\begin{array}{l}\text { Reconstruct } \\
\text { intersection }\end{array}$ & & & & & & & & & & & & & & & & & & & & \\
\hline $\begin{array}{l}\text { Redesign intersection } \\
\text { approaches }\end{array}$ & & & & & & & & & & & & & & & & & & & & \\
\hline $\begin{array}{l}\text { Reduce speed limit } \\
\text { on approaches }\end{array}$ & & & & & & & & & & & $\begin{array}{l}20 \\
(45 \\
) \\
\end{array}$ & $\begin{array}{l}20 \\
(45 \\
1\end{array}$ & 20 & 20 & & & & & & \\
\hline $\begin{array}{l}\text { Relocate of transit } \\
\text { stops from the near } \\
\text { side to the far side of } \\
\text { the intersection }\end{array}$ & & & & & & & & & & & & & & & & & & & & \\
\hline $\begin{array}{l}\text { Relocate signal } \\
\text { hardware out of clear } \\
\text { zone }\end{array}$ & & & & & & & & & & & & & & & & & & & & \\
\hline Relocate signals & & & & & & & & & & & & & & & & & & & & \\
\hline Relocate transit stop & & & & & & & & & & & & & & & & & & & & \\
\hline $\begin{array}{l}\text { Remove constriction } \\
\text { as parked vehicles }\end{array}$ & & & & & & & & & & & & & & & & & & & & \\
\hline $\begin{array}{l}\text { Remove deflection in } \\
\text { through-vehicle trave } \\
\text { path }\end{array}$ & & & & & & & & & & & & & & & & & & & & \\
\hline $\begin{array}{l}\text { Remove distracting } \\
\text { commercial lights }\end{array}$ & & & & & & & & & & & & & & & & & & & & \\
\hline $\begin{array}{l}\text { Remove late } \\
\text { night/early morning } \\
\text { flash }\end{array}$ & & & & & & & & & & & & & & & & & & & & \\
\hline $\begin{array}{l}\text { Remove or relocate } \\
\text { sign }\end{array}$ & & & & & & & & & & & & & & & & & & & & \\
\hline $\begin{array}{l}\text { Remove or re-locate } \\
\text { the median object }\end{array}$ & & & & & & & & & & & & & & & & & & & & \\
\hline
\end{tabular}




\begin{tabular}{|c|c|c|c|c|c|c|c|c|c|c|c|c|c|c|c|c|c|c|c|c|}
\hline & \multicolumn{2}{|c|}{ S1 } & \multicolumn{2}{|c|}{$\mathbf{S 2}$} & \multicolumn{2}{|c|}{ S3 } & \multicolumn{2}{|c|}{ S4 } & \multicolumn{2}{|c|}{ S5 } & \multicolumn{2}{|c|}{ S6 } & \multicolumn{2}{|c|}{ S7 } & \multicolumn{2}{|c|}{ S8 } & \multicolumn{2}{|c|}{ S9,S10 } & \multicolumn{2}{|c|}{ S11-S21 } \\
\hline & $\mathbf{R}$ & $\mathbf{U}$ & $\mathbf{R}$ & $\mathbf{U}$ & $\mathbf{R}$ & $\mathbf{U}$ & $\mathbf{R}$ & $\mathbf{U}$ & $\mathbf{R}$ & $\mathbf{U}$ & $\mathbf{R}$ & $\mathbf{U}$ & $\mathbf{R}$ & $\mathbf{U}$ & $\mathbf{R}$ & $\mathbf{U}$ & $\mathbf{R}$ & $\mathbf{U}$ & $\mathbf{R}$ & $\mathbf{U}$ \\
\hline $\begin{array}{l}\text { obstructing sight of } \\
\text { opposite traffic }\end{array}$ & & & & & & & & & & & & & & & & & & & & \\
\hline $\begin{array}{l}\text { Remove or relocate } \\
\text { trees }\end{array}$ & & & & & & & & & & & & & & & & & & & $\begin{array}{l}66 \\
(16 \\
)\end{array}$ & \\
\hline $\begin{array}{l}\text { Remove or relocate } \\
\text { utility poles }\end{array}$ & & & & & 38 & 38 & & & & & & & & & & & & & 32 & 32 \\
\hline $\begin{array}{l}\text { Remove sight } \\
\text { obstructions }\end{array}$ & $\begin{array}{l}12 \\
(3)\end{array}$ & & & & & & & & & & & & & & & & & & & \\
\hline $\begin{array}{l}\text { Remove the object } \\
\text { obstructing the sight } \\
\text { triangle }\end{array}$ & $\begin{array}{l}12 \\
(3) \\
\end{array}$ & & & & & & $\begin{array}{l}25 \\
(46 \\
1\end{array}$ & $\begin{array}{l}25 \\
(46 \\
1\end{array}$ & & & & & & & & & & & & \\
\hline $\begin{array}{l}\text { Remove unwarranted } \\
\text { signal }\end{array}$ & & & 75 & 75 & & & & & & & 53 & 53 & & & & & & 24 & & \\
\hline $\begin{array}{l}\text { Removed or relocate } \\
\text { unnecessary signs } \\
\text { "visual clutter" }\end{array}$ & & & & & & & & & & & & & & & & & & & & \\
\hline $\begin{array}{l}\text { Replace poorly } \\
\text { designed drain grates } \\
\text { with bicycle-safe } \\
\text { types }\end{array}$ & & & & & & & & & & & & & & & & & & & & \\
\hline $\begin{array}{l}\text { Restrict and prohibit } \\
\text { turning maneuver by } \\
\text { channelization or } \\
\text { signing (enforcement } \\
\text { may be required in } \\
\text { the first stage) }\end{array}$ & & & & & & & & & & & & & & & & & & & & \\
\hline $\begin{array}{l}\text { Restrict left-turn } \\
\text { maneuver }\end{array}$ & & & & & 45 & 45 & & & & & & & & & & & & & & \\
\hline $\begin{array}{l}\text { Restrict or eliminate } \\
\text { parking near corners }\end{array}$ & & & & & & & & & & & $\begin{array}{l}35 \\
(10 \\
) \\
\end{array}$ & $\begin{array}{l}35 \\
(10 \\
)\end{array}$ & & & & & & & 10 & 10 \\
\hline $\begin{array}{l}\text { Restrict or eliminate } \\
\text { turning maneuver }\end{array}$ & & & & & & & & & & & & & & & & & & & & \\
\hline Restrict parking & & & & & 22 & 22 & & & & & $\begin{array}{l}35 \\
(10 \\
\end{array}$ & $\begin{array}{l}35 \\
(10 \\
\end{array}$ & 8 & 8 & & & & & 10 & 10 \\
\hline
\end{tabular}




\begin{tabular}{|c|c|c|c|c|c|c|c|c|c|c|c|c|c|c|c|c|c|c|c|c|}
\hline & \multicolumn{2}{|c|}{ S1 } & \multicolumn{2}{|c|}{ S2 } & \multicolumn{2}{|c|}{ S3 } & \multicolumn{2}{|c|}{$\mathbf{S 4}$} & \multicolumn{2}{|c|}{ S5 } & \multicolumn{2}{|c|}{ S6 } & \multicolumn{2}{|c|}{ S7 } & \multicolumn{2}{|c|}{ S8 } & \multicolumn{2}{|c|}{ S9,S10 } & \multicolumn{2}{|c|}{ S11-S21 } \\
\hline & $\mathbf{R}$ & $\mathbf{U}$ & $\mathbf{R}$ & $\mathbf{U}$ & $\mathbf{R}$ & $\mathbf{U}$ & $\mathbf{R}$ & $\mathbf{U}$ & $\mathbf{R}$ & $\mathbf{U}$ & $\mathbf{R}$ & $\mathbf{U}$ & $\mathbf{R}$ & $\mathbf{U}$ & $\mathbf{R}$ & $\mathbf{U}$ & $\mathbf{R}$ & $\mathbf{U}$ & $\mathbf{R}$ & $\mathbf{U}$ \\
\hline & & & & & & & & & & & ) & ) & & & & & & & & \\
\hline $\begin{array}{l}\text { Restrict parking near } \\
\text { corners }\end{array}$ & & & & & & & & & & & & & & & & & & & 10 & 10 \\
\hline $\begin{array}{l}\text { Re-strip shoulder to } \\
\text { provide right turn lane } \\
\text { (assure adequate } \\
\text { distance to fix object) }\end{array}$ & & & & & & & & & & & & & & & & & & & & \\
\hline $\begin{array}{l}\text { Review signal timing } \\
\text { (consider timing } \\
\text { optimalization) }\end{array}$ & & & & & 10 & 10 & $\begin{array}{l}15 \\
(47 \\
)\end{array}$ & $\begin{array}{l}15 \\
(47 \\
)\end{array}$ & & & & & 10 & 10 & & & & & & \\
\hline $\begin{array}{l}\text { Shield drivers from } \\
\text { poles in hazardous } \\
\text { locations }\end{array}$ & & & & & & & & & & & & & & & & & & & & \\
\hline $\begin{array}{l}\text { Signed and marked } \\
\text { crosswalks }\end{array}$ & & & & & & & & & & & 25 & 25 & & & & & & & & \\
\hline Trim vegetation & & & & & & & & & & & & & & & & & & & & \\
\hline Upgrade signing & & & & & $\begin{array}{l}5 \\
(47 \\
) \\
\end{array}$ & $\begin{array}{l}5 \\
(47 \\
) \\
\end{array}$ & & & & & & & & & & & & & & \\
\hline $\begin{array}{l}\text { Use "Slippery when } \\
\text { wet" sign (temporary) }\end{array}$ & & & & & & & & & & & & & & & & & & & & \\
\hline $\begin{array}{l}\text { Use advance guide } \\
\text { signs }\end{array}$ & & & 25 & 25 & & & & & & & & & & & & & & & & \\
\hline $\begin{array}{l}\text { Use advance street } \\
\text { name signs }\end{array}$ & & & & & & & & & & & 25 & 25 & & & & & & & & \\
\hline $\begin{array}{l}\text { Use automated } \\
\text { enforcement of } \\
\text { approach speeds }\end{array}$ & & & & & & & & & & & & & & & & & & & & \\
\hline $\begin{array}{l}\text { Use break-away } \\
\text { devices }\end{array}$ & & & & & 30 & 30 & & & & & & & & & & & & & & \\
\hline $\begin{array}{l}\text { Use flashing lights in } \\
\text { advance on approach }\end{array}$ & & & & & 50 & 50 & & & & & & & & & & & & & & \\
\hline $\begin{array}{l}\text { Use other traffic } \\
\text { calming applications } \\
\text { to reduce vehicle } \\
\text { speeds or traffic }\end{array}$ & & & & & & & & & & & & & & & & & & & & \\
\hline
\end{tabular}




\begin{tabular}{|c|c|c|c|c|c|c|c|c|c|c|c|c|c|c|c|c|c|c|c|c|}
\hline & \multicolumn{2}{|c|}{ S1 } & \multicolumn{2}{|c|}{ S2 } & \multicolumn{2}{|c|}{ S3 } & \multicolumn{2}{|c|}{ S4 } & \multicolumn{2}{|c|}{ S5 } & \multicolumn{2}{|c|}{ S6 } & \multicolumn{2}{|c|}{ S7 } & \multicolumn{2}{|c|}{ S8 } & \multicolumn{2}{|c|}{ S9,S10 } & \multicolumn{2}{|c|}{$\mathrm{S} 11-\mathrm{S} 21$} \\
\hline & $\mathbf{R}$ & $\mathbf{U}$ & $\mathbf{R}$ & $\mathbf{U}$ & $\mathbf{R}$ & $U$ & $\mathbf{R}$ & $\mathbf{U}$ & $\mathbf{R}$ & U & $\mathbf{R}$ & $\mathbf{U}$ & $\mathbf{R}$ & $\mathbf{U}$ & $\mathbf{R}$ & U & $\mathbf{R}$ & $\mathbf{U}$ & $\mathbf{R}$ & $U$ \\
\hline $\begin{array}{l}\text { volumes on } \\
\text { intersection } \\
\text { approaches. }\end{array}$ & & & & & & & & & & & & & & & & & & & & \\
\hline $\begin{array}{l}\text { Use post-mounted } \\
\text { delineation }\end{array}$ & & & & & & & & & & & & & $\begin{array}{l}25 \\
(48)\end{array}$ & $\begin{array}{l}25 \\
(48 \\
1\end{array}$ & & & & & & \\
\hline Use protected phase & & $\begin{array}{l}70 \\
(49 \\
1\end{array}$ & 27 & 27 & & & & 25 & & & & & & & & $\begin{array}{l}70 \\
(49 \\
1\end{array}$ & & & & \\
\hline $\begin{array}{l}\text { Use } \\
\text { Protected/Permitted } \\
\text { phase }\end{array}$ & & & $\begin{array}{l}10 \\
(50 \\
1\end{array}$ & $\begin{array}{l}10 \\
(50 \\
1\end{array}$ & & & 10 & 10 & & & & & & & & & & & & \\
\hline $\begin{array}{l}\text { Use salt to prevent } \\
\text { snow or ice from } \\
\text { forming or from } \\
\text { sticking to the road } \\
\text { surface }\end{array}$ & & & & & & & & & & & & & & & & & & & & \\
\hline $\begin{array}{l}\text { Use sand to increase } \\
\text { pavement friction }\end{array}$ & & & & & & & & & & & & & & & & & & & & \\
\hline Use split phases & & & & & & & & & & & & & & & & & & & & \\
\hline $\begin{array}{l}\text { Use two red signal } \\
\text { sections }\end{array}$ & & & & & & & & & & & & & & & & & & & & \\
\hline $\begin{array}{l}\text { Widen the outside } \\
\text { through lanes or add } \\
\text { bike lanes }\end{array}$ & & & & & & & & & & & & & & & & & & & & \\
\hline
\end{tabular}




\section{COMMENTS}

(1) - all type of crashes

(2) - channelization $-\mathrm{CRF}=50 \%$ for Rear End, Head On, Sideswipe Same Direction, Sideswipe Opposite Direction, Left Turn, and Run-off-the-Road crashes.

(3) - the following reduction applies: in 1 quadrant - 5\%, in 2 quadrant - $9 \%$, in 3 quadrant $13 \%$, in 4 quadrant $-17 \%$

(4) - provide left turn with signal

(5) - for single lane approach; multiple lane approach: 5\% only urban

(6) - queue detection ramp location

(7) - relocate object fatal only $\mathrm{CRF}=60 \%$

(8) - conflict elimination

(9) - crashes with emergency vehicle

(10) - eliminate parking

(11) - right angle collisions

(12) - for left turn; if new $\mathrm{CRF}=50 \%$, existing $\mathrm{CRF}=30 \%$, no left lane $\mathrm{CRF}=20 \%$

(13) - provide illumination for intersection

(14) - provide delineation

(15) - total injury

(16) - fatal crashes only

(17) - install object marker

(18) - only for pedestrian

(19) - fatal only: install signal heads

(20) - install signal backplates

(21) - provide illumination for Intersection

(22) - for intersection only

(23) - general warning sign

(24) - general warning (sign/other)

(25) -12 inch

(26) - crash type: turning, angle, rear-end, sideswipe - over

(27) - general install rumble strips 
(28) - rumble strip general

(29) - general variable message sign

(30) - general information massages

(31) - provide illumination for intersection

(32) - width: from $2 \mathrm{ft}$ to $8 \mathrm{ft}$ : $12 \%$, from $4 \mathrm{ft}$ to $8 \mathrm{ft}: 9 \%$, from $6 \mathrm{ft}$ to $8 \mathrm{ft}: 5 \%$ (only for paved shoulder)

(33) - general length turning lanes

(34) - all: $8 \%$, rear-end $(-12 \%)$, angle - $4 \%$, pedestrian $34 \%$

(35) - by $5 \mathrm{ft}, 8 \mathrm{ft}, 10 \mathrm{ft}, 15 \mathrm{ft}, 20 \mathrm{ft} \mathrm{CRF}$ is respectively: $13 \%, 21 \%, 25 \%, 35 \%, 44 \%$

(36) - speed reduction (not crashes)

(37) - widen pavement

(38) - 10ft-12ft

(39) - only crosswalk (no school zone) data for Israel

(40) - improve clearance interval al

(41) - provide offset

(42) - exclusive pedestrian phase

(43) - 4 leg both approaches

(44) - urban multilane

(45) - modify speed limit

(46) - clear sight triangle

(47) - general upgrade signing

(48) - for horizontal curve only

(49) - change from permitted to protected

(50) - add protected/permissive left turn phase 


\section{All-Way Stop-Controlled Intersection}

\begin{tabular}{|c|c|c|c|c|c|c|c|c|c|c|c|c|c|c|c|c|c|c|c|c|}
\hline & \multicolumn{2}{|c|}{ S1 } & \multicolumn{2}{|c|}{ S2 } & \multicolumn{2}{|c|}{ S3 } & \multicolumn{2}{|c|}{ S4 } & \multicolumn{2}{|c|}{ s5 } & \multicolumn{2}{|c|}{ s6 } & \multicolumn{2}{|c|}{ s7 } & \multicolumn{2}{|c|}{ s8 } & \multicolumn{2}{|c|}{ S9,S10 } & \multicolumn{2}{|c|}{ S11-S21 } \\
\hline & $\mathbf{R}$ & $\mathbf{U}$ & $\mathbf{R}$ & $\mathbf{U}$ & $\mathbf{R}$ & $\mathbf{U}$ & $\mathbf{R}$ & $\mathbf{U}$ & $\mathbf{R}$ & $\mathbf{U}$ & $\mathbf{R}$ & $\mathbf{U}$ & $\mathbf{R}$ & $\mathbf{U}$ & $\mathbf{R}$ & $\mathbf{U}$ & $\mathbf{R}$ & $\mathbf{U}$ & $\mathbf{R}$ & $\mathbf{U}$ \\
\hline $\begin{array}{l}\text { Add overhead stop-signs } \\
\text { if the percent of tall } \\
\text { vehciles is considerable }\end{array}$ & & & & & & & & & & & & & & & & & & & & \\
\hline Add special signing & & & & & & & & & & & & & & & & & & & & \\
\hline $\begin{array}{l}\text { Change } \\
\text { horizontal/vertical } \\
\text { alignment }\end{array}$ & & & 58 & 58 & & & & & & & 50 & 50 & 50 & 50 & & & & & 10 & 10 \\
\hline $\begin{array}{l}\text { Chip and seal or slurry } \\
\text { seal approaches }\end{array}$ & & & & & & & & & & & & & & & & & & & & \\
\hline Close curb lanes & & & & & & & & & & & & & & & & & & & & \\
\hline $\begin{array}{l}\text { Close or relocate the } \\
\text { driveways }\end{array}$ & & & 50 & 50 & & & & & & & & & & & & & & & & \\
\hline $\begin{array}{l}\text { Construct pedestrian } \\
\text { refuge islands and } \\
\text { raised medians }\end{array}$ & & 46 & $\begin{array}{l}60 \\
(4)\end{array}$ & $\begin{array}{l}60 \\
(4)\end{array}$ & 32 & 23 & & & & & & & & & & 46 & & & & \\
\hline $\begin{array}{l}\text { Eliminate screening by } \\
\text { physical objects }\end{array}$ & & & & & & & & & & & & & $\begin{array}{l}50 \\
(7) \\
\end{array}$ & $50(7$ & & & & & $\begin{array}{l}76 \\
(8) \\
\end{array}$ & $\begin{array}{l}76 \\
(8) \\
\end{array}$ \\
\hline $\begin{array}{l}\text { Eliminate shoulder drop- } \\
\text { off }\end{array}$ & & & & & & & & & & & 25 & 25 & & & & & & & $\begin{array}{l}30 \\
(9)\end{array}$ & $\begin{array}{l}30 \\
(9)\end{array}$ \\
\hline $\begin{array}{l}\text { Enforce stop sign } \\
\text { compliance }\end{array}$ & & & & & & & & & & & & & & & & & & & & \\
\hline $\begin{array}{l}\text { Extend "no parking" } \\
\text { zone. }\end{array}$ & & & & & 22 & 22 & & & & & $\begin{array}{l}35 \\
(10 \\
) \\
\end{array}$ & $35(1$ & & & & & & & 10 & 10 \\
\hline $\begin{array}{l}\text { Groove pavement } \\
\text { surface }\end{array}$ & & & 21 & 21 & & & 14 & 14 & & & & & & & & & & & 46 & 46 \\
\hline $\begin{array}{l}\text { Implement road } \\
\text { narrowing measures }\end{array}$ & & & & & & & & & & & & & & & & & & & & \\
\hline Improve alignment/grade & & & & & & & & & & & & & & & & & & & & \\
\hline $\begin{array}{l}\text { Improve } \\
\text { channelization/delineatio }\end{array}$ & & & & & & & & & & & $\begin{array}{l}30 \\
(11\end{array}$ & $\begin{array}{l}30 \\
(11\end{array}$ & 40 & 40 & & & & & 15 & \\
\hline
\end{tabular}




\begin{tabular}{|c|c|c|c|c|c|c|c|c|c|c|c|c|c|c|c|c|c|c|c|c|}
\hline & \multicolumn{2}{|c|}{ S1 } & \multicolumn{2}{|c|}{ S2 } & \multicolumn{2}{|c|}{ S3 } & \multicolumn{2}{|c|}{ S4 } & \multicolumn{2}{|c|}{ s5 } & \multicolumn{2}{|c|}{ s6 } & \multicolumn{2}{|c|}{ s7 } & \multicolumn{2}{|c|}{ s8 } & \multicolumn{2}{|c|}{ S9,S10 } & \multicolumn{2}{|c|}{ S11-S21 } \\
\hline & $\mathbf{R}$ & $\mathbf{U}$ & $\mathbf{R}$ & $\mathbf{U}$ & $\mathbf{R}$ & $\mathbf{U}$ & $\mathbf{R}$ & $\mathbf{U}$ & $\mathbf{R}$ & $\mathbf{U}$ & $\mathbf{R}$ & $\mathbf{U}$ & $\mathbf{R}$ & $\mathbf{U}$ & $\mathbf{R}$ & $\mathbf{U}$ & $\mathbf{R}$ & $\mathbf{U}$ & $\mathbf{R}$ & $\mathbf{U}$ \\
\hline $\mathrm{n}$ & & & & & & & & & & & ) & ) & & & & & & & & \\
\hline Improve clear zone & & & & & $\begin{array}{l}25 \\
(12 \\
)\end{array}$ & \multicolumn{2}{|c|}{$25(12)$} & & & & & & & & & & & & & \\
\hline $\begin{array}{l}\text { Improve existing street } \\
\text { lights }\end{array}$ & 30 & 30 & & & 50 & 50 & & & & & & & & & & & & & & \\
\hline Improve lanes width & $8(13$ & & & & & & & & & & & & & & 8 & & & & & \\
\hline $\begin{array}{l}\text { Improve pavement } \\
\text { marking in the median } \\
\text { opening }\end{array}$ & & & 21 & 21 & & & & & & & & & & & & & & & & \\
\hline $\begin{array}{l}\text { Improve signage and/or } \\
\text { marking of the crosswalk }\end{array}$ & & & & & & & & & & & 25 & 25 & & & & & & & & \\
\hline Improve skid resistance & & & 9 & 9 & & & & & & & & & & & & & & & & \\
\hline $\begin{array}{l}\text { Improve winter } \\
\text { maintenance methods }\end{array}$ & & & & & & & & & & & & & & & & & & & & \\
\hline $\begin{array}{l}\text { Improve winter } \\
\text { maintenance } \\
\text { preparedness (shorter } \\
\text { response time) }\end{array}$ & & & & & & & & & & & & & & & & & & & & \\
\hline $\begin{array}{l}\text { Improve/install reflective } \\
\text { signs }\end{array}$ & & & 15 & 15 & & & & & & & & & & & & & & & & \\
\hline $\begin{array}{l}\text { Improve/install } \\
\text { reflectorized pavement } \\
\text { markers }\end{array}$ & & & & & & & & & & & & & & & & & & & & \\
\hline $\begin{array}{l}\text { Increase width of the } \\
\text { shoulders }\end{array}$ & $9(16$ & & 14 & 14 & & & & & & & 20 & 20 & & & 9 & & & & 14 & \\
\hline $\begin{array}{l}\text { Install a barrier curb or a } \\
\text { guardrail }\end{array}$ & $\begin{array}{l}45 \\
(17 \\
)\end{array}$ & $\begin{array}{l}45 \\
(17 \\
) \\
\end{array}$ & 11 & 11 & & & $\begin{array}{l}47 \\
(18 \\
)\end{array}$ & 47 & & & & & & 4 & & & $\begin{array}{l}44 \\
(7)\end{array}$ & 44( & & \\
\hline $\begin{array}{l}\text { Install a pedestrian } \\
\text { overpass or underpass }\end{array}$ & & & 86 & 86 & & & & & & & & & & & & & & & 91 & 91 \\
\hline Install a stop sign & & & 35 & 35 & & & & & & & 39 & 39 & & & & & & & & \\
\hline $\begin{array}{l}\text { Install a triangular island } \\
\text { to relocate the right- }\end{array}$ & & & & & & & & & & & & & & & & & & & & \\
\hline
\end{tabular}




\begin{tabular}{|c|c|c|c|c|c|c|c|c|c|c|c|c|c|c|c|c|c|c|c|c|}
\hline & \multicolumn{2}{|c|}{$\mathrm{S} 1$} & \multicolumn{2}{|c|}{$\mathbf{S 2}$} & \multicolumn{2}{|c|}{ S3 } & \multicolumn{2}{|c|}{$\mathbf{S 4}$} & \multicolumn{2}{|c|}{ s5 } & \multicolumn{2}{|c|}{ s6 } & \multicolumn{2}{|c|}{ s7 } & \multicolumn{2}{|c|}{ s8 } & \multicolumn{2}{|c|}{ S9,S10 } & \multicolumn{2}{|c|}{ S11-S21 } \\
\hline & $\mathbf{R}$ & $\mathbf{U}$ & $\mathbf{R}$ & $\mathbf{U}$ & $\mathbf{R}$ & $\mathbf{U}$ & $\mathbf{R}$ & $\mathbf{U}$ & $\mathbf{R}$ & $\mathbf{U}$ & $\mathbf{R}$ & $\mathbf{U}$ & $\mathbf{R}$ & $\mathbf{U}$ & $\mathbf{R}$ & $\mathbf{U}$ & $\mathbf{R}$ & $\mathbf{U}$ & $\mathbf{R}$ & $\mathbf{U}$ \\
\hline $\begin{array}{l}\text { turning movement away } \\
\text { from other movements }\end{array}$ & & & & & & & & & & & & & & & & & & & & \\
\hline $\begin{array}{l}\text { Install adequate traffic } \\
\text { control device for } \\
\text { bicycles (i.e. stop sign) }\end{array}$ & & & & & & & & & & & & & & & & & & & & \\
\hline $\begin{array}{l}\text { Install advance warning } \\
\text { sign }\end{array}$ & 20 & 20 & 25 & 25 & & & $\begin{array}{l}25 \\
(19 \\
)\end{array}$ & \multicolumn{2}{|c|}{$25(19)$} & & & & 30 & 40 & & & & & & \\
\hline Install an object marker & & & & & & & 16 & 16 & & & & & & & & & & & & \\
\hline $\begin{array}{l}\text { Install channelization of } \\
\text { the driveways }\end{array}$ & & & & & & & & & $\begin{array}{l}50 \\
(20 \\
1 \\
\end{array}$ & \multicolumn{2}{|c|}{$50(20)$} & & & & & & & & & \\
\hline Install corner mirrors & & & & & & & & & & & & & & & & & & & & \\
\hline $\begin{array}{l}\text { Install curbing to define } \\
\text { driveway location }\end{array}$ & & & & & & & & & & & & & & & & & & & & \\
\hline Install curb-ramps & & & & & & & & & & & & & & & & & & & & \\
\hline Install flashing beacons & 30 & 30 & & & 50 & 50 & $\begin{array}{l}30 \\
(21 \\
) \\
\end{array}$ & \multicolumn{2}{|c|}{$30(21)$} & & 30 & 30 & 24 & 24 & & & & & & \\
\hline $\begin{array}{l}\text { Install fog - warning } \\
\text { signs }\end{array}$ & & & & & & & $\begin{array}{l}25 \\
(19 \\
)\end{array}$ & \multicolumn{2}{|c|}{$25(19)$} & & & & & & & & & & & \\
\hline Install guardrails & & & & & 20 & 20 & 47 & 47 & & & $\begin{array}{l}65 \\
(4)\end{array}$ & \multicolumn{2}{|c|}{$65(4)$} & 4 & & & $\begin{array}{l}44 \\
\text { (4) }\end{array}$ & \multicolumn{2}{|c|}{$44(4)$} & \\
\hline $\begin{array}{l}\text { Install larger regulatory } \\
\text { and warning signs at } \\
\text { and in advance of } \\
\text { intersections }\end{array}$ & & & & & & & & & & & $\begin{array}{l}15 \\
(23 \\
)\end{array}$ & $\begin{array}{l}15 \\
(23 \\
)\end{array}$ & 40 & 30 & & & & & & \\
\hline Install lighting & 30 & 30 & 33 & 33 & $\begin{array}{l}50 \\
(25 \\
) \\
\end{array}$ & \multicolumn{2}{|c|}{$50(25)$} & & & & 30 & 30 & 37 & 37 & & & & & $\begin{array}{l}37 \\
(26 \\
) \\
\end{array}$ & $\begin{array}{l}37 \\
(26 \\
) \\
\end{array}$ \\
\hline Install median divider & $\begin{array}{l}15 \\
(27 \\
) \\
\end{array}$ & \multicolumn{2}{|c|}{$15(27)$} & & & & & & & & & & & & & & & & & \\
\hline
\end{tabular}




\begin{tabular}{|c|c|c|c|c|c|c|c|c|c|c|c|c|c|c|c|c|c|c|c|c|}
\hline & \multicolumn{2}{|c|}{ S1 } & \multicolumn{2}{|c|}{ S2 } & \multicolumn{2}{|c|}{ S3 } & \multicolumn{2}{|c|}{$\mathbf{S 4}$} & \multicolumn{2}{|c|}{ s5 } & \multicolumn{2}{|c|}{ s6 } & \multicolumn{2}{|c|}{ s7 } & \multicolumn{2}{|c|}{ s8 } & \multicolumn{2}{|c|}{ S9,S10 } & \multicolumn{2}{|c|}{ S11-S21 } \\
\hline & $\mathbf{R}$ & $\mathbf{U}$ & $\mathbf{R}$ & $\mathbf{U}$ & $\mathbf{R}$ & $\mathbf{U}$ & $\mathbf{R}$ & $\mathbf{U}$ & $\mathbf{R}$ & $\mathbf{U}$ & $\mathbf{R}$ & $\mathbf{U}$ & $\mathbf{R}$ & $\mathbf{U}$ & $\mathbf{R}$ & $\mathbf{U}$ & $\mathbf{R}$ & $\mathbf{U}$ & $\mathbf{R}$ & $\mathbf{U}$ \\
\hline $\begin{array}{l}\text { Install overhead flashing } \\
\text { beacon lights }\end{array}$ & 30 & 30 & & & 50 & 50 & $\begin{array}{l}30 \\
(21 \\
) \\
\end{array}$ & 30 & & & 30 & 30 & 24 & 24 & & & & & & \\
\hline $\begin{array}{l}\text { Install pavement } \\
\text { markings }\end{array}$ & 10 & 10 & 21 & 21 & & & & & 21 & 21 & & & & & & & & & 10 & 10 \\
\hline $\begin{array}{l}\text { Install pedestrian } \\
\text { actuated signals }\end{array}$ & & & & & & & & & & & & & & & & & & & & \\
\hline $\begin{array}{l}\text { Install pedestrian } \\
\text { barriers }\end{array}$ & & & & & & & & & & & & & & & & & & & & \\
\hline $\begin{array}{l}\text { Install pedestrian } \\
\text { crosswalk }\end{array}$ & & & 25 & 25 & & & & & 25 & 25 & & & & & & & & & & \\
\hline Install raised crosswalk & & & & & & & & & & & & & & & & & & & & \\
\hline $\begin{array}{l}\text { Install reflectorized } \\
\text { pavement marking }\end{array}$ & & & & & & & & & & & & & & & & & & & & \\
\hline $\begin{array}{l}\text { Install regulatory signs } \\
\text { on the both side of the } \\
\text { road }\end{array}$ & & & & & & & & & & & & & & & & & & & & \\
\hline Install rumble strips & & & 32 & 32 & 28 & 28 & & & & & $\begin{array}{l}25 \\
(23 \\
)\end{array}$ & $25(2$ & & & 21 & & & & 50 & 50 \\
\hline $\begin{array}{l}\text { Install school crossing } \\
\text { sign }\end{array}$ & & & 18 & 18 & & & & & & & 14 & 14 & & & & & & & & \\
\hline $\begin{array}{l}\text { Install school zone } \\
\text { markings }\end{array}$ & & & & & & & & & & & & & & & & & & & & \\
\hline $\begin{array}{l}\text { Install sidewalk set- } \\
\text { backs }\end{array}$ & & & & & & & & & & & & & & & & & & & & \\
\hline Install speed limit sign & & & & & & & & & & & & & & & & & & & & \\
\hline $\begin{array}{l}\text { Install static or variable } \\
\text { message signs } \\
\text { displaying weather } \\
\text { information }\end{array}$ & & & 15 & 15 & & & & & & & $\begin{array}{l}15 \\
(23 \\
)\end{array}$ & $15(2$ & & & & & & & & \\
\hline Install street lights & 30 & 30 & & & $\begin{array}{l}50 \\
(25 \\
)\end{array}$ & $50(2$ & & & & & & & & & & & & & & \\
\hline
\end{tabular}




\begin{tabular}{|c|c|c|c|c|c|c|c|c|c|c|c|c|c|c|c|c|c|c|c|c|}
\hline & \multicolumn{2}{|c|}{ S1 } & \multicolumn{2}{|c|}{ S2 } & \multicolumn{2}{|c|}{ S3 } & \multicolumn{2}{|c|}{ S4 } & \multicolumn{2}{|c|}{ s5 } & \multicolumn{2}{|c|}{ s6 } & \multicolumn{2}{|c|}{ s7 } & \multicolumn{2}{|c|}{ s8 } & \multicolumn{2}{|c|}{ S9,S10 } & \multicolumn{2}{|c|}{ S11-S21 } \\
\hline & $\mathbf{R}$ & $\mathbf{U}$ & $\mathbf{R}$ & $\mathbf{U}$ & $\mathbf{R}$ & $\mathbf{U}$ & $\mathbf{R}$ & $\mathbf{U}$ & $\mathbf{R}$ & $\mathbf{U}$ & $\mathbf{R}$ & $\mathbf{U}$ & $\mathbf{R}$ & $\mathbf{U}$ & $\mathbf{R}$ & $\mathbf{U}$ & $\mathbf{R}$ & $\mathbf{U}$ & $\mathbf{R}$ & $\mathbf{U}$ \\
\hline $\begin{array}{l}\text { Install streets name } \\
\text { signs }\end{array}$ & & & & & & & & & & & 25 & 25 & & & & & & & & \\
\hline Install traffic signals & & 14 & $\begin{array}{l}30 \\
(29 \\
)\end{array}$ & \multicolumn{2}{|c|}{$30(29)$} & & & & & & 28 & 28 & 28 & 28 & & 23 & $\begin{array}{l}23 \\
(30 \\
)\end{array}$ & \multicolumn{2}{|c|}{$23(30)$} & \\
\hline $\begin{array}{l}\text { Install/Improve lighting at } \\
\text { the access points }\end{array}$ & & & $\begin{array}{l}33 \\
(31 \\
) \\
\end{array}$ & \multicolumn{2}{|c|}{$33(31)$} & & & & & & 30 & 30 & 37 & 37 & & & & & $\begin{array}{l}37 \\
(26 \\
) \\
\end{array}$ & $\begin{array}{l}37 \\
(26 \\
) \\
\end{array}$ \\
\hline $\begin{array}{l}\text { Installing snow screens } \\
\text { in areas exposed to } \\
\text { snowdrifts }\end{array}$ & & & & & & & & & & & & & & & & & & & & \\
\hline $\begin{array}{l}\text { Move driveway to side } \\
\text { street }\end{array}$ & & & $\begin{array}{l}50 \\
(32 \\
1 \\
\end{array}$ & $\begin{array}{l}50 \\
(32 \\
) \\
\end{array}$ & 15 & 15 & & & & & & & & & & & & & & \\
\hline Overlay pavement & & & 17 & 17 & 18 & 18 & 27 & 27 & & & & & & & & & & & & \\
\hline $\begin{array}{l}\text { Post an adequate speed } \\
\text { limit }\end{array}$ & & & & & 28 & 28 & & & & & & & 20 & 20 & & & & & & \\
\hline $\begin{array}{l}\text { Post dynamic message } \\
\text { sign to display the speed } \\
\text { of approaching vehicles. }\end{array}$ & & & & & & & & & & & & & & & & & $\begin{array}{l}74 \\
(33 \\
) \\
\end{array}$ & 74( & & \\
\hline $\begin{array}{l}\text { Provide adequate } \\
\text { median width that will } \\
\text { not limited visibility }\end{array}$ & & & & & & & & & & & & & & & & & & & & \\
\hline $\begin{array}{l}\text { Provide "slippery when } \\
\text { wet" signs }\end{array}$ & & & & & & & & & & & & & & & & & & & & \\
\hline $\begin{array}{l}\text { Provide a double yellow } \\
\text { centerline on the median } \\
\text { opening of a divided } \\
\text { highway at intersections }\end{array}$ & & & & & & & & & & & & & & & & & & & & \\
\hline $\begin{array}{l}\text { Provide acceleration and } \\
\text { deceleration lanes }\end{array}$ & & & 26 & 26 & & & & & & & $\begin{array}{l}10 \\
(11 \\
) \\
\end{array}$ & $\begin{array}{l}10 \\
(11 \\
)\end{array}$ & 10 & 10 & & & & & & \\
\hline $\begin{array}{l}\text { Provide adequate } \\
\text { delineation for left-turns }\end{array}$ & & & & & & & & & & & 30 & 30 & & & & & & & & \\
\hline
\end{tabular}




\begin{tabular}{|c|c|c|c|c|c|c|c|c|c|c|c|c|c|c|c|c|c|c|c|}
\hline & \multicolumn{2}{|c|}{ S1 } & \multicolumn{2}{|c|}{ S2 } & \multicolumn{2}{|c|}{ S3 } & S4 & \multicolumn{2}{|c|}{ s5 } & \multicolumn{2}{|c|}{ s6 } & \multicolumn{2}{|c|}{ s7 } & \multicolumn{2}{|c|}{ s8 } & \multicolumn{2}{|c|}{ S9,S10 } & \multicolumn{2}{|c|}{ S11-S21 } \\
\hline & $\mathbf{R}$ & $\mathbf{U}$ & $\mathbf{R}$ & $\mathbf{U}$ & $\mathbf{R}$ & $\mathbf{U}$ & $\mathbf{U}$ & $\mathbf{R}$ & $\mathbf{U}$ & $\mathbf{R}$ & $\mathbf{U}$ & $\mathbf{R}$ & $\mathbf{U}$ & $\mathbf{R}$ & $\mathbf{U}$ & $\mathbf{R}$ & $\mathbf{U}$ & $\mathbf{R}$ & $\mathbf{U}$ \\
\hline $\begin{array}{l}\text { at the intersection } \\
\text { (markers or lines) }\end{array}$ & & & & & & & & & & & & & & & & & & & \\
\hline $\begin{array}{l}\text { Provide adequate } \\
\text { drainage }\end{array}$ & & & 20 & 20 & & & & & & & & & & & & & & & \\
\hline $\begin{array}{l}\text { Provide independent } \\
\text { bicycle path where } \\
\text { necessary }\end{array}$ & & & & & & & & & & & & & & & & & & & \\
\hline Provide median refuges & & 46 & & & & & & & & & & & & & & & & & \\
\hline $\begin{array}{l}\text { Provide on-pavement } \\
\text { horizontal signing }\end{array}$ & & & & & & & & & & & & & & & & & & & \\
\hline $\begin{array}{l}\text { Provide pavement } \\
\text { marking (stop sign, } \\
\text { chevron, etc.) }\end{array}$ & & & 21 & 21 & $\begin{array}{l}35 \\
(37 \\
) \\
\end{array}$ & $35(37)$ & & & & & & 35 & 35 & & & 25 & & & \\
\hline $\begin{array}{l}\text { Provide smooth paved } \\
\text { shoulders }\end{array}$ & & & 15 & 15 & & & & & & & & & $53(3$ & & & & & 14 & \\
\hline Provide splitter islands & & & & & & & & & & & & & & & & & & & \\
\hline $\begin{array}{l}\text { Provide targeted speed } \\
\text { enforcement }\end{array}$ & & & & & & & & & & & & & & & & & & & \\
\hline $\begin{array}{l}\text { Provide traffic calming } \\
\text { on intersection } \\
\text { approaches through a } \\
\text { combination of } \\
\text { geometric and traffic } \\
\text { control devices }\end{array}$ & & & & & & & & & & & & & & & & & & & \\
\hline Provide visible stop bars & & & & & & & & & & & & & & & & & & & \\
\hline $\begin{array}{l}\text { Provide/Improve } \\
\text { pavement surface } \\
\text { (improve transitions, } \\
\text { drop offs, fixed pothols, } \\
\text { rutting,etc.) }\end{array}$ & & & & & & & & & & & & & & & & & & & \\
\hline Reduce speed limit & & & & & & & & & & & & 20 & 20 & & & & & & \\
\hline $\begin{array}{l}\text { Reduce the number of } \\
\text { lanes if allowed by } \\
\text { capacity }\end{array}$ & & & & & & & & & & & & & & & & & & & \\
\hline
\end{tabular}




\begin{tabular}{|c|c|c|c|c|c|c|c|c|c|c|c|c|c|c|c|c|c|c|c|c|}
\hline & \multicolumn{2}{|c|}{ S1 } & \multicolumn{2}{|c|}{ S2 } & \multicolumn{2}{|c|}{ S3 } & \multicolumn{2}{|c|}{ S4 } & \multicolumn{2}{|c|}{ s5 } & \multicolumn{2}{|c|}{ s6 } & \multicolumn{2}{|c|}{ s7 } & \multicolumn{2}{|c|}{ s8 } & \multicolumn{2}{|c|}{ S9,S10 } & \multicolumn{2}{|c|}{ S11-S21 } \\
\hline & $\mathbf{R}$ & $\mathbf{U}$ & $\mathbf{R}$ & $\mathbf{U}$ & $\mathbf{R}$ & $\mathbf{U}$ & $\mathbf{R}$ & $\mathbf{U}$ & $\mathbf{R}$ & $\mathbf{U}$ & $\mathbf{R}$ & $\mathbf{U}$ & $\mathbf{R}$ & $\mathbf{U}$ & $\mathbf{R}$ & $\mathbf{U}$ & $\mathbf{R}$ & $\mathbf{U}$ & $\mathbf{R}$ & $\mathbf{U}$ \\
\hline Relocate islands & & & & & & & & & & & & & & & & & & & & \\
\hline $\begin{array}{l}\text { Relocate or split the } \\
\text { stopping line }\end{array}$ & & & & & & & & & & & & & & & & & & & & \\
\hline Relocate stop bar & & & & & & & & & & & & & & & & & & & & \\
\hline Relocate stop sign & & & & & & & & & & & & & & & & & & & & \\
\hline $\begin{array}{l}\text { Relocate the sign to } \\
\text { make it visible }\end{array}$ & & & & & & & & & & & & & & & & & & & & \\
\hline $\begin{array}{l}\text { Remove distracting } \\
\text { commercial lighting or } \\
\text { other source of glare }\end{array}$ & & & & & & & & & & & 15 & 15 & & & & & & & & \\
\hline $\begin{array}{l}\text { Remove or relocate the } \\
\text { object }\end{array}$ & & & & & 60 & 60 & $\begin{array}{l}29 \\
(44 \\
)\end{array}$ & 29( & & & 30 & 30 & $\begin{array}{l}50 \\
(7)\end{array}$ & 50 & & & & & & \\
\hline $\begin{array}{l}\text { Remove sight } \\
\text { obstructions }\end{array}$ & $12(42$ & & & & & & & & & & & & & & & & & & & \\
\hline $\begin{array}{l}\text { Remove the object } \\
\text { obstructing sight of the } \\
\text { warning sign }\end{array}$ & & & & & & & & & & & & & & & & & & & & \\
\hline $\begin{array}{l}\text { Remove the object } \\
\text { obstructing the sight of } \\
\text { the stop sign }\end{array}$ & & & & & & & & & & & & & & & & & & & & \\
\hline $\begin{array}{l}\text { Remove the object } \\
\text { obstructing the sight } \\
\text { triangle }\end{array}$ & $12(42$ & & & & & & 25 & 25 & & & & & & & & & & & 10 & 10 \\
\hline $\begin{array}{l}\text { Remove the object } \\
\text { obstructing visibility from } \\
\text { the median }\end{array}$ & & & & & & & & & & & & & & & & & & & & \\
\hline $\begin{array}{l}\text { Replace poorly designed } \\
\text { drain grates with bicycle- } \\
\text { safe types }\end{array}$ & & & & & & & & & & & & & & & & & & & & \\
\hline $\begin{array}{l}\text { Replace, repair or clean } \\
\text { the warning signs }\end{array}$ & & & & & 20 & 20 & 25 & 25 & & & & & & & & & & & & \\
\hline $\begin{array}{l}\text { Reroute pedestrian } \\
\text { paths }\end{array}$ & & & & & & & & & & & & & & & & & & & & \\
\hline
\end{tabular}




\begin{tabular}{|c|c|c|c|c|c|c|c|c|c|c|c|c|c|c|c|c|c|c|c|c|}
\hline & \multicolumn{2}{|c|}{ S1 } & \multicolumn{2}{|c|}{ S2 } & \multicolumn{2}{|c|}{ S3 } & \multicolumn{2}{|c|}{$\mathbf{S 4}$} & \multicolumn{2}{|c|}{ s5 } & \multicolumn{2}{|c|}{ s6 } & \multicolumn{2}{|c|}{ s7 } & \multicolumn{2}{|c|}{ s8 } & \multicolumn{2}{|c|}{ S9,S10 } & \multicolumn{2}{|c|}{ S11-S21 } \\
\hline & $\mathbf{R}$ & $\mathbf{U}$ & $\mathbf{R}$ & $\mathbf{U}$ & $\mathbf{R}$ & $\mathbf{U}$ & $\mathbf{R}$ & $\mathbf{U}$ & $\mathbf{R}$ & $\mathbf{U}$ & $\mathbf{R}$ & $\mathbf{U}$ & $\mathbf{R}$ & $\mathbf{U}$ & $\mathbf{R}$ & $\mathbf{U}$ & $\mathbf{R}$ & $\mathbf{U}$ & $\mathbf{R}$ & $\mathbf{U}$ \\
\hline $\begin{array}{l}\text { Restrict left-turning at } \\
\text { the access points }\end{array}$ & & & & & & & & & & & & & & & & & & & 100 & 47) \\
\hline $\begin{array}{l}\text { Restrict parking near the } \\
\text { driveway }\end{array}$ & & & & & $\begin{array}{l}22 \\
(48 \\
)\end{array}$ & \multicolumn{2}{|c|}{$22(48)$} & & & & 35 & 35 & 8 & 8 & & & & & 10 & 10 \\
\hline $\begin{array}{l}\text { Restrict parking on the } \\
\text { approach }\end{array}$ & & & & & & & & & & & & & & & & & & & 10 & 10 \\
\hline $\begin{array}{l}\text { Signals to alert motorists } \\
\text { that pedestrians are } \\
\text { crossing }\end{array}$ & & & & & & & & & & & & & & & & & & & & \\
\hline Trim vegetation & & & & & & & & & & & & & & & & & & & & \\
\hline $\begin{array}{l}\text { Trim vegetation on the } \\
\text { median }\end{array}$ & & & & & & & & & & & & & & & & & & & & \\
\hline Upgrade signing & & & & & $\begin{array}{l}5 \\
(45 \\
)\end{array}$ & $5(45$ & & & & & 24 & 24 & & & & & & & & \\
\hline $\begin{array}{l}\text { Use "Slippery when wet" } \\
\text { sign (temporary) }\end{array}$ & & & & & & & & & & & & & & & & & & & & \\
\hline Use break-away devices & & & & & 30 & 30 & & & & & & & & & & & & & & \\
\hline $\begin{array}{l}\text { Use salt to prevent snow } \\
\text { or ice from forming or } \\
\text { from sticking to the road } \\
\text { surface }\end{array}$ & & & & & & & & & & & & & & & & & & & & \\
\hline $\begin{array}{l}\text { Use sand to increase } \\
\text { pavement friction }\end{array}$ & & & & & & & & & & & & & & & & & & & & \\
\hline $\begin{array}{l}\text { Use school crossing } \\
\text { guards }\end{array}$ & & & & & & & & & & & & & & & & & & & & \\
\hline $\begin{array}{l}\text { Widen the outside } \\
\text { through lanes or add } \\
\text { bike lanes }\end{array}$ & & & & & & & & & & & & & & & & & & & & \\
\hline
\end{tabular}




\section{COMMENTS}

(1) - added painted lane

(2) - rural both approaches, 4 legs

(3) - four legs intersection

(4) - reduction in pedestrians fatal only

(5) - for single lane approach; multiple lane approach $\mathrm{CRF}=5 \%$ only urban

(6) - single lane

(7) - fatal only

(8) - conflict elimination

(9) - shoulder treatment

(10) - eliminate parking

(11) - post delineation

(12) - widen by 5,8,10,15,20 [ft] CRF is respectively: $13 \%, 21 \%, 25 \%, 35 \%, 44 \%$

(13) - widen lanes: 9ft-12ft CRF $=12 \%$; $10 \mathrm{ft}-12 \mathrm{ft} \mathrm{CRF}=8 \%$; $11 \mathrm{ft}-12 \mathrm{ft} \mathrm{CRF}=2 \%$

(14) - provide illumination for intersection

(15) - general any turn lane

(16) - for width: $2 \mathrm{ft}-8 \mathrm{ft}: 12 \%, 4 \mathrm{ft}-8 \mathrm{ft}: 9 \%$, $6 \mathrm{ft}-8 \mathrm{ft}: 5 \%$ (only for paved shoulder)

(17) - fatal $\mathrm{CRF}=44 \%$; injury $\mathrm{CRF}=47 \%$

(18)- total injury crashes (incl. fatal)

(19) - warning general

(20) - channelization $-\mathrm{CRF}=50 \%$ for Rear End, Head On, Sideswipe Same Direction, Sideswipe Opposite Direction, Left Turn, and Run-off-the-Road crashes.

(21) - for intersection only

(22) - provide left turn lane without signal

(23) - general warning and large sign

(24) - 4 leg intersection

(25) - provide illumination for intersection

(26) - CRF between 25\%-50\%

(27) - add mountable median 
(28) - turning, angle, rear-end, sideswipe - over

(29) - 3 leg: all types $\mathrm{CRF}=14 \%$; angle $\mathrm{CRF}=34 \%$, rear-end $\mathrm{CRF}=(-50 \%), 4$ leg: all types $\mathrm{CRF}=23 \%$; angle $\mathrm{CRF}=67 \%$, rear-end $\mathrm{CRF}=(-38 \%)$

(30) - 4 leg all crashes

(31) - treat as intersection

(32) - relocate driveway entrance: left, right, angle $=10 \%$; rear, head $=20 \%$

(33) - speed reduction (not crashes)

(34) - pavement markings

(35) - edge line markings

(36) - edge lines

(37) - chevron

(38) - urban multilane

(39) - 4 leg intersection

(40)- 4 leg both approaches

(41) - modify speed limit

(42) - the following reduction applies: in 1 quadrant - 5\%, in 2 quadrant - 9\%, in 3 quadrant 13\%, in 4 quadrant $-17 \%$

(43) - 3 quadrant

(44) - removed fixed object (relocate $=35 \%$ )

(45) - general upgrade signing

(46) - prohibit turning

(47) - only turning crashes

(48) - prohibit on street parking

(49) - pavement widening

(50) - widening and resurfacing or widening alone

(51) - average from $1 \mathrm{ft}$ and $2 \mathrm{ft}$

(52) $-10 \mathrm{ft}-12 \mathrm{ft}$

(53) - average from width: $4 \mathrm{ft}, 10 \mathrm{ft}, 20 \mathrm{ft}$ 


\section{The final CRF values}

\section{TWSC}

\begin{tabular}{|c|c|c|c|c|c|c|c|c|c|c|c|c|c|c|c|c|c|c|c|c|c|}
\hline & \multicolumn{2}{|c|}{ S1 } & \multicolumn{2}{|c|}{ S2 } & \multicolumn{2}{|c|}{ S3 } & \multicolumn{2}{|c|}{ S4 } & \multicolumn{2}{|c|}{ S5 } & \multicolumn{2}{|c|}{ S6 } & \multicolumn{2}{|c|}{ S7 } & \multicolumn{2}{|c|}{ S8 } & \multicolumn{2}{|c|}{ S9,S10 } & \multicolumn{2}{|c|}{ S11-S21 } & \multirow[t]{2}{*}{ FINAL } \\
\hline & $\mathbf{R}$ & $\mathbf{U}$ & $\mathbf{R}$ & $\mathbf{U}$ & $\mathbf{R}$ & $\mathbf{U}$ & $\mathbf{R}$ & $\mathbf{U}$ & $\mathbf{R}$ & $\mathbf{U}$ & $\mathbf{R}$ & U & $\mathbf{R}$ & $\mathbf{U}$ & $\mathbf{R}$ & U & $\mathbf{R}$ & U & $\mathbf{R}$ & $\mathbf{U}$ & \\
\hline $\begin{array}{l}\text { Add overhead stop-signs if } \\
\text { the percent of tall vehicles } \\
\text { is considerable }\end{array}$ & & & & & & & & & & & & & & & & & & & & & 20 \\
\hline Add special signing & & & & & & & & & & & & & & & & & & & & & 10 \\
\hline $\begin{array}{l}\text { Add stop-lines in the } \\
\text { median opening }\end{array}$ & & & & & & & & & & & & & & & & & & & & & 10 \\
\hline $\begin{array}{l}\text { Adjust signal offsets at the } \\
\text { upstream signalized } \\
\text { intersections to create } \\
\text { more gaps }\end{array}$ & & & & & & & & & & & & & & & & & & & & & 20 \\
\hline $\begin{array}{l}\text { Change horizontal/vertical } \\
\text { alignment }\end{array}$ & & & 58 & 58 & & & & & & & 50 & 50 & 50 & 50 & & & & & 10 & 10 & 42 \\
\hline $\begin{array}{l}\text { Chip and seal or slurry } \\
\text { seal approaches }\end{array}$ & & & & & & & & & & & & & & & & & & & & & 10 \\
\hline Close curb lanes & & & & & & & & & & & & & & & & & & & & & 25 \\
\hline $\begin{array}{l}\text { Close or relocate the } \\
\text { driveways }\end{array}$ & & & 50 & 50 & & & & & & & & & & & & & & & & & 50 \\
\hline Consider indirect left turns & & & 34 & 34 & & & 35 & 35 & 25 & 25 & 41 & 41 & 32 & 32 & & & 48 & 47 & 24 & & 35 \\
\hline $\begin{array}{l}\text { Consider intersection type } \\
\text { "J" (no directly left turn) }\end{array}$ & & & & & & & & & & & & & & & & & & & & & 20 \\
\hline $\begin{array}{l}\text { Consider moving the right- } \\
\text { turn lanes on the major } \\
\text { road laterally }\end{array}$ & & & & & & & & & & & & & & & & & & & & & 20 \\
\hline $\begin{array}{l}\text { Consider traffic signals } \\
\text { with protected left-turn } \\
\text { phase }\end{array}$ & & & & & & & & & & & & & & & & & & & & & 40 \\
\hline $\begin{array}{l}\text { Construct Pedestrian } \\
\text { Refuge Islands and }\end{array}$ & & 46 & 60 & 60 & 32 & 23 & & & & & & & & & & 46 & & & & & 45 \\
\hline
\end{tabular}




\begin{tabular}{|c|c|c|c|c|c|c|c|c|c|c|c|c|c|c|c|c|c|c|c|c|c|}
\hline & \multicolumn{2}{|c|}{ S1 } & \multicolumn{2}{|c|}{ S2 } & \multicolumn{2}{|c|}{ S3 } & \multicolumn{2}{|c|}{ S4 } & \multicolumn{2}{|c|}{ S5 } & \multicolumn{2}{|c|}{ S6 } & \multicolumn{2}{|c|}{ S7 } & \multicolumn{2}{|c|}{ S8 } & \multicolumn{2}{|c|}{ S9,S10 } & \multicolumn{2}{|c|}{ S11-S21 } & \multirow[t]{2}{*}{ FINAL } \\
\hline & $\mathbf{R}$ & $\mathbf{U}$ & $\mathbf{R}$ & $\mathbf{U}$ & $\mathbf{R}$ & $\mathbf{U}$ & $\mathbf{R}$ & $\mathbf{U}$ & $\mathbf{R}$ & $\mathbf{U}$ & $\mathbf{R}$ & $\mathbf{U}$ & $\mathbf{R}$ & $\mathbf{U}$ & $\mathbf{R}$ & $\mathbf{U}$ & $\mathbf{R}$ & $\mathbf{U}$ & $\mathbf{R}$ & $\mathbf{U}$ & \\
\hline \multicolumn{22}{|l|}{ Raised Medians } \\
\hline $\begin{array}{l}\text { Convert the intersection } \\
\text { into a roundabout }\end{array}$ & 58 & 72 & & & & & 40 & 40 & 40 & 40 & & & & & 58 & 72 & 58 & 72 & 38 & 38 & 52 \\
\hline $\begin{array}{l}\text { Eliminate screening by } \\
\text { physical objects }\end{array}$ & & & & & & & & & & & & & 50 & 50 & & & & & 76 & 76 & 63 \\
\hline $\begin{array}{l}\text { Eliminate shoulder drop- } \\
\text { off }\end{array}$ & & & & & & & & & & & 25 & 25 & & & & & & & 30 & 30 & 28 \\
\hline $\begin{array}{l}\text { Enforce stop sign } \\
\text { compliance }\end{array}$ & & & & & & & & & & & & & & & & & & & & & 70 \\
\hline Extend "no parking" zone. & & & & & 22 & 22 & & & & & 35 & 35 & & & & & & & 10 & 10 & 22 \\
\hline Groove pavement surface & & & 21 & 21 & & & 14 & 14 & & & & & & & & & & & 46 & 46 & 27 \\
\hline $\begin{array}{l}\text { Implement Road } \\
\text { Narrowing Measures }\end{array}$ & & & & & & & & & & & & & & & & & & & & & 30 \\
\hline Improve alignment/grade & & & & & & & & & & & & & & & & & & & & & 35 \\
\hline $\begin{array}{l}\text { Improve } \\
\text { channelization/delineation }\end{array}$ & & & & & & & & & & & 30 & 30 & 40 & 40 & & & & & 15 & & 31 \\
\hline Improve clear zone & & & & & 25 & 25 & & & & & & & & & & & & & & & 25 \\
\hline $\begin{array}{l}\text { Improve existing street } \\
\text { lights }\end{array}$ & 30 & 30 & & & 50 & 50 & & & & & & & & & & & & & & & 40 \\
\hline Improve lanes width & 8 & & & & & & & & & & & & & & 8 & & & & & & 8 \\
\hline $\begin{array}{l}\text { Improve pavement } \\
\text { marking }\end{array}$ & & & 21 & 21 & & & & & & & & & & & & & & & & & 21 \\
\hline $\begin{array}{l}\text { Improve pavement } \\
\text { marking in the median } \\
\text { opening }\end{array}$ & & & 21 & 21 & & & & & & & & & & & & & & & & & 21 \\
\hline $\begin{array}{l}\text { Improve signage and/or } \\
\text { marking of the crosswalk }\end{array}$ & & & & & & & & & & & 25 & 25 & & & & & & & & & 25 \\
\hline Improve skid resistance & & & 9 & 9 & & & & & & & & & & & & & & & & & 9 \\
\hline $\begin{array}{l}\text { Improve visibility from } \\
\text { median toward major } \\
\text { approach }\end{array}$ & & & & & & & & & & & & & & & & & & & 10 & 10 & 10 \\
\hline $\begin{array}{l}\text { Improve visibility of } \\
\text { intersections by providing }\end{array}$ & & & & & & & & & & & 30 & 30 & & & & & & & & & 30 \\
\hline
\end{tabular}




\begin{tabular}{|c|c|c|c|c|c|c|c|c|c|c|c|c|c|c|c|c|c|c|c|c|c|}
\hline & \multicolumn{2}{|c|}{ S1 } & \multicolumn{2}{|c|}{$\mathrm{S2}$} & \multicolumn{2}{|c|}{ S3 } & \multicolumn{2}{|c|}{ S4 } & \multicolumn{2}{|c|}{ S5 } & \multicolumn{2}{|c|}{ S6 } & \multicolumn{2}{|c|}{ S7 } & \multicolumn{2}{|c|}{ S8 } & \multicolumn{2}{|c|}{ S9,S10 } & \multicolumn{2}{|c|}{ S11-S21 } & \multirow[t]{2}{*}{ FINAL } \\
\hline & $\mathbf{R}$ & $\mathbf{U}$ & $\mathbf{R}$ & $\mathbf{U}$ & $\mathbf{R}$ & $\mathbf{U}$ & $\mathbf{R}$ & $\mathbf{U}$ & $\mathbf{R}$ & $\mathbf{U}$ & $\mathbf{R}$ & $\mathbf{U}$ & $\mathbf{R}$ & $\mathbf{U}$ & $\mathbf{R}$ & $\mathbf{U}$ & $\mathbf{R}$ & $\mathbf{U}$ & $\mathbf{R}$ & $\mathbf{U}$ & \\
\hline $\begin{array}{l}\text { enhanced signing and } \\
\text { delineation }\end{array}$ & & & & & & & & & & & & & & & & & & & & & \\
\hline $\begin{array}{l}\text { Improve visibility of the } \\
\text { intersection by providing } \\
\text { lighting }\end{array}$ & 30 & 30 & 33 & 33 & 50 & 50 & & & & & 30 & 30 & 37 & 37 & & & & & 37 & 37 & 36 \\
\hline $\begin{array}{l}\text { Improve winter } \\
\text { maintenance methods }\end{array}$ & & & & & & & & & & & & & & & & & & & & & 25 \\
\hline $\begin{array}{l}\text { Improve winter } \\
\text { maintenance } \\
\text { preparedness (shorter } \\
\text { response time) }\end{array}$ & & & & & & & & & & & & & & & & & & & & & 20 \\
\hline $\begin{array}{l}\text { Improve/install reflective } \\
\text { signs }\end{array}$ & & & 15 & 15 & & & & & & & & & & & & & & & & & 15 \\
\hline $\begin{array}{l}\text { Improve/install } \\
\text { reflectorized pavement } \\
\text { markers }\end{array}$ & & & & & & & & & & & & & & & & & & & & & 12 \\
\hline $\begin{array}{l}\text { Increase length of the right } \\
\text { turn lanes }\end{array}$ & & & 28 & 28 & & & 15 & 15 & & & 28 & 28 & & & & & & & & & 24 \\
\hline $\begin{array}{l}\text { Increase radius of the } \\
\text { corner curve }\end{array}$ & & & 13 & 13 & & & & & & & 15 & 15 & & & & & & & & & 14 \\
\hline $\begin{array}{l}\text { Increase the crosswalk } \\
\text { setback }\end{array}$ & & & & & & & & & & & & & & & & & & & & & 15 \\
\hline $\begin{array}{l}\text { Increase the length of the } \\
\text { left turn lanes }\end{array}$ & & & 28 & 28 & & & 15 & 15 & & & 28 & 28 & & & & & & & & & 24 \\
\hline $\begin{array}{l}\text { Increase the turning path } \\
\text { radius }\end{array}$ & & & 13 & 13 & & & & & & & 15 & 15 & & & & & & & & & 14 \\
\hline $\begin{array}{l}\text { Increase width of the } \\
\text { shoulders }\end{array}$ & 9 & & 14 & 14 & & & & & & & 20 & 20 & & & 9 & & & & 14 & & 14 \\
\hline $\begin{array}{l}\text { Install a barrier curb or a } \\
\text { guardrail }\end{array}$ & 45 & 45 & 11 & 11 & & & 47 & 47 & & & & & & 4 & & & 44 & 44 & & & 33 \\
\hline $\begin{array}{l}\text { Install a pedestrian } \\
\text { overpass or underpass }\end{array}$ & & & 86 & 86 & & & & & & & & & & & & & & & 91 & 91 & 89 \\
\hline Install a stop sign & & & 35 & 35 & & & & & & & 39 & 39 & & & & & & & & & 37 \\
\hline Install a triangular island to & & & & & & & & & & & & & & & & & & & & & 25 \\
\hline
\end{tabular}




\begin{tabular}{|c|c|c|c|c|c|c|c|c|c|c|c|c|c|c|c|c|c|c|c|c|c|}
\hline & \multicolumn{2}{|c|}{ S1 } & \multicolumn{2}{|c|}{ S2 } & \multicolumn{2}{|c|}{ S3 } & \multicolumn{2}{|c|}{ S4 } & \multicolumn{2}{|c|}{ S5 } & \multicolumn{2}{|c|}{ S6 } & \multicolumn{2}{|c|}{ S7 } & \multicolumn{2}{|c|}{ S8 } & \multicolumn{2}{|c|}{ S9,S10 } & \multicolumn{2}{|c|}{ S11-S21 } & \multirow[t]{2}{*}{ FINAL } \\
\hline & $\mathbf{R}$ & $\mathbf{U}$ & $\mathbf{R}$ & $\mathbf{U}$ & $\mathbf{R}$ & $\mathbf{U}$ & $\mathbf{R}$ & $\mathbf{U}$ & $\mathbf{R}$ & $\mathbf{U}$ & $\mathbf{R}$ & $\mathbf{U}$ & $\mathbf{R}$ & $\mathbf{U}$ & $\mathbf{R}$ & $\mathbf{U}$ & $\mathbf{R}$ & $\mathbf{U}$ & $\mathbf{R}$ & $\mathbf{U}$ & \\
\hline $\begin{array}{l}\text { relocate the right-turning } \\
\text { movement away from } \\
\text { other movements }\end{array}$ & & & & & & & & & & & & & & & & & & & & & \\
\hline $\begin{array}{l}\text { Install acceleration lanes } \\
\text { sufficiently long for trucks }\end{array}$ & & & 26 & 26 & & & & & & & & & & & & & & & & & 26 \\
\hline $\begin{array}{l}\text { Install advance warning } \\
\text { sign }\end{array}$ & 20 & 20 & 25 & 25 & & & 25 & 25 & & & & & 30 & 40 & & & & & & & 26 \\
\hline Install an acceleration lane & & & 26 & 26 & & & & & & & 10 & 10 & 10 & 10 & & & & & & & 15 \\
\hline Install an object marker & & & & & & & 16 & 16 & & & & & & & & & & & & & 16 \\
\hline $\begin{array}{l}\text { Install channelization of } \\
\text { the driveways }\end{array}$ & & & & & & & & & 50 & 50 & & & & & & & & & & & 50 \\
\hline Install corner mirrors & & & & & & & & & & & & & & & & & & & & & 10 \\
\hline $\begin{array}{l}\text { Install curbing to define } \\
\text { driveway location }\end{array}$ & & & & & & & & & & & & & & & & & & & & & 20 \\
\hline Install curb-ramps & & & & & & & & & & & & & & & & & & & & & 15 \\
\hline Install flashing beacons & 30 & 30 & & & 50 & 50 & 30 & 30 & & & 30 & 30 & 24 & 24 & & & & & & & 33 \\
\hline $\begin{array}{l}\text { Install flashing beacons at } \\
\text { stop-controlled } \\
\text { intersections }\end{array}$ & & & & & 50 & 50 & 30 & 30 & & & & & 24 & 24 & & & & & & & 35 \\
\hline Install fog - warning signs & & & & & & & 25 & 25 & & & & & & & & & & & & & 25 \\
\hline Install guardrails & & & & & 20 & 20 & 47 & 47 & & & 65 & 65 & & 4 & & & 44 & 44 & & & 40 \\
\hline $\begin{array}{l}\text { Install indirect left-turn } \\
\text { lanes }\end{array}$ & & & 34 & 34 & & & 35 & 35 & & & & & & & & & & & & & 35 \\
\hline $\begin{array}{l}\text { Install larger regulatory } \\
\text { and warning signs at and } \\
\text { in advance of intersections }\end{array}$ & & & & & & & & & & & 15 & 15 & 40 & 30 & & & & & & & 25 \\
\hline $\begin{array}{l}\text { Install larger regulatory } \\
\text { and warning signs at } \\
\text { intersections }\end{array}$ & & & & & & & & & & & 15 & 15 & & & & & & & & & 15 \\
\hline $\begin{array}{l}\text { Install left turn acceleration } \\
\text { lane }\end{array}$ & & & 26 & 26 & & & & & & & 10 & 10 & & & & & & & & & 18 \\
\hline Install left turn bays & 28 & 27 & & & 20 & 20 & & & & & & & & & 28 & 27 & & & & & 25 \\
\hline Install lighting & 30 & 30 & 33 & 33 & 50 & 50 & & & & & 30 & 30 & 37 & 37 & & & & & 37 & 37 & 36 \\
\hline
\end{tabular}




\begin{tabular}{|c|c|c|c|c|c|c|c|c|c|c|c|c|c|c|c|c|c|c|c|c|c|}
\hline & \multicolumn{2}{|c|}{ S1 } & \multicolumn{2}{|c|}{ S2 } & \multicolumn{2}{|c|}{ S3 } & \multicolumn{2}{|c|}{ S4 } & \multicolumn{2}{|c|}{ S5 } & \multicolumn{2}{|c|}{ S6 } & \multicolumn{2}{|c|}{ S7 } & \multicolumn{2}{|c|}{ S8 } & \multicolumn{2}{|c|}{ S9,S10 } & \multicolumn{2}{|c|}{ S11-S21 } & \multirow[t]{2}{*}{ FINAL } \\
\hline & $\mathbf{R}$ & $\mathbf{U}$ & $\mathbf{R}$ & $\mathbf{U}$ & $\mathbf{R}$ & $\mathbf{U}$ & $\mathbf{R}$ & $\mathbf{U}$ & $\mathbf{R}$ & $\mathbf{U}$ & $\mathbf{R}$ & $\mathbf{U}$ & $\mathbf{R}$ & $\mathbf{U}$ & $\mathbf{R}$ & $\mathbf{U}$ & $\mathbf{R}$ & $\mathbf{U}$ & $\mathbf{R}$ & $\mathbf{U}$ & \\
\hline Install median divider & 15 & 15 & & & & & & & & & & & & & & & & & & & 15 \\
\hline $\begin{array}{l}\text { Install overhead flashing } \\
\text { beacon lights }\end{array}$ & 30 & 30 & & & 50 & 50 & 30 & 30 & & & 30 & 30 & 24 & 24 & & & & & & & 33 \\
\hline Install pavement markings & 10 & 10 & 21 & 21 & & & & & 21 & 21 & & & & & & & & & 10 & 10 & 16 \\
\hline $\begin{array}{l}\text { Install pedestrian actuated } \\
\text { signals }\end{array}$ & & & & & & & & & & & & & & & & & & & & & 35 \\
\hline Install pedestrian barriers & & & & & & & & & & & & & & & & & & & & & 60 \\
\hline $\begin{array}{l}\text { Install pedestrian } \\
\text { crosswalk }\end{array}$ & & & 25 & 25 & & & & & 25 & 25 & & & & & & & & & & & 25 \\
\hline Install raised crosswalk & & & 25 & 25 & & & & & 25 & 25 & & & & & & & 46 & & & & 29 \\
\hline $\begin{array}{l}\text { Install reflectorized } \\
\text { pavement marking }\end{array}$ & & & & & & & & & & & & & & & & & & & & & 57 \\
\hline $\begin{array}{l}\text { Install regulatory signs on } \\
\text { the both side of the road }\end{array}$ & & & & & & & & & & & & & & & & & & & & & 20 \\
\hline $\begin{array}{l}\text { Install right turn bays if the } \\
\text { number of vehicle turning } \\
\text { right is considerable }\end{array}$ & & & & & & & & & & & & & & & & & & & & & 25 \\
\hline Install right turn lane & & 14 & 25 & 25 & & & 25 & 25 & & & 24 & 24 & & & 14 & & 26 & & 10 & & 21 \\
\hline Install rumble strips & & & 32 & 32 & 28 & 28 & & & & & 25 & 25 & & & 21 & & & & 50 & 50 & 32 \\
\hline Install school crossing sign & & & 18 & 18 & & & & & & & 14 & 14 & & & & & & & & & 16 \\
\hline $\begin{array}{l}\text { Install school zone } \\
\text { markings }\end{array}$ & & & & & & & & & & & & & & & & & & & & & 20 \\
\hline $\begin{array}{l}\text { Install shoulder rumble } \\
\text { strips }\end{array}$ & 21 & & & & & & 29 & 29 & 30 & 30 & 25 & 25 & & & 21 & & 25 & 25 & 35 & 35 & 28 \\
\hline Install sidewalk set-backs & & & & & & & & & & & & & & & & & & & & & 20 \\
\hline Install speed limit sign & & & & & & & & & & & & & & & & & & & & & 15 \\
\hline $\begin{array}{l}\text { Install static or variable } \\
\text { message signs displaying } \\
\text { weather information }\end{array}$ & & & 15 & 15 & & & & & & & 15 & 15 & & & & & & & & & 15 \\
\hline Install street lights & 30 & 30 & & & 50 & 50 & & & & & & & & & & & & & & & 40 \\
\hline Install streets name signs & & & & & & & & & & & 25 & 25 & & & & & & & & & 25 \\
\hline Install traffic signals & & 14 & 30 & 30 & & & & & & & 28 & 28 & 28 & 28 & & 23 & 23 & 23 & & & 26 \\
\hline
\end{tabular}




\begin{tabular}{|c|c|c|c|c|c|c|c|c|c|c|c|c|c|c|c|c|c|c|c|c|c|}
\hline & \multicolumn{2}{|c|}{ S1 } & \multicolumn{2}{|c|}{ S2 } & \multicolumn{2}{|c|}{ S3 } & \multicolumn{2}{|c|}{ S4 } & \multicolumn{2}{|c|}{ S5 } & \multicolumn{2}{|c|}{ S6 } & \multicolumn{2}{|c|}{ S7 } & \multicolumn{2}{|c|}{ S8 } & \multicolumn{2}{|c|}{ S9,S10 } & \multicolumn{2}{|c|}{ S11-S21 } & \multirow[t]{2}{*}{ FINAL } \\
\hline & $\mathbf{R}$ & $\mathbf{U}$ & $\mathbf{R}$ & $\mathbf{U}$ & $\mathbf{R}$ & $\mathbf{U}$ & $\mathbf{R}$ & $\mathbf{U}$ & $\mathbf{R}$ & $\mathbf{U}$ & $\mathbf{R}$ & $\mathbf{U}$ & $\mathbf{R}$ & $\mathbf{U}$ & $\mathbf{R}$ & $\mathbf{U}$ & $\mathbf{R}$ & $\mathbf{U}$ & $\mathbf{R}$ & $\mathbf{U}$ & \\
\hline $\begin{array}{l}\text { Install/Improve lighting at } \\
\text { the access points }\end{array}$ & & & 33 & 33 & & & & & & & 30 & 30 & 37 & 37 & & & & & 37 & 37 & 34 \\
\hline $\begin{array}{l}\text { Install/improve signing or } \\
\text { marking of pedestrian } \\
\text { crosswalks }\end{array}$ & & & & & & & & & & & 15 & 15 & & & & & 16 & 16 & & & 16 \\
\hline $\begin{array}{l}\text { Install/improve warning } \\
\text { signs }\end{array}$ & & & 25 & 25 & 20 & 20 & 25 & 25 & & & 25 & 25 & & & & & & & & & 24 \\
\hline $\begin{array}{l}\text { Installing snow screens in } \\
\text { areas exposed to } \\
\text { snowdrifts }\end{array}$ & & & & & 53 & 53 & & & & & & & & & & & & & & & 53 \\
\hline $\begin{array}{l}\text { Move driveway to side } \\
\text { street }\end{array}$ & & & 50 & 50 & 15 & 15 & & & & & & & & & & & & & & & 33 \\
\hline Overlay pavement & & & 17 & 17 & 18 & 18 & 27 & 27 & & & & & & & & & & & & & 21 \\
\hline $\begin{array}{l}\text { Post an adequate speed } \\
\text { limit }\end{array}$ & & & & & 28 & 28 & & & & & & & 20 & 20 & & & & & & & 24 \\
\hline $\begin{array}{l}\text { Post dynamic message } \\
\text { sign to display the speed } \\
\text { of approaching vehicles. }\end{array}$ & & & & & & & & & & & & & & & & & 74 & 74 & & & 74 \\
\hline $\begin{array}{l}\text { Properly maintain the } \\
\text { striping }\end{array}$ & & & & & & & & & & & & & & & & & & & & & 10 \\
\hline $\begin{array}{l}\text { Provide "Slippery when } \\
\text { wet" signs }\end{array}$ & & & & & & & & & & & & & & & & & & & & & 25 \\
\hline $\begin{array}{l}\text { Provide a bypass lane on } \\
\text { the approach with left-turn } \\
\text { traffic }\end{array}$ & & & & & & & & & & & & & & & & & & & & & 10 \\
\hline $\begin{array}{l}\text { Provide a double yellow } \\
\text { centerline on the median } \\
\text { opening of a divided } \\
\text { highway at intersections }\end{array}$ & & & & & & & & & & & & & & & & & & & & & 5 \\
\hline $\begin{array}{l}\text { Provide acceleration and } \\
\text { deceleration lanes }\end{array}$ & & & 26 & 26 & & & & & & & 10 & 10 & 10 & 10 & & & & & & & 15 \\
\hline $\begin{array}{l}\text { Provide adequate } \\
\text { delineation for left-turns at } \\
\text { the intersection (markers }\end{array}$ & & & & & & & & & & & 30 & 30 & & & & & & & & & 30 \\
\hline
\end{tabular}




\begin{tabular}{|c|c|c|c|c|c|c|c|c|c|c|c|c|c|c|c|c|c|c|c|c|c|}
\hline & \multicolumn{2}{|c|}{ S1 } & \multicolumn{2}{|c|}{ S2 } & \multicolumn{2}{|c|}{ S3 } & \multicolumn{2}{|c|}{ S4 } & \multicolumn{2}{|c|}{ S5 } & \multicolumn{2}{|c|}{ S6 } & \multicolumn{2}{|c|}{ S7 } & \multicolumn{2}{|c|}{ S8 } & \multicolumn{2}{|c|}{ S9,S10 } & \multicolumn{2}{|c|}{ S11-S21 } & \multirow[t]{2}{*}{ FINAL } \\
\hline & $\mathbf{R}$ & $\mathbf{U}$ & $\mathbf{R}$ & $\mathbf{U}$ & $\mathbf{R}$ & $\mathbf{U}$ & $\mathbf{R}$ & $\mathbf{U}$ & $\mathbf{R}$ & $\mathbf{U}$ & $\mathbf{R}$ & $\mathbf{U}$ & $\mathbf{R}$ & $\mathbf{U}$ & $\mathbf{R}$ & $\mathbf{U}$ & $\mathbf{R}$ & $\mathbf{U}$ & $\mathbf{R}$ & $\mathbf{U}$ & \\
\hline \multicolumn{22}{|l|}{ or lines) } \\
\hline $\begin{array}{l}\text { Provide adequate } \\
\text { drainage }\end{array}$ & & & 20 & 20 & & & & & & & & & & & & & & & & & 20 \\
\hline $\begin{array}{l}\text { Provide adequate lanes } \\
\text { signing or marking }\end{array}$ & & & & & 21 & 21 & & & & & & & & & & & & & & & 21 \\
\hline $\begin{array}{l}\text { Provide adequate turning } \\
\text { markers or pavement } \\
\text { markings }\end{array}$ & 10 & 10 & 21 & 21 & & & & & & & & & & & & & & & 10 & 10 & 14 \\
\hline $\begin{array}{l}\text { Provide dashed markings } \\
\text { (extended left edgelines) } \\
\text { for major-road continuity } \\
\text { across the median } \\
\text { opening at divided } \\
\text { highway intersections }\end{array}$ & & & & & 24 & 24 & & & & & 30 & 30 & 11 & 11 & & & & & & & 22 \\
\hline $\begin{array}{l}\text { Provide independent } \\
\text { bicycle path where } \\
\text { necessary }\end{array}$ & & & & & & & & & & & & & & & & & & & & & 20 \\
\hline Provide median refuges & & 46 & & & & & & & & & & & & & & & & & & & 46 \\
\hline $\begin{array}{l}\text { Provide offset for left turn } \\
\text { lanes on the opposite } \\
\text { approaches }\end{array}$ & & & & & & & 10 & 10 & & & & & & & & & & & & & 10 \\
\hline $\begin{array}{l}\text { Provide on-pavement } \\
\text { horizontal signing }\end{array}$ & & & & & & & & & & & & & & & & & & & & & 10 \\
\hline $\begin{array}{l}\text { Provide pavement marking } \\
\text { (stop sign, chevron, etc.) }\end{array}$ & & & 21 & 21 & 35 & 35 & & & & & & & 35 & 35 & & & 25 & & & & 30 \\
\hline $\begin{array}{l}\text { Provide roadside markers } \\
\text { or pavement markings to } \\
\text { assist drivers in judging } \\
\text { the suitability of available } \\
\text { gaps for making turning } \\
\text { and crossing maneuvers }\end{array}$ & & & & & & & & & & & & & & & & & & & & & 10 \\
\hline $\begin{array}{l}\text { Provide smooth paved } \\
\text { shoulders }\end{array}$ & & & 15 & 15 & & & & & & & & & & 53 & & & & & 14 & & 24 \\
\hline Provide splitter islands & & & & & & & & & & & & & & & & & & & & & 35 \\
\hline
\end{tabular}




\begin{tabular}{|c|c|c|c|c|c|c|c|c|c|c|c|c|c|c|c|c|c|c|c|c|c|}
\hline & \multicolumn{2}{|c|}{ S1 } & \multicolumn{2}{|c|}{ S2 } & \multicolumn{2}{|c|}{ S3 } & \multicolumn{2}{|c|}{ S4 } & \multicolumn{2}{|c|}{ S5 } & \multicolumn{2}{|c|}{ S6 } & \multicolumn{2}{|c|}{ S7 } & \multicolumn{2}{|c|}{ S8 } & \multicolumn{2}{|c|}{ S9,S10 } & \multicolumn{2}{|c|}{ S11-S21 } & \multirow[t]{2}{*}{ FINAL } \\
\hline & $\mathbf{R}$ & $\mathbf{U}$ & $\mathbf{R}$ & $\mathbf{U}$ & $\mathbf{R}$ & $\mathbf{U}$ & $\mathbf{R}$ & $\mathbf{U}$ & $\mathbf{R}$ & $\mathbf{U}$ & $\mathbf{R}$ & $\mathbf{U}$ & $\mathbf{R}$ & $\mathbf{U}$ & $\mathbf{R}$ & $\mathbf{U}$ & $\mathbf{R}$ & $\mathbf{U}$ & $\mathbf{R}$ & $\mathbf{U}$ & \\
\hline Provide stop signs & & & 35 & 35 & & & & & & & 39 & 39 & & & & & & & & & 37 \\
\hline $\begin{array}{l}\text { Provide targeted speed } \\
\text { enforcement }\end{array}$ & & & & & & & & & & & & & & & & & & & & & 70 \\
\hline $\begin{array}{l}\text { Provide traffic calming on } \\
\text { intersection approaches } \\
\text { through a combination of } \\
\text { geometric and traffic } \\
\text { control devices }\end{array}$ & & & & & & & & & & & & & & & & & & & & & 20 \\
\hline $\begin{array}{l}\text { Provide traffic signal if } \\
\text { warranted }\end{array}$ & & & & & & & & & & & 28 & 28 & & & & 23 & 23 & 23 & & & 25 \\
\hline Provide visible stop bars & & & & & & & & & & & & & & & & & & & & & 5 \\
\hline $\begin{array}{l}\text { Provide/Improve } \\
\text { pavement surface } \\
\text { (improve transitions, drop } \\
\text { offs, fixed pothols, rutting, } \\
\text { etc) }\end{array}$ & & & & & & & & & & & & & & & & & & & & & 28 \\
\hline Reconstruct the radius & & & 13 & 13 & & & & & & & 15 & 15 & & & & & & & & & 14 \\
\hline $\begin{array}{l}\text { Reduce speed on the } \\
\text { major road }\end{array}$ & & & & & & & & & & & 20 & 20 & 20 & 20 & & & & & & & 20 \\
\hline Reduce speed limit & & & & & & & & & & & & & 20 & 20 & & & & & & & 20 \\
\hline $\begin{array}{l}\text { Reduce the number of } \\
\text { lanes on the minor } \\
\text { approach if allowed by } \\
\text { capacity }\end{array}$ & & & & & & & & & & & & & & & & & & & & & 15 \\
\hline Relocate crosswalk & & & & & & & & & & & & & & & & & & & & & 15 \\
\hline Relocate islands & & & & & & & & & & & & & & & & & & & & & 10 \\
\hline $\begin{array}{l}\text { Relocate or split the } \\
\text { stopping line }\end{array}$ & & & & & & & & & & & & & & & & & & & & & 10 \\
\hline Relocate stop bar & & & & & & & & & & & & & & & & & & & & & 10 \\
\hline Relocate stop sign & & & & & & & & & & & & & & & & & & & & & 15 \\
\hline $\begin{array}{l}\text { Relocate the sign to make } \\
\text { it visible }\end{array}$ & & & & & & & & & & & & & & & & & & & & & 20 \\
\hline Remove distracting & & & & & & & & & & & 15 & 15 & & & & & & & & & 15 \\
\hline
\end{tabular}




\begin{tabular}{|c|c|c|c|c|c|c|c|c|c|c|c|c|c|c|c|c|c|c|c|c|c|}
\hline & \multicolumn{2}{|c|}{ S1 } & \multicolumn{2}{|c|}{ S2 } & \multicolumn{2}{|c|}{ S3 } & \multicolumn{2}{|c|}{ S4 } & \multicolumn{2}{|c|}{ S5 } & \multicolumn{2}{|c|}{ S6 } & \multicolumn{2}{|c|}{ S7 } & \multicolumn{2}{|c|}{ S8 } & \multicolumn{2}{|c|}{ S9,S10 } & \multicolumn{2}{|c|}{ S11-S21 } & \multirow[t]{2}{*}{ FINAL } \\
\hline & $\mathbf{R}$ & $\mathbf{U}$ & $\mathbf{R}$ & $\mathbf{U}$ & $\mathbf{R}$ & $\mathbf{U}$ & $\mathbf{R}$ & $\mathbf{U}$ & $\mathbf{R}$ & $\mathbf{U}$ & $\mathbf{R}$ & $\mathbf{U}$ & $\mathbf{R}$ & $\mathbf{U}$ & $\mathbf{R}$ & $\mathbf{U}$ & $\mathbf{R}$ & $\mathbf{U}$ & $\mathbf{R}$ & $\mathbf{U}$ & \\
\hline $\begin{array}{l}\text { commercial lighting or } \\
\text { other source of glare }\end{array}$ & & & & & & & & & & & & & & & & & & & & & \\
\hline $\begin{array}{l}\text { Remove or re-locate the } \\
\text { median object obstructing } \\
\text { sight of opposite traffic }\end{array}$ & 12 & & & & & & & & & & & & & & 13 & & & & 10 & 10 & 11 \\
\hline $\begin{array}{l}\text { Remove or relocate the } \\
\text { object }\end{array}$ & & & & & 60 & 60 & 29 & 29 & & & 30 & 30 & 50 & 50 & & & & & & & 42 \\
\hline Remove sight obstructions & 12 & & & & & & & & & & & & & & 13 & & & & & & 13 \\
\hline $\begin{array}{l}\text { Remove the object } \\
\text { obstructing sight of the } \\
\text { warning sign }\end{array}$ & & & & & & & & & & & & & & & & & & & & & 20 \\
\hline $\begin{array}{l}\text { Remove the object } \\
\text { obstructing the sight of the } \\
\text { stop sign }\end{array}$ & & & & & & & & & & & & & & & & & & & & & 30 \\
\hline $\begin{array}{l}\text { Remove the object } \\
\text { obstructing the sight } \\
\text { triangle }\end{array}$ & 12 & & & & & & 25 & 25 & & & & & & & 13 & & & & 10 & 10 & 16 \\
\hline $\begin{array}{l}\text { Replace poorly designed } \\
\text { drain grates with bicycle- } \\
\text { safe types }\end{array}$ & & & & & & & & & & & & & & & & & & & & & 10 \\
\hline $\begin{array}{l}\text { Replace, repair, or clean } \\
\text { the sign }\end{array}$ & & & & & 5 & 5 & & & & & & & & & & & & & & & 5 \\
\hline $\begin{array}{l}\text { Replace, repair, or clean } \\
\text { the warning signs }\end{array}$ & & & & & 20 & 20 & 25 & 25 & & & & & & & & & & & & & 23 \\
\hline Reroute pedestrian paths & & & & & & & & & & & & & & & & & & & & & 30 \\
\hline Restrict left-turn maneuver & & & & & 40 & & & & & & & & & & & & & & & & 40 \\
\hline $\begin{array}{l}\text { Restrict left-turning at the } \\
\text { access points }\end{array}$ & & & & & & & & & & & & & & & & & & & 100 & & 100 \\
\hline $\begin{array}{l}\text { Restrict parking near the } \\
\text { driveway }\end{array}$ & & & & & 22 & 22 & & & & & 35 & 35 & 8 & 8 & & & & & 10 & 10 & 19 \\
\hline $\begin{array}{l}\text { Restrict parking on the } \\
\text { approach }\end{array}$ & & & & & & & & & & & & & & & & & & & 10 & 10 & 10 \\
\hline $\begin{array}{l}\text { Signals to alert motorists } \\
\text { that pedestrians are }\end{array}$ & & & & & & & & & & & & & & & & & 16 & 16 & & & 16 \\
\hline
\end{tabular}




\begin{tabular}{|c|c|c|c|c|c|c|c|c|c|c|c|c|c|c|c|c|c|c|c|c|c|}
\hline & \multicolumn{2}{|c|}{ S1 } & \multicolumn{2}{|c|}{ S2 } & \multicolumn{2}{|c|}{ S3 } & \multicolumn{2}{|c|}{ S4 } & \multicolumn{2}{|c|}{ S5 } & \multicolumn{2}{|c|}{ S6 } & \multicolumn{2}{|c|}{ S7 } & \multicolumn{2}{|c|}{ S8 } & \multicolumn{2}{|c|}{ S9,S10 } & \multicolumn{2}{|c|}{ S11-S21 } & \multirow[t]{2}{*}{ FINAL } \\
\hline & $\mathbf{R}$ & $\mathbf{U}$ & $\mathbf{R}$ & $\mathbf{U}$ & $\mathbf{R}$ & $\mathbf{U}$ & $\mathbf{R}$ & $\mathbf{U}$ & $\mathbf{R}$ & $\mathbf{U}$ & $\mathbf{R}$ & $\mathbf{U}$ & $\mathbf{R}$ & $\mathbf{U}$ & $\mathbf{R}$ & $\mathbf{U}$ & $\mathbf{R}$ & $\mathbf{U}$ & $\mathbf{R}$ & $\mathbf{U}$ & \\
\hline \multicolumn{22}{|l|}{ crossing } \\
\hline Trim vegetation & & & & & & & & & & & & & & & & & & & & & 35 \\
\hline Upgrade signing & & & & & 5 & 5 & & & & & 24 & 24 & & & & & & & & & 15 \\
\hline $\begin{array}{l}\text { Use "Slippery when wet" } \\
\text { signs }\end{array}$ & & & & & & & & & & & & & & & & & & & & & 15 \\
\hline Use break-away devices & & & & & 30 & 30 & & & & & & & & & & & & & & & 30 \\
\hline $\begin{array}{l}\text { Use salt to prevent snow } \\
\text { or ice from forming or from } \\
\text { sticking to the road } \\
\text { surface }\end{array}$ & & & & & & & & & & & & & & & & & & & & & 20 \\
\hline $\begin{array}{l}\text { Use sand to increase } \\
\text { pavement friction }\end{array}$ & & & & & & & & & & & & & & & & & & & & & 20 \\
\hline $\begin{array}{l}\text { Use school crossing } \\
\text { guards }\end{array}$ & & & & & & & & & & & & & & & & & & & & & 50 \\
\hline $\begin{array}{l}\text { Vehicles in the right-turn } \\
\text { lane on the major road } \\
\text { block the minor-road } \\
\text { drivers' view of traffic } \\
\text { approaching on the major } \\
\text { road }\end{array}$ & & & & & & & & & & & & & & & & & & & & & 15 \\
\hline $\begin{array}{l}\text { Widen lanes to adequate } \\
\text { width }\end{array}$ & & & 40 & 40 & 38 & & 22 & 22 & 30 & 30 & 25 & 25 & 23 & 23 & 8 & & & & 13 & 13 & 25 \\
\hline $\begin{array}{l}\text { Widen the median to } \\
\text { provide sufficient } \\
\text { protection to crossing } \\
\text { vehicles }\end{array}$ & & & & & & & & & & & & & & & & & & & & & 5 \\
\hline Widen the median width & & & & & & & & & & & & & 13 & 13 & & & & & & & 13 \\
\hline $\begin{array}{l}\text { Widen the outside through } \\
\text { lanes or add bike lanes }\end{array}$ & & & & & & & & & & & & & & & & & & & & & 15 \\
\hline
\end{tabular}




\section{AWSC}

\begin{tabular}{|c|c|c|c|c|c|c|c|c|c|c|c|c|c|c|c|c|c|c|c|c|c|}
\hline & \multicolumn{2}{|c|}{ S1 } & \multicolumn{2}{|c|}{$\mathbf{S 2}$} & \multicolumn{2}{|c|}{ S3 } & \multicolumn{2}{|c|}{ S4 } & \multicolumn{2}{|c|}{ S5 } & \multicolumn{2}{|c|}{ S6 } & \multicolumn{2}{|c|}{ S7 } & \multicolumn{2}{|c|}{ S8 } & \multicolumn{2}{|c|}{ S9-S10 } & \multicolumn{2}{|c|}{ S11-S21 } & \multirow[t]{2}{*}{ FINAL } \\
\hline & $\mathbf{R}$ & $\mathbf{U}$ & $\mathbf{R}$ & $\mathbf{U}$ & $\mathbf{R}$ & $\mathbf{U}$ & $\mathbf{R}$ & $\mathbf{U}$ & $\mathbf{R}$ & $\mathbf{U}$ & $\mathbf{R}$ & $\mathbf{U}$ & $\mathbf{R}$ & $\mathbf{U}$ & $\mathbf{R}$ & $\mathbf{U}$ & $\mathbf{R}$ & $\mathbf{U}$ & $\mathbf{R}$ & $\mathbf{U}$ & \\
\hline $\begin{array}{l}\text { Add overhead stop-signs if } \\
\text { the percent of tall vehicles } \\
\text { is considerable }\end{array}$ & & & & & & & & & & & & & & & & & & & & & 20 \\
\hline Add special signing & & & & & & & & & & & & & & & & & & & & & 10 \\
\hline $\begin{array}{l}\text { Change horizontal/vertical } \\
\text { alignment }\end{array}$ & & & 58 & 58 & & & & & & & 50 & 50 & 50 & 50 & & & & & 10 & 10 & 42 \\
\hline $\begin{array}{l}\text { Chip and seal or slurry } \\
\text { seal approaches }\end{array}$ & & & & & & & & & & & & & & & & & & & & & 10 \\
\hline Close curb lanes & & & & & & & & & & & & & & & & & & & & & 10 \\
\hline $\begin{array}{l}\text { Close or relocate the } \\
\text { driveways }\end{array}$ & & & 50 & 50 & & & & & & & & & & & & & & & & & 50 \\
\hline $\begin{array}{l}\text { Construct pedestrian } \\
\text { refuge islands and raised } \\
\text { medians }\end{array}$ & & 46 & 60 & 60 & 32 & 23 & & & & & & & & & & 46 & & & & & 45 \\
\hline $\begin{array}{l}\text { Eliminate screening by } \\
\text { physical objects }\end{array}$ & & & & & & & & & & & & & 50 & 50 & & & & & 76 & 76 & 63 \\
\hline Eliminate shoulder drop-off & & & & & & & & & & & 25 & 25 & & & & & & & 30 & 30 & 28 \\
\hline $\begin{array}{l}\text { Enforce stop sign } \\
\text { compliance }\end{array}$ & & & & & & & & & & & & & & & & & & & & & 70 \\
\hline Extend "no parking" zone & & & & & 22 & 22 & & & & & 35 & 35 & & & & & & & 10 & 10 & 22 \\
\hline Groove pavement surface & & & 21 & 21 & & & 14 & 14 & & & & & & & & & & & 46 & 46 & 27 \\
\hline $\begin{array}{l}\text { Implement road narrowing } \\
\text { measures }\end{array}$ & & & & & & & & & & & & & & & & & & & & & 30 \\
\hline Improve alignment/grade & & & & & & & & & & & & & & & & & & & & & 35 \\
\hline $\begin{array}{l}\text { Improve } \\
\text { channelization/delineation }\end{array}$ & & & & & & & & & & & 30 & 30 & 40 & 40 & & & & & 15 & & 31 \\
\hline Improve clear zone & & & & & 25 & 25 & & & & & & & & & & & & & & & 25 \\
\hline $\begin{array}{l}\text { Improve existing street } \\
\text { lights }\end{array}$ & 30 & 30 & & & 50 & 50 & & & & & & & & & & & & & & & 40 \\
\hline Improve lanes width & 8 & & & & & & & & & & & & & & 8 & & & & & & 8 \\
\hline
\end{tabular}




\begin{tabular}{|c|c|c|c|c|c|c|c|c|c|c|c|c|c|c|c|c|c|c|c|c|c|}
\hline & \multicolumn{2}{|c|}{ S1 } & \multicolumn{2}{|c|}{ S2 } & \multicolumn{2}{|c|}{ S3 } & \multicolumn{2}{|c|}{ S4 } & \multicolumn{2}{|c|}{ S5 } & \multicolumn{2}{|c|}{ S6 } & \multicolumn{2}{|c|}{ S7 } & \multicolumn{2}{|c|}{ S8 } & \multicolumn{2}{|c|}{ S9-S10 } & \multicolumn{2}{|c|}{ S11-S21 } & \multirow[b]{2}{*}{$\begin{array}{r}\text { FINAL } \\
\\
21\end{array}$} \\
\hline $\begin{array}{l}\text { Improve pavement } \\
\text { marking in the median } \\
\text { opening }\end{array}$ & $\mathbf{R}$ & $\mathbf{U}$ & $\begin{array}{l}\mathbf{R} \\
21 \\
\end{array}$ & $\begin{array}{l}U \\
21\end{array}$ & $\mathbf{R}$ & $\mathbf{U}$ & $\mathbf{R}$ & $\mathbf{U}$ & $\mathbf{R}$ & $\mathbf{U}$ & $\mathbf{R}$ & $\mathbf{U}$ & $\mathbf{R}$ & $\mathbf{U}$ & $\mathbf{R}$ & $\mathbf{U}$ & $\mathbf{R}$ & $\mathbf{U}$ & $\mathbf{R}$ & $\mathbf{U}$ & \\
\hline $\begin{array}{l}\text { Improve signage and/or } \\
\text { marking of the crosswalk }\end{array}$ & & & & & & & & & & & 25 & 25 & & & & & & & & & 25 \\
\hline Improve skid resistance & & & 9 & 9 & & & & & & & & & & & & & & & & & 9 \\
\hline $\begin{array}{l}\text { Improve winter } \\
\text { maintenance methods }\end{array}$ & & & & & & & & & & & & & & & & & & & & & 25 \\
\hline $\begin{array}{l}\text { Improve winter } \\
\text { maintenance } \\
\text { preparedness (shorter } \\
\text { response time) }\end{array}$ & & & & & & & & & & & & & & & & & & & & & 20 \\
\hline $\begin{array}{l}\text { Improve/install reflective } \\
\text { signs }\end{array}$ & & & 15 & 15 & & & & & & & & & & & & & & & & & 15 \\
\hline $\begin{array}{l}\text { Improve/install } \\
\text { reflectorized pavement } \\
\text { markers }\end{array}$ & & & & & & & & & & & & & & & & & & & & & 12 \\
\hline $\begin{array}{l}\text { Increase width of the } \\
\text { shoulders }\end{array}$ & 9 & & 14 & 14 & & & & & & & 20 & 20 & & & 9 & & & & 14 & & 14 \\
\hline $\begin{array}{l}\text { Install a barrier curb or a } \\
\text { guardrail }\end{array}$ & 45 & 45 & 11 & 11 & & & 47 & 47 & & & & & & 4 & & & 44 & 44 & & & 33 \\
\hline $\begin{array}{l}\text { Install a pedestrian } \\
\text { overpass or underpass }\end{array}$ & & & 86 & 86 & & & & & & & & & & & & & & & 91 & 91 & 89 \\
\hline Install a stop sign & & & 35 & 35 & & & & & & & 39 & 39 & & & & & & & & & 37 \\
\hline $\begin{array}{l}\text { Install a triangular island to } \\
\text { relocate the right-turning } \\
\text { movement away from } \\
\text { other movements }\end{array}$ & & & & & & & & & & & & & & & & & & & & & 25 \\
\hline $\begin{array}{l}\text { Install adequate traffic } \\
\text { control device for bicycles } \\
\text { (i.e. stop sign) }\end{array}$ & & & & & & & & & & & & & & & & & & & & & 10 \\
\hline $\begin{array}{l}\text { Install advance warning } \\
\text { sign }\end{array}$ & 20 & 20 & 25 & 25 & & & 25 & 25 & & & & & 30 & 40 & & & & & & & 26 \\
\hline Install an object marker & & & & & & & 16 & 16 & & & & & & & & & & & & & 16 \\
\hline
\end{tabular}




\begin{tabular}{|c|c|c|c|c|c|c|c|c|c|c|c|c|c|c|c|c|c|c|c|c|c|}
\hline & \multicolumn{2}{|c|}{ S1 } & \multicolumn{2}{|c|}{ S2 } & \multicolumn{2}{|c|}{ S3 } & \multicolumn{2}{|c|}{ S4 } & \multicolumn{2}{|c|}{ S5 } & \multicolumn{2}{|c|}{ S6 } & \multicolumn{2}{|c|}{ S7 } & \multicolumn{2}{|c|}{ S8 } & \multicolumn{2}{|c|}{ S9-S10 } & \multicolumn{2}{|c|}{ S11-S21 } & \multirow[t]{2}{*}{ FINAL } \\
\hline $\begin{array}{l}\text { Install channelization of the } \\
\text { driveways }\end{array}$ & $\mathbf{R}$ & $\mathbf{U}$ & $\mathbf{R}$ & $\mathbf{U}$ & $\mathbf{R}$ & $\mathbf{U}$ & $\mathbf{R}$ & $\mathbf{U}$ & $\begin{array}{l}\mathbf{R} \\
50\end{array}$ & $\begin{array}{l}\mathbf{U} \\
50\end{array}$ & $\mathbf{R}$ & $\mathbf{U}$ & $\mathbf{R}$ & $\mathbf{U}$ & $\mathbf{R}$ & $\mathbf{U}$ & $\mathbf{R}$ & $\mathbf{U}$ & $\mathbf{R}$ & $\mathbf{U}$ & \\
\hline Install corner mirrors & & & & & & & & & & & & & & & & & & & & & 10 \\
\hline $\begin{array}{l}\text { Install curbing to define } \\
\text { driveway location }\end{array}$ & & & & & & & & & & & & & & & & & & & & & 20 \\
\hline Install curb-ramps & & & & & & & & & & & & & & & & & & & & & 15 \\
\hline Install flashing beacons & 30 & 30 & & & 50 & 50 & 30 & 30 & & & 30 & 30 & 24 & 24 & & & & & & & 33 \\
\hline Install fog - warning signs & & & & & & & 25 & 25 & & & & & & & & & & & & & 25 \\
\hline Install guardrails & & & & & 20 & 20 & 47 & 47 & & & 65 & 65 & & 4 & & & 44 & 44 & & & 40 \\
\hline $\begin{array}{l}\text { Install larger regulatory } \\
\text { and warning signs at and } \\
\text { in advance of intersections }\end{array}$ & & & & & & & & & & & 15 & 15 & 40 & 30 & & & & & & & 25 \\
\hline Install lighting & 30 & 30 & 33 & 33 & 50 & 50 & & & & & 30 & 30 & 37 & 37 & & & & & 37 & 37 & 36 \\
\hline Install median divider & 15 & 15 & & & & & & & & & & & & & & & & & & & 15 \\
\hline $\begin{array}{l}\text { Install overhead flashing } \\
\text { beacon lights }\end{array}$ & 30 & 30 & & & 50 & 50 & 30 & 30 & & & 30 & 30 & 24 & 24 & & & & & & & 33 \\
\hline Install pavement markings & 10 & 10 & 21 & 21 & & & & & 21 & 21 & & & & & & & & & 10 & 10 & 16 \\
\hline $\begin{array}{l}\text { Install pedestrian actuated } \\
\text { signals }\end{array}$ & & & & & & & & & & & & & & & & & & & & & 35 \\
\hline Install pedestrian barriers & & & & & & & & & & & & & & & & & & & & & 60 \\
\hline $\begin{array}{l}\text { Install pedestrian } \\
\text { crosswalk }\end{array}$ & & & 25 & 25 & & & & & 25 & 25 & & & & & & & & & & & 25 \\
\hline Install raised crosswalk & & & & & & & & & & & & & & & & & & & & & 29 \\
\hline $\begin{array}{l}\text { Install reflectorized } \\
\text { pavement marking }\end{array}$ & & & & & & & & & & & & & & & & & & & & & 57 \\
\hline $\begin{array}{l}\text { Install regulatory signs on } \\
\text { the both side of the road }\end{array}$ & & & & & & & & & & & & & & & & & & & & & 20 \\
\hline Install rumble strips & & & 32 & 32 & 28 & 28 & & & & & 25 & 25 & & & 21 & & & & 50 & 50 & 32 \\
\hline Install school crossing sign & & & 18 & 18 & & & & & & & 14 & 14 & & & & & & & & & 16 \\
\hline $\begin{array}{l}\text { Install school zone } \\
\text { markings }\end{array}$ & & & & & & & & & & & & & & & & & & & & & 20 \\
\hline Install sidewalk set-backs & & & & & & & & & & & & & & & & & & & & & 20 \\
\hline
\end{tabular}




\begin{tabular}{|c|c|c|c|c|c|c|c|c|c|c|c|c|c|c|c|c|c|c|c|c|c|}
\hline \multirow[b]{2}{*}{ Install speed limit sign } & \multicolumn{2}{|c|}{ S1 } & \multicolumn{2}{|c|}{ S2 } & \multicolumn{2}{|c|}{ S3 } & \multicolumn{2}{|c|}{ S4 } & \multicolumn{2}{|c|}{ S5 } & \multicolumn{2}{|c|}{ S6 } & \multicolumn{2}{|c|}{ S7 } & \multicolumn{2}{|c|}{ S8 } & \multicolumn{2}{|c|}{ S9-S10 } & \multicolumn{2}{|c|}{ S11-S21 } & \multirow{2}{*}{$\begin{array}{r}\text { FINAL } \\
15\end{array}$} \\
\hline & $\mathbf{R}$ & $\mathbf{U}$ & $\mathbf{R}$ & $\mathbf{U}$ & $\mathbf{R}$ & $\mathbf{U}$ & $\mathbf{R}$ & $\mathbf{U}$ & $\mathbf{R}$ & $\mathbf{U}$ & $\mathbf{R}$ & $\mathbf{U}$ & $\mathbf{R}$ & $\mathbf{U}$ & $\mathbf{R}$ & $\mathbf{U}$ & $\mathbf{R}$ & $\mathbf{U}$ & $\mathbf{R}$ & $\mathbf{U}$ & \\
\hline $\begin{array}{l}\text { Install static or variable } \\
\text { message signs displaying } \\
\text { weather information }\end{array}$ & & & 15 & 15 & & & & & & & 15 & 15 & & & & & & & & & 15 \\
\hline Install street lights & 30 & 30 & & & 50 & 50 & & & & & & & & & & & & & & & 40 \\
\hline Install streets name signs & & & & & & & & & & & 25 & 25 & & & & & & & & & 25 \\
\hline Install traffic signals & & 14 & 30 & 30 & & & & & & & 28 & 28 & 28 & 28 & & 23 & 23 & 23 & & & 26 \\
\hline $\begin{array}{l}\text { Install/Improve lighting at } \\
\text { the access points }\end{array}$ & & & 33 & 33 & & & & & & & 30 & 30 & 37 & 37 & & & & & 37 & 37 & 34 \\
\hline $\begin{array}{l}\text { Installing snow screens in } \\
\text { areas exposed to } \\
\text { snowdrifts }\end{array}$ & & & & & & & & & & & & & & & & & & & & & 53 \\
\hline $\begin{array}{l}\text { Move driveway to side } \\
\text { street }\end{array}$ & & & 50 & 50 & 15 & 15 & & & & & & & & & & & & & & & 33 \\
\hline Overlay pavement & & & 17 & 17 & 18 & 18 & 27 & 27 & & & & & & & & & & & & & 21 \\
\hline $\begin{array}{l}\text { Post an adequate speed } \\
\text { limit }\end{array}$ & & & & & 28 & 28 & & & & & & & 20 & 20 & & & & & & & 24 \\
\hline $\begin{array}{l}\text { Post dynamic message } \\
\text { sign to display the speed } \\
\text { of approaching vehicles. }\end{array}$ & & & & & & & & & & & & & & & & & 74 & 74 & & & 74 \\
\hline $\begin{array}{l}\text { Provide adequate median } \\
\text { width that will not limited } \\
\text { visibility }\end{array}$ & & & & & & & & & & & & & & & & & & & & & 15 \\
\hline $\begin{array}{l}\text { Provide "slippery when } \\
\text { wet" signs }\end{array}$ & & & & & & & & & & & & & & & & & & & & & 15 \\
\hline $\begin{array}{l}\text { Provide a double yellow } \\
\text { centerline on the median } \\
\text { opening of a divided } \\
\text { highway at intersections }\end{array}$ & & & & & & & & & & & & & & & & & & & & & 5 \\
\hline $\begin{array}{l}\text { Provide acceleration and } \\
\text { deceleration lanes }\end{array}$ & & & 26 & 26 & & & & & & & 10 & 10 & 10 & 10 & & & & & & & 15 \\
\hline $\begin{array}{l}\text { Provide adequate } \\
\text { delineation for left-turns at }\end{array}$ & & & & & & & & & & & 30 & 30 & & & & & & & & & 30 \\
\hline
\end{tabular}




\begin{tabular}{|c|c|c|c|c|c|c|c|c|c|c|c|c|c|c|c|c|c|c|c|c|c|}
\hline \multirow[b]{2}{*}{$\begin{array}{l}\text { the intersection (markers } \\
\text { or lines) }\end{array}$} & \multicolumn{2}{|c|}{ S1 } & \multicolumn{2}{|c|}{ S2 } & \multicolumn{2}{|c|}{ S3 } & \multicolumn{2}{|c|}{ S4 } & \multicolumn{2}{|c|}{ S5 } & \multicolumn{2}{|c|}{ S6 } & \multicolumn{2}{|c|}{ S7 } & \multicolumn{2}{|c|}{ S8 } & \multicolumn{2}{|c|}{ S9-S10 } & \multicolumn{2}{|c|}{ S11-S21 } & \multirow[t]{2}{*}{ FINAL } \\
\hline & $\mathbf{R}$ & $\mathbf{U}$ & $\mathbf{R}$ & $\mathbf{U}$ & $\mathbf{R}$ & $\mathbf{U}$ & $\mathbf{R}$ & $\mathbf{U}$ & $\mathbf{R}$ & $\mathbf{U}$ & $\mathbf{R}$ & $\mathbf{U}$ & $\mathbf{R}$ & $\mathbf{U}$ & $\mathbf{R}$ & $\mathbf{U}$ & $\mathbf{R}$ & $\mathbf{U}$ & $\mathbf{R}$ & $\mathbf{U}$ & \\
\hline Provide adequate drainage & & & 20 & 20 & & & & & & & & & & & & & & & & & 20 \\
\hline $\begin{array}{l}\text { Provide independent } \\
\text { bicycle path where } \\
\text { necessary }\end{array}$ & & & & & & & & & & & & & & & & & & & & & 20 \\
\hline Provide median refuges & & 46 & & & & & & & & & & & & & & & & & & & 46 \\
\hline $\begin{array}{l}\text { Provide on-pavement } \\
\text { horizontal signing }\end{array}$ & & & & & & & & & & & & & & & & & & & & & 10 \\
\hline $\begin{array}{l}\text { Provide pavement marking } \\
\text { (stop sign, chevron, etc.) }\end{array}$ & & & 21 & 21 & 35 & 35 & & & & & & & 35 & 35 & & & 25 & & & & 30 \\
\hline $\begin{array}{l}\text { Provide smooth paved } \\
\text { shoulders }\end{array}$ & & & 15 & 15 & & & & & & & & & & 53 & & & & & 14 & & 24 \\
\hline Provide splitter islands & & & & & & & & & & & & & & & & & & & & & 35 \\
\hline $\begin{array}{l}\text { Provide targeted speed } \\
\text { enforcement }\end{array}$ & & & & & & & & & & & & & & & & & & & & & 70 \\
\hline $\begin{array}{l}\text { Provide traffic calming on } \\
\text { intersection approaches } \\
\text { through a combination of } \\
\text { geometric and traffic } \\
\text { control devices }\end{array}$ & & & & & & & & & & & & & & & & & & & & & 20 \\
\hline Provide visible stop bars & & & & & & & & & & & & & & & & & & & & & 5 \\
\hline $\begin{array}{l}\text { Provide/Improve pavement } \\
\text { surface (improve } \\
\text { transitions, drop offs, fixed } \\
\text { pothols, rutting,etc.) }\end{array}$ & & & & & & & & & & & & & & & & & & & & & 28 \\
\hline Reduce speed limit & & & & & & & & & & & & & 20 & 20 & & & & & & & 20 \\
\hline $\begin{array}{l}\text { Reduce the number of } \\
\text { lanes if allowed by } \\
\text { capacity }\end{array}$ & & & & & & & & & & & & & & & & & & & & & 15 \\
\hline Relocate islands & & & & & & & & & & & & & & & & & & & & & 10 \\
\hline $\begin{array}{l}\text { Relocate or split the } \\
\text { stopping line }\end{array}$ & & & & & & & & & & & & & & & & & & & & & 10 \\
\hline
\end{tabular}




\begin{tabular}{|c|c|c|c|c|c|c|c|c|c|c|c|c|c|c|c|c|c|c|c|c|c|}
\hline \multirow[b]{2}{*}{ Relocate stop bar } & \multicolumn{2}{|c|}{ S1 } & \multicolumn{2}{|c|}{ S2 } & \multicolumn{2}{|c|}{ S3 } & \multicolumn{2}{|c|}{ S4 } & \multicolumn{2}{|c|}{ S5 } & \multicolumn{2}{|c|}{ S6 } & \multicolumn{2}{|c|}{ S7 } & \multicolumn{2}{|c|}{ S8 } & \multicolumn{2}{|c|}{ S9-S10 } & \multicolumn{2}{|c|}{ S11-S21 } & \multirow{2}{*}{$\begin{array}{r}\text { FINAL } \\
10 \\
\end{array}$} \\
\hline & $\mathbf{R}$ & $\mathbf{U}$ & $\mathbf{R}$ & $\mathbf{U}$ & $\mathbf{R}$ & $\mathbf{U}$ & $\mathbf{R}$ & $\mathbf{U}$ & $\mathbf{R}$ & $\mathbf{U}$ & $\mathbf{R}$ & $\mathbf{U}$ & $\mathbf{R}$ & $\mathbf{U}$ & $\mathbf{R}$ & $\mathbf{U}$ & $\mathbf{R}$ & $\mathbf{U}$ & $\mathbf{R}$ & $\mathbf{U}$ & \\
\hline Relocate stop sign & & & & & & & & & & & & & & & & & & & & & 15 \\
\hline $\begin{array}{l}\text { Relocate the sign to make } \\
\text { it visible }\end{array}$ & & & & & & & & & & & & & & & & & & & & & 20 \\
\hline $\begin{array}{l}\text { Remove distracting } \\
\text { commercial lighting or } \\
\text { other source of glare }\end{array}$ & & & & & & & & & & & 15 & 15 & & & & & & & & & 15 \\
\hline $\begin{array}{l}\text { Remove or relocate the } \\
\text { object }\end{array}$ & & & & & 60 & 60 & 29 & 29 & & & 30 & 30 & 50 & 50 & & & & & & & 42 \\
\hline Remove sight obstructions & 12 & & & & & & & & & & & & & & 13 & & & & & & 13 \\
\hline $\begin{array}{l}\text { Remove the object } \\
\text { obstructing sight of the } \\
\text { warning sign }\end{array}$ & & & & & & & & & & & & & & & & & & & & & 20 \\
\hline $\begin{array}{l}\text { Remove the object } \\
\text { obstructing the sight of the } \\
\text { stop sign }\end{array}$ & & & & & & & & & & & & & & & & & & & & & 30 \\
\hline $\begin{array}{l}\text { Remove the object } \\
\text { obstructing the sight } \\
\text { triangle }\end{array}$ & 12 & & & & & & 25 & 25 & & & & & & & 13 & & & & 10 & 10 & 16 \\
\hline $\begin{array}{l}\text { Remove the object } \\
\text { obstructing visibility from } \\
\text { the median }\end{array}$ & & & & & & & & & & & & & & & & & & & & & 20 \\
\hline $\begin{array}{l}\text { Replace poorly designed } \\
\text { drain grates with bicycle- } \\
\text { safe types }\end{array}$ & & & & & & & & & & & & & & & & & & & & & 10 \\
\hline $\begin{array}{l}\text { Replace, repair or clean } \\
\text { the warning signs }\end{array}$ & & & & & 20 & 20 & 25 & 25 & & & & & & & & & & & & & 23 \\
\hline Reroute pedestrian paths & & & & & & & & & & & & & & & & & & & & & 30 \\
\hline $\begin{array}{l}\text { Restrict left-turning at the } \\
\text { access points }\end{array}$ & & & & & & & & & & & & & & & & & & & 100 & & 90 \\
\hline $\begin{array}{l}\text { Restrict parking near the } \\
\text { driveway }\end{array}$ & & & & & 22 & 22 & & & & & 35 & 35 & 8 & 8 & & & & & 10 & 10 & 19 \\
\hline $\begin{array}{l}\text { Restrict parking on the } \\
\text { approach }\end{array}$ & & & & & & & & & & & & & & & & & & & 10 & 10 & 10 \\
\hline
\end{tabular}




\begin{tabular}{|c|c|c|c|c|c|c|c|c|c|c|c|c|c|c|c|c|c|c|c|c|c|}
\hline \multirow[b]{2}{*}{$\begin{array}{l}\text { Signals to alert motorists } \\
\text { that pedestrians are } \\
\text { crossing }\end{array}$} & \multicolumn{2}{|c|}{ S1 } & \multicolumn{2}{|c|}{ S2 } & \multicolumn{2}{|c|}{ S3 } & \multicolumn{2}{|c|}{ S4 } & \multicolumn{2}{|c|}{ S5 } & \multicolumn{2}{|c|}{ S6 } & \multicolumn{2}{|c|}{ S7 } & \multicolumn{2}{|c|}{ S8 } & \multicolumn{2}{|c|}{ S9-S10 } & \multicolumn{2}{|c|}{ S11-S21 } & \multirow[t]{2}{*}{ FINAL } \\
\hline & $\mathbf{R}$ & $\mathbf{U}$ & $\mathbf{R}$ & $\mathbf{U}$ & $\mathbf{R}$ & $\mathbf{U}$ & $\mathbf{R}$ & $\mathbf{U}$ & $\mathbf{R}$ & $\mathbf{U}$ & $\mathbf{R}$ & $\mathbf{U}$ & $\mathbf{R}$ & $\mathbf{U}$ & $\mathbf{R}$ & $\mathbf{U}$ & $\mathbf{R}$ & $\mathbf{U}$ & $\mathbf{R}$ & $\mathbf{U}$ & \\
\hline Trim vegetation & & & & & & & & & & & & & & & & & & & & & 35 \\
\hline $\begin{array}{l}\text { Trim vegetation on the } \\
\text { median }\end{array}$ & & & & & & & & & & & & & & & & & & & & & 25 \\
\hline Upgrade signing & & & & & 5 & 5 & & & & & 24 & 24 & & & & & & & & & 15 \\
\hline $\begin{array}{l}\text { Use "Slippery when wet" } \\
\text { sign (temporary) }\end{array}$ & & & & & & & & & & & & & & & & & & & & & 15 \\
\hline Use break-away devices & & & & & 30 & 30 & & & & & & & & & & & & & & & 30 \\
\hline $\begin{array}{l}\text { Use salt to prevent snow } \\
\text { or ice from forming or from } \\
\text { sticking to the road surface }\end{array}$ & & & & & & & & & & & & & & & & & & & & & 20 \\
\hline $\begin{array}{l}\text { Use sand to increase } \\
\text { pavement friction }\end{array}$ & & & & & & & & & & & & & & & & & & & & & 20 \\
\hline $\begin{array}{l}\text { Use school crossing } \\
\text { guards }\end{array}$ & & & & & & & & & & & & & & & & & & & & & 50 \\
\hline $\begin{array}{l}\text { Widen the outside through } \\
\text { lanes or add bike lanes }\end{array}$ & & & & & & & & & & & & & & & & & & & & & 15 \\
\hline
\end{tabular}

RRC

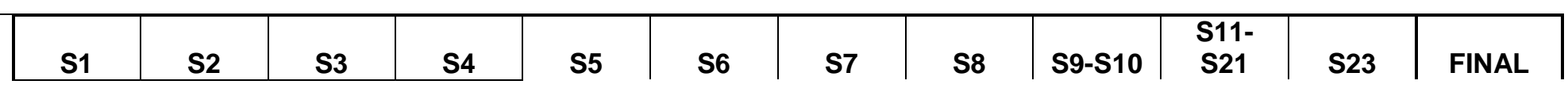




\begin{tabular}{|c|c|c|c|c|c|c|c|c|c|c|c|c|c|c|c|c|c|c|c|c|c|c|c|}
\hline & $\mathbf{R}$ & $\mathbf{U}$ & $\mathbf{R}$ & $\mathbf{U}$ & $\mathbf{R}$ & $\mathbf{U}$ & $\mathbf{R}$ & $\mathbf{U}$ & $\mathbf{R}$ & $\mathbf{U}$ & $\mathbf{R}$ & $\mathbf{U}$ & $\mathbf{R}$ & $\mathbf{U}$ & $\mathbf{R}$ & $\mathbf{U}$ & $\mathbf{R}$ & $\mathbf{U}$ & $\mathbf{R}$ & $\mathbf{U}$ & $\mathbf{R}$ & $\mathbf{U}$ & \\
\hline $\begin{array}{l}\text { Add overhead stop-signs } \\
\text { if the percent of tall } \\
\text { vehicles is considerable }\end{array}$ & & & & & & & & & & & & & & & & & & & & & & & 20 \\
\hline $\begin{array}{l}\text { Change } \\
\text { horizontal/vertical } \\
\text { alignment (skewed-angle } \\
\text { crossing) }\end{array}$ & & & & & & & & & & & & & & & & & & & & & 45 & 45 & 45 \\
\hline $\begin{array}{l}\text { Chip and seal or slurry } \\
\text { seal approaches }\end{array}$ & & & & & & & & & & & & & & & & & & & & & & & 10 \\
\hline Close or install overpass & & & & & & & & & & & & & & & & & & & & & & & 90 \\
\hline $\begin{array}{l}\text { Close rail-road crossing } \\
\text { point }\end{array}$ & & & & & & & & & & & & & & & & & & & & & & & 90 \\
\hline $\begin{array}{l}\text { Eliminate screening by } \\
\text { physical objects }\end{array}$ & & & & & & & & & & & & & & & & & & & & & & & 63 \\
\hline Extend "no parking" zone. & & & & & & & & & & & & & & & & & & & & & & & 22 \\
\hline Groove pavement surface & & & & & & & & & & & & & & & & & & & & & & & 27 \\
\hline $\begin{array}{l}\text { Improve } \\
\text { channelization/delineation }\end{array}$ & & & & & & & & & & & 30 & 30 & 40 & 40 & & & 75 & 75 & 15 & & & & 44 \\
\hline $\begin{array}{l}\text { Improve existing street } \\
\text { lights at the rail-road } \\
\text { crossing points }\end{array}$ & 30 & 30 & & & 50 & 50 & & & & & & & & & & & & & & & & & 40 \\
\hline $\begin{array}{l}\text { Improve gates } \\
\text { visibility/reflectivity } \\
\text { (flashing lights along the } \\
\text { gate) }\end{array}$ & & & 75 & 75 & & & & & & & & & & & & & & & & & & & 75 \\
\hline $\begin{array}{l}\text { Improve maintenance of } \\
\text { rail infrastructure (Motion } \\
\text { Sensitive Devices, } \\
\text { Constant Warning Time) }\end{array}$ & & & & & & & & & & & & & & & & & & & & & 50 & 50 & 50 \\
\hline $\begin{array}{l}\text { Improve maintenance of } \\
\text { train detective devices }\end{array}$ & & & & & & & & & & & & & & & & & & & & & & & 10 \\
\hline $\begin{array}{l}\text { Improve maintenance of } \\
\text { visual/audio signals }\end{array}$ & & & & & & & & & & & & & & & & & & & & & & & 15 \\
\hline $\begin{array}{l}\text { Improve } \\
\text { maintenance/work of }\end{array}$ & & & & & & & & & & & & & & & & & & & & & & & 35 \\
\hline
\end{tabular}




\begin{tabular}{|c|c|c|c|c|c|c|c|c|c|c|c|c|c|c|c|c|c|c|c|c|c|c|c|}
\hline \multirow[b]{3}{*}{ existing gates } & \multicolumn{2}{|c|}{ S1 } & \multicolumn{2}{|c|}{ S2 } & \multicolumn{2}{|c|}{ S3 } & \multicolumn{2}{|c|}{ S4 } & \multicolumn{2}{|c|}{ S5 } & \multicolumn{2}{|c|}{ S6 } & \multicolumn{2}{|c|}{ S7 } & \multicolumn{2}{|c|}{ S8 } & \multicolumn{2}{|c|}{ S9-S10 } & \multicolumn{2}{|c|}{$\begin{array}{l}\text { S11- } \\
\text { S21 }\end{array}$} & \multicolumn{2}{|c|}{ S23 } & \multirow[t]{3}{*}{ FINAL } \\
\hline & $\mathbf{R}$ & $\mathbf{U}$ & $\mathbf{R}$ & $\mathbf{U}$ & $\mathbf{R}$ & $\mathbf{U}$ & $\mathbf{R}$ & $\mathbf{U}$ & $\mathbf{R}$ & $\mathbf{U}$ & $\mathbf{R}$ & $\mathbf{U}$ & $\mathbf{R}$ & $\mathbf{U}$ & $\mathbf{R}$ & $\mathbf{U}$ & $\mathbf{R}$ & $\mathbf{U}$ & $\mathbf{R}$ & $\mathbf{U}$ & $\mathbf{R}$ & $\mathbf{U}$ & \\
\hline & & & & & & & & & & & & & & & & & & & & & & & \\
\hline $\begin{array}{l}\text { Improve pavement } \\
\text { markings and texturing }\end{array}$ & & & & & & & & & & & & & & & & & & & & & & & 16 \\
\hline $\begin{array}{l}\text { Improve signage of the } \\
\text { crosswalk }\end{array}$ & & & & & & & & & & & 25 & 25 & & & & & & & & & & & 25 \\
\hline Improve skid resistance & & & 9 & 9 & & & & & & & & & & & & & & & & & & & 9 \\
\hline $\begin{array}{l}\text { Improve winter } \\
\text { maintenance methods }\end{array}$ & & & & & & & & & & & & & & & & & & & & & & & 25 \\
\hline $\begin{array}{l}\text { Improve winter } \\
\text { maintenance } \\
\text { preparedness (shorter } \\
\text { response time) }\end{array}$ & & & & & & & & & & & & & & & & & & & & & & & 20 \\
\hline $\begin{array}{l}\text { Improve/install reflective } \\
\text { signs }\end{array}$ & & & 15 & 15 & & & & & & & & & & & & & & & & & & & 15 \\
\hline $\begin{array}{l}\text { Improve/install } \\
\text { reflectorized pavement } \\
\text { markers }\end{array}$ & & & & & & & & & & & & & & & & & & & & & & & 12 \\
\hline $\begin{array}{l}\text { Install a bicycles } \\
\text { overpass or underpass }\end{array}$ & & & & & & & & & & & & & & & & & & & & & & & 90 \\
\hline $\begin{array}{l}\text { Install a fence or chicane } \\
\text { to prevent bicycles } \\
\text { running across the track }\end{array}$ & & & & & & & & & & & & & & & & & & & & & & & 30 \\
\hline $\begin{array}{l}\text { Install a fence or chicane } \\
\text { to prevent pedestrians } \\
\text { running across the track }\end{array}$ & & & & & & & & & & & & & & & & & & & & & & & 30 \\
\hline $\begin{array}{l}\text { Install a pedestrian } \\
\text { overpass or underpass }\end{array}$ & & & 86 & 86 & & & & & & & & & & & & & & & & & & & 86 \\
\hline $\begin{array}{l}\text { Install active control } \\
\text { devices }\end{array}$ & 50 & 50 & & & & & & & & & & & & & & & & & & & & & 50 \\
\hline $\begin{array}{l}\text { Install adequate control } \\
\text { device. }\end{array}$ & & & & & & & & & & & & & & & & & & & & & & & 20 \\
\hline $\begin{array}{l}\text { Install advance warning } \\
\text { sign }\end{array}$ & 20 & 20 & 25 & 25 & & & 25 & 25 & 40 & 40 & & & 30 & 40 & & & & & & & 50 & 50 & 33 \\
\hline
\end{tabular}




\begin{tabular}{|c|c|c|c|c|c|c|c|c|c|c|c|c|c|c|c|c|c|c|c|c|c|c|c|}
\hline \multirow[b]{3}{*}{$\begin{array}{l}\text { Install audible signals } \\
\text { (warning bell) }\end{array}$} & \multicolumn{2}{|c|}{ S1 } & \multicolumn{2}{|c|}{ S2 } & \multicolumn{2}{|c|}{ S3 } & \multicolumn{2}{|c|}{ S4 } & \multicolumn{2}{|c|}{ S5 } & \multicolumn{2}{|c|}{ S6 } & \multicolumn{2}{|c|}{ S7 } & \multicolumn{2}{|c|}{ S8 } & \multicolumn{2}{|c|}{ S9-S10 } & \multicolumn{2}{|c|}{$\begin{array}{l}\text { S11- } \\
\text { S21 }\end{array}$} & \multicolumn{2}{|c|}{ S23 } & \multirow{3}{*}{$\begin{array}{r}\text { FINAL } \\
\\
50\end{array}$} \\
\hline & $\mathbf{R}$ & $\mathbf{U}$ & $\mathbf{R}$ & $\mathbf{U}$ & $\mathbf{R}$ & $\mathbf{U}$ & $\mathbf{R}$ & $\mathbf{U}$ & $\mathbf{R}$ & $\mathbf{U}$ & $\mathbf{R}$ & $\mathbf{U}$ & $\mathbf{R}$ & $\mathbf{U}$ & $\mathbf{R}$ & $\mathbf{U}$ & $\mathbf{R}$ & $\mathbf{U}$ & $\mathbf{R}$ & $\mathbf{U}$ & $\mathbf{R}$ & $\mathbf{U}$ & \\
\hline & & & & & & & & & 50 & 50 & & & & & & & & & & & & & \\
\hline $\begin{array}{l}\text { Install automated } \\
\text { bicycles/pedestrians } \\
\text { gates }\end{array}$ & & & & & & & & & & & & & & & & & & & & & 50 & 50 & 50 \\
\hline $\begin{array}{l}\text { Install automated } \\
\text { pedestrian gates }\end{array}$ & & & & & & & & & & & & & & & & & & & & & & & 30 \\
\hline Install automatic gates & & & 75 & 75 & & & & & & & & & & & & & & & & & & & 75 \\
\hline Install bicycles signals & & & & & & & & & & & & & & & & & & & & & & & 20 \\
\hline $\begin{array}{l}\text { Install cantilever flashing- } \\
\text { light signal }\end{array}$ & & & & & & & & & & & & & & & & & & & & & & & 33 \\
\hline Install corner mirrors & & & & & & & & & & & & & & & & & & & & & & & 20 \\
\hline Install crossbuck & & & & & & & & & 60 & 60 & & & & & & & & & & & & & 60 \\
\hline $\begin{array}{l}\text { Install DO NOT STOP } \\
\text { ON TRACKS signal }\end{array}$ & & & & & & & & & & & & & & & & & & & & & & & 10 \\
\hline $\begin{array}{l}\text { Install fixed message } \\
\text { signs }\end{array}$ & & & & & & & & & & & & & & & & & & & & & & & 10 \\
\hline Install flashers & & & 75 & 75 & & & & & & & & & & & & & & & & & & & 75 \\
\hline Install flashing beacons. & 30 & 30 & 65 & 65 & 50 & 50 & 30 & 30 & 60 & 60 & 58 & 58 & 24 & 24 & & & & & & & & & 45 \\
\hline $\begin{array}{l}\text { Install flashing light } \\
\text { activated by queuing } \\
\text { traffic in the exit lane(s) } \\
\text { from the crossing }\end{array}$ & & & & & & & & & & & & & & & & & & & & & & & 25 \\
\hline Install fog - warning signs & & & & & & & 25 & 25 & & & & & & & & & & & & & & & 25 \\
\hline $\begin{array}{l}\text { Install four-quadrant gate } \\
\text { arms }\end{array}$ & & & & & & & & & & & & & & & & & & & & & 55 & 55 & 55 \\
\hline Install gates & & & 65 & 65 & & & & & & & & & & & & & & & & & 46 & 46 & 56 \\
\hline $\begin{array}{l}\text { Install gates with self } \\
\text { deploying extension } \\
\text { sections }\end{array}$ & & & & & & & & & & & & & & & & & & & & & & & 20 \\
\hline Install lighting & 30 & 30 & 33 & 33 & 50 & 50 & & & & & 30 & 30 & 37 & 37 & & & & & 37 & 37 & 45 & 45 & 37 \\
\hline Install median barriers & & & & & & & & & & & & & & & & & & & & & & & 35 \\
\hline
\end{tabular}




\begin{tabular}{|c|c|c|c|c|c|c|c|c|c|c|c|c|c|c|c|c|c|c|c|c|c|c|c|}
\hline \multirow{3}{*}{$\begin{array}{l}\text { Install median } \\
\text { retroreflective post } \\
\text { delineators on the } \\
\text { approach }\end{array}$} & \multicolumn{2}{|c|}{ S1 } & \multicolumn{2}{|c|}{ S2 } & \multicolumn{2}{|c|}{ S3 } & \multicolumn{2}{|c|}{ S4 } & \multicolumn{2}{|c|}{ S5 } & \multicolumn{2}{|c|}{ S6 } & \multicolumn{2}{|c|}{ S7 } & \multicolumn{2}{|c|}{ S8 } & \multicolumn{2}{|c|}{ S9-S10 } & \multicolumn{2}{|c|}{$\begin{array}{l}\text { S11- } \\
\text { S21 }\end{array}$} & \multicolumn{2}{|c|}{ S23 } & \multirow[t]{2}{*}{ FINAL } \\
\hline & $\mathbf{R}$ & $\mathbf{U}$ & $\mathbf{R}$ & $\mathbf{U}$ & $\mathbf{R}$ & $\mathbf{U}$ & $\mathbf{R}$ & $\mathbf{U}$ & $\mathbf{R}$ & $\mathbf{U}$ & $\mathbf{R}$ & $\mathbf{U}$ & $\mathbf{R}$ & $\mathbf{U}$ & $\mathbf{R}$ & $\mathbf{U}$ & $\mathbf{R}$ & $\mathbf{U}$ & $\mathbf{R}$ & $\mathbf{U}$ & $\mathbf{R}$ & $\mathbf{U}$ & \\
\hline & & & & & & & & & & & & & & & & & & & & & & & 15 \\
\hline $\begin{array}{l}\text { Install non-mountable } \\
\text { curb islands }\end{array}$ & & & & & & & & & & & & & & & & & & & & & & & 20 \\
\hline Install overhead signs & & & & & & & & & & & & & & & & & & & & & & & 10 \\
\hline $\begin{array}{l}\text { Install pavement } \\
\text { markings }\end{array}$ & 10 & 10 & 21 & 21 & & & & & 21 & 21 & & & & & 10 & 10 & & & 10 & 10 & & & 14 \\
\hline Install pedestrian barriers & & & & & & & & & & & & & & & & & & & & & & & 25 \\
\hline Install pedestrian signals & & & & & & & & & & & & & & & & & & & & & & & 15 \\
\hline Install pre-signals & & & & & & & & & & & & & & & & & & & & & 50 & 50 & 50 \\
\hline Install rumble strips & & & 32 & 32 & 28 & 28 & & & & & 25 & 25 & & & 21 & & & & 50 & 50 & & & 32 \\
\hline $\begin{array}{l}\text { Install signs on both sides } \\
\text { of highway }\end{array}$ & & & & & & & & & & & & & & & & & & & & & & & 20 \\
\hline Install speed humps & & & & & & & & & & & & & & & & & & & & & 40 & 40 & 40 \\
\hline $\begin{array}{l}\text { Install static or variable } \\
\text { message signs displaying } \\
\text { weather information }\end{array}$ & & & 15 & 15 & & & & & & & 15 & 15 & & & & & & & & & & & 15 \\
\hline Install stop sign & & & 35 & 35 & & & & & & & 39 & 39 & & & & & & & & & 46 & 46 & 40 \\
\hline Install street lights & 30 & 30 & & & 50 & 50 & & & & & & & & & & & & & & & & & 40 \\
\hline Install swing gates & & & & & & & & & & & & & & & & & & & & & & & 25 \\
\hline $\begin{array}{l}\text { Install symbol storage } \\
\text { space sign }\end{array}$ & & & & & & & & & & & & & & & & & & & & & & & 10 \\
\hline $\begin{array}{l}\text { Install variable message } \\
\text { signs and blank out signs }\end{array}$ & & & & & & & & & & & & & & & & & & & & & & & 20 \\
\hline $\begin{array}{l}\text { Install vehicle-arresting } \\
\text { barriers }\end{array}$ & & & & & & & & & & & & & & & & & & & & & & & 25 \\
\hline $\begin{array}{l}\text { Install warning signs in } \\
\text { advance of rail-road } \\
\text { crossing }\end{array}$ & & & & & & & & & 40 & 40 & & & & & & & & & & & 50 & 50 & 45 \\
\hline Install yield sign & & & & & & & & & & & & & & & & & & & & & & & 10 \\
\hline
\end{tabular}




\begin{tabular}{|c|c|c|c|c|c|c|c|c|c|c|c|c|c|c|c|c|c|c|c|c|c|c|c|}
\hline \multirow{3}{*}{$\begin{array}{l}\text { Installing snow screens in } \\
\text { areas exposed to } \\
\text { snowdrifts }\end{array}$} & \multicolumn{2}{|c|}{ S1 } & \multicolumn{2}{|c|}{ S2 } & \multicolumn{2}{|c|}{ S3 } & \multicolumn{2}{|c|}{ S4 } & \multicolumn{2}{|c|}{ S5 } & \multicolumn{2}{|c|}{ S6 } & \multicolumn{2}{|c|}{ S7 } & \multicolumn{2}{|c|}{ S8 } & \multicolumn{2}{|c|}{ S9-S10 } & \multicolumn{2}{|c|}{$\begin{array}{l}\text { S11- } \\
\text { S21 }\end{array}$} & \multicolumn{2}{|c|}{ S23 } & \multirow[t]{2}{*}{ FINAL } \\
\hline & $\mathbf{R}$ & $\mathbf{U}$ & $\mathbf{R}$ & $\mathbf{U}$ & $\mathbf{R}$ & $\mathbf{U}$ & $\mathbf{R}$ & $\mathbf{U}$ & $\mathbf{R}$ & $\mathbf{U}$ & $\mathbf{R}$ & $\mathbf{U}$ & $\mathbf{R}$ & $\mathbf{U}$ & $\mathbf{R}$ & $\mathbf{U}$ & $\mathbf{R}$ & $\mathbf{U}$ & $\mathbf{R}$ & $\mathbf{U}$ & $\mathbf{R}$ & $\mathbf{U}$ & \\
\hline & & & & & & & & & & & & & & & & & & & & & & & 53 \\
\hline $\begin{array}{l}\text { Overlay pavement } \\
\text { (friction course) }\end{array}$ & & & 17 & 17 & 18 & 18 & 27 & 27 & & & & & & & & & & & & & & & 21 \\
\hline $\begin{array}{l}\text { Post a lower speed limit } \\
\text { for cars and/or trains }\end{array}$ & & & & & & & & & & & & & & & & & & & & & & & 20 \\
\hline $\begin{array}{l}\text { Post an adequate speed } \\
\text { limit }\end{array}$ & & & & & 28 & 28 & & & & & & & 20 & 20 & & & & & & & & & 24 \\
\hline $\begin{array}{l}\text { Post dynamic message } \\
\text { sign to display the speed } \\
\text { of approaching vehicles. }\end{array}$ & & & & & & & & & & & & & & & & & 74 & 74 & & & & & 74 \\
\hline Provide adequate delay & & & & & & & & & & & & & & & & & & & & & 33 & 33 & 33 \\
\hline $\begin{array}{l}\text { Provide adequate } \\
\text { drainage }\end{array}$ & & & & & & & & & & & & & & & & & & & & & & & 20 \\
\hline $\begin{array}{l}\text { Provide adequate gate } \\
\text { interval }\end{array}$ & & & & & & & & & & & & & & & & & & & & & 50 & 50 & 50 \\
\hline $\begin{array}{l}\text { Provide adequate } \\
\text { information to train } \\
\text { operator (for example: } \\
\text { location of the whistle } \\
\text { post) }\end{array}$ & & & & & & & & & & & & & & & & & & & & & & & 15 \\
\hline $\begin{array}{l}\text { Provide information of the } \\
\text { crossing type }\end{array}$ & & & & & & & & & & & & & & & & & & & & & & & 10 \\
\hline $\begin{array}{l}\text { Provide pavement } \\
\text { marking }\end{array}$ & & & & & 35 & 35 & & & & & 10 & 10 & 35 & 35 & & & & & & & & & 27 \\
\hline $\begin{array}{l}\text { Provide photo video } \\
\text { enforcement }\end{array}$ & & & & & & & & & & & & & & & & & & & & & 56 & 56 & 56 \\
\hline $\begin{array}{l}\text { Provide targeted speed } \\
\text { enforcement }\end{array}$ & & & & & & & & & & & & & & & & & & & & & & & 70 \\
\hline $\begin{array}{l}\text { Provide traffic calming on } \\
\text { approaches through a } \\
\text { combination of geometric }\end{array}$ & & & & & & & & & & & & & & & & & & & & & & & 20 \\
\hline
\end{tabular}




\begin{tabular}{|c|c|c|c|c|c|c|c|c|c|c|c|c|c|c|c|c|c|c|c|c|c|c|c|}
\hline \multirow[b]{3}{*}{ and traffic control devices } & \multicolumn{2}{|c|}{ S1 } & \multicolumn{2}{|c|}{ S2 } & \multicolumn{2}{|c|}{ S3 } & \multicolumn{2}{|c|}{ S4 } & \multicolumn{2}{|c|}{ S5 } & \multicolumn{2}{|c|}{ S6 } & \multicolumn{2}{|c|}{ S7 } & \multicolumn{2}{|c|}{ S8 } & \multicolumn{2}{|c|}{ S9-S10 } & \multicolumn{2}{|c|}{$\begin{array}{l}\text { S11- } \\
\text { S21 }\end{array}$} & \multicolumn{2}{|c|}{ S23 } & \multirow[t]{3}{*}{ FINAL } \\
\hline & $\mathbf{R}$ & $\mathbf{U}$ & $\mathbf{R}$ & $\mathbf{U}$ & $\mathbf{R}$ & $\mathbf{U}$ & $\mathbf{R}$ & $\mathbf{U}$ & $\mathbf{R}$ & $\mathbf{U}$ & $\mathbf{R}$ & $\mathbf{U}$ & $\mathbf{R}$ & $\mathbf{U}$ & $\mathbf{R}$ & $\mathbf{U}$ & $\mathbf{R}$ & $\mathbf{U}$ & $\mathbf{R}$ & $\mathbf{U}$ & $\mathbf{R}$ & $\mathbf{U}$ & \\
\hline & & & & & & & & & & & & & & & & & & & & & & & \\
\hline $\begin{array}{l}\text { Provide/Improve } \\
\text { pavement surface } \\
\text { (improve transitions, drop } \\
\text { offs, fixed potholes, } \\
\text { rutting, etc) }\end{array}$ & & & & & & & & & & & 34 & 34 & & & & & & & & & & & 34 \\
\hline Relocate control device & & & & & & & & & & & & & & & & & & & & & & & 20 \\
\hline $\begin{array}{l}\text { Relocate stop bar (If stop } \\
\text { sign present) }\end{array}$ & & & & & & & & & & & & & & & & & & & & & & & 10 \\
\hline Relocate stop sign & & & & & & & & & & & & & & & & & & & & & & & 15 \\
\hline $\begin{array}{l}\text { Relocate the crossing } \\
\text { section }\end{array}$ & & & & & & & & & & & & & & & & & & & & & & & 30 \\
\hline $\begin{array}{l}\text { Remove distracting } \\
\text { commercial lights }\end{array}$ & & & & & & & & & & & 15 & 15 & & & & & & & & & & & 15 \\
\hline $\begin{array}{l}\text { Remove the object } \\
\text { obstructing the sight of } \\
\text { the control device }\end{array}$ & & & & & & & & & & & & & & & & & & & & & & & 20 \\
\hline $\begin{array}{l}\text { Remove the object } \\
\text { obstructing the sight of } \\
\text { the stop sign }\end{array}$ & & & & & & & & & & & & & & & & & & & & & 47 & 47 & 47 \\
\hline $\begin{array}{l}\text { Remove the object } \\
\text { obstructing the sight } \\
\text { triangle }\end{array}$ & 12 & & & & & & 25 & 25 & & & & & & & 13 & & & & 10 & 10 & 50 & 50 & 24 \\
\hline $\begin{array}{l}\text { Remove the object } \\
\text { obstructing view from the } \\
\text { approach }\end{array}$ & & & & & & & & & & & & & & & & & & & & & 50 & 50 & 50 \\
\hline Replace / repair signals & & & & & & & & & & & & & & & & & & & & & & & 10 \\
\hline $\begin{array}{l}\text { Replace, repair, or clean } \\
\text { the control device }\end{array}$ & & & & & & & & & & & & & & & & & & & & & & & 20 \\
\hline $\begin{array}{l}\text { Replace, repair, or clean } \\
\text { the sign }\end{array}$ & & & & & 5 & 5 & & & & & & & & & & & & & & & & & 5 \\
\hline Trim vegetation & & & & & & & & & & & & & & & & & & & & & & & 35 \\
\hline
\end{tabular}




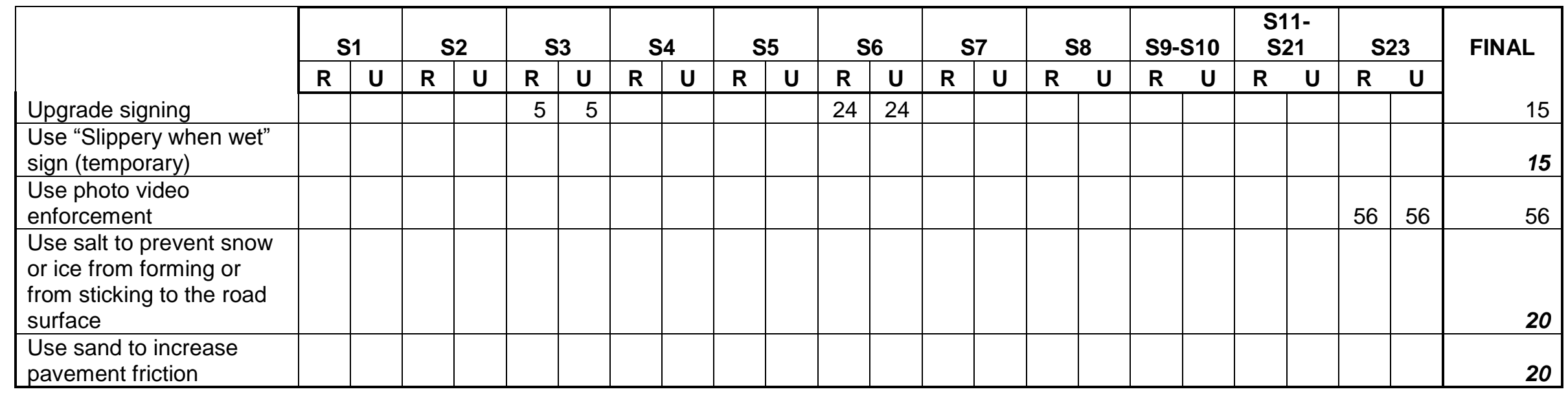

\section{RS}

\begin{tabular}{|c|c|c|c|c|c|c|c|c|c|c|c|c|c|c|c|c|c|c|c|c|c|}
\hline & \multicolumn{2}{|c|}{ S1 } & \multicolumn{2}{|c|}{ S2 } & \multicolumn{2}{|c|}{ S3 } & \multicolumn{2}{|c|}{ S4 } & \multicolumn{2}{|c|}{ S5 } & \multicolumn{2}{|c|}{ S6 } & \multicolumn{2}{|c|}{ S7 } & \multicolumn{2}{|c|}{ S8 } & \multicolumn{2}{|c|}{ S9-S10 } & \multicolumn{2}{|c|}{ S11-S21 } & \multirow[t]{2}{*}{ FINAL } \\
\hline & $\mathbf{R}$ & $\mathbf{U}$ & $\mathbf{R}$ & $\mathbf{U}$ & $\mathbf{R}$ & $\mathbf{U}$ & $\mathbf{R}$ & $\mathbf{U}$ & $\mathbf{R}$ & $\mathbf{U}$ & $\mathbf{R}$ & U & $\mathbf{R}$ & U & $\mathbf{R}$ & U & $\mathbf{R}$ & $\mathbf{U}$ & $\mathbf{R}$ & $\mathbf{U}$ & \\
\hline Add special signing & & & & & & & & & & & & & & & & & & & & & 10 \\
\hline $\begin{array}{l}\text { Centerline rumble } \\
\text { strips for two-lane } \\
\text { roads }\end{array}$ & & & 32 & 32 & 32 & 32 & & & & & 25 & 25 & & & 12 & & 22 & 22 & & & 26 \\
\hline $\begin{array}{l}\text { Chip and seal or slurry } \\
\text { seal approaches }\end{array}$ & & & & & & & & & & & & & & & & & & & & & 10 \\
\hline $\begin{array}{l}\text { Close or relocate the } \\
\text { driveways }\end{array}$ & & & 50 & 50 & 15 & 15 & & & & & & & & & & & & & & & 33 \\
\hline $\begin{array}{l}\text { Construct pedestrian } \\
\text { refuge islands and } \\
\text { raised medians }\end{array}$ & & 46 & & & & & & & & & & & & & & & & & & & 46 \\
\hline $\begin{array}{l}\text { Create additional } \\
\text { parking spaces }\end{array}$ & & & & & & & & & & & & & & & & & & & & & 10 \\
\hline
\end{tabular}




\begin{tabular}{|c|c|c|c|c|c|c|c|c|c|c|c|c|c|c|c|c|c|c|c|c|c|}
\hline & \multicolumn{2}{|c|}{ S1 } & \multicolumn{2}{|c|}{ S2 } & \multicolumn{2}{|c|}{ S3 } & \multicolumn{2}{|c|}{$\mathbf{S 4}$} & \multicolumn{2}{|c|}{ S5 } & \multicolumn{2}{|c|}{ S6 } & \multicolumn{2}{|c|}{ S7 } & \multicolumn{2}{|c|}{ S8 } & \multicolumn{2}{|c|}{ S9-S10 } & \multicolumn{2}{|c|}{ S11-S21 } & \multirow[t]{2}{*}{ FINAL } \\
\hline & $\mathbf{R}$ & $\mathbf{U}$ & $\mathbf{R}$ & $\mathbf{U}$ & $\mathbf{R}$ & $\mathbf{U}$ & $\mathbf{R}$ & $\mathbf{U}$ & $\mathbf{R}$ & $\mathbf{U}$ & $\mathbf{R}$ & $\mathbf{U}$ & $\mathbf{R}$ & $\mathbf{U}$ & $\mathbf{R}$ & $\mathbf{U}$ & $\mathbf{R}$ & $\mathbf{U}$ & $\mathbf{R}$ & $\mathbf{U}$ & \\
\hline $\begin{array}{l}\text { Cut trees and other } \\
\text { roadside vegetation } \\
\text { obstruct the view }\end{array}$ & & & & & 25 & 25 & & & & & & & & & & & & & & & 25 \\
\hline $\begin{array}{l}\text { Design safer slopes } \\
\text { and ditches to prevent } \\
\text { rollovers }\end{array}$ & & & & & 35 & 35 & & & & & & & & & & & & & & & 35 \\
\hline $\begin{array}{l}\text { Eliminate screening by } \\
\text { physical objects }\end{array}$ & & & & & 60 & 60 & & & & & & & 50 & 50 & & & & & 76 & 76 & 62 \\
\hline $\begin{array}{l}\text { Eliminate shoulder } \\
\text { drop-offs }\end{array}$ & & & & & & & & & & & 25 & 25 & & & & & & & 30 & 30 & 28 \\
\hline $\begin{array}{l}\text { Eliminating compound } \\
\text { curves }\end{array}$ & & & & & & & & & & & & & & & & & & & & & 25 \\
\hline $\begin{array}{l}\text { Enhance delineation of } \\
\text { sharp curves }\end{array}$ & & & & & 16 & 16 & & & & & & & 17 & 17 & & & 15 & & 15 & & 16 \\
\hline $\begin{array}{l}\text { Enhance delineation of } \\
\text { sharp curves segment }\end{array}$ & & & & & 16 & 16 & & & & & & & & & & & 15 & & 15 & & 16 \\
\hline $\begin{array}{l}\text { Groove pavement } \\
\text { surface }\end{array}$ & & & 21 & 21 & & & 14 & 14 & & & & & & & & & & & 46 & 46 & 27 \\
\hline $\begin{array}{l}\text { Identify and treat truck } \\
\text { crash roadway } \\
\text { segments-signing }\end{array}$ & & & & & & & & & & & & & & & & & & & & & 26 \\
\hline $\begin{array}{l}\text { Implement lighting / } \\
\text { illumination measures }\end{array}$ & 23 & 23 & 28 & 28 & & & & & & & 25 & 25 & 37 & 37 & & & & & 37 & 37 & 30 \\
\hline $\begin{array}{l}\text { Implement road } \\
\text { narrowing measures }\end{array}$ & & & & & & & & & & & & & & & & & & & & & 30 \\
\hline Improve alignment & & & & & & & & & & & & & & & & & & & & & 35 \\
\hline $\begin{array}{l}\text { Improve } \\
\text { channelization/delineati } \\
\text { on }\end{array}$ & & & & & & & & & & & 30 & 30 & 40 & 40 & & & & & & & 35 \\
\hline Improve clear zone & & & & & 25 & 25 & & & & & & & & & & & & & & & 25 \\
\hline $\begin{array}{l}\text { Improve existing street } \\
\text { lights }\end{array}$ & 30 & 30 & & & 50 & 50 & & & & & & & & & & & & & & & 40 \\
\hline Improve lanes width & 9 & & & & & & & & & & 25 & 25 & 23 & 23 & 8 & & & & & & 19 \\
\hline
\end{tabular}




\begin{tabular}{|c|c|c|c|c|c|c|c|c|c|c|c|c|c|c|c|c|c|c|c|c|c|}
\hline & \multicolumn{2}{|c|}{ S1 } & \multicolumn{2}{|c|}{$\mathrm{S2}$} & \multicolumn{2}{|c|}{ S3 } & \multicolumn{2}{|c|}{ S4 } & \multicolumn{2}{|c|}{ S5 } & \multicolumn{2}{|c|}{ S6 } & \multicolumn{2}{|c|}{ S7 } & \multicolumn{2}{|c|}{ S8 } & \multicolumn{2}{|c|}{ S9-S10 } & \multicolumn{2}{|c|}{ S11-S21 } & \multirow[t]{2}{*}{ FINAL } \\
\hline & $\mathbf{R}$ & $\mathbf{U}$ & $\mathbf{R}$ & $\mathbf{U}$ & $\mathbf{R}$ & $\mathbf{U}$ & $\mathbf{R}$ & $\mathbf{U}$ & $\mathbf{R}$ & $\mathbf{U}$ & $\mathbf{R}$ & $\mathbf{U}$ & $\mathbf{R}$ & $\mathbf{U}$ & $\mathbf{R}$ & $\mathbf{U}$ & $\mathbf{R}$ & $\mathbf{U}$ & $\mathbf{R}$ & $\mathbf{U}$ & \\
\hline $\begin{array}{l}\text { Improve or restore } \\
\text { superelevation }\end{array}$ & 11 & & 28 & 28 & & & & & & & 40 & 40 & & & 11 & & & & & & 26 \\
\hline $\begin{array}{l}\text { Improve pavement } \\
\text { markings }\end{array}$ & & & 21 & 21 & 10 & 10 & & & 21 & 21 & & & & & & & & & 10 & 10 & 16 \\
\hline $\begin{array}{l}\text { Improve pavements } \\
\text { resistant }\end{array}$ & & & & & & & & & & & & & & & & & & & & & 9 \\
\hline $\begin{array}{l}\text { Improve } \\
\text { reflectorization/conspic } \\
\text { uity of pedestrians }\end{array}$ & & & & & & & & & & & & & & & & & & & & & 20 \\
\hline $\begin{array}{l}\text { Improve signage } \\
\text { and/or marking of the } \\
\text { crosswalk }\end{array}$ & & & & & 5 & 5 & & & & & 25 & 25 & & & & & & & & & 15 \\
\hline $\begin{array}{l}\text { Improve skid } \\
\text { resistance }\end{array}$ & & & 9 & 9 & & & & & & & & & & & & & & & 20 & 20 & 15 \\
\hline $\begin{array}{l}\text { Improve skid } \\
\text { resistance along the } \\
\text { segment }\end{array}$ & & & 9 & 9 & & & & & & & & & & & & & & & & & 9 \\
\hline $\begin{array}{l}\text { Improve winter } \\
\text { maintenance methods }\end{array}$ & & & & & & & & & & & & & & & & & & & & & 25 \\
\hline $\begin{array}{l}\text { Improve winter } \\
\text { maintenance } \\
\text { preparedness (shorter } \\
\text { response time) }\end{array}$ & & & & & & & & & & & & & & & & & & & & & 20 \\
\hline $\begin{array}{l}\text { Improve/install } \\
\text { reflective signs }\end{array}$ & & & 15 & 15 & & & & & & & & & & & & & & & & & 15 \\
\hline $\begin{array}{l}\text { Improve/install } \\
\text { reflectorized pavement } \\
\text { markers }\end{array}$ & & & & & & & & & & & & & & & & & & & & & 57 \\
\hline $\begin{array}{l}\text { Incorporate rumble } \\
\text { strips into new and } \\
\text { existing roadways }\end{array}$ & & & 32 & 32 & 32 & 32 & & & & & 25 & 25 & & & & & & & & & 30 \\
\hline $\begin{array}{l}\text { Increase efficiency of } \\
\text { use of existing parking } \\
\text { spaces }\end{array}$ & & & & & & & & & & & & & & & & & & & & & 10 \\
\hline
\end{tabular}




\begin{tabular}{|c|c|c|c|c|c|c|c|c|c|c|c|c|c|c|c|c|c|c|c|c|c|}
\hline & \multicolumn{2}{|c|}{ S1 } & \multicolumn{2}{|c|}{ S2 } & \multicolumn{2}{|c|}{ S3 } & \multicolumn{2}{|c|}{ S4 } & \multicolumn{2}{|c|}{ S5 } & \multicolumn{2}{|c|}{ S6 } & \multicolumn{2}{|c|}{ S7 } & \multicolumn{2}{|c|}{ S8 } & \multicolumn{2}{|c|}{ S9-S10 } & \multicolumn{2}{|c|}{ S11-S21 } & \multirow[t]{2}{*}{ FINAL } \\
\hline & $\mathbf{R}$ & $\mathbf{U}$ & $\mathbf{R}$ & $\mathbf{U}$ & $\mathbf{R}$ & $\mathbf{U}$ & $\mathbf{R}$ & $\mathbf{U}$ & $\mathbf{R}$ & $\mathbf{U}$ & $\mathbf{R}$ & $\mathbf{U}$ & $\mathbf{R}$ & $\mathbf{U}$ & $\mathbf{R}$ & $\mathbf{U}$ & $\mathbf{R}$ & $\mathbf{U}$ & $\mathbf{R}$ & $\mathbf{U}$ & \\
\hline $\begin{array}{l}\text { Increase width of the } \\
\text { shoulders }\end{array}$ & & & 14 & 14 & & & 8 & 8 & & & 20 & 20 & & & 9 & & & & & & 13 \\
\hline $\begin{array}{l}\text { Install “Cross only at } \\
\text { Crosswalk" signs }\end{array}$ & & & & & & & & & & & & & & & & & & & & & 5 \\
\hline $\begin{array}{l}\text { Install a barrier curb or } \\
\text { a guardrail }\end{array}$ & & & 11 & 11 & 20 & 20 & 47 & 47 & & & & & & 4 & & & 44 & 44 & & & 28 \\
\hline $\begin{array}{l}\text { Install a bridge warning } \\
\text { sign }\end{array}$ & & & 25 & 25 & & & 25 & 25 & & & 34 & 34 & & & & & & & & & 28 \\
\hline $\begin{array}{l}\text { Install a pedestrian } \\
\text { overpass or underpass }\end{array}$ & & & 86 & 86 & & & & & & & & & & & & & & & 91 & 91 & 89 \\
\hline Install a tree marker & & & & & 16 & 16 & & & & & & & & & & & & & & & 16 \\
\hline $\begin{array}{l}\text { Install advance } \\
\text { warning signs }\end{array}$ & & & 25 & 25 & 36 & & 25 & 25 & & & & & & & & & & & & & 27 \\
\hline $\begin{array}{l}\text { Install advisor speed } \\
\text { sign }\end{array}$ & & & & & & & & & & & 26 & 26 & & & & & & & & & 26 \\
\hline $\begin{array}{l}\text { Install anutility polet } \\
\text { marker }\end{array}$ & & & & & 16 & 16 & & & & & & & & & & & & & & & 16 \\
\hline $\begin{array}{l}\text { Install central rumble } \\
\text { strips }\end{array}$ & 12 & & & & & & 14 & 14 & & & 25 & 25 & & & 12 & & 22 & 22 & 54 & 54 & 25 \\
\hline $\begin{array}{l}\text { Install channelization of } \\
\text { the driveways }\end{array}$ & & & & & & & & & 50 & 50 & & & & & & & & & & & 50 \\
\hline $\begin{array}{l}\text { Install curbing to define } \\
\text { driveway location }\end{array}$ & & & & & & & & & & & & & & & & & & & & & 10 \\
\hline Install curb-ramps & & & & & & & & & & & & & & & & & & & & & 15 \\
\hline Install flashing beacons & 30 & 30 & & & 50 & 50 & & & & & 30 & 30 & 54 & 54 & & & & & & & 41 \\
\hline $\begin{array}{l}\text { Install fog - warning } \\
\text { signs }\end{array}$ & & & & & & & 25 & 25 & & & & & & & & & & & & & 25 \\
\hline $\begin{array}{l}\text { Install Horizontal } \\
\text { alignment signs (Turn, } \\
\text { Curve, Reverse Turn, } \\
\text { Winding Road) }\end{array}$ & 30 & 30 & & & & & & & & & & & & & & & & & & & 30 \\
\hline $\begin{array}{l}\text { Install in-pavement } \\
\text { luminaires }\end{array}$ & & & & & & & & & & & & & & & & & & & & & 20 \\
\hline
\end{tabular}




\begin{tabular}{|c|c|c|c|c|c|c|c|c|c|c|c|c|c|c|c|c|c|c|c|c|c|}
\hline & \multicolumn{2}{|c|}{ S1 } & \multicolumn{2}{|c|}{ S2 } & \multicolumn{2}{|c|}{ S3 } & \multicolumn{2}{|c|}{ S4 } & \multicolumn{2}{|c|}{ S5 } & \multicolumn{2}{|c|}{ S6 } & \multicolumn{2}{|c|}{ S7 } & \multicolumn{2}{|c|}{ S8 } & \multicolumn{2}{|c|}{ S9-S10 } & \multicolumn{2}{|c|}{ S11-S21 } & \multirow[t]{2}{*}{ FINAL } \\
\hline & $\mathbf{R}$ & $\mathbf{U}$ & $\mathbf{R}$ & $\mathbf{U}$ & $\mathbf{R}$ & $\mathbf{U}$ & $\mathbf{R}$ & $\mathbf{U}$ & $\mathbf{R}$ & $\mathbf{U}$ & $\mathbf{R}$ & $\mathbf{U}$ & $\mathbf{R}$ & $\mathbf{U}$ & $\mathbf{R}$ & $\mathbf{U}$ & $\mathbf{R}$ & $\mathbf{U}$ & $\mathbf{R}$ & $\mathbf{U}$ & \\
\hline $\begin{array}{l}\text { Install interactive truck } \\
\text { rollover signing }\end{array}$ & & & & & & & & & & & & & & & & & & & & & 10 \\
\hline $\begin{array}{l}\text { Install larger regulatory } \\
\text { and warning signs at } \\
\text { and in advance of } \\
\text { hazardous location }\end{array}$ & & & & & & & & & & & 32 & 32 & & & & & & & & & 32 \\
\hline $\begin{array}{l}\text { Install LED barrier- } \\
\text { mounted guidance } \\
\text { tubes }\end{array}$ & & & & & & & & & & & & & & & & & & & & & 20 \\
\hline $\begin{array}{l}\text { Install light-emitting } \\
\text { diode (LED) }\end{array}$ & & & & & & & & & & & & & & & & & & & & & 20 \\
\hline Install lighting & 30 & 30 & 23 & 23 & 50 & 50 & & & & & 25 & 25 & 37 & 37 & & & & & 37 & 37 & 34 \\
\hline Install median barriers & 60 & & & & 61 & 61 & & & 63 & 63 & & & 90 & 90 & 60 & & & & & & 69 \\
\hline Install median divider & & & 15 & 15 & & & & & & & & & & & & & & & & & 15 \\
\hline $\begin{array}{l}\text { Install midlane rumble } \\
\text { strips }\end{array}$ & & & 32 & 32 & & & 14 & 14 & & & 25 & 25 & & & 12 & & 22 & 22 & 54 & 54 & 28 \\
\hline $\begin{array}{l}\text { Install overhead } \\
\text { flashing beacon lights }\end{array}$ & 30 & 30 & & & 50 & 50 & & & & & 30 & 30 & 54 & 54 & & & & & & & 41 \\
\hline $\begin{array}{l}\text { Install } \\
\text { Overpasses/Underpas } \\
\text { ses }\end{array}$ & & & 86 & 86 & & & & & & & & & & & & & & & & & 86 \\
\hline $\begin{array}{l}\text { Install pavement } \\
\text { markings }\end{array}$ & & & 21 & 21 & 10 & 10 & & & 21 & 21 & & & & & & & & & 10 & 10 & 16 \\
\hline $\begin{array}{l}\text { Install pedestrian } \\
\text { actuated signals }\end{array}$ & & & & & & & & & & & & & & & & & & & & & 22 \\
\hline $\begin{array}{l}\text { Install pedestrian } \\
\text { barriers }\end{array}$ & & & & & & & & & & & & & & & & & & & & & 60 \\
\hline $\begin{array}{l}\text { Install pedestrian } \\
\text { crosswalk }\end{array}$ & & & 25 & 25 & & & & & 25 & 25 & & & & & & & & & & & 25 \\
\hline Install raised crosswalk & & & & & & & & & & & & & & & & & 46 & & & & 46 \\
\hline $\begin{array}{l}\text { Install reflectorized } \\
\text { pavement markings }\end{array}$ & 10 & 10 & 10 & 10 & & & & & & & & & & & & & & & & & 10 \\
\hline Install regulatory signs & & & & & & & & & & & & & & & & & & & & & 20 \\
\hline
\end{tabular}




\begin{tabular}{|c|c|c|c|c|c|c|c|c|c|c|c|c|c|c|c|c|c|c|c|c|c|}
\hline & \multicolumn{2}{|c|}{ S1 } & \multicolumn{2}{|c|}{ S2 } & \multicolumn{2}{|c|}{ S3 } & \multicolumn{2}{|c|}{ S4 } & \multicolumn{2}{|c|}{ S5 } & \multicolumn{2}{|c|}{ S6 } & \multicolumn{2}{|c|}{ S7 } & \multicolumn{2}{|c|}{ S8 } & \multicolumn{2}{|c|}{ S9-S10 } & \multicolumn{2}{|c|}{ S11-S21 } & \multirow[t]{2}{*}{ FINAL } \\
\hline & $\mathbf{R}$ & $\mathbf{U}$ & $\mathbf{R}$ & $\mathbf{U}$ & $\mathbf{R}$ & $\mathbf{U}$ & $\mathbf{R}$ & $\mathbf{U}$ & $\mathbf{R}$ & $\mathbf{U}$ & $\mathbf{R}$ & $\mathbf{U}$ & $\mathbf{R}$ & $\mathbf{U}$ & $\mathbf{R}$ & $\mathbf{U}$ & $\mathbf{R}$ & $\mathbf{U}$ & $\mathbf{R}$ & $\mathbf{U}$ & \\
\hline $\begin{array}{l}\text { on the both side of the } \\
\text { road }\end{array}$ & & & & & & & & & & & & & & & & & & & & & \\
\hline Install rumble strips & & & 32 & 32 & & & & & & & 25 & 25 & & & 21 & & & & & & 27 \\
\hline $\begin{array}{l}\text { Install school crossing } \\
\text { sign }\end{array}$ & & & 18 & 18 & & & & & & & 14 & 14 & & & & & & & & & 16 \\
\hline $\begin{array}{l}\text { Install school zone } \\
\text { markings }\end{array}$ & & & & & & & & & & & & & & & & & & & & & 20 \\
\hline $\begin{array}{l}\text { Install shoulder rumble } \\
\text { strips }\end{array}$ & 21 & & & & & & 29 & 29 & 30 & 30 & 25 & 25 & & & 21 & & 25 & 25 & 35 & 35 & 28 \\
\hline $\begin{array}{l}\text { Install sidewalk set- } \\
\text { backs }\end{array}$ & & & & & & & & & & & & & & & & & & & & & 20 \\
\hline Install sidewalks & & & 74 & 74 & & & & & & & 65 & 65 & & & & & & & 70 & 70 & 70 \\
\hline Install speed limit sign & & & & & & & & & & & & & & & & & & & & & 25 \\
\hline $\begin{array}{l}\text { Install static or variable } \\
\text { message signs } \\
\text { displaying weather } \\
\text { information }\end{array}$ & & & 15 & 15 & & & & & & & 15 & 15 & & & & & & & & & 15 \\
\hline Install street lights & 30 & 30 & & & 50 & 50 & & & & & & & & & & & & & & & 40 \\
\hline $\begin{array}{l}\text { Install warning signs } \\
\text { (can be install on both } \\
\text { side of the road) }\end{array}$ & & & 25 & 25 & 36 & & 25 & 25 & & & & & & & & & & & & & 27 \\
\hline $\begin{array}{l}\text { Install/Improve lighting } \\
\text { at the access points }\end{array}$ & & & 33 & 33 & & & & & & & 25 & 25 & 37 & 37 & & & & & 37 & 37 & 33 \\
\hline $\begin{array}{l}\text { Installing snow screens } \\
\text { in areas exposed to } \\
\text { snowdrifts }\end{array}$ & & & & & 53 & 53 & & & & & & & & & & & & & & & 53 \\
\hline $\begin{array}{l}\text { Modify horizontal } \\
\text { alignment }\end{array}$ & & & 45 & 45 & 40 & & 66 & 66 & & & 40 & 40 & & & & & & & 64 & 64 & 52 \\
\hline $\begin{array}{l}\text { Modify roadside clear } \\
\text { zone }\end{array}$ & & & & & 25 & 25 & & & & & & & & & & & & & & & 25 \\
\hline $\begin{array}{l}\text { Modify speed limits } \\
\text { and increase } \\
\text { enforcement to reduce } \\
\text { truck and other vehicle }\end{array}$ & & & & & & & & & & & 20 & 20 & & & & & & & & & 20 \\
\hline
\end{tabular}




\begin{tabular}{|c|c|c|c|c|c|c|c|c|c|c|c|c|c|c|c|c|c|c|c|c|c|}
\hline & \multicolumn{2}{|c|}{ S1 } & \multicolumn{2}{|c|}{ S2 } & \multicolumn{2}{|c|}{ S3 } & \multicolumn{2}{|c|}{ S4 } & \multicolumn{2}{|c|}{ S5 } & \multicolumn{2}{|c|}{ S6 } & \multicolumn{2}{|c|}{ S7 } & \multicolumn{2}{|c|}{ S8 } & \multicolumn{2}{|c|}{ S9-S10 } & \multicolumn{2}{|c|}{ S11-S21 } & \multirow[t]{2}{*}{ FINAL } \\
\hline & $\mathbf{R}$ & $\mathbf{U}$ & $\mathbf{R}$ & $\mathbf{U}$ & $\mathbf{R}$ & $\mathbf{U}$ & $\mathbf{R}$ & $\mathbf{U}$ & $\mathbf{R}$ & $\mathbf{U}$ & $\mathbf{R}$ & $\mathbf{U}$ & $\mathbf{R}$ & $\mathbf{U}$ & $\mathbf{R}$ & $\mathbf{U}$ & $\mathbf{R}$ & $\mathbf{U}$ & $\mathbf{R}$ & $\mathbf{U}$ & \\
\hline speeds & & & & & & & & & & & & & & & & & & & & & \\
\hline $\begin{array}{l}\text { Move driveway to side } \\
\text { street }\end{array}$ & & & 50 & 50 & 15 & 15 & & & & & & & & & & & & & & & 33 \\
\hline $\begin{array}{l}\text { Overlay pavement } \\
\text { (friction course) }\end{array}$ & & & 17 & 17 & 18 & 18 & 27 & 27 & & & & & 13 & 34 & & & & & & & 21 \\
\hline Pave shoulders & & & 15 & 15 & & & & & & & 15 & 15 & & 53 & & & & & & & 23 \\
\hline $\begin{array}{l}\text { Place utilities } \\
\text { underground }\end{array}$ & & & & & & & & & & & & & & & & & & & & & 60 \\
\hline $\begin{array}{l}\text { Placed delineation on } \\
\text { guardrail located } \\
\text { outside the roadway } \\
\text { shoulder, along the } \\
\text { curve }\end{array}$ & & & & & 16 & 16 & & & & & 30 & 30 & 17 & 17 & & & 15 & & 15 & & 20 \\
\hline $\begin{array}{l}\text { Post an adequate } \\
\text { speed limit }\end{array}$ & & & & & 28 & 28 & & & & & & & 20 & 20 & & & & & & & 24 \\
\hline $\begin{array}{l}\text { Post dynamic message } \\
\text { sign to display the } \\
\text { speed of approaching } \\
\text { vehicles. }\end{array}$ & & & & & & & & & & & & & & & & & 74 & 74 & & & 74 \\
\hline $\begin{array}{l}\text { Profiled thermoplastic } \\
\text { stripes for centerline }\end{array}$ & & & & & & & & & & & & & & & & & & & & & 10 \\
\hline $\begin{array}{l}\text { Prohibit/Restrict trucks } \\
\text { with very Long } \\
\text { semitrailers on roads } \\
\text { with horizontal curves } \\
\text { that cannot } \\
\text { accommodate truck } \\
\text { offtracking }\end{array}$ & & & & & & & & & & & & & & & & & & & & & 60 \\
\hline $\begin{array}{l}\text { Provide acceleration } \\
\text { and deceleration lanes }\end{array}$ & & & 26 & 26 & & & & & & & 10 & 10 & & & & & & & & & 18 \\
\hline $\begin{array}{l}\text { Provide adequate } \\
\text { drainage }\end{array}$ & & & 20 & 20 & & & & & & & & & & & & & & & & & 20 \\
\hline $\begin{array}{l}\text { Provide adequate } \\
\text { speed limit for trucks }\end{array}$ & & & & & & & & & & & 20 & 20 & & & & & & & & & 20 \\
\hline
\end{tabular}




\begin{tabular}{|c|c|c|c|c|c|c|c|c|c|c|c|c|c|c|c|c|c|c|c|c|c|}
\hline & \multicolumn{2}{|c|}{ S1 } & \multicolumn{2}{|c|}{ S2 } & \multicolumn{2}{|c|}{ S3 } & \multicolumn{2}{|c|}{ S4 } & \multicolumn{2}{|c|}{ S5 } & \multicolumn{2}{|c|}{ S6 } & \multicolumn{2}{|c|}{ S7 } & \multicolumn{2}{|c|}{ S8 } & \multicolumn{2}{|c|}{ S9-S10 } & \multicolumn{2}{|c|}{ S11-S21 } & \multirow[t]{2}{*}{ FINAL } \\
\hline & $\mathbf{R}$ & $\mathbf{U}$ & $\mathbf{R}$ & $\mathbf{U}$ & $\mathbf{R}$ & $\mathbf{U}$ & $\mathbf{R}$ & $\mathbf{U}$ & $\mathbf{R}$ & $\mathbf{U}$ & $\mathbf{R}$ & $\mathbf{U}$ & $\mathbf{R}$ & $\mathbf{U}$ & $\mathbf{R}$ & $\mathbf{U}$ & $\mathbf{R}$ & $\mathbf{U}$ & $\mathbf{R}$ & $\mathbf{U}$ & \\
\hline $\begin{array}{l}\text { Provide adequate } \\
\text { vertical curve design } \\
\text { (crest/redesign) }\end{array}$ & & & 87 & 87 & 88 & & 40 & 40 & 40 & 40 & 40 & 40 & 45 & 45 & & & & & & & 54 \\
\hline $\begin{array}{l}\text { Provide advance } \\
\text { warning of unexpected } \\
\text { changes in horizontal } \\
\text { alignment }\end{array}$ & & & & & & & 25 & 25 & & & & & 30 & 30 & & & & & 18 & 18 & 24 \\
\hline $\begin{array}{l}\text { Provide advance } \\
\text { warning of unexpected } \\
\text { changes in } \\
\text { horizontal/vertical } \\
\text { alignment }\end{array}$ & & & & & & & 25 & 25 & & & 32 & 32 & 30 & 30 & & & & & & & 29 \\
\hline $\begin{array}{l}\text { Provide advance } \\
\text { warning of unexpected } \\
\text { changes in vertical } \\
\text { alignment }\end{array}$ & & & & & & & 25 & 25 & & & 32 & 32 & 30 & 30 & & & & & & & 29 \\
\hline $\begin{array}{l}\text { Provide breakaway } \\
\text { and crashworthy mail } \\
\text { box }\end{array}$ & & & & & & & & & & & & & & & & & & & & & 15 \\
\hline $\begin{array}{l}\text { Provide breakaway } \\
\text { and crashworthy sign } \\
\text { supports }\end{array}$ & & & & & 30 & 30 & & & & & & & & & & & & & & & 30 \\
\hline Provide chevrons & & & & & 35 & 35 & & & & & 30 & 30 & 35 & 35 & & & 25 & & & & 32 \\
\hline $\begin{array}{l}\text { Provide dynamic curve } \\
\text { warning system }\end{array}$ & & & & & & & & & & & & & & & & & & & $\begin{array}{r}10 \\
0\end{array}$ & & 100 \\
\hline $\begin{array}{l}\text { Provide enhanced } \\
\text { pavement markings }\end{array}$ & & & 10 & 10 & 10 & 10 & & & & & & & & & & & & & 10 & 10 & 10 \\
\hline $\begin{array}{l}\text { Provide grooved } \\
\text { pavement }\end{array}$ & & & 21 & 21 & & & 14 & 14 & & & & & & & & & & & 46 & 46 & 27 \\
\hline $\begin{array}{l}\text { Provide independent } \\
\text { bicycle path where } \\
\text { necessary }\end{array}$ & & & & & & & & & & & & & & & & & & & & & 40 \\
\hline $\begin{array}{l}\text { Provide median } \\
\text { refuges }\end{array}$ & & 46 & & & & & & & & & & & & & & & & & & & 46 \\
\hline
\end{tabular}




\begin{tabular}{|c|c|c|c|c|c|c|c|c|c|c|c|c|c|c|c|c|c|c|c|c|c|}
\hline & \multicolumn{2}{|c|}{ S1 } & \multicolumn{2}{|c|}{ S2 } & \multicolumn{2}{|c|}{ S3 } & \multicolumn{2}{|c|}{ S4 } & \multicolumn{2}{|c|}{ S5 } & \multicolumn{2}{|c|}{ S6 } & \multicolumn{2}{|c|}{ S7 } & \multicolumn{2}{|c|}{ S8 } & \multicolumn{2}{|c|}{ S9-S10 } & \multicolumn{2}{|c|}{ S11-S21 } & \multirow[t]{2}{*}{ FINAL } \\
\hline & $\mathbf{R}$ & $\mathbf{U}$ & $\mathbf{R}$ & $\mathbf{U}$ & $\mathbf{R}$ & $\mathbf{U}$ & $\mathbf{R}$ & $\mathbf{U}$ & $\mathbf{R}$ & $\mathbf{U}$ & $\mathbf{R}$ & $\mathbf{U}$ & $\mathbf{R}$ & $\mathbf{U}$ & $\mathbf{R}$ & $\mathbf{U}$ & $\mathbf{R}$ & $\mathbf{U}$ & $\mathbf{R}$ & $\mathbf{U}$ & \\
\hline $\begin{array}{l}\text { Provide on-pavement } \\
\text { horizontal signing }\end{array}$ & & & & & & & & & & & & & & & & & 10 & & & & 10 \\
\hline $\begin{array}{l}\text { Provide post-mounted } \\
\text { delineators }\end{array}$ & & & & & 16 & 16 & & & & & 30 & 30 & 25 & 25 & & & 15 & & 15 & & 22 \\
\hline $\begin{array}{l}\text { Provide smooth paved } \\
\text { shoulders }\end{array}$ & & & 15 & 15 & & & 8 & 8 & & & & & & 53 & & & & & & & 20 \\
\hline $\begin{array}{l}\text { Provide targeted speed } \\
\text { enforcement }\end{array}$ & & & & & & & & & & & & & & & & & & & & & 70 \\
\hline $\begin{array}{l}\text { Provide traffic calming } \\
\text { through a combination } \\
\text { of geometric and traffic } \\
\text { control devices }\end{array}$ & & & & & & & & & & & & & & & & & & & & & 20 \\
\hline $\begin{array}{l}\text { Provide wider cross } \\
\text { sections on two-lane } \\
\text { roads }\end{array}$ & & & & & & & & & & & & & & & & & & & & & 20 \\
\hline $\begin{array}{l}\text { Provide/Improve } \\
\text { pavement surface } \\
\text { (improve transitions, } \\
\text { drop offs, fixed } \\
\text { potholes, rutting, etc) }\end{array}$ & & & & & & & & & & & & & & & & & & & & & 28 \\
\hline $\begin{array}{l}\text { Provide/Improve } \\
\text { pavement surface } \\
\text { along the segment } \\
\text { (improve transitions, } \\
\text { drop offs, fixed } \\
\text { potholes, rutting, etc) }\end{array}$ & & & & & & & & & & & & & & & & & & & & & 28 \\
\hline $\begin{array}{l}\text { Providing spiral } \\
\text { transition curves }\end{array}$ & & & & & & & & & & & & & & & & & & & & & 20 \\
\hline $\begin{array}{l}\text { Raised pavement } \\
\text { markers }\end{array}$ & & & & & 9 & 9 & & & & & 10 & 10 & 4 & 4 & & & 9 & 9 & & & 8 \\
\hline $\begin{array}{l}\text { Reallocate total two- } \\
\text { lane roadway width } \\
\text { (lane and shoulder) to } \\
\text { include a narrow }\end{array}$ & & & & & & & & & & & & & & & & & & & 50 & 50 & 50 \\
\hline
\end{tabular}




\begin{tabular}{|c|c|c|c|c|c|c|c|c|c|c|c|c|c|c|c|c|c|c|c|c|c|}
\hline & \multicolumn{2}{|c|}{ S1 } & \multicolumn{2}{|c|}{ S2 } & \multicolumn{2}{|c|}{ S3 } & \multicolumn{2}{|c|}{ S4 } & \multicolumn{2}{|c|}{ S5 } & \multicolumn{2}{|c|}{ S6 } & \multicolumn{2}{|c|}{ S7 } & \multicolumn{2}{|c|}{ S8 } & \multicolumn{2}{|c|}{ S9-S10 } & \multicolumn{2}{|c|}{ S11-S21 } & \multirow[t]{2}{*}{ FINAL } \\
\hline & $\mathbf{R}$ & $\mathbf{U}$ & $\mathbf{R}$ & $\mathbf{U}$ & $\mathbf{R}$ & $\mathbf{U}$ & $\mathbf{R}$ & $\mathbf{U}$ & $\mathbf{R}$ & $\mathbf{U}$ & $\mathbf{R}$ & $\mathbf{U}$ & $\mathbf{R}$ & $\mathbf{U}$ & $\mathbf{R}$ & $\mathbf{U}$ & $\mathbf{R}$ & $\mathbf{U}$ & $\mathbf{R}$ & $\mathbf{U}$ & \\
\hline "buffer median" & & & & & & & & & & & & & & & & & & & & & \\
\hline $\begin{array}{l}\text { Relocate the sign to } \\
\text { make it visible }\end{array}$ & & & & & & & & & & & & & & & & & & & & & 20 \\
\hline $\begin{array}{l}\text { Remove distracting } \\
\text { commercial lights }\end{array}$ & & & & & & & & & & & & & & & & & & & & & 15 \\
\hline $\begin{array}{l}\text { Remove or relocate } \\
\text { mail box }\end{array}$ & & & & & 29 & 29 & & & & & 30 & 30 & & & & & & & 27 & 27 & 29 \\
\hline $\begin{array}{l}\text { Remove or relocate } \\
\text { sign }\end{array}$ & & & & & 29 & 29 & & & & & & & & & & & & & & & 29 \\
\hline $\begin{array}{l}\text { Remove or relocate } \\
\text { trees }\end{array}$ & & & & & 25 & 25 & & & & & & & & & & & & & 66 & 66 & 46 \\
\hline $\begin{array}{l}\text { Remove or relocate } \\
\text { utility Poles }\end{array}$ & & & & & 38 & 38 & & & & & & & & & & & & & 32 & & 36 \\
\hline $\begin{array}{l}\text { Remove sight } \\
\text { obstructions }\end{array}$ & & & & & & & & & & & & & & & & & & & & & 20 \\
\hline $\begin{array}{l}\text { Remove the object } \\
\text { obstructing sight of the } \\
\text { warning sign }\end{array}$ & & & & & & & & & & & & & & & & & & & & & 20 \\
\hline $\begin{array}{l}\text { Removed other } \\
\text { obstructions }\end{array}$ & & & & & 29 & 29 & & & & & & & & & & & & & & & 29 \\
\hline $\begin{array}{l}\text { Replace poorly } \\
\text { designed drain grates } \\
\text { with bicycle-safe types }\end{array}$ & & & & & & & & & & & & & & & & & & & & & 10 \\
\hline $\begin{array}{l}\text { Replace, repair, or } \\
\text { clean the warning } \\
\text { signs }\end{array}$ & & & & & & & 25 & 25 & & & 24 & 24 & 30 & 40 & & & & & & & 28 \\
\hline $\begin{array}{l}\text { Restrict left-turning at } \\
\text { the access points }\end{array}$ & & & & & 45 & 45 & & & & & & & & & & & & & & & 45 \\
\hline Restrict parking & & & & & & & & & & & 35 & 35 & 8 & 8 & & & & & 10 & 10 & 18 \\
\hline $\begin{array}{l}\text { Restrict parking near } \\
\text { the driveway }\end{array}$ & & & & & & & & & & & & & & & & & & & & & 19 \\
\hline $\begin{array}{l}\text { Shield drivers from } \\
\text { poles in hazardous } \\
\text { locations }\end{array}$ & & & & & & & & & & & & & & & & & & & & & 20 \\
\hline
\end{tabular}




\begin{tabular}{|c|c|c|c|c|c|c|c|c|c|c|c|c|c|c|c|c|c|c|c|c|c|}
\hline & \multicolumn{2}{|c|}{ S1 } & \multicolumn{2}{|c|}{ S2 } & \multicolumn{2}{|c|}{ S3 } & \multicolumn{2}{|c|}{ S4 } & \multicolumn{2}{|c|}{ S5 } & \multicolumn{2}{|c|}{ S6 } & \multicolumn{2}{|c|}{ S7 } & \multicolumn{2}{|c|}{ S8 } & \multicolumn{2}{|c|}{ S9-S10 } & \multicolumn{2}{|c|}{ S11-S21 } & \multirow[t]{2}{*}{ FINAL } \\
\hline & $\mathbf{R}$ & $\mathbf{U}$ & $\mathbf{R}$ & $\mathbf{U}$ & $\mathbf{R}$ & $\mathbf{U}$ & $\mathbf{R}$ & $\mathbf{U}$ & $\mathbf{R}$ & $\mathbf{U}$ & $\mathbf{R}$ & $\mathbf{U}$ & $\mathbf{R}$ & $\mathbf{U}$ & $\mathbf{R}$ & $\mathbf{U}$ & $\mathbf{R}$ & $\mathbf{U}$ & $\mathbf{R}$ & $\mathbf{U}$ & \\
\hline $\begin{array}{l}\text { Signals to alert } \\
\text { motorists that } \\
\text { pedestrians are } \\
\text { crossing }\end{array}$ & & & & & & & & & & & & & & & & & & & & & 10 \\
\hline Upgrade signing & & & & & 5 & 5 & & & & & 24 & 24 & & & & & & & & & 15 \\
\hline $\begin{array}{l}\text { Use "Slippery when } \\
\text { wet" sign (temporary) }\end{array}$ & & & & & & & & & & & & & & & & & & & & & 15 \\
\hline $\begin{array}{l}\text { Use alternating } \\
\text { passing lanes or four- } \\
\text { lane sections at key } \\
\text { locations }\end{array}$ & & & & & 30 & 30 & 25 & 25 & & & & & & & & & 35 & 35 & 25 & 25 & 29 \\
\hline $\begin{array}{l}\text { Use break-away } \\
\text { devices }\end{array}$ & & & & & 30 & 30 & & & & & & & & & & & & & & & 30 \\
\hline $\begin{array}{l}\text { Use salt to prevent } \\
\text { snow or ice from } \\
\text { forming or from sticking } \\
\text { to the road surface }\end{array}$ & & & & & & & & & & & & & & & & & & & & & 20 \\
\hline $\begin{array}{l}\text { Use sand to increase } \\
\text { pavement friction }\end{array}$ & & & & & & & & & & & & & & & & & & & & & 20 \\
\hline $\begin{array}{l}\text { Use school crossing } \\
\text { guards }\end{array}$ & & & & & & & & & & & & & & & & & & & & & 50 \\
\hline $\begin{array}{l}\text { Use transverse striping } \\
\text { treatments }\end{array}$ & & & & & & & & & & & & & & & & & & & & & 10 \\
\hline $\begin{array}{l}\text { Widen and/or pave } \\
\text { shoulders }\end{array}$ & 9 & & 14 & 14 & & & 8 & 8 & & & 20 & 20 & & 53 & & & & & 19 & 19 & 18 \\
\hline $\begin{array}{l}\text { Widen the outside } \\
\text { through lanes or add } \\
\text { bike lanes }\end{array}$ & & & & & & & & & & & & & & & & & & & & & 15 \\
\hline
\end{tabular}




\section{SIGN}

\begin{tabular}{|c|c|c|c|c|c|c|c|c|c|c|c|c|c|c|c|c|c|c|c|c|c|}
\hline & \multicolumn{2}{|c|}{ S1 } & \multicolumn{2}{|c|}{ S2 } & \multicolumn{2}{|c|}{ S3 } & \multicolumn{2}{|c|}{ S4 } & \multicolumn{2}{|c|}{ S5 } & \multicolumn{2}{|c|}{ S6 } & \multicolumn{2}{|c|}{ S7 } & \multicolumn{2}{|c|}{ S8 } & \multicolumn{2}{|c|}{ S9-S10 } & \multicolumn{2}{|c|}{ S11-S21 } & \multirow[t]{2}{*}{ FINAL } \\
\hline & $\mathbf{R}$ & $\mathbf{U}$ & $\mathbf{R}$ & $\mathbf{U}$ & $\mathbf{R}$ & $\mathbf{U}$ & $\mathbf{R}$ & $U$ & $\mathbf{R}$ & $\mathbf{U}$ & $\mathbf{R}$ & $\mathbf{U}$ & $\mathbf{R}$ & $\mathbf{U}$ & $\mathbf{R}$ & $\mathbf{U}$ & $\mathbf{R}$ & $U$ & $\mathbf{R}$ & $\mathbf{U}$ & \\
\hline $\begin{array}{l}\text { Add } \\
\text { illuminated/reflectorized } \\
\text { name signs }\end{array}$ & 23 & 23 & 28 & 28 & & & & & & & & & & & & & & & & & 26 \\
\hline Add special signing & & & & & & & & & & & & & & & & & & & & & 10 \\
\hline $\begin{array}{l}\text { Adjust minimum green } \\
\text { or extension time }\end{array}$ & & & & & & & & & & & & & & & & & & & & & 10 \\
\hline $\begin{array}{l}\text { Adjust phase time and } \\
\text { cycle time }\end{array}$ & & & & & & & & & & & & & & & & & & & & & 15 \\
\hline $\begin{array}{l}\text { Adjust yellow change } \\
\text { interval }\end{array}$ & & & & & & & & & & & & & & & & 8 & & 8 & & & 8 \\
\hline $\begin{array}{l}\text { Change } \\
\text { horizontal/vertical } \\
\text { alignment }\end{array}$ & & & 58 & 58 & & & & & & & 50 & 50 & 50 & 50 & & & & & & & 53 \\
\hline
\end{tabular}




\begin{tabular}{|c|c|c|c|c|c|c|c|c|c|c|c|c|c|c|c|c|c|c|c|c|c|}
\hline & \multicolumn{2}{|c|}{ S1 } & \multicolumn{2}{|c|}{$\mathbf{S 2}$} & \multicolumn{2}{|c|}{ S3 } & \multicolumn{2}{|c|}{ S4 } & \multicolumn{2}{|c|}{ S5 } & \multicolumn{2}{|c|}{ S6 } & \multicolumn{2}{|c|}{ S7 } & \multicolumn{2}{|c|}{ S8 } & \multicolumn{2}{|c|}{ S9-S10 } & \multicolumn{2}{|c|}{ S11-S21 } & \multirow[t]{2}{*}{ FINAL } \\
\hline & $\mathbf{R}$ & $\mathbf{U}$ & $\mathbf{R}$ & $\mathbf{U}$ & $\mathbf{R}$ & $\mathbf{U}$ & $\mathbf{R}$ & $\mathbf{U}$ & $\mathbf{R}$ & $\mathbf{U}$ & $\mathbf{R}$ & $\mathbf{U}$ & $\mathbf{R}$ & $\mathbf{U}$ & $\mathbf{R}$ & $\mathbf{U}$ & $\mathbf{R}$ & $\mathbf{U}$ & $\mathbf{R}$ & $\mathbf{U}$ & \\
\hline Channelize intersections & & & & & & & & & 50 & 50 & & & 70 & 70 & & & & & & & 60 \\
\hline $\begin{array}{l}\text { Chip and seal or slurry } \\
\text { seal approaches }\end{array}$ & & & & & & & & & & & & & & & & & & & & & 10 \\
\hline Clear sight triangles & 12 & & & & & & 25 & 25 & & & & & & & & & & & & & 21 \\
\hline $\begin{array}{l}\text { Close intersection leg or } \\
\text { convert to one-way } \\
\text { street }\end{array}$ & & & & & & & & & & & & & & & & & & & & & 40 \\
\hline $\begin{array}{l}\text { Consider indirect left } \\
\text { turns }\end{array}$ & & & 28 & 28 & & & 25 & 25 & 25 & 25 & 22 & 22 & & & & & & 37 & & & 26 \\
\hline Construct interchanges & & & & & & & & & & & & & & & & & & & & & 50 \\
\hline $\begin{array}{l}\text { Convert a four-leg } \\
\text { intersection to two } \mathrm{T} \\
\text { intersection }\end{array}$ & & & & & & & & & & & & & & & & & & & & & 57 \\
\hline $\begin{array}{l}\text { Convert intersection to } \\
\text { roundabouts }\end{array}$ & 58 & 72 & & & & & 40 & 40 & 40 & 40 & & & & & 58 & 72 & 58 & 72 & 38 & 38 & 52 \\
\hline $\begin{array}{l}\text { Convert two } \mathrm{T} \\
\text { intersection to one four- } \\
\text { leg intersection }\end{array}$ & & & & & & & & & & & & & & & & & & & & & 15 \\
\hline $\begin{array}{l}\text { Convert two-way streets } \\
\text { to a one-way pair }\end{array}$ & & & & & & & & & & & & & & & & & & & & & 35 \\
\hline $\begin{array}{l}\text { Coordinate the railroad } \\
\text { and traffic signal to } \\
\text { provide preemption to } \\
\text { the traffic signal when } \\
\text { trains are approaching } \\
\text { the intersection }\end{array}$ & & & & & & & 30 & 30 & & & & & & & & & & & 25 & 25 & 28 \\
\hline Create one way streets & & & & & & & & & & & & & & & & & & & & & 45 \\
\hline Delineate turn path & & & & & & & & & & & 30 & 30 & & & & & & & & & 30 \\
\hline $\begin{array}{l}\text { Eliminate screening by } \\
\text { physical objects }\end{array}$ & & & & & 60 & 60 & & & & & & & 50 & 50 & & & & & 76 & 76 & 62 \\
\hline $\begin{array}{l}\text { Employ emergency } \\
\text { vehicle preemption }\end{array}$ & & & & & & & & & & & & & & & & & & & 70 & 70 & 70 \\
\hline Encourage safe speeds & & & & & & & & & & & & & & & & & & & & & 20 \\
\hline
\end{tabular}




\begin{tabular}{|c|c|c|c|c|c|c|c|c|c|c|c|c|c|c|c|c|c|c|c|c|c|}
\hline & \multicolumn{2}{|c|}{ S1 } & \multicolumn{2}{|c|}{$\mathbf{S 2}$} & \multicolumn{2}{|c|}{ S3 } & \multicolumn{2}{|c|}{ S4 } & \multicolumn{2}{|c|}{ S5 } & \multicolumn{2}{|c|}{ S6 } & \multicolumn{2}{|c|}{ S7 } & \multicolumn{2}{|c|}{ S8 } & \multicolumn{2}{|c|}{ S9-S10 } & \multicolumn{2}{|c|}{ S11-S21 } & \multirow[t]{2}{*}{ FINAL } \\
\hline & $\mathbf{R}$ & $\mathbf{U}$ & $\mathbf{R}$ & $\mathbf{U}$ & $\mathbf{R}$ & $\mathbf{U}$ & $\mathbf{R}$ & $\mathbf{U}$ & $\mathbf{R}$ & $\mathbf{U}$ & $\mathbf{R}$ & $\mathbf{U}$ & $\mathbf{R}$ & $\mathbf{U}$ & $\mathbf{R}$ & $\mathbf{U}$ & $\mathbf{R}$ & $\mathbf{U}$ & $\mathbf{R}$ & $\mathbf{U}$ & \\
\hline through design & & & & & & & & & & & & & & & & & & & & & \\
\hline $\begin{array}{l}\text { Extend "no parking" } \\
\text { zone. }\end{array}$ & & & & & 22 & 22 & & & & & 35 & 35 & 8 & 8 & & & & & 10 & 10 & 19 \\
\hline $\begin{array}{l}\text { Groove pavement } \\
\text { surface }\end{array}$ & & & 21 & 21 & & & 14 & 14 & & & & & & & & & & & 46 & 46 & 27 \\
\hline $\begin{array}{l}\text { Implement automated } \\
\text { enforcement of red-light } \\
\text { running (cameras) }\end{array}$ & & 9 & & & & & & & & & & & & & & 9 & & 25 & & & 14 \\
\hline $\begin{array}{l}\text { Improve (add) } \\
\text { coordinating signals }\end{array}$ & & & & & & & & & & & & & & & & & & & 25 & 25 & 25 \\
\hline $\begin{array}{l}\text { Improve (add) phases } \\
\text { (length) }\end{array}$ & & & & & 30 & 30 & & & & & & & & & & & & & & & 30 \\
\hline $\begin{array}{l}\text { Improve } \\
\text { alignment/grade }\end{array}$ & & & & & & & & & & & & & & & & & & & & & 35 \\
\hline $\begin{array}{l}\text { Improve existing street } \\
\text { lights }\end{array}$ & 30 & 30 & & & 50 & 50 & & & & & & & & & & & & & & & 40 \\
\hline $\begin{array}{l}\text { Improve intersection } \\
\text { angle }\end{array}$ & & & & & & & & & & & & & & & & & & & & & 30 \\
\hline $\begin{array}{l}\text { Improve intersection } \\
\text { skew angle }\end{array}$ & & & & & & & 40 & 40 & & & & & & & & & & & & & 40 \\
\hline $\begin{array}{l}\text { Improve left-turn lane } \\
\text { geometry }\end{array}$ & & & & & & & & & & & & & & & & & & & & & 10 \\
\hline $\begin{array}{l}\text { Improve right-turn lane } \\
\text { geometry }\end{array}$ & & & & & & & & & & & & & & & & & & & & & 10 \\
\hline $\begin{array}{l}\text { Improve signing and } \\
\text { delineation }\end{array}$ & & & & & & & & & & & 30 & 30 & 17 & 17 & & & & & 15 & & 22 \\
\hline Improve skid resistance & & & 9 & 9 & & & & & & & & & & & & & & & & & 9 \\
\hline $\begin{array}{l}\text { Improve winter } \\
\text { maintenance methods }\end{array}$ & & & & & & & & & & & & & & & & & & & & & 25 \\
\hline $\begin{array}{l}\text { Improve winter } \\
\text { maintenance } \\
\text { preparedness (shorter } \\
\text { response time) }\end{array}$ & & & & & & & & & & & & & & & & & & & & & 20 \\
\hline Improve/install reflective & & & 15 & 15 & & & & & & & & & & & & & & & & & 15 \\
\hline
\end{tabular}




\begin{tabular}{|c|c|c|c|c|c|c|c|c|c|c|c|c|c|c|c|c|c|c|c|c|c|}
\hline & \multicolumn{2}{|c|}{ S1 } & \multicolumn{2}{|c|}{ S2 } & \multicolumn{2}{|c|}{ S3 } & \multicolumn{2}{|c|}{ S4 } & \multicolumn{2}{|c|}{ S5 } & \multicolumn{2}{|c|}{ S6 } & \multicolumn{2}{|c|}{ S7 } & \multicolumn{2}{|c|}{ S8 } & \multicolumn{2}{|c|}{ S9-S10 } & \multicolumn{2}{|c|}{ S11-S21 } & \multirow[t]{2}{*}{ FINAL } \\
\hline & $\mathbf{R}$ & $\mathbf{U}$ & $\mathbf{R}$ & $\mathbf{U}$ & $\mathbf{R}$ & $\mathbf{U}$ & $\mathbf{R}$ & $\mathbf{U}$ & $\mathbf{R}$ & $\mathbf{U}$ & $\mathbf{R}$ & $\mathbf{U}$ & $\mathbf{R}$ & $\mathbf{U}$ & $\mathbf{R}$ & $\mathbf{U}$ & $\mathbf{R}$ & $\mathbf{U}$ & $\mathbf{R}$ & $\mathbf{U}$ & \\
\hline signs & & & & & & & & & & & & & & & & & & & & & \\
\hline $\begin{array}{l}\text { Improve/install } \\
\text { reflectorized pavement } \\
\text { markers }\end{array}$ & & & & & & & & & & & & & & & & & & & & & 12 \\
\hline $\begin{array}{l}\text { Increase the crosswalk } \\
\text { setback }\end{array}$ & & & & & & & & & & & & & & & & & & & & & 15 \\
\hline $\begin{array}{l}\text { Install a barrier curb or a } \\
\text { guardrail }\end{array}$ & & & 11 & 11 & 20 & 20 & 47 & 47 & & & & & & 4 & & & & 44 & & & 26 \\
\hline Install a tree marker & & & & & 16 & 16 & & & & & & & & & & & & & & & 16 \\
\hline $\begin{array}{l}\text { Install additional signal } \\
\text { head }\end{array}$ & & & & & 50 & 50 & & & & & & & 31 & 31 & & & & & & & 41 \\
\hline $\begin{array}{l}\text { Install adequate } \\
\text { signings }\end{array}$ & & & 30 & 30 & & & & & & & & & & & & & & & & & 30 \\
\hline $\begin{array}{l}\text { Install an utility poles } \\
\text { marker }\end{array}$ & & & & & 16 & 16 & & & & & & & & & & & & & & & 16 \\
\hline Install backplates & & & & & & & 5 & 5 & & & 24 & 24 & & & & & & & & & 15 \\
\hline $\begin{array}{l}\text { Install bicycles traffic } \\
\text { lights }\end{array}$ & & & & & & & & & & & & & & & & & & & & & 20 \\
\hline Install curb-ramps & & & & & & & & & & & & & & & & & & & & & 15 \\
\hline Install flashing beacons & 30 & 30 & & & 50 & 50 & 30 & 30 & & & 30 & 30 & 24 & 24 & & & & & & & 33 \\
\hline $\begin{array}{l}\text { Install fog - warning } \\
\text { signs }\end{array}$ & & & 25 & 25 & & & 25 & 25 & & & & & 24 & 24 & & & & & & & 25 \\
\hline $\begin{array}{l}\text { Install larger signal } \\
\text { lenses }\end{array}$ & & & 10 & 10 & & & & & & & 12 & 12 & 10 & 10 & & & & & & & 11 \\
\hline Install larger signs & & & & & & & & & & & & & & & & & & & & & 25 \\
\hline Install left-turn lane & & & 28 & 28 & & & & & 25 & 25 & & & & & & & & & & & 27 \\
\hline Install median driver & & & & & & & & & & & & & & & & & & & & & 30 \\
\hline $\begin{array}{l}\text { Install multi-dialer } \\
\text { controller }\end{array}$ & & & & & & & & & & & & & & & & & & & & & 10 \\
\hline $\begin{array}{l}\text { Install overhead street } \\
\text { signing }\end{array}$ & & & & & & & & & & & & & & & & & & & & & 78 \\
\hline $\begin{array}{l}\text { Install painted/raised } \\
\text { islands (provide }\end{array}$ & & & 23 & 23 & & & & & & & 10 & 10 & 10 & 10 & & & & & & & 14 \\
\hline
\end{tabular}




\begin{tabular}{|c|c|c|c|c|c|c|c|c|c|c|c|c|c|c|c|c|c|c|c|c|c|}
\hline & \multicolumn{2}{|c|}{ S1 } & \multicolumn{2}{|c|}{ S2 } & \multicolumn{2}{|c|}{ S3 } & \multicolumn{2}{|c|}{ S4 } & \multicolumn{2}{|c|}{ S5 } & \multicolumn{2}{|c|}{ S6 } & \multicolumn{2}{|c|}{ S7 } & \multicolumn{2}{|c|}{ S8 } & \multicolumn{2}{|c|}{ S9-S10 } & \multicolumn{2}{|c|}{ S11-S21 } & \multirow[t]{2}{*}{ FINAL } \\
\hline & $\mathbf{R}$ & $\mathbf{U}$ & $\mathbf{R}$ & $\mathbf{U}$ & $\mathbf{R}$ & $\mathbf{U}$ & $\mathbf{R}$ & $\mathbf{U}$ & $\mathbf{R}$ & $\mathbf{U}$ & $\mathbf{R}$ & $\mathbf{U}$ & $\mathbf{R}$ & $\mathbf{U}$ & $\mathbf{R}$ & $\mathbf{U}$ & $\mathbf{R}$ & $\mathbf{U}$ & $\mathbf{R}$ & $\mathbf{U}$ & \\
\hline $\begin{array}{l}\text { adequate visibility of the } \\
\text { island) }\end{array}$ & & & & & & & & & & & & & & & & & & & & & \\
\hline $\begin{array}{l}\text { Install pedestrian } \\
\text { overpasses }\end{array}$ & & & 86 & 86 & & & & & & & & & & & & & & & 91 & 91 & 89 \\
\hline $\begin{array}{l}\text { Install physical barriers } \\
\text { to restrict pedestrian } \\
\text { crossing maneuvers at } \\
\text { higher-risk locations }\end{array}$ & & & & & & & & & & & & & & & & & & & & & 60 \\
\hline $\begin{array}{l}\text { Install queue detection } \\
\text { system }\end{array}$ & & & & & & & & & & & & & & & & & & & & & 22 \\
\hline Install raised crosswalk & & & 25 & 25 & & & & & 25 & 25 & & & & & & & & 46 & & & 29 \\
\hline Install right-turn lane & & 4 & 17 & 17 & & & & & & & & & & & & & & & & & 13 \\
\hline $\begin{array}{l}\text { Install rumble strips on } \\
\text { approach }\end{array}$ & & & 32 & 32 & & & & & & & 25 & 25 & & & & & & & 50 & 50 & 36 \\
\hline $\begin{array}{l}\text { Install static or variable } \\
\text { message signs } \\
\text { displaying weather } \\
\text { information }\end{array}$ & & & 15 & 15 & & & & & & & 15 & 15 & & & & & & & & & 15 \\
\hline Install street lights & 30 & 30 & & & 50 & 50 & & & & & & & & & & & & & & & 40 \\
\hline $\begin{array}{l}\text { Install traffic actuated } \\
\text { signal }\end{array}$ & & & & & & & & & & & 22 & 22 & & & & & & & & & 22 \\
\hline Install warning sign & & & 25 & 25 & 20 & 20 & 25 & 25 & & & & & 20 & 15 & & & & & & & 22 \\
\hline $\begin{array}{l}\text { Install/improve center } \\
\text { line markings }\end{array}$ & & & 33 & 33 & 33 & 33 & & & & & 35 & 35 & & & & & & & & & 34 \\
\hline $\begin{array}{l}\text { Install/improve } \\
\text { Intersection lighting }\end{array}$ & 30 & 30 & 33 & 33 & 50 & 50 & & & & & 30 & 30 & 37 & 37 & & & & & 37 & 37 & 36 \\
\hline $\begin{array}{l}\text { Installing snow screens } \\
\text { in areas exposed to } \\
\text { snowdrifts }\end{array}$ & & & & & 53 & 53 & & & & & & & & & & & & & & & 53 \\
\hline Lengthen left-turn lane & 9 & & 28 & 28 & & & 15 & 15 & & & & & & & & & & & & & 19 \\
\hline Lengthen right-turn lane & 9 & & 28 & 28 & & & 15 & 15 & & & & & & & & & & & & & 19 \\
\hline $\begin{array}{l}\text { Lengthening clearance } \\
\text { intervals }\end{array}$ & & 8 & 15 & 15 & & & & & & & & & & & & & & 8 & & & 12 \\
\hline
\end{tabular}




\begin{tabular}{|c|c|c|c|c|c|c|c|c|c|c|c|c|c|c|c|c|c|c|c|c|c|}
\hline & \multicolumn{2}{|c|}{ S1 } & \multicolumn{2}{|c|}{ S2 } & \multicolumn{2}{|c|}{ S3 } & \multicolumn{2}{|c|}{ S4 } & \multicolumn{2}{|c|}{ S5 } & \multicolumn{2}{|c|}{ S6 } & \multicolumn{2}{|c|}{ S7 } & \multicolumn{2}{|c|}{ S8 } & \multicolumn{2}{|c|}{ S9-S10 } & \multicolumn{2}{|c|}{ S11-S21 } & \multirow[t]{2}{*}{ FINAL } \\
\hline & $\mathbf{R}$ & $\mathbf{U}$ & $\mathbf{R}$ & $\mathbf{U}$ & $\mathbf{R}$ & $\mathbf{U}$ & $\mathbf{R}$ & $\mathbf{U}$ & $\mathbf{R}$ & $\mathbf{U}$ & $\mathbf{R}$ & $\mathbf{U}$ & $\mathbf{R}$ & $\mathbf{U}$ & $\mathbf{R}$ & $\mathbf{U}$ & $\mathbf{R}$ & $\mathbf{U}$ & $\mathbf{R}$ & $\mathbf{U}$ & \\
\hline $\begin{array}{l}\text { Modify roadside clear } \\
\text { zone }\end{array}$ & & & & & 25 & 25 & & & & & & & & & & & & & 9 & 9 & 17 \\
\hline $\begin{array}{l}\text { Optimize clearance } \\
\text { interval }\end{array}$ & & & & & & & & & & & & & & & & & & & 9 & 9 & 9 \\
\hline $\begin{array}{l}\text { Overlay pavement } \\
\text { (friction course) }\end{array}$ & & & 17 & 17 & 18 & 18 & 27 & 27 & & & & & 13 & 34 & & & & & & & 21 \\
\hline $\begin{array}{l}\text { Place utilities } \\
\text { underground }\end{array}$ & & & & & & & & & & & & & & & & & & & & & 60 \\
\hline $\begin{array}{l}\text { Post an adequate speed } \\
\text { limit }\end{array}$ & & & & & 28 & 28 & & & & & & & 20 & 20 & & & & & & & 24 \\
\hline $\begin{array}{l}\text { Post dynamic message } \\
\text { sign to display the } \\
\text { speed of approaching } \\
\text { vehicles. }\end{array}$ & & & & & & & & & & & & & & & & & 74 & 74 & & & 74 \\
\hline Prohibit right turn on red & & & 20 & 20 & 45 & 45 & & & & & & & & & & & & & & & 33 \\
\hline $\begin{array}{l}\text { Prohibit turning } \\
\text { movements }\end{array}$ & & & & & 40 & & & & & & 45 & 45 & & & & & & & & & 43 \\
\hline $\begin{array}{l}\text { Provide additional } \\
\text { pavement }\end{array}$ & & & & & & & & & & & & & & & & & & & & & 45 \\
\hline $\begin{array}{l}\text { Provide adequate } \\
\text { channelization and } \\
\text { dealination }\end{array}$ & & & 22 & 22 & & & & & 50 & 50 & & & & & & & & & & & 36 \\
\hline $\begin{array}{l}\text { Provide adequate } \\
\text { drainage }\end{array}$ & & & 20 & 20 & & & & & & & & & & & & & & & & & 20 \\
\hline $\begin{array}{l}\text { Provide adequate lane } \\
\text { width }\end{array}$ & 9 & & & & & & & & & & 25 & 25 & & & & 8 & & & & & 17 \\
\hline $\begin{array}{l}\text { Provide adequate left- } \\
\text { turn lane width }\end{array}$ & 9 & & & & & & & & & & & & & & & 8 & & & & & 9 \\
\hline $\begin{array}{l}\text { Provide adequate right- } \\
\text { turn lane width }\end{array}$ & 9 & & & & & & & & & & & & & & & 8 & & & & & 9 \\
\hline $\begin{array}{l}\text { Provide adequate } \\
\text { signals for handicapped }\end{array}$ & & & & & & & & & & & & & & & & & & & & & 20 \\
\hline $\begin{array}{l}\text { Provide adequate } \\
\text { signing }\end{array}$ & & & 30 & 30 & & & & & & & & & & & & & & & & & 30 \\
\hline
\end{tabular}




\begin{tabular}{|c|c|c|c|c|c|c|c|c|c|c|c|c|c|c|c|c|c|c|c|c|c|}
\hline & \multicolumn{2}{|c|}{ S1 } & \multicolumn{2}{|c|}{$\mathbf{S 2}$} & \multicolumn{2}{|c|}{ S3 } & \multicolumn{2}{|c|}{ S4 } & \multicolumn{2}{|c|}{ S5 } & \multicolumn{2}{|c|}{ S6 } & \multicolumn{2}{|c|}{ S7 } & \multicolumn{2}{|c|}{ S8 } & \multicolumn{2}{|c|}{ S9-S10 } & \multicolumn{2}{|c|}{ S11-S21 } & \multirow[t]{2}{*}{ FINAL } \\
\hline & $\mathbf{R}$ & $\mathbf{U}$ & $\mathbf{R}$ & $\mathbf{U}$ & $\mathbf{R}$ & $\mathbf{U}$ & $\mathbf{R}$ & $\mathbf{U}$ & $\mathbf{R}$ & $\mathbf{U}$ & $\mathbf{R}$ & $\mathbf{U}$ & $\mathbf{R}$ & $\mathbf{U}$ & $\mathbf{R}$ & $\mathbf{U}$ & $\mathbf{R}$ & $\mathbf{U}$ & $\mathbf{R}$ & $\mathbf{U}$ & \\
\hline $\begin{array}{l}\text { Provide adequate signs } \\
\text { and markings }\end{array}$ & & & 30 & 30 & & & & & & & & & & & & & & & & & 30 \\
\hline $\begin{array}{l}\text { Provide all-red } \\
\text { clearance interval }\end{array}$ & & & 15 & 15 & & & & & & & & & & & & & & & & & 15 \\
\hline $\begin{array}{l}\text { Provide appropriate } \\
\text { intervals for crossings } \\
\text { and minimize wait time }\end{array}$ & & & & & & & & & & & & & & & & & & & & & 35 \\
\hline Provide bicycle box & & & & & & & & & & & & & & & & & & & & & 10 \\
\hline $\begin{array}{l}\text { Provide breakaway and } \\
\text { crashworthy sign } \\
\text { supports }\end{array}$ & & & & & 30 & 30 & & & & & & & & & & & & & & & 30 \\
\hline Provide channelization & & & 22 & 22 & & & & & 50 & 50 & & & & & & & & & & & 36 \\
\hline $\begin{array}{l}\text { Provide continuous } \\
\text { sidewalks }\end{array}$ & & & 74 & 74 & & & & & & & 65 & 65 & & & & & & & & & 70 \\
\hline $\begin{array}{l}\text { Provide crossing guards } \\
\text { for school children }\end{array}$ & & & & & & & & & & & & & & & & & & & & & 50 \\
\hline $\begin{array}{l}\text { Provide far-side left-turn } \\
\text { signal }\end{array}$ & & & & & & & & & & & & & & & & & & & & & 28 \\
\hline $\begin{array}{l}\text { Provide grooved } \\
\text { pavement }\end{array}$ & & & 21 & 21 & & & 14 & 14 & & & & & & & & & & & 46 & 46 & 27 \\
\hline $\begin{array}{l}\text { Provide independent } \\
\text { bicycle path where } \\
\text { necessary }\end{array}$ & & & & & & & & & & & & & & & & & & & & & 40 \\
\hline $\begin{array}{l}\text { Provide indirect left turn } \\
\text { (can be for high left turn } \\
\text { volume) }\end{array}$ & & & 28 & 28 & & & 25 & 25 & 25 & 25 & 22 & 22 & & & & & & & & & 25 \\
\hline $\begin{array}{l}\text { Provide intersection } \\
\text { lighting }\end{array}$ & 30 & 30 & 33 & 33 & 50 & 50 & & & & & 30 & 30 & 37 & 37 & & & & 37 & 37 & 37 & 36 \\
\hline $\begin{array}{l}\text { Provide lights in } \\
\text { crosswalks in schools } \\
\text { zones }\end{array}$ & & & & & & & & & & & & & & & & & & & 43 & 43 & 43 \\
\hline $\begin{array}{l}\text { Provide longer } \\
\text { clearance intervals }\end{array}$ & & & 15 & 15 & & & & & & & & & & & & & & & & & 15 \\
\hline Provide louvers, visors, & & & & & & & & & & & & & & & & & & & & & 10 \\
\hline
\end{tabular}




\begin{tabular}{|c|c|c|c|c|c|c|c|c|c|c|c|c|c|c|c|c|c|c|c|c|c|}
\hline & \multicolumn{2}{|c|}{ S1 } & \multicolumn{2}{|c|}{$\mathbf{S 2}$} & \multicolumn{2}{|c|}{ S3 } & \multicolumn{2}{|c|}{ S4 } & \multicolumn{2}{|c|}{ S5 } & \multicolumn{2}{|c|}{ S6 } & \multicolumn{2}{|c|}{ S7 } & \multicolumn{2}{|c|}{ S8 } & \multicolumn{2}{|c|}{ S9-S10 } & \multicolumn{2}{|c|}{ S11-S21 } & \multirow[t]{2}{*}{ FINAL } \\
\hline & $\mathbf{R}$ & $\mathbf{U}$ & $\mathbf{R}$ & $\mathbf{U}$ & $\mathbf{R}$ & $\mathbf{U}$ & $\mathbf{R}$ & $\mathbf{U}$ & $\mathbf{R}$ & $\mathbf{U}$ & $\mathbf{R}$ & $\mathbf{U}$ & $\mathbf{R}$ & $\mathbf{U}$ & $\mathbf{R}$ & $\mathbf{U}$ & $\mathbf{R}$ & $\mathbf{U}$ & $\mathbf{R}$ & $\mathbf{U}$ & \\
\hline $\begin{array}{l}\text { or special lenses so } \\
\text { drivers are able to view } \\
\text { signals only for their } \\
\text { approach }\end{array}$ & & & & & & & & & & & & & & & & & & & & & \\
\hline Provide median refuges & & 46 & & & & & & & & & & & & & & & & & & & 46 \\
\hline $\begin{array}{l}\text { Provide offset for left } \\
\text { turn lanes on the } \\
\text { opposite approaches }\end{array}$ & & & & & & & 37 & 37 & & & & & & & & & & & & & 37 \\
\hline $\begin{array}{l}\text { Provide pavement } \\
\text { markings }\end{array}$ & & & 21 & 21 & 10 & 10 & & & 21 & 21 & & & & & & & & & 10 & 10 & 16 \\
\hline $\begin{array}{l}\text { Provide pedestrian push } \\
\text { buttons }\end{array}$ & & & & & & & & & & & & & & & & & & & & & 15 \\
\hline $\begin{array}{l}\text { Provide pedestrian-only } \\
\text { phase or pedestrian- } \\
\text { lead phase during signal } \\
\text { operation }\end{array}$ & & & 20 & 20 & & & & & & & 23 & 23 & & & & & & & 50 & 50 & 31 \\
\hline $\begin{array}{l}\text { Provide positive offset } \\
\text { for left-turn lanes }\end{array}$ & & & & & & & 37 & 37 & & & & & & & & & & & & & 37 \\
\hline Provide right turn lane & & & 25 & 25 & & & 25 & 25 & & & 24 & 24 & & & & 4 & & 8 & & & 20 \\
\hline $\begin{array}{l}\text { Provide roadside } \\
\text { delineators }\end{array}$ & & & & & & & & & & & 30 & 30 & & & & & & & 15 & & 25 \\
\hline $\begin{array}{l}\text { Provide sidewalk set- } \\
\text { backs }\end{array}$ & & & & & & & & & & & & & & & & & & & & & 20 \\
\hline $\begin{array}{l}\text { Provide smooth paved } \\
\text { shoulders }\end{array}$ & & & 15 & 15 & & & & & & & 15 & 15 & & 53 & & & & & & & 23 \\
\hline $\begin{array}{l}\text { Provide target } \\
\text { conventional } \\
\text { enforcement of traffic } \\
\text { laws }\end{array}$ & & & & & & & & & & & & & & & & & & & & & 70 \\
\hline $\begin{array}{l}\text { Provide target } \\
\text { enforcement }\end{array}$ & & & & & & & & & & & & & & & & & & & & & 70 \\
\hline $\begin{array}{l}\text { Provide targeted speed } \\
\text { enforcement }\end{array}$ & & & & & & & & & & & & & & & & & & & & & 70 \\
\hline Provide traffic calming & & & & & & & & & & & & & & & & & & & & & 20 \\
\hline
\end{tabular}




\begin{tabular}{|c|c|c|c|c|c|c|c|c|c|c|c|c|c|c|c|c|c|c|c|c|c|}
\hline & \multicolumn{2}{|c|}{ S1 } & \multicolumn{2}{|c|}{$\mathbf{S 2}$} & \multicolumn{2}{|c|}{ S3 } & \multicolumn{2}{|c|}{ S4 } & \multicolumn{2}{|c|}{ S5 } & \multicolumn{2}{|c|}{ S6 } & \multicolumn{2}{|c|}{ S7 } & \multicolumn{2}{|c|}{ S8 } & \multicolumn{2}{|c|}{ S9-S10 } & \multicolumn{2}{|c|}{ S11-S21 } & \multirow[t]{2}{*}{ FINAL } \\
\hline & $\mathbf{R}$ & $\mathbf{U}$ & $\mathbf{R}$ & $\mathbf{U}$ & $\mathbf{R}$ & $\mathbf{U}$ & $\mathbf{R}$ & $\mathbf{U}$ & $\mathbf{R}$ & $\mathbf{U}$ & $\mathbf{R}$ & $\mathbf{U}$ & $\mathbf{R}$ & $\mathbf{U}$ & $\mathbf{R}$ & $\mathbf{U}$ & $\mathbf{R}$ & $\mathbf{U}$ & $\mathbf{R}$ & $\mathbf{U}$ & \\
\hline $\begin{array}{l}\text { on intersection } \\
\text { approaches through a } \\
\text { combination of } \\
\text { geometric and traffic } \\
\text { control devices }\end{array}$ & & & & & & & & & & & & & & & & & & & & & \\
\hline $\begin{array}{l}\text { Provide visors to shade } \\
\text { signal lenses from } \\
\text { sunlight }\end{array}$ & & & & & & & & & & & & & & & & & & & & & 20 \\
\hline $\begin{array}{l}\text { Provide/Improve } \\
\text { pavement surface } \\
\text { (improve transitions, } \\
\text { drop offs, fixed potholes, } \\
\text { rutting, etc) }\end{array}$ & & & & & & & & & & & & & & & & & & & & & 28 \\
\hline $\begin{array}{l}\text { Provide/improve } \\
\text { progression through a } \\
\text { set of signalized } \\
\text { intersections }\end{array}$ & & & & & & & & & & & & & & & & & & & & & 25 \\
\hline Reconstruct intersection & & & & & & & & & & & & & & & & & & & & & 45 \\
\hline $\begin{array}{l}\text { Redesign intersection } \\
\text { approaches }\end{array}$ & & & & & & & & & & & & & & & & & & & & & 35 \\
\hline $\begin{array}{l}\text { Reduce speed limit on } \\
\text { approaches }\end{array}$ & & & & & & & & & & & 20 & 20 & 20 & 20 & & & & & & & 20 \\
\hline $\begin{array}{l}\text { Relocate signal } \\
\text { hardware out of clear } \\
\text { zone }\end{array}$ & & & & & & & & & & & & & & & & & & & & & 62 \\
\hline Relocate signals & & & & & & & & & & & & & & & & & & & & & 20 \\
\hline Relocate transit stop & & & & & & & & & & & & & & & & & & & & & 15 \\
\hline $\begin{array}{l}\text { Remove constriction as } \\
\text { parked vehicles }\end{array}$ & & & & & & & & & & & & & & & & & & & & & 19 \\
\hline $\begin{array}{l}\text { Remove deflection in } \\
\text { through-vehicle travel } \\
\text { path }\end{array}$ & & & & & & & & & & & & & & & & & & & & & 20 \\
\hline $\begin{array}{l}\text { Remove distracting } \\
\text { commercial lights }\end{array}$ & & & & & & & & & & & & & & & & & & & & & 45 \\
\hline
\end{tabular}




\begin{tabular}{|c|c|c|c|c|c|c|c|c|c|c|c|c|c|c|c|c|c|c|c|c|c|}
\hline & \multicolumn{2}{|c|}{ S1 } & \multicolumn{2}{|c|}{ S2 } & \multicolumn{2}{|c|}{ S3 } & \multicolumn{2}{|c|}{ S4 } & \multicolumn{2}{|c|}{ S5 } & \multicolumn{2}{|c|}{ S6 } & \multicolumn{2}{|c|}{ S7 } & \multicolumn{2}{|c|}{ S8 } & \multicolumn{2}{|c|}{ S9-S10 } & \multicolumn{2}{|c|}{ S11-S21 } & \multirow[t]{2}{*}{ FINAL } \\
\hline & $\mathbf{R}$ & $\mathbf{U}$ & $\mathbf{R}$ & $\mathbf{U}$ & $\mathbf{R}$ & $\mathbf{U}$ & $\mathbf{R}$ & $\mathbf{U}$ & $\mathbf{R}$ & $\mathbf{U}$ & $\mathbf{R}$ & $\mathbf{U}$ & $\mathbf{R}$ & $\mathbf{U}$ & $\mathbf{R}$ & $\mathbf{U}$ & $\mathbf{R}$ & $\mathbf{U}$ & $\mathbf{R}$ & $\mathbf{U}$ & \\
\hline $\begin{array}{l}\text { Remove late night/early } \\
\text { morning flash }\end{array}$ & & & & & & & & & & & & & & & & & & & & & 20 \\
\hline Remove or relocate sign & & & & & & & & & & & & & & & & & & & & & 15 \\
\hline $\begin{array}{l}\text { Remove or re-locate the } \\
\text { median object } \\
\text { obstructing sight of } \\
\text { opposite traffic }\end{array}$ & & & & & & & & & & & & & & & & & & & & & 45 \\
\hline $\begin{array}{l}\text { Remove or relocate } \\
\text { trees }\end{array}$ & & & & & & & & & & & & & & & & & & & 66 & & 66 \\
\hline $\begin{array}{l}\text { Remove or relocate } \\
\text { utility poles }\end{array}$ & & & & & 38 & 38 & & & & & & & & & & & & & 32 & 32 & 35 \\
\hline $\begin{array}{l}\text { Remove sight } \\
\text { obstructions }\end{array}$ & 12 & & & & & & & & & & & & & & & & & & & & 12 \\
\hline $\begin{array}{l}\text { Remove the object } \\
\text { obstructing the sight } \\
\text { triangle }\end{array}$ & 12 & & & & & & 25 & 25 & & & & & & & & & & & & & 21 \\
\hline $\begin{array}{l}\text { Remove unwarranted } \\
\text { signal }\end{array}$ & & & 75 & 75 & & & & & & & 53 & 53 & & & & & & 24 & & & 56 \\
\hline $\begin{array}{l}\text { Removed or relocate } \\
\text { unnecessary signs } \\
\text { "visual clutter" }\end{array}$ & & & & & & & & & & & & & & & & & & & & & 30 \\
\hline $\begin{array}{l}\text { Replace poorly } \\
\text { designed drain grates } \\
\text { with bicycle-safe types }\end{array}$ & & & & & & & & & & & & & & & & & & & & & 25 \\
\hline $\begin{array}{l}\text { Restrict and prohibit } \\
\text { turning maneuver by } \\
\text { channelization or } \\
\text { signing (enforcement } \\
\text { may be required in the } \\
\text { first stage) }\end{array}$ & & & & & & & & & & & & & & & & & & & & & 60 \\
\hline $\begin{array}{l}\text { Restrict left-turn } \\
\text { maneuver }\end{array}$ & & & & & 45 & 45 & & & & & & & & & & & & & & & 45 \\
\hline $\begin{array}{l}\text { Restrict or eliminate } \\
\text { parking near corners }\end{array}$ & & & & & & & & & & & 35 & 35 & & & & & & & 10 & 10 & 23 \\
\hline
\end{tabular}




\begin{tabular}{|c|c|c|c|c|c|c|c|c|c|c|c|c|c|c|c|c|c|c|c|c|c|}
\hline & \multicolumn{2}{|c|}{ S1 } & \multicolumn{2}{|c|}{ S2 } & \multicolumn{2}{|c|}{ S3 } & \multicolumn{2}{|c|}{ S4 } & \multicolumn{2}{|c|}{ S5 } & \multicolumn{2}{|c|}{ S6 } & \multicolumn{2}{|c|}{ S7 } & \multicolumn{2}{|c|}{ S8 } & \multicolumn{2}{|c|}{ S9-S10 } & \multicolumn{2}{|c|}{ S11-S21 } & \multirow[t]{2}{*}{ FINAL } \\
\hline & $\mathbf{R}$ & $\mathbf{U}$ & $\mathbf{R}$ & $\mathbf{U}$ & $\mathbf{R}$ & $\mathbf{U}$ & $\mathbf{R}$ & $\mathbf{U}$ & $\mathbf{R}$ & $\mathbf{U}$ & $\mathbf{R}$ & $\mathbf{U}$ & $\mathbf{R}$ & $\mathbf{U}$ & $\mathbf{R}$ & $\mathbf{U}$ & $\mathbf{R}$ & $\mathbf{U}$ & $\mathbf{R}$ & $\mathbf{U}$ & \\
\hline $\begin{array}{l}\text { Restrict or eliminate } \\
\text { turning maneuver }\end{array}$ & & & & & & & & & & & & & & & & & & & & & 60 \\
\hline Restrict parking & & & & & 22 & 22 & & & & & 35 & 35 & 8 & 8 & & & & & 10 & 10 & 19 \\
\hline $\begin{array}{l}\text { Restrict parking near } \\
\text { corners }\end{array}$ & & & & & & & & & & & & & & & & & & & 10 & 10 & 10 \\
\hline $\begin{array}{l}\text { Re-strip shoulder to } \\
\text { provide right turn lane } \\
\text { (assure adequate } \\
\text { distance to fix object) }\end{array}$ & & & & & & & & & & & & & & & & & & & & & 40 \\
\hline $\begin{array}{l}\text { Review signal timing } \\
\text { (consider timing } \\
\text { optimalization) }\end{array}$ & & & & & 10 & 10 & 15 & 15 & & & & & 10 & 10 & & & & & & & 12 \\
\hline $\begin{array}{l}\text { Shield drivers from } \\
\text { poles in hazardous } \\
\text { locations }\end{array}$ & & & & & & & & & & & & & & & & & & & & & 30 \\
\hline $\begin{array}{l}\text { Signed and marked } \\
\text { crosswalks }\end{array}$ & & & & & & & & & & & 25 & 25 & & & & & & & & & 25 \\
\hline Trim vegetation & & & & & & & & & & & & & & & & & & & & & 40 \\
\hline Upgrade signing & & & & & 5 & 5 & & & & & & & & & & & & & & & 5 \\
\hline $\begin{array}{l}\text { Use "Slippery when wet" } \\
\text { sign (temporary) }\end{array}$ & & & & & & & & & & & & & & & & & & & & & 15 \\
\hline $\begin{array}{l}\text { Use advance guide } \\
\text { signs }\end{array}$ & & & 25 & 25 & & & & & & & & & & & & & & & & & 25 \\
\hline $\begin{array}{l}\text { Use advance street } \\
\text { name signs }\end{array}$ & & & & & & & & & & & 25 & 25 & & & & & & & & & 25 \\
\hline $\begin{array}{l}\text { Use automated } \\
\text { enforcement of } \\
\text { approach speeds }\end{array}$ & & & & & & & & & & & & & & & & & & & & & 25 \\
\hline Use break-away devices & & & & & 30 & 30 & & & & & & & & & & & & & & & 30 \\
\hline $\begin{array}{l}\text { Use flashing lights in } \\
\text { advance on approach }\end{array}$ & & & & & 50 & 50 & & & & & & & & & & & & & & & 50 \\
\hline $\begin{array}{l}\text { Use other traffic calming } \\
\text { applications to reduce } \\
\text { vehicle speeds or traffic }\end{array}$ & & & & & & & & & & & & & & & & & & & & & 20 \\
\hline
\end{tabular}




\begin{tabular}{|c|c|c|c|c|c|c|c|c|c|c|c|c|c|c|c|c|c|c|c|c|c|}
\hline & \multicolumn{2}{|c|}{ S1 } & \multicolumn{2}{|c|}{ S2 } & \multicolumn{2}{|c|}{ S3 } & \multicolumn{2}{|c|}{ S4 } & \multicolumn{2}{|c|}{ S5 } & \multicolumn{2}{|c|}{ S6 } & \multicolumn{2}{|c|}{ S7 } & \multicolumn{2}{|c|}{ S8 } & \multicolumn{2}{|c|}{ S9-S10 } & \multicolumn{2}{|c|}{ S11-S21 } & \multirow[t]{2}{*}{ FINAL } \\
\hline & $\mathbf{R}$ & $\mathbf{U}$ & $\mathbf{R}$ & $\mathbf{U}$ & $\mathbf{R}$ & $\mathbf{U}$ & $\mathbf{R}$ & U & $\mathbf{R}$ & $\mathbf{U}$ & $\mathbf{R}$ & U & $\mathbf{R}$ & $\mathbf{U}$ & $\mathbf{R}$ & $U$ & $\mathbf{R}$ & $\mathbf{U}$ & $\mathbf{R}$ & $\mathbf{U}$ & \\
\hline $\begin{array}{l}\text { volumes on intersection } \\
\text { approaches. }\end{array}$ & & & & & & & & & & & & & & & & & & & & & \\
\hline $\begin{array}{l}\text { Use post-mounted } \\
\text { delineation }\end{array}$ & & & & & & & & & & & & & 25 & 25 & & & & & & & 25 \\
\hline Use protected phase & & 70 & 27 & 27 & & & & 25 & & & & & & & & 70 & & & & & 44 \\
\hline $\begin{array}{l}\text { Use Protected/Permitted } \\
\text { phase }\end{array}$ & & & 10 & 10 & & & 10 & 10 & & & & & & & & & & & & & 10 \\
\hline $\begin{array}{l}\text { Use salt to prevent snow } \\
\text { or ice from forming or } \\
\text { from sticking to the road } \\
\text { surface }\end{array}$ & & & & & & & & & & & & & & & & & & & & & 20 \\
\hline $\begin{array}{l}\text { Use sand to increase } \\
\text { pavement friction }\end{array}$ & & & & & & & & & & & & & & & & & & & & & 20 \\
\hline Use split phases & & & & & & & & & & & & & & & & & & & & & 25 \\
\hline $\begin{array}{l}\text { Use two red signal } \\
\text { sections }\end{array}$ & & & & & & & & & & & & & & & & & & & & & 36 \\
\hline $\begin{array}{l}\text { Widen the outside } \\
\text { through lanes add bike I. }\end{array}$ & & & & & & & & & & & & & & & & & & & & & 15 \\
\hline
\end{tabular}

